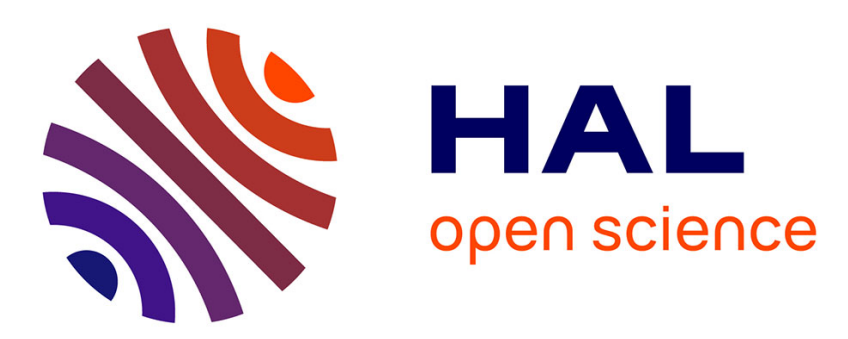

\title{
Réversibilité possible de la sarcopénie chez des patients porteurs de BPCO
}

L. Perrot, A. Greil, N. Farigon, F. Costes, D. Caillaud, A. Mulliez, Yves Boirie

\section{To cite this version:}

L. Perrot, A. Greil, N. Farigon, F. Costes, D. Caillaud, et al.. Réversibilité possible de la sarcopénie chez des patients porteurs de BPCO. JFN 2018, Journées Francophones de Nutrition, Nov 2018, Nice, France. hal-01927455

\section{HAL Id: hal-01927455 \\ https://hal.science/hal-01927455}

Submitted on 2 Jun 2020

HAL is a multi-disciplinary open access archive for the deposit and dissemination of scientific research documents, whether they are published or not. The documents may come from teaching and research institutions in France or abroad, or from public or private research centers.
L'archive ouverte pluridisciplinaire HAL, est destinée au dépôt et à la diffusion de documents scientifiques de niveau recherche, publiés ou non, émanant des établissements d'enseignement et de recherche français ou étrangers, des laboratoires publics ou privés. 


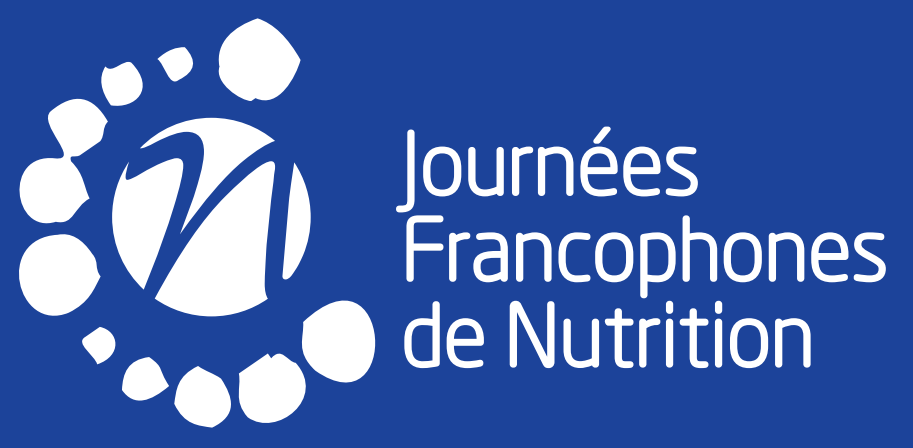

\section{LIVRE DES ABSTRACTS}

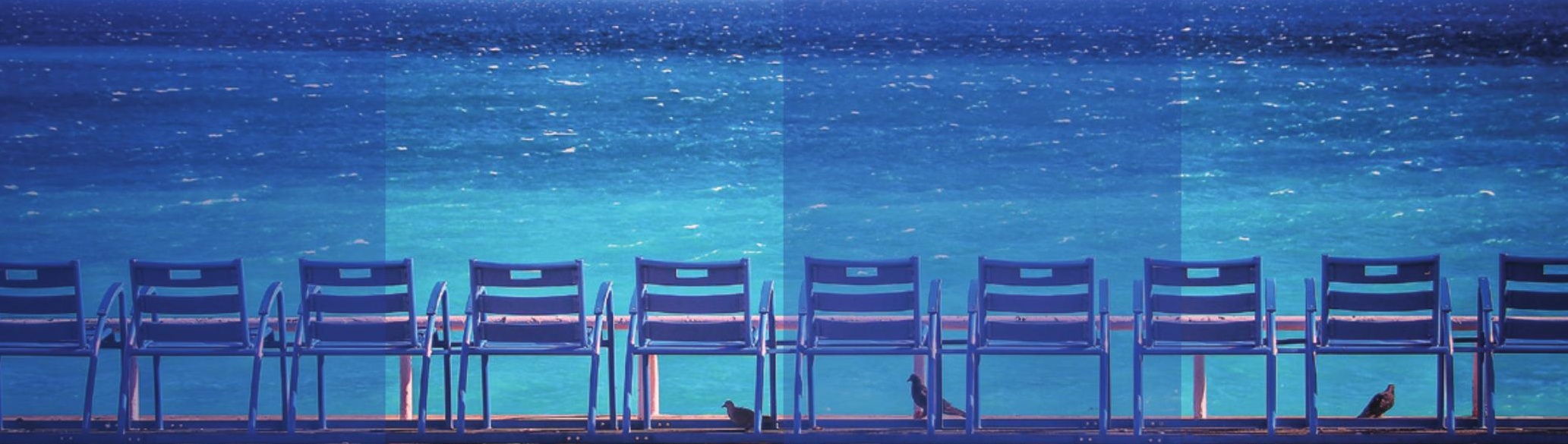

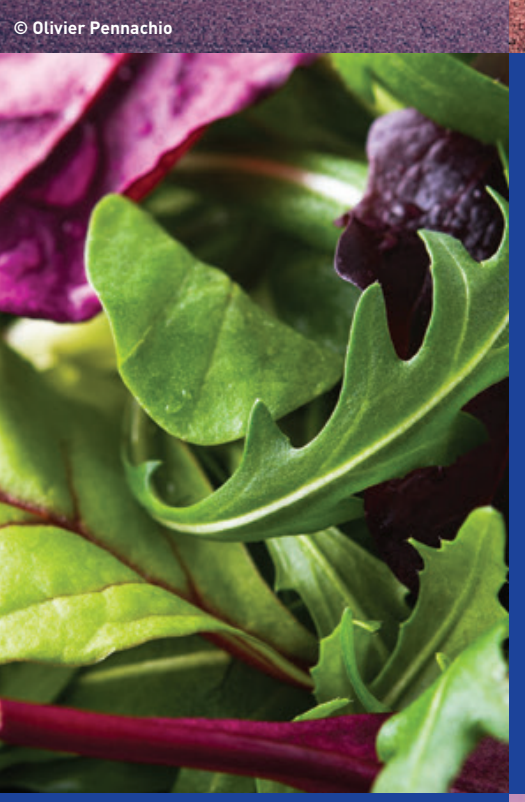

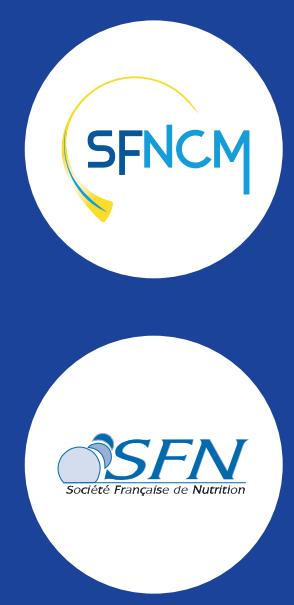

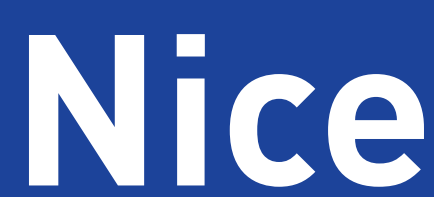

Acropolis

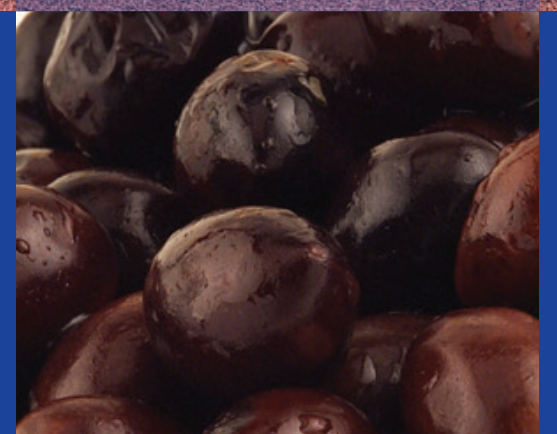

$28-30$ novembre 2018

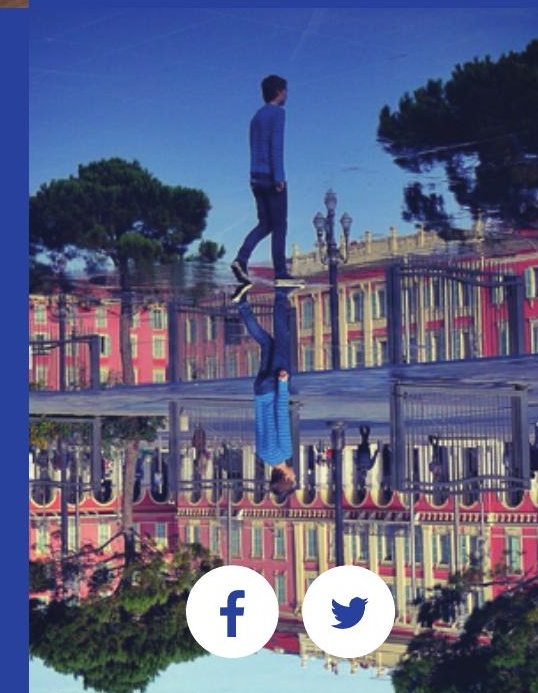




\section{SOMMAIRE}

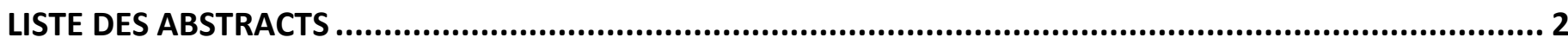

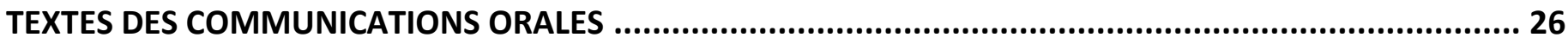

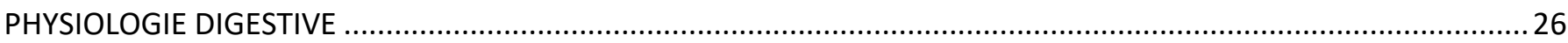

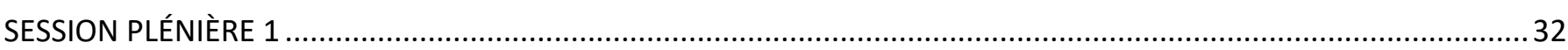

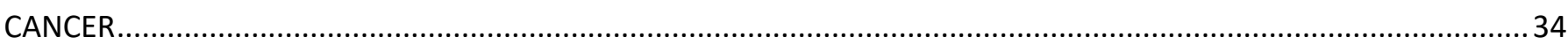

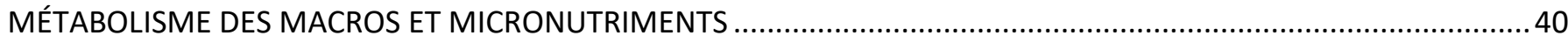

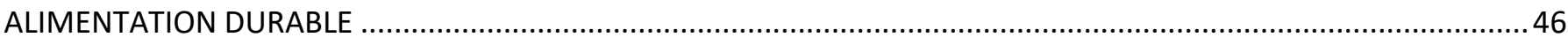

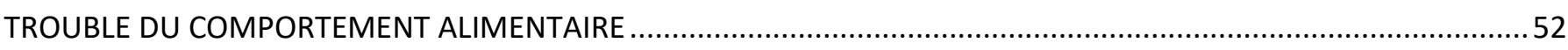

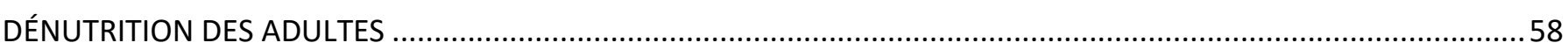

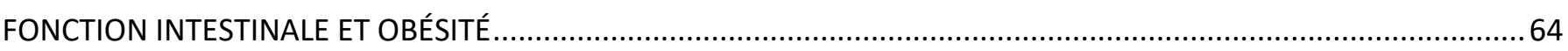

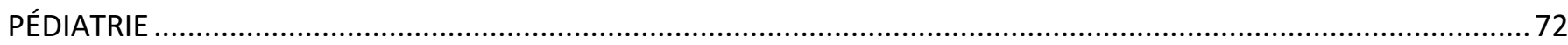

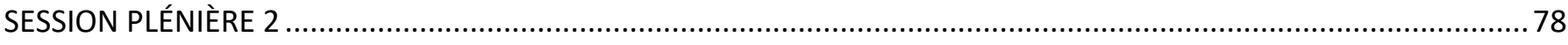

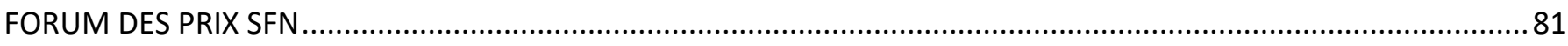

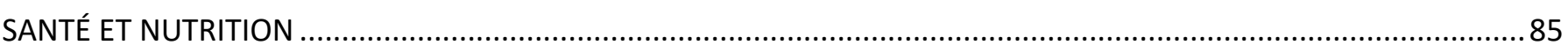

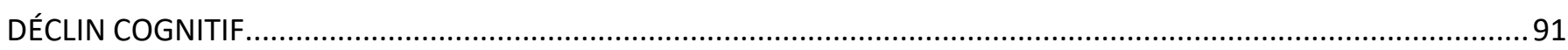

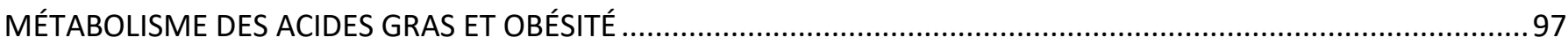

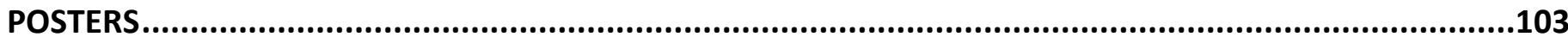

ALIMENTATION DURABLE - ALIMENTATION ANIMALE ET QUALITE DES PRODUITS TECHNOLOGIE ALIMENTAIRE.103

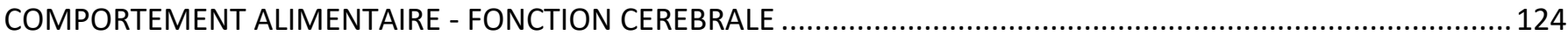

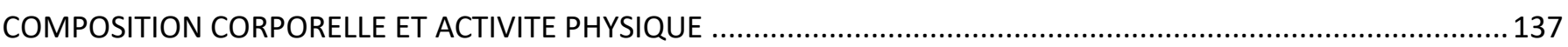

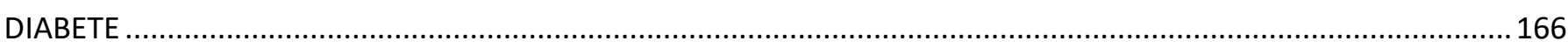

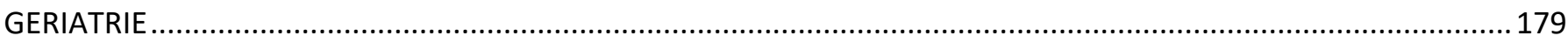

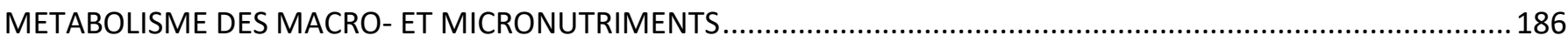

NUTRITION EN PATHOLOGIE : CANCER, INSUFFISANCE D'ORGANES, REANIMATION, PATHOLOGIES DIGESTIVES... (n)

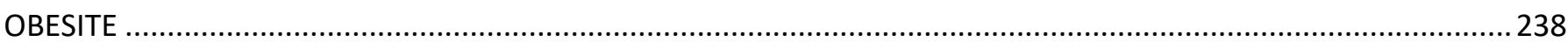

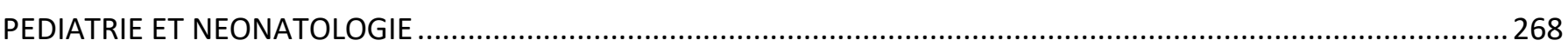

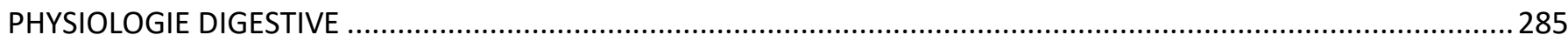

SANTE PUBLIQUE

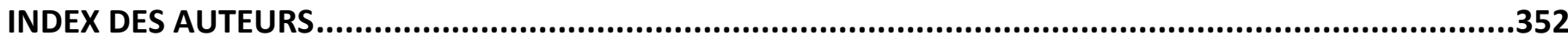




\section{LISTE DES ABSTRACTS}

\section{Communications orales}

\section{PHYSIOLOGIE DIGESTIVE}

CO01 Associations entre profils metabolomiques plasmatiques RMN et composition du microbiote intestinal au sein d'une population d'adultes francais en bonne sante V. Partula*, S. Mondot, M. Torres, E. Kesse-Guyot, L. Lécuyer, M. Deschasaux, K. Assmann, P. Latino-Martel, C. Buscail, C. Julia, P. Galan, S. Hercberg, A. Victor-Bala, N. Bouchemal, M. Triba, P. Savarin, V. Rouilly, S. Thomas, L. Quintana-Murci, M. Albert, O. Lantz, D. Duffy, M. Touvier et Consortium Milieu Intérieur (Bobigny)

CO02 | Le microbiote intestinal : un acteur cle dans les effets metaboliques de l'inuline chezles patients obeses J. Rodriguez*, A. M. Neyrinck, T. Le Roy, S. Leclercq, S. Hiel, P. D. Cani, N. Lanthier, J. P. Thissen, L. Bindels, N. M. Delzenne (Bruxelles, Belgique)

CO03 | Chirurgie bariatrique de l'adolescent obese : premiere serie francaise incluantles 3 techniques chirurgicales S. Mayer Lacrosniere*, B. Dubern, A. Karsenty, H. Chantereau, L. Oderda, J. L. Bouillot, P. Tounian (Paris)

CO04 | Evolution de la detection oro-sensorielle du saccharose chez des souris obeses apres Sleeve gastrectomie ou By-pass Roux en $Y$ modifie

A. Bernard*, C. Le May, A. Dastugue, A. Ayer, C. Blanchard, B. Cariou, S. Ledoux, X. Collet, A. Dhaussy, F. Respondek, L. Lagrost, P. Besnard (Dijon)

CO05 | Impact extra-intestinal de l'inflammation colique aigue sur le metabolisme proteique dans un modele murin de colitechimio induite

S. Vidal*, N. Khodorova, M. Piscuc, S. Mekiri, C. Gaudichon, F. Blachier, A. Lan (Paris)

CO06 | Role du CD36 enterocytaire dans l'adaptation intestinale a un regime riche en lipides

S. Moreira*, V. Barbet, M.-C. Monnot, K. Gaire, H. Poirier, I. Niot (Dijon)

\section{SESSION PLÉNIÈRE 1}

CO07 | Evolution de la perception orosensorielle du sucre et du gras apres une chirurgie bariatrique restrictive ou malabsorptive chez la femme adulte

A. Bernard*, S. Ledoux, L. Radoi, M. Coupaye, O. Sami, N. Casanova, C. Le May, X. Collet, A. Dhaussy, F. Respondek, L. Lagrost, P. Besnard (Dijon)

C008 I Impact de l'anorexie mentale sur l'integrite fonctionnelle et structurelle hypothalamique au cours de la prise alimentaire V. Florent*, M. Baroncini, P. Jissendi, J. VIGNAU, M. Pigeyre, I. Nilsson, V. Prevot (Arras)

\section{CANCER}

CO09 | Qualite nutritionnelle des aliments definie par le score FSAm-NPS sous-tendant le logo Nutri-Score et risque de cancer en Europe : resultats de la cohorte EPIC M. Deschasaux*, I. Huybrechts, N. Murphy, C. Julia, S. Hercberg, B. Srour, E. Kesse-Guyot, P. Latino-Martel, C. Biessy, C. Casagrande, M. Jenab, H. Ward, E. Weiderpass, P. Ferrari, E. Riboli, M. Gunter, M. Touvier (Bobigny)

C010 | Mesure pre operatoire de l'indice de masse musculaire en L3 en chirurgie thoracique : evaluation de l'equivalence de deux logiciels disponibles

G. Gayraud* (Clermont Ferrand) 
C011 | La sarcopenie est sous-diagnostiquee chez les patients atteints de cancer metastatique : resultats de l'etude SCAN B. Raynard*, F. Pigneur, H. Simon, V. Tissot, D. Vansteene, P. Meingan, M. Di Palma, A. Tolédano, F. Goldwasser (Villejuif)

C012 | Interet des outils cliniques pour predire la sarcopenie chez les patients atteints de cancer metastatique : resultats de l'etude SCAN

B. Raynard*, F. Pigneur, M. Di Palma, A. Tolédano, F. Goldwasser (Villejuif)

C013 | Evaluation de l'etat nutritionnel chez les patients ages atteints de cancer : une enquete transversale nationale (NutriAgeCancer)

E. Paillaud*, D. Heitz, A.-L. Couderc, R. Boulahssass, A. Chah Wakilian, F. Pamoukdjian, L. De Decker, S. Valero, A. Rouaud, A. Raynaud-Simon, C. Mertens, P. Caillet, V. Antoine, L. Cristol, I. Palayer, H. Solem Laviec (Paris)

C014 | Validation de l'outil EPAR chez les patients en phase palliative d'un cancer : resultats de l'etude ALIM-K G. Grodard*, V. Royer-Garabige, A. Anota, C. Bouleuc, R. Aubry (Besançon)

\section{MÉTABOLISME DES MACROS ET MICRONUTRIMENTS}

C015 | Impact de la structure des aliments sur la biodisponibilite des micronutriments lipophiles : etude in vivo chez I'homme B. Gleize, M. Hiolle*, N. Meunier, B. Pereira, R. Richard, I. Savary-Auzeloux, C. Buffière, C. Caris-Veyrat, D. Dupont, F. Nau, E. Reboul (Rennes)

C016 | Biodisponibilite des acides amines d'un isolat proteique de tournesol marque au $15 \mathrm{~N}$ et au $2 \mathrm{H}$ chez le rat R. Tessier*, N. Khodorova, J. Calvez, R. Kapel, F. Cacérès, A. Dinar, J. Piedcoq, D. Tomé, C. Gaudichon (Paris)

C017 | ABCB1 (P-glycoproteine) contribue a la regulation de l'absorption et a l'efflux transintestinalde vitamine $D$ M. Margier, X. Collet, C. Le May, C. Desmarchelier, F. André, C. Lebrun, C. Defoort, A. Bluteau, P. Borel, A. Lespine, E. Reboul* (Marseille)

C018 Q Quel est le role de la synthese intestinale de citrulline dans l'homeostasie proteique ? J. P. De Bandt*, M. Juchet-Martin, M. Aboubacar, S. Magassa, I. Dubail, L. Cynober (Paris)

C019 | La consommation d'un regime riche en fructose induit une augmentation des niveaux plasmatiques d'acides amines a chaine ramifiee chez le rat insulino-resistant et non obese

J. David, L. Mosoni, D. Dardevet, I. Savary-Auzeloux, S. Polakof* (Saint-Gènes-Champanelle)

CO20 | La pathologie de l'aorte ascendante en reponse a programmation foetale par la deficience en donneurs de methyle B. Balint*, R.-M. Rodriguez-Gueant, J.-L. Gueant (Vandoeuvre-Les-Nancy)

\section{ALIMENTATION DURABLE}

CO21 | Effets du mode de production sur l'impact environnemental de differents menus : importance du choix de l'unite fonctionnelle

G. Mairesse*, H. M. G. Van Der Werf, G. Chesneau, N. Kerhoas (Combourtille)

CO22 | NUTRI-SCORE : quelle place pour les eleveurs engages dans l'amelioration de la qualite nutritionnelle des produits animaux?

G. Mairesse*, G. Chesneau, N. Kerhoas (Combourtille)

C023 | Les coacervats de proteines ameliorent la protection et la biodisponibilite de nutriments : exemple de la vitamine B9 S. Bouhallab*, A.-L. Chapeau, P. Hamon, N. Bertrand (Rennes)

CO24 | Les proprietes anti-inflammatoires de la bacterie probiotique Propionibacterium freudenreichii revelee de l'in vitro a l'in vivo grace au fromage

G. Jan* et Consortium du projet ANR «Surfing» (Rennes) 
CO25 | Effets favorables de l'introduction de graines de lin extrudees dans la ration sur les performances et la sante des vaches laitieres

G. Chesneau, T. Meignan, A. Madouasse, F. Beaudeau, V. Chatellier, G. Mairesse*, N. Bareille (Combourtille)

CO26 | Amelioration de l'autonomie proteique alimentaire du poulet de chair par l'incorporation de graines oleoproteagineuses en substitution du tourteau de soja d'importation

M. Guillevic, M. Lessire, A. Germain, H. Juin, G. Mairesse*, G. Chesneau (Combourtille)

\section{TROUBLE DU COMPORTEMENT ALIMENTAIRE}

CO27 | Caracterisation des perturbations biochimiques dans un modele murin d'anorexie (ABA) par resonance magnetique nucleaire - Etude metabonomique

J. Breton*, N. Giallourou, S. Nobis, A. Morin, N. Achamrah, A. Goichon, L. Belmonte, P. Déchelotte, J.-L. do Rego, M. Coëffier, J. Swann (Rouen)

CO28 Impact d'une depletion du microbiote intestinal sur la reponse au modele murin d'anorexie associee a l'activite physique P. Tirelle*, W. Bahlouli, C. L'Huillier, E. Salameh, A. Amamou, M. Jarbeau, C. Guérin, A. Goichon, J. C. Do Rego, P. Déchelotte,

D. Ribet, M. Coëffier (Rouen)

CO29 | Etude des neuropeptides plasmatiques et de leurs immunoglobulines respectives chez 120 patients atteints de troubles du comportement alimentaire

M. Galmiche*, C. Deroissart, M.-A. Le Solliec, J. Rondeaux, S. Azhar, N. Achamrah, L. Belmonte, A. Lamarre, S. Grigioni, V. Folope, A. Rimbert, T. Saillard, J. Delay, M. P. Tavolacci, M. Quillard, G. Lambert, P. Dechelotte, N. Lucas, R. Legrand (Rouen)

CO30 | Validite des equations d'impedancemetrie bioelectrique pour la mesure de la composition corporelle chez des patientes anorexiques

M. Coëffier*, M. Gaté, A. Rimbert, A. Petit, V. Folope, S. Grigioni, P. Déchelotte, N. Achamrah (Rouen)

C031 | Depistage des troubles du comportement alimentaire a l'aide du SCOFF-F chez 964 actifs et etudiants en Haute-Vienne M. Arnal*, P. Fayemendy, B. Morin, M. Perrier, M. Gravelat, G. Magne, C. Le Flahec, V. Sabourdy, S. Depoux, M. Szopinski, D. Mathe,

M. Vergonjeanne, J. C. Desport, P. Jésus (Limoges)

CO32 | Comparaison des facteurs et comportements associes des trois categories de trouble du comportement alimentaire : restrictif, boulimique et conpulsif

Y. Sirejacob*, M. Galmiche, N. Achamrah, L. E. Belmonte, A. Lamarre, S. Grigioni, V. Foloppe, A. Rimbert, T. Saillard, J. Delay, P. Déchelotte, M.-P. Tavolacci (Rouen)

\section{DÉNUTRITION DES ADULTES}

C033 | Reversibilite possible de la sarcopenie chez des patients porteurs de BPCO

L. Perrot*, A. Greil, N. Farigon, F. Costes, D. Caillaud, A. Mulliez, Y. Boirie (Clermont-Ferrand)

C034 | Apport preoperatoire d'une boisson glucidique et reponse au stress operatoire apres resection intestinale chez le rat N. Tennoune-El Hafaia*, Z. Desmazières, M. Juchet-Martin, P. Jegatheesan, S. Magassa, N. Neveux, S. Nakib, G. Sarfati, M. Aboubacar, R. Ramassamy, L. Cynober, J.-P. De Bandt (Paris)

CO35 | La compliance a la complementation nutritionnelle orale diminue le risque d'hospitalisation chez les patients ages denutris vivant a domicile, sans augmenter les couts : etude ENNIGME

D. Seguy*, H. Hubert, J. Robert, J.-P. Meunier, O. Guérin, A. Raynaud-Simon (Lille) 
C036 | Impact de la gastrostomie sur la survie des patients atteints de la sclerose laterale amyotophique (SLA), en fonction de leur perte ponderale au cours du temps

M. Vergonjeanne, P. Fayemendy, B. Marin, M. Nicol, G. Lautrette, H. Sourisseau, P. M. Preux, J. C. Desport, P. Couratier, P. Jésus* (Limoges)

CO37 | Epidemiologie de la Nutrition Parenterale a Domicile (NPD) chez l'Adulte en France (etude preliminaire) D. Lescut*, F. Diebold, J. Gonneau-Lejeune, A. De Luca, C. Bouteloup, N. Danel, A. Léké, D. Barnoud, M.-F. Vaillant (Lille)

C038 | Evaluation de la fonction renale chez les patients en nutrition parenterale pour insuffisance intestinale M. Lauverjat*, L. Dubourg, S. Ait, D. Barnoud, C. Bergoin, C. Peraldi, C. Chambrier (Pierre-Bénite)

\section{FONCTION INTESTINALE ET OBÉSITÉ}

C039 | Frequence des mutations des genes de la voie leptine/melanocortines dans l'obesite severe

J. Le Beyec-Le Bihan*, C. Christine Poitou-Bernert, A. Karsenty, V. Pelloux, J. M. Lacorte, P. Tounian, J. M. Oppert, K. Clement, B. Dubern (Paris)

CO40 | Effet de la modulation de la digestibilite de l'amidon sur la variabilite glycemique chez les diabetiques de type 2 A.-E. Breyton*, A. Goux, S. Lambert-Porcheron, A. Meynier, M. Sothier, L. Van Den Berghe, S. Normand, E. Disse, M. Laville, S. Vinoy,

J.-A. Nazare (Pierre-Bénite)

C041 | Lors du developpement nutritionnel du syndrome metabolique, le metabolisme proteique est differemment affecte dans le foie et les muscles

J.-F. Huneau*, O. L. Mantha, V. Mathé, D. Hermier, N. Khodorova, F. Mariotti, H. Fouillet (Paris)

C042 | La chirurgie bariatrique affecte les besoins en proteines lies a l'obesite

C. Guillet*, A. Masgrau, A. Mishellany-Dutour, A. Blot, B. Pereira, K. Slim, M. Robert, E. Disse, N. Feugier, P. Leruyet, C. Louvet, M. Miolanne, N. Farigon, J. Goudable, M. Laville, Y. Boirie (Clermont-Ferrand)

C043 | Fonctions intestinales endocrines chez des patients a deux ans d'une chirurgie bariatrique de type bypass gastrique en Omega versus Roux en $Y$

D. De Bandt*, L. Morreau, D. Bergerot, M. Le Gall, J. M. Lacorte, S. Czernichow, A. Blanchard, J. Le Beyec, C. Carette (Paris)

CO44 | dentification de nouvelles bacteries probiotiques contre le developpement de desordres metaboliques dans un modele de souris soumises a une diete obesogene

M. Le Barz*, N. Daniel, T. V. Varin, S. Naimi, V. Demers-Mathieu, G. Pilon, J. Audy, E. Laurin, D. Roy, M. C. Urdaci, D. St-Gelais, I. Fliss, A. Marette (Québec, Canada)

CO45 | Un nouveau probiotique, Hafnia alvei, reduit le gain de poids dans deux modeles murins d'obesite en agissant sur les voies centrales et peripheriques de l'homeostasie energetique

N. Lucas*, S. Azhar, C. Deroissart, M.-A. Le Solliec, M. Dominique, J. Rondeaux, S. Nobis, C. Guérin, F. Léon, J.-C. Do Rego,

P. Déchelotte, S. Fetissov, G. Lambert, R. Legrand (Rouen)

C046 | Role de la proteine bacterienne ClpB et d'un de ses fragments peptidique dans la regulation de la prise alimentaire M. Dominique*, I. Boulete, C. Bole-Feysot, F. Leon, J.-C. Do Rego, S. O Fetissov, P. Déchelotte, G. Lambert, R. Legrand, N. Lucas (Rouen)

\section{PÉDIATRIE}

CO47 | Inegalites sociales dans la supplementation prenatale en acide folique : resultats de la cohorte de naissance ELFE A. Camier*, M. Kadawathagedara, S. Lioret, C. Bois, M. Cheminat, M.-N. Dufourg, M. A. Charles, B. De Lauzon-Guillain (Villejuif) 
CO48 Allaitement dans la premiere annee de vie et trajectoires d'infections et de symptomes allergiques dans la cohorte mereenfant EDEN

C. Davisse-Paturet, B. Heude, A. Forhan, M.-A. Charles, B. De Lauzon-Guillain* (Villejuif)

C049 | L'abondance naturelle en $15 \mathrm{~N}$ dans les cheveux est un marqueur d'exposition alimentaire et de malnutrition aigue chez l'enfant en bas age

T. Dailey-Chwalibóg*, H. Fouillet, F. Mariotti, V. Mathé, N. Khodorova, B. Guesdon, J.-F. Huneau (Paris)

CO50 | Lors de la prise en charge nutritionnelle de la malnutrition aigue severe chez l'enfant en bas age, l'evolution de l'abondance naturelle en $15 \mathrm{~N}$ le long des cheveux signe l'historique des gains anthropometriques

T. Dailey-Chwalibóg*, J.-F. Huneau, F. Mariotti, V. Mathé, N. Khodorova, B. Guesdon, H. Fouillet (Paris)

CO51 | Epidemiologie de la nutrition artificielle a domicile en pediatrie dans la region des Hauts de France en 2016 : etude preliminaire

R. Dehak*, D. Guimber, D. Ley, S. Coopman, L. Michaud, F. Gottrand, D. Lescut (Lille)

C052 | Insuffisance intestinale chronique : quand les enfants deviennent adultes

L. Schubert*, L. Billiauws, V. Boehm, F. Joly (Clichy)

\section{SESSION PLÉNIÈRE 2}

C053 | Des (nano)particules de dioxyde de titane (TiO2) issues de l'additif alimentaire E171 franchissent la barrière placentaire chez l'Homme: une étude ex vivo sur placentas isolés et perfusés

A. Guillard*, E. Gaultier, C. Cartier, L. Devoille, C. Coméra, F. Grandin, A. Cazanave, A. De Place, M. Morin, C. Vayssière,

N. Feltin, F. de La Farge, V. Gayrard, N. Picard-Hagen, E. Houdeau (Toulouse)

CO54 | Exposition prénatale aux acides gras polyinsaturés et quotient intellectuel à 5-6 ans des enfants de la cohorte EDEN E. Petit, M. Armand, M. De Agostini, A. Forhan, M.-A. Charles, B. Heude, J. Y. Bernard* (Villejuif)

CO55 | Les lipides polaires laitiers diminuent les facteurs lipidiques du risque cardiovasculaire chez des femmes ménopausées C. Vors, L. Joumard-Cubizolles, E. Combe, L. Ouchchane, J. Drai, K. Raynal, P. Gaborit, F. Joffre, A. Blot, G. Gesan-Guiziou, M. Laville, S. Lambert-Porcheron, C. Malpuech-Brugère, M.-C. Michalski* (Villeurbanne)

\section{FORUM DES PRIX SFN : RELATION NUTRITION/SANTÉ : NOUVEAUX REGARDS SUR LE RÔLE DE LA VARIABILITÉ INDIVIDUELLE}

C056 | Actions synergiques d'une restriction calorique et de l'activité physique sur la santé et la longévité J. Royo, F. Pifferi* (Brunoy)

C057 | Effets de la variabilité interindividuelle de la salive humaine (flux et composition) sur la libération et perception d'arômes chez le sujet âgé

C. Munoz*, M. Brule, C. Martin, C. Septier, G. Feron, F. Canon (Dijon)

C059 | Influence du genre sur l'évolution des stéatohépatites métaboliques : Rôle hépatocytaire du récepteur aux oestrogènes $\mathrm{ER} \alpha$

S. Smati-Grangeon, B. Tramunt, A. Polizzi, M. Régnier, H. Guillou, P. Gourdy, A. Montagner* (Toulouse)

C060 | Impacts d'un régime hyper-protéiné chez le mâle, sur l'épigénome de ses spermatozoïdes et sur la santé métabolique de sa descendance

S. Bottini*, J. Movassat, V. Grandjean (Nice) 


\section{SANTÉ ET NUTRITION}

C061 | Consommation d'aliments ultra-transformes et risque de maladies cardiovasculaires dans la cohorte NutriNet-Sante B. Srour*, L. K. Fezeu, E. Kesse-Guyot, B. Allès, C. Méjean, R. M. Andrianasolo, E. Chazelas, M. Deschasaux, S. Hercberg, P. Galan, C. A. Monteiro, C. Julia, M. Touvier (Bobigny)

C062 | Un faible statut en acides gras omega-3 a longue chaine en debut de grossesse est associe a un risque plus eleve de depression post-partum

A. Hoge*, M. Nisolle, M. Guillaume, V. Castronovo, S. Degée, V. Tabar, M. Timmermans, A.-F. Donneau, N. Dardenne (Liège, Belgique)

C063 | Effet de l'arrivee d'un premier enfant dans le foyer sur les evolutions sur 7 ans des consommations alimentaires et du poids des adultes (Etude NutriNet-Sante)

C. Mejean*, P. Rollet, W. Si Hassen, C. Tichit, A. Lampuré, S. Hercberg, M. Touvier, K. Castetbon (Montpellier)

CO64 | L'outil d'evaluation de la prise alimentaire EPAR (ou SEFIR) comme test de depistage de la denutrition en medecine generale: etude prospective non interventionnelle multicentrique

G. Bouëtté*, M. Esvan, K. Apel, R. Thibault (Rennes)

C065 | Analyse de la composition corporelle sur coupe scannographique transversale passant par la 3eme vertebre lombaire (L3) en reanimation : impact pronostique, reproductibilite interobservateur, et prevalence de la sarcopenie

E. Jali*, A. Mulliez, L. Lacaze, E. Lascouts, N. Rotovnik Kozjek, A. -M. Makhlouf, I. Ceniceros Rozalen, J.-C. Preiser, Z. Krznaric,

K. Kupczyk, N. Cano, C. Pichard, R. Thibault Et Phase Angle Project Investigators (Rennes)

C066 | Hydratation et nutrition enterale a domicile

P. Fayemendy*, D. Barnoud, S. Schneider, B. Morin-Boulogne, P. Jésus, J. C. Desport et Comité de Nutrition à Domicile de la SFNCM (Limoges)

\section{DÉCLIN COGNITIF}

C067 | Trajectoires de facteurs de mode de vie depuis la quarantaine et declin cognitif apres 70 ans chez la femme M. Wagner*, F. Grodstein, C. Proust-Lima, C. Samieri (Bordeaux)

C068 | Metabolomique, metabolome alimentaire et declin cognitif du sujet age

S. Lefevre-Arbogast*, D. Low-Yanwen, B. P. Hejblum, C. Helmer, P. Micheau, C. Proust-Lima, S. Thuret, C. Manach, C. Samieri et Consortium Européen D-Cogplast (JPI HDHL) (Bordeaux)

C069 | Signature lipidique precoce du declin cognitif chez le sujet age S. Lefevre-Arbogast*, S. Thuret, C. Helmer, C. Proust-Lima, B. P. Hejblum, C. Samieri Et Consortium Européen D-Cogplast (JPI $H D H L)$ (Bordeaux)

CO70 | Les apports en acides gras insatures au milieu de la vie sont positivement associes au fonctionnement cognitif mesure 13 ans plus tard, avec un effet modulateur d'une supplémentation en antioxydants

K. E. Assmann, M. Adjibade*, S. Hercberg, P. Galan, E. Kesse-Guyot (Bobigny)

C071 | Association entre statut plasmatique en vitamine D et connectivite cerebrale fonctionnelle chez le sujet age - cohorte des 3-Cites - Bordeaux

A. Thomas*, C. Féart, C. Delcourt, C. Helmer, M. Edde, G. Catheline, C. Samieri (Bordeaux)

C072 | Carotenoides plasmatiques, biomarqueurs du risque de degenerescence maculaire : I'etude Alienor B. M. Merle*, A. Cougnard-Grégoire, J.-F. Korobelnik, W. Schalch, S. Etheve, C. Féart, C. Samieri, M.-N. Delyfer, C. Delcourt et The Eye-Risk Consortium (Bordeaux) 


\section{MÉTABOLISME DES ACIDES GRAS ET OBÉSITÉ}

C073 | Controle de l'adipogenese par les metabolites des acides gras

C. Colson*, E.-Z. Amri (Nice)

C074 | Impact de l'acide trans-palmitoleique alimentaire sur les composantes du syndrome metabolique : etude chez la souris C57BL/6N

E. J. Guillocheau*, G. Drouin, C. Penhoat, G. Ghukasyan, A. Fautrel, M. Marty, V. Quillien, D. Catheline, P. Legrand, V. Rioux (Rennes)

C075 | Impact de lipides bioactifs, les FAHFAs, sur les desordres metaboliques associes a l'obesite M. Benlebna*, L. Balas, B. Bonafos, L. Pessemesse, G. Fouret, S. Gaillet, T. Durand, C. Coudray, F. Casas, C. Feillet-Coudray (Montpellier)

C076 | Influence de l'auto-immunite dans la resistance au modele murin d'induction d'une obesite par une diete riche en lipides R. Legrand*, S. Azhar, C. Deroissart, M.-A. Le Solliec, M. Dominique, F. Léon, J.-C. Do Rego, N. Lucas (Rouen)

CO77 | Les acides gras poly-insatures de type omega3 inhibent la production d'IL-17A mediee par les cellules souches du tissu adipeux, en diminuant l'expression d'ICAM-1, chez le sujet obese M. Chehimi, R. Ward, H. Vidal, A. Eljaafari* (Lyon)

C078 | Ghreline linguale agit comme un facteur autocrine pour moduler la signalisation cellulaire par les acides gras dans les cellules gustatives humaines

A. Khan*, L. Brissard, A. Hichami, H. Ozdener, N. A. Khan (Dijon) 


\section{Posters}

\section{ALIMENTATION DURABLE - ALIMENTATION ANIMALE ET QUALITE DES PRODUITS TECHNOLOGIE ALIMENTAIRE}

P001 | Etude de la destructuration et du confort en bouche de deux produits cerealiers enrichis en proteines vegetales chez les personnes agees : impact du statut dentaire et du flux salivaire

C. Septier*, M. Assad-Bustillos, C. Tournier, J. Palier, M. Vandenberghe-Descamps, G. Della Valle, G. Feron (Dijon)

P002 | Evaluation du degre de transformation de 15100 produits alimentaires etiquetes selon le score holistique Siga A. Fardet*, K. Frank, S. Davidou, A. Christodoulou (Clermont-Ferrand-Theix)

P003 | Risques lies a la consommation de certaines denrees alimentaires : bilan a 9 ans du dispositif national de Nutrivigilance unique en Europe

G. Vo Van-Regnault*, I. Margaritis et groupe de travail «Nutrivigilance» (2015-2018) de L'Anses (Maisons-Alfort)

P006 | es caracteristiques epidemiologiques des TIAC dans les provinces (Kenitra, Sidi Kacem, Sidi Slimane) au Nord-Ouest du Maroc entre 2013 et 2017

B. Mohamed, Y. Aboussaleh* (Kenitra, Maroc)

P007 | Developpement et validation d'un index d'evaluation de la durabilite des regimes alimentaires au sein de la cohorte NutriNet-Sante

L. Seconda*, J. Baudry, P. Pointereau, C. Lacour, B. Langevin, S. Hercberg, D. Lairon, B. Allès, E. Kesse-Guyot (Bobigny)

P008 | Les jardins collectifs, outils de promotion de modes de vie durables ? Protocole de l'etude JArDinS

M. Tharrey*, M. Perignon, C. Mejean, N. Darmon (Montpellier)

P009 | La cinetique de digestion chez le Senior des proteines d'une emulsion de viande type knack differe de celle de l'adulte : cartographie des proteines modulees

S. Sante-Lhoutellier*, T. Sayd, J. Pinguet, D. Richard, C. Chambon, M. A. Peyron (Saint-Genès-Champanelle)

P010 | Impact de la structure du bol alimentaire des seniors sur la bioaccesibilite des proteines de pois au cours de la digestion in vitro d'une genoise

M. Assad-Bustillos*, J. Palier, H. Rabesona, Y. Choiset, G. Della-Valle, G. Feron (Nantes)

P011 | Caracterisation du recours aux aliments issus de l'agriculture biologique dans les premiers mois de vie F. Gaudfernau, C. Davisse-Paturet, A. Camier, S. Lioret, S. Nicklaus, M. A. Charles, B. De Lauzon-Guillain* (Villejuif)

P012 | Effets des graines de lin (Linum usitatissimum) sur les chiffres tensionnels, le statut redox et l'activite de la paraoxonase (PON1), chez des rats rendus hypertendus par l'ouabaine

S. Berzou*, D. Krouf, N. Dida-Taleb, A. Guenzet (Oran, Algérie)

P013 | Evaluation de la qualite bacteriologique de la viande rouge dans les boucheries du gouvernorat de Tunis S. Ben Youssef*, G. Dali Temala, I. Sebai, N. Jaziri, S. Ennigrou (Tunis, Tunisie)

P014 | Modifier les apports proteiques pour ameliorer l'adequation nutritionnelle en visant systematiquement ou non davantage de proteines vegetales : impacts sur la durabilite

E. De Gavelle*, P. Leroy, M. Perrimon, J.-F. Huneau, C. Orset, H. Fouillet, L.-G. Soler, F. Mariotti (Paris)

P015 | UNI-VERT : mise au point de produits alimentaires contenant de la spiruline et repondant aux besoins et attentes des beneficiaires de l'aide alimentaire

A.-E. Le Minous*, S. Harnay, M. Dameron, P. Mahe, C. Deschamp, J. Leuranguer (Quimper) 
P016 | Conditions de conservation des aliments de complement du jeune enfant au Niger : un risque pour la qualite des produits?

M. Chapron, C. Mouquet-Rivier, G. Pianelli, I. Laoualy, H. Moussa, M. Denizeau, Y. M. Hemery* (Montpellier)

P017 | 《L'arbre en ville 》: quand le safou, un produit forestier non ligneux, regale les consommateurs des villes et des champs, au Cameroun

C. Icard-Vernière*, S. Zoungrana, H. Womeni, C. Mouquet-Rivier (Montpellier)

P018 | Interet pour la truie et sa portee de l'apport en acides gras n-3 dans l'alimentation gestante et allaitante : impact de la duree de distribution

M. Guillevic*, G. Mairesse, G. Chesneau (Combourtille)

P019 | Facteurs nutritionnels et non nutritionnels du taux de cholesterol de l'oeuf M. Colin*, C. Riou, J. Delarue, A. Y. Prigent, N. Guriec, M. Van Lissum (Ploudalmezeau)

P020 | Determinants sensoriels a l'origine des perceptions saine, durable et naturelle d'un produit ultra transforme : les pizzas A. Saint-Eve*, B. Maurice, J. Delarue, L.-G. Soler, I. Souchon (Thiverval-Grignon)

P021 | Possibilite de couvrir les besoins journaliers de l'Homme en DHA grace a des menus principalement composes de produits provenant d'animaux terrestres enrichis naturellement

M. Colin*, L. Caillaud, A. Boutinon, J. Delarue, A. Y. Prigent, M. Van Lissum (Ploudalmezeau)

P022 | Habitudes alimentaires en matiere de consommation du lait et produits laitiers chez la population algerienne M. Bencharif*, M. Bentaleb (Constantine, Algérie)

P023 | Enrichissement et preservation de la qualite hygienique du yaourt par l'extrait aqueux de curcuma, le gel d'aloe vera et I'huile essentielle du romarin

H. Boughellout*, W. Salmia, F. Adoui (Constantine, Algérie)

\section{COMPORTEMENT ALIMENTAIRE - FONCTION CEREBRALE}

P024 | Effet du jeune du ramadan sur l'alimentation et la corpulence des adultes au niveau de deux regions algeriennes (2017) O. Allam*, H. Oulamara, A. N. Agli (Constantine, Algérie)

P025 | Traitement cerebral des odeurs subliminales : une etude en IRM fonctionnelle C. Mignot*, D. Gounot, S. Chambaron, G. Gaeta, I. Kontaris, L. Marlier (Strasbourg)

P026 | Apports en macronutriments chez les adultes francais souffrant de migraines ou de cephalees non migraineuses V. A. Andreeva*, F. Szabo De Edelenyi, N. Druesne-Pecollo, M. Touvier, S. Hercberg, P. Galan (Bobigny)

P027 | L'acceptabilite d'un repas est affectee par la variation de sa composition par rapport au repas precedent L. Boros, F. Gaudfernau, R. Giraudet, S. Raoul, N. Darcel, O. Davidenko* (Paris)

P028 | Le vieillissement et les parametres physiologiques oraux impactent la liberation in vivo d'aromes pendant la consommation d'un aliment

P. Jeltsch*, A. Laguerre, C. Septier, H. Labouré, G. Feron (Dijon)

P029 | Les determinants de la prise de poids des patients atteints de troubles de l'humeur, depressifs ou bipolaires C. Quéré, A.-K. Illner*, J.-L. Damelincourt, H. Younes (Beauvais)

P030 | Comment la participation d'un enfant a un atelier culinaire influence-t-elle sa capacite à cuisiner a court et a moyen terme?

L. Thiriet, P. Fernandez, E. Castagna, L. Depezay* (Montréal, Canada) 
P031 | Profil de consommation proteique et attitudes vis-a-vis des proteines animales des vegetariens, flexitariens et omnivores d'une population représentative française

E. De Gavelle*, O. Davidenko, H. Fouillet, J. Delarue, N. Darcel, J.-F. Huneau, F. Mariotti (Paris)

P032 | La consommation reguliere de snacks de pistaches n'altere ni la regulation ponderale, ni la composition corporelle M. Fantino*, F. Bellisle, A. Carughi, M. Feeney (Lyon)

P033 | Impact des determinants sensoriels et des informations nutritionnelles sur le comportement alimentaire des consommateurs francais a risque de diabete de type 2

A. Saint-Eve*, E. Boukaïba, B. Maurice, L.-G. Soler, I. Souchon, J. Delarue (Thiverval-Grignon)

P034 | Epilepsie et diabete de type 1 : Est- ce que l'hypoglycemie est le seul facteur incrimine ?

N. El Ouarradi*, G. El Mghari, N. El Ansari (Marrakech, Maroc)

P035 | Prevalence des troubles du comportement alimentaire chez un groupe d'obeses tunisiens M. Khiari*, R. Mizouri, F. Boukhayatia, S. Zribi, F. Mahjoub, O. Berriche, H. Jamoussi (Tunis, Tunisie)

P036 | Etudiants Africains installes en Tunisie, attention a la malbouffe !

R. Serairi Beji*, K. Ayed, S. Jameleddine (Tunis, Tunisie)

\section{COMPOSITION CORPORELLE ET ACTIVITE PHYSIQUE}

P038 | La composition corporelle predit la vitesse de course chez plus de 3000 coureurs a pied

J. Mareschal*, F. Herrmann, C. Graf, V. Karsegard, N. Achamrah, M. Delsoglio, M. Schindler, C. Pichard, L. Genton (Genève, Suisse)

P039 | Alteration du maintien de la masse musculaire et effets immuno-metaboliques induits par un traitement long avec un ligand activateur de PPAR $\beta$

I. Mothe-Satney, V. Guidal, D. Lepousé, S. Le Garf, J. Murdaca, B. Sibille, J. Neels, A.-S. Rousseau* (Nice)

P040 | Cancer du sein metastatique et masse musculaire : role de l'activite physique dans la prevention de la sarcopenie et des toxicites

L. Delrieu*, A. Martin, B. Fervers, M. Morelle, O. Febvey-Combes, O. Pérol, D. Freyssenet, P. Bachmann, O. Trédan, F. Pilleul, M. Touillaud, V. Pialoux (Lyon)

P041 | Effets du jeune du ramadan sur le profil nutritionnel chez des footballeurs amateurs

F. Chiha, H. Djemai*, P. Noirez, F. Desgorces, Y. Benkara (Paris)

P042 | Deficit energetique induit par differentes modalites de pedalage en immersion : etude pilote L. Metz*, D. Thivel, P. Genin, A. Fillon, M. Duclos (Aubière)

P043 | L'activite physique et le comportement sedentaire chez une population representative de la ville Casablanca, Maroc : etude GPAQ

R. Msaad, K. Mohtadi, N. Benalioua, Y. Elkardi, R. Essadik, H. Lebrazi, A. Jafri, A. Derouiche, A. Kettani*, H. Taki, R. Saile* (Casablanca, Maroc)

\section{DENUTRITION HOSPITALIERE - CLAN - NUTRITION A DOMICILE}

P044 | Nutrition enterale : une bonne transition a la norme ENFIT ?

C. Chabut*, R. Gervais, G. Foy, X. Deviot (Saint Denis)

P045 | Le changement d'offre du petit dejeuner ameliore la satisfaction du patient C. Meyer*, L. Joly, A. Gaude, S. Langlais, C. Lirot, E. Fontaine (Grenoble) 
P046 | Prevalence de la denutrition en consultation plaies et cicatrisation au CHU de Montpellier C. Gourc*, L. Teot, S. Pujol et UTN / Consultation plaies et cicatrisation (Montpellier)

P047 | Influence de la polymedication sur l'etat nutritionnel des hypertendus H. N. Merad-Boudia*, Y. Kachekouche, M. Dali-Sahi, N. Medjati-Dennouni, A. Meziane (Sba, Algérie)

P048 | La Dyslipidemie, et risque de denutrition chez les patients ages hypertendus Y. Kachekouche, M. Dali-Sahi*, N. Medjati-Dennouni, H. N. Merad Boudia, A. Meziane (Tlemcen, Algérie)

P049 | Peser les patients habilles induit un risque de surestimation de leur statut nutritionnel Y. Nasser*, P. Fayemendy, M. Arnal, H. Sourisseau, S. Bonhommo, J.-C. Desport, B. Morin, P. Jésus (Limoges)

P050 | Vers une amelioration des prescriptions medicamenteuses chez les patients ayant des troubles de la deglutition et/ou une sonde nasogastrique au $\mathrm{CHI}$ de Villeneuve-Saint-Georges

J. Potier, S. Fondaneche, N. Robquin, G. Goujon, C. Martin, J. Naturel, F. Chaumel, C. Burnat, E. Barsam* (Villeneuve-SaintGeorges)

P051 | Evaluation de la consommation des plateaux repas et de la couverture des objectifs caloriques et protidiques au sein de plusieurs unites du CHU de Strasbourg

E. Merkt*, L. Parizet, A. Botz, E. Riehl, S. Dekaj, L. Trasrieux, A. Pradignac (Strasbourg)

P052 | Projet de teleconsultation dietetique dans le suivi de la personne agee denutrie a domicile N. Masseboeuf*, F. Diebold, V. Attalin (Fréjus)

P053 | Evaluation de la qualite et de la pertinence des prescriptions de nutrition parenterale en oncologie medicale au sein du centre de lutte contre le cancer de Nantes au cours des annees 2015 et 2016

C. Saintes, F. Dayot, S. Dauffy, S. Folliard, H. Lusson, E. Perrien, H. Senellart, D. Vansteene* (Nantes)

P054 | Le supplement nutritionnel oral : impact en terme de compliance du patient et en terme financier lorsque celui-ci est prescrit via une prescription medicale informatisee

M. Lardinois, M. D'Alu, C. Michel, G. Remy, V. Vanesse, D. Noël, D. Lacrosse* (Yvoir, Belgique)

P055 | Impact des soins infirmiers sur le suivi nutritionnel des patients inclus dans un programme dedie au 《long sejour $\gg$ : analyse de 120 patients de soins intensifs

A. Martinez*, E. Favre, T. Kelevina, G. Bagnoud, M. Charrière, D. Favre, O. Pantet, M. Berger, P. Eckert (Lausanne, Suisse)

P056 | Second audit de prescription, d'administration et de suivi des alimentations parenterales au Centre Hospitalier Universitaire de Mont-Godinne. Interet de l'implication d'une dieteticienne

G. Dauw, D. Noël, D. Lacrosse, V. Vanesse, J.-D. Hecq, L. Soumoy, C. Michel* (Yvoir, Belgique)

P057 | Impact de la denutrition sur la survenue des infections nosocomiales au sein de deux poles du CHU de Strasbourg C. Dos Reis*, T. Lavigne, D. Mutter, D. Christmann, A. Pradignac (Strasbourg)

P058 | La prevalence de la denutrition chez les patientes atteintes le cancer du sein T. Salmi, M. Dali-Sahi*, Y. Kachkouche, N. Medjati-Dennouni (Tlemcen, Algérie)

P059 | Validation in vitro d'un nouveau calorimetre indirect a des reglages FI02 eleves Y. M. Dupertuis*, T. Oshima, M. Delsoglio, N. Achamrah, C.-P. Heidegger, C. Pichard et étude multicentrique Icalic sponsorisée par

I'ESICM et ESPEN (Genève, Suisse)

P060 | Validation in vitro d'un nouveau calorimetre indirect pour des niveaux de consommation d'O2 et de production de $\mathrm{CO} 2$ tres faibles

Y. M. Dupertuis*, T. Oshima, M. Delsoglio, N. Achamrah, C.-P. Heidegger, C. Pichard et étude multicentrique ICALIS sponsorisée par I'ESICM et ESPEN (Genève, Suisse) 
P061 | Evaluation des pratiques professionnelles : prise en charge nutritionnelle des patients atteints de cancer des voies aerodigestives superieures traites par radiotherapie ou radio-chimiotherapie dans un Centre francais de Lutte contre le Cancer D. Vansteene*, M. Pons, P. Bachmann, B. Raynard et Interclan Unicancer (Nantes)

P062 | Profil socio-demographique, clinique et paraclinique d'une cohorte de patients adultes atteints d'anorexie mentale (AM) hospitalises dans une unite de Nutrition Clinique-Trouble du Comportement Alimentaire (TCA)

M. Guinhut*, J.-C. Melchior, N. Godart, M. Hanachi (Garches)

P063 | Realisation d'un e-learning sur le branchement - debranchement de la nutrition parenterale dans un centre hospitalier A. Monribot*, J. Bataille, S. Peyronnet, V. Bonal, S. Nérome, L. Billiauws, F. Joly, J. Le Grand (Clichy)

P064 | Etude des scores de denutrition chez l'enfant hospitalise

O. Zaghet*, R. Enaud, H. Clouzeau, L. Rebouissoux, T. Lamireau (Bordeaux)

P065 | Evaluation de l'etat nutritionnel chez l'enfant polyhandicape (etude Polynut)

O. Zaghet*, R. Enaud, H. Clouzeau, L. Rebouissoux, T. Lamireau (Bordeaux)

P066 | L'echelle des prises alimentaires (EPAR) permet elle en consultation dietetique ambulatoire de cancerologie d'apprecier le niveau d'apport calorique et de predire l'echec de prise en charge dietetique orale ?

J. Rieubon, P. Roux, I. Fiorletta*, C. Fingal, B. Poirée, C. Occhipinti, V. Garabige, S. Buzzo, M. Pons, P. Senesse, B. Raynard,

$P$. Bachmann et Interclan Crlcc - Unicancer (Lyon)

\section{DIABETE}

P067 | Evaluation du risque de survenue de la denutrition chez des sujets diabetiques hypertendus M. Dali-Sahi*, N. Dennouni-Medjati, Y. Kachkouche, H. N. Merad Boudia (Tlemcen, Algérie)

P068 | Prise en charge des patients diabetiques dans la region de Chlef (Algerie)

A. Ghouini*, A. Boukoufa (Blida, Algérie)

P069 | Effet de l'entrainement en resistance chez les femmes post-menopausees, obeses et saines sur la sensibilite a l'insuline P. Noirez*, S. Berhaiem, A. D. Karelis, M. Aubertin-Leheudre, J.-F. Brun (Paris)

P071 | Analyse du statut en viamine $E$ chez les diabetiques de type 1

F. Z. Meziane*, A. Bahar, M. Dali-Sahi, N. Medjati-Dennouni, Y. Benslama (Tlemcen, Algérie)

P072 | Apport alimentaire du selenium chez des diabetiques de type 1 de la ville de Tlemcen (extreme ouest Algerien)

B. Ammaria, N. Dennouni*, M. Dali-Sahi, Y. Benslama, M. Belhadj, Y. Kachecouche, Y. Harek (Tlemcen, Algérie)

P073 | Apport nutritionnel en Magnesium des diabetiques de type 1 dans la population de Tlemcen (extreme ouest Algerien) Y. Benslama, A. Behar, N. Dennouni*, M. Dali-Sahi, Y. Harek (Tlemcen, Algérie)

P074 | Effet a 3 ans sur le poids et l'equilibre glycemique de l'exercice en endurance cible sur l'oxydation des lipides (LIPOXmax) chez des diabetiques de type 2 compares a des non-diabetiques

J.-M. Nguyen, M. Richou, F. Bughin, J. Mercier, E. Raynaud De Mauverger, J.-F. Brun* (Montpellier)

P075 | Les grignons d'olive diminuent la glycemie et ameliorent l'activite antioxydante tissulaire, chez le rat rendu diabetique par injection a la streptozotocine

H. Cherrad*, S. Bouderbala (Oran, Algérie)

P076 | Linum usitatissimum previent, le diabete, la dyslipidemie et les dommages oxydatifs en ameliorant les activites des PON1 et LCAT chez les rats rendus diabetiques par la streptozotocine

F. Z. Alachaher* (Oran, Algérie)

P077 | Effet des grignons d'olive sur la peroxydation lipidique serique et tissulaire, chez le rat rendu diabetique H. Cherrad*, S. Bouderbala, M. Bekhtaoui (Oran, Algérie) 
P078 | Les proteines de sardine attenuent l'hyperglycemie, l'insulinoresistance ainsi que l'inflammation, au cours du diabete de type 2, chez le rat Wistar

N. Benaicheta, F. O. Boukortt* (Oran, Algérie)

P079 | La consommation alimentaire et l'activite physique chez les diabetiques de la region Oujda Angad

A. Hsaini*, Y. Aboussaleh (Kenitra, Maroc)

P080 | Les habitudes alimentaires chez les diabetiques de type 1 au cours du mois de Ramadan

N. El Ouarradi*, G. El Mghari, N. El Ansari (Marrakech, Maroc)

\section{GERIATRIE}

P081 | Impact du 《manger-mains》 chez le resident institutionnalise ne mangeant pas seul, en raison de troubles cognitifs et/ou physiques, a travers l'interdisciplinarite soignants/cuisiniers d'une recherche en EHPAD

S. Verbrugghe*, E. Coulon-Bidet, J. Dimet (La Roche-Sur-Yon)

P082 | Lors d'une inflammation intestinale a bas bruit, S. Thermophilus CRZ170 permet de limiter les pertes de masse maigre chez l'age

I. Savary-Auzeloux, M. Jarzaguet, J. David, M. De Avezedo, J.-M. Chatel, D. Dardevet* (Ceyrat)

P083 | Effets combines d'une supplementation en citrulline et d'un entrainement par intervalles de haute intensite sur le profil corporel, musculaire et fonctionnel de personnes agees : influence de l'apport proteique initial

M. Aubertin-Leheudre*, L. P. Carvalho, M. C. Dulac, G. El Hajj Boutros, P. Noirez, J. A. Morais, V. Marcangeli (Montreal, Canada)

P084 | Etat nutritionnel des patients ages d'un Etablissement d'Hebergement pour Personnes Agees Dependantes (EHPAD) et liens entre la denutrition, la consommation de complements oraux et le service a l'assiette

F. Desbordes*, S. Jouveau, A. Broussaudier, V. Noailletas, P. Fayemendy, B. Morin, B. Misset, F. Rebière, J. C. Desport, P. Jésus (Limoges)

P085 | Le blocage du systeme endocannabinoide stimule la synthese proteique via un mecanisme mTOR et calcium dependant dans un modele de myotubes en culture

O. Le Bacquer*, K. Lanchais, Y. Boirie, S. Walrand (Clermont-Ferrand)

P086 | Auton'Al 60 : Programme de Prevention de la perte d'autonomie des seniors de l'Oise

L. Lavefve, J. Branchu, B. Breil, F. Depeint, P. Pouillart, C.-N. Niamba, H. Younes, C. Buche-Foissy, A.-K. IIIner* (Beauvais)

P087 | Le risque cardio-metabolique du sujet age diabetique

R. Mizouri*, I. Rezgani, F. Boukhayatia, K. Ben Naceur, H. Zahra, M. Khiari, Y. Hatira, A. Temssek, B. M. Faika (Tunis, Tunisie)

\section{METABOLISME DES MACRO- ET MICRONUTRIMENTS}

P088 | Impact d'un regime hyperlipidique sur la biochimie et l'ultrastructure cardiaque de modele prepubere femelle D. Sibouakaz, K. Othmani-Mecif*, A. Fernane, A. Taghlit, S. Ait-Benali, Y. Benazzoug (Alger, Algérie)

P089 | Relation entre apports nutritionnels et composition du microbiote dans une cohorte de femmes en bonne sante C. Bobin-Dubigeon*, T. Luu Huyen, E.-H. Nazih, S. Leuillet, T. Carton, F. Le Vacon, J. M. Bard (Nantes)

P090 | Relations entre nutrition avant la grossesse, polymorphisme du gene FADS et statut en acides gras polyinsatures pendant la grossesse dans la cohorte EDEN

E. Petit, M. Armand, M. De Agostini, A. Forhan, M.-A. Charles, B. Heude, J. Y. Bernard* (Villejuif) 
P091 | Analyse des relations entre la consommation de produits laitiers et le syndrome metabolique chez l'homme par metabolomique

F. Capel*, V. Bongard, C. Malpuech-Brugère, E. Karoly, G. Michelotti, J.-P. Rigaudière, C. Jouve, J. Ferrieres, C. Marmonier, J.-L. Sebedio (Clermont-Ferrand)

P092 | Un apport eleve d'Amidon Lentement Digestible diminue les reponses glycemique et insulinemique de facon equivalente dans la population caucasienne et asiatique

A. Goux*, L. Neufcourt, O. Brack, F. Atkinson, S. Vinoy (Saclay)

P093 | Pharmacocinetique d'une nouvelle forme galenique de vitamine D3 $100000 \mathrm{UI}$ en capsule molle

P. Fardellone*, R. Mentaverri, G. Brami, J. C. Souberbielle (Amiens)

P094 | Identification de biomarqueurs de sources proteiques deficientes en acides amines indispensables : lysine et threonine J. Moro*, N. Khodorova, C. Chaumontet, P. Even, A. Blais, J. Piedcoq, C. Gaudichon, D. Tomé, D. Azzout-Marniche (Paris)

P095 | Cooccurrence du surpoids et d'un apport inadequat en iode chez les enfants tunisiens d'age scolaire en 2012 R. Doggui*, M. El Ati-Hellal, P. Traissac, J. El Ati (Tunis, Tunisie)

P096 | Indices de qualite nutritionnelle d'un isolat de proteines de pois seul ou complemente chez le rat F. M. Guillin*, J. Calvez, L. Guérin-Deremaux, C. Lefranc-Millot, D. Azzout-Marniche, N. Khodorova, C. Gaudichon (Paris)

P097 | Analyse des traits cliniques et genetiques de 100 hypertriglyceridemies majeures J.-M. Lecerf*, F. Deseure, L. Boidin-Ceschini, P.-Y. Hatron, P. Benlian (Lille)

P098 | Apports alimentaires et statut biologique en magnesium dans la population de diabetiques de type 2 H. Zahra*, O. Berriche, R. Mizouri, M. Khiari, F. Boukhayatia, R. Ben Othman, F. Mahjoub, H. Jamoussi (Tunis, Tunisie)

P099 | Effets du monosodium glutamate sur l'os en situation de restriction proteique M. Moreau, G. Y. Rochefort, J. Calvez, F. Blachier, A. Blais* (Paris)

P100 | Evaluation du statut en calcium chez des enfants Marocains par l'utilisation de l'excretion urinaire de 24-heures A. Bouziani*, N. Saeid, M. Idrissi, A. Rami, A. El Hamdouchi, Y. Taboz, M. El Mzibri, K. El Kari, H. Aguenaou, H. Benkirane (Kenitra, Maroc)

\section{NUTRITION EN PATHOLOGIE : CANCER, INSUFFISANCE D'ORGANES, REANIMATION, PATHOLOGIES DIGESTIVES...}

P101 | Evaluation de l'etat nutritionnel des hemodialyses chroniques M. Fadli*, N. Kennab, A. Ghouini, K. Khelfat, M. Boulghiti, N. Ayad, N. Kassa (Alger, Algérie)

P102 | Interet du rapport Creatinine/Cystatine C et de l'impedancemetrie bioelectrique (BIA) dans l'evaluation de la composition corporelle chez le patient atteint de cancer

G. Ulmann*, J. Kai, J. P. Durand, N. Neveux, A. Jouinot, J. P. De Bandt, F. Goldwasser, L. Cynober (Paris)

P103 | Retrouver le plaisir de manger apres un cancer ORL

M. Burgevin* (Toulouse)

P104 | Exces de methionine et alteration de la structure renale chez le rat des sables, Psammomys obesus

B. Chaouad, A. Ghoul, F. Zerrouk, A. Moulahoum, K. Othmani-Mecif*, B. Yasmina (Alger, Algérie)

P105 | Correlations entre les parametres biologiques et la composition corporelle dans la sclerose laterale amyotrophique P. Jésus*, F. Patin, S. E. Bakkouche, S. Beltran, C. R. Andrès, P. Vourc'H, H. Blasco, P. Corcia (Limoges) 
P106 | Caracteristiques nutritionnelles des patients sejournant plus de 2 semaines en reanimation : identification de facteurs de risque liés au pronostic chez 120 patients

G. E. Bagnoud*, A. Martinez, E. Favre, M. Charrière, D. Favre, T. Kelevina, O. Pantet, P. Eckert, M. M. Berger (Lausanne, Suisse)

P107 | Le regime pauvre en FODMAPs, sans consultation dietetique, dans la prise en charge des patients souffrant du syndrome de l'intestin irritable

P. Van Ouytsel*, A. Szalai, M. Arvanitakis, A. Van Gossum, J.-C. Preiser, H. Louis (Bruxelles, Belgique)

P108 | L'evaluation de la sarcopenie avant greffe hepatique pourrait contribuer a determiner le pronostic post-greffe des patients en ACLF 2 et 3

S. Testu* (Lyon)

P109 | Valeur predictive de la sarcopenie et de la lymphopenie preoperatoires sur la survie sans recidive dans l'adenocarcinome pancreatique operable

J. Raillat, J. Grillot*, A. Vienot, D. Vernerey, F. Fein, C. Turco, B. Heyd, S. Koch, L. Vuitton, C. D'Engremont, C. Borg (Besancon)

P110 | Evaluation des parametres nutritionnels preoperatoires dans une cohorte d'adenocarcinome pancreatique resecable J. Grillot*, A. Vienot, J. Raillat, D. Cazaux, C. Prothe, C. Turco, S. Borot, C. D’Engremont (Besancon)

P111 | Etude de la nature des allergies alimentaires en consultation au Laboratoire National de reference de Casablanca-Maroc L. Slimani*, S. Souat, J. El Bakkouri, A. Jafri, F. Dehbi (Casablanca, Maroc)

P112 | Complications cardiometaboliques et deficits cognitifs chez les survivants de la leucemie lymphoblastique aigue pediatrique : associations avec l'inflammation peripherique?

P. Léveillé*, A. Boulet-Craig, J. Laniel, A. Franco, S. Morel, S. Drouin, F. Rodier, M. Krajinovic, C. Laverdière, D. Sinnett, P. Robaey, S. Sultan, E. Levy, V. Marcil, S. Lippé (Montréal, Canada)

P113 | DHA des spermatozoides et infertilite : revue de la litterature et implications physiopathologiques F. Saur, C. Ingueneau, L. Bujan, S. Caspar-Bauguil*, S. Hamdi (Toulouse)

P114 | Grossesse et carences en vitamines et fer : des indicateurs de prevalence inquietants A. Galinier, C. Ingueneau*, M. Bertrand, W. Fillali, M. Coustols-Valat, O. Parant, E. Lobinet (Toulouse)

P115 | Evaluation nutritionnelle des patients sous traitement anti neoplasique en Algerie M. A. Melzi*, Z. Derbouz, Z. Bachiri, A. Bounedjar (Blida, Algérie)

P116 | Effets de la N-carbamoyl-putrescine sur le metabolisme proteique musculaire du rat age denutri P. Jegatheesan*, N. Akir, N. El-Hafaia, R. Ramassamy, M. Aboubacar, S. Nakib, N. Neveux, C. Loï, L. Cynober, J. P. De Bandt (Paris)

P117 | La spasticite est-elle un facteur predictif de l'etat nutritionnel des patients en etat de conscience alteree ? M. Fadeur*, C. Ippoliti, C. Malherbe, A.-M. Verbrugge, O. Gosseries, J. De Flines, A. Thibaut, S. Laureys, N. Paquot (Liège, Belgique)

P118 | Mise en place de sonde naso-gastrique video-assistee par l'equipe infirmiere de reanimation : experience locale G. Crohin*, S. Waeytens, R. Sand, N. Bailly, M. De Mahieu, J.-C. Preiser (Brussels, Belgique)

P119 | Interet d'un depistage systematique de la dysphagie sur l'incidence des pneumonies acquises aux soins intensifs P. Stalins*, M. Devroey, I. Koube, J.-C. Preiser (Brussels, Belgique)

P120 | Le cancer et la chimiotherapie affecte la fonction mitochondriale hepatique dans un modele murin de cancer colique B. Martin*, F. Lamarche, E. Fontaine, C. Moinard, C. Breuillard (Grenoble)

P121 | Effets d'un regime a haute teneur en proteines dans un contexte de surnutrition lipidique et calorique sur la synthese proteique musculaire et l'infiltration lipidique chez les rats adultes et ages

E. Poggiogalle*, A. Carayon, J. Salles, C. Giraudet, J.-P. Rigaudière, S. De Saint-Vincent, P. Sanchez, O. Lebacquer, F. Capel,

S. Walrand, Y. Boirie, C. Guillet (Clermont-Ferrand) 
P122 | Interet du hand grip test dans la prise en charge dietetique du patient cirrhotique complique de decompensation oedemato-ascitique

B. Sainton*, A. Paillard, J. Capelle, R. Piquemal (Blois)

P123 | Analyse longitudinale du support nutritionnel chez des patients primo traites pour un cancer des voies aerodigestives superieures (VADS) au Centre Antoine Lacassagne

C. Hebert*, C. Michel, J. Sicurani, Y. Chateau, J. Viotti, M.-N. Falewee (Nice)

P124 | Supplements oraux d'Acides Amines Essentiels (AAE) et d'ellagitannins chez le patient hemodialyse

S. Treille*, F. Dewez, S. Asta, A. Benahmed, A. Legrand, B. Guillaume (Lodelinsart, Belgique)

P125 | Prevalence de la denutrition a l'admission et impact sur la mortalite en reanimation, experience dans un service de reanimation polyvalente

P.-A. Rogghe*, V. Pages, F. Rousseau, T. Vanderlinden (Lomme-Les-Lille)

P126 | Protection contre le developpement futur de l'atherosclerose via l'administration perinatale d'un extrait liquide de spiruline chez la souris APO E -/-

M. Coué*, B. Castellano, J. Falewée, A. Aguesse, O. Lépine, K. Ouguerram (Nantes)

P127 | L'activite physique spontanee en condition d'obesite ralentit la croissance tumorale en modulant les signaux hormonaux tissulaires

D. Le Guennec*, V. Hatte, M. Goepp, M.-C. Farges, S. Rougé, F. Caldefie-Chezet, M.-P. Vasson, A. Rossary (Clermont-Ferrand)

P128 | Signature postprandiale des microARN circulants et association a l'hypertriglyceridemie

R. Daclat*, P. Giral, J.-E. Salem, M. Guérin, J.-M. Lacorte (Paris)

P129 | L'apport proteique en cancerologie : limite-t-il la malnutrition ou favorise-t-il la croissance tumorale ?

B. Martin*, K. Couturier, C. Moinard, C. Breuillard (Grenoble)

P130 | Profil alimentaire selenie des cardiopathes de la population de Tlemcen (extreme Ouest Algerien) M. Belhadj, N. Dennouni*, A. Behar, M. Dali-Sahi, Y. Harek (Tlemcen, Algérie)

P131 | Traitement des tumeurs des voies aerodigestives superieures : faut-il mettre une sonde de gastrostomie prophylactique ?

M. M. Van Gossum, A. Yanni*, D. Dequanter, A. Barik, V. Pierart, P. Eisendrath (Bruxelles, Belgique)

P132 | La prevalence de la boulimie chez les adolescents au Maroc : Region de Fes

M. Makdad*, K. Harraqui, Z. Hannoun, A. Bour (Kénitra, Maroc)

P133 | Risque de syndrome de renutrition en post-operatoire d'une chirurgie sus-mesocolique majeure M. Geraud*, E. Vastel, J. Lubrano, M.-A. Piquet (Caen)

P134 | Hyperadiponectinemie chez les patients diabetiques denutris hospitalises pour traitement de lesions du pied R. Charchour*, F. Phan, S. Fourati, E. Ben Salah, F. Mestari, O. Bourron, J.-M. Lacorte (Paris)

P135 | Impact et faisabilite de la nutrition enterale chez les patients autogreffes

F. Piquemal*, A. Banos, C. Araujo, J. Gay, C. Capdupuy, C. Duclos, M. A. Piquet (Bayonne)

P136 | Les parametres de composition corporelle (CC) sont-ils associés au devenir clinique de patients atteints d'un cancer du pancreas avance traites par chimiotherapie a base de fluoropyrimidine?

M. Som*, C. Brac De La Perrière, J. Viaud, B. Campillo-Gimenez, M. Thomas, L. Lacaze, J. Edeline, R. Thibault (Rennes)

P137 | Evaluation de pratique : Nutrition enterale en Reanimation

A. Robin, M. Kaidomar*, N. Masseboeuf, F. Diebold, V. Bertolino, O. Fleuriet Et Service Réanimation (Fréjus) 
P138 | Impact de l'immunonutrition orale sur la morbidite postoperatoire en chirurgie oncologique digestive : une etude de cohorte a l'echelle nationale

C. Rives-Lange*, A. Challine, D. Danoussou, S. Katsahian, A. Ait Boudaoud, S. Gaujoux, B. Dousset, C. Carette, A. Lazzati,

S. Czernichow (Paris)

P139 | Impact du statut nutritionnel sur l'efficacite et la tolerance du NIVOLUMAB dans les cancers broncho-pulmonaires T. Marchal*, V. Royer-Garabige, B. Raynard, N. Girard (Paris)

\section{OBESITE}

P140 | Effet de la 20-hydroxyecdysone sur l'obesite viscerale induite par un stress nutritionnel chez un modele du syndrome metabolique « Gerbillus tarabuli» : Implication du cortisol

Z. Bellahreche*, N. Semiane, A. Mallek, Y. Dahmani (Alger, Algérie)

P141 | Prise en charge et depistage de l'obesite infantile: Pratiques et attentes des Medecins Generalistes et des Pediatres liberaux du Limousin

H. Guibert, A. Lienhardt-Roussie, P. Fayemendy, B. Morin, A. Voisin, H. Thibault, J. C. Desport, P. Jésus* (Limoges)

P142 | Preference aux aliments apportant le gout gras et surcharge ponderale

O. Allam*, H. Oulamara, A. N. Agli (Constantine, Algérie)

P143 | Insulinoresistance et Inflammation : effets de la spiruline en prevention primaire chez Psammomys obesus A. Ghennai, E. H. A. Koceir, S. Bouderba* (Alger, Algérie)

P144 | Effets de la spiruline enrichie en silicium sur les composantes majeures du syndrome metabolique chez le rat Zucker obese

J. Vidé, B. Bonafos, G. Fouret, N. Jouy, C. Coudray, S. Gaillet, C. Feillet-Coudray* (Montpellier)

P145 | Effets de la spiruline enrichie en silicium sur les desordres metaboliques associes a l'obesite chez le rat J. Vidé, B. Bonafos, G. Fouret, N. Jouy, C. Feillet-Coudray*, S. Gaillet, C. Coudray (Montpellier)

P146 | Inflammation et stress oxydant au cours du developpement de l'intolerance au glucose et de la steatose hepatique chez des rats nourris avec un regime obesogene

C. Feillet-Coudray*, G. Fouret, C. Vigor, B. Bonafos, B. Jover, A. Blachnio-Zabielska, J. Rieusset, S. Gaillet, T. Durand, C. Coudray (Montpellier)

P147 | Impact d'un programme d'education therapeutique 《 reflexif 》 dans l'obesite N. Debossu*, B. Gavlak, S. Vatin, I. Gaubil-Kaladjian, J. Grulet, P. Humbert, E. Bertin (Reims)

P148 | Association prospective entre les comportements alimentaires durables evalues par l'index generique de durabilite des regimes (SDI), la prise de masse corporelle et le risque d'obesite dans la cohorte NutriNet-Sante

L. Seconda*, J. Baudry, M. Egnell, C. Julia, S. Hercberg, P. Pointereau, D. Lairon, B. Allès, E. Kesse-Guyot (Bobigny)

P149 | Indicateurs anthropometriques et risque cardiovasculaire dans la population adulte Oranaise - Etude ISOR

L. Houti*, S. Mediene-Benchekor, H. Ouhaibi-Djellouli, S. Lardjam-Hetraf, I. Hamani-Medjaoui (Oran, Algérie)

P150 | Consequences immuno-metaboliques d'une activation de la voie PPARbeta dans le traitement de l'obesite chez la souris S. Le Garf*, J. Murdaca, I. Mothe-Satney, G. Le Menn, B. Sibille, J. Neels, A.-S. Rousseau (Nice)

P151 | Peut-on utiliser le Quotient de Satiete comme predicteur de la prise alimentaire chez l'adolescent en situation d'obesite ? A. Fillon, M. Miguet, M. Khammassi, J. Masurier, M. Duclos, Y. Boirie, A. Tremblay, V. Drapeau, M.-E. Mathieu, D. Thivel* (Clermont-Ferrand)

P152 | Sentiment de satisfaction apres chirurgie bariatrique : quels predicteurs a un an post-operatoire ?

B. Gaudrat*, S. Andrieux, V. Florent, A. Rousseau (Lille) 
P153 | Un nouvel effet secondaire de la chirurgie bariatrique : la rhinorrhee post-prandiale M. Pouget*, C. Lemaire, L.-A. Gendre, M. Riquelme, E. Gentes, N. Farigon, C. Palmier-Forestier, M. Miolanne, K. Slim, Y. Boirie (Clermont-Ferrand)

P154 | Interet d'un suivi en SSR specialise sur l'evolution ponderale et la survenue de complications post chirurgie bariatrique D. Ruillier*, M. Vix, S. Rohr, P. Grosjean, T. Leroi, V. Fontaa, M. Riegert, A. Pradignac (Strasbourg)

P155 | Prevalence de l'obesite et du surpoids chez des ecolieres et ecoliers de Casablanca : Maroc H. Makhlouki*, S. Lougda, A. Jafri, F. Achibane, M. El Arbaoui, Y. Elkardi, H. Taki, R. Saile, A. Derouiche (Casablanca, Maroc)

P156 | La prevalence de la surcharge ponderale et les facteurs sociodemographiques associes a Casablanca, Maroc K. Mohtadi, R. Msaad, N. Benalioua, Y. Elkardi, R. Essadik, H. Lebrazi, A. Jafri, A. Derouiche, E. H. Tahri, A. Kettani*, H. Taki, R. Saile* (Casablanca, Maroc)

P157 | Un regime a teneur faible en proteine et en methionine induit une augmentation de la prise alimentaire, de la depense energetique et de FGF21 et une baisse de I'IGF-1 et affecte peu l'adiposite chez les souris femelle BalbC P. Even*, C. Chaumontet, D. Azzout-Marniche, A. Blais, J. Piedcoq, D. Tomé, C. Gaudichon (Paris)

P158 | Accompagnement familial a domicile et de PROXimite de l'OBesite infanto-juvenile PROXOB : etude pilote de faisabilite en recherche interventionnelle en sante

R. Rigondet, A. Rigal, C. Desblès, Q. Lesaichot, J. Masurier, C. Cardenoux, D. Thivel, B. Pereira, C. Lambert, Y. Boirie*, M. Miolanne (Clermont-Ferrand)

P159 | Etude pilote chez 45 patients obeses : evaluation d'un traitement innovant ambulatoire pluridisciplinaire d'aide a la gestion de l'impulsivite alimentaire

H. Lelandais*, T. Saillard, E. Diologent, M. Riguet, C. Meret, V. Risselin, C. Lemiere, M. Valentino, D. Hazard, P. Prié, G. Le Foll, A. Raimbault, J. Dijoux, P. Dechelotte, S. Grigioni, V. Folope (Rouen)

P160 | Influence d'un amorcage olfactif chez des adultes de statut ponderal different M. Mas*, S. Chambaron, C. Chabanet, M.-C. Brindisi (Dijon)

P161 | Variabilite de l'expression d'ER $\beta$ en fonction de la sensibilite à prendre ou perdre du poids chez le rat Wistar L. Metz*, M. Gerbaix, C. Guillet, S. Walrand, N. Boisseau, Y. Boirie, D. Courteix (Aubière)

P162 | L'addiction alimentaire diagnostiquee par la Yale Food Addiction Scale version 2.0 est frequente chez les patients obeses mais n'est pas associee aux complications de l'obesite M. Som*, D. Val-Laillet, A. Constant, R. Moirand, R. Thibault (Rennes)

P163 | Effets benefiques d'une prise en charge pluridisciplinaire sur l'inflammation systemique : impact de l'endurance cardiorespiratoire chez des adolescents obeses presentant des troubles du sommeil J. Roche, L. Isacco, D. Thivel*, F. Perret, G. Dumoulin, V. Gillet, F. Mougin (Clermont-Ferrand)

P164 | Evaluation de la sante sexuelle des patientes obeses par les medecins generalistes nutritionnistes M. Lavenat*, A.-M. Guedj, D. Costa, P. Mares, V. Taillard (Nîmes)

P165 | Cout materiel d'une ration journaliere d'un obese : proposition d'une ration equilibree a moindre cout R. Serairi Beji*, J. Boumaiza, S. Ennaifer, K. Ayed, R. Ksouri, S. Jameleddine (Tunis, Tunisie)

P166 | Une nouvelle methode pour etudier les determinants gustatifs chez la souris : etude de l'impact d'une obesite nutritionnelle sur la perception du sucre

A. Dastugue*, J.-F. Merlin, G. Maquart, A. Bernard, P. Besnard (Dijon)

P167 | Explorations fonctionnelles digestives en post chirurgie bariatrique M. Massicard*, E. Vastel, F. Vasseur, C. Beche, K. Lacombe, M.-A. Piquet (Caen)

P168 | Le Yucatan Minipig comme futur modele d'etudes precliniques en denutrition et obesite sarcopenique L. Lacaze*, K. Boudjema, J. Georges, J. Delamarre, F. Le-Gouevec, A. Chauvin, D. Val-Laillet, R. Thibault (Rennes) 
P169 | Efficacite de la prise en charge dans la filiere bariatrique du centre Hospitalier de Blois A. Paillard*, J. Capelle, L. Dalmasso, R. Piquemal (Blois)

\section{PEDIATRIE ET NEONATOLOGIE}

P170 | Exposition perinatale a un regime de quantite et de qualite variables en proteines chez le rat- croissance, preferences alimentaires et risque de surpoids chez la descendance femelle adulte

G. Carlin*, C. Chaumontet, C. Delteil, N. Darcel, B. Van De Heijning, A. Kodde, D. Tome, A.-M. Davila (Paris)

P171 | Nouvelles courbes de croissance \& diagnostic de l'etat nutritionnel chez l'enfant, quels impacts ? V. Harivel*, D. Grey, R. Gandy et Unité Transversale De Nutrition (UTN) (Montpellier)

P172 | Changement de sources proteiques des formules infantiles : degre d'hydrolyse et bioaccessibilite des acides amines L. Le Roux*, F. Nau, P. Schuck, R. Chacon, R. Jeantet, A. Deglaire, D. Dupont (Rennes)

P173 | Caracterisation de l'alimentation des peres avant la conception et lien avec le poids de naissance dans la cohorte nationale ELFE

S. Krinitzki, S. Lioret, M. A. Charles, B. De Lauzon-Guillain* (Villejuif)

P174 | Teneur en matieres grasses et profil en acides gras des laits de suite commercialises en officine de pharmacie a Abidjan K.-A. Kouassi, A. N'bra, J. S. Yao, S. Dibi, M. Aké* (Abidjan, Côte d'Ivoire)

P175 | Introduction des aliments textures dans l'alimentation des enfants de 4 a 36 mois : descriptions des pratiques en France et facteurs associes

C. Tournier*, L. Demonteil, E. Ksiazek, H. Weenen, M. Dusoulier, A. Marduel, S. Nicklaus (Dijon)

P176 | Les nourrissons dont l'alimentation est exclusivement lactee sont-ils capables de compenser des modifications de la densite energetique du lait lors du repas suivant ? (Prix SFN 2015)

C. Schwartz*, P. Brugaillères, S. Issanchou, S. Nicklaus (Dijon)

P177 | Degradation de N-acetyle-cysteine en N, N-diacetyle-cystine dans les nutritions parenterales pediatriques I. Sommer*, H. Schwebel, V. Adamo, L. Bouchoud, P. Bonnabry, F. Sadeghipour (Lausanne, Suisse)

P178 | Pancreatite aigue secondaire a une hypertriglyceridemie chez l'enfant : une cause rare a evoquer J. Lemale*, S. Keloua, B. Dubern, J.-P. Girardet, P. Tounian (Paris)

P179 | e-Pinut 2018 - Donnees de croissance chez l'enfant hospitalise

V. Raoult, L. Bouvart, D. Guimber, N. Peretti, H. Piloquet, R. Hankard, A. De Luca* et The Pediatric Nutritional Assessment Network (www.epinut.fr) (Tours)

P180 | Influence des apports nutritionnels durant les premieres semaines de vie du premature sur la croissance et le developpement psychomoteur jusqu'a l'age de 2 ans

P. Poidevin, A. Leke* (Amiens)

P181 | Evaluation des pratiques du depistage de la denutrition chez l'enfant au cabinet du medecin generaliste. V. Bertrand*, B. Pichot, P. Déchelotte (Montivilliers)

P182 | Exposition prenatale aux omega 6 et omega 3 et sante cardio-metabolique a 5 ans des enfants de la cohorte EDEN M. Armand*, J. Y. Bernard, É. Petit, A. Forhan, M. Bernard, M.-A. Charles, B. Heude et groupe de l'étude EDEN (Marseille)

P183 | Les suspicions d'allergies au lait de vache non IgE-mediee sont rarement confirmees

A. Lemoine*, J. Aroulandom, C. O'Neill, J. Lemale, P. Tounian (Paris)

P184 | Velocite de croissance des nouveau-nes prematures : comparaison du modele exponentiel de Patel avec les variations de Z-score de poids

L. Simon*, M. Hanf, A. Frandas-Chauty, D. Darmaun, G. Gascoin, C. Flamant, J.-C. Rozé (Nantes) 
P185 | La matiere grasse laitiere et le probiotique L. fermentum incorpores dans des preparations pour nourrissons modulent leur digestion ainsi que la composition du microbiote et les proprietes barriere de l'intestin chez le mini-porc

M. Lemaire, G. Boudry, O. Ménard, P. Le Ruyet, C. Baudry, P. Gérard, D. Dupont, A. Deglaire, I. Le Huërou-Luron*, S. Blat (Rennes)

P186 | Frequence de la malnutrition (denutrition, surpoids/obesite) et prevalence de la carence martiale chez les nourrissons de I'Ouest algerien

K. Bouziane-Nedjadi*, A. Hadj Kadi, A. Hamous, S. Benadouda, N. Dida, I. Boureghda, M. Bouchenak (Oran, Algérie)

\section{PHYSIOLOGIE DIGESTIVE}

P187 | Impact des lipides polaires laitiers sur le gain de poids, le microbiote et des marqueurs de la barriere intestinale in vivo et role de la sphingomyeline in vitro

M. Milard*, A. Penhoat, V. Sauvinet, A. Durand, E. Meugnier, E. Loizon, C. Buisson, L. Bindels, A. Neyrinck, N. Delzenne,

F. Laugerette, M.-C. Michalski (Lyon)

P188 | L'inflammation intestinale de rats carences en folate et vitamine B12 et exposes au DSS produit une steatohepatite nonalcoolique (NASH) liee a l'augmentation de MCP-1 et IL-1beta

Z. Harb*, L. Lagrost, A. Marchal Bressenot, J. Raso, J.-M. Alberto, R. Umoret, J.-P. Bronowicki, J.-L. Guéant (Nancy)

P189 | L'effet antiproliferatif du cholest-4-en-3-one, un metabolite du microbiote intestinal, est associe a une diminution de la lipogenese et a une desorganisation des radeaux lipidiques dans deux modeles de cellules cancereuses mammaires J. Elia*, D. Carbonnelle, C. Gaudin, J.-M. Huvelin, A. Burghelea, M. Tannoury, M. Diab-Assaf, K. Petit, H. Nazih (Nantes)

P190 | La taurine previent la peroxydation lipidique chez les souris sensibilisees a la $\beta$-Lactoglobuline S. Ainad Tabet*, A. Haddi, H. Negaoui, O. Kheroua, D. Saidi (Oran, Algérie)

P191 | Mise en place d'une hyperphagie associee a une adaptation jejunale et colique dans un modele de grele court chez le rat A. Le Moigne, A.-C. Jarry, L. Ribeiro-Parenti, M. Le Gall, A. Bado, F. Joly, J. Le Beyec - Le Bihan* (Paris)

P192 | Evaluation de la flore bacterienne intestinale chez le rat wistar malnutris : impact de la realimentation par un ble fermente type Hamoum

D. Yssaad*, B. M. Benakriche, D. Saidi, O. Kheroua (Oran, Algérie)

P193 | Etude du metabolisme central et peripherique du tryptophane et de la kynurenine dans un modele de denutrition secondaire a une inflammation aigue

H. Hounkponou, P. Tirelle, E. Salameh, C. L'Huillier, W. Bahlouli, C. Guérin, C. Bôle-Feysot, J. C. Do Rego, A. Goichon, P. Déchelotte, R. Marion-Letellier, N. Achamrah, M. Coëffier* (Rouen)

P194 | La supplementation en glutamine restaure la fonction de barriere intestinale dans un modele murin d'anorexie C. L'Huillier*, M. Jarbeau, L. Belmonte, S. Nobis, A. Goichon, É. Salameh, W. Bahlouli, J.-C. Do Rego, P. Déchelotte, M. Coëffier (Rouen)

P195 | Effet d'un regime riche en sel sur la fibrose intestinale dans un modele murin de colite chronique A. Amamou*, M. Rouland, E. Salameh, L. Yaker, M. Jarbeau, C. Guérin, A. Goichon, G. Savoye, R. Marion-Letellier (Rouen)

P196 | Etude de l'interaction entre des derives du tryptophane et le recepteur aryl hydrocarbone dans un modele in vitro de fibrose intestinale

A. Amamou*, L. Yaker, C. Bôle-Feysot, G. Savoye, R. Marion-Letellier (Rouen)

P197 | Identification de la proteine ClpB (caseinolytic peptidase B), mimetique du neuropeptide anorexigene $\alpha$-MSH ( $\alpha$ melanocyte-stimulating hormone) chez la souche Hafnia alvei 4597 par technique LC-MS/MS DIA

N. Lucas, M. Dominique, C. Deroissart, J. Vowinckel, K. Novy, T. Treiber, P. Déchelotte, G. Lambert, R. Legrand* (Rouen)

P198 | Impact d'un by-pass Roux-en-Y sur la morphologie et les fonctions endocrines gastro-intestinales chez un modele de miniporc Yucatan obese

B. Mohamed-Elarbi, D. Bergeat*, G. Randuineau, A. Cahu, Y. Gautier, R. Thibault, D. Val-Laillet (St Gilles) 
P199 | Evaluation du tissu adipeux visceral et des parametres glycemiques en post chirurgie bariatrique N. C. Dagher*, R. Damade, A. Monier, C. De Brauer, S. Antoun (Chartres)

P200 | Effet d'un mois de sevrage en hormone de croissance (GH) chez des patients deficitaires en GH : etude du role de la GH dans la regulation du metabolisme des lipoproteines riches en triglycerides (LRT)

S. Béliard, R. Valéro, J. Dupont-Roussel, A. Calabrese, M. Maraninchi* (Marseille)

P201 | Impact nutritionnel d'un ble fermente type Hamoum sur la translocation bacterienne intestinale chez le rat malnutri en phase de realimentation

B. Ben Mehel*, S. Bousbahi, P. Gérard Et Soumia Bousbahi (Oran, Algérie)

\section{SANTE PUBLIQUE}

P202 | Des dispositifs de cuisine connectes pour influencer l'homeostasie nutritionnelle : un essai controle randomise de faisabilite a domicile

M.-F. Vaillant*, H. Terrisse, S. Artemova, C. Rolland, M. Joyeux-Faure, J.-C. Borel, O. Marion, P. Dancer, M. Sicard,

P. Casas-Agustench, C. Andres-Lacueva, A. J. Lloyd, J. Draper, C. Pison, C. Moinard (Grenoble)

P203 | Il est possible de reduire la teneur en sel des menus des restaurants scolaires grace a des interventions coordonnees aupres des personnels de cuisine et des fournisseurs d'aliments industriels

J. C. Desport*, A. Baptiste, C. Villemonteix, L. Darthou-Pouchard, C. Soulefour, M. Guitard, S. De Magalhaes, V. Javerliat,

C. Bachelerie, C. Jalladeau, A. Lecointre, P. Fayemendy, P. Jésus (Limoges)

P204 | Adherence au regime Mediterraneen, indice de masse corporelle et risque de diabete de type 2 : resultats de l'etude UK Biobank

P. M. Andre*, G. Proctor, F. Rodriguez-Artalejo, E. Lopez-Garcia, D. Gomez-Cabrero, P. Bros, E. Neyraud, E. Garcia-Esquinas, M. Morzel, C. Féart (Bordeaux)

P205 | Le selenium plasmatique chez des tabagiques Tlemceniens (ouest Algerie)

N. Dennouni Medjati*, M. Dali Sahi, Y. Harek (Tlemcen, Algérie)

P206 | Association entre profils metabolomiques plasmatiques par RMN et risque a long terme de developper un cancer de la prostate

L. Lécuyer*, A. Victor Bala, N. Bouchemal, M. Nawfal Triba, A. Demidem, A. Rossary, P. Galan, S. Hercberg, V. Partula,

L. Le Moyec, P. Latino-Martel, E. Kesse-Guyot, M. Deschasaux, M.-P. Vasson, P. Savarin, M. Touvier (Bobigny)

P207 | Association prospective entre le potentiel inflammatoire du regime alimentaire et le risque de symptomes depressifs incidents

M. Adjibade*, C. Lemogne, M. Touvier, S. Hercberg, P. Galan, K. E. Assmann, C. Julia, E. Kesse-Guyot (Bobigny)

P208 | Association prospective entre la consommation d'aliments ultra-transformes et le risque de symptomes depressifs incidents

M. Adjibade*, C. Julia, B. Allès, M. Touvier, C. Lemogne, B. Srour, S. Hercberg, P. Galan, K. E. Assmann, E. Kesse-Guyot (Bobigny)

P209 | Association entre consommation de charcuterie et symptomes d'asthme

R. M. Andrianasolo*, S. Hercberg, M. Touvier, N. Druesne-Pecollo, M. Adjibade, E. Kesse-Guyot, P. Galan, R. Varraso (Bobigny)

P210 | Association entre le score individuel FSA mesurant la qualite globale de l'alimentation et les symptomes d'asthme R. M. Andrianasolo*, C. Julia, R. Varraso, M. Egnell, M. Touvier, E. Kesse-Guyot, S. Hercberg, P. Galan (Bobigny)

P211 | Les technologies innovantes pour l'evaluation de la prise alimentaire par capture d'image : un outil pour la recherche clinique et epidemiologique?

L. Albaladejo*, M.-F. Vaillant, P. Gillois, J.-L. Bosson (Grenoble)

P212 | Developpement et validation d'un SCore d'Alimentation SAine : SCASA

M. Kruseman, E. Farina, I. Carrard, J. Cela*, I. Guessous, P. Marques Vidal (Carouge, Suisse)

JFN 2018 - Livre des résumes | Page 22 
P213 | PNNS-GS2 : Developpement et validation d'un score de qualite dietetique refletant les recommandations nutritionnelles francaises de 2017

D. Chaltiel*, M. Adjibade, V. Deschamps, M. Touvier, S. Hercberg, C. Julia, E. Kesse-Guyot (Bobigny)

P214 | Analyse de l'association prospective entre le score d'adequation aux recommandations nutritionnelles francaises de 2017 (PNNS-GS2) et l'apparition de surpoids et d'obesite dans la cohorte NutriNet-Sante

D. Chaltiel*, V. Deschamps, M. Touvier, S. Hercberg, C. Julia, E. Kesse-Guyot (Bobigny)

P215 | L'impact de l'education nutritionnelle sur l'evolution des habitudes alimentaires et la composition corporelle des salaries de l'industrie agroalimentaire au Maroc

A. Derouiche*, H. Makhlouki, A. Belhouari, K. Mouhtadi, Y. Elkardi, A. Jafri, C. Martin (Casablanca, Maroc)

P216 | Evaluation de la consommation de l'iode et du sodium dans une population d'adultes Marocains agee de 18 à 25 ans A. Derouiche*, M. Elarbaoui, F. Achibane, H. Makhlouki, S. Loukda, Y. Elkardi, H. Taki, R. Saile, A. Jafri, B. Ellahi (Casablanca, Maroc)

P217 | L'exclusion du gluten dans la population francaise : caracteristiques socio-demographiques, motivations et profils alimentaires

L. Perrin, B. Allès*, C. Buscail, S. Hercberg, C. Julia, E. Kesse-Guyot (Bobigny)

P218 | Modelisation de l'impact de differents logos nutritionnels en face avant des emballages sur la mortalite par maladie chronique

M. Egnell*, P. Crosetto, T. D’Almeida, E. Kesse-Guyot, M. Touvier, B. Ruffieux, S. Hercberg, L. Muller, C. Julia (Bobigny)

P219 | Comprehension objective de differents formats de logos nutritionnels en face avant des emballages : resultats d'une etude comparative experimentale internationale

M. Egnell*, S. Pettigrew, Z. Talati, S. Hercberg, C. Julia (Bobigny)

P220 | Signatures metabolomiques associes à des profils alimentaires specifiques dans la cohorte SU.VI.MAX

L. Lécuyer*, C. Dalle, P. Micheau, M. Pétéra, D. Centeno, B. Lyan, C. Morand, P. Galan, S. Hercberg, A. Rossary, A. Demidem, M.-P. Vasson, V. Partula, M. Deschasaux, B. Srour, P. Latino-Martel, E. Kesse-Guyot, S. Durand, E. Pujos-Guillot, C. Manach,

T. Mathilde (Bobigny)

P221 Impact des logos nutritionnels en face avant des emballages sur la selection des tailles de portions : etude experimentale M. Egnell*, E. Kesse-Guyot, P. Galan, M. Touvier, M. Rayner, J. Jewell, J. Breda, S. Hercberg, C. Julia (Bobigny)

P222 | La prevalence du syndrome metabolique comparee a l'activite physique chez la population de Marrakech, au sud-ouest du Maroc

Z. Hannoun*, I. Samara, K. Harraqui, R. Attoumane, O. Bensmail, F. El Arabi, A. Bour (Kenitra, Maroc)

P223 | Rapport au plaisir alimentaire chez les mangeurs urbains en France

S. Thiron* (Montpellier)

P224 | Consommation de boissons sucrees et risque de cancer : resultats de la cohorte prospective NutriNet- Sante E. Chazelas*, B. Srour, E. Desmetz, E. Kesse-Guyot, C. Julia, V. Deschamps, N. Druesne-Pecollo, P. Galan, S. Hercberg, P. Latino-Martel, M. Deschasaux, M. Touvier (Bobigny)

P225 | Effets des logos nutritionnels en face avant des emballages sur la qualite nutritionnelle des achats des etudiants : essai randomise controle a trois bras

M. Egnell*, I. Boutron, S. Péneau, P. Ducrot, M. Touvier, P. Galan, C. Buscail, R. Porcher, P. Ravaud, S. Hercberg, E. Kesse-Guyot, C. Julia (Bobigny)

P226 | Risque et benefice nutritionnels associes a la consommation de viande rouge en France J. De Oliveira Mota*, P. Tounian, S. Guillou, F. Pierre, J.-M. Membré (Nantes) 
P227 | Apports en macro- et micronutriments et caracteristiques sociodemographiques et cliniques des consommateurs de produits laitiers chez les personnes agees de la cohorte Trois-Cites-Bordeaux H. Pellay*, C. Helmer, C. Boyer, C. Marmonier, C. Samieri, C. Féart (Bordeaux)

P228 | Evaluation du statut en iode et determination des carences iodes chez des enfants Marocains ages de 6 à 18 ans A. Rami*, N. Saeid, M. Idrissi, M. Taghzaoui, A. El Hamdouchi, F. Z. Mouzouni, K. El Kari, M. El Mzibri, H. Aguenaou (Rabat, Maroc)

P229 | La qualite de l'alimentation contribue-t-elle a expliquer les differences socioeconomiques de la prevalence du syndrome metabolique dans les Antilles Francaises?

Z. Colombet*, M. Pérignon, B. Salanave, E. Landais, Y. Martin-Prével, B. Allès, S. Drogué, M.-J. Amiot-Carlin, C. Méjean (Montpellier)

P230 | Estimation des facteurs de risque des maladies cardiovasculaires dans une population representative de Casablanca K. Mohtadi*, R. Msaad, R. Essadik, H. Lebrazi, A. Kettani, H. Taki, R. Saile (Casablanca, Maroc)

P231 | Apports alimentaires en sodium et en potassium chez des adultes Tunisiens : enquete pilote realisee dans le cadre de l'evaluation de la strategie nationale visant a reduire la consommation de sel

R. Doggui*, M. El Ati-Hellal, P. Traissac, A. Mensi, S. Sassi, M. M. Abassi, H. Ben Gharbia, M. Thabet, J. El Ati (Tunis, Tunisie)

P232 | Apports en sucres libres de la population francaise et qualite de l'alimentation des forts-consommateurs : resultats de l'enquete CCAF 2016

C. Deshayes, E. Sauvage, P. Reiser*, P. Hebel (Paris)

P233 | Modelisation de l'impact du respect des reperes alimentaires du HCSP sur les apports nutritionnelsdes adultes francais M. Maillot, N. Darmon* (Montpellier)

P234 | Association prospective entre divers scores nutritionnels et les pathologies cardiovasculaires : l'adequation au regime Mediterraneen est-elle davantage associee aux pathologies cardiovasculaires que l'adequation aux recommandations nutritionnelles?

A. Trébuchet, C. Julia, L. Fézeu, M. Touvier, D. Chaltiel, S. Hercberg, P. Galan, M. Adjibade*, E. Kesse-Guyot (Bobigny)

P235 | Prevalence des troubles du comportement alimentaire au cours de la periode 2000-2018 : une revue systematique de la litterature

M. Galmiche*, P. Déchelotte, G. Lambert, M. P. Tavolacci (Rouen)

P236 | Restructurer la consommation de sources proteiques par des modifications de tailles de portion pour augmenter l'adequation nutritionnelle : ce qui fonctionne pour tout le monde et ce qui depend du profil de consommation initial E. De Gavelle*, J.-F. Huneau, H. Fouillet, F. Mariotti (Paris)

P237 | Vecu, ressenti et prise en charge des differentes intolerances alimentaires non-allergiques (gluten, lactose, FODMAP) en medecine generale. Une etude descriptive aupres de 188 medecins generalistes en Rhone-Alpes-Auvergne M. Cart-Tanneur, P.-L. Braud, B. Prost* (Lyon)

P238 | Developpement d'un modele d'estimation de l'excretion urinaire de sodium de 24-heures en utilisant le rappel alimentaire de 24-heures chez des enfants marocains M. Idrissi*, N. Saeid, A. Rami, T. Marouan, A. El Hamdouchi, S. Mounach, M. El Mzibri, K. El Kari, H. Aguenaou (Rabat, Maroc)

P239 | Niveau d'activite physique et qualite de vie d'un groupe de femmes libanaises agees entre 40 et 60 ans A. El Hajj, N. Wardy, S. Haidar, E.O. Nada, L. Khabbaz, T. Wahanian Papazian* (Beirut, Liban)

P240 | Consommation des produits ultra-transformes chez les pesco-vegetariens, les vegetariens et les veganes et les determinants sociodemographiques associes dans l'etude de cohorte Nutri-Net-Sante J. Gehring*, M. Touvier, J. Baudry, C. Julia, C. Buscail, S. Hercberg, E. Kesse-Guyot, B. Allès (Bobigny)

P241 | Effet du chomage sur les consommations alimentaires des adultes sur 5 ans (Etude Nutri-Net-Sante) C. Mejean*, P. Rollet, W. Si Hassen, A. Lampuré, S. Hercberg, M. Touvier, K. Castetbon (Montpellier) 
P242 | Des changements alimentaires pluriels et evolutifs pour les mangeurs nouvellement 《 parents 》 A. Rochedy* et cette étude est dirigée par Jean-Louis Lambert Et Silvy Auboiron (Toulouse)

P243 | Influence des disparites socioeconomiques sur la qualite de l'alimentation dans les Antilles francaises Z. Colombet*, M. Pérignon, B. Salanave, E. Landais, Y. Martin-Prével, B. Allès, M.-J. Amiot-Carlin, N. Darmon, C. Méjean (Montpellier)

P244 | Identification des caracteristiques individuelles associees a la sous-declaration dans les Antilles Francaises Z. Colombet*, M. Pérignon, B. Salanave, E. Landais, Y. Martin-Prével, B. Allès, M.-J. Amiot-Carlin, N. Darmon, C. Méjean (Montpellier)

P245 | Effet de l'alimentation maternelle sur le gain de poids au cours de la grossesse F. Tebbani*, H. Oulamara, A. Agli (Constantine, Algérie)

P246 | Le role des herbes et epices sur l'appreciation de plats à base de legumineuses M. Vannereux*, A. Dougkas, A. Giboreau (Ecully)

P247 | Facteurs influant sur le gain ponderal insuffisant au cours de la grossesse F. Tebbani*, H. Oulamara, A. Agli (Constantine, Algérie)

P248 | Effet de l'alimentation maternelle sur le poids du nouveau-ne F. Tebbani, H. Oulamara*, A. Agli (Constantine, Algérie)

P249 | Les acides gras trans monoinsatures dans l'alimentation des Francais : une etude qualitative E. J. Guillocheau*, C. Penhoat, A. Godet, D. Catheline, P. Legrand, V. Rioux (Rennes)

P251 | Consommation d'Alcool et Risque de Parodontite : resultats d'une enquete menee aupres de 35390 adultes francais M. C. Carra, L. Detzen, Z. Hamdi, S. Fessi, S. Hercberg, C. Julia, P. Bouchard, V. Andreeva* (Paris)

P252 | Inventaire de plats et mets traditionnels consommes durant le mois de Ramadan M. Bencharif*, E. Boudjouada, Y. Benabbas (Constantine, Algérie)

P253 | Utilisation d'un journal alimentaire numerique a champ libre : evaluation preliminaire de l'exploitabilite des donnees recoltees avec une approche semi-supervisee

S. Akkoyunlu*, N. Darcel, C. Manfredotti, A. Cornuéjols, F. Delaere (Paris)

P254 | Statut nutritionnel des adolescents algeriens

M. Bencharif*, F. Z. Boutata (Constantine, Algérie) 


\section{TEXTES DES COMMUNICATIONS ORALES}

\section{PHYSIOLOGIE DIGESTIVE}

CO01

Associations entre profils métabolomiques plasmatiques RME et composition du microbiote intestinal au sein d'une population d'adultes français en bonne santé

V. Partula ${ }^{1,}{ }^{*}$, S. Mondot ${ }^{2}$, M. Torres ${ }^{3}$, E. Kesse-Guyot ${ }^{1}$, L. Lécuyer ${ }^{1}$, M. Deschasaux ${ }^{1}$, K. Assmann ${ }^{1}$, P. Latino-Martel $^{1}$, C. Buscail ${ }^{1}$, C. Julia ${ }^{1}$, P. Galan ${ }^{1}$, S. Hercberg ${ }^{1}$, A. Victor-Bala ${ }^{4}$, N. Bouchemal ${ }^{4}$, M. Triba ${ }^{4}$, P. Savarin ${ }^{4}$, V. Rouilly ${ }^{5}$, S. Thomas $^{6}$, L. Qunintana-

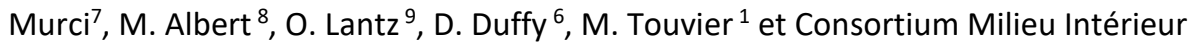

${ }^{1}$ EREN CRESS, INSERM/INRA/CNAM/U.Paris-XIII, Bobigny, ${ }^{2}$ MICALIS, INRA/AgroParisTech, Jouy-en-Josas, ${ }^{3}$ ESEN, CRESS/Santé Publique France/U.Paris-XIII, ${ }^{4}$ SBMB CSPBAT, CNRS/U.Paris-XIII, Bobigny, ${ }^{5}$ Center of Bioinformatics, Biostatistics and Integrative Biology, Institut Pasteur, ${ }^{6}$ Immunobiology of Dendritic Cells, INSERM/Institut Pasteur, ${ }^{7}$ Human Evolutionary Genetics, CNRS/Institut Pasteur, Paris, France, ${ }^{8}$ Department of Cancer Immunology, Genentech Inc., San Fransisco, États-Unis, ${ }^{9}$ Institut Curie/U.PSL, Paris, France

Introduction et but de l'étude : Le co-métabolisme hôte-microbiote est à l'origine d'un très grand nombre de molécules intégrées au sein d'axes métaboliques complexes. De nombreuses études se sont attachées à la caractérisation fonctionnelle spécifique de certaines de ces molécules (AGCC, BCAA, TMAO, etc.), mais les études envisageant plus globalement les relations métaboliques entre l'hôte et son microbiote intestinal restent rares. À ce titre, l'étude globale des métabolites endogènes et exogènes présents dans le plasma par métabolomique non ciblée semble prometteuse.

L'objectif de cette étude était de caractériser les associations entre profils métabolomiques plasmatiques et composition du microbiote intestinal dans une population d'adultes en bonne santé.

Matériel et méthodes : La composition du microbiote intestinal a été déterminée dans les selles (séquençage du gène ARNr16S, les matrices de Jaccard et Bray-Curtis ont été déterminées) et les profils métabolomiques ont été générés en utilisant les séquences RMN CPMG et NOESY sur des échantillons de plasma, chez 846 individus de la population Milieu Intérieur.

La co-structure globale des données $16 \mathrm{~S}$ et RMN a été évaluée par co-inertie. Les associations entre variables métabolomiques d'une part et matrices de $\beta$-diversité ou abondance des taxons d'autre part ont été calculées par PERMANOVA ou MaAsLin ajustés sur le sexe, l'âge, I'IMC, le statut tabagique et l'activité physique (PERMANOVA également ajustées sur la profondeur de séquençage). Une correction de Benjamini-Hochberg (FDR-10\%) a été appliquée.

Résultats et Analyse statistique : La co-inertie globale des données microbiote et métabolomique était limitée $(R V \leq 0.05)$. En revanche, des associations spécifiques ont été détectées. Les matrices de Jaccard et de Bray-Curtis étaient significativement et respectivement associées à 51 et 3 variables CPMG, dont certaines ont d'ores et déjà été identifiées (pyruvate, tyrosine, choline, glucose, etc.). Des associations entre le pyruvate et 3 genres bactériens (positives avec Catabacter et Acholeplasma, négative avec Faecalibacterium) ont été mises en évidence. L'analyse des associations entre variables métabolomiques NOESY et données microbiote $16 \mathrm{~S}$, et l'identification des métabolites discriminants sont en cours.

Conclusion : Ces résultats préliminaires permettent de mettre en évidence des associations entre le profil métabolomique RMN déterminé sur le plasma de l'hôte et la composition du microbiote intestinal. Toutefois, cette étude ne permet pas de conclure sur une potentielle relation causale, et d'autres études sont nécessaires.

Remerciements : Université Paris-VII Diderot. ED474 Frontières du Vivant (FdV) - Programme Bettencourt. LabEx Milieu Intérieur.

Conflits d'intérêts : Aucun conflit à déclarer 
Le microbiote intestinal : un acteur clé dans les effets métaboliques de l'inuline chez les patients obèses.

J. Rodriguez ${ }^{1, *}$, A. M. Neyrinck ${ }^{1}$, T. Le Roy ${ }^{1}$, S. Leclercq ${ }^{1}$, S. Hiel ${ }^{1}$, P. D. Cani ${ }^{1}$, N. Lanthier ${ }^{2}$, J. P. Thissen $^{3}$, L. Bindels ${ }^{1}$, N. M. Delzenne ${ }^{1}$

${ }^{1}$ Louvain Drug Research Institute, ${ }^{2}$ Laboratory of Hepatogastroenterology, Institut de recherche expérimentale et clinique, ${ }^{3}$ Pole of Endocrinology, Diabetes and Nutrition, Institut de Recherches Expérimentales et Cliniques IREC, Université Catholique de Louvain, Bruxelles, Belgique

Introduction et but de l'étude : Il a été précédemment démontré qu'une supplémentation avec des prébiotiques, de type inuline, pouvait améliorer les désordres métaboliques associés à l'obésité chez certains patients. En utilisant un modèle de souris " humanisées " grâce à un transfert de microbiote intestinal provenant de patients obèses, nous avons tenté de répondre à la question suivante : la composition du microbiote intestinal influence-t-elle la réponse à l'inuline ?

Matériel et méthodes : Le microbiote intestinal de souris C57BL/6Jrj âgées de 4 semaines a été déplété à l'aide d'une antibiothérapie à large spectre permettant une élimination de plus de 99,8\% des bactéries totales. Une semaine plus tard, les souris ont été recolonisées avec les selles provenant de quatre patients obèses (hum-ob), choisis en raison de profils différents du microbiote intestinal (index de diversité bactérienne, abondance de Bifidobacterium spp.,...). Les souris hum-ob, ainsi qu'un groupe de souris conventionnelles ont ensuite été soumises à un régime riche en graisses durant quatre semaines, et supplémentées ou non avec $0.2 \mathrm{~g}$ / souris/ jour d'inuline native (Cosucra, Belgium) ( $\mathrm{n}=6-9$ souris par groupe).

Résultats et Analyse statistique : Même si les souris hum-ob tendaient à prendre plus de poids après le régime riche en graisses, comparées aux souris conventionnelles, des différences de réponse à la diète obésogène entre les quatre donneurs étaient clairement visibles. De plus, la supplémentation en inuline a largement réduit la prise de poids chez un des quatre donneurs (environ 30\%, p <0.05), Cet effet a été récapitulé seulement chez les souris transplantées avec le microbiote de ce donneur, ce qui a permis d'analyser les mécanismes en jeu et les conséquences métaboliques de la sélectivité de réponse (augmentation de la thermogenèse dans le tissu adipeux brun, diminution des lipides intramusculaires, réduction de l'adiposité et de la stéatose hépatique). L'augmentation des bifidobactéries, signature de la réponse du microbiote à l'inuline, n'a pas été retrouvée chez tous les donneurs, suggérant que la diversité de réponse est liée à l'impact sur la composition du microbiote intestinal. Les données d'analyse transcriptomiques sont en cours en vue d'analyser les changements bactériens en lien avec les modifications d'expression de gènes clés dans les tissus de l'hôte.

Conclusion : Nos travaux, basés sur le modèle de transfert de microbiote intestinal de patients obèses chez la souris, permettent d'analyser le mécanisme moléculaire en jeu dans la réponse différentielle à une approche nutritionnelle visant à améliorer l'obésité chez le patient, un contexte particulièrement important dans la prise en charge personnalisée du patient.

Conflits d'intérêts : Aucun conflit à déclarer 
Introduction et but de l'étude : Chaque année en France une centaine d'adolescents obèses bénéficient d'une chirurgie bariatrique. Jusqu'à ce jour, les seules séries publiées concernaient l'anneau gastrique ajustable (AGA) ou la sleeve gastrectomie (SG). Notre objectif était de rapporter la première série française d'adolescents obèses ayant bénéficié de l'une des 3 techniques disponibles : bypass gastrique (BPG), SG et AGA.

Matériel et méthodes : Tous les adolescents obèses âgés de 13 à 19 ans et ayant bénéficié d'une chirurgie bariatrique dans notre service depuis septembre 2008 avec un recul post-opératoire d'au moins 2 ans ont été inclus. L'évolution des données anthropométriques et des comorbidités, ainsi que la survenue de complications ont été analysées.

Résultats et Analyse statistique : Depuis septembre 2008, 78 adolescents obèses se sont présentés pour une demande de chirurgie bariatrique. Parmi eux, seuls 27 (35\%) ont bénéficié d'une chirurgie bariatrique : 21 BPG, 4 SG, 2 AGA, dont 20 (15 BPG, 4 SG, 1 AGA) avec un recul d'au moins 2 ans. Au moment de l'intervention, ces 20 adolescents obèses étaient âgés de 16,6 $\pm 1,6$ ans et avaient un IMC de 53,1 $\pm 8,9 \mathrm{~kg} / \mathrm{m} 2$. Deux ans après l'intervention, l'IMC moyen était de 36,3 $\pm 10,4 \mathrm{~kg} / \mathrm{m} 2, \mathrm{soit}$ une diminution de $-31,5 \%$. Tous les patients sauf 2 (1 SG, 1BPG) avaient diminué leur IMC. Les comorbidités sévères suivantes ont disparu : hypertension artérielle (2/2), diabète (1/2), syndrome d'apnées du sommeil nécessitant une ventilation non invasive $(1 / 3)$ et hypertension intracrânienne idiopathique (1/1). Se sont également normalisées : I'hypertriglycéridémie (3/3), I'hyper-LDLcholestérolémie (3/3), l'insulinorésistance $(8 / 13)$ et la stéatose hépatique (6/7). Les complications suivantes sont survenues : anémie $(n=3)$, hypoalbuminémie $(n=4)$ et carence martiale $(n=4) .3$ patients ont nécessité une cœlioscopie pour exploration de douleurs abdominales, sans cause retrouvée dans les 3 cas.

Conclusion : Seul un tiers des adolescents obèses demandant une chirurgie bariatrique sont finalement opérés. Notre série principalement constituée d'adolescents obèses ayant bénéficié d'un BPG montre une efficacité de cette technique sur la réduction pondérale et des comorbidités. Les complications nutritionnelles ou mécaniques restent rares. Ces résultats sont tout à fait comparables à ceux obtenus chez l'adulte.

Conflits d'intérêts : Aucun conflit à déclarer 
Evolution de la détection oro-sensorielle du saccharose chez des souris obèses après Sleeve gastrectomie ou By-pass Roux en $Y$ modifié.

A. Bernard ${ }^{1,}{ }^{*}$, C. Le May ${ }^{2}$, A. Dastugue ${ }^{1}$, A. Ayer ${ }^{2}$, C. Blanchard ${ }^{2}$, B. Cariou ${ }^{2}$, S. Ledoux ${ }^{3}$, X. Collet ${ }^{4}$, A. Dhaussy $^{5}$, F. Respondek $^{6}$, L. Lagrost ${ }^{7}, \mathrm{P}$. Besnard ${ }^{1}$

${ }^{1}$ NUTox, UMR 1231 INSERM/AgroSup Dijon/UBFC, DIJON, ${ }^{2}$ UMR 1087 INSERM/6291 CNRS, Nantes, ${ }^{3}$ Hôpital Louis Mourier (APHP), Colombes, ${ }^{4} 4$ UMR 1048 INSERM/Univ Toulouse III, Toulouse, ${ }^{5}$ Lesieur groupe Avril, Asniere sur Seine, ${ }^{6}$ TEREOS, MARCKOSLSHEIM, ${ }^{7}$ Lipness, UMR 1231 INSERM/AgroSup Dijon/UBFC, Dijon, France

Introduction et but de l'étude : La surconsommation d'aliments riches en sucres et en graisses est un des facteurs de risque d'obésité. Ces aliments s'avèrent fortement attractifs probablement en raison de la satisfaction hédonique qu'ils procurent. Ce constat suppose l'existence d'une détection précoce et sélective de ces nutriments dès la mise en bouche des aliments orientant les choix alimentaires. Chez le rat, le fait que des animaux rendus obèses par régime surconsomment les aliments denses en énergie suggère que leur détection oro-sensorielle des sucres et lipides pourrait être perturbée. Ce phénomène est réversible puisque des rats initialement obèses ayant subi un by-pass gastrique Roux en $Y$ délaissent les aliments riches en sucres et graisses au profit d'un régime pauvre en énergie. Les mécanismes à l'origine de ces modifications comportementales sont actuellement mal connus. Pour tenter de les élucider, nous avons transposé chez la souris deux chirurgies bariatriques largement pratiquées chez l'Homme : la sleeve gastrectomie (SG, chirurgie restrictive) et le by-pass gastrique Roux en Y (RYGBm) connues pour impacter les choix alimentaires. Utilisant ces modèles, nous avons tenté de répondre à la question suivante : Quelle est l'évolution de la détection oro-sensorielle d'un sucre (le saccharose) chez des souris obèses ayant subies une SG ou un RYGBm ?

Matériel et méthodes : Cette étude a été validée par les comités éthiques ad hoc. Pour chaque modèle de chirurgie, 3 groupes de souris ont été constitués : des témoins opérés à blanc en régime standard (T-Std) ou rendus obèses par régime gras (T-HFD) et des souris obèses opérées ( $S G$ ou RYGBm) maintenues en régime HFD après opération pour cibler uniquement l'impact de la chirurgie. La composition corporelle (RMN quantitative) et l'intolérance au glucose (OGTT) ont été évaluées avant et après chirurgie. La sensibilité orosensorielle du sucré a été analysée au moyen d'un gustomètre permettant l'analyse de la réponse (lapées) des animaux à différentes concentrations de saccharose.

Résultats et Analyse statistique : La SG et le RYGBm induisent une diminution significative de la masse grasse et améliorent la sensibilité au glucose des souris initialement obèses en dépit du fait qu'elles soient maintenues en régime HFD. Globalement, la chute du nombre total de lapées par session de $30 \mathrm{~min}$, du nombre de lapées/10sec en fonction de la concentration de saccharose et du seuil de détection oro-sensoriel du saccharose observé chez les T-HFD vs T-Std est partiellement corrigée par les 2 chirurgies, le RYGBm s'avérant plus efficace que la SG.

Conclusion : Ces données montrent que la chirurgie bariatrique améliore significativement la perception oro-sensorielle du sucré chez la souris initialement obèse en dépit de son maintien sur un régime obésogène. Cette observation, inédite pour cette espèce, ouvre de nouvelles perspectives d'étude du changement du comportement alimentaire au cours de l'obésité.

Remerciements : Ces Travaux ont bénéficié du soutien financier de Téréos, Avril et du Labex LipSTIC (ANR-11-LABX-0021-LipSTIC).

Conflits d'intérêts : A. Bernard: Aucun conflit à déclarer, C. Le May: Aucun conflit à déclarer, A. Dastugue: Aucun conflit à déclarer, A. Ayer: Aucun conflit à déclarer, C. Blanchard: Aucun conflit à déclarer, B. Cariou: Aucun conflit à déclarer, S. Ledoux: Aucun conflit à déclarer, X. Collet: Aucun conflit à déclarer, A. Dhaussy est employé(e) de Lesieur groupe Avril, F. Respondek est employé(e) de Téréos, L. Lagrost: Aucun conflit à déclarer, P. Besnard: Aucun conflit à déclarer 
S. Vidal ${ }^{1, *}$, N. Khodorova ${ }^{1}$, M. Piscuc ${ }^{1}$, S. Mekiri ${ }^{1}$, C. Gaudichon ${ }^{1}$, F. Blachier ${ }^{1}$, A. Lan ${ }^{1}$

${ }^{1}$ Physiologie de la Nutrition et du Comportement Alimentaire, INRA-AgroParisTech, Paris, France

Introduction et but de l'étude : Chez les patients atteints de maladies inflammatoires chroniques intestinales, les manifestations extra-digestives sont fréquentes et pourraient résulter d'une altération du métabolisme protéique en réponse à l'inflammation intestinale. Le but de cette étude est d'évaluer l'impact d'un épisode inflammatoire colique aigu sur le métabolisme protéique de différents organes extra-intestinaux chez la souris pendant la phase d'inflammation et au cours de la résolution de la colite.

Matériel et méthodes : Afin d'induire une poussée inflammatoire intestinale aiguë, 54 souris C57BL/6 ont été traitées avec du dextran-sulfate de sodium à J0 (DSS, 3,5\%, 5 jours) puis euthanasiées à différents temps (J7, J10, J13 et J28). Une étude de suivi du poids, composition corporelle, prise alimentaire et de marqueurs inflammatoires plasmatiques a été réalisée en comparaison avec un groupe contrôle $(n=12)$ n'ayant pas reçu de DSS. La vitesse de synthèse protéique des organes a été déterminée en administrant une dose massive de valine ${ }^{13} \mathrm{C} 20 \mathrm{~min}$ avant le sacrifice et l'incorporation du traceur a été mesurée par spectrométrie de masse.

Résultats et Analyse statistique : L'inflammation colique induit une perte de poids pour l'ensemble des souris traitées au DSS (à $\mathrm{J} 7,-14 \%$ du poids initial, $\mathrm{p}<0,001)$ et a un impact sévère sur la composition corporelle. A J7, l'augmentation des concentrations plasmatiques des marqueurs inflammatoires IL-6 (J7: $92 \pm 16 \mathrm{pg} / \mathrm{mL}, p<0,001$ vs J0) et Amyloïde Sérique A ( $x 3,5$ vs J0, $p<0,0001$ ) indique un impact systémique de l'inflammation colique. La concentration plasmatique de "LPS-binding protein », un marqueur d'endotoxémie, reste élevée jusqu'à $\mathrm{J28}$, témoignant d'altérations périphériques à long terme malgré la résolution de la colite (J28: 4,58 $\pm 0,30$ vs J0 1,97 $\pm 0,24 \mu \mathrm{g} / \mathrm{mL}, \mathrm{p}<0,001)$. En réponse à l'inflammation colique, le renouvellement protéique est doublé dans le foie (FSR J0 : $161 \pm 9,0$ vs J7 : $307 \pm 25 \% / j, p<0,0001$ ) et la rate (FSR J0: $110 \pm 9,7$ vs J7 : 273 $\pm 39 \% / j, p<0,001$ ) tandis qu'il est diminué dans les reins (FSR J0 : $151 \pm 5,8$ vs J7 : $109 \pm 13 \% / \mathrm{j}, \mathrm{p}=0,0024$ ) et le cæcum (FSR J0 : $196 \pm 16$ vs J7: $131 \pm 17 \% / \mathrm{j}$, $\mathrm{p}=0,0077$ ). Entre $\mathrm{J} 10$ et $\mathrm{J} 13$, les taux absolus de synthèse protéique du foie et de la rate restent encore élevés (NS vs J7), contrairement aux reins et au cæcum où ces taux reviennent aux valeurs basales dès J10. A J28. Bien que l'inflammation colique soit résolue, les altérations extra-digestives sur le métabolisme protéique persistent dans le foie (J28:199 $\pm 21 \% / j$, p=0,023 vs J0) et la rate (J28: $206 \pm 22, p=0,0021$ vs J0).

Conclusion : Un épisode inflammatoire colique aigu induit par le DSS provoque des altérations locales et systémiques en perturbant le métabolisme protéique du cæcum, des reins, de la rate et du foie. L'évolution de la vitesse de synthèse protéique dans les tissus périphériques en cas d'inflammation colique aiguë pourrait être reliée à l'implication de ces tissus dans la réponse inflammatoire.

Remerciements : SFN, Association François Aupetit, Idex ALIAS

Conflits d'intérêts : Aucun conflit à déclarer 
${ }^{1}$ Equipe NUTOX, Inserm U1231/UB/AgrosupDijon, Dijon, France

Introduction et but de l'étude : CD36 est un récepteur ubiquitaire aux acides gras à longue chaine (AGLC), exprimé sur les microvillosités des entérocytes différenciés du jéjunum, site majeur de l'absorption des lipides. L'activation du CD36 intestinal par les AGLC déclenche la synthèse de protéines clés de la formation des chylomicrons (CM) (Tran et al 2011). Puisque les CM véhiculent également les lipopolysaccharides bactériens (LPS), CD36 pourrait aussi contrôler l'endotoxémie postprandiale. II a été montré qu'en cas de régime hyperlipidique $(\mathrm{HL})$, l'intestin adapte ses capacités d'absorption en induisant l'expression des gènes favorisant la formation de gros CM, bien dégradés dans la circulation (Petit et al 2007). Or, un défaut d'adaptation intestinale en régime $\mathrm{HL}$ est associé à une susceptibilité à l'obésité accrue (Kondo et al 2006). L'hypothèse est donc que le CD36 entérocytaire permet la formation de gros CM mieux dégradés dans la circulation sanguine et limite l'endotoxémie et l'obésité induites par un régime HL. Pour vérifier l'hypothèse, les effets d'un régime $\mathrm{HL}$ ont été comparés à ceux d'un régime normolipidique (NL) chez des souris CD36Ent(-/-) délétées spécifiquement en CD36 entérocytaire

Matériel et méthodes : Des souris femelles CD36Ent(-/-) et CD36Ent(+/+) (CTL) (Cifarelli et al 2016) sont nourries pendant 21j avec un régime contenant $3 \%(\mathrm{NL})$ ou $40 \% \mathrm{~m} / \mathrm{m}(\mathrm{HL})$ d'huile, ( $\mathrm{n}=8 /$ groupe). La masse et la composition corporelles (EchoMRI) et les pertes fécales en lipides (Méthode Folch) sont mesurées. Les souris sont sacrifiées $4 \mathrm{~h}$ après un gavage avec $0.5 \mathrm{ml} \mathrm{d}$ 'huile du régime et un traitement préalable avec un inhibiteur de la lioproteine lipase (Tyloxapol, $500 \mu \mathrm{g} / \mathrm{g}$ de masse corporelle). La sécrétion postprandiale des triglycérides (TG) dans le sang (kit Biomérieux) et la taille des CM est mesurée par Dynamic Light Scattering. L'expression des gènes de la muqueuse jéjunale est mesurée par qPCR. Les résultats sont exprimés en moyenne +/- SEM. La significativité des différences entre groupes NL et $H L$ est déterminée par des tests de Student $(*=p<0,05)$

Résultats et Analyse statistique : En régime HL, la prise de masse grasse est de 54,7\%+/-11,6* chez les CD36Ent(-/-) alors qu'elle est de $49,5 \%+/-8,2$ chez les CTL sans variation de la prise alimentaire. La proportion de TG excrétés dans les lipides des fécès est de $39,94 \%$ chez les CD36Ent(-/-) et de $60 \% *$ chez les CTL. 4 h après le gavage, le régime HL induit une sécrétion de TG plus faible de $30 \% *$ chez les CD36Ent(-/-) alors qu'il n'a pas d'effet chez les CTL. L'augmentation de la taille des CM par le HL ne se fait que $4 \mathrm{~h}$ après le gavage chez les CD36Ent(-/-)* alors qu'elle se fait dès $2 \mathrm{~h}$ chez les $\mathrm{CTL}$ *. Enfin, $4 \mathrm{~h}$ après le gavage l'expression des gènes impliqués dans la sécrétion des CM n'est pas induite chez les CD36Ent(-/-) alors qu'elle l'est chez les CTL*. Ces effets sont associés à une augmentation du LPS sanguin chez les CD36Ent(-/-)* absente chez les CTL

Conclusion : En conclusion, l'absence de CD36 entérocytaire en régime HL crée un retard de sécrétion de gros CM. Le CD36 entérocytaire est donc un élément clé de l'adaptation intestinale à un régime $\mathrm{HL}$, qui protège de l'effet obésogène et de l'endotoxémie souvent rapportés en cas d'excès de lipides, en limitant l'efficacité de l'absorption des TG mais en favorisant la sécrétion de gros $\mathrm{CM}$ mieux dégradés au niveau sanguin

Conflits d'intérêts : Aucun conflit à déclarer 
COO7

Evolution de la perception orosensorielle du sucré et du gras après une chirurgie bariatrique restrictive ou malabsorptive chez la femme adulte.

A. Bernard ${ }^{1,}{ }^{*}$, S. Ledoux ${ }^{2}$, L. Radoi ${ }^{2}$, M. Coupaye ${ }^{2}$, O. Sami ${ }^{2}$, N. Casanova ${ }^{2}$, C. Le May ${ }^{3}$, X. Collet ${ }^{4}$, A. Dhaussy ${ }^{5}$, F. Respondek ${ }^{6}$, L. Lagrost ${ }^{7}$, P. Besnard ${ }^{1}$

${ }^{1}$ NUTox, UMR 1231 INSERM/AgroSup Dijon/UBFC, Dijon, ${ }^{2}$ Hôpital Louis Mourier (APHP), Colombes, ${ }^{3}$ UMR 1087 INSERM/6291 CNRS, Nantes, ${ }^{4} 4$ UMR 1048 INSERM/Univ Toulouse III, Toulouse, ${ }^{5}$ Lesieur groupe Avril, Asniere sur Seine, ${ }^{6}$ TEREOS, MARCKOSLSHEIM, ${ }^{7}$ UMR 1231 INSERM/AgroSup Dijon/UBFC, Dijon, France

Introduction et but de l'étude : L'augmentation de la prévalence de l'obésité est l'un des défis majeurs de santé publique. En cas d'obésité morbide, la chirurgie bariatrique, qu'elle soit restrictive (Sleeve gastrectomie - SG) ou malabsorptive (court-circuit gastrique - CCG), entraîne une réduction à long terme du poids et des co-morbitités. Ces interventions s'accompagnent généralement d'un changement comportemental privilégiant les aliments peu palatables pauvres en sucres et en graisses. Cette observation suscite deux questions complémentaires : la chirurgie bariatrique affecte-t-elle la détection oro-sensorielle du sucré et du gras ? Quel est l'impact respectif de la SG et de la CCG sur ce paramètre ?

Matériel et méthodes : $L^{\prime}$ inclusion des patients a été réalisée après consentement éclairé et validation du comité de protection des personnes. Les seuils de détection du saccharose (S) puis de l'acide linoléique (AL) ont été étudiés au moyen de tests triangulaires ( 3 alternative-forced choice) chez des femmes adultes souffrant d'une obésité sévère $\left(I M C>43 \mathrm{~kg} / \mathrm{m}^{2}\right) 2$ semaines avant SG $(n=32)$ ou CCG $(n=13)$ puis 6 mois après la chirurgie. Huit et 18 concentrations croissantes de $S$ et de AL ont été respectivement testées le matin chez les sujets à jeun ayant consommés la veille au soir un repas standardisé. Les solutions témoins étaient de l'eau de Volvic seule (S) ou texturée avec $5 \%$ de gomme d'acacia, $5 \%$ d'huile de paraffine et 0,01\% d'EDTA (AL). Résultats et Analyse statistique : La perte moyenne de poids à 6 mois post-chirurgie était respectivement de $31 \%$ (SG) et $36.5 \%$ (CCG). Pour le sucré, le seuil de détection du $S$ était similaire avant et 6 mois après chirurgie quelle que soit la technique utilisée (SG: $0,54 \%$ vs $0,56 \%, p=0,44$; CCG : $0,65 \%$ vs $0,51 \%, p=0,09$, test Wilcoxon-Pratt), même si une tendance à la baisse était observable après CCG. Aucun changement significatif du seuil de détection de l'AL après $S G(0,64 \mathrm{mM}$ vs $0,50 \mathrm{mM}, p=0,46)$ n'a été observé. En revanche, la CCG a provoqué une diminution significative du seuil de détection lipidique $(0,90 \mathrm{mM} v s 0,41 \mathrm{mM}, p=0,01)$. Conclusion : SG et la CCG ne semblent pas affecter la détection oro-sensorielle du sucré contrairement au "gras » dont la sensibilité semble améliorée après CCG (i.e. détection à partir d'une plus faible concentration). Ces résultats inédits indiquent que SG et CCG n'ont pas le même impact sur la détection du sucré et du gras. Ils montrent également que la sensibilité de ces 2 modalités gustatives peut être régulée de façon indépendante l'une de l'autre. Ces observations sont paradoxalement discordantes avec le ressenti des patientes qui décrivent une augmentation de l'intensité des goûts " gras » et sucré dans plus de $2 / 3$ des cas après intervention quel que soit le type de chirurgie. Le changement des choix alimentaires consécutifs à la chirurgie bariatrique est donc un phénomène complexe ne se limitant pas à la sensibilité gustative.

Remerciements : Ces Travaux ont bénéficié du soutien financier de Téréos, Avril et du Labex LipSTIC (ANR-11-LABX-0021-LipSTIC).

Conflits d'intérêts : A. Bernard: Aucun conflit à déclarer, S. Ledoux: Aucun conflit à déclarer, L. Radoi: Aucun conflit à déclarer, M. Coupaye: Aucun conflit à déclarer, O. Sami: Aucun conflit à déclarer, N. Casanova: Aucun conflit à déclarer, C. Le May: Aucun conflit à déclarer, X. Collet: Aucun conflit à déclarer, A. Dhaussy est employé(e) de Lesieur groupe Avril, F. Respondek est employé(e) de Téréos, L. Lagrost: Aucun conflit à déclarer, P. Besnard: Aucun conflit à déclarer 
Impact de l'anorexie mentale sur l'intégrité fonctionnelle et structurelle hypothalamique au cours de la prise alimentaire V. Florent ${ }^{12^{*}}$, M. Baroncini ${ }^{3}$, P. Jissendi ${ }^{4}$, J. VIGNAU ${ }^{5}$, M. Pigeyre ${ }^{6}$, I. Nilsson ${ }^{7}$, V. Prevot ${ }^{2}$

${ }^{1}$ Nutrition CSO, CHG Arras, Arras, ${ }^{2}$ Centre de recherche Jean Pierre Aubert, INSERM U1172, ${ }^{3}$ Neurochirurgie, CHRU Lille, Lille, France, ${ }^{4}$ Neuroradiologie, CHU Saint Pierre, ULB, Brussels, Belgique, ${ }^{5}$ Psychiatrie TCA, ${ }^{6}$ Nutrition, CHRU Lille, Lille, France, ${ }^{7}$ Karolinska institutet, Stockholm, Suède

Introduction et but de l'étude : L'hypothalamus contient différents systèmes neuronaux coordonnant la prise alimentaire, la dépense énergétique ou encore la reproduction. De nombreuses approches en imagerie par résonnance magnétique cérébrale chez l'Homme ont émergé dans l'étude des troubles du comportement alimentaire comme l'anorexie mentale (AM), principalement dans des zones corticales. Aucune d'entre d'elles n'a encore porté sur une étude fonctionnelle de l'AM à l'échelle ultrastructurelle des noyaux hypothalamiques.

Matériel et méthodes : Notre étude explore ainsi la connectivité fonctionnelle et structurelle des noyaux hypothalamiques en IRM multimodale in vivo chez des patientes présentant une AM restrictive comparativement à des témoins ajustés en poids (maigreur constitutive) et des témoins normopondéraux. Ces 30 volontaires ont bénéficié de séquences anatomiques, de spectroscopie en résonnance magnétique et de séquences de diffusion avec algorithme de tractographie, à jeun et après prise alimentaire calibrée. Les variations neurobiochimiques à l'échelle hypothalamique et la connectivité structurelle à l'échelle de chaque noyau hypothalamique ont pu être étudiées.

Résultats et Analyse statistique : Nous avons mis en évidence une activité basale du réseau glutamatergique exacerbée avant prise alimentaire qui décroit paradoxalement après prise alimentaire, à contrario des sujets contrôle. Cette réponse inverse de neurotransmission est associée à un nombre de fibres nerveuses passant par le noyau arqué plus faible chez les AM mais également à une connectivité accrue de l'aire hypothalamique latérale, deux régions fortement impliquées dans le contrôle de la prise alimentaire.

Conclusion : Cette étude en imagerie cérébrale non invasive met en lumière différents dysfonctionnements hypothalamiques et ouvre la porte à de nouvelles stratégies à la fois diagnostiques et thérapeutiques dans cette pathologie sévère aux traitements limités et au pronostic sombre.

Remerciements : aux Dr Baroncini et Dr Prevot

à l'équipe d'ingénieurs de I'IRM recherche du CHRU de Lille

à l'équipe de Psychiatrie du CHRU de Lille

à l'équipe de Nutrition du $\mathrm{CH}$ Arras

Conflits d'intérêts : Aucun conflit à déclarer 


\section{CO09}

Qualité nutritionnelle des aliments définie par le score FSAm-NPS sous-tendant le logo Nutri-Score et risque de cancer en Europe : résultats de la cohorte EPIC

M. Deschasaux ${ }^{1,}{ }^{*}$, I. Huybrechts ${ }^{2}$, N. Murphy ${ }^{2}$, C. Julia ${ }^{13}$, S. Hercberg ${ }^{13}$, B. Srour ${ }^{1}$, E. Kesse-Guyot ${ }^{1}$, P. Latino-Martel ${ }^{1}$, C. Biessy ${ }^{2}$, C. Casagrande ${ }^{2}$, M. Jenab ${ }^{2}$, H. Ward ${ }^{4}$, E. Weiderpass ${ }^{5}$, P. Ferrari ${ }^{2}$, E. Riboli ${ }^{4}$, M. Gunter ${ }^{2}$, M. Touvier ${ }^{1}$ au nom de l'ensemble des collaborateurs EPIC

${ }^{1}$ Equipe de Recherche en Epidémiologie Nutritionnelle (CRESS-EREN), U1153 Inserm, U1125 Inra, Cnam, Université Paris 13, Bobigny, ${ }^{2}$ Nutrition and Metabolism Section, International Agency for Research on Cancer, Lyon, ${ }^{3}$ Département de Santé Publique, Hôpital Avicenne, Bobigny, France, ${ }^{4}$ Faculty of Medicine, School of Public Health, Imperial College London, Londres, Royaume-Uni, ${ }^{5}$ Department of Medical Epidemiology and Biostatistics, Karolinska Institutet, Stockholm, Suède

Introduction et but de l'étude : Orienter les consommateurs vers des choix alimentaires plus sains est un enjeu important pour la prévention des cancers et des autres maladies chroniques. Dans différents pays, les politiques envisagent la mise en place d'un étiquetage simplifié reflétant la qualité nutritionnelle des aliments. En France, le logo à 5 couleurs "Nutri-Score" basé sur le score FSAm-NPS a obtenu le soutien officiel du Ministère de la Santé. Son application reste toutefois optionnelle du fait de la réglementation européenne.

L'impact de la consommation d'aliments avec un score FSAm-NPS plus ou moins élevé sur le risque de cancer a été étudié en France et au Royaume-Uni. Notre objectif est donc d'étendre ces études à un contexte européen au sein d'une large population représentant divers pays.

Matériel et méthodes : Cette analyse prospective inclut 471495 adultes de la cohorte européenne EPIC (10 pays, 1992-2014, suivi médian:15,3 ans), parmi lesquels 49794 cas de cancer incidents ont été diagnostiqués (principales localisations: sein, $n=12063$; prostate, $n=6745$; côlon-rectum, $n=5806$ ). Les prises alimentaires usuelles ont été estimées à l'aide d'outils standardisés spécifiques à chaque pays. Le score FSAm-NPS est calculé pour chaque aliment à partir de sa teneur /100g en énergie, sucres, acides gras saturés, sodium, fibres, protéines, et fruits et légumes. La moyenne de l'ensemble des scores FSAm-NPS associés aux aliments habituellement consommés par un individu permet d'obtenir le score FSAm-NPS DI. Des modèles de Cox multi-ajustés ont été utilisés pour les analyses.

Résultats et Analyse statistique : Un score FSAm-NPS DI plus élevé, reflétant une moindre qualité nutritionnelle des aliments consommés était associé à un risque accru de cancer (RRo5vs.01=1,07 (1,03-1,10), $P$-tendance $<0,001)$. Les individus avec les scores FSAm-NPS DI les plus élevés (Q5) et les plus faibles (Q1) présentaient des taux d'incidence de 81,4 et 69,5 cas de cancer/10000 personnes-années, respectivement. En particulier, des scores FSAm-NPS DI plus élevés étaient associés à des risques accrus de cancers du côlon-rectum, des voies aérodigestives supérieures et de l'estomac, du poumon chez les hommes, du foie et du sein (post-ménopause) chez les femmes ( $P<0,05$ pour tous).

Conclusion : Au sein de cette large cohorte européenne, la consommation d'aliments avec un score FSAm-NPS plus élevé (qualité nutritionnelle moindre) était associée à un risque accru de cancer. Ces résultats contribuent à montrer la pertinence du score FSAm-NPS comme outil sous-tendant des mesures nutritionnelles de santé publique telles que le Nutri-Score.

Références : Deschasaux $M$ et al. Nutritional quality of food as represented by the FSAm-NPS nutrient profiling system underlying the Nutri-Score label and cancer risk in Europe: Results from the EPIC prospective cohort study. PLOS Medicine. In press

Remerciements : Partenaires EPIC

INCa-Cancéropôle lle-de-France (2017-1-PL SHS-01-INSERM ADR 5-1)

Label NACRe Partenariat (https://www6.inra.fr/nacre/)

Conflits d'intérêts : Aucun conflit à déclarer 
$\mathrm{CO} 10$

Mesure pré opératoire de l'indice de masse musculaire en $L 3$ en chirurgie thoracique : Évaluation de l'équivalence de deux logiciels disponibles

G. Gayraud ${ }^{1, *}$, M. P. Vasson ${ }^{2}$, A. Da Costa ${ }^{2}$

${ }^{1}$ Anesthésie Réanimation, ${ }^{2}$ Centre Jean Perrin, Clermont Ferrand, France

Introduction et but de l'étude : Le diagnostic de dénutrition et l'évaluation du statut nutritionnel est recommandé en pré opératoire de chirurgie oncologique. La mesure de la masse appendiculaire en pratique clinique courante n'est pas tout le temps disponible. Le diagnostic de sarcopénie par mesure l'indice de masses musculaire squelettique (IMMS) sur coupe scanner en L3 pourrait présenter un intérêt complémentaire. Plusieurs logiciels équivalents sont disponibles pour réaliser cette mesure mais jusqu'ici réservés à des protocoles de recherche fondamentale ou clinique. Nous avons souhaité vérifier l'équivalence des logiciels SliceOmatic ${ }^{\circledR}$ et ImageJ ${ }^{\circledR}$ avant d'envisager une mesure systématique pré opératoire.

Matériel et méthodes : Après vérification de la disponibilité d'une coupe scanner pré opératoire en $L 3$, nous avons sélectionné de manière rétrospective et alétaoire 51 dossiers de patients opérés d'une résection pulmonaire pour cancer dans notre établissement. Deux opérateurs ont été formés à la mesure de l'IMMS sur les deux logiciels SliceOmatic ${ }^{\circledR}$ et Image ${ }^{\circledR}$. Nous avons d'abord conduit une étude de répétabilité et de reproductibilité pour vérifier l'absence de variabilité intra et inter observateur sur 10 premiers dossiers. L'équivalence des logiciels était ensuite vérifiée par une étude de reproductibilité portant sur 51 mesures d'IMMS permettant de détecter un effet de taille de 0,4.

Résultats et Analyse statistique : Concernant la varibilité intra opérateur, les hypothèses de normalité et d'homoscédasticité étaient vérifiées pour les deux opérateurs : $p=0,92$ et 0,85 (test de student apparié) et coefficient de corélation intra classe (ICC) $=0,999$ et 0,998 . II n'y avait pas différence significative entre les mesures des deux opérateurs : $p=0,25$ (test de student apparié et ICC = 0,998 (95\% IC 0,991-0,999). Concernant la variabilité interlogiciel, 102 mesures ont été réalisée au total, 2 mesures extrèmes ont été rétirées, ainsi les hypothèses de normalité et d'homoscédasticité étaient vérifiées. Nous avons retrouvé une différence signficative entre les mesures effectuées sur les deux logiciels $p<0,001$ (test de Student apparié). Le biais était égale à 1,049 (95\% IC 0,831-1,267) même si L'ICC restait dans l'intervalle de confiance(95\% IC 0,981-0,994). Dans notre effectif global, cela se traduit cliniquement sur le diagnostic de sarcopénie : 9/51 avce le logiciel SliceOmatic ${ }^{\circledR}$ et 5 avec ImageJ ${ }^{\circledR}$.

Conclusion : L'équivalence des logiciels Image ${ }^{\circledR}$ et SliceOmatic ${ }^{\circledR}$ n'a pas été vérifiée dans notre travail. Le diagnostic de sarcopénie est aussi clinique (force musculaire diminuée ou performance physique diminuée). En pratique, la mesure de l'IMMS en L3 sur scanner nécessite la disponibilté de la coupe et la compétence d'un opérateur. L'utilisation de cette technique complémentaire nécessite une validation préalable de la méthode de mesure et la recherche d'un éventule biais logiciel à corriger localement avant de généraliser son application.

Références : van Vugt JLA \& al, 2017, Journal of Cachexia, Sarcopenia and Muscle 8: 285-297

Gomez-Perez SL \& al, 2016, Journal of Parenteral and Enteral Nutrition 40: 308-318

Mourtzakis M, \& al, 2008, Applied Physiology, Nutrition, and Metabolism 33: 997-1006

Conflits d'intérêts : Aucun conflit à déclarer 
CO11

La sarcopénie est sous-diagnostiquée chez les patients atteints de cancer métastatique : résultats de l'étude SCAN

B. Raynard ${ }^{1,}{ }^{*}$, F. Pigneur ${ }^{2}$, H. Simon ${ }^{3}$, V. Tissot ${ }^{4}$, D. Vansteene ${ }^{5}$, P. Meingan ${ }^{6}$, M. Di Palma ${ }^{7}$, A. Tolédano ${ }^{8}$, F. Goldwasser $^{9}$

${ }^{1}$ UTDN, Gustave Roussy, Villejuif, ${ }^{2}$ Radiologie, Hôpital Henri Mondor, Créteil, ${ }^{3}$ Oncologie médicale, ${ }^{4}$ Radiologie, Hôpital Morvan, Brest, ${ }^{5}$ Oncologie médicale, Institut de Cabcérologie de l'Ouest, ${ }^{6}$ Radiologie, Institut de Cancérologie de l'Ouest, Saint-Herblain, ${ }^{7}$ Direction médicale, Hôpital Américain de Paris, ${ }^{8}$ Radiothérapie, Clinique Hartmann, Neuilly sur Seine, ${ }^{9}$ Oncologie médicale, Hôpital Cochin, Paris, France

Introduction et but de l'étude : L'étude SCAN est une étude nationale visant à mesurer la prévalence de la sarcopénie chez les patients atteints d'un cancer métastatique (pts) et à étudier la relation entre la sarcopénie et la survenue de toxicités des traitements.

Matériel et méthodes : L'étude a été réalisée sur un mois en septembre-octobre 2017 et a inclus des pts atteints d'un cancer métastatique du côlon, poumon, prostate, sein ou rein, avec un traitement anticancéreux continu, et disposant d'un scanner permettant de mesurer l'indice musculaire squelettique (SMI) en L3. Les caractéristiques cliniques comprenaient l'état fonctionnel, l'échelle visuelle analogique des ingesta (échelle 0-10) et la mesure de la force musculaire à l'aide d'un dynamomètre hydraulique manuel JAMAR. l'évaluation par l'oncologue était enregistrée indépendamment du résultat du scanner. Toute toxicité entraînant une interruption du traitement était considérée comme une toxicité limitante aiguë (ALT).

Résultats et Analyse statistique : 779 pts ont été inclus (âge moyen 65,1 \pm 11,8; 34\% supérieur à 69 ans). Le site tumoral était le côlon (37\%), le poumon (26\%), le sein (22\%), le rein ( $8 \%$ ) et la prostate (7\%). 19\% étaient PS2 +. Ils étaient sous chimiothérapie cytotoxique (69\%), thérapie ciblée (40\%) ou immunothérapie (15\%). À l'inclusion, $28 \%$ étaient en surpoids et $12 \%$ obèses et $61 \%$ avaient perdu du poids depuis le diagnostic du cancer. La prévalence de la sarcopénie était de $68 \%$ avec une prévalence spécifique du côlon $70 \%$, du poumon $70 \%$, du sein $54 \%$, du rein $77 \%$ et de la prostate $93 \%$, et $36 \%$ chez les patients obèses. La présence de sarcopénie était associée à une amyotrophie clinique de la cuisse $(43,8 \pm 7,1$ vs $47,6 \pm 7,6, p<0,01)$, une circonférence faible $(26,9$ $\pm 4,2$ vs $29,0 \pm 4,5 \mathrm{~cm} ; p<0,01)$ et des apports oraux $(7,8 \pm 2,4$ vs $8,4 \pm 2,1, p<0,01)$. L'évaluation de la sarcopénie par l'oncologue était médiocre ( $49 \%$ de concordance avec le scanner; $53 \%$ chez les pts obèses). La survenue d'ALT était de $11 \%$ chez les patients sarcopéniques contre $7 \%$ chez les patients non sarcopéniques ( $p=$ NS). Et les toxicités de grade 3 ou 4 ont tendance à être plus fréquentes $(8 \%$ contre $6 \% ; p=N S)$. Les conseils diététiques et le soutien nutritionnel étaient très insuffisants chez les sarcopéniques (34\% et $28 \%$ respectivement).

Conclusion : La sarcopénie était très fréquente chez les patients atteints de cancer métastatique sans association statistique à la survenue d'événements liés au traitement. Cependant, la sarcopénie est souvent non détectée et insuffisamment prise en charge. Une tomodensitométrie L3 et une étroite collaboration entre oncologue et radiologue pourraient permettre de détecter plus tôt la sarcopénie.

Conflits d'intérêts : B. Raynard est consultant chez Fresenius-Kabi, F. Pigneur est consultant chez Fresenius-Kabi, H. Simon: Aucun conflit à déclarer, V. Tissot: Aucun conflit à déclarer, D. Vansteene: Aucun conflit à déclarer, P. Meingan: Aucun conflit à déclarer, M. Di Palma est consultant chez Fresenius-Kabi, A. Tolédano est consultant chez Fresenius-Kabi, F. Goldwasser est consultant chez Fresenius-Kabi 
Intérêt des outils cliniques pour prédire la sarcopénie chez les patients atteints de cancer métastatique : résultats de l'étude SCAN

B. Raynard ${ }^{1,{ }^{*}}$ au nom de Groupe investigateur SCAN, F. Pigneur ${ }^{2}$, M. Di Palma ${ }^{3}$, A. Tolédano ${ }^{4}$, F. Goldwasser $^{5}$

${ }^{1}$ Unité Transversale de Diététique et de Nutrition, GUSTAVE ROUSSY, Villejuif, ${ }^{2}$ Radiologie, Hôpital Henri Mondor, Créteil, ${ }^{3}$ Direction médicale, Hôpital américain, ${ }^{4}$ Radiothérapie, Clinique Hartmann, Neuilly sur Seine, ${ }^{5}$ Oncologie médicale, Hôpital Cochin, Paris, France

Introduction et but de l'étude : L'étude SCAN est une étude nationale visant à mesurer la prévalence de la sarcopénie chez les patients atteints d'un cancer métastatique (pts) et à comparer le scanner aux autres outils d'évaluation de l'état nutritionnel.

Matériel et méthodes : L'étude a été réalisée sur un mois en septembre-octobre 2017 et a inclus des pts atteints d'un cancer métastatique du côlon, poumon, prostate, sein ou rein, avec un traitement anticancéreux continu, et disposant d'un scanner permettant de mesurer l'indice musculaire squelettique (SMI) en L3, ainsi que la surface totale des 2 psoas (so mme des surfaces des 2 psoas sur la coupe tomodensitométrique en L3) (STP). Les caractéristiques cliniques comprenaient l'état fonctionnel, l'échelle visuelle analogique des ingesta (échelle 0-10) et la mesure de la force musculaire à l'aide d'un dynamomètre hydraulique manuel JAMAR (seuil pathologique défini : $30 \mathrm{~kg}$ pour les hommes et $20 \mathrm{~kg}$ pour les femmes), le périmètre brachial (PB) et le périmètre de cuisse du côté dominant, et l'estimation de l'amyotrophie au niveau du quadriceps par l'oncologue.

Résultats et Analyse statistique : 779 pts ont été inclus (âge moyen 65,1 $\pm 11,8 ; 34 \%$ supérieur à 69 ans). Le site tumoral était le côlon (37\%), le poumon (26\%), le sein (22\%), le rein ( $8 \%$ ) et la prostate (7\%). À l'inclusion, $28 \%$ étaient en surpoids, $12 \%$ obèses et $7 \%$ dénutris selon I'IMC. La prévalence de la sarcopénie évaluée par le SMI était de $68 \%$. Les oncologues ont déclaré une atrophie quadricipitale chez $33 \%$ des patients. Le périmètre brachial moyen était de $27,53 \mathrm{~cm}( \pm 4,47)(20 \%$ avaient un PB $<25 \mathrm{~cm})$ et le périmètre de cuisse moyen $45,07 \mathrm{~cm}( \pm 2,44) .26 \%$ des pts avec une EVA des ingesta entre 5 et 7 , et $8 \%$ entre 0 et $4.33 \%$ des hommes et $36 \%$ des femmes avaient une force de préhension basse. La valeur prédictive positive de sarcopénie (définie par le SMI) était respectivement de $81 \%$ pour le PB (en dessous de $21 \mathrm{~cm}$ ), $85 \%$ pour le périmètre de cuisse (en dessous de $39 \mathrm{~cm}$ ), 76 $\%$ pour la force de préhension basse, $81 \%$ pour EVA des ingesta entre 0 et 4 (75\% entre 0 et 7), $83 \%$ pour la présence d'une atrophie quadricipitale, $90 \%$ pour un IMC définissant la dénutrition. La corrélation entre SMI et STP était bonne, et la VVP de la STP indexée $\leq 5 \mathrm{~cm} 2 / \mathrm{m} 2$ était de $82 \%$.

Conclusion : L'IMC, la mesure du PB, du périmètre de cuisse ou l'objectivation d'une atrophie quadricipitale permettent de bien repérer les patients sarcopéniques. Une altération des ingesta est aussi un bon outil de dépistage dans ce contexte. Des outils simples d'évaluation nutritionnelle ou anthropométrique pourraient permettre de guider le praticien dans le répérage des patients sarcopéniques ou cachectiques, et rendre leur PEC nutritionnelle plus précoce.

Conflits d'intérêts : B. Raynard est consultant chez Fresenius-Kabi, F. Pigneur est consultant chez Fresenius-Kabi, M. Di Palma est consultant chez Fresenius-Kabi, A. Tolédano est consultant chez Fresenius-Kabi, F. Goldwasser est consultant chez Fresenius-Kabi 
$\mathrm{CO} 13$

Évaluation de l'état nutritionnel chez les patients âgés atteints de cancer: une enquête transversale nationale (NutriAgeCancer) E. Paillaud ${ }^{1,}$, , D. Heitz ${ }^{2}$, A.-L. Couderc ${ }^{3}$, R. Boulahssass ${ }^{4}$ au nom de Boulahssass, A. Chah Wakilian ${ }^{5}$, F. Pamoukdjian ${ }^{6}$, L. De Decker 7 ,

S. Valero ${ }^{8}$, A. Rouaud ${ }^{9}$, A. Raynaud-Simon ${ }^{10}$, C. Mertens ${ }^{11}$, P. Caillet ${ }^{12}$, V. Antoine ${ }^{13}$, L. Cristol ${ }^{14}$, I. Palayer ${ }^{15}$, H. Solem Laviec ${ }^{16}$ ${ }^{1}$ Departement de gériatrie, HOPITAL EUROPEEN GEORGES POMPIDOU, Paris, ${ }^{2}$ Unité de coordination OncoGeriatrie, Hopital de Hautepierre, Strasbourg, '3UCOG PACA Ouest, Hopital Sainte Marguerite, Marseille, ${ }^{4}$ UCOG PACA Est, Hôpital de Cimiez, Nice, ${ }^{5}$ UCOG Paris Ouest, Hopital Broca, Paris, ${ }^{6}$ UCOG Paris Seine Saint Denis, CHU Avicenne, Avicenne, ${ }^{7}$ UCOG Pays de la Loire, CHU , Nantes, ${ }^{8}$ UCOG Poitou-Charentes, CHU Poitiers, Poitiers, ${ }^{9}$ UCOG Pays de la Loire, CHU Nantes, Nantes, ${ }^{10}$ UCOG Paris Nord, Hopital Bichat, Paris, ${ }^{11}$ UCOG, Institut Bergonié, Bordeaux, ${ }^{12}$ UCOG Paris Ouest, Hopital Européen Georges Pompidou, Paris, ${ }^{13}$ Service Gériatrie, CHU Nimes, Nimes, ${ }^{14}$ UCOG, ICM Val d'AurelleMontpellier, Montpellier, ${ }^{15}$ Oncogeriatrie, hopital privé Toulon Hyères, Toulon, ${ }^{16}$ UCOG Normandie, Centre François Baclesse, Caens, France

Introduction et but de l'étude : La perte de poids est fréquente chez les patients âgés atteints de cancer et fortement associée à des événements péjoratifs. Notre objectif était d'évaluer la dénutrition et la perte de poids chez les patients âgés atteints de cancer

Matériel et méthodes : Une enquête nationale transversale (44 centres d'expertise oncogeriatrique, France) de patients atteints de cancer âgés de 70 ans ou plus a été menée de novembre à décembre 2017. Des données démographiques et cliniques ont été recueillies et une évaluation nutritionnelle a été réalisée par un gériatre. Le critère de jugement principal a été la perte de poids au cours des six derniers mois précédant le traitement anticancéreux. Les patients ont été ainsi classés en trois groupes selon le pourcentage de perte de poids ( $\% \mathrm{PDP})$ : minime $(\leq 5 \%)$; modérée $(>5 \%$ et $<10 \%)$; et sévère $(\geq 10 \%)$. La dénutrition est également définie selon les critères de la Haute Autorité de Santé (HAS) avec le Mini Nutritional Assessment (MNA), I'indice de masse corporelle (IMC), la concentration d'albumine sérique et le \% PDP.

Résultats et Analyse statistique : 571 patients étaient inclus dans l'étude (âge médian: 83 ans [intervalle interquartile: 79-87], $50 \%$ des hommes, $43 \%$ avec des métastases). Les principaux types de cancer ont été le cancer du sein (17\%), colorectal (14\%) et tractus digestif haut/pancréas (10\%) Au total, 124 patients (22\%) avaient une PDP modéré (entre 2,5 et 11kg; 5,2 en moyenne) et 146 (26\%) avaient une PDP sévère (entre 5 et $35 \mathrm{~kg} ; 12,1$ en moyenne). En analyse univariée, les patients présentant une perte de poids modérée ou sévère avaient le plus souvent un score de comorbidités plus élevé (Index de Charlson ajusté ; $P=0,018$ ), un cancer du tractus digestif haut/pancréas et du poumon $(P=0,001)$, des métastases $(P=0,016)$, un statut de performance altéré (PS $\geq 2 ; P<0,0001$ ), des troubles dépressifs (jugement clinique et test du Mini-Geriatric Depression Scale $\geq 1$ ) ; $P=0,005$ ), des troubles cognitifs (jugement clinique et test du Mini Mental State Examination $\leq 24 ; P=0,038$ ), de dépendance dans les activités de la vie quotidienne $(P<0,0001)$, une mobilité altérée (test Get up and Go ; $P<0,0001$ ) et sarcopénie (questionnaire SARC-F ; $P$ $<0,0001)$, par rapport aux patients sans perte de poids ou PDP minime.

Conclusion : La moitié de la population étudiée avait perdu plus de $5 \%$ de son poids au cours des six derniers mois. L'enquête se poursuit et sera renouvelée en 2018 permettant une analyse finale multivariée.

Remerciements : NUTRICIA pour son soutien

Conflits d'intérêts : Aucun conflit à déclarer 


\section{$\mathrm{CO} 14$}

Validation de l'outil EPA ${ }^{\circledR}$ chez les patients en phase palliative d'un cancer : résultats de l'étude ALIM-K.

G. Grodard ${ }^{1,}{ }^{*}$, V. Royer-Garabige ${ }^{2}$, A. Anota $^{3}$, C. Bouleuc ${ }^{4}$, R. Aubry ${ }^{5}$

${ }^{1}$ Diététique/CIC INSERM 1431, CHRU Besançon, Besançon, ${ }^{2}$ Diététique et réadaptation fonctionnelle, Institut Curie, Paris, ${ }^{3}$ Unité de méthodologie et de qualité de vie en cancérologie, CHRU Besançon, Besançon cedex, ${ }^{4}$ Département de Soins de Support, Institut Curie, Paris, ${ }^{5}$ Pôle Autonomie Handicap/CIC INSERM 1431 Axe "Ethique et Progrès Médical", CHRU Besançon, Besançon, France

Introduction et but de l'étude : L'outil d'évaluation de la prise alimentaire EPA ${ }^{\circledR}$ est recommandé depuis 2012 en cancérologie ${ }^{[1],[2]}$. Mais, s'il est indiqué pour le dépistage précoce et systématique de la cachexie chez les patients ambulatoires et hospitalisés ${ }^{[3],[4]}$, il n'existe pas de données démontrant son intérêt en soins palliatifs, qui plus est chez les personnes en phase avancée de cancer. Les résultats de l'étude ALIM-K (essai prospectif, multicentrique, contrôlé randomisé, évaluant l'efficacité de la nutrition parentérale sur la qualité de vie et la survie globale chez des patients en phase palliative d'un cancer[5]) permettent d'en discuter l'intérêt dans ces contextes.

Matériel et méthodes : ALIM-K incluait des patients dénutris. Une consultation diététique a été réalisée à l'inclusion. $L^{\prime} E P A^{\circledR}$ était recueillie en plus d'une évaluation des ingesta (rappel des 24h), des besoins énergétiques (Harris\&Benedict) et protéiques $(1,2 \mathrm{~g} / \mathrm{kg} / \mathrm{j})$. La passation d'un questionnaire alimentaire a permis de recueillir l'EPA ${ }^{\circledR}$ au cours du temps : entre J1 et J60 et J60 jusqu'au décès. Une analyse descriptive a été réalisée. La corrélation entre l'EPA ${ }^{\circledR}$ et la différence entre besoins énergétiques et apports réels a été étudiée. Idem avec la différence entre besoins protidiques et apports réels. Ces analyses ont été complétées par un modèle de régression linéaire.

Résultats et Analyse statistique : 111 patients ont été inclus sur une période de 5 ans dans 13 CHU et centres anticancéreux français. Parmi eux, 109 ont bénéficié d'une consultation diététique au début de l'étude (entre J-7 et J28). Les besoins énergétiques théoriques étaient en moyenne de $1864,4 \mathrm{kcal} / \mathrm{j}(\mathrm{SD}=326,5)$ et l'estimation quantitative des ingesta était en moyenne de $991,1 \mathrm{kcal} / \mathrm{j}(\mathrm{SD}=489,7)$. L'estimation des besoins protidiques était en moyenne de $71,3 \mathrm{~g} / \mathrm{j}(\mathrm{SD}=17,76)$ et la part protéique des ingesta était en moyenne de $37,6 \mathrm{~g}(\mathrm{SD}=21,8)$. La corrélation entre $\mathrm{EPA}^{\circledR}{ }^{\circledR}$ et la différence entre besoins et apports énergétiques réels était de $0,56(p<0,001)$. La corrélation entre EPA ${ }^{\circledR}$ et la différence entre besoins protidiques et apports réels était de $0,48(p<0,001)$. Le modèle de régression a permis de valider l'outil.

Conclusion : L'outil EPA ${ }^{\circledR}$ est une méthode fiable d'identification de la sous-consommation alimentaire (en termes d'énergie globale et de protéines) chez les patients atteints d'un cancer en phase avancée. Cet outil à toute sa place dans la prise en soin nutritionnelle de ces malades.

Références : [1] Thibault R, Guex E, Pichard C. Comment évaluer l'état et le risque nutritionnel ? Quelle stratégie d'intervention ? Questions de Nutrition Clinique de l'Adulte, Ed. SFNEP, chap. 5:55-79.

[2] Senesse P et al. Recommandations professionnelles. Nutrition chez le patient adulte atteint de cancer : textes courts. Nutr Clin Metab 2012;26:151-158.

[3] Kondrup J et al. ESPEN guidelines for nutrition screening 2002. Clin Nutr 2003;22:415-21.

[4] Thibault R, et al. Use of 10-point analogue scales to estimate dietary intake: A prospective study in patients nutritionally atrisk. Clin Nutr 2009;28:134-40.

[5] Pazart L, et al. Parenteral nutrition at the palliative phase of advanced cancer: the ALIM-K study protocol for a randomized controlled trial. Trials 2014, 15:370.

Conflits d'intérêts : Aucun conflit à déclarer 


\section{$\mathrm{CO} 15$}

Impact de la structure des aliments sur la biodisponibilité des micronutriments lipophiles : étude in vivo chez l'homme

B. Gleize ${ }^{1}$, M. Hiolle ${ }^{2,}$, N. Meunier ${ }^{3}$, B. Pereira ${ }^{4}$, R. Richard ${ }^{3}$, I. Savary-Auzeloux ${ }^{5}$, C. Buffière ${ }^{5}$, C. Caris-Veyrat $^{1}$, D. Dupont ${ }^{2}$, F. Nau ${ }^{2}$, E. Reboul ${ }^{6}$

${ }^{1}$ UMR 408 INRA-Université d'Avignon SQPOV, Avignon, ${ }^{2}$ UMR 1253 INRA-Agrocampus Ouest STLO, Rennes, ${ }^{3}$ Centre de Recherche en Nutrition Humaine d'Auvergne, ${ }^{4} \mathrm{CHU}$ de Clermont-Ferrand, ${ }^{5} \mathrm{UMR} 1019$ INRA-Université d'Auvergne UNH, Clermont-Ferrand, ${ }^{6}$ UMR 1260 INRA-INSERM-Université Aix-Marseille, Marseille, France

Introduction et but de l'étude : Un des défis majeurs auquel sera prochainement confrontée l'industrie agroalimentaire consistera à produire des aliments sains et durables tout en optimisant leur qualité nutritionnelle. Alors que la prévalence de l'obésité s'accentue, les carences en micronutriments continuent elles aussi d'augmenter. En particulier, la carence en vitamine D touche encore $50 \%$ de la population mondiale. De même, la DMLA est la première cause de handicap visuel chez les plus de 50 ans, alors qu'il a récemment été confirmé que la supplémentation en composés xanthophylles diminue le risque de progression de cette pathologie.

Afin de pallier ces carences, une des stratégies utilisées actuellement est l'enrichissement des aliments. Étant donné que les caractéristiques structurelles des aliments influencent le processus de désintégration pendant la digestion, il semble pertinent d'évaluer l'impact de la structure des aliments sur la biodisponibilité des micronutriments qu'ils contiennent.

Matériel et méthodes : Une étude clinique a été réalisée en utilisant quatre aliments modèles enrichis en lutéine et en vitamine D. Ces produits ont été conçus avec une composition strictement identique (sur matière sèche) mais avec des structures et textures différentes (crème, flan, génoise et biscuit). Ils ont été caractérisés par microscopie confocale et analyse de texture. Douze sujets mâles en bonne santé ont été recrutés pour cette étude monocentrique, contrôlée et randomisée, selon un plan expérimental croisé. Après un jeûne d'une nuit, l'absorption des micronutriments a été enregistrée par leur dosage dans les chylomicrons durant les 8 heures suivant la consommation de chaque produit.

Résultats et Analyse statistique : Deux paramètres ont été déterminés à partir des cinétiques d'absorption des micronutriments : le temps du pic d'absorption maximale et l'aire totale sous la courbe.

\begin{tabular}{|l|l|l|l|l|}
\hline Matrices & \multicolumn{2}{|c|}{$\begin{array}{c}\text { Temps du pic d'absorption } \\
\text { maximale (min) }\end{array}$} & $\begin{array}{r}\text { Aire sous la courbe } \\
\text { (nmol.h/L par mg de } \\
\text { micronutriment ingéré) }\end{array}$ \\
\cline { 2 - 5 } & Lutéine & Vitamine D & Lutéine & Vitamine D \\
\hline Biscuit & $250 \pm 56(a)$ & $250 \pm 56(a)$ & $260 \pm 172(a, b)$ & $768 \pm 232(a)$ \\
\hline Génoise & $210 \pm 48(b)$ & $215 \pm 48(b)$ & $318 \pm 226(a)$ & $891 \pm 418(a, b)$ \\
\hline Flan & $215 \pm 54(b)$ & $215 \pm 48(b)$ & $191 \pm 135(b)$ & $881 \pm 366(a, b)$ \\
\hline Crème & $202 \pm 40(b)$ & $207 \pm 41(b)$ & $319 \pm 260(a)$ & $1037 \pm 420(b)$ \\
\hline
\end{tabular}

Dans chaque colonne, les matrices associées à une lettre différente sont significativement différentes $(p<0,05)$.

Le biscuit présente le pic d'absorption le plus tardif pour les deux micronutriments (250 vs. 202 à 215 min). L'aire sous la courbe, indicative de la biodisponibilité, la plus élevée est quant à elle obtenue avec la crème et la génoise pour la lutéine, et avec la crème pour la vitamine D.

Conclusion : Les résultats suggèrent que la structure des aliments module l'apparition des micronutriments dans le plasma, du point de vue cinétique et quantitatif. Cela pourrait être dû à des différences de désintégration des matrices durant leur digestion. Une étude complémentaire utilisant un modèle de digestion in vitro visera à confirmer cette hypothèse.

Conflits d'intérêts : Aucun conflit à déclarer 


\section{CO16}

Biodisponibilité des acides aminés d'un isolat protéique de tournesol marqué au $15 \mathrm{~N}$ et au $2 \mathrm{H}$ chez le rat R. Tessier ${ }^{1,}{ }^{*}$, N. Khodorova ${ }^{1}$, J. Calvez ${ }^{1}$, R. Kapel ${ }^{2}$, F. Cacérès ${ }^{3}$, A. Dinar ${ }^{3}$, J. Piedcoq ${ }^{1}$, D. Tomé ${ }^{1}$, C. Gaudichon $^{1}$ ${ }^{1}$ PNCA, AgroParisTech, INRA, Université Paris-Saclay, Paris, ${ }^{2}$ LRGP, UMR CNRS 7274, Université de Lorraine, Plateforme SVS, Vandoeuvre-les-Nancy, ${ }^{3}$ Terres Inovia, 1 avenue Lucien Brétignières, Thierval-Grignon, France

Introduction et but de l'étude : Les protéines d'oléagineux sont de bons candidats à une utilisation alimentaire chez l'Homme de par leur composition en acides aminés (AA) et les surfaces agricoles déjà existantes. Il existe peu d'études sur la biodisponibilité des protéines du tournesol. Le but de l'étude est d'évaluer la digestibilité chez le rat des protéines et des AA indispensables (AAI) $d^{\prime}$ un isolat de tournesol intrinsèquement marqué avec 2 isotopes stables : le ${ }^{15} \mathrm{~N}$, usuellement utilisé, et le ${ }^{2} \mathrm{H}$, jusqu'alors non utilisé pour cette application.

Matériel et méthodes : Le marquage du tournesol a été réalisé en serre par administration de 3 apports de ${ }^{15} \mathrm{NH}_{4}{ }^{15} \mathrm{NO}_{3}$ marqué à $99 \%$ et, au moment de la floraison, de 5 apports successifs de ${ }^{2} \mathrm{H}_{2} \mathrm{O}$ marqué à $20 \%$ ( (er $^{\mathrm{er}}$ apport) puis à $10 \%$. Les isolats protéiques ont été obtenus après extraction et purification sur membranes. Le marquage obtenu était de $1 \%$ en ${ }^{15} \mathrm{~N}$ et de $0.15 \%$ en ${ }^{2} \mathrm{H}$, soit respectivement 3 et 8 fois l'abondance naturelle.

Vingt-deux rats Wistar mâles reçoivent un repas comprenant $0.47 \mathrm{~g}$ de cet isolat protéique. Les rats sont sacrifiés $3 \mathrm{~h}$ ( $\mathrm{n}=7$ ) ou $6 \mathrm{~h}$ $(n=15)$ après le repas. Les contenus de l'estomac, intestin grêle, iléon, cæcum et côlon sont collectés. L'azote ${ }^{15} \mathrm{~N}$ est déterminé dans les digestas par un analyseur élémentaire couplé à un spectromètre de masse à ratio isotopique (EA-IRMS). Les AAI sont dosés par UPLC dans le cæcum et leurs enrichissements en ${ }^{15} \mathrm{~N}$ et en ${ }^{2} \mathrm{H}$ sont mesurés par GC couplé à un IRMS via l'interface de combustion $\left({ }^{15} \mathrm{~N}\right)$ ou pyrolyse $\left({ }^{2} \mathrm{H}\right)$. Les résultats sont exprimés en moyennes ( $\left.\pm E S M\right)$ et les différences entre les 2 marquages sont analysées par un t-test apparié $(P<0,05)$.

Résultats et Analyse statistique : La quantité de $\mathrm{N}$ alimentaire dans les digestas indique une digestion incomplète à $3 \mathrm{~h}(2,7 \pm 0,8$ et 2,6 $\pm 0,4 \%$ de l'azote ingéré dans l'estomac et l'intestin grêle respectivement) alors qu'après $6 \mathrm{~h}$, la majorité du $\mathrm{N}$ ingéré est dans le cæcum et le côlon. La digestibilité oro-fécale du $\mathrm{N}$ des protéines ingérées mesurée $6 \mathrm{~h}$ après le repas est de $93,8 \pm 0,5 \%$. Tableau : digestibilité des AAI (en \%) obtenue avec les deux isotopes :

\begin{tabular}{|l|c|c|c|c|c|c|c|}
\hline & val & leu & ile & thr & phe & lys & met \\
\hline${ }^{15} \mathbf{N}$ & $94,2 \pm 0,6$ & $94,4 \pm 0,5$ & $94,9 \pm 0,5$ & $96,0 \pm 0,6$ & $94,7 \pm 0,5$ & $94,7 \pm 0,5$ & $96,0 \pm 0,4$ \\
\hline${ }^{2} \mathbf{H}$ & $95,5 \pm 0,5$ & $95,2 \pm 0,5$ & $96,0 \pm 0,5$ & $95,8 \pm 0,7$ & $94,9 \pm 0,5$ & $96,6 \pm 0,3$ & $96,2 \pm 0,4$ \\
\hline T test & $p<0,001$ & $p<0,01$ & $p<0,001$ & $N S$ & $N S$ & $p<0,001$ & $N S$ \\
\hline
\end{tabular}

La digestibilité des AAl est significativement plus grande avec le ${ }^{2} \mathrm{H}$ qu'avec le ${ }^{15} \mathrm{~N}$, sauf pour la thréonine, phénylalanine et méthionine. Le DIAAS (Digestible Indispensable Amino Acid Score) du tournesol, calculé sur la base des AAI ${ }^{15} \mathrm{~N}$ dans le cæcum, est de 0,93 $\pm 0,1$ pour la lysine et il est supérieur à 1 pour tous les autres AA mesurés.

Conclusion : Les valeurs obtenues avec le ${ }^{2} \mathrm{H}$ sont supérieures par rapport au ${ }^{15} \mathrm{~N}$ pour tous les AAl excepté trois, mais les différences étant modestes, le ${ }^{2} \mathrm{H}$ s'avère néanmoins un traceur approprié pour cette application. La qualité de l'isolat de tournesol se caractérise par une digestibilité de $94 \%$, plus faible que celle de la caséine (97\%) mais plus élevée que celle d'autres protéines végétales (90-91\%), mesurée avec la même méthodologie. Le tournesol est légèrement déficient en lysine mais répond aux besoins pour les autres AAI mesurés.

Conflits d'intérêts : R. Tessier: Aucun conflit à déclarer, N. Khodorova: Aucun conflit à déclarer, J. Calvez: Aucun conflit à déclarer, R. Kapel: Aucun conflit à déclarer, F. Cacérès est employé(e) de Terres Inovia, A. Dinar est employé(e) de Terres Inovia, J. Piedcoq: Aucun conflit à déclarer, D. Tomé: Aucun conflit à déclarer, C. Gaudichon: Aucun conflit à déclarer 
$A B C B 1$ (P-glycoprotéine) contribue à la régulation de l'absorption et à l'efflux transintestinal de vitamine $D$

M. Margier ${ }^{1}$, X. Collet $^{2}$, C. Le May ${ }^{3}$, C. Desmarchelier ${ }^{1}$, F. André ${ }^{4}$, C. Lebrun ${ }^{5}$, C. Defoort ${ }^{1}$, A. Bluteau ${ }^{5}$, P. Borel ${ }^{1}$, A. Lespine ${ }^{5}$, E. Reboul ${ }^{1, *}$

${ }^{1}$ C2VN, Marseille, ${ }^{2}$ UMR 1048, Toulouse, ${ }^{3}$ Institut du thorax, Nantes, ${ }^{4}$ I2BC, Gif-sur-Yvette, ${ }^{5}$ INTHERES, Toulouse, France

Introduction et but de l'étude : La vitamine $D$ est une molécule essentielle à l'homéostasie phosphocalcique. Elle jouerait également un rôle clé dans la prévention d'un certain nombre de pathologies immunitaires et métaboliques, ainsi que vis-à-vis de certains cancers. Pourtant, les carences et les subcarences en vitamine $D$ restent un problème majeur de santé public, aussi bien dans les pays en voie de développement que dans les pays industrialisés.

Mieux comprendre le transport intestinal de la vitamine D pourrait permettre d'optimiser le statut des individus. Nous avons donc étudié l'implication de l'ATP binding cassette B1 (ABCB1) dans l'efflux intestinal de vitamine D.

Matériel et méthodes : Pour mener à bien cette étude, nous avons mis en place une approche translationnelle de la protéine à I'homme en combinant des modèles in silico, in vitro, ex vivo et in vivo chez la souris - notamment chez des souris déficientes en ABCB1 ( $A b c b 1-/-)$, et clinique. Ces modèles nous ont permis d'évaluer l'efflux de vitamine $D$ néo-absorbée, mais aussi le transfert de la vitamine $D$ du plasma vers la lumière intestinale.

Résultats et Analyse statistique : Les efflux apicaux de cholécalciférol (vitamine $D$ alimentaire) et de 25-hydroxycholécalciférol (vitamine $D$ circulante) étaient diminués par inhibition chimique dans les cellules Caco-2 TC7 (jusqu'à $-34,9 \%, p<0,05$ ), et augmentés par la surexpression d'ABCB1 dans les Griptites ou les cellules MDCKII (jusqu'à $+167,1 \%, p<0,05$ ).

Les souris Abcb1 -/- présentaient une accumulation significative de 25 -hydroxycholécalciférol dans le plasma $(42,6 \%)$, les intestins $(+43,0 \%)$, le cerveau $(+58,2 \%)$, le foie $(+59,2 \%)$ et les reins $(+20,6 \%)$, ainsi qu'une réponse postprandiale en cholécalciférol accrue après gavage $\left(3,43\right.$ and $6,71 \mu \mathrm{mol} . \mathrm{h} / \mathrm{g}$ de lipides pour les souris $A b c b 1^{\%}$ et témoins, respectivement $(\mathrm{p}<0.05)$.

L'efflux de 25- hydroxycholécalciférol par des explants intestinaux issus de souris $A b c b 1$-/- étaient réduit de moitié par rapport aux explants témoins $(p<0,05)$. Cette réduction du transfert de 25 -hydroxycholécalciférol du plasma à la lumière intestinale a été confirmée in vivo chez des souris perfusées in situ au niveau intestinale $(-36,6 \%$ chez les souris transgéniques par rapport au témoins, $p<0,05)$.

Des expériences in silico ont permis d'établir que le cholécalciférol et le 25-hydroxycholécalciférol pouvaient tous deux se lier avec une grande affinité à la P-glycoprotéine de Caenorhabditis elegans, utilisée comme modèle ABCB1.

Enfin, dans un groupe de 39 hommes adultes en bonne santé, un SNP dans ABCB1 (rs17064) était significativement associé à la concentration plasmatique en 25 -hydroxycholécalciférol à jeun $(35,94 \pm 4,50 \mathrm{nmol} / \mathrm{L}, \mathrm{n}=9 \mathrm{vs} 56,07 \pm 3,00 \mathrm{nmol} / \mathrm{L}, \mathrm{n}=30 ; \mathrm{p}$ ajustée $=0,041$, t-test avec correction de Benjamini-Hochberg).

Conclusion : Nous avons ainsi montré pour la première fois qu'ABCB1 est impliqué dans l'efflux de vitamine $D$ néo-absorbés par les entérocytes, et qu'ABCB1 contribue également à l'excrétion transintestinale de vitamine $D$.

Conflits d'intérêts : Aucun conflit à déclarer 


\section{$\mathrm{CO} 18$}

Quel est le rôle de la synthèse intestinale de citrulline dans l'homéostasie protéique ?

J. P. De Bandt ${ }^{12}{ }^{*}$, M. Juchet-Martin ${ }^{2}$, M. Aboubacar ${ }^{2}$, S. Magassa ${ }^{2}$, I. Dubail ${ }^{3}$, L. Cynober ${ }^{12}$

${ }^{1}$ Service de Biochimie, Hopital Cochin, Hopitaux Universitaires Paris CentreAPHP, ${ }^{2}$ EA4466 PRETRAM, ${ }^{3}$ UMRS 1140, Faculté de Pharmacie de Paris, Université Paris Descartes, Sorbonne Paris Cité, Paris, France

Introduction et but de l'étude : La concentration plasmatique de citrulline (Cit) est essentiellement déterminée par l'équilibre entre sa production intestinale et sa conversion rénale en arginine. Cette production intestinale dépend d'une enzyme, l'ornithine carbamyl transférase (OCT), qui permet de convertir l'ornithine en Cit. Nos travaux ont montré qu'à dose pharmacologique la Cit active la synthèse protéique musculaire. Notre hypothèse était que l'OCT intestinale, en contrôlant la disponibilité de la Cit, pourrait être un élément clé de l'homéostasie protéique musculaire, hypothèse que nous avons voulu tester par une invalidation de l'enzyme. Toutefois, l'OCT est également exprimée par les hépatocytes et la déficience en OCT à la naissance est associée à une mortalité précoce. Nous avons donc eu recours à un modèle d'invalidation conditionnelle de l'OCT intestinale (OCT Cre-Lox) permettant d'induire une délétion spécifique de l'enzyme intestinale chez des souris adultes.

Matériel et méthodes : Des souris $O C T^{\text {Cre-Lox }}$ ont été obtenues en croisant des souris transgéniques OCT ${ }^{\text {Lox }}$ avec des souris transgéniques exprimant une protéine de fusion formée par la recombinase Cre et le domaine de liaison au tamoxifène sous le contrôle d'un promoteur spécifique de l'intestin (villine). Seules des souris sauvages mâles (Wt, $n=6)$ et $O C T^{\text {Cre-Lox }}(n=12)$ ont été utilisées pour nos expériences. Les souris Wt et la moitié des souris $\mathrm{OCT}^{\text {Cre-Lox }}$ (oct) ont reçu une administration quotidienne IP de tamoxifène $(75 \mathrm{mg} / \mathrm{kg} / \mathrm{j}$ dans de l'huile de maïs) pendant 5 jours. Les autres souris OCT Cre-Lox $(O C T)$ ont reçu de I'huile de maïs IP. Les souris ont été ensuite suivies pendant 2 mois sur le plan de l'évolution pondérale, de la consommation alimentaire et de la citrullinémie. Lors de l'euthanasie, nous avons déterminé l'aminoacidogramme plasmatique et la masse maigre (poids de la carcasse). Statistiques : ANOVA suivi d'un test PLSD de Fisher ; significativité $p<0,05$.

Résultats et Analyse statistique : Dès J7 et jusqu'à la fin de l'étude, les souris oct ont présenté une concentration plasmatique très faible de Cit (Wt : $44 \pm 13 \mu \mathrm{mol} / \mathrm{I}, \mathrm{OCT}: 33 \pm 10 \mu \mathrm{mol} / \mathrm{l}$, oct : $4 \pm 2 \mu \mathrm{mol} / \mathrm{l} ; \mathrm{p}<0,05 \mathrm{Wt}$ et OCT vs oct). La consommation alimentaire, l'évolution pondérable, la masse maigre globale (Wt : 13,3 $\pm 0,8 \mathrm{~g}$, OCT : $16 \pm 3 \mathrm{~g}$, oct : 12,3 $\pm 0,5 \mathrm{~g}$; NS), et l'amino acidogramme (en dehors d'une légère diminution de la phénylalaninémie dans le groupe oct) ne différaient pas entre les groupes lors de l'euthanasie.

Conclusion : Nos résultats ne sont pas en faveur d'un rôle majeur de la production intestinale de Cit dans la régulation physiologique de l'homéostasie des protéines musculaires. Il est à noter que les effets pharmacologiques de Cit sont observés à des concentrations plasmatiques de l'ordre de $1000 \mu \mathrm{mol} / \mathrm{l}$.

Conflits d'intérêts : J. P. De Bandt est actionnaire de Citrage, M. Juchet-Martin: Aucun conflit à déclarer, M. Aboubacar: Aucun conflit à déclarer, S. Magassa: Aucun conflit à déclarer, I. Dubail: Aucun conflit à déclarer, L. Cynober est actionnaire de Citrage 
CO19

La consommation d'un régime riche en fructose induit une augmentation des niveaux plasmatiques d'acides aminés à chaîne ramifiée chez le rat insulino-résistant et non obèse

J. David ${ }^{1}$, L. Mosoni ${ }^{1}$, D. Dardevet ${ }^{1}$, I. Savary-Auzeloux ${ }^{1}$, S. Polakof ${ }^{1, *}$

${ }^{1}$ Unité de Nutrition Humaine, INRA, Saint-Gènes-Champanelle, France

Introduction et but de l'étude : Des études récentes ont montré que des taux élevés d'acides aminés à chaîne ramifiée (AACR) plasmatiques sont observés chez les sujets et les animaux de laboratoire obèses et résistants à l'insuline (IR). Parmi les hypothèses proposées, une capacité réduite du tissu adipeux à cataboliser les AACR a été observée lors de l'apparition de l'IR (1), notamment chez les sujets obèses, les animaux génétiquement modifiés ou nourris avec des régimes hyperlipidiques. II reste néanmoins à élucider si l'élévation des AACR est la cause ou la conséquence du phénotype IR, et quels sont les tissus responsables d'une telle augmentation (2). Afin de mieux comprendre le rôle précis de chaque organe et le rôle de l'obésité, nous avons utilisé un modèle de rat IR à un stade où l'obésité n'est pas installée.

Matériel et méthodes : Deux groupes de rats ont été nourris avec un régime standard, contenant comme source de glucides (63\%) de l'amidon ou du fructose pendant 45 jours. Après 5, 12, 30 ou 45 jours d'alimentation, huit animaux par groupe ont été euthanasiés à l'état post-absorptif. Le métabolisme des AACR a été exploré dans le plasma, le foie et le muscle gastrocnémien par biochimique et biologie moléculaire. Le poids et la composition corporelle ont été suivis de façon hebdomadaire. Les données plasmatiques (cinétique) ont été analysées par une ANOVA à deux voies et les données tissulaires (point final) par un test-t de Student. Le seuil de signification a été fixé à 0.05 .

Résultats et Analyse statistique : Nous montrons pour la première fois que des niveaux élevés d'AACR dans le plasma (+36\%, $p=0.023$ ) peuvent également être observés dans un modèle de rongeur IR (rats nourris au fructose, $+300 \% H O M A-I R, p<0.001$ ). Niveaux d'AACR et indice HOMA-IR sont d'ailleurs fortement corrélés (corr. $0.66 ; p=0.002$ ). Nos résultats laissent penser que cela pourrait résulter d'un défaut d'oxydation des AACR dans le muscle (réduction des potentiels de transamination (-12\%, $p=0.032$ ) et de désamination oxydative $(-40 \%, p<0.001)$ dans ce tissu, plutôt que de modifications au niveau du tissu adipeux : la composition corporelle et le poids ne sont pas modifiés, les animaux n'accumulent pas de masse grasse. De plus, la voie de signalisation mTOR était activée dans le foie $(+64 \%, p<0.001)$ et le muscle $(+64 \%, p<0.001)$ des rats nourris avec le régime fructose, puisque la stimulation persistante de cette voie par les taux élevés d'AACR circulants a été montré comme potentiel contributeur au développement de I'IR.

Conclusion : Les taux élevés de AACR chez le rat nourri avec un régime riche en fructose en l'absence de modification de la composition corporelle semble minimiser le rôle du tissue adipeux alors que l'altération du métabolisme des AACR dans le muscle squelettique pourrait être clé. Ce modèle pourrait donc fournir de nouveaux éléments dans les relations causes/conséquences du tissu adipeux dans la séquence métabolique d'installation du phénotype IR et de dissocier le rôle des différents organes dans l'élévation des niveaux des AACR lors de l'installation de l'IR.

Références : (1) Herman et al. 2010. J Biol Chem 285:11348-11356; (2) Lynch et Adams. 2014. Nat Rev Endocrinol 10:723-736

Conflits d'intérêts : Aucun conflit à déclarer 
$\mathrm{CO} 20$

La pathologie de l'aorte ascendante en réponse à programmation fœtale par la déficience en donneurs de méthyle B. Balint ${ }^{1}{ }^{*}$, R.-M. Rodriguez-Gueant ${ }^{1}$, J.-L. Gueant ${ }^{1}$

${ }^{1}$ INSERM, Université de Lorraine, Vandoeuvre-les-Nancy, France

Introduction et but de l'étude : La déficience en donneurs de méthyle (CDM, carence en vitamine B12 et folate) pendant la gestation et la lactation est fréquente chez l'homme et produit des effets de programmation fœtale associés aux pathologies chroniques liées à l'âge, notamment métaboliques, hépatiques, myocardiques et neurologiques. L'hyperhomocystéinémie produite par la CDM pourrait contribuer à ces pathologies. Cependant, les manifestations vasculaires et leurs mécanismes moléculaires à long terme pouvant potentiellement être liés à la programmation fœtale de la CDM n'ont pas été étudiés. Le but de cette étude était d'évaluer les paramètres structuraux et moléculaires de l'aorte ascendante chez des rats âgés nés de mères carencées pendant la gestation et l'allaitement (iCDM).

Matériel et méthodes : Nous avons étudié les effets de la programmation fœtale sur l'aorte ascendante de rats âgés nés de mères iCDM comparés à un groupe contrôle né de mères non carencées. Les deux groupes ont été soumis à un régime alimentaire normal après 21 jours jusqu'au sacrifice (J400). La pression artérielle et les prélèvements ont été déterminés quotidiennement de J365 à J400. Au moment du sacrifice, une partie de l'aorte ascendante a été fixée dans du formol et incluse dans la paraffine et ont permis d'évaluer le remodelage pathologique par histologie. Une autre a été traitée pour des analyses protéomiques ont été effectuées pour évaluer les altérations d'expression en réponse à l'iCDM.

Résultats et Analyse statistique : La pression artérielle et les taux d'angiotensine Il était significativement augmentée chez les rats du groupe iCDM vs contrôles ( $p<0,0001$ ). L'examen histologique des coupes de tissus colorés au Trichrome et au Picrosirius a révélé une augmentation significative de la fibrose aortique en réponse à la programmation foetale par l'iCDM (augmentation de $21,1 \%, p=0,0025$ et de 52,9\%, p=0,0001, respectivement). L'épaisseur intima-média du groupe iCDM était augmentée 1,4 fois $(p=0,046)$. La pression artérielle était positivement corrélée à l'étendue de la fibrose et à l'épaisseur médiale de l'aorte $(p<0,05)$. De plus, l'examen en immunofluorescence révélait une augmentation significative du marquage de l'homocystéine dans l'aorte dans le groupe iCDM vs contrôles $(p<0,0001)$. Le changement protéomique majeur était une diminution de 9 fois du groupe de protéines impliquées dans l'adhésion cellule-cellule dans le groupe iCDM (FDR=0,002). En concordance avec ce résultat, nous avons constaté une augmentation de la localisation nucléaire de la $\mathrm{N}$-cadhérine.

Conclusion : Nos résultats révèlent un effet de la programmation fœtale par l'iCDM sur l'aorte, au cours du vieillissement chez les rats de première génération. Cet effet correspond à un remodelage pathologique de l'aorte ascendante, y compris par fibrose et épaississement de l'intima-média, qui est corrélé à l'augmentation de la pression sanguine. En outre, l'augmentation de l'expression de l'homocystéine dans les aortes des animaux iCDM pourrait jouer un rôle dans les changements observés dans l'adhésion cellule-cellule. Ces résultats suggèrent que le fœtal programming lié à l'iCDM favorise le vieillissement vasculaire pathologique indépendamment de tout autre facteur de risque nutritionnel.

Conflits d'intérêts : Aucun conflit à déclarer 


\section{ALIMENTATION DURABLE}

\section{$\mathrm{CO} 21$}

Effets du mode de production sur l'impact environnemental de différents menus : importance du choix de l'unité fonctionnelle G. Mairesse ${ }^{1,{ }^{*}}$, H. M. G. van der Werf ${ }^{2}$, G. Chesneau ${ }^{1}$, N. Kerhoas ${ }^{3}$

${ }^{1}$ R\&D, VALOREX, Combourtille, ${ }^{2}$ UMR SAS, INRA/Agrocampus Ouest, Rennes, ${ }^{3}$ Association Bleu-Blanc-Coeur, Combourtille, France

Introduction et but de l'étude : L'unité fonctionnelle (UF) en Analyse de Cycle de Vie (ACV) quantifie la fonction d'un produit. Dans le cas d'un menu, différentes UF sont possibles, telles que " $100 \mathrm{kcal}$ d'aliments ingérées " ou "100g d'aliments ingérés ". Cependant, une autre UF peut être employée: "l'apport d'une quantité de nutriments conforme aux recommandations nutritionnelles par adulte et par jour ». L'étude présentée propose un indice synthétique (QUALIP) permettant d'évaluer le niveau de compliance des apports en acides gras par rapport aux Apports Nutritionnels Conseillés (ANC) et vise à exprimer l'impact environnemental de menus selon différentes unités fonctionnelles.

Matériel et méthodes : Trois menus ont été modélisés : un menu « moyen », un menu « PNNS » répondant aux recommandations du PNNS et un menu "végétarien » (Schmitt et al., 2018). Chacun de ces 3 menus a été décliné avec des produits animaux issus de la filière standard (STD) ou Bleu-Blanc-Cœur (BBC). Un bilan nutritionnel de ces 6 menus a été réalisé à partir duquel un indice "QUALIP » a été construit à partir du score NRF 9.3 (Fulgoni et al., 2009) :

QUALIP $=\left[\left(A L A / A N C_{A L A}+L A / A N C_{L A}+E P A / A N C_{E P A}+D H A / A N C_{D H A}+\right.\right.$ Oléique $\left./ A N C_{O l e ́ i q}\right)-\left[\left(1-\left(A N C_{C 12-14-16 / A G C 12-14-16)}\right)+(1-\right.\right.$ $\left.\left.\left.\left(A N C_{L A / A L A} /(L A / A L A)\right)\right)\right]\right] *$ Lip/ANCLip

Cet indice, entre 0 et 5 , indique respectivement une adéquation très faible ou parfaite aux ANC acides gras. L'évaluation environnementale des menus a été réalisée par ACV (Coelho et al., 2016).

Résultats et Analyse statistique : Le menu ayant la plus faible adéquation avec les ANC acides gras est le menu végétarien (QUALIP le plus faible), tandis que le menu PNNS possède l'indice QUALIP le plus élevé. D'autre part, l'indice QUALIP est supérieur pour les menus BBC traduisant le fait qu'ils permettent de se rapprocher des ANC pour les acides gras.

Tableau 1. Indice QUALIP et Impact Changement Climatique (CC) des menus

\begin{tabular}{|l|c|c|c|c|c|c|}
\hline & \multicolumn{2}{|c|}{ Menu Moyen } & \multicolumn{2}{c|}{ Menu PNNS } & \multicolumn{2}{c|}{$\begin{array}{c}\text { Menu } \\
\text { Végétarien }\end{array}$} \\
\hline & STD & BBC & STD & BBC & STD & BBC \\
\hline QUALIP & 2,15 & 3,68 & 3,65 & 4,01 & 1,54 & 3,19 \\
\hline CC (g CO2 eq/100g) & 324 & 322 & 230 & 229 & 169 & 168 \\
\hline CC (g CO2 eq/100kcal) & 260 & 256 & 215 & 212 & 168 & 166 \\
\hline CC (kg CO 2 eq/unité QUALIP) & 2,78 & 1,61 & 1,35 & 1,22 & 2,38 & 1,14 \\
\hline
\end{tabular}

L'impact changement climatique exprimé selon $100 \mathrm{~g}$ de menu ou $100 \mathrm{kcal}$ est le plus faible pour le menu végétarien et le plus élevé pour le menu Moyen, essentiellement du fait de la consommation de produits carnés. Néanmoins, cette classification est différente lorsque l'impact est exprimé par unité QUALIP. En version standard, le menu PNNS possède alors le meilleur résultat, en version $B B C$ c'est le menu végétarien qui présente le plus faible impact. D'autre part, l'alimentation BBC permet, quel que soit le menu de fournir à un cout environnemental réduit, la meilleure adéquation vis-à-vis de ANC lipidiques.

Conclusion : Cette étude montre l'importance du choix de l'unité fonctionnelle dans l'évaluation de la durabilité d'un menu. A ce titre, le choix du mode de production Bleu-Blanc-Cœur est l'une des solutions permettant de répondre à la fonction d'un menu qui est d'apporter des nutriments d'intérêt et conformes aux recommandations nutritionnelles.

Références : Coelho C. R. et al. (2016). PLoS One, 11(8), 1-1

Fulgoni et al. (2009). J. Nutr, 139, 1549-1554

Schmitt B. et al. (2018). OCL, 25(2), D205

Remerciements : Ces travaux ont été financés par l'ANR 12-ALID-0003, AGRALID.

Conflits d'intérêts : G. Mairesse est employé(e) de VALOREX, H. M. G. van der Werf: Aucun conflit à déclarer, G. Chesneau est employé(e) de VALOREX, N. Kerhoas est employé(e) de Bleu-Blanc-Coeur 
NUTRI-SCORE : quelle place pour les éleveurs engagés dans l'amélioration de la qualité nutritionnelle des produits animaux ? G. Mairesse ${ }^{1,},{ }^{*}$, G. Chesneau ${ }^{1}$, N. Kerhoas ${ }^{2}$

${ }^{1}$ R\&D, VALOREX, ${ }^{2}$ Association Bleu-Blanc-Coeur, Combourtille, France

Introduction et but de l'étude : L'amélioration de la composition en acides gras (AG) des produits animaux peut intervenir dès la phase d'élevage au travers notamment d'une alimentation spécifique, comme le propose la filière Bleu-Blanc-Cœur (BBC) à partir d'une teneur minimale en AGn-3, AG largement déficitaires pour 99.1\% des français (ANSES, 2015). II existe en effet un lien étroit entre la composition en AG des régimes et des produits animaux, les autres compartiments (protéines, glucides) étant peu ou pas impactés (Mourot et al., 2015). L'objectif de ce travail est d'étudier l'impact de ces pratiques d'alimentation en élevages sur le Nutri-Score.

Matériel et méthodes : Les étiquettes nutritionnelles ont été relevées en août 2018, sur 6 laits $1 / 2$ écrémés (4 en filière standards (STD), 2 en BBC), 3 boites d'œufs (2 STD, 1 BBC) et 4 paquets de jambons Torchon sans couenne (2 STD, 2 BBC). Le Nutri-Score a été calculé selon la méthodologie officielle (agriculture.gouv.fr/telecharger/87510?token=537276c9f22122955 add7292abbcce0d), par la soustraction de deux composantes : i) les facteurs nutritionnels à limiter en cas de consommation excessive : énergie ( $\mathrm{kJ} / 100 \mathrm{~g})$, AG saturés (AGS) $(\mathrm{g} / 100 \mathrm{~g})$, sucres simples $(\mathrm{g} / 100 \mathrm{~g})$ et sel $(\mathrm{mg} / 100 \mathrm{~g})$; ii) les facteurs nutritionnels à favoriser : fibres $(\mathrm{g} / 100 \mathrm{~g})$, protéines $(\mathrm{g} / 100 \mathrm{~g})$, teneur en fruits et légumes, légumineuses et fruits à coque $(\mathrm{g} / 100 \mathrm{~g})$.

Résultats et Analyse statistique : Pour les produits bruts, lait et œufs, le Nutri-Score obtenus est $\mathrm{A}$, avec respectivement un score de 0 et -1. Il ne discrimine donc pas l'amélioration avérée du profil lipidique de ces produits, notamment en Oméga 3 (BBC vs STD) (Mourot et al., 2015), car il ne prend en compte que les seuls AGS totaux dans son évaluation, pour lesquels il n'existe pas d'Apports Nutritionnels Conseillés.

Comme attendu, le Nutri-Score permet d'identifier des différences entre jambons, même si elles s'expriment davantage au niveau des scores que des classes. Alors que les jambons STD sont tous classés en $C$ (notes allant de 3 à 5 ), les jambons BBC à teneur en sel réduite permettent une amélioration de la qualité nutritionnelle (classe $B$ ). Néanmoins, cette amélioration est expliquée exclusivement par la réduction de la teneur en sel, le score AGS restant inchangé par rapport au produit STD.

Tableau 1 : Nutri-Score des jambons

\begin{tabular}{|l|l|l|c|c|c|c|c|}
\hline Marque & Filière & Recette & $\begin{array}{l}\text { AGS } \\
\mathbf{( g / 1 0 0 g})\end{array}$ & $\begin{array}{l}\text { Point } \\
\text { AGS }\end{array}$ & $\begin{array}{l}\text { Sodium } \\
\text { (mg/100g) }\end{array}$ & $\begin{array}{l}\text { Point } \\
\text { Sodium }\end{array}$ & $\begin{array}{l}\text { Score - Classe } \\
\text { Nutri-Score }\end{array}$ \\
\hline A & STD & & 1 & $\mathbf{0}$ & 720 & $\mathbf{7}$ & $\mathbf{3}-\mathbf{C}$ \\
\hline A & BBC & Teneur en sel réduite & 1 & $\mathbf{0}$ & 560 & $\mathbf{6}$ & $\mathbf{2}-$ B \\
\hline B & STD & & 1,6 & $\mathbf{1}$ & 760 & $\mathbf{8}$ & $\mathbf{5}-$ C \\
\hline B & BBC & Teneur en sel réduite & 1,3 & $\mathbf{1}$ & 560 & $\mathbf{6}$ & $\mathbf{3 - C}$ \\
\hline
\end{tabular}

Conclusion : Bien que le Nutri-Score représente une avancée importante sur le plan de l'affichage nutritionnel, il s'avère être un outil de notation des pratiques industrielles et non des pratiques d'élevage à l'origine de la matière première. Ainsi, la complémentarité du Nutri-Score à d'autres signes de différenciation représentatifs des efforts réalisés par les producteurs prend tout son sens pour engager une dynamique d'amélioration nutritionnelle globale des menus tant à l'étape de l'élaboration des produits transformés qu'à l'étape de la production des produits bruts dont ils sont issus (Schmitt et al. 2018).

Références : ANSES (2015). Apports en acides gras de la population vivant en France [...], 244p.

Mourot J. et al. (2015). OCL, 22(6), D610.

Schmitt B. et al. (2018). OCL, 25(2), D205

Conflits d'intérêts : G. Mairesse est employé(e) de VALOREX, G. Chesneau est employé(e) de VALOREX, N. Kerhoas est employé(e) de Bleu-Blanc-Coeur 
${ }^{1}$ UMR1253, STLO, INRA, Agrocampus-ouest, Rennes, France, ${ }^{2}$ Faculte of pharmacy, Université Laval, Québec, Canada

Introduction et but de l'étude : L'encapsulation de nutriments et de bioactifs pour la formulation d'aliments fonctionnels représente une voie à fort potentiel pour l'industrie agro-alimentaire. La coacervation complexe, séparation de phase liquideliquide obtenue par mélange de protéines et de polysaccharides de charges opposées, est un procédé doux et adéquat pour l'encapsulation. Nous démontrons que les coacervats exclusivement de protéines (coacervats hétéroprotéiques) présentent la même efficacité en tant que vecteur d'encapsulation.

Matériel et méthodes : La lactoferrine et la ß-lactoglobuline, deux protéines laitières, ont été utilisées pour former les coacervats hétéroprotéiques. La capacité et l'efficacité de ces coacervats à encapsuler la vitamine B9, nutriment modèle, ont été étudié en conditions optimales de $\mathrm{pH}$ et de force ionique, en faisant varier les concentrations en protéines et en B9. Le pouvoir protecteur des coacervats vis-à-vis de la dégradation de B9 par différents traitements utilisés lors de la transformation et du stockage des aliments a été déterminé. La biodisponibilité de B9 ainsi encapsulée a été évaluée in vivo par une étude pharmacocinétique après administration orale chez le rat. Le dosage de la vitamine B9 par ELISA, garantissant la détection exclusive de la forme native, a été effectué sur des prélèvements sanguins réalisés jusqu'à $10 \mathrm{~h}$ post administration.

Résultats et Analyse statistique : Nous avons établi la preuve de concept de la formation des coacervats de protéines en présence de B9. Des hauts rendements à la fois de coacervation et d'encapsulation de B9 ont pu être obtenus. Ainsi, en conditions optimales, nous obtenions l'encapsulation de $4 \mathrm{mg}$ de B9/g de protéines. Par ailleurs, comparativement à la B9 seule en solution, la B9 encapsulée est plus résistante aux traitements physiques ou chimiques tels que la lumière, la lyophilisation ou encore lors d'une oxydation accélérée en présence d $d^{\prime} \mathrm{H}_{2} \mathrm{O}_{2}$. Outre la protection, le principal objectif de l'encapsulation est de favoriser la libération contrôlée de la molécule encapsulée. L'étude pharmacocinétique chez le rat met en évidence une concentration plasmatique supérieure en B9 lorsque celle-ci est administrée sous forme de coacervats, en comparaison à l'administration de B9 seule ou simplement complexée à une des deux protéines [1]. La biodisponibilité améliorée par les coacervats est attribuée à leur effet protecteur améliorant ainsi la solubilité de la vitamine lors du transit gastro-intestinal.

Conclusion : Ce travail constitue une première preuve de concept prometteuse pour l'utilisation potentielle des coacervats de protéines pour l'encapsulation de bioactifs. Leur usage pour formuler des produits sans additifs est une des applications. Des études sur la stabilité de la vitamine B9 dans une matrice alimentaire ainsi que sur le devenir de la vitamine et des protéines des coacervats après traitement physique (thermique) ou biologique (digestion) sont en cours. L'usage des coacervats hétéroprotéiques pour l'encapsulation de molécules d'intérêt pour d'autres secteurs est également envisagé.

Références : [1] Chapeau A.L. et al. Journal of Functional Food, 2017; 38:197-204.

Remerciements : Ce travail a été réalisé dans le cadre du projet PROFIL financé par les régions Bretagne et Pays de Loire, coordonné par I'INRA (J. Léonil) et géré par l'association BBA.

Conflits d'intérêts : Aucun conflit à déclarer 

grâce au fromage

G. Jan ${ }^{1, *}$ et consortium du projet ANR "SURFING"

${ }^{1}$ STLO, INRA, Rennes, France

Introduction et but de l'étude : Les maladies inflammatoires chronique de l'intestin (MICl) sont reliées à divers facteurs, incluant le fond génétique, le système immunitaire, l'alimentation et d'autres facteurs environnementaux. Le microbiote intestinal est impliqué dans les $\mathrm{MICl}$. La consommation de bactéries probiotiques sélectionnées peut induire ou prolonger des rémissions dans le cas de $\mathrm{MICl}$ telles que la RCH (rectocolite hémorragique). L'effet des bactéries consommées massivement dans les aliments fermentés est encore peu pris en compte. L'actinobactérie Propionibacterium freudenreichii est couramment consommée dans les fromages à pâte pressée cuite, ainsi que dans des compléments alimentaires probiotiques. Un grand nombre de souches sont disponibles pour cette espèce. Le but de cette étude était de 1) rechercher l'aptitude de certaines souches à induire la cytokine modulatrice IL-10, au niveau de cellules immunitaires humaines (PBMC), 2) développer un fromage expérimental « monosouche » avec la souche la plus immunomodulatrice de $P$. freudenreichii et 3 ) de tester l'impact de la consommation de ce fromage dans le contexte de la colite induite chez la souris.

Matériel et méthodes : Les propionibactéries ont été cultivées sur ultrafiltrat de lait de vache supplémenté en peptone de caséine puis conservées à $-20^{\circ} \mathrm{C}$ dans du PBS glycérolé ( 1 milliard de bactéries par $\mathrm{mL}$ ). La colite aigue a été induite par administration intra-rectale de TNBS (100 mg/kg de poids corporel) et les animaux euthanasiés après 3 jours d'induction. L'inflammation a été quantifiée par mesure des score macroscopique Wallace et microscopique Ameho. Les résultats sont exprimés comme des moyennes \pm SEM.

Résultats et Analyse statistique : La consommation de ce fromage a protégé les souris de la colite aigue induite. L'induction de marqueurs locaux et systémiques de l'inflammation était réduite par la consommation du fromage. IL-6: $366,17 \pm 134$ versus $1726,7 \pm 548$ pour le placebo $(p<0,05)$. SAA : $29812 \pm 2909$ versus $9535 \pm 2909$ pour le placebo $(p<0,05)$. La consommation de fromage a également conduit à une forte diminution de l'expression colique des gènes $/ / 6$, Cox 2 et $\mathrm{Hmox}$, des scores cliniques et anatomopathologiques. En revanche, cette consommation a restauré l'expression de Pparg et de Zo1. Globalement, ces résultats démontrent un effet protecteur du fromage fermenté par $P$. freudenreichii, et pas du fromage placébo stérile, vis-à-vis de l'induction de la colite aigüe.

Conclusion : Cette étude confirme le potentiel probiotique précédemment décrit pour la bactérie alimentaire $P$. freudenreichii. Elle propose un nouveau produit laitier fermenté, et ouvre de nouvelles perspectives, pour des études pré-cliniques et cliniques ultérieures, visant la prévention et/ou l'aide au traitement de maladies inflammatoires.

Conflits d'intérêts : Aucun conflit à déclarer 
Introduction et but de l'étude : La graine de lin extrudée (GLE), riche en acide $\alpha$-linolénique, est incorporée aux rations des vaches laitières depuis quelques années. De nombreux essais expérimentaux ont démontré que la GLE modifiait le profil en acides gras du lait au bénéfice de la santé humaine. Cependant, globalement la voie de l'expérimentation n'a pas permis de montrer les effets de la GLE sur les performances et la santé animale, vraisemblablement du fait d'une part de leur manque de puissance statistique et d'autre part de quantités apportées souvent trop importantes. Dans ces conditions, la voie de l'épidémiologie peut représenter une plus-value en s'appuyant sur des apports modérés, de grands échantillons et en contrôlant les facteurs de confusion. Ainsi, l'objectif de cette étude rétrospective exposé-non exposé était de quantifier les effets de l'utilisation de la GLE sur les performances et la santé des vaches laitières en conditions de terrain.

Matériel et méthodes : En l'absence de connaissance de la composition exacte des rations, la mesure de l'exposition à la GLE a été estimée sur la base des informations des livraisons opérées par 22 entreprises d'aliments du bétail entre 2008 et 2015 en France. Pour les élevages ayant utilisé la GLE, l'ajout des données du système national d'information génétique a permis de distinguer les vaches exposées à la GLE (avec 4 niveaux d'exposition, de $30 \mathrm{~g}$ à $800 \mathrm{~g} / \mathrm{j}$ ) et les vaches non exposées à la GLE (population de référence), au sein des mêmes élevages. Les performances de reproduction ont été étudiées sur 423605 inséminations artificielles (IA) issues de 158125 vaches dans 1096 troupeaux en mobilisant des modèles de Cox pour les intervalles post-partum et de régression logistique pour la fertilité. Les performances laitières et la santé de la mamelle ont été étudiées sur 1997763 contrôles laitiers issus de 194056 vaches dans 1291 troupeaux grâce à des modèles linéaires avec structure d'autocorrélation d'ordre 1 et de régression logistique. Tous les modèles ont été ajustés des facteurs de variation connus de la performance à étudier et d'un effet aléatoire troupeau.

Résultats et Analyse statistique : L'exposition à la GLE était associée de façon dose-effet à une amélioration de la production laitière $(+0,4$ à $+1,3 \mathrm{~kg} / \mathrm{j}$ selon la parité), et une légère réduction des taux de matières utiles $(-0,03$ à $-0,20 \mathrm{~g} / \mathrm{kg}$ de taux protéique et $-0,1$ à $-0,9 \mathrm{~g} / \mathrm{kg}$ de taux de matières grasse selon la parité). L'intervalle vêlage-fécondation était significativement réduit chez les vaches ayant reçu de la GLE depuis le vêlage quelle que soit la dose que chez celles n'en ayant pas reçu (de -2,9 à -6,6 jours selon la quantité de GLE), sans effet sur la fertilité. Les chances de guérison des mammites subcliniques étaient améliorées chez les vaches recevant a minima $50 \mathrm{~g} / \mathrm{j}$ de GLE (Odds Ratio de 1,03 à 1,10).

Conclusion : Cette $1^{\text {ère }}$ étude épidémiologique en nutrition animale a permis de montrer qu'en moyenne les éleveurs retirent des bénéfices sur les performances de production, de reproduction et de santé du troupeau à l'apport de quantités modestes de GLE. Elle ouvre des perspectives d'exploration des liens alimentation-performances-santé chez la vache laitière en conditions de terrain.

Conflits d'intérêts : G. Chesneau est employé(e) de VALOREX, T. Meignan est employé(e) de VALOREX, A. Madouasse: Aucun conflit à déclarer, F. Beaudeau: Aucun conflit à déclarer, V. Chatellier est employé(e) de VALOREX, G. Mairesse est employé(e) de VALOREX,

N. Bareille: Aucun conflit à déclarer 
Amélioration de l'autonomie protéique alimentaire du poulet de chair par l'incorporation de graines oléo-protéagineuses en substitution du tourteau de soja d'importation

M. Guillevic ${ }^{1}$, M. Lessire ${ }^{2}$, A. Germain ${ }^{1}$, H. Juin ${ }^{3}$, G. Mairesse $^{1,{ }^{*}}$, G. Chesneau $^{1}$

${ }^{1}$ VALOREX, Combourtille, ${ }^{2}$ UMR BOA, INRA, Nouzilly, ${ }^{3}$ UE EASM, INRA, Surgeres, France

Introduction et but de l'étude : Afin de répondre aux attentes sociétales, les filières avicoles cherchent à s'affranchir de l'importation de tourteau de soja. Une des solutions consisterait à substituer ces protéines importées par des matières premières produites en France, non OGM et tracées. Cependant, pour rendre cette démarche pérenne, il convient de répondre aux attentes tant des producteurs que des consommateurs. Dans cet objectif, nous avons déterminé les impacts zootechniques, environnementaux et économiques d'un mélange de graines oléo-protéagineuses sélectionnées et traitées spécifiquement incorporé dans les aliments de poulet de chair.

Matériel et méthodes : L'essai a été mené à l'INRA sur des poulets mâles ROSS PM3 recevant des aliments où les principales sources protéiques étaient du tourteau de soja d'importation (TEMOIN) ou bien un mélange (90-10) de graines sélectionnées de féverole et de soja qui avaient subi un traitement thermomécanique adapté (ESSAI). Ce mélange etait incorporé à hauteur de $15 \%$ dans l'aliment croissance (EM : $2950 \mathrm{kcal} / \mathrm{kg} ;$ LYSd : 11g/kg) et 20\% dans l'aliment finition (EM : $3000 \mathrm{kcal} / \mathrm{kg} ; \mathrm{LYSd}: 10 \mathrm{~g} / \mathrm{kg}$ ). Ces aliments présentaient les mêmes caractéristiques nutritionnelles que le TEMOIN. Les performances zootechniques ont été relevées, les impacts environnementaux (ECOALIM) et économiques (conjoncture 2018) de ces produits ont été déterminés.

Résultats et Analyse statistique : Les performances de croissance des poulets des lots TEMOIN et ESSAI sont identiques. Le poids des poulets à la fin de l'essai $(1,732$ versus $1,793 \mathrm{~kg}$ ) ainsi que l'indice de consommation $(1,456$ versus 1,444$)$ ne sont pas significativement différents.

L'impact environnemental de la production de poulet avec une alimentation à base de graines oléo-protéagineuses est, positif sur le changement climatique (-41\%), la consommation de phosphore (-36\%), la consommation d'énergie fossile (-22\%) et l'acidification (-18\%); neutre sur l'eutrophisation (-2\%) et négatif sur l'occupation des sols (+13\%).

L'impact économique de la production de poulet de chair, évalué au travers de la part liée à l'alimentation qui représente environ les deux tiers des coûts de production, est de 40,20 € et de 39,45€/ $100 \mathrm{~kg}$ de poids vif soit une baisse de $-1,9 \%$. Sur la base de la consommation française de poulet qui est de $19,0 \mathrm{~kg} / \mathrm{an}$, l'impact est tout autant neutre puisqu'il permet d'économiser $0,14 €$ /an.

Conclusion : Ces travaux ont permis de mettre en évidence qu'il existe, par l'acte d'achat du consommateur et au travers de l'alimentation du poulet, une solution pérenne pour lutter contre la déforestation importée. Cette solution est viable pour la filière avicole grâce à des performances de croissance conservées et à un coût alimentaire stable; mais elle est aussi durable sociétalement puisque l'impact sur l'environnement est significativement amélioré pour un coût de revient identique.

Conflits d'intérêts : Aucun conflit à déclarer 


\section{$\mathrm{CO} 27$}

Caractérisation des perturbations biochimiques dans un modèle murin d'anorexie (ABA) par résonance magnétique nucléaire -Etude métabonomique-

J. Breton ${ }^{12,{ }^{*}}$, N. Giallourou ${ }^{3}$, S. Nobis ${ }^{12}$, A. Morin ${ }^{12}$, N. Achamrah ${ }^{124}$, A. Goichon ${ }^{12}$, L. Belmonte ${ }^{2}$, P. Déchelotte ${ }^{12} 4$, J.-L. do Rego ${ }^{15}$, M. Coëffier ${ }^{124}$, J. Swann ${ }^{3}$

${ }^{1}$ Institute for Research and Innovation in Biomedicine (IRIB), Normandie univ, UNIROUEN, ${ }^{2}$ Nutrition, Gut and Brain Laboratory, INSERM U1073 NORMANDY UNIVERSITY, Rouen, France, ${ }^{3}$ Division of Computational and Systems Medicine, Imperial College London, Londres, Royaume-Uni, ${ }^{4}$ Nutrition Department, Rouen University Hospital, ${ }^{5}$ Animal Behavior Platform SCAC, Normandie univ, UNIROUEN, Rouen, France

Introduction et but de l'étude : I'Anorexie Mentale (AM) est un trouble psychologique sévère du comportement alimentaire. Les souris 'activity-based anorexia' (ABA) semblent tout à fait adaptées pour étudier la physiopathologie de l'AM puisque ce modèle associe, une activité physique (très fréquemment retrouvée dans la pathologie) à un accès progressivement limité à la nourriture. Cependant, malgré l'intérêt reconnu de ce modèle murin d'anorexie, peu de choses sont connues sur les perturbations métaboliques associées.

Matériel et méthodes : Dans cette présente étude, les phénotypes métaboliques urinaires, plasmatiques et fécaux ont été réalisé chez des animaux contrôles avec ou sans activité physique (CTPA et CT, respectivement), des animaux dont l'alimentation a été progressivement limitée (LFA), et chez les souris anorexiques (ABA). Les phénotypes métaboliques des souris furent analysés par spectroscopie à résonance magnétique nucléaire $(R M N){ }^{1} \mathrm{H}$ afin de mieux comprendre les mécanismes biochimiques adaptatifs en réponse à la restriction calorique " auto-motivée ». Les spectres $\mathrm{RMN}{ }^{1} \mathrm{H}$ ont été convertis et analysé via le logiciel statistique MATLAB. Des composantes en analyse principale (PCA) et des régressions PLS discriminantes (OPLS-DA) ont été appliquées sur les spectres RMN urinaires, plasmatiques et fécaux dans le but d'identifier les métabolites discriminant les groupes d'animaux.

Résultats et Analyse statistique : Au niveau fécal, 18 métabolites permettaient la discrimination des groups principalement selon l'activité physique avec des métabolites impliqués principalement dans le métabolisme des acides aminés et des acides gras à chaines courtes. Au niveau urinaire et plasmatique, 24 et 8 métabolites respectivement, ont été identifiés pour différencier les groupes principalement en fonction de l'état nutritionnel des animaux, avec des informations pertinentes liées à la fonte musculaire, la dégradation des acides aminés et le métabolisme des acides gras

Conclusion : Ce travail apporte pour la première fois une signature métabolique des souris ABA et donc une meilleure compréhension des adaptations biologiques lors d'une restriction calorique " auto-motivée " associée à une activité physique dans un modèle murin d'anorexie. Ces résultats devront par la suite être confirmés en clinique chez les patients anorexiques.

Conflits d'intérêts : Aucun conflit à déclarer 

P. Déchelotte ${ }^{13}$, D. Ribet ${ }^{1}, M$. Coëffier ${ }^{13}$

${ }^{1}$ INSERM UMR1073, ${ }^{2}$ Plateforme d'Analyse Comportementale SCAC, Université de Rouen Normandie, ${ }^{3}$ Département de Nutrition, $\mathrm{CHU}$, Rouen, France

Introduction et but de l'étude : L'axe microbiote-intestin-cerveau semble impliqué dans la régulation du comportement alimentaire et dans les troubles anxio-dépressifs. Cependant, son rôle au cours de l'anorexie mentale n'est pas complètement élucidé. Le but de ce travail a été d'étudier les conséquences d'une déplétion du microbiote intestinal sur la réponse au modèle murin d'anorexie, Activity-Based Anorexia (ABA).

Matériel et méthodes : Des souris mâles $\mathrm{C} 57 \mathrm{BI} / 6$ ont été soumises au modèle $A B A$ de $\mathrm{J} 1$ à $\mathrm{J} 18$, associant l'accès à une roue d'activité et une limitation progressive du temps d'accès à l'alimentation jusqu'à 3 heures/jour. Les souris contrôles (CT) avaient un accès libre à l'alimentation, sans roue d'activité. Les souris ont reçu ( $A B A+A T B$ et $C T+A T B, n=16 / g r o u p e)$ ou non ( $A B A$ et $C T$, $n=16 / g r o u p e)$ par gavage ( 2 fois/jour de J-9 à J17) une combinaison antifongique/antibiotiques permettant $d^{\prime}$ induire une déplétion du microbiote intestinal, vérifiée par quantification de l'ADN bactérien total au niveau fécal. Au cours du modèle ABA, des tests comportementaux (Open field) et des mesures de composition corporelle (MiniSpec) ont été réalisés à J17. Les résultats ont été comparés par ANOVA à 2 voies ( $A B A$ x Déplétion) avec des post-tests de Bonferroni.

Résultats et Analyse statistique : Entre J-9 et J1, les souris ABA+ATB et CT+ATB présentaient une perte de 99,99\% de la quantité d'ADN bactérien dans les fèces, confirmant la forte déplétion du microbiote intestinal en réponse au traitement administré. A J17, la prise alimentaire était diminuée de façon similaire dans les groupes $A B A$ et $A B A+A T B$ par rapport aux souris $C T$ et $C T+A T B$ $(p<0,0001)$. Les groupes $A B A$ et $A B A+A T B$ présentaient une perte de poids par rapport aux groupes $C T$ et $C T+A T B(p<0,0001)$. Cependant, la perte de poids était moins marquée dans le groupe $A B A+A T B(10,0 \pm 6,5 \%$ vs J6) par rapport au groupe $A B A(15,1 \pm$ $6,0 \%$ vs $J 6, p<0,01)$. Les souris $A B A+A T B$ présentaient une augmentation de masse grasse et une diminution de masse maigre par rapport aux souris $A B A(p<0,001)$. Le groupe $A B A$ présentait une augmentation de l'activité physique anticipatoire à l'accès à la nourriture entre $\mathrm{J} 5$ et $\mathrm{J} 16(p<0,0001)$, augmentation qui était moins marquée dans le groupe $A B A+A T B$ ( $p<0,05$ vs $A B A)$. De plus, les activités physiques nocturne et totale à $J 17$ étaient diminuées dans le groupe $A B A+A T B(p<0,0001$ vs $A B A)$. Lors du test de l'Open field, une diminution de la distance parcourue par les souris $A B A+A T B$ à la périphérie $(p<0,05$ vs $C T+A T B ; p<0,001$ vs $C T)$ et au centre ( $p<0,05$ vs $C T$ ) était observée. Une augmentation du temps d'immobilité était observée chez les souris $A B A+A T B$ $(p<0,01$ vs $C T+A T B$ et $C T)$, mais pas chez les souris $A B A$.

Conclusion : Dans le modèle $A B A$, une déplétion du microbiote intestinal est associée à une limitation de la perte de poids, de l'activité physique et de l'activité exploratoire et à une modification de la composition corporelle, sans modification de la prise alimentaire. D’autres études sont nécessaires pour préciser les mécanismes périphériques et/ou centraux impliqués.

Conflits d'intérêts : Aucun conflit à déclarer 
Etude des neuropeptides plasmatiques et de leurs immunoglobulines respectives chez 120 patients atteints de troubles du comportement alimentaire.

M. Galmiche ${ }^{12} 3^{3}{ }^{*}$, C. Deroissart ${ }^{1}$, M.-A. Le Solliec ${ }^{1}$, J. Rondeaux ${ }^{2}$, S. Azhar ${ }^{1}$, N. Achamrah ${ }^{234}{ }^{3}$, L. Belmonte ${ }^{234}$, A. Lamarre ${ }^{4}$, S. Grigioni ${ }^{2} 34$, V. Folope 234 , A. Rimbert ${ }^{234}$, T. Saillard 234 , J. Delay ${ }^{234}$, M. P. Tavolacci ${ }^{235}$, M. Quillard $^{5}$, G. Lambert $^{1}$, P. Dechelotte ${ }^{234}$, N. Lucas $^{1}$, R. Legrand ${ }^{1}$

${ }^{1}$ Targedys SA, ${ }^{2}$ Inserm UMR1073, ${ }^{3}$ Université de Rouen, Institute for Research and Innovation in Biomedicine (IRIB), ${ }^{4}$ Nutrition, ${ }^{5} \mathrm{CIC}-\mathrm{CRB} 1204$ INSERM, CHU-Hôpitaux de Rouen, Rouen, France

Introduction et but de l'étude : Les troubles du comportement alimentaires (TCA) sont des pathologies fréquentes, dont la physiopathologie implique l'axe microbiote-intestin-cerveau. Plusieurs neuropeptides et hormones sont impliqués dans la régulation du comportement alimentaire et du métabolisme énergétique. Des études ont mis en évidence au cours des TCA la présence d'autoanticorps (AutoAc) dirigés contre les neuropeptides orexigènes et anorexigènes, avec des différences de profil (taux/affinité) qui pourraient participer à la dérégulation neurobiologique du comportement alimentaire. Evaluer les principaux neuropeptides impliqués dans la régulation du comportement alimentaire et les anticorps correspondants au cours des différents TCA : anorexie mentale (AN), boulimie nerveuse (BN) et hyperphagie boulimique (BED).

Matériel et méthodes : Les analyses plasmatiques ont été réalisées chez 120 patients (34 AN, 12 BN et 74 BED) inclus avant traitement dans la cohorte EDILS (Eating Disorder Inventory Longitudinal Study). Les taux de leptine, GLP-1, PYY et insuline ont été analysés par Milliplex et ceux d'acyl ghréline, des-acyl ghréline et $\alpha$-MSH par Elisa. L'affinité des IgG reconnaissant ces peptides a été mesurée par résonnance plasmonique de surface et le taux des IgG analysés par ELISA. Le test de Kruskal-Wallis et de Spearman ont été utilisés.

Résultats et Analyse statistique : La leptinémie était corrélée positivement avec l'IMC et plus élevée dans le groupe BED (AN vs $B E D$ et $B N$ vs $B E D: p<0,001)$. L'insulinémie était positivement corrélée avec l'IMC et plus élevée chez les patients $B E D$ vs $A N$ $(p<0,001)$. Les taux de GLP-1 et PYY étaient augmentés chez les patients BED : AN vs BED (GLP-1, $p=0,004)$ et AN vs BED (PYY, $p=0,02)$. Le taux d' $\alpha-M S H$ était plus élevé chez les patients $A N$ vs BED $(p=0,02)$. La ghréline totale était corrélée négativement avec I'IMC $(p<0,001)$. Cette corrélation négative était également constatée avec les taux de des-acyl ghréline $(p<0,001)$ et les taux d'acyl ghréline $(p<0,001)$. Le taux d'IgG anti-GLP-1 était augmenté chez les patients AN vs BED $(p=0,008)$, ainsi que le taux d'autoAc PYY (AN vs BED : $p=0,03$ ). Le taux des IgG anti-insuline était aussi plus élevé chez les patients AN vs $B N(p=0,02)$.

Conclusion : L'élévation des taux de leptine, PYY et GLP-1 au cours de l'hyperphagie soulignent la résistance aux signaux anorexigènes au cours de ce TCA. Inversement, le taux d' $\alpha$-MSH est plus élevé au cours de l'AN vs BED et associé à une élévation de la ghréline totale, de l'acyl ghréline et de la des-acyl ghréline qui semble donc être impliquée dans la ghrélinorésistance au cours de I'AN. Ces analyses seront étendues sur un plus grand nombre de patients, pour préciser les profils de dérégulation neuropeptidergique au cours des TCA et identifier des cibles thérapeutiques.

Conflits d'intérêts : M. Galmiche: Aucun conflit à déclarer, C. Deroissart: Aucun conflit à déclarer, M.-A. Le Solliec: Aucun conflit à déclarer, J. Rondeaux: Aucun conflit à déclarer, S. Azhar: Aucun conflit à déclarer, N. Achamrah: Aucun conflit à déclarer, L. Belmonte: Aucun conflit à déclarer, A. Lamarre: Aucun conflit à déclarer, S. Grigioni: Aucun conflit à déclarer, V. Folope: Aucun conflit à déclarer, A. Rimbert: Aucun conflit à déclarer, T. Saillard: Aucun conflit à déclarer, J. Delay: Aucun conflit à déclarer, M. P. Tavolacci: Aucun conflit à déclarer, M. Quillard: Aucun conflit à déclarer, G. Lambert est employé(e) de Targedys, P. Dechelotte est actionnaire de Targedys, N. Lucas est employé(e) de Targedys, R. Legrand est employé(e) de Targedys 
$\mathrm{CO} 30$

Validité des équations d'impédancemétrie bioélectrique pour la mesure de la composition corporelle chez des patientes anorexiques

M. Coëffier ${ }^{123,{ }^{*},}$, M. Gaté ${ }^{1}$, A. Rimbert ${ }^{1}$, A. Petit ${ }^{1}$, V. Folope ${ }^{1}$, S. Grigioni ${ }^{1}$, P. Déchelotte ${ }^{123}$, N. Achamrah ${ }^{123}$

${ }^{1}$ Département de Nutrition, ${ }^{2} \mathrm{CIC}$ 1404, INSERM, CHU, ${ }^{3}$ INSERM UMR1073, Université de Rouen Normandie, Rouen, France

Introduction et but de l'étude : L'impédancemétrie bioélectrique (BIA) est une technique simple et rapide pour évaluer la composition corporelle. Cependant chez des patients présentant des indices de masse corporelle (IMC) extrêmes, notamment dans l'anorexie mentale sévère, la validité de la BIA est controversée. Différentes équations de BIA ont été développées mais il n'existe à l'heure actuelle pas de consensus quant à leur utilisation chez les patientes anorexiques. Nous avons donc comparé les résultats de composition corporelle obtenus par BIA en fonction de ces différentes équations à ceux obtenus par absorptiométrie biphotonique à rayons $X$ (DEXA), technique de référence.

Matériel et méthodes : Huit équations de BIA (Deuremberg et al, Kyle et al, Sun et al, Bedogni et al, Roubenoff et al, Scalfi-1 et al, Scalfi-2 et al, Kushner et al) ont été appliquées, rétrospectivement, sur les paramètres électriques mesurées par BIA chez des patientes anorexiques ayant un IMC $<16 \mathrm{~kg} / \mathrm{m}^{2}$. Les mesures de BIA (Bodystat Quadscan 4000) et par DEXA (Lunar Prodigy Advance) ont été réalisées après une période de jeûne de $12 \mathrm{~h}$. Les résultats ont été analysés par la technique de Bland-Altman, le test de corrélation de Pearson et le test de concordance de Lin.

Résultats et Analyse statistique : Les données de 115 patientes anorexiques $\left(32,3 \pm 14,5\right.$ ans ; 14,6 $\left.\pm 1,2 \mathrm{~kg} \cdot \mathrm{m}^{-2}\right)$ ont été incluses dans l'étude. Mesurées par DEXA, la masse grasse (MG) était de 4,2 $\pm 2.4 \mathrm{~kg}$ et la masse non grasse (MNG) de 35,4 $\pm 3,8 \mathrm{~kg}$. L'équation de Sun et al donnait les meilleurs résultats en BIA avec, respectivement pour la MNG et la MG, un facteur de corrélation de Pearson $r=0,86$ et 0,86, un coefficient de concordance de Lin $r=0,84$ et 0,81 et un biais moyen de 0,706 $\mathrm{kg}$ et 0,548 $\mathrm{kg}$. Cependant, l'intervalle de confiance à 95\% (IC 95\%) était important (de -4,55 à 3,13 kg pour la MNG et de $-2,73$ à 3,83 kg pour la MG). Les autres équations présentaient également des IC 95\% élevés (tableau 1).

\begin{tabular}{|l|l|l|l|l|l|l|l|}
\hline (en kg) & Deuremberg & Kyle & Bedogni & Roubenoff & Scalfi-1 & Scalfi-2 & Kushner \\
\hline IC 95\% MNG & $-2,589 / 6,831$ & $-2,447 / 5,534$ & $-5,972 / 6,671$ & $-2,069 / 5,918$ & $-1,394 / 7,633$ & $-6,594 / 5,547$ & $-7,032 / 1,608$ \\
\hline Biais MNG & 2,121 & 1,543 & 0,349 & 1,925 & 3,119 & $-0,523$ & $-2,712$ \\
\hline IC 95\% MG & $-6,511 / 1,954$ & $-5,189 / 1,787$ & $-6,596 / 5,582$ & $-5,611 / 1,446$ & $-7,141 / 0,587$ & $-5,522 / 6,254$ & $-1,367 / 6,476$ \\
\hline Biais MG & $-2,278$ & $-1,701$ & $-0,507$ & $-2,082$ & $-3,277$ & 0,366 & 2,555 \\
\hline
\end{tabular}

Conclusion : Chez des patientes présentant une anorexie mentale sévère $\left(\mathrm{IMC}<16 \mathrm{~kg} / \mathrm{m}^{2}\right)$ aucune des équations de $\mathrm{BIA}$ testées ne semble adaptée pour évaluer précisément la composition corporelle à l'échelle de l'individu.

Conflits d'intérêts : Aucun conflit à déclarer 


\section{CO31}

Dépistage des troubles du comportement alimentaire à l'aide du SCOFF-F chez $\mathbf{9 6 4}$ actifs et étudiants en Haute-Vienne.

M. Arnal ${ }^{1,}{ }^{*}$, P. Fayemendy ${ }^{12}$, B. Morin ${ }^{1}$, M. Perrier ${ }^{3}$, M. Gravelat ${ }^{4}$, G. Magne ${ }^{5}$, C. Le Flahec ${ }^{6}$, V. Sabourdy $^{6}$, S. Depoux $^{7}$, M. Szopinski ${ }^{8}$, D. Mathe ${ }^{9}$, M. Vergonjeanne ${ }^{2}$, J. C. Desport ${ }^{12}$, P. Jésus ${ }^{12}$

${ }^{1}$ Unité de Nutrition, CHU Limoges, ${ }^{2}$ Inserm, UMR 1094, Faculté de Médecine de Limoges, ${ }^{3}$ AIST87, ${ }^{4}$ Service Médical inter-entreprise des organismes de la Sécurité Sociale du régime général de Haute-Vienne, ${ }^{5}$ Service de Santé au travail de la MSA de la HauteVienne, ${ }^{6}$ Service Médical autonome des établissements Legrand, ${ }^{7}$ Service de Médecine du Travail, CHS Esquirol, ${ }^{8}$ Service de Santé au Travail de l'AMCOBTP, ${ }^{9}$ SUMPPS de Limoges, Limoges, France

Introduction et but de l'étude : Les troubles du comportement alimentaire (TCA) dont l'anorexie mentale (AM), la boulimie nerveuse (BN), l'hyperphagie boulimique (HB) et l'hyperphagie nocturne (HN) sont de pathologies psychiatriques sévères. Le dépistage des TCA peut se faire à l'aide du SCOFF-F. L'objectif de notre travail était i) de dépister les TCA à l'aide du SCOFF-F chez des étudiants et travailleurs actifs de la Haute-Vienne ii) de rechercher les facteurs associés à un SCOFF-F positif.

Matériel et méthodes : Cette étude prospective a été menée pendant 5 mois dans 6 services de Médecine du Travail et un service de Médecine Universitaire. A l'aide d'un auto-questionnaire anonyme des données déclaratives concernant l'âge, l'IMC, la présence de TCA, l'activités physique ont été recueillies. Le SCOFF-F était aussi réalisé (positif si 2 réponses positives sur 5). L'analyse statistique comprenait les tests $t$ de Student, du Chi2 et la régression logistique.

Résultats et Analyse statistique : 964 personnes d'un âge moyen de 34,3 $\pm 12,6$ ans ont été inclus. L'IMC moyen était de $24,4 \pm$ $4,6 \mathrm{~kg} / \mathrm{m}^{2}$, avec $4,7 \%$ de dénutris et $12,2 \%$ d'obèses. $26,7 \%$ étaient étudiants. Les personnes déclaraient avoir une $A M$, une $B N$, une $\mathrm{HB}$ et une $\mathrm{HN}$ dans $2,5 \%, 4,0 \%, 3,6 \%$, et $2,8 \%$ des cas respectivement. Le SCOFF-F était positif dans $13,7 \%$ des cas, et de façon plus importante chez les étudiants $(46,9 \%$ vs $23,6 \%, p<0.0001)$. En analyse multivariée, un âge plus faible, les femmes, un IMC plus haut, une activité sportive à haut niveau, la qualité de vie altérée par les troubles et les TCA déclarés étaient positivement associées à un SCOFF-F positif $(O R=1,03, p=0,01 ; O R=2,17, p=0,009 ; O R=1,09, p=0,002 ; O R=3,00, p=0,002 ; O R=5,3, p<0,0001$ et $\mathrm{OR}=4,20, p<0,0001$ respectivement). Pour les TCA déclarés un SCOFF-F positif était plus spécifiquement associé à la BN et l'HN (OR=4,6, $p=0,001$ et $O R=5,64, p=0,002$ respectivement).

Conclusion : Notre étude fournit les premières données sur le dépistage des TCA chez des adultes en Haute-Vienne. Le SCOFF-F a dépisté prêt de $14 \%$ de TCA, ce qui est cohérent avec la littérature. Dans notre population, il semble plus spécifiquement positif en cas de BN et d'HN déclarées. Même si nous n'avons pas retrouvé d'association en fonction du statut socioprofessionnel, les étudiants restent une population à risque qu'il est facile de dépister en Médecine Universitaire à l'aide du SCOFF-F.

Conflits d'intérêts : Aucun conflit à déclarer 
Comparaison des facteurs et comportements associes des trois catégories de trouble du comportement alimentaire : restrictif, boulimique et compulsif

Y. Sirejacob ${ }^{1, *}$, M. Galmiche ${ }^{234}$, N. Achamrah ${ }^{5}$, L. E. Belmonte ${ }^{5}$, A. Lamarre ${ }^{5}$, S. Grigioni $^{5}$, V. Foloppe ${ }^{5}$, A. Rimbert $^{5}$, T. Saillard $^{5}$, J. Delay ${ }^{5}$, P. Déchelotte ${ }^{56}$, M.-P. Tavolacci ${ }^{16}$

${ }^{1}$ CIC-CRB 1404, Centre Hospitalier Universitaire de Rouen, ${ }^{2}$ Targedys SA, ${ }^{3}$ INSERM 1073, ${ }^{4}$ Institute for Research and Innovation in Biomedicine (IRIB), Université de Rouen, ${ }^{5}$ Nutrition, Centre Hospitalier Universitaire de Rouen, ${ }^{6}$ INSERM U1073, Rouen, France

Introduction et but de l'étude : De multiples études se sont intéressées aux facteurs associés aux Troubles du Comportement Alimentaire (TCA) mais très peu ont comparé simultanément les 3 principales catégories de TCA : Restrictif $(R)$, Boulimique $(B)$ et Compulsif (C).

Le but de l'étude était de comparer les facteurs de survenue et les comportements associés aux 3 groupes de TCA.

Matériel et méthodes : Tous les nouveaux patients avec un TCA du service de Nutrition du CHU de Rouen entre Mai 2013 et Mai 2018 ont complété un auto-questionnaire recueillant les caractéristiques sociodémographiques, les comportements à risque (tabac, alcool, cannabis, cyberaddiction), la survenue d'événements majeurs au cours de la vie, la dépression et l'anxiété, les scores EDI-2 et le ROME III. Les patients ont été classés en 3 catégories larges de TCA : R, B et C. L'analyse univariée a été réalisée avec les tests de Student, du Chi2, exact de Fisher et ANOVA, l'analyse multivariée à l'aide de modèles de régression logistique.

Résultats et Analyse statistique : Le groupe R comprend 71 patients (28,4\%), B $22(8,8 \%)$ et C $157(62,8 \%)$. Les patients R et B sont significativement plus jeunes que les patients $C(R: 31,1$ ans $; B: 32,0 ; C: 38,8 ; p<0,001)$. La proportion d'hommes est plus élevée chez les patients $C$ que chez les patients $R$ et $B(R: 4,2 \% ; B: 4,5 \% ; C: 18,5 \% ; p<0,005)$. II n'existe pas de différence significative pour la consommation de tabac (total : $24,1 \% ; p=0,44$ ), d'alcool (total : 13,8\%; $p=0,13$ ), de cannabis (total : $1,6 \% ; p=1$ ) et dans I'utilisation d'internet (score d'Orman) (total : 1,$9 ; p=0,82$ ). Concernant les événements majeurs liés à la vie, après ajustement sur l'âge et le sexe, plus de patients $C$ ont vécu une perte d'emploi (AOR(IC95\%): 2,26(1,14-4,67); $p=0,02$ ) et ont eu une ou plusieurs naissances ( $A O R(I C 95 \%): 2,38(1,16-4,96) ; p=0,02)$ que les patients R. L'anxiété est plus fréquente chez les patients $R$ et $B$ que les patients $C(R: 66,2 \% ; B: 68,2 \% ; C: 45,2 \% ; p<0,005)$. Il n'existe pas de différence significative pour la dépression ( $R$ : $32,4 \% ; B: 36,4 \% ; C: 31,6 \% ; p=0,85)$. Les patients $R$ sont plus perfectionnistes que les patients $B$ et $C(R: 7,2 ; B: 6,2 ; C: 5,2$; $p<0,01)$ et ont plus peur de la maturité que les patients $C(R: 6,5 ; B: 5,9 ; C: 5,3 ; p<0,05)$ selon le score EDI-2. Les patients $R$ et $B$ sont plus susceptibles de présenter le syndrome de l'intestin irritable $(R: 35,2 \% ; B: 50,0 \% ; C: 14,8 \% ; p<0,001)$.

Conclusion : Dans cette étude, la prévalence de plusieurs facteurs diffère selon le type de TCA. Si aucune différence significative n'existe pour les comportements addictifs, la survenue d'événements majeurs est généralement plus fréquente chez les patients C. A l'inverse, l'anxiété est plus fréquente chez les patients $R$, pouvant être liée à leur profil psychologique, plus perfectionnistes et avec une peur de la maturité plus fréquente.

Remerciements : Targedys

Conflits d'intérêts : Y. Sirejacob: Aucun conflit à déclarer, M. Galmiche a reçu une subvention/soutien de Targedys, N. Achamrah: Aucun conflit à déclarer, L. Belmonte: Aucun conflit à déclarer, A. Lamarre: Aucun conflit à déclarer, S. Grigioni: Aucun conflit à déclarer, V. Foloppe: Aucun conflit à déclarer, A. Rimbert: Aucun conflit à déclarer, T. Saillard: Aucun conflit à déclarer, J. Delay: Aucun conflit à déclarer, P. Déchelotte est actionnaire de Targedys, M.-P. Tavolacci: Aucun conflit à déclarer 


\section{DÉNUTRITION DES ADULTES}

\section{$\mathrm{CO} 33$}

Réversibilité possible de la sarcopénie chez des patients porteurs de BPCO

L. Perrot ${ }^{1, *}$, A. Greil ${ }^{1}$, N. Farigon ${ }^{2}$, F. Costes ${ }^{3}$, D. Caillaud ${ }^{1}$, A. Mulliez ${ }^{4}$, Y. Boirie $^{2}$

${ }^{1}$ Pneumologie, ${ }^{2}$ Nutrition, ${ }^{3}$ Médecine du sport et explorations fonctionnelles, ${ }^{4}$ Délégation à la Recherche Clinique et Innovation, CHU Gabriel-Montpied, Clermont-Ferrand, France

Introduction et but de l'étude : La perte de masse musculaire squelettique peut apparaître précocement dans les maladies chroniques telles que la BPCO. Associée à une diminution des performances physiques, elle définit la sarcopénie. Cependant, son évolution peut être très variable en fonction de différents facteurs d'aggravation à déterminer. L'objectif de cette étude est de décrire la prévalence de la sarcopénie chez des patients ayant une insuffisance respiratoire chronique obstructive pendant une hospitalisation pour exacerbation aiguë de leur maladie. L'évolution de la sarcopénie à six mois, les facteurs l'influençant et la survie à un an de ces patients ont également été étudiés.

Matériel et méthodes : Nous avons inclus prospectivement des patients hospitalisés et mesuré leur composition corporelle, leur force de préhension et leur fonction respiratoire, dont la pression inspiratoire maximale (PIM) représentative de la fonction diaphragmatique. Les mêmes mesures ont été réalisées à six mois en consultation. La sarcopénie était définie par un indice de masse musculaire squelettique (IMS) bas mesuré par impédancemétrie, et une force de préhension (FP) diminuée, sur la base des critères du groupe de travail européen sur la sarcopénie chez les personnes âgées (EWGSOP). Les données de survie ont été collectées dix-huit mois après l'hospitalisation.

Résultats et Analyse statistique : Nous avons analysé les données de cinquante-quatre patients, âgés de $68 \pm 9$ ans et d'IMC 26,9 $\pm 7,8 \mathrm{~kg} / \mathrm{m}^{2}$, avec un VEMS moyen de 1,13 \pm 0,49 L ( $45 \pm 16 \%$ ). Une sarcopénie était observée chez $48 \%$ des patients lors de I'hospitalisation et chez $30 \%$ en consultation. A six mois, l'IMS s'est amélioré davantage chez les hommes que chez les femmes $(+5 \%$ vs $-3 \%, p=0,01)$, il tend à s'améliorer plus chez les patients prenant des compléments nutritionnels oraux ( $+13 \%$ vs $+1 \%$, $p=0,05)$. La FP tend à augmenter plus chez les patients physiquement actifs $(+324 \% v s+7 \%, p=0,05)$. En analyse multivariée, IMS et PIM étaient liés $(p=0,03)$. A un an, le taux de survie était plus faible chez les patients sarcopéniques $(65 \%$ vs $86 \%, p=$ $0,03)$.

Conclusion : La sarcopénie dans l'insuffisance respiratoire chronique obstructive est fréquente lors d'une exacerbation et à l'état stable. II s'agit d'un processus potentiellement réversible nécessitant une intervention ciblée multimodale. Sa prise en charge pourrait réduire la mortalité dans cette population.

Conflits d'intérêts : Aucun conflit à déclarer 


\section{$\mathrm{CO} 34$}

Apport préopératoire d'une boisson glucidique et réponse au stress opératoire après résection intestinale chez le rat N. Tennoune-El Hafaia ${ }^{1,}{ }^{*}$, Z. Desmazières ${ }^{1}$, M. Juchet-Martin ${ }^{1}$, P. Jegatheesan ${ }^{1}$, S. Magassa ${ }^{1}$, N. Neveux ${ }^{12}$, S. Nakib ${ }^{2}$, G. Sarfati $^{2}$, M. Aboubacar ${ }^{1}$, R. Ramassamy ${ }^{1}$, L. Cynober ${ }^{12}$, J.-P. De Bandt ${ }^{12}$

${ }^{1}$ EA 4466 PRETRAM, Faculté de pharmacie, Université Paris Descartes, ${ }^{2}$ Service de Biochimie, Hôpitaux Universitaires Paris Centre, AP-HP, Paris, France

Introduction et but de l'étude : La stratégie ERAS (réhabilitation améliorée après chirurgie) vise à améliorer la récupération postopératoire des patients en minimisant le stress métabolique induit par l'intervention. Elle repose sur la limitation du jeûne préopératoire en administrant des solutions glucidiques jusqu'à $2 \mathrm{~h}$ avant l'intervention. Si cette stratégie réduit la morbidité et la durée de séjour des patients, les données relatives aux mécanismes sous-jacents sont peu nombreuses. L'objectif de notre travail était d'évaluer l'effet de l'administration préopératoire d'une solution glucidique sur l'homéostasie glucidique, la synthèse protéique et la réponse inflammatoire chez le rat opéré.

Matériel et méthodes : Vingt-neuf rats mâles Sprague-Dawley ont été utilisés. La veille de la chirurgie, les animaux ont été mis à jeun et ont reçu soit une solution glucidique $\left(4,4 \mathrm{~g}\right.$ dans de l'eau ; PréLoad ${ }^{\circledR}$, Nestlé Health Science) pour le groupe ERAS ( $\left.n=15\right)$, soit de l'eau ad libitum pour le groupe contrôle $(C ; n=14)$. Le matin suivant, les animaux ont subi une résection intestinale de 5 $\mathrm{cm}$ réalisée à $1 \mathrm{~cm}$ de l'angle de Treitz. Leur prise alimentaire, poids et température corporelle ont été mesurés. Le lendemain, après une nuit de jeûne, les animaux ont été mis à mort soit directement (état de jeûne) soit $2 \mathrm{~h}$ après gavage (état nourri) et injection intrapéritonéale de puromycine (méthode SUNSET) pour la mesure de la synthèse protéique. Le foie, les muscles, la rate, le thymus, le sang et les urines ont été récupérés. Statistiques : ANOVA double voies; seuil de significativité $p<0,05$.

Résultats et Analyse statistique : Le poids des organes, la prise alimentaire postopératoire et le bilan azoté étaient similaires entre les groupes $C$ et ERAS. La synthèse protéique postprandiale dans le tibialis et le soleus était significativement ( $\leq \leq 0.05)$ plus importante dans le groupe ERAS (+135\%). Dans le groupe C, la glycémie était significativement plus basse avant la chirurgie comparée au groupe ERAS ; après chirurgie, cette différence disparaissait. De plus, tandis que dans le groupe $C$ l'insulinémie plasmatique augmentait significativement $(p \leq 0.05)$ après chirurgie, dans le groupe ERAS l'insulinémie comme la glycémie restaient constantes et le score HOMA-IR était significativement $(p \leq 0.05)$ plus faible par rapport au groupe $C$. La réponse inflammatoire était similaire entre les deux groupes.

Conclusion : L'administration préopératoire d'une solution glucidique a un effet bénéfique sur la synthèse protéique $24 \mathrm{~h}$ après l'opération probablement via une amélioration de l'insulinosensibilité, et indépendamment de la réponse inflammatoire à la chirurgie.

Remerciements : Prix Baxter SFNEP 2017

Conflits d'intérêts : N. Tennoune-El Hafaia: Aucun conflit à déclarer, Z. Desmazières: Aucun conflit à déclarer, M. Juchet-Martin: Aucun conflit à déclarer, P. Jegatheesan: Aucun conflit à déclarer, S. Magassa: Aucun conflit à déclarer, N. Neveux: Aucun conflit à déclarer, S. Nakib: Aucun conflit à déclarer, G. Sarfati: Aucun conflit à déclarer, M. Aboubacar: Aucun conflit à déclarer, R. Ramassamy: Aucun conflit à déclarer, L. Cynober est consultant chez Nestlé Health Science, J.-P. De Bandt a reçu une subvention/soutien de Nestlé Health Science 
La compliance à la complémentation nutritionnelle orale diminue le risque d'hospitalisation chez les patients âgés dénutris vivant à domicile, sans augmenter les coûts : Etude ENNIGME

D. Seguy ${ }^{1, *}$, H. Hubert ${ }^{2}$, J. Robert ${ }^{3}$, J.-P. Meunier ${ }^{4}$, O. Guérin ${ }^{5}$, A. Raynaud-Simon ${ }^{6}$

${ }^{1}$ Service de Nutrition, CHU de Lille - Université de Lille, ${ }^{2}$ Département de Santé Publique, Université de Lille, Lille, ${ }^{3}$ Cemka Eval, Bourg-La-Reine, ${ }^{4}$ Axonal-Biostatem, Nanterre, ${ }^{5}$ Service de Gériatrie, CHU de Nice - Université de Nice Sophia Antipolis, Nice, ${ }^{6}$ Service de Médecine Gériatrique, Hôpitaux Bichat et Beaujon - Université Paris Diderot, Paris, France

Introduction et but de l'étude : La dénutrition affecte 5 à $10 \%$ des sujets âgés vivant à domicile. Une intervention nutritionnelle pourrait engendrer des économies de coûts. L'étude ENNIGME a comparé les coûts de santé chez les patients âgés et dénutris vivant à domicile en fonction de la prescription ou non de compléments nutritionnels oraux (CNO).

Matériel et méthodes : Cette étude observationnelle prospective multicentrique a inclus des patients âgés de 70 ans et plus, dénutris (critères HAS) vivant à domicile. Les CNOs étaient prescrits par les médecins généralistes selon leur pratique habituelle. Les coûts de santé et d'hospitalisations ont été enregistrés sur une période de six mois. Les autres données recueillies étaient I'IMC, la perte de poids, l'appétit, les comorbidités, la dépendance, la perception de l'état de santé actuel, la qualité de vie et la compliance aux CNO.

Résultats et Analyse statistique : 191 patients ont été analysés. À l'inclusion, les 133 patients ayant reçu des CNO étaient plus dépendants $(p<0,001)$, avaient un appétit $(p<0,001)$ et une qualité de vie $(p=0,04)$ plus faibles et une moins bonne perception de leur santé $(p=0,02)$ que les 58 patients sans CNO. À 1 mois, l'observance était de 83,5\%. À 6 mois, l'appétit s'est davantage amélioré dans le groupe CNO $(p=0,001)$. Il n'y avait pas de différence de coûts entre les groupes avec et sans CNO (2732 $€$ vs. $2345 €, p=0,48)$. Après ajustement par la méthode du score de propension, les coûts de santé étaient inférieurs dans le groupe CNO, lorsque la consommation d'énergie provenant des CNO était $\geq 500 \mathrm{kcal} / \mathrm{j}$ vs. $<500 \mathrm{kcal} / \mathrm{j}(1389 €$ vs. $3502 €, \mathrm{p}=0,04)$. Les patients avec CNO étaient plus souvent hospitalisés que les patients sans CNO (OR = 2,52 [IC 95\% 1,09 - 5,83], p = 0,03). Mais lorsque que dans le groupe $\mathrm{CNO}$, la consommation des $\mathrm{CNO}$ était $\geq 30 \mathrm{~g}$ de protéines/jour ou $\geq 500 \mathrm{kcal} / \mathrm{j}$, le risque d'hospitalisation était réduit $(O R=0,320$ [IC 95\% 0,121 - 0,845], $p=0,02$ ou $O R=0.185$ [IC95\% 0,063 - 0,547], $p=0,002)$.

Conclusion : Cette étude observationnelle montre que les médecins généralistes prescrivent des CNO chez les sujets âgés dénutris les plus dépendants et les plus anorexiques, avec le plus grand risque d’hospitalisation. La prescription de CNO ne génère pas de coût supplémentaire et une bonne compliance au traitement (apports énergétiques et protidiques élevés) est associée à une réduction du risque d'hospitalisation et des coûts de santé.

Remerciements : Aux autres investigateurs qui ont été impliqués dans cette étude: Dr. Jacques Marty; les membres de l'Alliance 7: Elodie Sebag, Julie Avrillier, Dr. Julien Gautry (Nestlè), Dr. Catherine Dive-Pouletty (Nestlè), Dr. Cécile Bonhomme (Lactalis), Dr. Claire Coplo (Nutricia), Dr. Claude Yvon et Dr. Marc Poterre (Fresenius-Kabi) pour leur contribution et leur aide; Antoine Lafuma (Cemka Eval, Bourg-La-Reine) pour la validation de l'étude économique; Muriel Tounsi (Axonal-Biostatem, Nanterre) pour l'analyse statistique.

Conflits d'intérêts : D. Seguy est consultant chez Alliance 7, Paris, France, H. Hubert est consultant chez Alliance 7, Paris, France, J. Robert est consultant chez Alliance 7, Paris, France, J.-P. Meunier a reçu une subvention/soutien de Alliance 7, Paris, France, O. Guérin est consultant chez Alliance 7, Paris, France, A. Raynaud-Simon est consultant chez Alliance 7, Paris, France 
CO36

Impact de la gastrostomie sur la survie des patients atteints de la sclérose latérale amyotrophique (SLA), en fonction de leur perte pondérale au cours du temps.

M. Vergonjeanne ${ }^{12, *}$, P. Fayemendy ${ }^{123}$, B. Marin ${ }^{124}$, M. Nicol ${ }^{12} 5$, G. Lautrette ${ }^{5}$, H. Sourisseau ${ }^{3}$, P. M. Preux ${ }^{12} 4$, J. C. Desport ${ }^{123}$, P. Couratier ${ }^{125}$, P. Jésus ${ }^{123}$

${ }^{1}$ INSERM UMR 1094, Neuroépidémiologie Tropicale, Faculté de Médecine de Limoges, ${ }^{2}$ Institut de Neuroépidémiologie et Neurologie Tropicale, CNRS FR 3503 GEIST, Université de Limoges, ${ }^{3}$ Unité de Nutrition, ${ }^{4}$ Centre d’Epidémiologie, de Biostatistique et de Méthodologie de la Recherche, ${ }^{5}$ Service de Neurologie, Centre SLA, CHU de Limoges, Limoges, France

Introduction et but de l'étude : La dénutrition est un facteur pronostic péjoratif retrouvé dans 8 à $55 \%$ des patients atteints de Sclérose Latérale Amyotrophique (SLA). Une gastrostomie est recommandée en cas de perte pondérale $>10 \%$, de fausses routes répétées, d'un temps de repas $>45$ min oud'une capacité vitale forcée (CVF) $<50 \%$. L'impact de la gastrostomie sur la survie des patients SLA n'est actuellement pas claire. Les objectifs de notre étude étaient d'évaluer à la fois l'impact de la perte de poids et de la gastrostomie sur la survie, puis de rechercher les potentiels facteurs associés à l'indication pour la pose d'une gastrostomie. Matériel et méthodes : Les patients SLA suivis au centre expert SLA après Avril 2006 ont bénéficiés au diagnostic et au cours du suivi d'évaluation neurologique (forme de début, échelle fonctionnelle bulbaire (EFB), nutritionnelle (poids, indice de masse corporelle (IMC), la composition corporelle en impédancemétrie) et respiratoire (CVF, SNIFF test) étaient relevées. La mise en place d'une gastrotomie et d'une ventilation non invasive, la date de décès ou de trachéotomie étaient aussi notées. L'analyse statistique utilisait les tests de Mann Whitney ou du Chi2. L'analyse de survie utilisait le modèle de Cox. L'analyse multivariée des facteurs associés à la gastrostomie utilisait la régression logistique

Résultats et Analyse statistique : Deux cent quatre vingt quatorze patients ont été inclus, avec un âge au diagnostic de 74,6 ans $[64,8-83,6]$ et un sex-ratio H/F de 1,17. Une gastrostomie était indiquée dans $65,0 \%$ des cas dont $62,8 \%$ avaient accepté la pose du dispositif. Une perte pondérale de $5 \%$ augmentait significativement le risque de décès de $17 \%($ IC95\% 1,09-1,26) $(p<0,0001)$. En revanche la pose d'une gastrostomie n'avait pas d'impact sur la survie des patients (HRa = 1,25 [IC95\% 0,88-1,79]; $p=0,216)$. Lors de la consultation diagnostique, la perte d'un point d'IMC était positivement associée à la mise en place d'une gastrostomie $(\mathrm{ORa}=1,17$ [IC95\% 1,01-1,35] ; $p=0,032)$, de même que la perte d'un point sur l'EFB $(\mathrm{ORa}=1,17$ [IC95\% 1,06-1,32] ; $\mathrm{p}=0,003)$.

Conclusion : Au cours de la SLA, une prise en charge nutritionnelle précoce est indispensable, la perte pondérale étant un facteur péjoratif de survie. Dans notre travail, la gastrostomie n'avait pas d'impact sur la survie, pouvant suggérer une pose trop tardive chez des patients avec une maladie trop avancée. L'indication de la gastrostomie décidée par l'expertise médicale reste un choix pour le patient, pour qui la qualité de vie est un élément indispensable à ne pas perdre de vue.

Remerciements : Association ALAIR

Conflits d'intérêts : Aucun conflit à déclarer 

Calais, Lille, ${ }^{7}$ Délégué SFNCM, Picardie, Amiens, ${ }^{8}$ délégué SFNCM, Rhône Alpes, Lyon, ${ }^{9}$ Comité des Délégués régionaux, SFNCM, Grenoble, France

Introduction et but de l'étude : Les données épidémiologiques concernant la NPD en France sont méconnues et peu précises. L'arrêté de 2014 (1) imposant une prestation pour toute NPD, avec plusieurs forfaits de prise en charge correspondant à des codes de la Liste des Produits et Prestations (LPP), permet actuellement d'obtenir des données fiables.

Matériel et méthodes : Selon la méthode utilisée pour les données épidémiologiques de Nutrition Entérale à Domicile (NED) (2) et à l'aide des codes de LPP et des données des Affections de Longue durée (ALD), il a été demandé aux Directions Régionales du Service Médical de l'Assurance Maladie (DRSM) de l'ensemble du territoire de communiquer $1 /$ le nombre de nouveaux patients pris en charge en NPD en 2016 (ce qui rapporté à la population permet le calcul de l'incidence) 2/le nombre total de patient pris en charge en NPD en 2016 (pour le calcul de la prévalence) 3/ de croiser ces résultats avec les fichiers d'ALD. Les patients ont été regroupés en 3 tranches d'âge (15 à 39 ans, 40 à 65 ans et > 65 ans) et en 5 catégories (cancérologie, neurologie, Maladies intestinales chroniques inflammatoires ( $\mathrm{MICl}$ ), insuffisance d'organe, autres).

Résultats et Analyse statistique : 5 DRSM ont actuellement répondu (Auvergne - Rhöne Alpes, Centre, Hauts de France, ProvenceAlpes-Côte d’Azur - Corse, Réunion) correspondant à une population totale de 14577618 personnes.

\begin{tabular}{|l|c|c|c|c|c|c|c|}
\hline & Incidence & $\begin{array}{c}\text { Préval } \\
\text { Globale } \\
/>12 \text { sem } \\
(/ 100000)\end{array}$ & Cancéro & Neuro & $\mathrm{MICl}$ & Insuf organe & Autres \\
\hline 15 à 39 ans & 3,4 & $4,8 / 2,4$ & $37,5 \%$ & $14,4 \%$ & $28,1 \%$ & $7,5 \%$ & $12,5 \%$ \\
\hline 40 à 64 ans & 23,9 & $32,1 / 7,5$ & $85,6 \%$ & $11,7 \%$ & $4,5 \%$ & $21,3 \%$ & $10,4 \%$ \\
\hline$>65$ ans & 67,7 & $84,0 / 18,1$ & $85,9 \%$ & $10,7 \%$ & $1,1 \%$ & $46,2 \%$ & $17,9 \%$ \\
\hline Total adultes & 25,8 & $33,4 / 10,4$ & $83,6 \%$ & $11,3 \%$ & $3,6 \%$ & $34,6 \%$ & $14,7 \%$ \\
\hline
\end{tabular}

Conclusion : Cette étude effectuée sur une population de près de 15 millions d'habitants de plus de 15 ans retrouve en 2016 une incidence annuelle moyenne de la NPD chez l'adulte de 25,8/100 000 habitants et une prévalence moyenne globale de 33,4/ 100 000 habitants. La prévalence moyenne de NPD > 12 semaines est de 10,4/100 000 habitants. A partir de ces données une estimation nationale de prévalence en France ( 59 millions habitants $>15$ ans) serait d'environ 19700 patients adultes/an pris en charge en NPD dont 6100 pendant plus de 12 semaines. La cancérologie est la première des indications de NPD (85\%). Les MICI sont la seconde cause de NPD dans la tranche d'âge 15 à 39 ans (28\%). Alors que la NPD doit être réservée à l'insuffisance intestinale (1), ces données évoquent une vraisemblable utilisation excessive de la NPD au dépends de la NED notamment en cancérologie.

Références : 1- Arrêté du 16/06/2014. Journal Officiel de la République française, texte 20, 18 juin 2014

2- D.Lescut et al., Nutr Clin Metabol 2013;27:171-177

Remerciements : Les auteurs remercient les DRSM qui ont participé à ce travail

Conflits d'intérêts : Aucun conflit à déclarer 
Evaluation de la fonction rénale chez les patients en nutrition parentérale pour insuffisance intestinale M. Lauverjat ${ }^{1,}{ }^{*}$, L. Dubourg ${ }^{2}$, S. Ait ${ }^{1}$, D. Barnoud ${ }^{1}$, C. Bergoin ${ }^{1}$, C. Peraldi ${ }^{1}$, C. Chambrier ${ }^{1}$

${ }^{1}$ Nutrition Clinique Intensive, Hospices Civils de Lyon, Pierre Bénite, ${ }^{2}$ Explorations fonctionnelles rénales, Hôpital Edouard Herriot, Lyon, France

Introduction et but de l'étude : Les patients en nutrition parentérale à domicile (NPAD) sont à haut risque d'insuffisance rénale (déshydratation chronique, lithiase). Plusieurs formules permettent d'estimer le débit de filtration glomérulaire (DFG) mais elles ne semblent pas toujours fiables pour ces patients. Le but de cette étude est de comparer les valeurs du DFG calculées selon différentes équations et la clairance mesurée (inuline ou iohexol) chez les patients en NPAD.

Matériel et méthodes : étude rétrospective sur 20 ans. Recueil de l'âge, poids, IMC, durée de la NPAD, créatinine et urée plasmatiques. Mesure du DFG par la clairance de l'inuline/iohexol et estimation par les formules Cockroft et Gault (CG), CKD EPI et MDRD. Le CG n'est plus recommandé pour l'estmation du DFG mais encore couramment utilisé. Classification selon les stades d'évolution de la maladie rénale chronique. Les résultats sont exprimés en moyenne \pm SD. Les comparaisons sont réalisées par des tests ANOVA et des tests t appariés, les corrélations avec le coefficient de Pearson. La concordance est analysée par le test de Bland Altman. Calcul de l'exactitude à 30\% (P30\% : pourcentage de valeurs calculées situées dans un intervalle de 70 à $130 \%$ de la valeur mesurée) pour évaluer la précision des équations.

Résultats et Analyse statistique : 125 patients inclus: $46,4 \%$ d'hommes, âge : 53,9 $\pm 17,8$ ans, poids : $57,7 \pm 14,9 \mathrm{~kg}$, IMC : $21,3 \pm$ $4,7,76 \%$ de grêles courts, durée de NP : 75,8 $\pm 75,7$ mois. Le DFG mesuré est de $68,9 \pm 27,0 \mathrm{ml} / \mathrm{min} / 1,73 \mathrm{~m}^{2}$. Le DFG mesuré par les équations de CG, CKD EPI et MDRD est respectivement de 85,3 $\pm 41,6(p<0.001), 87,3 \pm 33,5$ ( $p<0.001)$ et $92,9 \pm 45,1$ $\mathrm{mL} / \mathrm{min} / 1,73 \mathrm{~m}^{2}$ (p<0.001). L'exactitude des formules est faible puisque le P30\% est de 56,8 \% pour le CKD EPI, 51,2\% pour le MDRD et $56 \%$ pour le CG. Avec les formules, la gravité de l'IRC est sous-estimée dans 33,6\% (CG), 43,2\% (CKD EPI) et 38,4\% (MDRD), essentiellement pour les stades calculés 1 et 2 d'IRC. Le biais moyen ( $\left.\mathrm{mL} / \mathrm{min} / 1,73 \mathrm{~m}^{2}\right)$ entre clairance mesurée et calculée est de $16,7 \pm 28,5$ pour $C G, 18,4 \pm 20,4$ pour CKD EPI et $24,0 \pm 30,6$ pour MDRD $(p<0.001)$. Il est inversement corrélé à l'âge ( $p<0.001)$, à la créatininémie $(p<0.001)$ et à l'urémie (CKD EPI et MDRD : $p<0.001, C G: p=0,002)$ pour toutes les formules. L'IMC $n^{\prime}$ a pas d'influence. Le stade de l'IRC n'est pas correctement estimé dans 41,6\%(CG), 45,6\% (CKD EPI) et 42,4\% (MDRD) car ces patients ont souvent des créatininémies basses (moyenne à 55,6 $\mu \mathrm{mol} / \mathrm{L}$ pour les stades 1 et 67,9 $\mu \mathrm{mol} / \mathrm{L}$ pour les stades 2 mesurés).

Conclusion : Chez les patients en NPAD présentant une insuffisance intestinale, les équations de calcul du DFG sont imprécises et n'estiment correctement le stade de l'IRC que pour un peu plus d'un cas sur deux. Le DFG est très souvent surestimé, notamment chez les sujets jeunes pour lesquels les formules retrouvent un stade 1 ou 2, suggérant qu'il faut réaliser chez ces patients des mesures régulières de la fonction rénale par une clairance de l’iohexol.

Conflits d'intérêts : Aucun conflit à déclarer 
CO39

Fréquence des mutations des gènes de la voie leptine/mélanocortines dans l'obésité sévère

J. Le Beyec -Le Bihan ${ }^{1}$, C. Christine Poitou-Bernert ${ }^{23}$, A. Karsenty ${ }^{34}$, V. Pelloux ${ }^{5}$, J. M. Lacorte ${ }^{1}$, P. Tounian $^{34}$, J. M. Oppert $^{23}$, K. Clement ${ }^{23}$, B. Dubern ${ }^{34, *}$

${ }^{1}$ UF génétique obésité et Dyslipidémies, Biochimie Endocrinienne et Oncologique, ${ }^{2}$ Nutrition, Pitié Salpétrière, ${ }^{3} \mathrm{CRMR}$ Syndrome de Prader-Willi et syndromes avec troubles du comportement alimentaire, APHP, Paris Sorbonne Université/ Inserm, Nutriomics, ${ }^{4}$ Nutrition et gastroentérologie pédiatrique, Hopital Trousseau, ${ }^{5}$ Paris Sorbonne Université/ Inserm, Nutriomics, Paris, France

Introduction et but de l'étude : La voie leptine/mélanocortines joue un rôle critique dans le contrôle hypothalamique de la prise alimentaire. Des mutations sur les gènes de cette voie, comme leptine (LEP) et son récepteur (LEPR), proopiomélanocortine (POMC), proconvertase 1 (PCSK1) sont associées à une obésité précoce et sévère avec anomalies endocriniennes notamment en cas d'homozygotie. Les mutations du récepteur de type 4 aux mélanocortines (MC4R) hétérozygotes sont associées à une augmentation du risque d'obésité sévère. L'objectif est de décrire la fréquence des mutations homozygotes et hétérozygotes dans les gènes de la voie leptine/mélanocortine chez des enfants et adultes obèses consultant dans un centre spécialisé.

Matériel et méthodes : Les exons codants des gènes $L E P, L E P R, P O M C, P C S K 1$ et $M C 4 R$ ont été séquencés par la méthode SANGER. Les 6100 patients inclus avaient une obésité sévère $\left(I M C>35 \mathrm{~kg} / \mathrm{m}^{2}\right.$ pour les adultes et Zscore de l'IMC $>+2,5$ DS pour les enfants). MC4R a été séquencé chez 5815 sujets, LEPR chez 1180 patients, LEP chez 800 patients, POMC chez 556 patients et PCSK1 chez 288 patients.

Résultats et Analyse statistique : La fréquence des variants homozygotes était $\leq 1 \%$ avec 13 (1.02\%) variants $L E P R$ (p.C604G, p.L786P, p.H800_N831del, p.Y422H p.T711N, p.535-1G>A, p.P166CfsX7,p.Met1X), 3 (0.4\%) variants $L E P(p . Q 55 X, p . R 105 W$, p.V94M), 1 variant (0.4\%) PCSK1 (c.286-2A>G), 3 variants (0.6\%) POMC (p.R75Profs*44; p.E214G; p.D53G) et 7 variants (0.12\%) MC4R (p.R165Q, p.S127L, p.C277X, p.V166I, p.C271R, p.I170V). Les patients homozygotes avaient une obésité précoce débutée avant 3 ans, une hyperphagie majeure dans la petite enfance et d'autres phénotypes notamment endocriniens associés. La fréquence des variants hétérozygotes était de 2-3\% pour POMC, MC4R et $L E P R$ alors qu'elle était inférieure à $2 \%$ pour PCSK1 $(1,4 \%)$ et $L E P(0,5 \%)$. La présence d'un variant hétérozygote avec conséquence fonctionnelle dans ces gènes était toujours associée à une obésité débutée avant 10 ans sans anomalie endocrine. Quatre patients étaient porteurs de variants combinés hétérozygotes sur au moins deux gènes de la voie (POMC p.D53G et LEP p.V94M $(\mathrm{n}=1)$; LEPR p.W699M et LEP p.V94M $(\mathrm{n}=1)$; MC4R p.S58C et LEPR p.W699M $(\mathrm{n}=2)$ ) avec possible effet cumulatif.

Conclusion : Ce travail confirme l'implication des variants des gènes de la voie leptine/mélanocortines dans l'obésité précoce et sévère ( 2 à $3 \%$ des patients avec obésité sévère) et est actuellement étendu à d'autres gènes modulant cette voie. Ce dépistage est critique car aujourd'hui des options thérapeutiques efficaces par agoniste MC4R (setmelanotide) existent et permettent de restaurer l'activité de cette voie (Clément et al, Nature Med, 2018). Le développement d'outils cliniques aidant au diagnostic et de nouveaux tests moléculaires par NGS étendue aux autres gènes de la voie permettra de diagnostiquer les patients candidats à cette nouvelle thérapeutique.

Conflits d'intérêts : Aucun conflit à déclarer 


\section{CO40}

Effet de la modulation de la digestibilité de l’amidon sur la variabilité glycémique chez les diabétiques de type 2

A.-E. Breyton ${ }^{12, *}$, A. Goux ${ }^{1}$, S. Lambert-Porcheron ${ }^{2}$, A. Meynier ${ }^{1}$, M. Sothier ${ }^{2}$, L. Van Den Berghe ${ }^{2}$, S. Normand $^{2}$, E. Disse ${ }^{2}{ }^{3}$, M. Laville ${ }^{23}$, S. Vinoy ${ }^{1}$, J.-A. Nazare ${ }^{24}$

${ }^{1}$ R\&D Nutrition, Mondelez International, Saclay, ${ }^{2}$ CRNH Rhône-Alpes, CRNH Rhône-Alpes, ${ }^{3}$ Endocrinologie, Nutrition, Diabète, HCL, Pierre-Bénite, ${ }^{4}$ Physiologie, UCBL, Lyon, France

Introduction et but de l'étude : Chez les patients diabétiques de type 2 (DT2), la réduction de la variabilité glycémique et des excursions glycémiques postprandiales pourrait au-delà du niveau d'HbA1c être associée à un meilleur pronostic évolutif. L'alimentation reste la pierre angulaire de la prise en charge du DT2, même sous traitement hypoglycémiant. Des interventions nutritionnelles régulant la réponse glycémique postprandiale pourraient s'avérer pertinentes pour contrôler la variabilité glycémique de ces patients. En effet, il a été démontré que la modulation de la digestibilité de l'amidon chez le sujet sain ou intolérant au glucose permet d'obtenir des différences de réponses postprandiales glycémiques et insulinémiques. Le but de cette étude est de déterminer si l'augmentation de la teneur de l'alimentation en amidon lentement digestible (SDS) chez des DT2 permet de diminuer les hyperglycémies postprandiales et la variabilité glycémique par rapport à une alimentation classique, à faible teneur en amidon lentement digestible.

Matériel et méthodes : Pour cette étude pilote randomisée en cross-over, huit sujets diabétiques de type $2(6.5 \% \leq \mathrm{HbA} 1 \mathrm{c} \leq 8.5 \%$, $22 \leq \mathrm{IMC} \leq 37 \mathrm{~kg} / \mathrm{m}^{2}$, traités par metformine et sitagliptine) ont consommé à 2 reprises pendant une semaine un régime contrôlé contenant des produits amylacés riches ou pauvres en SDS (High-SDS vs Low-SDS, wash-out de 2 semaines). La teneur en SDS de tous les produits amylacés a été mesurée par la méthode SDS. Pendant chaque régime, le profil glycémique a été évalué pendant 6 jours grâce à un CGMS (Continuous Glucose Monitoring System). Les paramètres de variabilité glycémique suivants ont été calculés: MAGE (Amplitude Moyenne des Excursions Glycémiques), DS (Déviation Standard), CV (Coefficient de Variation), MODD (Moyenne des Différences inter-journalières), IQR50 (Interquartile 50) et les temps passés dans la cible (TDLC) en période postprandiale (4h après chaque repas).

Résultats et Analyse statistique : La teneur moyenne en SDS des 2 régimes était significativement différente (Low-SDS 11,4 vs High-SDS 61,4 g/jour ; $p<0,0001)$. La variabilité glycémique était significativement plus faible lors du régime High-SDS comparé au régime Low-SDS pour le MAGE (Low-SDS 79,6 vs High-SDS 61,6 mg/dL, p<0,01), la DS (Low-SDS 33,0 vs High-SDS 27,3 mg/dL, p<0,05), le CV (Low-SDS 24,6 vs High-SDS 20,4\%, p=0,001). Le TDLC post-prandial 140-180 mg/dL était significativement plus élevé lors du régime High-SDS (High-SDS $36 \%$ vs Low-SDS 29\%, p<0.05) alors que le temps passé entre $180-250 \mathrm{mg} / \mathrm{dL}$, était significativement plus élevé lors du régime Low-SDS (Low-SDS $20 \%$ vs High-SDS $15 \%$, p<0,05). Le MODD et l'IQR50 n'étaient pas significativement différents entre les 2 régimes.

Conclusion : Une semaine de régime enrichie en SDS chez le patient DT2 permet de diminuer la variabilité du profil glycémique et d'augmenter le temps passé dans une cible glycémique postprandiale adaptée. La modulation de la digestibilité de l'amidon dans l'alimentation pourrait être utilisée comme un outil nutritionnel simple chez le patient DT2 pour améliorer l'homéostasie glucidique au quotidien.

Conflits d'intérêts : A.-E. Breyton a un conflit d'intérêt avec Thèse CIFRE Mondelez, A. Goux est employé(e) de Mondelez International, S. Lambert-Porcheron: Aucun conflit à déclarer, A. Meynier est employé(e) de Mondelez International, M. Sothier: Aucun conflit à déclarer, L. Van Den Berghe: Aucun conflit à déclarer, S. Normand: Aucun conflit à déclarer, E. Disse: Aucun conflit à déclarer, M. Laville: Aucun conflit à déclarer, S. Vinoy est employé(e) de Mondelez International, J.-A. Nazare: Aucun conflit à déclarer 
CO41

Lors du développement nutritionnel du syndrome métabolique, le métabolisme protéique est différemment affecté dans le foie et les muscles

J.-F. Huneau ${ }^{1,}{ }^{*}$, O. L. Mantha ${ }^{1}$, V. Mathé ${ }^{1}$, D. Hermier ${ }^{1}$, N. Khodorova ${ }^{1}$, F. Mariotti ${ }^{1}$, H. Fouillet $^{1}$

${ }^{1}$ UMR PNCA, AgroParisTech, INRA, Université Paris-Saclay, 75005, Paris, France

Introduction et but de l'étude : On méconnait les altérations du métabolisme protéique tissulaire lors du développement nutritionnel du syndrome métabolique (SM), associant obésité et insulino-résistance. Dans cette étude, en exploitant la variabilité interindividuelle de réponse métabolique à un régime gras et sucré, nous avons exploré les liens entre niveaux d’altérations métaboliques et de protéosynthèses tissulaires.

Matériel et méthodes : 42 rats Wistar mâles, initialement de même poids, ont été nourris avec un régime riche en lipides et sucres (en $\%$ de l'énergie : $21 \%$ protéines, $54 \%$ lipides et $25 \%$ glucides dont $14 \%$ amidon et $11 \%$ saccharose) pendant 4 mois puis euthanasiés pour mesurer leurs masses protéiques et taux de protéosynthèses tissulaires. Pour estimer le niveau d'altérations métaboliques, un score de SM a été calculé comme la moyenne des Zscores de ses différentes composantes (poids, adiposités corporelle et viscérale, HOMA-IR, triglycérides plasmatiques et hépatiques). Pour mesurer la synthèse protéique, après surcharge initiale, les rats ont été exposés au ${ }^{2} \mathrm{H}_{2} \mathrm{O}$ dans l'eau de boisson pendant $2 \mathrm{j}$ avant l'euthanasie, et les enrichissements en ${ }^{2} \mathrm{H}$ ont été mesurés dans l'alanine libre et liée aux protéines tissulaires par GC-MS, pour calculer les taux relatifs (FSR) et absolus (ASR) de protéosynthèse. Les liens entre score de SM et taux tissulaires de protéosynthèse ont été analysés par régression linéaire.

Résultats et Analyse statistique : Après 4 mois de régime d'induction, il y avait des rats minces sains, obèses sains et en SM ( $1 / 3$ de la population pour chacun de ces 3 phénotypes globaux), et le score de SM était continu sur la population (de -1,3 à 1,6). Dans le foie, la synthèse de protéines constitutives et exportées augmentait avec le score de $\mathrm{SM}$, comme attesté par ses associations positives avec l'ASR des protéines hépatiques $(\beta=13,8 \pm 1,8)$ et l'ASR $(\beta=17,1 \pm 6,3)$ et le FSR $(\beta=0,074 \pm 0,025)$ des protéines plasmatiques. Les masses et ASR des protéines hépatiques étaient ainsi $\sim 40 \%$ plus élevées chez les rats en SM que minces ou obèses sains. L'ASR des protéines rénales était aussi positivement associé au score de $\operatorname{SM}(\beta=3,7 \pm 0,8)$. Au contraire, les taux de protéosynthèse intestinaux et musculaires étaient peu ou pas associés au score de SM, avec aucune association pour l'intestin, le cœur et le muscle tibialis et uniquement de faibles associations positives avec l'ASR $(\beta=0,66 \pm 0,24)$ et le FSR $(\beta=0,004 \pm 0,002)$ pour le muscle gastrocnémien.

Conclusion : Les protéosynthèses hépatiques et rénales, mais pas musculaires ni intestinales, augmentent avec le score de SM, sous l'effet des altérations métaboliques et non de l'obésité. Ces différences pourraient s'expliquer par l'entrée de certains tissus en résistance anabolique : l'anabolisme protéique pourrait être d'abord stimulé dans tous les tissus lors de la phase précoce de développement de l'obésité et du SM, qui s'accompagne d'un gain de masses grasse et maigre, avant que l'insulino-résistance ne le limite dans certains tissus. Nous serions ici, à 4 mois d'induction, dans une phase intermédiaire du développement du SM où l'anabolisme protéique pourrait être toujours stimulé dans le foie mais plus dans les muscles et l'intestin, qui entreraient les premiers dans une phase de résistance anabolique.

Conflits d'intérêts : Aucun conflit à déclarer 
C. Guillet ${ }^{1,}{ }^{*}$, A. Masgrau ${ }^{1}$, A. Mishellany-Dutour ${ }^{1}$, A. Blot ${ }^{2}$, B. Pereira ${ }^{3}$, K. Slim $^{4}$, M. Robert ${ }^{5}$, E. Disse ${ }^{6}$, N. Feugier $^{6}$, P. Leruyet $^{7}$, C. Louvet $^{8}$, M. Miolanne ${ }^{9}$, N. Farigon ${ }^{9}$, J. Goudable $^{6}$, M. Laville ${ }^{6}$, Y. Boirie $^{19}$

${ }^{1}$ UNH UMR 1019, Université Clermont Auvergne, ${ }^{2} \mathrm{CRNH}$ Auvergne, ${ }^{3} \mathrm{DRCl}$, ${ }^{4}$ Chirurgie digestive, CHU Clermont-Ferrand, ClermontFerrand, ${ }^{5}$ Chirurgie digestive, Hôpital Edouard Herriot - Hospices Civiles de Lyon, ${ }^{6}$ Endocrinologie Diabétologie et Nutrition, Hôpital Edouard Herriot - Hospices Civiles de Lyon- CRNH Rhône-Alpes, Lyon, ${ }^{7}$ Recherche \& Développement, Lactalis, Retiers, ${ }^{8} \mathrm{CH}$ Vichy, Vichy, ${ }^{9}$ Nutrition Clinique, CHU Clermont-Ferrand, Clermont-Ferrand, France

Introduction et but de l'étude : Après chirurgie bariatrique, un déficit en protéines est fréquemment rapporté chez les patients souffrant d'obésité morbide alors que les besoins en protéines n’ont pas été précisément déterminés dans cette population. L'objectif de cette étude est d'évaluer le besoin protéique moyen (BPM) chez les sujets obèses, avant, 3 mois et 12 mois après la chirurgie bariatrique en utilisant la méthode de bilan azoté.

Matériel et méthodes : Une étude longitudinale prospective, incluant 21 patients en obésité morbide (IMC $\left.43,9 \pm 1,4 \mathrm{~kg} / \mathrm{m}^{2}\right)$ a été mise en place pour évaluer le bilan azoté calculé à partir des mesures des apports alimentaires et des pertes d'azote dans les urines et les selles collectées durant 3 jours avant (M0), 3 mois (M3) et 12 mois (M12) après sleeve gastrectomie ou by-pass gastrique. Une régression linéaire entre les apports en protéines et le bilan azoté a été utilisée pour déterminer la valeur du BPM. Une étude transversale plus large a été réalisée pour valider le BPM chez des patients obèses appariés non opérés $(n=106)$. Les valeurs de BPM sont exprimées en moyenne [Intervalle de confiance (IC) à 95\%] et les autres valeurs en moyenne \pm écart-type à la moyenne.

Résultats et Analyse statistique : Avant la chirurgie, le BPM des patients obèses était de 0,76 [IC à 95\%, 0,66-0,92] g/kg de poids corporel/j dans le groupe expérimental et de $0,74[0,70-0,80] \mathrm{g} / \mathrm{kg} / \mathrm{j}$ dans le groupe de validation. II était de 0,62 [0,51-0,75] g/ $\mathrm{kg} / \mathrm{j}$ à $\mathrm{M} 3$ et de $0,87[0,75-0,98] \mathrm{g} / \mathrm{kg} / \mathrm{j}$ à $\mathrm{M} 12$, sans différence entre les procédures chirurgicales. Les apports spontanés en protéines étaient respectivement de $0,80 \pm 0,05 ; 0,43 \pm 0,03 ; 0,71 \pm 0,04 \mathrm{~g} / \mathrm{kg} / \mathrm{j}$ respectivement à $\mathrm{M} 0, \mathrm{M} 3$ et $\mathrm{M} 12$.

Conclusion : Cette étude indique un changement temporel des besoins en protéines après chirurgie bariatrique quel que soit le type de chirurgie. Les apports spontanés après chirurgie bariatrique ne couvrent pas les besoins en protéines de la plupart des patients, ce qui suggère que des recommandations spécifiques sur les protéines alimentaires doivent être adaptées chez les patients obèses candidats à une chirurgie bariatrique.

Identifiant Clinical Trials: NCT01249326

Conflits d'intérêts : Aucun conflit à déclarer 
Fonctions intestinales endocrines chez des patients à deux ans d'une chirurgie bariatrique de type bypass gastrique en Oméga versus Roux en Y.

D. De Bandt ${ }^{1 *}$, L. Morreau ${ }^{2}$, D. Bergerot ${ }^{3}$, M. Le Gall ${ }^{2}{ }^{4}$, J. M. Lacorte ${ }^{56}$, S. Czernichow ${ }^{17}$, A. Blanchard ${ }^{37}$, J. Le Beyec ${ }^{256}$, C. Carette ${ }^{13}$

${ }^{1}$ Service de nutrition, CSO, HEGP, ${ }^{2}$ U1149, INSERM, ${ }^{3} \mathrm{CIC}, \mathrm{HEGP},{ }^{4}$ Université Paris $7,{ }^{5}$ Biochimie endocrinienne, Pitié-Salpêtrière, ${ }^{6}$ Université Paris 6, ${ }^{7}$ Université Paris 5, Paris, -

Introduction et but de l'étude : La chirurgie bariatrique connaît un essor considérable dans le traitement de l'obésité. Les deux interventions de référence sont la Sleeve Gastrectomie (SG) et le Bypass Gastrique Roux-en-Y (RYGB). Une troisième procédure est de plus en plus réalisée en France, il s'agit du bypass gastrique en oméga (OLGB) techniquement moins difficile à réaliser mais dont on ne sait pas encore clairement si elle est équivalente au RYGB. Le succès du RYGB est en partie associé à un changement des profils de sécrétion des hormones entéro-insulaires, notamment du GLP-1. L'objectif de notre étude est d'explorer les profils de sécrétion de ces hormones chez des patients opérés d'OLGB en les comparant aux profils observés chez des patients opérés de RYGB.

Matériel et méthodes : II s'agit d'une étude clinique : Code projet : K171007J/ N IDRCB : 2017-A02816-47 avec un financement par la fondation ANTADIR (JFN 2017). Au total, il est prévu d'inclure 30 patients non traités par hypoglycémiants à deux ans de leur chirurgie (15 RYGB et 15 OLGB). Des prélèvements sanguins à jeun puis après repas test calibré (à 15, 30, 60, 90 et 120 min) seront réalisés pour mesurer les taux de glycémie et d'hormones entéro-insulaires (insuline, peptide C, glucagon, GLP-1, GIP, GLP2 , glicentine). L'analyse statistique sera faite avec un test non paramétrique de Mann Whitney pour comparer les variables cliniques, biologiques et les AUC moyen (Area Under the Curve) pour chaque paramètre mesuré. Des tests Anova 2 way avec correction de Bonferroni permettront de comparer les cinétiques d'évolution des variables cliniques et biologiques.

Résultats et Analyse statistique : Actuellement, nous avons inclus 12 patients opérés de OLGB et 6 de RYGB. La perte de poids moyenne n'est pas statistiquement différente entre les deux groupes de patients $(p=0,254)$. Il en est de même concernant la moyenne des glycémies à jeun et post repas test et avec les valeurs moyennes des AUC glycémiques similaire ( $p=0,958)$. Les cinétiques de sécrétions de l'insuline, du peptide $C$, de la glicentine et du GLP-1 ont été évaluées chez 4 RYGB et 5 OLGB. Comme attendu, nous observons un pic de sécrétion postprandiale de ces hormones dans les deux groupes de patients sans différence statistique ( $p=0,190 ; p=0,413 ; p=0,905 ; p=1$ RYGB).

Conclusion : II s'agit de la seule étude clinique ayant analysé les secrétions hormonales responsables de l'homéostasie glucidique chez des patients opérés de OLGB en les comparant à l'intervention de référence qu'est le RYGB. Nous n'avons pour le moment pas mis en évidence de différence statistique entre les deux interventions. Ces résultats bien que préliminaires montrent que I'OLGB peut permettre de restaurer des secrétions de GLP-1 notamment de façon comparable au RYGB.

Conflits d'intérêts : Aucun conflit à déclarer 
Identification de nouvelles bactéries probiotiques contre le développement de désordres métaboliques dans un modèle de souris soumises à une diète obésogène

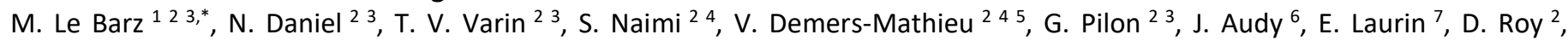
M. C. Urdaci ${ }^{1}$, D. St-Gelais ${ }^{245}$, I. Fliss ${ }^{24}$, A. Marette 23

${ }^{1}$ Laboratoire de Microbiologie et Biochimie Appliquée, Bordeaux Science Agro, Bordeaux, France, ${ }^{2}$ Institut sur la Nutrition et les Aliments Fonctionnels, ${ }^{3} \mathrm{CRIUCPQ},{ }^{4}$ Centre de recherche STELA, Université Laval, Québec, ${ }^{5}$ Centre en recherche et développement de Ste-Hyacinthe, Agriculture et Agroalimentaire Canada, Ste-Hyacinthe, ${ }^{6}$ Agropur Coopérative, Saint-Hubert, ${ }^{7}$ Ultima Foods, Granby, Canada

Introduction et but de l'étude : Le tractus gastro-intestinal représente l'interface entre l'alimentation et l'organisme hôte. Chez l'individu obèse, cet écosystème est altéré et associé à une augmentation de l'inflammation et des désordres métaboliques. Dans ce contexte, l'objectif est d'étudier in vivo les effets potentiellement anti-obésité de souches bactériennes préalablement isolées, testées in vitro et sélectionnées selon leur propriétés anti-inflammatoires.

Matériel et méthodes : Des souris mâles C57BL/6 ont été soumises à un régime riche en gras et en sucre (HFHS) et gavées avec le véhicule ou l'une des 6 souches suivantes : P35 (Propionibacterium freudenreichii), Lb38 (Lactobacillus plantarum), Lb79 (Lactobacillus paracasei/casei), Lb102 (Lactobacillus rhamnosus), Bf26 et Bf141 (2 souches de Bifidobacterium animalis spp lactis) à $10^{9} \mathrm{ufc} /$ jour pendant 8 semaines. Au cours du protocole, des tests physiologiques ont été réalisés, la prise de poids et de nourriture ont été mesurées 3 fois/semaine, et des échantillons sanguins, fécaux et tissulaires ont été collectés.

Résultats et Analyse statistique : L'ANOVA à une voie, comparant les effets de chaque souche par rapport au groupe contrôle HFHS (test post hoc Dunnett), montre que les souches P35, Lb38, Lb102 et Bf141 préviennent significativement la prise de poids normalement induite par la diète HFHS, sans affecter la prise alimentaire. Ces souches améliorent également la tolérance au glucose et la sensibilité à l'insuline, et leurs effets sont associés à une diminution de l'accumulation de graisse viscérale (eWAT). La souche P35 a particulièrement permis de prévenir le dépôt de graisse ectopique au niveau du tissu adipeux brun, qui est impliqué dans la thermogenèse. L'analyse du statut inflammatoire du eWAT a mis en évidence une baisse significative de la production de MCP-1, chimiokine impliquée dans l'infiltration des macrophages. D'autre part, l'analyse de l'ARNm au niveau du côlon montre que la souche Lb102 augmente significativement l'expression des mucines et des protéines de jonction serrées, suggérant une diminution de la perméabilité intestinale induite par la diète HFHS. De plus, P35, Lb102 et Bf141 diminuent l'expression du récepteur $c b 1$ du système endocannabinoïde, démontrant une amélioration de la santé intestinale. De manière intéressante, aucune des souches bactériennes n'a permis de renverser la dysbiose du microbiote intestinal induite par la diète HFHS, au niveau des grands groupes bactériens, ce qui est en accord avec la littérature récente.

Conclusion : Par des analyses comparatives à la fois in vitro et in vivo, 3 souches bactériennes d'intérêt ont finalement été identifiées pour leurs effets probiotiques dans la prévention du développement de l'obésité, ainsi que des désordres métaboliques et inflammatoires associés.

Remerciements : Nous remercions Agropur Coopérative, Ultima Foods et le FRQ-NT pour leur soutien financier.

Conflits d'intérêts : M. Le Barz: Aucun conflit à déclarer, N. Daniel: Aucun conflit à déclarer, T. Varin: Aucun conflit à déclarer, S. Naimi: Aucun conflit à déclarer, V. Demers-Mathieu: Aucun conflit à déclarer, G. Pilon: Aucun conflit à déclarer, J. Audy est employé(e) de Agropur Coopérative qui a participé aux financements de l'étude, Julie Audy a participé à la sélection des souches bactériennes et à la production de celles-ci pour l'étude, a pris part aux discussions en amont du projet in vivo quant aux contraintes industrielles mais n'a pas pris part dans l'interprétation des résultats, E. Laurin est employé(e) de Ultima Foods Agropur Coopérative qui a participé aux financements de l'étude, Emilie Laurin a pris part aux discussions en amont du projet in vivo quant aux contraintes industrielles mais n'a pas pris part dans l'interprétation des résultats, D. Roy: Aucun conflit à déclarer, M. Urdaci: Aucun conflit à déclarer, D. St-Gelais: Aucun conflit à déclarer, I. Fliss: Aucun conflit à déclarer, A. Marette: Aucun conflit à déclarer 
Un nouveau probiotique, Hafnia alvei, réduit le gain de poids dans deux modèles murins d'obésité en agissant sur les voies centrales et périphériques de l'homéostasie énergétique.

N. Lucas ${ }^{1}$, S. Azhar ${ }^{1}$, C. Deroissart ${ }^{123}$, M.-A. Le Solliec ${ }^{123}$, M. Dominique ${ }^{123}$, J. Rondeaux ${ }^{123}$, S. Nobis ${ }^{34}$, C. Guérin $^{23}$, F. Léon ${ }^{34}$, J.-C. do Rego ${ }^{4}$, P. Déchelotte ${ }^{1235}$, S. Fetissov ${ }^{123}$, G. Lambert $^{1}$, R. Legrand ${ }^{1, *}$

${ }^{1}$ TargEDys SA, ${ }^{2}$ Inserm UMR1073 - nutrition, inflammation et dysfonction de l'axe intestin-cerveau, ${ }^{3}$ Institute for Research and Innovation in Biomedicine (IRIB), ${ }^{4}$ Service Commun d'Analyse Comportementale (SCAC), ${ }^{5}$ Centre Hospitalier Universitaire Charles Nicolle, Normandie Université, Rouen, France

Introduction et but de l'étude : La prévalence du surpoids et l'obésité est en sévère augmentation à travers le Monde et expose à de nombreuses comorbidités. Dans l'apparition et l'évolution de ces pathologies multifactorielles, le microbiote intestinal pourrait avoir un rôle clé. En effet, des protéines bactériennes pourraient interagir avec l'hôte en présentant un mimétisme avec des facteurs impliqués dans le contrôle de la prise alimentaire. Sécrétée par les entérobactéries, la protéine ClpB (pour caseinolytic protease $B$ ) possède notamment une réaction croisée avec les auto-anticorps naturels dirigés contre le neuropeptide anorexigène

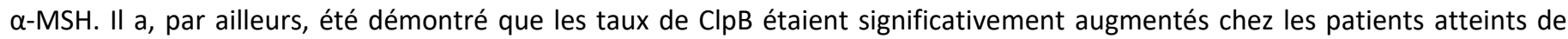
troubles du comportement alimentaire, soulignant leur rôle crucial dans l'homéostasie énergétique. L'objectif de cette étude est donc d'évaluer les effets d'une entérobactérie alimentaire optimisée, Hafnia alvei 4597, sur le poids corporel de deux modèles murins d'obésité.

Matériel et méthodes : Des souris mâles $\mathrm{C} 57 \mathrm{Bl} / 6$ ont été soumises à une induction de 15 semaines à une diète riche en lipides (D12451, Research Diets) puis traitées pendant 6 semaines par gavage intragastrique quotidien de la souche Hafnia alvei 4597 en culture $(n=24)$ et comparées au groupe contrôle $(n=22)$. Parallèlement, des souris $O b / O b(n=15)$ ont été traitées de la même façon pendant 3 semaines. A la fin des deux protocoles expérimentaux, les variations de poids corporel et de masse grasse ont été analysés. Les taux de ClpB et d'autoanticorps dirigés contre l' $\alpha-\mathrm{MSH} / \mathrm{ClpB}$ ont été mesurés par méthode ELISA, l'expression hypothalamique d'AgRP a été évaluée par RT-PCR et la lipolyse adipocytaire par mesure de la production de pHSL (hormonesensitive lipase phosphorylée) par western blot.

Résultats et Analyse statistique : Les résultats montrent que le traitement par Hafnia alvei diminuait significativement ( 50\%, $p<0,01$ ) le gain de poids et améliorait la composition corporelle dans les deux modèles (réduction du rapport masse grasse / masse maigre). Au niveau plasmatique, l'augmentation des taux de ClpB était est associée à une stimulation de la réponse auto-immune anti- $\alpha-M S H$. Plus encore, il a été observé une inhibition de la voie hypothalamique orexigène dépendant de l'AgRP ( $<<0,05)$ associée à une diminution significative $(p<0,001)$ de la prise alimentaire cumulée des souris hyperphage Ob/Ob. Enfin, une activation de la lipolyse a été mise en évidence chez les souris Ob/Ob mais pas dans le modèle régime hyperlipidique.

Conclusion : Ces résultats permettent de conclure que la souche optimisée Hafnia alvei 4597 est un bon candidat pour le développement d'un complément alimentaire probiotique innovant visant à réduire la prise de poids en agissant à la fois au niveau central et périphérique sur l’homéostasie énergétique.

Conflits d'intérêts : N. Lucas est employé(e) de TargEDys SA, S. Azhar est employé(e) de TargEDys SA, C. Deroissart est employé(e) de TargEDys SA, M.-A. Le Solliec est employé(e) de TargEDys SA, M. Dominique: Aucun conflit à déclarer, J. Rondeaux est employé(e) de TargEDys SA, S. Nobis: Aucun conflit à déclarer, C. Guérin: Aucun conflit à déclarer, F. Léon: Aucun conflit à déclarer, J.-C. do Rego: Aucun conflit à déclarer, P. Déchelotte est consultant chez TargEDys SA, S. Fetissov est consultant chez TargEDys SA, G. Lambert est employé(e) de TargEDys SA, R. Legrand est employé(e) de TargEDys SA 


\section{CO46}

Rôle de la protéine bactérienne ClpB et d'un de ses fragments peptidique dans la régulation de la prise alimentaire

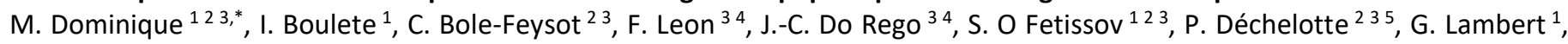
R. Legrand ${ }^{1}, \mathrm{~N}$. Lucas $^{1}$

${ }^{1}$ TargEDys SA, ${ }^{2}$ Nutrition, Inflammation et Dysfonctions Intestin Cerveau, INSERM U1073, ${ }^{3}$ IRIB, ${ }^{4}$ Service Commun d'Analyse Comportementale (SCAC), ${ }^{5}$ Nutrition, CHU Charles Nicolle, Rouen, France

Introduction et but de l'étude : L'obésité et les Troubles du Comportement Alimentaire (TCA) sont des problèmes majeurs de santé publique, dont la physiopathologie est multifactorielle. La régulation du comportement alimentaire implique des boucles neurohormonales liées à l'axe microbiote-intestin-cerveau; une dysbiose intestinale peut donc contribuer aux modifications des signaux de faim ou de satiété. Au cours de l'anorexie mentale, une augmentation des Enterobacteriaceae est observée. Parmi elles, la bactérie Escherichia coli ( $E$. coli) produit une protéine présentant une séquence mimétique avec le neuropeptide anorexigène $\alpha$-melanocyte-stimulating hormone $(\alpha-\mathrm{MSH})$ : la caseinolytic protease $B(\mathrm{ClpB})$. Des études récentes ont montré que la $\mathrm{ClpB}$ possède un effet inhibiteur sur la prise alimentaire, pouvant être lié à l'activation de récepteurs aux mélanocortines au niveau des cellules entéroendocrines intestinales entraînant la libération de peptides satiétogènes (peptide YY, PYY et glucagonlike peptide 1, GLP-1). A l'état physiologique, elle peut être clivée en plusieurs fragments dont les effets biologiques sont méconnus. L'objectif de cette étude a été de rechercher si des fragments de ClpB exercent un effet satiétogène.

Matériel et méthodes : Afin d'évaluer les effets de la ClpB et de ses fragments sur la production de PYY, une culture primaire de cellules intestinales issus de colons de rats mâles Sprague-Dawley a été établie. Des protéines extraites d'E. coli $W T$ et d'E. coli $\triangle \mathrm{ClpB}$ ainsi que des concentrations croissantes de ClpB recombinante ont été incubées sur cette culture. Le dosage de PYY a été mesurée par ELISA. Les différents fragments de ClpB ont été identifiés par Western-Blot à l'aide d'anticorps polyclonaux anti$\alpha-\mathrm{MSH}$ ou anti-ClpB au niveau hypothalamique, intestinal et plasmatique chez le Rat et la Souris. Enfin, l'impact de l'administration intrapéritonéale de la protéine totale et de son fragment à $25 \mathrm{kDa}$ sur la prise alimentaire a été mesuré chez des souris mâles C57BI/6.

Résultats et Analyse statistique : Les résultats montrent que la ClpB stimule la sécrétion de PYY par des cellules primaires intestinales de Rat (Test de Mann et Whitney, $p<0.001, C \mid p B>13 n M)$. L'étude par Western Blot révèle la présence d'un fragment à $25 \mathrm{kDa}$ qui est retrouvé au niveau plasmatique, hypothalamique et colique chez les rongeurs et l'Homme. Les injections intrapéritonéales révèlent un effet aigu avec une diminution de la prise alimentaire perdurant $2 \mathrm{~h}$ après l'injection de la Clp $\mathrm{B}$ totale et de son fragment chez la souris $\mathrm{C} 57 \mathrm{BI} / 6$ ainsi que chez la souris génétiquement obèse $O b / O b(A N O V A$ 2,p<0.01,p<0.001, 90 à 120 min et $\mathrm{p}<0.05$ à $120 \mathrm{~min}$ respectivement).

Conclusion : En conclusion, ce travail conforte le rôle de la protéine ClpB dans la régulation de la satiété au sein de l'axe microbioteintestin-cerveau. La ClpB stimule positivement la production de PYY et son fragment 25kDa a des effets inhibiteurs comparables sur la prise alimentaire. Ces résultats ouvrent des perspectives d'applications thérapeutiques.

Conflits d'intérêts : M. Dominique: Aucun conflit à déclarer, I. Boulete: Aucun conflit à déclarer, C. Bole-Feysot: Aucun conflit à déclarer, F. Leon: Aucun conflit à déclarer, J.-C. Do Rego: Aucun conflit à déclarer, S. O Fetissov est consultant chez TargEDys SA, P. Déchelotte est consultant chez TargEDys SA, G. Lambert est employé(e) de TargEDys SA, R. Legrand est employé(e) de TargEDys SA, N. Lucas est employé(e) de TargEDys SA 
Introduction et but de l'étude : La plupart des organisations professionnelles et nationales, y compris l'Organisation mondiale de la santé et, en France, la Haute Autorité de Santé, recommandent une supplémentation en acide folique pour les femmes en période périconceptionnelle (4 semaines avant la conception et 8 semaines après la conception). Les résultats de diverses études européennes ont montré des niveaux élevés de non-suivi de cette recommandation. L'objectif de la présente étude était d'identifier les caractéristiques sociodémographiques liées au suivi de la recommandation concernant la supplémentation en acide folique.

Matériel et méthodes : Les analyses ont reposé sur les données de 14156 femmes de la cohorte de naissance nationale ELFE, collectées en 2011. Le moment de la supplémentation en acide folique a été évalué lors de l'entretien en face-à-face mené à la maternité et les caractéristiques sociodémographiques détaillées ont été recueillies après deux mois. L'association entre le respect de la recommandation sur la supplémentation en acide folique (pas de supplémentation ; supplémentation pendant la période périconceptionnelle : avant la conception ou durant les deux premiers mois de grossesse ; supplémentation seulement après la période périconceptionnelle) et les caractéristiques sociodémographiques a été étudiée en utilisant une régression logistique multinomiale.

Résultats et Analyse statistique : Seulement $26 \%$ des femmes enceintes ont reçu une supplémentation en acide folique pendant la période périconceptionnelle, $10 \%$ pendant la grossesse mais après la période périconceptionnelle, et $64 \%$ des femmes n'ont reçu aucune supplémentation. Dans les analyses multivariées, un jeune âge maternel, un faible niveau d'études, un faible revenu familial, la multiparité, un surpoids maternel et un tabagisme maternel pendant la grossesse étaient associés à une plus faible probabilité de supplémentation en acide folique pendant la période périconceptionnelle $\left(O R_{<25}\right.$ ans vs. $25-29$ ans $=0,74[0,67 ; 0,82]$; OR études primaires vs. $\mathrm{BAC}+5=0,43[0,35 ; 0,52] ; \mathrm{OR}$ 4ème enfant vs. 1er enfant $=0,41[0,33 ; 0,51] ; \mathrm{OR}$ obésité vs. poids normal $=0,74[0,65 ; 0,85]$; $\mathrm{OR}$ fumeuse toute la grossesse vs. non-fumeuse $=0,71[0,63 ; 0,79])$. A l'inverse, les femmes qui ont arrêté de fumé juste avant leur grossesse avaient une probabilité plus élevée de recevoir une supplémentation en acide folique pendant la période périconceptionnelle $(O R$ fumeuse avant grossesse vs. non-fumeuse $=1,10[1,01 ; 1,20])$. Ces associations persistaient après exclusion des grossesses non planifiées Les femmes immigrées et présentant une insuffisance pondérale étaient plus susceptibles de recevoir une supplémentation en acide folique uniquement après la période périconceptionnelle. (OR immigrée vs. née en France=1,33 $[1,12 ; 1,57] ; O R$ insuffisance pondérale vs. poids normal= $1,30[1,07 ; 1,58])$.

Conclusion : Notre étude confirme un faible suivi de la recommandation concernant la supplémentation en acide folique au cours de la période périconceptionnelle en France et de grandes disparités sociales dans le suivi de cette recommandation.

Conflits d'intérêts : Aucun conflit à déclarer 
Introduction et but de l'étude : Le lait maternel véhicule des éléments du système immunitaire maternel au nourrisson, permettant à celui-ci de développer le sien, encore immature. L'objectif de ce travail était d'étudier les liens entre l'allaitement et les trajectoires d'infections jusqu'à 2 ans (rhumes - rhinopharyngites, diarrhées, otites, bronchites - bronchiolites) d'une part, et les trajectoires de symptômes allergiques jusqu'à 8 ans (sifflements, éruptions cutanés, crises d'asthme) d'autre part.

Matériel et méthodes : Les données de ce travail ont été extraites de la cohorte mère-enfant EDEN. L'allaitement, collecté à la maternité, 4 mois, 8 mois, 12 mois et 2 ans, a été traité de deux façons différentes ; 1 / l'enfant a reçu du lait maternel (oui vs non) 2/ durée d'allaitement (moins d'1 mois, [1 - 4 mois[, 4 mois ou plus). Les trajectoires de rhumes - rhinopharyngites et de diarrhées ont été modélisées de 4 à 12 mois, celles d'otites et de bronchites - bronchiolites de 4 mois à 2 ans, celles de sifflements et d'éruptions cutanées ont été modélisées de 8 mois à 8 ans et celles d'asthme de 2 ans à 8 ans, à partir des auto-questionnaires parentaux posés à 4 mois, 8 mois, 12 mois, 2 ans, 3 ans, 4 ans, 5 ans et 8 ans. Les associations entre l'allaitement et les trajectoires d'infections et de symptômes allergiques ont été étudiées à l'aide de régressions logistiques multinomiales ajustées notamment sur la parité, l'âge d'entrée en garde collective, le sexe, l'âge gestationnel, le mode d'accouchement et les antécédents familiaux d'allergie (parents et fratrie), ainsi que sur des variables sociodémographiques de la famille (centre, niveau d'étude maternel, revenus, ...). Les enfants ayant moins de deux points de suivi pour un des symptômes considérés ou des données manquantes sur une des variables d'ajustement ont été exclus des analyses. Les analyses ont été menées en considérant d'une part les infections $(n=1603)$ et d'autre part les symptômes allergiques $(n=1377)$ jusqu'à 8 ans.

Résultats et Analyse statistique : Concernant les infections jusqu'à 2 ans, les enfants n'ayant jamais été allaités avaient un risque plus élevé d'avoir des diarrhées dans les premiers mois de vie que ceux qui avaient été allaités. Par rapport aux enfants allaités au moins 4 mois, les enfants non allaités ou allaités moins de 4 mois étaient plus à risque d'avoir des diarrhées dans les premiers mois de vie avec une relation dose-effet inverse selon la durée d'allaitement. Aucune association n'a été retrouvée entre l'allaitement et les autres infections étudiées (rhumes - rhinopharyngites, otites et bronchites - bronchiolites), que l'allaitement soit considéré comme une variable binaire ou classé selon sa durée.

Concernant les symptômes allergiques jusqu'à 8 ans, aucune association n'a été retrouvée entre l'allaitement et les trajectoires de symptômes allergiques dans l'enfance, même après stratification sur les antécédents familiaux d'allergie.

Conclusion : Dans la cohorte mère-enfant EDEN, un rôle protecteur de l'allaitement maternel vis-à-vis des diarrhées dans les premiers mois de vie a été suggéé mais pas vis-à-vis des autres infections infantiles considérées ou des symptômes allergiques dans l'enfance.

Conflits d'intérêts : Aucun conflit à déclarer 
L'abondance naturelle en $15 \mathrm{~N}$ dans les cheveux est un marqueur d'exposition alimentaire et de malnutrition aiguë chez l'enfant en bas âge

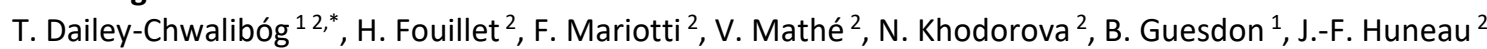

${ }^{1}$ Action Contre la Faim (ACF), ${ }^{2}$ UMR PNCA, AgroParisTech, INRA, Université Paris-Saclay, Paris, France

Introduction et but de l'étude : L'enrichissement naturel en ${ }^{15} \mathrm{~N}\left(\delta^{15} \mathrm{~N}\right)$ des cheveux est un marqueur reconnu d'exposition alimentaire aux protéines d'origine animale, mais plusieurs études suggèrent qu'il augmente aussi lors d'épisodes de dénutrition. Ce travail visait à évaluer les liens entre $\delta^{15} \mathrm{~N}$ des cheveux, exposition alimentaire et statut nutritionnel chez de jeunes enfants.

Matériel et méthodes : L'étude a pris appui sur une enquête transversale plus large menée par ACF sur un échantillon de 657 enfants représentatif de la population d'enfants de 6 à 60 mois vivant dans le district de Cox's Bazar, au Bangladesh. Le poids, la taille et le périmètre médio-brachial $(\mathrm{PMB})$ ont été mesurés et utilisés pour objectiver la présence d'une malnutrition aiguë ( $\mathrm{z}$ score du PMB ou de l'indice poids-pour-taille) ou chronique (z-score de l'indice taille-pour-âge). La présence de signes cliniques d'émaciation a été recherchée et les mères ont été interrogées sur l'évolution du poids, de la consommation alimentaire et sur la fréquence des diarrhées et vomissements au cours des 15 derniers jours. L'alimentation des enfants (allaitement et diversité alimentaire sur 7 points) a été caractérisée. Sur 329 enfants aléatoirement sélectionnés dans l'échantillon initial (fraction d'échantillonnage de 50\%), 254 présentaient des cheveux qui ont été prélevés pour mesurer le $\delta^{15} \mathrm{~N}$ de la section de $1 \mathrm{~cm}$ la plus proche du scalp par spectrométrie de masse à ratio isotopique.

Résultats et Analyse statistique : Parmi ces 254 enfants (34 \pm 15 mois), 32,5\% étaient allaités partiellement ou totalement, $21 \%$ présentaient une malnutrition aiguë (7,5\% sévère et $13,5 \%$ modérée) et $38 \%$ une malnutrition chronique ( $11 \%$ sévère et $27 \%$ modérée). Le $\delta^{15} \mathrm{~N}$ des cheveux $\left(8,74 \pm 1,23 \%\right.$ o était négativement associé à l'âge $\left(7,70+4,49 * \exp \left(-0,05^{*}\right.\right.$ âge $\left.), P<0,0001\right)$. Après ajustement pour l'âge, le $\delta^{15} \mathrm{~N}$ était plus élevé chez les enfants allaités que non allaités $\left(\delta^{15} \mathrm{~N}=9,85 \%\right.$ ovs $8,18 \%$ o). Le score de diversité alimentaire reflétant le niveau de diversification était négativement associé au $\delta^{15} \mathrm{~N}$ chez les enfants allaités $(\beta=-0,22 \%$, $P<0,01)$, mais pas non allaités.

Le $\delta^{15} \mathrm{~N}$ tendait à être plus élevé chez les enfants présentant une malnutrition aiguë $\left(\delta^{15} \mathrm{~N}=8,97\right.$ vs $\left.8,65, P=0,1\right)$ ou ceux présentant des signes cliniques d'émaciation ( $\delta^{15} \mathrm{~N}=8,98$ vs $8,64, P=0,07$ ) par rapport aux enfants sans malnutrition aiguë ou émaciation, respectivement. Le $\delta^{15} \mathrm{~N}$ était également associé positivement à la diminution de la consommation alimentaire, à la perte de poids, à la fréquence des diarrhées et des vomissements rapportées par les mères. La présence d'une malnutrition chronique n'était pas associée à une modification du $\delta^{15} \mathrm{~N}$.

Conclusion : L'âge et l'allaitement sont les principaux facteurs explicatifs du $\delta^{15} \mathrm{~N}$ des cheveux chez l'enfant de 6 à 60 mois. Dans une moindre mesure, le $\delta^{15} \mathrm{~N}$ est affecté par la présence d'une malnutrition aiguë objectivée par des critères anthropométrique ou cliniques et/ou des facteurs pouvant conduire à une malnutrition aiguë. Ces données suggèrent que le $\delta^{15} \mathrm{~N}$ des cheveux est un marqueur d'exposition alimentaire et de statut nutritionnel chez l'enfant en bas âge, et appellent des études complémentaires avec suivi longitudinal des enfants.

Conflits d'intérêts : Aucun conflit à déclarer 
Lors de la prise en charge nutritionnelle de la malnutrition aiguë sévère chez l'enfant en bas âge, l'évolution de l'abondance naturelle en $15 \mathrm{~N}$ le long des cheveux signe l'historique des gains anthropométriques

T. Dailey-Chwalibóg ${ }^{12,{ }^{*}}$, J.-F. Huneau ${ }^{1}$, F. Mariotti ${ }^{1}$, V. Mathé ${ }^{1}$, N. Khodorova ${ }^{1}$, B. Guesdon ${ }^{2}$, H. Fouillet $^{1}$

${ }^{1}$ UMR PNCA, AgroParisTech, INRA, Université Paris-Saclay, ${ }^{2}$ Action Contre la Faim (ACF), Paris, France

Introduction et but de l'étude : Comme l'abondance naturelle en ${ }^{15} \mathrm{~N}\left(\delta^{15} \mathrm{~N}\right)$ dans l'organisme dépend de l'exposition alimentaire et du statut nutritionnel, les variations de $\delta^{15} \mathrm{~N}$ le long de la tige capillaire pourraient servir à retracer leurs historiques. Cette étude visait à en apporter la preuve de concept dans le contexte de la prise en charge nutritionnelle de la malnutrition aiguë sévère (MAS) chez le jeune enfant.

Matériel et méthodes : L'étude a pris appui sur une étude longitudinale menée par Action Contre la Faim (ACF) à Cox's Bazar, au Bangladesh, sur un échantillon de 144 jeunes enfants souffrant de MAS sans complications médicales. La MAS a été diagnostiquée selon les 2 indicateurs anthropométriques de l'OMS : le périmètre médio-brachial (PMB, < $115 \mathrm{~mm}$ ) et/ou l'indice poids-pourtaille (IPT, < -3 écart-type de la médiane de référence). Les enfants MAS ont été traités selon le modèle de prise en charge ambulatoire, en recevant une ration adaptée d'un aliment thérapeutique prêt à l'emploi (ATPE) chaque semaine pendant 2 mois, jusqu'à la guérison. À l'admission et toutes les deux semaines, des mesures anthropométriques ont été effectuées (poids, taille, $\mathrm{PMB} .$. ) et une mèche de cheveux a été rasée dans une même zone prédéfinie, de manière à prélever les cheveux présents à l'admission puis ayant repoussés lors de chaque quinzaine. Le $\delta^{15} \mathrm{~N}$ de chaque section de cheveux a été mesuré par spectrométrie de masse à ratio isotopique.

Résultats et Analyse statistique : Chez les 68 enfants analysés jusqu'ici (56\% de sexe féminin, âge de $19 \pm 14$ mois, poids de 6,7 $\pm 2,1 \mathrm{~kg}$, taille de $72,6 \pm 12,2 \mathrm{~cm}$ et PMB de $114 \pm 8,6 \mathrm{~mm}$ à l'admission), au cours de la prise en charge nutritionnelle, le $\delta^{15} \mathrm{~N}$ des cheveux baissait modérément lors de la $1^{\text {ère }}$ quinzaine $(-0,12 \pm 0,34 \%, P<0,01)$, de manière plus marquée lors de la $2^{\text {ème }}$ quinzaine $(-0,33 \pm 0,43 \%$ o, $P<0,0001)$, puis marginalement lors de la $3^{\text {ème }}$ quinzaine $(-0,09 \pm 0,36 \%$ o, $P=0,053)$ et ne variait plus lors de la $4^{\text {ème }}$ quinzaine. Cette évolution du $\delta^{15} \mathrm{~N}$ était inversement corrélée à l'évolution de l'indice poids-pour-âge (IPA, $\left.r=-0,30, P=0,04\right)$, de l'indice de masse corporelle (IMC)-pour-âge (IMCA, $r=-0,25, P=0,10)$ et de I'IPT $(r=-0,25, P=0,10)$. Ces corrélations inverses entre variations isotopiques et gains anthropométriques étaient trouvées aussi bien sur l'ensemble de la période de prise en charge nutritionnelle que sa période la plus efficace (les plus grands gains anthropométriques de la $1^{\text {ère }}$ quinzaine se reflétant dans la plus grande baisse de $\delta^{15} \mathrm{~N}$ de la $2^{\text {ème }}$ quinzaine, du fait du délai de pousse).

Conclusion : Après la dénutrition qui avait probablement induit une augmentation du $\delta^{15} \mathrm{~N}$ du fait de la fonte protéique subie, la renutrition entraine une baisse (renormalisation) du $\delta^{15} \mathrm{~N}$ qui est bien proportionnelle à l'augmentation (renormalisation) de certains indicateurs anthropométriques. Les corrélations étaient plus marquées avec les indicateurs liés au poids qu'avec ceux liés à la masse musculaire comme le PMB. Ces résultats partiels, issus de l'analyse de $\sim 50 \%$ des enfants, seront complétés pour augmenter la puissance de l'étude.

Conflits d'intérêts : Aucun conflit à déclarer 
CO51

Épidémiologie de la nutrition artificielle a domicile en pédiatrie dans la région des Hauts-de-France en 2016 : étude préliminaire R. Dehak ${ }^{1}$, D. Guimber ${ }^{2,}{ }^{,}$, D. Ley ${ }^{2}$, S. Coopman ${ }^{2}$, L. Michaud ${ }^{2}$, F. Gottrand ${ }^{2}$, D. Lescut ${ }^{34}$

${ }^{1}$ Pédiatrie, ${ }^{2}$ Gastroentérologie et nutrition pédiatrique, ${ }^{3}$ Gastroentérologie et nutrition, CHU Lille, ${ }^{4}$ Comité des CLAN et des délégués régionaux de SFNCM, Lille, France

Introduction et but de l'étude : Les données épidémiologiques concernant la nutrition artificielle à domicile (NAD) pédiatrique en France sont peu connues et très peu de données ont été publiées. C'est en raison de l'évolution du cadre législatif concernant la NAD, notamment depuis 2014 pour la nutrition parentérale, que les études d'incidence et de prévalence sont devenues possibles en France.

Matériel et méthodes : Il s'agit d’une étude observationnelle rétrospective réalisée grâce aux directions régionales du service médical de l'assurance maladie (DRSM). L'objectif principal est de déterminer l'incidence et la prévalence de la NAD pédiatrique, dans la région des Hauts-de-France de manière fiable et de décrire au mieux les pathologies à l'origine des indications de NAD en s'aidant de la classification des Affections de Longue Durée (ALD) chez les enfants de moins de 15 ans.

Résultats et Analyse statistique : L'incidence de la nutrition entérale à domicile (NED) pédiatrique dans la région des Hauts-deFrance est de 17,9 enfants pour $100000 \mathrm{Hab} / a n$ et celle de la nutrition parentérale à domicile (NPD) pédiatrique est de 1,1 enfants pour $100000 \mathrm{Hab} / \mathrm{an}$. Les prévalences de la NED et de la NPD pédiatriques sont respectivement de 67,2 patients /100 000 hab/an et 2,7 patients/100 $000 \mathrm{Hab} / \mathrm{an}$.

Les indications de NED sont dominées par les affections neurologiques (34,6\%), les pathologies faisant partie des ALD hors liste (représentées en majorité par les pathologies digestives hors $\mathrm{MICl}$ ) (35,8\%) et les insuffisances d'organe (18,7\%) (Tableau I). Pour la NPD les données ALD n'ont pas été renseignées par la DRSM (trop peu d'effectif risquant l'identification des patients).

Tableau I : Répartition des pathologies des enfants recevant une NED

\begin{tabular}{|c|c|c|c|c|c|c|c|}
\hline \multicolumn{2}{|c|}{ Nombre de patients } & \multirow{2}{*}{$\begin{array}{c}\text { Affections } \\
\text { Neurologiques } \\
n(\%)\end{array}$} & \multirow{2}{*}{$\begin{array}{c}\text { Cancers et } \\
\text { hémopathie } \\
\text { s malignes } \\
\mathrm{n}(\%)\end{array}$} & \multirow[b]{2}{*}{$\begin{array}{l}\mathrm{MICl} \\
\mathrm{n}(\%)\end{array}$} & \multirow{2}{*}{$\begin{array}{c}\text { Insuffisance } \\
\text { d'organe } \\
\text { n (\%) }\end{array}$} & \multirow[b]{2}{*}{$\begin{array}{c}\text { Autres } \\
\mathrm{n}(\%)\end{array}$} & \multirow{2}{*}{$\begin{array}{c}\text { ALD hors } \\
\text { Listes } \\
\mathrm{n}(\%)\end{array}$} \\
\hline Age & Sexe & & & & & & \\
\hline$<15$ & $\mathrm{H}$ & 126 & 25 & 10 & 54 & 56 & 106 \\
\hline ans & $\mathrm{F}$ & 111 & 35 & 10 & 74 & 52 & 139 \\
\hline \multicolumn{2}{|c|}{ Total } & $\begin{array}{c}237 \\
(34,6 \%)\end{array}$ & $\begin{array}{c}60 \\
(8,7 \%)\end{array}$ & $\begin{array}{c}20 \\
(2,9 \%)\end{array}$ & $\begin{array}{c}128 \\
(18,7 \%)\end{array}$ & $\begin{array}{c}108 \\
(15,7 \%)\end{array}$ & $\begin{array}{c}245 \\
(35,8 \%)\end{array}$ \\
\hline
\end{tabular}

Conclusion : La projection de ces taux à la population générale amènerait à une estimation d'environ 7984 enfants pris en charge en France en NED et 264 enfants pris en charge en NPD.

Selon les données de l'Inserm de 2016, la région des Hauts-de-France est la $3^{\text {ème }}$ région en France en termes de nombre de naissance par mère et elle a le taux le plus élevé de naissances prématurées en France Métropolitaine soit 9,4\% des naissances. Le taux de natalité et de prématurité élevé peut, peut-être, expliquer cette prévalence élevée de NED dans notre région.

Les résultats de notre étude sont des résultats préliminaires, nous sommes en attente des données des DRSM des autres régions de France pour compléter notre étude.

Conflits d'intérêts : Aucun conflit à déclarer 
Introduction et but de l'étude : Des avancées majeures ont récemment amélioré la survie des patients atteints d'insuffisance intestinale chronique. Il y a très peu de données sur le futur et la transition d'enfants insuffisants intestinaux chroniques devenus adulte. Le but de ce travail a été de décrire cette population depuis l'existence de notre centre.

Matériel et méthodes : Dans un centre reférence de nutrition parentérale à domicile dédié à l'insuffisance intestinale chronique depuis 1984, nous avons collecté rétrospectivement toutes les données relatives aux patients traités par nutrition parentérale à domicile pendant l'enfance (au moins 4 ans avant la transition vers notre centre) puis transférés dans notre centre adulte depuis 1984. Nous avons recueilli des données démographiques et sociales, des données sur l'évolution de la prise en charge de l'insuffisance intestinale chronique à l'âge adulte et enfin sur les principales complications apparues. La date limite de collection des données fut le 1er Décembre 2017. Les résultats sont exprimés en médiane [ \pm écart type].

Résultats et Analyse statistique : Sur un total de 870 patients sous NPAD depuis 1984, 44 jeunes adultes (17F/27H) ont été transférés depuis 3 hopitaux pédiatriques franciliens. L'âge de transition était de $20 \pm 2$ ans. Les principales étiologies d'insuffisance intestinale chronique étaient la POIC $(n=24)$, le syndrome de grele court $(n=15)$ et les pathologies épithéliales $(n=5)$. A la fin de la période de suivie définie comme les dernieres nouvelles au 1er Décembre 2017 ou le décès, 7/44 patients étaient décédés (3 par sepsis, 3 dans les suites de transplantation intestinale et 1 d'accident), 3/44 ont été sevré de NP ( 2 grâce à un facteur de croissance, 1 grâce à la transplantation intestinale), 3 furent perdus de vue et $31 / 44$ étaient sous NPAD $(6 \pm 1,7$ perfusion/semaine; $2,2 \pm 1,3 \mathrm{~L} / \mathrm{j}$; $29 \pm 13 \mathrm{kcal} / \mathrm{kg} / \mathrm{j})$. Les apports oraux étaient de $2000 \pm 1085 \mathrm{kcal} / \mathrm{j}$ mais $9 / 44$ présentaient des troubles de l'oralité persistants. $17 / 44$ travaillaient régulièrement (35 $\pm 6,5$ heures/semaine). 23/44 vivaient chez leurs parents, $17 / 44$ vivaient en couple et $7 / 44$ avaient au moins un enfant.

Conclusion : En dépit des progrès dans la survie et l'amélioration de la qualité de vie sous NPAD, une majorité d'enfants devenus adultes restent sous nutrition parentérale. Beaucoup d'entre eux vivent chez leurs parents et ne travaillent pas. La transition requiert probablement une préparation tant sociale que psychologique et médicale si nous voulons améliorer le futur de ces patients.

Conflits d'intérêts : Aucun conflit à déclarer 


\section{$\mathrm{CO} 53$}

Des (nano)particules de dioxyde de titane (TiO2) issues de l'additif alimentaire E171 franchissent la barrière placentaire chez l'Homme : une étude ex vivo sur placentas isolés et perfusés

A. Guillard ${ }^{1,}{ }^{*}$, E. Gaultier ${ }^{1}$, C. Cartier ${ }^{1}$, L. Devoille ${ }^{2}$, C. Coméra ${ }^{1}$, F. Grandin ${ }^{3}$, A. Cazanave ${ }^{1}$, A. De Place ${ }^{4}$, M. Morin $^{4}$, C. Vayssière ${ }^{4}$, N. Feltin ${ }^{2}$, F. de La Farge ${ }^{1}$, V. Gayrard ${ }^{3}$, N. Picard-Hagen ${ }^{3}$, E. Houdeau ${ }^{1}$

${ }^{1}$ Toxalim (Centre de Recherche en Toxicologie Alimentaire), INRA, Toulouse, ${ }^{2}$ Equipe Nanométrologie, Laboratoire National de métrologie \& d'essais (LNE), Paris, ${ }^{3}$ Toxalim, ENVT, ${ }^{4}$ Service Obstétrique \& Gynécologie, Hôpital Paule de Viguier (CHU Toulouse), Toulouse, France

Introduction et but de l'étude : Le dioxyde de titane $\left(\mathrm{TiO}_{2}\right)$ est un colorant alimentaire (additif E171) couramment utilisé dans les confiseries, pâtisseries, sauces et plats préparés. Selon l'origine commerciale, le E171 peut contenir jusqu'à $44 \%$ de nanoparticles (NPs, <100nm). Si l'absorption intestinale est faible, l'exposition au E171 est cependant chronique et des $\mathrm{NPs}$ de $\mathrm{TiO}_{2} \mathrm{~s}^{\prime}$ accumulent dans des organes systémiques comme le foie et la rate. Chez l'animal, des NPs modèles de $\mathrm{TiO}_{2}$ (i.e., 100\% nano) passent le placenta, avec des conséquences " santé » dans la descendance. Le but de cette étude est d'évaluer si l'exposition maternelle à l'additif E171 peut conduire à une exposition fotale à des $\mathrm{NPs}_{\mathrm{de}} \mathrm{TiO}_{2}$.

Matériel et méthodes : Des placentas collectés au moment de l'accouchement (CHU Toulouse) après recueil du consentement écrit des mères ont été perfusés en double circuit ouvert pendant $30 \mathrm{~min}$ avec du milieu de Earle, suivi de $60 \mathrm{~min}(1)$ de milieu de Earle seul (contrôles, $n=2)$, ou (2) additionné de E171 $(15 \mu \mathrm{g} / \mathrm{mL}, \mathrm{n}=6)$ préalablement soniqué (16 min, amplitude $30 \%)$. La viabilité des placentas a été validée par le taux de transfert de l'antipyrine ( $\geq 20 \%)$. Le profil de passage materno-fœtal de particules a été déterminé sur la base des propriétés de réflexion laser des métaux en microscopie confocale sur des fractions d'exsudats ( $2 \mathrm{ml}$ ) collectées toutes les $5 \mathrm{~min}$. Leur composition chimique a été analysée en spectrométrie à dispersion d'énergie (EDX) couplée à la microscopie électronique à balayage (MEB). La taille des particules a été mesurée à l’aide du logiciel Image J.

Résultats et Analyse statistique : Des particules ont été détectées dans l'exsudat foetal dès 5-10 min après ajout de E171 dans la circulation maternelle, atteignant un plateau après 25-30 min de perfusion (4-8 particules/champ, 3 champs/fraction analysée). L'analyse EDX a confirmé la nature $\mathrm{TiO}_{2}$ des particules dans les exsudats fœtaux. Toutes présentaient une dimension submicronique (i.e., comprise entre 20 et $200 \mathrm{~nm}$ ), dont $70 \%$ étaient nanodimensionnées (<100nm). Pour un placenta, 100\% du $\mathrm{TiO}_{2}$ retrouvé dans la circulation fœtale était composé essentiellement de NPs.

Conclusion : En associant plusieurs méthodes de détection à haute sensibilité et résolution, nous avons mis en évidence un transfert materno-foetal de (nano)particules de $\mathrm{TiO}_{2}$ issues de l'additif alimentaire E171. Ce passage est limité à des particules de dimension inférieure à $200 \mathrm{~nm}$, voire essentiellement des NPs. Si le taux de transfert de $\mathrm{TiO}_{2}$ semble faible, l'exposition fœtale pourrait être chronique tout au long de la grossesse. Compte tenu de la toxicité chez le rongeur après exposition périnatale à des NPs modèles de $\mathrm{TiO}_{2}$ (retard de croissance intra-utérin, accumulation dans le cerveau, désordres comportementaux dans la descendance), ces travaux soulèvent la question du risque chez l'Homme lié à l'exposition au E171 pendant la grossesse.

Conflits d'intérêts : Aucun conflit à déclarer 
Exposition prénatale aux acides gras polyinsaturés et quotient intellectuel à 5-6 ans des enfants de la cohorte EDEN E. Petit ${ }^{1}$, M. Armand ${ }^{2}$, M. De Agostini ${ }^{1}$, A. Forhan ${ }^{1}$, M.-A. Charles ${ }^{1}$, B. Heude ${ }^{1}$, J. Y. Bernard ${ }^{1, *}$

${ }^{1}$ Centre de recherche en Epidemiologie et Statistique Sorbonne Paris Cité, Inserm, Villejuif, ${ }^{2}$ Centre de Résonance Magnétique Biologique et Médicale, Université Aix-Marseille, CNRS, Marseille, France

Introduction et but de l'étude : Le développement cérébral du fœtus nécessite un apport adéquat et équilibré en acides gras polyinsaturés (AGPI) oméga $6(n-6)$, tels les acides linoléique et arachidonique, et oméga 3 ( $n-3)$, notamment l'acide docosahexaénoïque (DHA). Le niveau d'exposition prénatal aux AGPI pourrait programmer le neurodéveloppement. Notre objectif était d'examiner les relations entre le statut maternel et fœtal en AGPI et le quotient intellectuel (QI) ultérieur des enfants.

Matériel et méthodes : L'étude EDEN est une cohorte mère-enfant française de 2002 femmes enceintes recrutées entre 2003 et 2006 dans les maternités de Poitiers et Nancy. Des échantillons de sang maternel (à 24-28 semaines de grossesse) et du cordon ombilical (à l'accouchement) ont été prélevés. Le profil en AGPI n-6 et n-3 des membranes des érythrocytes a été analysé par chromatographie en phase gazeuse. Le QI (total, performance et verbal) des enfants a été évalué à l'âge de 5-6 ans avec la batterie Wechsler Preschool and Primary Scale of Intelligence-III. Les relations entre les taux en AGPI (standardisés pour les rendre comparables puis exprimés en continu ou en quartile) et le QI ont été examinées par régression linéaire avec ajustement sur de nombreux facteurs de confusion potentiels.

Résultats et Analyse statistique : Des 1100 enfants ayant des mesures du QI, 1026 et 775 disposaient de données sur les AGPI maternels et fœtal, respectivement. Parmi les 773 couples mères-enfants ayant des données complètes, l'âge moyen ( \pm écarttype) des mères à l'accouchement était de $30,1( \pm 4,8)$ ans et leur durée d'instruction de $13,5( \pm 2,2)$ ans. Le QI total des enfants était de 103 ( \pm 13 ) points. Le taux d’AGPI n-6 maternel n'était pas associé au QI, tandis que celui en AGPI n-3 était associé positivement au QI total $(1,04$ [95 \% IC : 0,$14 ; 1,90]$ points pour une variation d'un écart-type du taux d'AGPI) et verbal $(1,15$ $[0,25 ; 2,05])$, mais pas au QI performance. Ces relations étaient principalement dues au DHA (67 \% des AGPI n-3 totaux). Le statut fœtal en AGPI n-3 était associé non linéairement au QI : seuls les enfants du quartile de DHA le plus élevé ( $\geq 6,8 \%)$ avaient un QI performance supérieur $(2,85[0,67: 5,03]$ points) à celui des autres enfants. Pour les AGPI n-6 totaux du cordon, les enfants du quartile le plus bas $(<25,6 \%)$ avaient un QI performance supérieur de $3,94[1,74 ; 6,14]$ points aux autres enfants. Aucune relation n'a été observée avec les AGPI n-6 pris individuellement.

Conclusion : Dans l'étude EDEN, l'exposition prénatale aux AGPI n-3 était associée positivement au QI des enfants à 5-6 ans. Le QI était associé négativement aux taux d'AGPI n-6 fœtaux, mais pas aux AGPI n-6 maternels. Notre étude suggère que l'exposition prénatale aux AGPI pourrait participer à la programmation du neurodéveloppement et influencer le QI jusqu'à 5-6 ans. L'emploi de méthodes d'inférence causale telles que la randomisation mendélienne permettra de déterminer si ces relations sont causales. Remerciements : Prix IDF/FRM pour les Sciences de l'Alimentation 2015.

Conflits d'intérêts : Aucun conflit à déclarer 
Les lipides polaires laitiers diminuent les facteurs lipidiques du risque cardiovasculaire chez des femmes ménopausées

C. Vors ${ }^{1}$, L. Joumard-Cubizolles ${ }^{2}$, E. Combe ${ }^{1}$, L. Ouchchane ${ }^{3}$, J. Drai ${ }^{4}$, K. Raynal ${ }^{5}$, P. Gaborit ${ }^{5}$, F. Joffre ${ }^{6}$, A. Blot ${ }^{7}$, G. Gesan-Guiziou ${ }^{8}$, M. Laville ${ }^{9}$, S. Lambert-Porcheron ${ }^{4}$, C. Malpuech-Brugère ${ }^{2}$, M.-C. Michalski ${ }^{10,}{ }^{*}$

${ }^{1}$ CarMeN, INRA, INSERM U1060, Oullins, ${ }^{2}$ UNH, INRA, UCA, ${ }^{3}$ SIGMA, UCA UMR6602, Clermont-Ferrand, ${ }^{4} \mathrm{CRNH}$ Rhône-Alpes, HCL, Oullins, ${ }^{5}$ ACTALIA, ENILIA, Surgères, ${ }^{6}$ ITERG, Canéjan, ${ }^{7}$ CRNH Auvergne, CHU Clermont-Ferrand, Clermont-Ferrand, ${ }^{8}$ STLO, INRA UMR1253, Rennes, ${ }^{9} \mathrm{CarMeN}$, UCBL, Oullins, ${ }^{10} \mathrm{CarMeN}$, INRA UMR1397, Villeurbanne, France

Introduction et but de l'étude : Des perturbations du métabolisme lipidique à jeun et en phase postprandiale constituent des facteurs de risque cardiovasculaire. La nutrition joue un rôle majeur dans leur modulation, notamment chez les femmes ménopausées qui constituent une population à risque. Un intérêt s'est récemment développé sur les effets bénéfiques potentiels des lipides polaires (LP) laitiers, qui sont riches en sphingomyéline. A ce jour, leurs effets chez l'Homme restent cependant à clarifier, car les rares études cliniques ont jusqu'à présent été réalisées chez des sujets sains et par supplémentation, augmentant ainsi l'apport énergétique. Nous avons donc testé l'hypothèse qu'un enrichissement isolipidique de l'alimentation avec des LP laitiers apportés par un produit laitier réaliste pourrait améliorer le profil cardiovasculaire de femmes ménopausées à risque.

Matériel et méthodes : Un essai clinique contrôlé aléatoire en double aveugle chez 58 femmes ménopausées en surpoids a été réalisé. Les volontaires ont été soumises à (i) 4 semaines d'intervention avec consommation quotidienne d'un fromage à tartiner contenant $12 \mathrm{~g}$ de matière grasse laitière incluant aléatoirement soit $0 \mathrm{~g}$ (contrôle, $n=19), 3 \mathrm{~g}(\mathrm{n}=19)$ ou $5 \mathrm{~g}(\mathrm{n}=20)$ de LP laitiers, et (ii) des tests d'exploration métabolique sur $8 \mathrm{~h}$, avant et après intervention ( 2 visites). Des marqueurs lipidiques ont été mesurés dans le sérum et dans la fraction riche en chylomicrons. L'effet de la dose de LP laitiers a été testé par un modèle linéaire mixte $\left(\mathrm{SAS}^{\circledR}\right)$ recherchant les différences entre visites et entre groupes d'intervention.

Résultats et Analyse statistique : La consommation du fromage à $5 \mathrm{~g}$-LP diminue les concentrations sériques à jeun et postprandiales du cholestérol total $(-0,33 \pm 0,03 \mathrm{mM}, \mathrm{p}<0,01)$ et des triglycérides $(T \mathrm{~T},-0,35 \pm 0,05 \mathrm{mM}, \mathrm{p}<0,01)$, diminue le cholestérol LDL à jeun $(-0,34 \pm 0,08 \mathrm{mM}, p<0,01)$, augmente le cholestérol $\mathrm{HDL}(+0,06 \pm 0,03 \mathrm{mM}, \mathrm{p}<0,05)$ et diminue le rapport ApoB/ApoA1 $(-0,07 \pm 0.01, p<0,01)$. Les effets sont différents de ceux du fromage contrôle $(p<0,05)$ qui $n^{\prime}$ induit pas de modification du profil lipidique. La dose de LP laitiers modifie le cholestérol et les TG des chylomicrons $(p<0,01)$ avec des concentrations diminuées dans le groupe $5 \mathrm{~g}-\mathrm{LP}(-0,07 \pm 0,02 \mathrm{mM}$ et $-0,26 \pm 0,09 \mathrm{mM}$, respectivement; $p<0,01)$, suggérant une moindre absorption intestinale et/ou une augmentation de l'épuration des résidus de chylomicrons.

Conclusion : Ces résultats suggèrent qu'une stratégie nutritionnelle ciblée sur la qualité des lipides laitiers, incluant les lipides polaires, pourrait contribuer à améliorer la santé cardiométabolique des femmes ménopausées.

Remerciements : Etude financée par I'ANR (projet VALOBAB, ANR-11-ALID-007-01, coord. MC Michalski), le PHRC-I ( ${ }^{\circ} 14-007$, coord. clinique S. Lambert-Porcheron) et le CNIEL.

Conflits d'intérêts : C. Vors: Aucun conflit à déclarer, L. Joumard-Cubizolles: Aucun conflit à déclarer, E. Combe: Aucun conflit à déclarer, L. Ouchchane: Aucun conflit à déclarer, J. Drai: Aucun conflit à déclarer, K. Raynal est employé(e) de Terra Lacta, P. Gaborit est employé(e) de ACTALIA Produits Laitiers, F. Joffre est employé(e) de ITERG, A. Blot: Aucun conflit à déclarer, G. GesanGuiziou: Aucun conflit à déclarer, M. Laville: Aucun conflit à déclarer, S. Lambert-Porcheron: Aucun conflit à déclarer, C. MalpuechBrugère: Aucun conflit à déclarer, M.-C. Michalski a reçu une subvention/soutien de CNIEL, est consultant chez CNIEL 


\section{CO56}

Actions synergiques d'une restriction calorique et de l'activité physique sur la santé et la longévité

J. Royo ${ }^{1}$, F. Pifferi $^{1, *}$

${ }^{1}$ UMR CNRS MNHN 7179, CNRS, Brunoy, France

Introduction et but de l'étude : Le bouleversement des pratiques nutritionnelles et des habitudes de vie qui a eu lieu au cours du siècle dernier conduit aujourd'hui à une augmentation dramatique des pathologies chroniques sur l'ensemble du globe. En effet, bien que la longévité n'ait cessé d'augmenter dans cette période, cette augmentation s'accompagne depuis plusieurs décennies d'une augmentation massive de pathologies chroniques liées à l'âge. De ce fait, le bénéfice du gain de longévité est gâché par l'épidémie de syndrome métabolique et de démences séniles. Nous postulons ici que la très grande majorité de ces pathologies pourrait être évitée car elles ne sont pas liées à l'âge mais à l'inadéquation entre des apports caloriques élevés et une activité physique très faible. En effet, l'apparition et l'évolution de l'Homme ont eu lieu dans un environnement dans lequel la nourriture n'était pas nécessairement abondante. Récolter et chasser sa nourriture impliquait une activité physique importante, activité à laquelle la physiologie et la morphologie de notre espèce est adaptée. Depuis la révolution industrielle, la quantité d'activité physique n'a cessé de diminuer, alors que la quantité de calories ingérées n'a cessé de croître. C'est pourquoi nous avons proposé de tester les effets d'une intervention multi-modale combinant les effets d'une restriction calorique chronique modérée et d'activité physique chronique modérée, sur la santé et la longévité.

Matériel et méthodes : Cette étude est réalisée chez le microcèbe (Microcebus murinus), un primate d'une longévité moyenne de 6 ans. Les animaux sont suivis depuis leur inclusion dans l'étude, vers l'âge de 3 ans, jusqu'à leur mort naturelle. Les traitements suivants ont été appliqués : restriction calorique modérée (CR) et/ou activité physique (ACT). Ces traitements sont testés séparément ( 2 groupes différents : CR ou ACT) et en interaction ACT+CR). Cela nous permettra comprendre les effets synergiques potentiels des deux traitements. Chaque groupe est comparé à un groupe contrôle (CTL) maintenu en condition d'élevage standard. Ce groupe reçoit un régime composé de $15 \mathrm{~g}$ de bouillie et $6 \mathrm{~g}$ de fruits frais par jour (soit $105 \mathrm{~kJ} . \mathrm{j}^{-1}$ en moyenne). Le groupe CR reçoit 15\% de calories en moins que le groupe CTL (soit $89 \mathrm{~kJ}^{-j^{-1}}$ en moyenne). Le groupe ACT effectue $2 \times 30$ minutes d'activité physique modérée par jour sur un tapis roulant (marche à une vitesse de $0,2 \mathrm{~m} \mathrm{~s}-1$ et $0^{\circ}$ d'inclinaison (soit $720 \mathrm{~m}$.j-1).

Résultats et Analyse statistique : Les premières données montrent une diminution significative du poids des animaux CR, ACT et $C R+A C T$. Des améliorations des performances cognitives chez les animaux du groupe $C R+A C T$ ont également été observées.

Conclusion : Une diminution des pathologies liées à l'âge est attendue avec les traitements par rapport aux contrôles, ce qui devrait s'accompagner d'une durée de vie prolongée, les effets les plus forts étant attendus dans le groupe recevant les deux interventions $(C R+A C T)$. Les premiers résultats de longévité seront disponibles après 2,5 ans d'étude, la durée nécessaire pour atteindre la durée de vie médiane des animaux CTL (5,7 ans). Cinq ans après le début de l'étude, 70\% des animaux CTL devraient être morts. De tels résultats contribueront à mieux comprendre et définir des niveaux optimaux d'apports caloriques et d'activité.

Conflits d'intérêts : Aucun conflit à déclarer 
Effets de la variabilité interindividuelle de la salive humaine (flux et composition) sur la libération et perception d'arômes chez le sujet âgé

C. Munoz ${ }^{1, *}$, M. Brule ${ }^{1}$, C. Martin ${ }^{1}$, C. Septier $^{1}$, G. Feron ${ }^{1}$, F. Canon ${ }^{1}$

${ }^{1}$ Centre des Sciences du Goût et de l'Alimentation, AgroSup Dijon, CNRS, INRA, Université Bourgogne Franche-Comté, Dijon, France

Introduction et but de l'étude : La population mondiale vieillit rapidement. Le vieillissement s'accompagne souvent d'une réduction des sécrétions salivaires qui peuvent entraîner des changements des habitudes alimentaires et de la consommation de certains aliments. A cet égard, la supplémentation des aliments avec des ingrédients fonctionnels comme les fibres prébiotiques et/ou les antioxydants (polyphénols) est de plus en plus proposée comme stratégie pour maintenir une bonne santé générale chez les seniors. Cependant, l'ajout de ces ingrédients peut entraîner une modification de la flaveur et changer l'appréciation du produit. En effet, ces composés peuvent d'une part stimuler per se les récepteurs gustatifs et d'autre part interagir avec les arômes présents dans les aliments et/ou les protéines salivaires, modifiant leur libération pendant la consommation et donc, sa perception. L'objectif de cette étude est de décrire pour la première fois le rôle de la salive (flux/composition) sur la libération et la perception d'arômes chez les personnes âgées, en tenant compte de l'ajout d'ingrédients fonctionnels (fibres prébiotiques, polyphénols).

Matériel et méthodes : Dans un première phase, la libération d'arômes in vivo a été mesuré chez un panel de personnes âgées $(n=73)$ représentatif de la population senior française. Des solutions d'arômes alimentaires seuls (contrôle) ou en présence des nutriments fonctionnels de matrices alimentaires (fibres prébiotiques, polyphénol) ont été consommées par l'ensemble du panel et la quantité des composés d'arôme atteignant la cavité nasale a été suivie en temps réel par spectrométrie de masse à transfert de proton (PTR-MS). Dans une deuxième phase, la perception d'arôme dans différentes matrices a été mesurée ( $n=26)$. Dans les deux phases de cette étude, la salive des participants a été collectée pour déterminer le flux ainsi que la composition salivaire (teneur totale en protéines, capacité antioxydante totale). Les donnes recollectés ont étés analysés par des statistiques descriptives, l'analyse de la variance (ANOVA) et des analyses de régression afin de mettre en évidence les effets de la composition et flux salivaire sur la libération et la perception d'arômes.

Résultats et Analyse statistique : Les résultats ont montré différences significatives sur la libération et la perception des arômes chez les personnes âgées, qui ont été corrélés à différents degrés de signification aux paramètres salivaires des participants de l'étude. De plus, des différences entre les composés aromatiques et les matrices alimentaires ont été observées. La présence de polyphénol a modifié la perception des arômes, au contraire que les fibres testées qui n'ont pas montré le même comportement. Conclusion : Cette étude a montré l'importance de considérer la variabilité interindividuelle de la salive humaine sur la libération et la perception d'arômes chez le sujet âgé. Cette compréhension nous aidera à proposer des solutions innovantes pour la formulation de produits alimentaires mieux adaptés aux besoins des personnes âgées tout en conservant leurs propriétés organoleptiques.

Remerciements : CMG remercie la SFN pour le prix de recherche qui l'a été attribué ainsi comme au programme Agreenskills+ pour son contrat N FP7-609398.

Conflits d'intérêts : Aucun conflit à déclarer 
CO59

Influence du genre sur l'évolution des stéatohépatites métaboliques : Rôle hépatocytaire du récepteur aux oestrogènes ER(alpha)

S. Smati-Grangeon ${ }^{1}$, B. Tramunt ${ }^{2}$, A. Polizzi ${ }^{1}$, M. Régnier ${ }^{1}$, H. Guillou ${ }^{1}$, P. Gourdy ${ }^{2}$, A. Montagner ${ }^{2, *}$

${ }^{1}$ INRA/ UMR1331 ToxAlim, ${ }^{2}$ INSERM/UMR 1048/ I2MC, Toulouse, France

Introduction et but de l'étude : Les maladies métaboliques du foie non liées à l'alcool ou NAFLD pour "Non Alcoholic Fatty Liver Disease" font partie des affections soumises à un dimorphisme sexuel. Leur prévalence progresse de façon rapide dans la plupart des pays industrialisés pour atteindre 20 à $35 \%$ de la population, une situation préoccupante compte-tenu du risque évolutif de ces affections qui peuvent conduire à la survenue de complications sévères telles qu'une cirrhose ou un hépatocarcinome. Ainsi, il est important de comprendre les mécanismes qui conditionnent la progression de la stéatose hépatique, stade initial et bénin des NAFLD, à la stéatohépatite afin de mieux identifier les patients présentant ce risque évolutif mais également de proposer de nouvelles cibles d'intervention préventive. Plusieurs données épidémiologiques et expérimentales ont montré que les oestrogènes, via l'activation de leur récepteur, le récepteur nucléaire ER $\square$, jouent un rôle protecteur sur le développement de la stéatose hépatique et sur le risque de transition vers la stéatohépatite.

Objectif : Notre objectif est de déterminer la fonction d'ER $\alpha$ hépatocytaire sur la protection de l'évolution de la stéatose en stéatohépatite.

Matériel et méthodes : Afin d'étudier l'influence du sexe sur l'installation et la progression des NAFLD, un modèle nutritionnel de stéatohépatite a été utilisé chez des souris mâles et femelles sauvages C57BL/6J ou invalidées pour le récepteur spécifiquement dans les hépatocytes (Alb-Cre $E R \alpha$ ). Le phénotype hépatique et métabolique a été déterminé en analysant l'histologie hépatique complétée par une analyse lipidique et transcriptomique par microrrays.

Résultats et Analyse statistique : Nos résultats montrent tout d'abord que seules les souris mâles sauvages soumises à un régime pro-stéatohépatite développent une obésité et une intolérance au glucose en comparaison aux souris femelles. De plus, les femelles sont protégées du développement d'une stéatohépatite et d'une fibrose. De façon intéressante, l'utilisation de souris invalidées pour ER $\square$ dans les hépatocytes suggère que si ER $\square$ hépatocytaire ne joue pas de rôle dans la prévention de la prise pondérale et le développement de la stéatose, il prévient l'installation d'une inflammation et d'une fibrose hépatique.

Conclusion : Ainsi, nos données suggèrent que l'activité hépatocytaire d'ER $\square$ est cruciale afin de limiter le développement d'une stéatohépatite et d'une fibrose par des mécanismes qui restent à être identifiés. La prévention des stéatohépatites et de leurs complications est considérée depuis quelques années comme un des enjeux majeurs de santé publique. Nos résultats pourraient ouvrir des perspectives intéressantes pour le développement de nouvelles stratégies d'activation d'ER $\alpha$ qui pourraient être restreintes au foie.

Conflits d'intérêts : Aucun conflit à déclarer 
Impacts d'un régime hyper-protéiné chez le mâle, sur l'épigénome de ses spermatozoïdes et sur la santé métabolique de sa descendance

S. Bottini ${ }^{1{ }^{*}}$, J. Movassat ${ }^{2}$, V. Grandjean ${ }^{1}$

${ }^{1} \mathrm{c} 3 \mathrm{~m}$ inserm, c3m inserm, nice, ${ }^{2}$ Biologie et Pathologie du Pancréas Endocrine, Unité BFA-Université Paris Diderot / CNRS - UMR 8251, Paris, France

Introduction et but de l'étude : Il est maintenant clairement établi que ce que nous mangeons à un impact direct sur notre santé. Mais ce qui est beaucoup moins bien établi, ce sont les conséquences de notre alimentation sur la santé de nos descendants. En utilisant des modèles expérimentaux murins nous avons démontré que les descendants de mâles nourris avec une alimentation riche en graisse développent à l'âge adulte des pathologies métaboliques similaires à celles de leur père alors qu'ils sont nourris eux-mêmes avec une nourriture normale. Nos précédentes études démontrent l'implication des petits ARN présents dans les spermatozoïdes dans le transfert héréditaire de ces nouvelles informations nouvellement acquises suite à des changements environnementaux. Ce processus largement analysé pour les régimes riches en graisse n'a pas encore été analysé pour des régimes hyper-protéinés pourtant très prisés chez les personnes sportives ou les personnes souhaitant perdre du poids, population souvent jeune et en âge de procréer. Une alimentation hyper-protéinée a-t-elle des conséquences sur le phénotype métabolique des descendants ? Si oui, les ARN présents dans les spermatozoïdes sont-ils impliqués ? Apporter des éléments de réponse à ces questions est l'objectif de notre projet.

Matériel et méthodes : Pour cela, nous avons nourri des rats mâles avec une nourriture hyper-protéinée pendant une durée de 3 mois. Après cette période et afin d'obtenir une descendance mâle et femelle, nous les avons croisés avec des femelles nourries avec un régime standard.

Résultats et Analyse statistique : L'analyse phénotypique de cette descendance révèle que la prise d'une nourriture hyperprotéinée chez le père a un impact sur la régulation du glucose chez la descendance mâle. Ceci indique que cette alimentation altère le patrimoine épigénétique des spermatozoïdes du mâle, altérations qui se répercutent sur le métabolisme de la descendance. Afin d'identifier les vecteurs potentiels de cette transmission, nous avons réalisé un séquençage exhaustif (RNAseq) des petits ARN présents dans les spermatozoïdes d'un mâle nourri avec une nourriture standard avec celle d'un mâle nourri avec une alimentation hyper-protéinée. L'analyse bioinformatique de ces données met en évidence plusieurs séquences potentiellement impliquées dans cette hérédité dont notamment les microRNA miR-195 et miR-126 connus pour être impliqués dans le développement du pancréas et/ou la régulation de la sécrétion de l'insuline.

Conclusion : L'ensemble de ces données indique que l'alimentation hyper-protéinée induit chez le mâle des modifications épigénétiques dans sa lignée germinale, modifications qui seraient transmises à sa descendance. Le vecteur de cette hérédité serait les molécules d'ARN spermatiques.

Remerciements : Les auteurs remercient la SFN pour son prix accorder à ce travail.

Conflits d'intérêts : Aucun conflit à déclarer 


\section{SANTÉ ET NUTRITION}

\section{CO61}

Consommation d'aliments ultra-transformés et risque de maladies cardiovasculaires dans la cohorte NutriNet-Santé

B. Srour ${ }^{1,}{ }^{*}$, L. K. Fezeu ${ }^{1}$, E. Kesse-Guyot ${ }^{1}$, B. Allès ${ }^{1}$, C. Méjean ${ }^{2}$, R. M. Andrianasolo ${ }^{1}$, E. Chazelas ${ }^{1}$, M. Deschasaux ${ }^{1}$, S. Hercberg ${ }^{13}$, P. Galan ${ }^{1}$, C. A. Monteiro ${ }^{4}$, C. Julia $^{13}$, M. Touvier ${ }^{1}$

${ }^{1}$ Equipe de Recherche en Epidémiologie Nutritionnelle EREN - UMR U1153 Inserm / U1125 Inra / Cnam / Université Paris 13 Centre de Recherche en Epidémiologie et Biostatistiques Sorbonne Paris Cité, Bobigny, ${ }^{2}$ UMR MOISA, INRA CIRAD, Montpellier, ${ }^{3}$ Département de Santé Publique, Hôpital Avicenne, Bobigny, France, ${ }^{4}$ Département de Nutrition, Université de Sao Paulo, Sao Paulo, Brésil

Introduction et but de l'étude : Les conséquences de l'augmentation récente de la consommation d'aliments ultra-transformés (AUT) sur la santé sont mal connues. Les AUT présentent en moyenne une moins bonne qualité nutritionnelle, contiennent des additifs alimentaires et peuvent contenir des substances provenant des emballages, ainsi que des composés néoformés. Des études épidémiologiques antérieures ont trouvé des associations entre la consommation d'AUT et une incidence plus élevée de certaines maladies chroniques. Récemment, la consommation d'AUT a été associée à des risques plus élevés de cancers dans la cohorte NutriNet-Santé. Certaines études mécanistiques suggèrent des effets cardiométaboliques pour plusieurs composants couramment trouvés dans ces aliments, cependant, les preuves épidémiologiques sont rares. Cette étude a pour objectif d'estimer les associations entre la consommation d'AUT et le risque de maladies cardiovasculaires.

Matériel et méthodes : Au total, 105159 participants âgés d'au moins 18 ans (âge médian 41,5 ans) de la cohorte NutriNet-Santé (2009-2018) ont été inclus. Les apports alimentaires ont été recueillis par des enregistrements alimentaires de $24 \mathrm{~h}$ répétés, conçus pour collecter la consommation habituelle des participants pour 3300 aliments différents. Ceux-ci ont été catégorisés en fonction de leur degré de transformation grâce à la classification NOVA. Les associations entre la consommation d'AUT et le risque de maladies cardiovasculaires, coronariennes et cérébro-vasculaires ont été évaluées par des modèles de Cox à risques proportionnels ajustés sur les facteurs de risque connus.

Résultats et Analyse statistique : La consommation d'AUT était associée à une augmentation du risque de maladies cardiovasculaires au global $(n=1409$ cas, Hazard Ratio pour une augmentation de $10 \%$ de la proportion d'AUT dans le régime = $1,12(1,05$ à 1,20); $p=0,0008)$, de maladies coronariennes ( $n=665$ cas, HR =1,13 $(1,02$ à 1,24), $p=0,02)$ et de maladies cérébrovasculaires $(n=829$ cas, $H R=1,11(1,01$ à 1,22), $p=0,02)$. Ces résultats restaient significatifs après ajustement sur plusieurs marqueurs de la qualité nutritionnelle de l'alimentation (acides gras saturés, sodium, sucres, fibres) ainsi que sur des patterns Healthy ou Western.

Conclusion : Dans cette large étude prospective, une consommation plus élevée d'AUT dans l'alimentation était associée à une augmentation de risques de maladies cardiovasculaires, coronariennes et cérébro-vasculaires. Différentes dimensions de la transformation alimentaire telles que la composition nutritionnelle du produit final, les additifs alimentaires, les matériaux de contact et les contaminants néoformés pourraient jouer un rôle dans ces associations ? D'autres études sont donc nécessaires afin de mieux comprendre leur contribution relative. Dans l'attente, la consommation d'aliments frais ou peu transformés est à privilégier, au nom du principe de précaution.

Conflits d'intérêts : Aucun conflit à déclarer 
Un faible statut en acides gras oméga-3 à longue chaine en début de grossesse est associé à un risque plus élevé de dépression post-partum

A. Hoge ${ }^{1,{ }^{*}}$, M. Nisolle ${ }^{2}$, M. Guillaume ${ }^{1}$, V. Castronovo ${ }^{3}$ et S. Degée, V. Tabar, M. Timmermans, A.-F. Donneau, N. Dardenne ${ }^{1}$ Département des Sciences de la Santé publique, ${ }^{2}$ Département de Gynécologie-Obstétrique, ${ }^{3}$ Département des Sciences biomédicales et précliniques, Université de Liège, Liège, Belgique

Introduction et but de l'étude : La dépression est l'une des complications du post-partum les plus fréquentes, avec une prévalence estimée entre 10 et $20 \%$ à travers le monde. Son dépistage, sa prévention et sa prise en charge sont indispensables pour éviter les conséquences négatives de cette pathologie, tant sur la mère que sur la relation mère-bébé et le développement neurocognitif et affectif de l'enfant. Le rôle des acides gras sur la santé mentale a été largement étudié en population générale. Dans le cadre du post-partum, des études observationnelles mettent en évidence une relation entre la consommation d'acides gras polyinsaturés oméga-3 (AGPI n-3) et la santé mentale maternelle; néanmoins, les preuves restent mitigées. Les recherches fondées sur les analyses sanguines montrent que la déplétion des réserves maternelles en DHA, observée au cours de la grossesse, et la lente normalisation du statut post-partum, augmenterait le risque de dépression post-partum (DPP) [1].

La présente étude vise à étudier l'association entre les AGPI n-3 érythrocytaires, mesurés en début de grossesse, et le risque de DPP.

Matériel et méthodes : Soixante-douze femmes enceintes de 6 à 18 semaines, ayant assisté à une consultation prénatale au Centre Régional de la Citadelle de Liège (Belgique), ont été inclues. La prise de sang a été effectuée lors du recrutement, et l'analyse des acides gras polyinsaturés érythrocytaires a été réalisée par chromatographie en phase gazeuse. L'index oméga-3 représente le pourcentage d'acides gras oméga-3 à longue chaine (EPA et DHA) sur l'ensemble des acides gras présents au niveau de la membrane des érythrocytes. La DPP a été déterminée un an après l'accouchement, lors d'une interview téléphonique se basant sur les critères diagnostiques du DSM-V (épisode dépressif majeur).

Résultats et Analyse statistique : Afin de tester l'impact des AGPI n-3 sur la survenue de la DPP, la méthode de régression logistique a été utilisée. Le modèle a été ajusté par plusieurs autres facteurs de risque tels que les caractéristiques sociodémographiques, les antécédents de dépression et les évènements stressants. Une association négative a été observée entre les taux de DHA et le risque de dépression post-partum $(p=0,044)$. Nos résultats ont révélé qu'un index oméga-3 inférieur à $5 \%$ (seuil préalablement proposé par [2]) augmente d'environ 5 fois le risque de DPP en comparaison à un index oméga-3 supérieur ou égal à 5\% (OR 4,75 [1,16-19,4]). Enfin, un rapport oméga-6/oméga-3 élevé a été significativement associé à un risque augmenté d'épisode dépressif majeur du post-partum $(p=0,023)$.

Conclusion : La DPP se révèle être un enjeu important de santé publique, de par sa prévalence élevée et ses conséquences à plus ou moins longs termes. Dans notre étude, un faible statut en acides gras oméga-3, seul ou combiné à un taux élevé d'acides gras oméga-6, a été associé à un risque plus élevé de DPP dans l'année qui a suivi l'accouchement. Les interventions nutritionnelles au cours de la période périnatale peuvent constituer une stratégie simple et rentable pour prévenir les déficits nutritionnels et réduire ainsi la prévalence de la DPP.

Références : [1]Otto SJ et al. Prostaglandins Leukot Essent Fatty Acids 2003;69.

[2]Markhus MW et al. PLoS One 2013;8(7).

Conflits d'intérêts : Aucun conflit à déclarer 
Effet de l'arrivée d'un premier enfant dans le foyer sur les évolutions sur $\mathbf{7}$ ans des consommations alimentaires et du poids des adultes (Etude NutriNet-Santé)

C. Mejean ${ }^{1,}{ }^{*}$, P. Rollet ${ }^{1}$, W. Si Hassen ${ }^{2}$, C. Tichit $^{3}$, A. Lampuré ${ }^{2}$, S. Hercberg ${ }^{2}$, M. Touvier ${ }^{2}$, K. Castetbon $^{4}$

${ }^{1}$ MOISA, Univ Montpellier, INRA, CIRAD, CIHEAM-IAMM, Montpellier SupAgro, Montpellier, ${ }^{2}$ Equipe de Recherche en Epidémiologie Nutritionnelle EREN , UMR U1153 Inserm / U1125 Inra / Cnam / Université Paris 13 Centre de Recherche en Epidémiologie et Biostatistiques Sorbonne Paris Cité, Bobigny, ${ }^{3} \mathrm{CMH}$, INRA, CNRS, ENS, EHESS, Paris, France, ${ }^{4}$ Université Libre de Bruxelles, Ecole de Santé publique, Centre de Recherche en Epidémiologie, Biostatistique et Recherche Clinique, Bruxelles, Belgique

Introduction et but de l'étude : Au cours de la vie, les transitions telles que la parentalité sont décrites, dans les études qualitatives, comme des périodes de changement éventuel dans les attitudes et pratiques de santé. Il a été montré que la présence d'enfants modifie les pratiques et rythmes alimentaires des adultes et que l'arrivée d'un enfant est notamment perçue comme étant liée aux à des changements des comportements alimentaires. Néanmoins, aucune étude quantitative n'en a évalué l'impact par une approche longitudinale. L'objectif de notre étude était d'examiner l'effet de l'arrivée d'un premier enfant dans un foyer sur les apports alimentaires et le poids des adultes pendant une période de suivi de 7 ans.

Matériel et méthodes : Cette étude a été menée auprès de 3955 adultes inclus dans l'étude NutriNet-Santé entre 2009 et 2010, et pour lesquels les consommations alimentaires ont été estimées à l'inclusion et chaque année de suivi, par des enregistrements alimentaires de $24 \mathrm{~h}$ répétés. Dans cette population, 185 personnes ont vécu l'arrivée d'un premier enfant dans leur foyer au cours des 7 années de suivi. Les évolutions du poids, évalué par questionnaire auto-administré chaque année, ont été analysées uniquement chez les femmes $(n=115)$. Les facteurs d'ajustement comme le sexe, l'âge et le niveau d'éducation ont été collectés à l'inclusion.

Résultats et Analyse statistique : Des modèles mixtes à effets aléatoires pour le temps et la période (avant/après l'arrivée de l'enfant) ajustés sur les facteurs d'ajustement et les apports en énergie ont été utilisés pour évaluer les changements alimentaires et de poids liés à cet événement. L'arrivée d'un premier enfant dans le foyer était associée à une diminution du score d'adéquation aux recommandations du PNNS (score PNNS, $\beta$ [IC 95\%]= $-0.45[-0.74 ;-0.16]$ ), des consommations de "fruits et légumes» et de «viande rouge, charcuterie, volaille» et des apports en vitamine $C$ (respectivement, $\beta=-0.30[-0.43 ;-0.17]$ ); $\beta=-0.20$ [-0.32; -0.17$]$ ) ; $\beta=-0.54[-0.88 ;-0.19])$ ) et à une augmentation des apports en acides gras saturés et sodium (respectivement, $\beta=1.49$ [0.51; 2.45$]$ ); $\beta=0.31[0.20 ; 0.43]))$. L'arrivée d'un premier enfant dans le foyer était aussi associée à une prise de poids chez les femmes ( $\beta=1.70$ $[1.07 ; 2.33])$.

Conclusion : Notre étude a mis en évidence que l'arrivée d'un premier enfant dans le foyer est associée à des changements plutôt vers des apports nutritionnels moins favorables à la santé. Ces résultats pourraient permettre d'orienter plus finement les interventions de santé publique se focalisant sur cette période de vie spécifique.

Conflits d'intérêts : Aucun conflit à déclarer 
L'outil d'évaluation de la prise alimentaire $\operatorname{EPA}^{\circledR}$ (ou $\mathrm{SEFI}^{\circledR}$ ) comme test de dépistage de la dénutrition en médecine générale : étude prospective non interventionnelle multicentrique

G. Bouëtté ${ }^{1, *}$, M. Esvan ${ }^{2}, K$. Apel ${ }^{1}$, R. Thibault ${ }^{34}$

${ }^{1}$ Département de Médecine Générale, Universtiré de Rennes $1,{ }^{2} \mathrm{DRCl}$, CHU de Rennes, ${ }^{3}$ Institut NuMeCan, INRA, INSERM, Univ Rennes, ${ }^{4}$ Unité de Nutrition, CHU de Rennes - Universtiré de Rennes 1, Rennes, France

Introduction et but de l'étude : La prévalence de la dénutrition est de $35 \%$ à l'hôpital, $30 \%$ en institution et d'au moins $5 \%$ en ambulatoire. Son diagnostic est souvent retardé. Le ScorE Fiable des Ingesta $\left(\mathrm{SEFI}^{\circledR}\right.$, anciennement $\left.\mathrm{EPA}^{\circledR}\right)$, échelle visuelle analogique (EVA) de 0 à 10, ou évaluation des portions consommées, permet une évaluation rapide de la prise alimentaire actuelle. II est validé et recommandé en milieu hospitalier et permet d'identifier précocement un risque de dénutrition lorsque le sore est $<7 / 10$ (1). En EPHAD, il permet de dépister la dénutrition si les portions consommées sont $\leq 50 \%$ du plat principal (2). Objectif principal: évaluer la fiabilité de $\mathrm{SEFI}^{\circledR}$ pour le dépistage de la dénutrition de l'adulte en médecine générale. Objectifs secondaires: évaluer la faisabilité de $\mathrm{SEFI}{ }^{\circledR}$ et les facteurs de risque de dénutrition.

Matériel et méthodes : Etude prospective non interventionnelle de 3 mois, en 2018, chez des patients consécutifs vus en consultation dans deux cabinets de médecine générale en France métropolitaine. Critères d'inclusion: adulte consultant en médecine générale auprès de l'investigateur principal, non opposé à participer à l'étude. Critères d'exclusion : grossesse, diurétiques, chirurgie de l'obésité, troubles cognitifs, impossibilité de peser ou de collecter les informations. Critère de jugement principal : positivité du test $\left(\mathrm{EVA} \mathrm{SEFI}^{\circledR}<7 / 10\right)$ comparée à la présence de dénutrition définie par : perte de poids en 6 mois $\geq 10 \%$ et/ou 1 mois $\geq 5 \%$ et/ou indice de masse corporelle (IMC) $\leq 18,5$ ou $<21$ si $\geq 70$ ans. Critère de jugement secondaire : proportion de patients pour lesquels un score EVA SEFI ${ }^{\circledR}$ a été recueilli. Analyse multivariée: seuil $\alpha=0.20$ lors des analyses univariées, sélection pas à pas descendante.

Résultats et Analyse statistique : Parmi 747 patients répondant aux critères d'inclusion, 505 patients ont été inclus : âge moy. $( \pm S D), 56 \pm 19$ ans, $23 \% \geq 70$ ans, $61 \%$ de femmes, $31 \%$ vivant seuls, $49 \%$ consultaient pour problème médical aigu et $37 \%$ pour renouvellement d'ordonnance. 80 patients avaient une EVA SEFI ${ }^{\circledR}<7 / 10(15,8 \%)$ et 32 patients $(6,3 \%)$ étaient atteints de dénutrition. La sensibilité de I'EVA SEFI ${ }^{\circledR}<7$ pour le diagnostic de dénutrition était de $34,4 \%$ (intervalle de confiance (IC) 95\% $17,9-$ $50,8)$, la spécificité de $85,4 \%(82,2-88,6)$, la valeur prédictive positive de $13,4 \%(7,7-19,2)$, et la valeur prédictive négative de $96,2 \%(93,7-97,7)$. Tous les patients inclus ont pu évaluer leur prise alimentaire selon I'EVA SEFI ${ }^{\circledR}$. En analyse multivariée, les facteurs de risque de dénutrition étaient: âge de 45 à 70 ans (odds ratio, 0,34 [IC95\%, 0,13; 0,87]), sexe féminin (4,72 [1,63; $13,66])$, cancer $(5,66[1,58 ; 20,29])$, et alcoolisme chronique $(8,60[1,46 ; 50,81]), P<0,05$.

Conclusion : Les spécificité et VPN élevées, ainsi que la faisabilité du test, en font un outil fiable pour le dépistage de la dénutrition en médecine générale. Un score négatif pourrait notamment faire écarter le diagnostic de dénutrition par le médecin généraliste dans les situations où la perte de poids récente ou l'IMC sont indéterminés.

Références : (1) Thibault et al. CLin Nutr 2009;28:134-140. (2) Mesbah et al. Communication orale. JFN 2017

Conflits d'intérêts : G. Bouëtté: Aucun conflit à déclarer, M. Esvan: Aucun conflit à déclarer, K. Apel: Aucun conflit à déclarer, R. Thibault est consultant chez Fresenius Kabi, Lactalis, Shire, Aguettant, Astra-Zeneca, Baxter, BBraun, Nestlé, Nutricia, a un conflit d'intérêt avec concepteur de I'EPA/SEFI 
Analyse de la composition corporelle sur coupe scannographique transversale passant par la 3ème vertèbre lombaire (L3) en réanimation : impact pronostique, reproductibilité interobservateur, et prévalence de la sarcopénie.

E. Jali ${ }^{1,}{ }^{*}$, A. Mulliez ${ }^{2}$, L. Lacaze ${ }^{13}$, E. Lascouts ${ }^{1}$, N. Rotovnik Kozjek ${ }^{4}$, A.-M. Makhlouf ${ }^{5}$, I. Ceniceros Rozalen ${ }^{6}$, J.-C. Preiser ${ }^{7}$, Z. Krznaric ${ }^{8}$, K. Kupczyk $^{9}$, N. Cano ${ }^{10}$, C. Pichard ${ }^{5}$, R. Thibault ${ }^{13}$ et Phase angle project Investigators

${ }^{1}$ Unité de Nutrition, CHU DE RENNES - UNIVERSITÉ DE RENNES 1, Rennes, ${ }^{2}$ DRCI, CHU Clermont-Ferrand, Clermont-Ferrand, ${ }^{3}$ Institut NuMeCan, INRA, INSERM, Univ Rennes, Rennes, France, ${ }^{4}$ Clinical Nutrition Unit, Institute of Oncology, Ljubljana, Slovénie, ${ }^{5}$ Unité de Nutrition, HUG, Genève, Suisse, ${ }^{6} 6$ Department of Intensive Care, Clinic USP Palmaplanas, Palma de Majorque, Espagne, ${ }^{7}$ Service de Soins Intensifs, Hôpital Universitaire Erasme, Bruxelles, Belgique, ${ }^{8}$ Department of Internal Medicine , University Hospital Center Zagreb, Zagreb, Croatie, ${ }^{9}$ Department of Anesthesiology and Intensive Care, University Hospital no 1 CM NCU, Bydgoszcz, Pologne, ${ }^{10} \mathrm{CRNH}, \mathrm{CHU}$ Clermont-Ferrand, Clermont-Ferrand, France

Introduction et but de l'étude : Une masse maigre basse à l'admission en réanimation, évaluée par l'angle de phase (1) ou l'index d'aire du psoas (2), prédit la mortalité à 28 jours (J28). Objectifs : évaluer : i) si, sur une coupe de scanner passant par L3, la composition corporelle: surfaces musculaire totale (SMT) (objectif principal), des psoas, des tissus adipeux sous-cutané (SAT), périviscéral (VAT), intra-musculaire (IMAT), intra-psoas (PAT) et total (TAT) (objectifs secondaires), prédisait la mortalité J28; ii) reproductibilité inter-observateur; iii) prévalence de la sarcopénie.

Matériel et méthodes : Etude ancillaire du PHASE ANGLE PROJECT ( $n=931)(1)$, multicentrique internationale. Critères d'inclusion : scanner abdominal de routine dans les 9 jours entourant l'admission. Analyse de la composition corporelle à l'aide du logiciel Image (NIH, USA). Statistiques: aire sous la courbe (AUC) ROC. Régression logistique multivariée: variables associées à la mortalité à J28. Analyse de 33 scanners en double lecture en aveugle. Sarcopénie définie par: SMT/taille ${ }^{2}<38,9$ (femmes (F)) ou $<55,4$ $\mathrm{cm}^{2} / \mathrm{m}^{2}$ (hommes $(\mathrm{H})$ ).

Résultats et Analyse statistique : 210 patients inclus : 55\% H, 62 \pm 17 ans, APACHE II 20 $\pm 9,50 / 50 \%$ méd./chir. Chez les $F$, augmentation de l'IMAT, du VAT et du TAT indexés à la taille étaient associées à la mortalité à $J 28$ ( $p=0,03 ; p=0,02 ; p=0,02$, respectivement). Chez les $\mathrm{H}$, diminution de la SMT et des psoas indexés à la taille, de la SMT et la surface du psoas non indexées à la taille étaient associées à la mortalité à $\mathrm{J} 28(\mathrm{p}=0,01 ; \mathrm{p}=0,003 ; p=0,003 ; p<0,001$, respectivement). La SMT non indexée à la taille était prédictive de la mortalité avec une meilleure fiabilité chez I'H $(A \cup C=0,74)$ que chez la $F(A \cup C=0,61)$. Analyse multivariée: $1^{\text {er }}$ quartile de SMT (<96 (F) ou <118 (H) $\mathrm{cm}^{2}$ ) (Odds ratio=2,5 [IC95\%, 1,0-6,1], p=0,04), âge $>65$ ans $(2,8[1,1-7,3], p=0,03)$, et angle de phase à l'admission $(2,71[1,1-6,6], p=0,03)$ associés à la mortalité J28. La reproductibilité inter-observateur était bonne avec pour les tissus adipeux viscéral et total (coefficient de corrélation $r=0,99)$ ), la surface musculaire totale $(r=0,72)$, et la surface du psoas ( $r=0,90)$. Prévalence de la sarcopénie: $57 \%$ (total); 35\% (F); $74 \%(H)$.

Conclusion : La surface musculaire totale exprimée en $\mathrm{cm}^{2}$ est associée à la mortalité à J28. II existe une bonne reproductibilité inter-observateur des paramètres de composition corporelle mesurés par scanner passant par L3. Plus de la moitié des patients de réanimation sont sarcopéniques. Cette étude confirme l'importance de la mesure du niveau de masse maigre pour évaluer le pronostic du patient de réanimation.

Références : (1) Thibault et al. Intensive Care Med 2016;42:1445-53 ; (2) Lascouts et al. JFN 2016, plénière, CO32

Conflits d'intérêts : Aucun conflit à déclarer 


\section{CO66}

\section{Hydratation et nutrition entérale à domicile}

P. Fayemendy ${ }^{12,}{ }^{*}$, D. Barnoud ${ }^{3}$, S. Schneider ${ }^{4}$, B. Morin-Boulogne ${ }^{1}$, P. Jésus ${ }^{12}$, J. C. Desport ${ }^{12}$ et Comité de Nutrition à domicile de la SFNCM

${ }^{1}$ Unité Transversale de Nutrition, CHU de Limoges, ${ }^{2}$ Inserm UMR 1094, Faculté de Médecine, Limoges, ${ }^{3}$ Nutrition clinique intensive, Hospices Civils de Lyon, LYON, ${ }^{4}$ Unité de support nutritionnel, CHU de NICE, Nice, France

Introduction et but de l'étude : II existe peu de données sur l'hydratation lors de la nutrition entérale à domicile (NEAD). Une enquête francophone d'opinion était réalisée, dont l'objectif était de faire le point sur les pratiques d'hydratation complémentaire en NEAD.

Matériel et méthodes : Cette enquête menée par la SFNCM s'adressait aux professionnels de santé impliqués dans la NEAD. Le questionnaire en ligne administré en avril et mai 2018, recueillait des données générales, de prescription, d'apports et de modalités d'hydratation. L'analyse statistique utilisait les tests du Chi ${ }^{2}$ et de Fisher. Le seuil de significativité était fixé à $p<0,05$.

Résultats et Analyse statistique : 295 professionnels répondaient. 91,2\% étaient français, 4,7\% belges et 2,1\% suisses. $62,7 \%$ exerçaient en établissement de santé (ES) et 37,3\% intervenaient au domicile. 63,7\% des répondants étaient diététiciens et $29,8 \%$ médecins. La file active médiane des patients suivis était de 40 (minimum $=1 ;$ maximum $=600$ ). Les répondants déclaraient être confrontés fréquemment $(44,3 \%)$ ou toujours $(8,3 \%)$ à des difficultés d'hydratation, quels que soient la profession, le lieu d'exercice ou l'activité.

Pour 76,6\% des répondants, les consignes d'hydratation relevaient d'une prescription médicale ou d'un conseil diététique. Le conseil diététique était plus souvent cité par les diététiciens $(p=0,0001)$.

D'après les répondants, une hydratation et le volume hydrique à administrer étaient systématiquement prescrits dans respectivement $54,3 \%$ et $40,5 \%$ des cas. Le type d'eau et le changement des dispositifs d'hydratation n'étaient jamais prescrits dans respectivement $59,1 \%$ et $56,1 \%$ des cas.

Les répondants déclaraient tenir compte des prises hydriques per os $(93,1 \%)$, de la situation clinique $(86,6 \%)$ et du volume de mélange nutritif $(80,8 \%)$ pour déterminer le volume hydrique à administrer. L'état d'hydratation clinique était plus souvent cité par les médecins $(94,3 \%$ vs $73,1 \%$ des diététiciens, $p<0,001)$ comme paramètre de surveillance de l'adéquation des apports. La surveillance du transit était plus fréquemment citée par les diététiciens ( $76,9 \%$ vs $51,1 \%$ des médecins, p<0,001) et le personnel des $\operatorname{ES}(70,6 \%$ vs $46,7 \%$ des intervenants au domicile, $p<0,001)$.

La majorité des répondants déclarait utiliser de l'eau du robinet ou en bouteille. $61,6 \%$ disaient ne jamais supplémenter en sel lors de la NEAD exclusive.

$90,0 \%$ des répondants déclaraient utiliser les poches à eau et $84,9 \%$ les seringues pour hydrater. Près d'un quart des répondants ( $27,0 \%$ pour les seringues et $23,4 \%$ pour les poches) disait ne pas connaitre la fréquence de changement des dispositifs, cette méconnaissance touchant plus souvent les médecins et le personnel d'ES $(p<0,001)$.

Le recours au régulateur de débit électrique était fréquemment cité, le plus souvent de façon concomitante à l'administration du mélange nutritif.

Les répondants étaient demandeurs d'une amélioration des prescriptions d'hydratation et de référentiels synthétiques à ce propos.

Conclusion : Les pratiques d'hydratation en NEAD sont disparates, et les problèmes fréquents. Les référentiels sont peu connus, dispersés, parfois non concordants. La création d'un document support unique et consensuel d'aide aux pratiques pourrait être utile.

Conflits d'intérêts : Aucun conflit à déclarer 


\section{DÉCLIN COGNITIF}

\section{CO67}

Trajectoires de facteurs de mode de vie depuis la quarantaine et déclin cognitif après 70 ans chez la femme M. Wagner ${ }^{1, *}$, F. Grodstein ${ }^{23}$, C. Proust-Lima ${ }^{1}$, C. Samieri ${ }^{1}$

${ }^{1}$ Université de Bordeaux, Bordeaux Population Health Research Center, UMR U1219, Bordeaux, France, ${ }^{2}$ Channing Division of Network Medicine, Department of Medicine, Brigham and Women's Hospital and Harvard Medical School, ${ }^{3} \mathrm{Harvard}$ T.H. Chan School of Public Health, Boston, États-Unis

Introduction et but de l'étude : Le mode de vie aux âges moyens (40-50 ans) a été associé à une diminution du déclin cognitif à des âges plus avancés, tandis que chez la personne âgée les associations restent contradictoires. L'objectif de cette étude était de décrire les trajectoires de l'indice de masse corporelle (IMC), d'un score d'adhérence au régime méditerranéen ( $A$-MeDi) et des niveaux d'activité physique (AP) depuis la quarantaine jusqu'à des âges avancés, et de contraster ces trajectoires entre des personnes ayant un déclin cognitif après 70 ans et des personnes maintenant une cognition plus stable.

Matériel et méthodes : La Nurses' Health Study est une cohorte prospective ayant inclus 121700 infirmières américaines âgées de 30-55 ans en 1976. Un questionnaire sur le mode de vie et la santé a été administré à l'inclusion puis tous les 2 ans; un fréquentiel alimentaire et un questionnaire sur l'AP ont été ajoutés en 1984 et 1986, respectivement. En 1995, les participantes de plus de 70 ans sans antécédent d'accident vasculaire cérébral ont répondu à une batterie de tests cognitifs par téléphone ( $n=19415)$, répétée trois fois à 2 ans d'intervalle. Cette étude cas-témoin nichée porte sur un échantillon cognitivement sain au début de l'étude cognitive et inclut 1460 cas dont la pente de déclin cognitif global après 70 ans était parmi les $10 \%$ les plus importantes, et 7299 témoins avec une pente de déclin cognitif supérieure à la médiane. Les trajectoires d'IMC, de score A-MeDi et d'AP depuis la quarantaine jusqu'à l'année précédant l'étude cognitive ont été modélisées par modèles mixtes à processus latent et comparées dans les deux groupes.

Résultats et Analyse statistique : Une prise de poids était généralement observée avec l'âge; toutefois, les cas présentaient un IMC plus élevé dès 19 ans (différence moyenne [DM] $\left.=0,62 \mathrm{~kg} / \mathrm{m}^{2} ; P<0,001\right)$ et jusqu'à 3 ans $\left(\mathrm{DM}=0,33 \mathrm{~kg} / \mathrm{m}^{2} ; P=0,006\right)$ avant le début de l'étude cognitive. Au cours des dernières années précédant l'étude cognitive, cette différence s'atténuait en raison d'une décélération du gain d'IMC ( $P<0,001$ pour l'interaction groupe $\mathrm{x}$ temps). La qualité alimentaire s'améliorait dans les âges jeunes, puis se stabilisait, de façon identique dans les deux groupes ( $P=0,543$ pour groupe $x$ temps). Le score A-MeDi chez les cas était constamment inférieur à celui des témoins (à -11 ans $D M=-0,14$ point; $P<0,01$ et à -1 an $D M=-0,19$ point; $P<0,001$ ). Enfin, les niveaux d'AP déclinaient avec l'âge mais de façon plus prononcée chez les cas $(P<0,01$ pour groupe $x$ temps). De plus, les niveaux d'AP étaient constamment plus faibles chez les cas de -9 ans ( $\mathrm{DM}=-1,37 \mathrm{MET}-\mathrm{h} / \mathrm{sem}$.; $P<0,001)$ à -1 an $(\mathrm{DM}=-2,54 \mathrm{MET}-\mathrm{h} / \mathrm{sem}$; $P<0,001)$.

Conclusion : Dans cette grande cohorte, les femmes avec un déclin cognitif élevé au-delà de 70 ans présentaient un IMC plus élevé, une plus faible adhérence à l'alimentation méditerranéenne et pratiquaient moins d'AP depuis la quarantaine, par rapport à celles maintenant une cognition plus stable. A l'approche de leurs 70 ans, les femmes avec un déclin cognitif présentaient un ralentissement dans la prise de poids et une diminution plus forte des niveaux d'AP. Ces modifications physiologiques/comportementales pourraient refléter les premiers signes d’une maladie cérébrale sous-jacente (comme une démence).

Conflits d'intérêts : Aucun conflit à déclarer 


\section{CO68}

Métabolomique, métabolome alimentaire et déclin cognitif du sujet âgé.

S. Lefevre-Arbogast ${ }^{1,}{ }^{*}$, D. Low-Yanwen ${ }^{2}$, B. P. Hejblum ${ }^{13}$, C. Helmer ${ }^{1}$, P. Micheau ${ }^{2}$, C. Proust-Lima ${ }^{1}$, S. Thuret ${ }^{4}$, C. Manach ${ }^{2}$, C. Samieri ${ }^{1}$ et Consortium européen D-CogPlast (JPI HDHL)

${ }^{1}$ Univ. Bordeaux, Inserm, Bordeaux Population Health, UMR1219, Bordeaux, ${ }^{2}$ Unité Nutrition Humaine, INRA Auvergne Rhône Alpes, Univ. Clermont Auvergne, Clermont-Ferrand, ${ }^{3}$ INRIA SISTM, Bordeaux Sud-Ouest, Bordeaux, France, ${ }^{4}$ Neurosciences fondamentales et cliniques, King's College London, Institut de psychiatrie, psychologie et neurosciences, Maurice Wohl, Londres, Royaume-Uni

\section{Introduction et but de l'étude : Introduction et But de l'étude}

Le vieillissement cognitif et les maladies associées comme la démence représentent une charge sociétale majeure, pour lesquels la prévention primaire est une piste à privilégier. Parmi les facteurs de risque à cibler pour la prévention, la nutrition, pilier de la santé vasculaire et métabolique, est prometteuse. Alors que l'alimentation apporte environ 30000 composés (et près de 100000 métabolites dérivés), très peu de bioactifs ont été étudiés dans le vieillissement cognitif. L'analyse du métabolome, notamment celui en lien avec l'alimentation, pourrait permettre d'identifier de nouvelles cibles préventives.

Notre objectif était d'investiguer, par une approche métabolomique non-ciblée (profilage agnostique de tous les métabolites quantifiables dans un biofluide) une signature métabolique précoce du déclin cognitif chez le sujet âgé - avec un intérêt particulier pour les métabolites dérivés de l'alimentation.

Matériel et méthodes : Nous avons construit un cas-témoin niché dans la cohorte des 3 Cités, Bordeaux (taille ciblée 400 participants) parmi les 1293 participants ( $\geq 65$ ans) non-déments ayant accepté un prélèvement sanguin à l'inclusion en 1999-2000 et suivis sur le plan cognitif jusqu'à 12 années. Nous avons i) estimé les pentes individuelles de déclin cognitif en appliquant un modèle linéaire mixte aux mesures répétées d'un score composite incluant 5 tests cognitifs; ii) identifié les 220 sujets avec les pentes les plus importantes (cas) ; iii) appariés avec succès 209 d'entre eux à 209 témoins avec une cognition plus stable (pente > pente médiane) et de même âge, sexe et niveau d'études. L'analyse métabolomique non ciblée a été réalisée par spectrométrie de masse (301 ions après prétraitement des données). Le profil métabolique le plus associé au déclin cognitif a été identifié par régression LASSO pour données appariées (ajustée sur les facteurs d'appariement, l'IMC, le nombre de médicaments régulièrement consommés, et le niveau cognitif à l'inclusion) combinée à un ré-échantillonnage bootstrap.

Résultats et Analyse statistique : Nous avons identifié une vingtaine de métabolites associés au déclin cognitif ; ajoutés à un modèle de facteurs cliniques standards, ces métabolites amélioraient la capacité discriminante d'un AUC cross-validé de 62\% (IC 95\%: 56-67\%) à 75\% (IC 95\%: 70-80\%). La confrontation des métabolites aux bases de données existantes et/ou standards a permis l'identification formelle ou probable de : (i) 3 biomarqueurs du café, notamment l'atractyligenin glucuronide associé à un moindre déclin ; (ii) un biomarqueur du jus d'orange, la proline bétaine, associé à un plus fort déclin ; (iii) un marqueur possible du chocolat, le cyclo(prolyl-valyl), associé à un moindre déclin. De plus, la signature incluait plusieurs métabolites endogènes.

Conclusion : Nous avons identifié des métabolites précocement associés au déclin cognitif, dont plusieurs marqueurs du métabolome alimentaire. Ces résultats nécessitent d'être validés dans une population externe. Néanmoins, ils offrent des pistes intéressantes pour établir de nouvelles formulations préventives du déclin cognitif.

Conflits d'intérêts : Aucun conflit à déclarer 

(JPI HDHL)

${ }^{1}$ Univ. Bordeaux, Inserm, Bordeaux Population Health, UMR1219, Bordeaux, France, ${ }^{2}$ Neurosciences fondamentales et cliniques, King's College London, Institut de Psychiatrie, Psychologie et Neurosciences, Maurice Wohl, Londres, Royaume-Uni, ${ }^{3}$ INRIA SISTM, Bordeaux Sud-Ouest, Bordeaux, France

Introduction et but de l'étude : Le tissu cérébral est constitué à $60 \%$ de lipides, en particulier de lipides polyinsaturés. Des anomalies lipidiques du tissu cérébral ont été rapportées dans la maladie d'Alzheimer et lors du vieillissement. Des variations du pool de lipides circulants, qui peuvent refléter à la fois des facteurs étiologiques (expositions environnementales, métabolisme) et les désordres neurobiologiques sous-jacents (comme la neuro-dégénérescence) ont également été associées au déclin cognitif. Le développement de la lipidomique offre de nouvelles perspectives pour mieux comprendre les perturbations lipidiques impliquées dans le vieillissement cérébral. A ce jour, la plupart des études, de par leur caractère transversal ou leur suivi court, ont plus vraisemblablement capturé des conséquences des désordres neurobiologiques plutôt que des facteurs étiologiques.

Notre objectif était d'identifier, chez des personnes âgées non-démentes de la cohorte des 3 cités (3C), une signature lipidique précoce d'un déclin cognitif survenant dans les 12 années suivant le dosage sanguin.

Matériel et méthodes : Nous avons construit un cas-témoin niché dans la cohorte 3C Bordeaux (taille d'échantillon ciblée 400 participants), parmi les 1293 participants ( $\geq 65$ ans) non-déments et ayant accepté un prélèvement sanguin à l'inclusion en 19992000 et suivis sur le plan cognitif jusqu'à 12 années. Nous avons estimé les pentes individuelles de déclin cognitif en appliquant un modèle linéaire mixte aux mesures répétées d'un score composite incluant 5 tests cognitifs, et nous avons identifié les 220 sujets avec les pentes les plus importantes; parmi eux, 209 participants ont été appariés avec succès à 209 témoins avec une cognition plus stable (pente> pente médiane) et de même âge, sexe et niveau d'études. L'analyse lipidomique a permis de quantifier 752 espèces de lipides appartenant à une quinzaine de classes différentes. Le profil lipidique le plus associé au déclin cognitif a été identifié par régression LASSO pour données appariées (ajusté sur l'IMC, le nombre de médicaments régulièrement consommés, et le niveau cognitif à l'inclusion) combinée à un rééchantillonage bootstrap.

Résultats et Analyse statistique : Nous avons identifié une signature lipidique du déclin cognitif incluant 31 lipides, principalement des glycérophospholipides (phosphatidylcholines (PC), phosphatidylethanolamines (PE), phosphatidylinositols (PI) et leurs dérivés -éthers), quelques sphingolipides, glycérolipides et esters de cholestérol. Malgré quelques espèces divergentes, nous avons observé une tendance à des niveaux sanguins augmentés en PE et PI et diminués en PC chez les cas avec déclin cognitif substantiel par rapport aux témoins. La signature lipidique améliorait la performance de discrimination cas/témoin d'un modèle clinique standard; I'AUC cross-validé passait de 68\% (IC 95\% 63-73\%) avec les facteurs cliniques standards à un AUC de $83 \%$ (80-87\%) en incluant en plus la signature lipidique.

Conclusion : Nous avons identifié une signature lipidique précoce d’un déclin cognitif ultérieur chez les personnes âgées nondémentes, suggérant une altération précoce des glycérophospholipides dans le vieillissement cognitif. Ce travail est en cours de validation dans une population externe (3C Dijon).

Conflits d'intérêts : Aucun conflit à déclarer 


\section{$\mathrm{CO} 70$}

Les apports en acides gras insaturés au milieu de la vie sont positivement associés au fonctionnement cognitif mesuré 13 ans plus tard, avec un effet modulateur d'une supplémentation en antioxydants

K. E. Assmann ${ }^{1}$, M. Adjibade ${ }^{1,}{ }^{*}$, S. Hercberg ${ }^{12}$, P. Galan ${ }^{1}$, E. Kesse-Guyot ${ }^{1}$

${ }^{1}$ Equipe de Recherche en Epidémiologie Nutritionnelle (EREN), Université Paris 13, Centre d'Epidémiologie et Statistiques Sorbonne Paris Cité, Inserm (U1153), Inra (U1125), Cnam, COMUE Sorbonne Paris Cité, ²Département de Santé Publique, Hôpital Avicenne, Bobigny, France

Introduction et but de l'étude : La littérature scientifique concernant le lien entre les apports en acides gras mono- et polyinsaturés est abondante, mais les résultats sont inconsistants. Notre objectif était d'étudier l'association entre les apports en acides gras insaturés au milieu de la vie et le fonctionnement cognitif mesuré 13 ans plus tard, et d'évaluer un effet modulateur potentiel d'une supplémentation en antioxydants.

Matériel et méthodes : Les apports en acides gras étaient estimés en utilisant des rappels alimentaires de 24h répétés (19941996), chez 3362 participants (âge moyen \pm déviation standard : $65.5 \pm 5.6$ ans) de l'étude SU.VI.MAX (SUpplémentation en VItamines et Minéraux AntioXydants). L'étude SU.VI.MAX incluait une phase d'intervention (1994-2002) pendant laquelle les participants étaient attribués, de manière randomisée, à un groupe "supplémentation en antioxydants à doses nutritionnelles " ou à un groupe placebo. Le supplément reçu chaque jour entre 1994 et 2002, était composé d'une combinaison de 120 mg de vitamine $C, 30 \mathrm{mg}$ de vitamine $E, 6 \mathrm{mg}$ de bêta-carotène, $100 \mu \mathrm{g}$ de sélénium et $20 \mathrm{mg}$ de zinc. Le fonctionnement cognitif était évalué uniquement à la fin du suivi (2007-2009), par une batterie de 6 tests neuropsychologiques. Un score cognitif global a été créé en calculant la somme de T-scores de ces 6 tests.

Résultats et Analyse statistique : Des modèles d'analyse de covariance (ANCOVA) ajustés sur les facteurs confondants potentiels ont été utilisés afin de calculer des différences moyennes ajustées du score cognitif global entre tertiles des apports en acides gras. Dans ces modèles multivariables, les apports en acides gras monoinsaturés (AGMI) totaux, polyinsaturés (AGPI) totaux, et polyinsaturés n- 6 étaient positivement associés avec le fonctionnement cognitif global. Les acides gras polyinsaturés $n-3$ montraient des relations positives avec le fonctionnement cognitif uniquement chez les participants supplémentés en antioxydants (différence moyenne Tertile3 versus Tertile1 $=1.40$; intervalle de confiance à $95 \%=0.30,2.51, P_{\text {trend }}=0.01, P_{\text {interaction }}=0.01$ ). Un moins bon fonctionnement cognitif global a été observé chez les sujets présentant des apports plus élevés en acide arachidonique uniquement au sein du groupe placebo (différence moyenne Tertile3 versus Tertile1=-1.38; intervalle de confiance à $95 \%=-2.57,-0.18$, $\left.P_{\text {trend }}=0.02, P_{\text {interaction }}=0.07\right)$.

Conclusion : Des apports élevés en AGMI totaux et en AGPI n-6 pourraient être bénéfiques pour maintenir une bonne santé cognitive au cours du vieillissement, des apports plus élevés en AGPI n-3 pourraient être bénéfiques seulement chez des individus ayant un statut adéquat en antioxydants. Ces résultats soulignent l'importance de ne pas seulement cibler des nutriments spécifiques dans le cadre de la prévention de démences, mais de considérer l'interaction complexe entre les nutriments consommés.

Conflits d'intérêts : Aucun conflit à déclarer 
Association entre statut plasmatique en vitamine D et connectivité cérébrale fonctionnelle chez le sujet âgé - cohorte des 3Cités - Bordeaux

A. Thomas ${ }^{1,}{ }^{*}$, C. Féart ${ }^{1}$, C. Delcourt ${ }^{1}$, C. Helmer ${ }^{1}$, M. Edde ${ }^{2}$, G. Catheline ${ }^{2}$, C. Samieri ${ }^{1}$

${ }^{1}$ Bordeaux Population Health Research Center, UMR 1219, Universite de Bordeaux, Inserm, ${ }^{2}$ Institut de Neurosciences Cognitives et Intégratives d'Aquitaine, UMR 5287, Universtite de Bordeaux, CNRS, Bordeaux, France

Introduction et but de l'étude : La plasticité du cerveau (définie comme sa capacité à modifier sa structure et son fonctionnement en réponse à divers stimuli) est altérée dans le cadre du vieillissement cérébral et peut être modulée par certaines expositions, dont la nutrition. Une association entre un statut plus élevé en vitamine $D$ et une meilleure connectivité cérébrale au niveau structurel a, par exemple, été démontrée dans quelques études épidémiologiques, mais n’a jamais été étudiée au niveau fonctionnel chez le sujet âgé.

L'objectif de cette étude observationnelle était d'évaluer l'association entre le statut plasmatique en vitamine D (25(OH)D) et la connectivité cérébrale fonctionnelle au repos, mesurée par Imagerie par Résonance Magnétique fonctionnelle (IRMf) dans deux réseaux fonctionnels d'intérêt (le réseau de mode par défaut (DMN) et le fronto-pariétal (FP)), chez la personne âgée.

Matériel et méthodes : Dans la cohorte des 3 Cités - Bordeaux (âge $\geq 65$ ans), 102 sujets non-déments ayant des mesures de 25(OH)D à l'inclusion, en 1999-2000, ont passé un examen IRMf au repos 10 ans plus tard. Les associations entre le niveau plasmatique en $25(\mathrm{OH}) \mathrm{D}$ (défini en trois catégories : suffisant, $>50 \mathrm{nmol} / \mathrm{L}$; insuffisant, $25-50 \mathrm{nmol} / \mathrm{L} ;$ carence, $<25 \mathrm{nmol} / \mathrm{L}$ ) et le niveau de connectivité fonctionnelle dans le FP et le DMN ont été évaluées par régressions linéaires multivariables (modèles ajustés sur l'âge, le sexe, la présence de l'allèle epsilon 4 de l'Apolipoprotéine $E$, le niveau d'études et la saison du prélèvement sanguin).

Résultats et Analyse statistique : Les participants avec une déficience ou une carence en vitamine D à l'inclusion (60\% et $26 \%$, respectivement) avaient une connectivité fonctionnelle significativement augmentée dans le FP, par rapport à ceux ayant des niveaux suffisants en vitamine $D$ (Tableau, p global=0,04). Aucune association n'a été trouvée dans le DMN.

\begin{tabular}{|c|c|c|c|}
\hline & $\mathrm{N}$ & $\begin{array}{l}\text { Différence moyenne de } \\
\text { connectivité estimée }(\beta)\end{array}$ & IC à $95 \%$ \\
\hline Carence (<25 nmol/L) & 27 & $4,8 \times 10^{-2}$ & {$\left[1,1 \times 10^{-2} ; 8,4 \times 10^{-2}\right]$} \\
\hline Insuffisance $(5-50 \mathrm{nmol} / \mathrm{L})$ & 61 & $4,1 \times 10^{-2}$ & {$\left[0,9 \times 10^{-2} ; 7,2 \times 10^{-2}\right]$} \\
\hline Suffisance (>50 nmol/L) & 14 & Ref & - \\
\hline
\end{tabular}

Conclusion : Dans cette étude, une carence ou une déficience en vitamine $D$ était associée à une connectivité moyenne augmentée dans le FP. Les études de connectivité fonctionnelle suggèrent la présence d'effets de compensation, notamment en frontal, avec une augmentation de la connectivité fonctionnelle parfois observée pour compenser des altérations cérébrales se développant en postérieur. La relation entre carence en vitamine $D$ et augmentation de la connectivité fonctionnelle dans le FP pourrait ainsi être interprétée comme le reflet de mécanismes de compensation dans le cerveau âgé.

Les associations entre la vitamine $D$ et la connectivité fonctionnelle apportent des arguments mécanistiques supplémentaires en faveur du rôle de cette vitamine dans le déclin cognitif lié à l'âge et la démence ; des études complémentaires restant nécessaires pour confirmer ces résultats.

Conflits d'intérêts : Aucun conflit à déclarer 
Caroténoïdes plasmatiques, biomarqueurs du risque de dégénérescence maculaire : l'étude Aliénor.

B. M. Merle ${ }^{1, *}$, A. Cougnard-Grégoire ${ }^{1}$, J.-F. Korobelnik ${ }^{1}$, W. Schalch ${ }^{2}$, S. Etheve ${ }^{2}$, C. Féart ${ }^{1}$, C. Samieri ${ }^{1}$, M.-N. Delyfer ${ }^{1}$, C. Delcourt ${ }^{1}$ et The EYE-RISK Consortium

${ }^{1}$ Lifelong Exposures Health and Aging (LEHA), Inserm U1219, Bordeaux, France, ${ }^{2}$ DSM Nutritional Products, Kaiseraugst, Suisse

Introduction et but de l'étude : La dégénérescence maculaire liée à l'âge (DMLA) est la $1^{\text {ère }}$ cause de malvoyance dans les pays industrialisés. Cette maladie du vieillissement de la rétine touche environ 600000 personnes en France. Généralement précédées de formes précoces asymptomatiques, les formes avancées entraînent une baisse de vision souvent rapide et importante, les traitements disponibles ne permettant au mieux que de stabiliser les lésions. Les principaux facteurs de risque identifiés sont des facteurs génétiques et le tabagisme. Les caroténoïdes, la lutéine notamment, s'accumulent spécifiquement dans la macula et filtrent la lumière bleue toxique pour la rétine. A ce jour, les études peu nombreuses et uniquement transversales ne permettent pas d'établir de relation causale entre statut en caroténoïdes et risque de DMLA. L'objectif de cette étude est d'évaluer les associations entre caroténoïdes plasmatiques et incidence de DMLA avancée à 6 ans dans la cohorte Aliénor.

Matériel et méthodes : En 2006-08, 963 personnes âgées de 73 ans et plus ont été inclues dans la cohorte Aliénor et sont suivies tous les 2 ans (dernier suivi 2015-17). A chaque suivi, un examen ophtalmologique complet, incluant des photographies de la rétine et de l'imagerie en Tomographie en Cohérence Optique de haute résolution (SD-OCT) est réalisé. Avec ces images, un comité détermine le stade de DMLA : avancée (atrophique ou néovasculaire) ou précoce (anomalies rétiniennes et/ou pigmentaires) ou absence. Les caroténoïdes plasmatiques (xanthophylles (lutéine+zéaxanthine+ $\beta$-cryptoxanthine) et carotènes ( $\beta$-carotène+ $\alpha$ carotène+lycopène)) ont été mesurés par chromatographie liquide haute performance à partir d'échantillons sanguins à jeun recueillis à l'inclusion. Les caroténoïdes plasmatiques, liés aux lipides circulants, ont été considérés selon le ratio caroténoïde/(cholestérol total (TC)+triglycérides (TG)). Les associations entre les ratios caroténoïde/(TC+TG) et l'incidence de DMLA avancée ont été évaluées par des modèles de Cox prenant en compte les données des deux yeux et leur corrélation. Les modèles ont été ajustés sur les facteurs sociodémographiques, oculaires, génétiques, médicaux, biologiques, alimentaires et de mode de vie.

Résultats et Analyse statistique : Après ajustement, les sujets ayant des niveaux élevés de caroténoïdes totaux présentaient un risque plus faible de développer une DMLA avancée (risque relatif pour l'augmentation d'un écart-type (RR) $=0,59$; intervalle de confiance $95 \%(I C 95 \%)=0,38-0,91 ; p=0,018)$, parmi eux la lutéine (RR=0,61; IC95 \%=0,42-0,89; $p=0,010)$, la zéaxanthine $(R R=0,70 ;$ IC95 \%=0,51-0,95; $p=0,024)$, le $\beta$-carotène ( $R R=0,60 ;$ IC95 \%=0,39-0,92; $p=0,021)$ et le lycopène ( $R R=0,65$; IC95 $\%=0,44-0,96 ; p=0,030)$ étaient significativement associés au risque de DMLA avancée

Conclusion : Cette étude originale suggère qu'en population âgée une concentration plasmatique de caroténoïdes plus élevée (notamment en lutéine et zéaxanthine) serait associée à un moindre risque de développer une DMLA avancée. Les biomarqueurs présentent l'avantage d'être plus objectifs que le recueil des consommations alimentaires et de prendre un compte la biodisponibilité des nutriments.

Remerciements : DSM Nutritional Products et Laboratoires Théa

Conflits d'intérêts : B. Merle a reçu une subvention/soutien de Voyages par Laboratoires Théa, A. Cougnard-Grégoire a reçu une subvention/soutien de Voyages par Laboratoires Théa, J.-F. Korobelnik est consultant chez Alcon, Allergan, Bayer, Baush\&Lomb, Beaver Visitec, Horus, Krys, Kanghong, NanoRetina, Novartis, Roche, Thea, Zeiss, W. Schalch est employé(e) de DSM Nutritional Products, S. Etheve est employé(e) de DSM Nutritional Products, C. Féart a reçu une subvention/soutien de Participation à des congrès par Danone Research et Nutricia, C. Samieri: Aucun conflit à déclarer, M.-N. Delyfer est consultant chez Allergan, Bausch + Lomb, Bayer, Novartis, Théa, C. Delcourt est consultant chez Allergan, Bausch+Lomb, Laboratoires Théa, Novartis et Roche 


\section{MÉTABOLISME DES ACIDES GRAS ET OBÉSITÉ}

\section{$\mathrm{CO} 73$}

Contrôle de l'adipogénèse par les métabolites des acides gras

C. Colson ${ }^{1, *}$, E.-Z. Amri ${ }^{1}$

${ }^{1}$ Institut de Biologie Valrose, UCA, CNRS, Inserm, Nice, France

Introduction et but de l'étude : L'augmentation de la prévalence du surpoids et de l'obésité a atteint un seuil qualifié d'épidémique. L'obésité est due à un déséquilibre de la balance énergétique et il n'existe actuellement pas de traitement efficace. La redécouverte d'un BAT fonctionnel chez l'adulte ouvre de nouvelles perspectives thérapeutiques, en particulier avec la découverte des adipocytes brite.

Les acides gras stockés dans ces adipocytes sont les principaux substrats de la thermogénèse mais participent également au développement et à la fonction du tissu adipeux. Les différences de composition et les quantités relatives d'acides gras polyinsaturés (AGPI) $\omega 3$ et $\omega 6$ participent au développement du tissu adipeux. Récemment, notre équipe a montré que l'acide arachidonique, un AGPI $\omega 6$, ainsi que certains de ses métabolites appelés oxylipines, sont capables d'inhiber ou d'induire l'activité thermogénique voire de favoriser la formation d'adipocytes brite, évènement appelé "brunissage". Notre étude vise à déterminer le rôle potentiel des métabolites des acides gras dans le contrôle du développement du tissu adipeux et la régulation de la thermogénèse.

Matériel et méthodes : Afin de déterminer le rôle potentiel des métabolites des acides gras, nous disposons de divers échantillons : 1) biopsies de BAT et WAT humain, 2) BAT et WAT de souris exposées i) à différents régimes alimentaires, ii) au froid ou iii) à un agoniste des récepteurs $\beta 3$-adrénergiques. Nous disposons également d'un modèle cellulaire unique : hMADS (human Multipotent Adipose-Derived Stem), capables de se différencier en adipocytes blancs et se convertir en adipocytes brite fonctionnels. Des profils lipidiques et transcriptomiques de ces échantillons ont été réalisés, nous permettant d'établir des corrélations entre certaines oxylipines et le niveau d'expression de messagers clefs de l'adipocyte thermogénique tel qu'UCP1. Plusieurs métabolites candidats ont été sélectionnés puis testés par le traitement in vitro des cellules hMADS et l'analyse de plusieurs paramètres : l'expression des messagers de gènes clefs, la fonction respiratoire et lipolytique des adipocytes.

Résultats et Analyse statistique : Jusqu'à présent, 2 candidats semblent être prometteurs : 9- et 13-Hydroxyoctodienoique (9- et 13-HODE), des métabolites de l'acide arachidonique. Les niveaux de 9- et 13-HODE sont statistiquement corrélés à l'expression du messager d'UCP1 dans les biopsies humaines ainsi que dans les tissus adipeux murins. Par ailleurs, les niveaux sont statistiquement augmentés dans i) les sérums humains (PET-scan + ), ii) les modèles murins exposés au froid $\left(4-6^{\circ} \mathrm{C}\right)$. Enfin, les niveaux des $9-$ et 13-HODE sont augmentés dans les milieux de sécrétion des adipocytes hMADS brite mais curieusement diminués dans leur contenu cellulaire. Le traitement des cellules hMADS par ces métabolites potentialise la fonction lipolytique des adipocytes sans toutefois induire l'expression du messager d'UCP1. Cependant, l'inhibition de leur voie de synthèse induit une forte inhibition des gènes de l'adipogenèse et de la thermogenèse et cette inhibition peut être reversée par l'ajout des composés.

Conclusion : Ces résultats montrent de manière intéressante que les composés 9- et 13-HODE semblent jouer un rôle direct et/ou indirect dans l'adipogenèse et le phénotype thermogénique. Des études complémentaires sont néanmoins nécessaires pour comprendre les mécanismes sous-jacents à leur fonction.

Conflits d'intérêts : Aucun conflit à déclarer 
Impact de l'acide trans-palmitoléique alimentaire sur les composantes du syndrome métabolique : étude chez la souris C57BL/6N.

\author{
E. J. Guillocheau ${ }^{12,{ }^{*}, \text { G. Drouin }}{ }^{1}$, C. Penhoat ${ }^{1}$, G. Ghukasyan ${ }^{3}$, A. Fautrel ${ }^{3}$, M. Marty ${ }^{4}$, V. Quillien ${ }^{4}$, D. Catheline ${ }^{1}$, P. Legrand ${ }^{1}$, \\ V. Rioux $^{1}$ \\ ${ }^{1}$ Laboratoire de Biochimie-Nutrition humaine, Agrocampus-Ouest, Rennes, ${ }^{2}$ Direction des Affaires Scientifiques et Techniques, \\ Centre national interprofessionnel de l'économie laitière (CNIEL), Paris, ${ }^{3}$ Plate-forme H2P2, Université de Rennes 1 , ${ }^{4}$ Centre \\ Eugène Marquis, Rennes, France
}

Introduction et but de l'étude : Plusieurs études épidémiologiques ont associé l'acide trans-palmitoléique (C16:1 n-7 trans, TPA) à un moindre risque de syndrome métabolique: plus ses taux circulants sont importants, moins le risque de syndrome métabolique est élevé. Le TPA est retrouvé dans la matière grasse du lait (0,04\% des acides gras totaux), en tant qu'acide gras trans naturel : ces résultats épidémiologiques font donc écho à la relation inverse entre consommation de produits laitiers et risque de syndrome métabolique. Toutefois, aucune étude de supplémentation en TPA n'a permis à ce jour de vérifier ses bénéfices vis-à-vis du syndrome métabolique : en effet, de telles quantités de TPA, conjuguées à une haute pureté (>95\%), ne sont pas disponibles commercialement.

Cette étude se propose donc de vérifier pour la première fois si le TPA, dans un contexte de syndrome métabolique, possède des bénéfices, tout en montrant que l'obtention chimique de quantités adéquates de TPA en grande pureté est possible.

Matériel et méthodes : Le TPA a été obtenu par synthèse chimique, à partir de son isomère de configuration, l'acide cispalmitoléique. Une étape d'isomérisation, puis de purification ont été nécessaires, pour obtenir $70 \mathrm{~g}$ de TPA à $99 \%$ de pureté sous forme d'ester éthylique, convenable à la supplémentation.

Des souris C57BL/6N ont été nourries à 6 semaines d'âge avec un régime riche en sucres et en graisses pendant 7 semaines, et supplémentées pendant toute la durée soit en TPA (4\% de l'énergie totale), soit en acide palmitique (4\% de l'énergie totale, contrôle positif). Un contrôle négatif (chow) a également été utilisé. En fin d'expérimentation, les tissus adipeux viscéral (TAV) et sous-cutané (TASC) ont été prélevés, pesés, et des coupes histologiques ont été réalisées. Le plasma a également été recueilli, et les paramètres métaboliques (insuline, glucose, leptine, résistine, cholestérol total, cholestérol-HDL, triglycérides) ont été déterminés. Une analyse de variance a été effectuée pour analyser les données.

Résultats et Analyse statistique : La supplémentation en TPA réduit les poids du TAV et du TASC de manière significative par rapport au contrôle positif. Les diamètres des adipocytes du TAV et du TASC sont également significativement plus petits par rapport au contrôle positif. De manière concordante, les taux plasmatiques de leptine et de résistine sont significativement plus bas chez les souris supplémentées en TPA par rapport au contrôle positif.

Conclusion : Notre étude montre pour la première fois le bénéfice d'une supplémentation en TPA dans un contexte de mise en place du syndrome métabolique, par le biais d'une action sur les tissus adipeux. Plus généralement, cet acide gras trans naturel pourrait en partie expliquer l'effet protecteur des produits laitiers sur le risque de syndrome métabolique. D'autres analyses sont en cours pour déterminer les mécanismes d'action précis du TPA.

Conflits d'intérêts : E. Guillocheau est employé(e) de Centre national interprofessionnel de l'économie laitière (CNIEL), G. Drouin: Aucun conflit à déclarer, C. Penhoat: Aucun conflit à déclarer, G. Ghukasyan: Aucun conflit à déclarer, A. Fautrel: Aucun conflit à déclarer, M. Marty: Aucun conflit à déclarer, V. Quillien: Aucun conflit à déclarer, D. Catheline: Aucun conflit à déclarer, P. Legrand: Aucun conflit à déclarer, V. Rioux: Aucun conflit à déclarer 
CO75

Impact de lipides bioactifs, les FAHFAs, sur les désordres métaboliques associés à l'obésité

M. Benlebna ${ }^{1,}{ }^{*}$, L. Balas ${ }^{2}$, B. Bonafos ${ }^{1}$, L. Pessemesse ${ }^{1}$, G. Fouret ${ }^{1}$, S. Gaillet ${ }^{1}$, T. Durand ${ }^{2}$, C. Coudray $^{1}$, F. Casas ${ }^{1}$, C. Feillet-Coudray ${ }^{1}$

${ }^{1}$ UMR 0866 DMEM, INRA, ${ }^{2}$ IBMM, Montpellier, France

Introduction et but de l'étude : Les esters d'acides gras hydroxylés (FAHFAs) sont une nouvelle classe de lipides endogènes récemment découverts par l'équipe du Pr Kahn (Yore et al, Cell, 2014), laquelle a pu montrer certains effets métaboliques et antiinflammatoires de ces lipides.

Les données d'une étude préliminaire in vitro réalisée dans notre laboratoire indiquent que ces FAHFAs modulent le fonctionnement de l'activité mitochondriale dans des myoblastes murins C2C12.

L'objectif de notre étude est d'explorer pour la première fois in vivo l'impact des FAHFAs sur le phénotype musculaire et sur l'activité mitochondriale de souris contrôles ou de souris nourries avec un régime obésogène.

Matériel et méthodes : Des souris mâles C57Bl6 âgées de 1 mois ( $n=10$ par groupe) ont été réparties dans deux lots de 30 souris chacun. Un lot a reçu un régime témoin et l'autre lot un régime obésogène. Deux groupes de chaque lot ont été traités avec le 9 OAHPA ou le 9-PAHPA (15 mg/kg de poids pondéral de souris) durant les 12 semaines de régimes. Le poids des souris, la composition corporelle et la dépense énergétique ont été évalués au long de l'étude, ainsi que différents paramètres de l'insulinorésistance, du métabolisme lipidique, de l'activité mitochondriale et du stress oxydant.

Résultats et Analyse statistique : Nos premiers résultats montrent que le 9-PAHPA diminue la prise de poids et la masse maigre des souris contrôles alors que le 9-OAHPA les augmente. Chez les souris sous régime obésogène, la prise de poids et de masse grasse augmente mais sans effet des FAHFAs. De plus, les FAHFAs modifient la consommation d'O2 et le RER (rapport d'échange respiratoire $\mathrm{VCO} / \mathrm{VO} 2$ ) chez les souris contrôles et obèses, mais différemment selon le FAHFAs considéré. Enfin, le régime obésogène diminue la sensibilité à l'insuline, alors que les FAHFAs l'augmentent aussi bien chez les souris contrôles que chez les souris obèses. Par contre, alors que le régime obésogène diminue la tolérance au glucose, les FAHFAs sont sans effet.

Conclusion : Dans l'ensemble, ces travaux indiquent que les FAHFAs modulent différents paramètres métaboliques, de façon différentielle selon le régime alimentaire (contrôle ou obésogène) et la nature du FAHFA (9-OAHPA ou 9-PAHPA).

Références : Yore et al. Discovery of a class of endogenous mammalian lipids with anti-diabetic and anti-inflammatory effects. Cell. 2014 Oct 9;159(2):318-32.

Conflits d'intérêts : Aucun conflit à déclarer 


\section{CO76}

Influence de l'auto-immunité dans la résistance au modèle murin d'induction d'une obésité par une diète riche en lipides R. Legrand ${ }^{1,}{ }^{*}$, S. Azhar ${ }^{1}$, C. Deroissart ${ }^{1}$, M.-A. Le Solliec ${ }^{123}$, M. Dominique ${ }^{123}$, F. Léon ${ }^{24}$, J.-C. do Rego ${ }^{24}$, N. Lucas ${ }^{1}$

${ }^{1}$ TargEDys SA, Université de Rouen, ${ }^{2}$ Institute for Research and Innovation in Biomedicine (IRIB), ${ }^{3}$ Inserm UMR1073, Nutrition, Inflammation et Dysfonction de l'axe Intestin-Cerveau, ${ }^{4}$ Service Commun d'Analyse Comportementale (SCAC), Normandie Université, Rouen, France

Introduction et but de l'étude : L'induction de l'obésité par une diète riche en lipides (diet-induced obesity ou DIO) est l'un des modèles les plus utilisés chez le Rongeur pour mimer les désordres métaboliques associés au surpoids. Néanmoins, si celui-ci a été critiqués sur de nombreux aspects expérimentaux (souche animale de référence, composition nutritionnelle de la diète, palatabilité, ...), l'exclusion systématique des animaux ne présentant un phénotype attendu à l'issue du protocole reste assez silencieuse au sein de la littérature scientifique ; une des causes majeures étant la méconnaissance des mécanismes sous-jacents à cette résistance. Cependant, la différence entre les animaux répondeurs (DIO) et résistants (DIO-R) au régime hyperlipidique pourraient, en partie, s'expliquer par la propriété des auto-anticorps naturellement produits chez l'Homme et le Rongeur à modifierles effets périphériques des peptides impliqués dans la régulation de l'homéostasie énergétique.

Matériel et méthodes : Cette étude s'intéresse donc à caractériser cette composante auto-immune dans un modèle DIO typique de 15 semaines chez la souris mâle C57BI/6 $(n=52)$. A l'issue du protocole, les animaux DIO-R ( $n=10-$ soit 19,2\% de l'effectif total) ont été détectés conformément aux cinétiques de poids corporel comparativement à des animaux DIO ; gain inférieur à $12 \%$ de la moyenne des contrôles ayant accès à une diète standard $(S D, n=8)$. Ensuite, les taux plasmatiques de leptine, ghréline, PYY, GLP1 et a-MSH ont été mesurés par Milliplex (Merck Millipore) ou technique ELISA. Les cinétiques d'affinité des auto-anticorps (IgG) dirigés contre ces peptides ont finalement été analysées par résonnance plasmonique de surface (BiaCore T-200, GE Healthcare). Résultats et Analyse statistique : En dépit d'un phénotype comparable aux animaux SD en terme de poids, les souris DIO-R présentaient un profil peptidique global intermédiaire entre les SD et DIO, avec notamment des taux de leptine plasmatique 1,8 fois inférieur au groupe DIO $(2880 \mathrm{pg} / \mathrm{mL}$ versus $5133 \mathrm{pg} / \mathrm{mL}$, Kruskal-Wallis test, $p<0,001)$ mais 6 fois supérieur au groupe SD (479 $\mathrm{pg} / \mathrm{mL}$, Kruskal-Wallis test, $\mathrm{p}<0,01)$. Plus encore, il a été observé une diminution de l'affinité des auto-anticorps dirigés contre les peptides orexigènes (Mann-Whitney test, $p<0,05$ ), alors que l'affinité des auto-anticorps dirigés contre les peptides anorexigènes était globalement augmentée.

Conclusion : Ces données suggèrent donc l'implication de l'auto-immunité dans le phénomène de résistance au modèle DIO murin et soulignent l'intérêt potentiel des autoanticorps contre les neuropeptides comme biomarqueurs prédictifs du développement de l'obésité.

Conflits d'intérêts : R. Legrand est employé(e) de TargEDys SA, S. Azhar est employé(e) de TargEDys SA, C. Deroissart est employé(e) de TargEDys SA, M.-A. Le Solliec: Aucun conflit à déclarer, M. Dominique: Aucun conflit à déclarer, F. Léon: Aucun conflit à déclarer, J.-C. do Rego: Aucun conflit à déclarer, N. Lucas est employé(e) de TargEDys SA 
Les acides gras poly-insaturés de type oméga3 inhibent la production d'IL-17A médiée par les cellules souches du tissu adipeux, en diminuant l'expression d'ICAM-1, chez le sujet obèse

M. Chehimi ${ }^{1}$, R. Ward ${ }^{2}$, H. Vidal $^{1}$, A. Eljaafari ${ }^{3, *}$

${ }^{1}$ Inserm unité 1060-Carmen, inserm, lyon, France, ${ }^{2}$ Nutrition dietetics and food department, utah state University, logan, ÉtatsUnis, ${ }^{3}$ Inserm unité 1060 -Carmen, Hospices Civils de Lyon, Iyon, France

Introduction et but de l'étude : Dans l'obésité, le tissu adipeux est infiltré par de nombreuses cellules immunitaires proinflammatoires, telles que les Th17, une sous-population de lymphocytes T secrétant l'IL-17A. Nous avons récemment démontré à l'aide d'un modèle de co-culture, qu'au sein du tissu adipeux, la polarisation des lymphocytes T vers la voie Th17 est sous la dépendance des cellules souches adipocytaires (ob-CSA) ou des adipocytes provenant de sujets obèses $(1,2)$. Comme les acides gras poly-insaturés de type oméga3 sont connus pour réduire l'inflammation du tissu adipeux et l'hyperplasie adipocytaire chez le sujet obèse, nous avons posé la question de savoir si cela pouvait être lié à l'inhibition de la production d'IL-17A, médiée par les ob-CSA.

Matériel et méthodes : : L'acide alpha linolenique (ALA), le précurseur des omégas 3, ou deux de ses métabolites; l'acide eicosapentaenoïque(EPA), ou l'acide docosahexaenoïque (DHA), ont été rajoutés à des co-cultures de cellules souches adipocytaires provenant de sujets obèses (ob-CSA) et de cellules mononucléées issues du sang périphérique (MNC). La phytohemagglutinine $A$, un mitogène lymphocytaire, a été utilisée pour activer les lymphocytes T dans ce contexte. La production d'IL-17A, et des autres cytokines pro-inflammatoires a été mesurée par ELISA. Les tests statistiques de type Anova, suivis de comparaison par Bonferroni, ont été utilisés pour valider les résultats

Résultats et Analyse statistique : Nos résultats ont montré que I'ALA, I'EPA ou la DHA induit une inhibition statistiquement significative de la sécrétion d'IL-17A par les lymphocytes T, sans pour autant altérer la sécrétion d'IL-1b, IL-6 ou TNFa par les monocytes, Sur le plan mécanistique, nous avons montré que TLR-4 est impliqué dans la production d'IL-17A dans notre modèle, à l'aide de Viper, un peptide inhibiteur, et que I'ALA inhibe son expression, ainsi que celle de Cox2. De plus l'activation de Stat-3, un facteur de transcription nécessaire à la production d'IL-17A a été également inhibé par l'ALA. Enfin, nous avons montré par QRTPCR, et cytométrie de flux que I'ALA inhibe l'expression d'ICAM-1, une molécule d'adhésion nécessaire aux contacts intercellulaires entre obCSA et MNC, et dont l'inhibition entraîne une diminution de la sécrétion d'IL-17A

Conclusion : En conclusion, nous démontrons dans ce travail que les acides gras poly-insaturés de type oméga3 inhibent la sécrétion d'IL-17A médiée par les ob-CSA, via l'inhibition de l'expression d'ICAM-1, et la réduction des contacts cellulaires entre CSA et MNC, entraînant ainsi une baisse de la signalisation biochimique nécessaire à la sécrétion d'IL-17A. Ces mécanimes pourraient expliquer les effets bénéfiques des omégas 3, observés dans certaines maladies liées à l'IL-17A $(3,4)$

Références : 1 .Eljaafari A, Robert M, Chehimi M, Chanon S, Durand C, Vial G, Bendridi N, Anne-Marie Madec A-M, Disse E, Laville M, Rieusset J, Lefai E, Vidal H, and Pirola L. 2015 Diabetes. 64:2477-88

2.Chehimi M, Robert M, Vial G, Rieusset J, Vidal H, Pirola L, Eljaafari A.2016. Adipocyte. 5(3):275-82

3. Monk J, Hou T, Turk H, Weeks B et al. Plos One.2012. 7:11.e49739

4. Shoda H, Yanai R, Yoshimura T, Nagai T et al. Plos One 2015, 10: 9. e0138241

Conflits d'intérêts : Aucun conflit à déclarer 
Ghréline linguale agit comme un facteur autocrine pour moduler la signalisation cellulaire par les acides gras dans les cellules gustatives humaines

A. Khan ${ }^{1,}{ }^{*}$, L. Brissard $^{1}$, A. Hichami ${ }^{1}$, H. Ozdener ${ }^{2}$, N. A. Khan ${ }^{1}$

${ }^{1}$ Equipe NUTOXE INSERM 1231, Université de Bourgogne Dijon, Dijon, France, ${ }^{2}$ Monell Chemical senses centerl, Pheladelphia, États-Unis

Introduction et but de l'étude : Il existe cinq modalités gustatives. Cependant, des preuves irréfutables récentes suggèrent qu'il pourrait y avoir une modalité gustative, destinée à la perception orosensorielle des lipides alimentaires. Les cellules gustatives, localisées dans les papilles linguales, expriment des récepteurs de goût du gras tels que CD36 et GPR120. Notre laboratoire a démontré que les agents paracrines, tels que le GLP-1, connus pour être libérés par les cellules pancréatiques, sont également libérés par les cellules des papilles gustatives et modulent la perception et la préférence des lipides alimentaires.

Matériel et méthodes : Les expériences ont été réalisées sur les cellules gustatives humaines, immortalisées au sein de notre laboratoire. Les ARNm codant le récepteur de la ghréline (GSH-R) ont été étudié par la technique du RT-PCR. La signalisation calcique a été étudiée, en utilisant la sonde fluorescente Fura-2/AM, sur ces cellules gustatives. Les dosages de la ghréline, dans le surnageant de cellules gustatives, ont été effectué par le kit (Millipore).

Résultats et Analyse statistique : Dans la présente étude, nous avons détecté les ARNm codant pour la synthèse de la ghréline et de son récepteur (GSH-R) dans les cellules des papilles gustatives humaines. Nous avons observé que la ghréline induisait la signalisation du $\mathrm{Ca}^{2+}$ via son récepteur dans ces cellules gustatives humaines. En outre, la signalisation calcique, déclenchée par un acide gras à longue chaîne, à savoir l'acide linoléique, était modulée par la ghréline exogène.

Conclusion : Dans ce rapport, nous démontrons, pour la première fois, que la ghréline, sécrétée par les cellules gustatives, peut interagir avec la perception orosensorielle des lipides alimentaires.

Conflits d'intérêts : Aucun conflit à déclarer 


\section{POSTERS}

\section{ALIMENTATION DURABLE - ALIMENTATION ANIMALE ET QUALITE DES PRODUITS TECHNOLOGIE ALIMENTAIRE}

P001

Etude de la déstructuration et du confort en bouche de deux produits céréaliers enrichis en protéines végétales chez les personnes âgées : Impact du statut dentaire et du flux salivaire

C. Septier ${ }^{1, *}$, M. Assad-Bustillos ${ }^{123}$, C. Tournier ${ }^{1}$, J. Palier ${ }^{2}$, M. Vandenberghe-Descamps ${ }^{1}$, G. Della Valle ${ }^{2}$, G. Feron $^{1}$

${ }^{1}$ Centre des Sciences du Goût et de I'Alimentation, AgroSup Dijon, CNRS, INRA, Univ. Bourgogne Franche-Comté, Dijon, ${ }^{2}$ INRA UR1268, Biopolymères Interactions et Assemblages, INRA, Nantes, ${ }^{3}$ CERELAB ${ }^{\circledR}$, Aiserey, France

Introduction et but de l'étude : Le vieillissement s'accompagne souvent de troubles buccaux tels que la perte de dents ou la diminution du flux salivaire qui peuvent impacter la prise alimentaire avec un risque de dénutrition et d'apport en protéines. Ainsi, il est important de développer des aliments répondant aux besoins nutritionnels tout en permettant le maintien du " plaisir à manger ». Dans ce contexte, deux produits céréaliers appréciés par les séniors ont été enrichis en protéines végétales, i.e. une brioche et une génoise formulées spécifiquement. L'objectif de l'étude était d'évaluer le plaisir à manger (en terme de confort) sur ces deux produits et de comprendre, lors de leur consommation respective, l'impact de l'état dentaire, du flux salivaire et des caractéristiques de formation du bol sur ce confort.

Matériel et méthodes : Deux groupes de séniors (>65ans) ont été sélectionnés selon leur nombre de dents fonctionnelles (Unités Fonctionnelles Postérieures ou UFP), $10(6 \mathrm{~F}, 4 \mathrm{H})$ avec une bonne dentition (BD, âge $=72 \pm 5$, UFP=9 \pm 1 , flux salivaire stimulé ( $F S S=2,1 \pm 0,7 \mathrm{ml} / \mathrm{min}$ ) et $10(5 \mathrm{~F}, 5 \mathrm{H})$ avec une mauvaise dentition ( $\mathrm{MD}$, âge $=77 \pm 4$, UFP $=3 \pm 1, \mathrm{FSS}=1,9 \pm 0,8 \mathrm{ml} / \mathrm{min}$ ). Le confort en bouche de 2 matrices brioche(B) et génoise(G) enrichies en protéines végétales a été évalué au moyen d'un questionnaire sensoriel spécifiquement développé ${ }^{1}$. La formation du bol (nombre de cycle et fréquence masticatoire) et ses propriétés à déglutition (taux de salive incorporée, viscosité, taille médiane et nombre de particules) ont été déterminées. Les résultats ont été analysés par ANCOVA avec interactions d'ordre 2 sur l'effet produit. Le protocole a été approuvé par le CPP EST-1 NIRB 2016A00916-45.

Résultats et Analyse statistique : Les deux produits ont été jugés confortables. Cependant, la génoise est notée plus confortable en bouche que la brioche $(F=16,54, p<0,001)$. Elle est plus facile à manger $(F=7,3, p=0,01)$, plus intense en goût $(F=8,6, p=0,008)$ et moins salée $(F=10,23, p=0,005)$. Les sujets $B D$ notent les deux produits plus confortables en bouche que les sujets $M D(F=4,3$, $\mathrm{p}<0,05)$, plus facile à mastiquer $(F=2,7, p<0,05)$ et à avaler $(F=3,7, p<0,01)$. Une tendance est observée pour le flux salivaire quelle que soit la dentition $(F=3,74, p=0,06)$ avec une augmentation du confort avec le flux. A l'inverse, les résultats ne montrent pas d'impact du comportement masticatoire et des propriétés du bol sur le confort en bouche et la facilité à avaler.

Conclusion : Les résultats montrent que l'enrichissement en protéines végétales peut conduire à des produits confortables en bouche et que la formulation employée influence très significativement le degré de confort. Ils montrent aussi que le confort dépend de l'état bucco-dentaire du sujet âgé. Cette étude démontre ainsi la faisabilité de cette stratégie d'enrichissement en termes de plaisir à manger sur des produits consommés par les personnes âgées. La suite de ce travail consistera à évaluer l'acceptabilité de ces produits chez le consommateur sénior.

Références : 1.Vandenberghe-Descamps, et al. (2017). Journal of Texture Studies, 48:280-287

Remerciements : Projet financé par l'ANR (AlimaSSenS ANR-14-CE20-0003-01)

Conflits d'intérêts : Aucun conflit à déclarer 
Evaluation du degré de transformation de 15100 produits alimentaires étiquetés selon le score holistique Siga

A. Fardet ${ }^{1, *}$, K. Frank ${ }^{2}$, S. Davidou ${ }^{3}$, A. Christodoulou ${ }^{2}$

${ }^{1}$ Nutrition Humaine, INRA, Clermont-Ferrand-Theix, ${ }^{2}$ Science des aliments et nutrition, Siga, ${ }^{3}$ Technologie alimentaire, CNAM, Paris, France

Introduction et but de l'étude : Jusqu'à aujourd'hui I des aliments a été évaluée sur la base de la seule composition nutritionnelle (approche réductionniste). Or l'aliment est beaucoup plus qu'une seule somme de nutriments (approche holistique). D'autres alternatives existent pour classer les aliments, comme la classification NOVA selon le degré de transformation. Par ailleurs de plus en plus d'études associent le risque de maladies chroniques avec l'augmentation du degré de transformation des aliments. Le projet Siga, basé sur les 4 groupes technologiques NOVA, propose une nouvelle classification holistique en 9 groupes technologiques. Le but de cette étude est d'évaluer le degré de transformation d'un grand nombre de produits alimentaires avec le score Siga.

Matériel et méthodes : Un aliment est défini comme ultra-transformé s'il contient au moins un ingrédient ultra-transformé (fractionnement excessive de sa matrice originelle) ou un additif cosmétique (colorant, texturant, exhausteur). A partir de la base Siga 15100 aliments étiquetés hors découpe (plusieurs enseignes de supermarchés, marques nationales et distributeurs) ont été testés pour leur degré de transformation. L'algorithme Siga distingue les aliments non transformés (A0), peu transformés (A1), transformés (B1), transformés riches en sucre, sel et/ou gras (B2, seuils de la Food Standard Agency), ultra-transformés (C0.1, C0.2, C1, C2 et C3 selon le degré de transformation des ingrédients industriels, les teneurs en sel/sucre/gras et le nombre, la nature et le risque potentiel des additifs).

Résultats et Analyse statistique : Sur les 15100 produits alimentaires, 3\% sont classés en A0, 14\% en A1, 8\% en B1, 5\% en B2, $12 \%$ en $\mathrm{C} 0,10 \%$ en $\mathrm{C} 1,14 \%$ en C2 et $33 \%$ en C3 ; soit au total $69 \%$ de produits ultra-transformés dont $12 \%$ acceptable en C0. En termes de catégories plus de 95\% des spiritueux, bouchées et biscuits apéritifs, glaces, confiseries chocolatées, viennoiseries, sirops et concentrés, chips, gâteaux et pâtisseries, biscuits secs, salaisons viande, confiseries sucrées, sandwichs et burgers, compléments alimentaires, boissons lactées et végétales aromatisées et laits pour bébé sont ultra-transformés.

Conclusion : L'offre alimentaire en supermarché présente un pourcentage élevé ("2/3) de produits ultra-transformés. On y trouve beaucoup de produits de type snacking. Le score Siga, par son approche à la fois scientifique et holistique, devrait permettre aux distributeurs et industriels de s'orienter vers une offre alimentaire moins transformée et moins délétère pour la santé. Des études épidémiologiques supplémentaires seront nécessaires pour calculer les risques de maladies chroniques en fonction du degré de transformation des aliments utilisant ce type de score holistique.

Références : Monteiro C, Cannon G, Levy R et al. (2016) The star shines bright. World Nutrition 7, 28-38.

Fardet A (2018) Vers une classification des aliments selon leur degré de transformation : approches holistique et/ou réductionniste de la technologie. Pratiques en Nutrition. Sous presse.

Fardet A (2018) Chapter 3 - Characterization of the degree of food processing in relation with its health potential and effects. Adv Food Nutr Res 85, 79-121.

Conflits d'intérêts : Aucun conflit à déclarer 
P003

Risques liés à la consommation de certaines denrées alimentaires : bilan à 9 ans du dispositif national de Nutrivigilance unique en Europe

G. Vo Van-Regnault ${ }^{1, *}$, I. Margaritis ${ }^{2}$ et Groupe de travail "Nutrivigilance" (2015-2018) de I'Anses

${ }^{1}$ Nutrivigilance, Anses, Maisons-Alfort, ${ }^{2}$ Unité d'évaluation des risques liés à la nutrition, Anses, Maisons Alfort, France

Introduction et but de l'étude : L'offre et la consommation de compléments alimentaires (CA) augmentent régulièrement, notamment du fait de leur distribution par internet. Dans un souci de protection de la santé publique, la loi Hôpital, Patients, Santé et Territoires a mis en place en 2009 un dispositif de vigilance spécifique, unique en Europe, coordonné par l'Anses : la Nutrivigilance.

L'objectif de cette communication est de présenter le fonctionnement et les principaux résultats du dispositif de Nutrivigilance, 9 ans après sa création.

Matériel et méthodes : La Nutrivigilance a collecté et analysé les effets indésirables (EI) des CA, des denrées destinées à une alimentation particulière (dont DADFMS), des aliments enrichis (dont les boissons énergisantes) et des nouveaux aliments. Pour chaque déclaration reçue et suffisamment documentée, l'imputabilité (probabilité que l'effet indésirable déclaré soit lié à la consommation d'un produit) et la sévérité (s'échelonnant du niveau 1 pour une sévérité faible au niveau 4 pour un décès) ont été déterminées, selon une procédure inspirée de la pharmacovigilance. Cette analyse a été réalisée par des experts médecins spécialistes puis soumise à un groupe de travail dédié composé de médecins et de pharmaciens.

Résultats et Analyse statistique : Entre le 13 novembre 2009 et le 30 juin 2018, 3612 déclarations ont été enregistrées dont 980 suffisamment documentées pour être exploitées. Ces déclarations sont principalement liées à la consommation de CA (71\% des cas exploitables). Les effets indésirables rapportés sont majoritairement d'ordre gastro-entérologique (214 cas, $22 \%$ ), cardiovasculaires (158 cas, 16\%) et dermatologiques (133 cas, 14\%) avec une sévérité élevée (niveau 3 ou 4 ) dans $28 \%$ des cas. Pour $38 \%$ de ces cas exploitables, l'imputabilité de l'effet au produit consommé était élevée (vraisemblable ou très vraisemblable). Concernant les consommateurs, $17 \%$ avaient moins de 20 ans, $39 \%$ de 20 à 49 ans, $27 \%$ de 50 à 69 ans et $14 \%$ plus de 70 ans. II s'agissait principalement de femmes (59\%).

L'analyse des déclarations a permis à l'Anses de renforcer ses évaluations des risques liés à la consommation de CA contenant certaines substances (spiruline, levure de riz rouge, mélatonine...) ou ciblant certaines catégories de consommateurs (sportifs, femmes enceintes ...). Le dispositif permet également d'identifier des situations à risque en mettant en évidence des cas d'adultération, de mésusage ou de confusion.

Conclusion : Les risques liés à la consommation de CA notamment conduisent l'Anses à émettre des recommandations à l'attention des professionnels de santé, des ministères de tutelle et des consommateurs. En France, selon l'étude INCA3, 29\% des adultes et $19 \%$ des enfants consomment au moins occasionnellement des CA. Il est avéré que ces produits, souvent perçus comme anodins et très consommés peuvent présenter des risques pour la santé. La déclaration des effets indésirables est cruciale afin de continuer à identifier des dangers liés à ces consommations. En ce sens, il est indispensable que les professionnels de santé (PS), acteurs clés de la Nutrivigilance, interrogent leurs patients sur leur consommation de CA et déclarent systématiquement tout effet indésirable observé.

Conflits d'intérêts : G. Vo Van-Regnault: Aucun conflit à déclarer, I. Margaritis est consultant chez Afnor, SFMES, SFN, Plant libra, Clymbol 
P006

Les caractéristiques épidémiologiques des TIAC dans les provinces (Kenitra, Sidi Kacem, Sidi Slimane) au Nord-Ouest du Maroc entre 2013 et 2017

B. Mohamed ${ }^{1}$, Y. Aboussaleh ${ }^{2, *}$

${ }^{1}$ Filière Infirmier Polyvalent, Institut de Professions Infirmières et Techniques de Santé, ${ }^{2}$ Département des Sciences de la Vie, Faculté des Sciences, Kenitra, Maroc

Introduction et but de l'étude : Les Toxi-Infections Alimentaires Collectives (TIAC) représentent un problème majeur de santé publique à l'échelle mondiale, pour leur fréquence de plus en plus préoccupante, ainsi que pour leur potentiel épidémique et leur gravité [1].Nous proposons une étude des caractéristiques épidémiologiques des TIAC afin d'évaluer le système de surveillance au niveau du Service des Réseaux des Etablissements de Santé (SRES) des provinces (Kenitra, Sidi Kacem, Sidi Slimane) au Maroc Matériel et méthodes : L'approche est de type descriptive des caractéristiques sociodémographiques et évolutives et une détermination des facteurs associés au risque des 392 cas de TIAC enregistrés sur la période du 1 Janvier 2013 au 31 Décembre 2017.

Résultats et Analyse statistique : Les principaux résultats montrent que la classe d'âge la plus touchée est inférieure à 10 ans. Elle concernait principalement les préadolescents, l'infection touche d'une manière aléatoire les deux sexes. Les fréquences des intoxications les plus élevées ont été enregistrées dans le milieu rural avec $85 \%$. Les signes cliniques étaient en majorité de première classe (douleurs abdominales (95\%), diarrhées (88\%), vomissements (87\%) et nausées (25\%)). L'aliment le plus incriminé était le poisson avec un taux de $53 \%$ des cas. Le taux d'hospitalisation est de $28 \%$. II faut noter que l'agent pathogène causal de TIAC dominant est le Staphylococus aureus avec 55\%. Les écoles sont les plus touchées avec 56\%. Les médecins des urgences sont la première source de déclaration avec $40 \%$. Ces résultats sont soutenus par l'étude réalisée par Chiguer au Maroc [2], mais diffèrent bien des résultats reportés dans la wilaya de Bejaiaen termes d'agent responsable [3].

Conclusion : En se référant à la situation sus décrite, et comptant sur le manque d'exhaustivité des données existantes, nous devons commenter l'état avec un esprit de réflexion. Toutefois, l'étude dont les résultats sont détaillés dans ce présent travail est consacrée à une étude épidémiologique rétrospective descriptive des cas de toxi-infections alimentaires collectives diagnostiqués. II serait souhaitable d'étaler cette étude sur une longue période, dans d'autres lieux, d'inclure d'autres paramètres tels que les facteurs favorisants la survenue de cette épidémie pour mieux la décrire.

Références : [1] Hamza. R, Hajjem.S, Hsairi.M ; Revue Santé Publique ; investigation d'une toxi-infection alimentaire collective : justifications et principes généraux, Tunisie ; 2012

[2] Chiguer.B ; toxi-infections alimentaires collectives, fléau mondial à surveiller (exemple du Maroc 2008-2012) ; p-31.Thèse de médecine 2014

[3] Belhadri A., Dilmi Bouras A. ; Taux des intoxications alimentaires et efficacité des contrôles microbiologiques ; les sixièmes journées de microbiologie ; 27-28 Octobre 2010 ; p-21.

Mots clés : Toxi-Infections-Alimentaires-Collectives-Kenitra-Sidi Kacem-Sidi Slimane

Remerciements : Remerciements aux Responsables des SRES qui ont contribué à la réalisation de cette étude ainsi qu'aux lecteurs d'avoir accepter de lire cette production

Conflits d'intérêts : Aucun conflit à déclarer 
Développement et validation d'un index d'évaluation de la durabilité des régimes alimentaires au sein de la cohorte NutriNetSanté

L. Seconda ${ }^{12, *}$, J. Baudry ${ }^{1}$, P. Pointereau ${ }^{3}$, C. Lacour ${ }^{1}$, B. Langevin ${ }^{3}$, S. Hercberg ${ }^{1}$, D. Lairon ${ }^{4}$, B. Allès ${ }^{1}$, E. Kesse-Guyot ${ }^{1}$ ${ }^{1}$ EREN, Université Paris 13 , Bobigny, ${ }^{2}$ ADEME, Angers, ${ }^{3}$ Agriculture, Solagro, Toulouse, ${ }^{4}$ Nort, Université Aix-Marseille, Marseille, France

Introduction et but de l'étude : En 2010, l'organisation mondiale de l'agriculture et de l'alimentation (FAO) a clarifié la définition de régime alimentaire durable. Ainsi ces régimes doivent répondre aux enjeux de sécurité alimentaire et nutritionnelle, de la protection des écosystèmes et de la biodiversité, d'une économie juste ainsi qu'au maintien du patrimoine culturel. Depuis cette date, de nombreux chercheurs ont travaillé pour évaluer la durabilité des régimes avec divers indicateurs. Cependant, il n'existe pas actuellement de score holistique pour évaluer la durabilité des régimes alimentaires au niveau individuel.

L'objectif de notre étude était de proposer un index individuel pour évaluer la durabilité des régimes alimentaires sur de multiples dimensions.

Matériel et méthodes : La synthèse de la littérature nous a amené à considérer 14 indicateurs, regroupés en quatre sous scores de poids équivalent reflétant: l'impact environnemental incluant des indicateurs relatifs à l'occupation des sols, les émissions de gaz à effet de serre, la consommation d'énergie, la contribution des aliments issus de l'agriculture biologique au régime, l'intérêt pour la saisonnalité et celui pour la réduction de l'usage des emballages; la nutrition qui comporte le score PANDiet d'évaluation de la qualité de l'alimentation, le ratio protéines animales / protéines totales, et l'adéquation calorique du régime; l'économie comporte la proportion du revenu dédiée à l'alimentation, l'intérêt pour l'éthique et pour le commerce équitable ; et enfin le score socio-culturel qui évalue les lieux d'achat et la consommation de plats préparés. Le score sur 12 points, nommé SDI est obtenu en sommant les quatre sous scores. II a été mesuré pour 29390 participants de l'étude NutriNet-Santé. La validité de contenu et de construit de l'index a été évaluée.

Résultats et Analyse statistique : Au sein de notre échantillon, le SDI (moyenne=5,72/12 [5,72-5,78]) est fortement corrélé à tous les sous scores dont chacun contribue au classement des participants. Le sous score environnemental est le plus corrélé au SDI alors que le sous score nutritionnel est le moins corrélé (les coefficients de Pearson sont de 0,73 et 0,59 respectivement). Les niveaux de consommations alimentaires chez les participants ayant un SDI le plus élevé sont cohérents avec d'autres régimes considérés comme durables et proposés par Afterre2050 ou Livewell 2030.

Conclusion : Les validations de contenu et de construit du SDI comme outil d'évaluation de la durabilité des régimes sont satisfaisantes. Ainsi, cet index pourrait être utile dans l'identification des modifications nécessaires à l'amélioration de la durabilité des régimes alimentaires, dans l'estimation des associations avec les événements de santé sur le long terme, ainsi que pour guider les futures politiques alimentaires et de santé publique.

Conflits d'intérêts : Aucun conflit à déclarer 
Introduction et but de l'étude : En plus de l'impact sur l'environnement, l'urbanisation de la société s'est accompagnée d'une profonde modification de nos modes de vie pour tendre vers des comportements pouvant être néfastes pour la santé (déséquilibres alimentaires, sédentarité, etc...). II est donc primordial de trouver des stratégies pour tendre vers gestion urbaine durable qui favorise la santé des individus. Les jardins collectifs, une forme d'agriculture en plein essor dans les villes, auraient de nombreux bénéfices sur la santé de ce qui les fréquentent, notamment en favorisant la consommation de fruits et légumes. Néanmoins, les études menées à ce jour sont majoritairement transversales et basées sur des déclarations, ne permettant pas d'explorer le lien de causalité entre l'accès à un jardin collectif et l'adoption de comportements favorables à la santé. De plus, ces études ayant été principalement réalisées dans des contextes Nord-Américains, les résultats restent à confirmer dans un contexte Français. L'objectif du projet JArDins est d'évaluer l'impact le rôle des jardins collectifs dans la promotion de modes de vie durables. Nous présentons ici le protocole de cette recherche.

Matériel et méthodes : L'étude est une expérimentation naturelle (accès à un jardin collectif) évaluée selon un design de type quasi-expérimental. Des personnes débutant l'activité de jardinage dans un jardin partagé $(n=80)$ seront recrutées sur la base sur volontariat dans les différents jardins de Montpellier. En parallèle, des personnes semblables aux jardiniers (appariés selon l'âge, le sexe, la structure et les revenus du foyer) et vivant près des jardins enquêtés, mais n'ayant pas accès à un jardin collectifs ( $n=80$ ) seront recrutées pour constituer un groupe témoin. Les participants seront enquêtés au moment de l'inclusion dans l'étude, puis 1 an après. La durabilité des modes de vie sera étudiée de manière globale en tenant compte de trois dimensions de la durabilité: 1) sociale/santé - mesurée par la qualité nutritionnelle des approvisionnements alimentaires et les achats en fruits et légumes, le niveau d'activité physique, le bien-être mental et le lien social.

2) environnementale - mesurée par l'impact environnemental des approvisionnements alimentaires et déplacements alimentaires associés, l'équilibre animal/végétal des approvisionnements alimentaires, la sensibilité au gaspillage alimentaire et la connexion à la nature.

3) économique - mesurée par les dépenses alimentaires du foyer et parts budgétaires des différents groupes d’aliments. Ces données seront collectées au moyen de trois outils : un carnet des approvisionnements alimentaires à remplir pendant un mois, le port d'un enregistreur de mouvements (Actigraph) pendant 9 jours et le remplissage d'un questionnaire en ligne.

Résultats et Analyse statistique : Après 1 an de suivi, l'évolution des variables mesurées sera comparée entre le groupe de jardiniers et le groupe contrôle pour évaluer l'effet du jardin collectif sur les différentes dimensions de la durabilité.

Conclusion : De par son design d'étude de type quasi expérimental permettant de tester la causalité, l'étude JArDinS apportera des réponses sur le rôle des jardins collectifs comme outils de promotion de modes de vie plus durables.

Conflits d'intérêts : Aucun conflit à déclarer 
P009

La cinétique de digestion chez le Sénior des protéines d'une émulsion de viande type knack diffère de celle de l'adulte : cartographie des protéines modulées.

S. Sante-Lhoutellier ${ }^{1,}$, T. Sayd $^{1}$, J. Pinguet ${ }^{2}$, D. Richard ${ }^{2}$, C. Chambon ${ }^{1}$, M. A. Peyron ${ }^{3}$

${ }^{1}$ QuaPA, INRA, Saint Genes Champanelle, 'Labo de Pharmacy, CHU, Clermont Ferrand, ${ }^{3} \mathrm{UNH}$, INRA, Saint Genes Champanelle, France

Introduction et but de l'étude : La population Sénior ne cesse d'augmenter avec des besoins nutritionnels spécifiques. La viande est une source de protéines de bonne qualité en termes de composition en acides aminés essentiels et de vitesse de digestion. Peu de données existent sur la biodisponibilité des protéines chez le Sénior en fonction de son statut oral. Ce travail a pour objectif de quantifier la bioaccessibilité des nutriments protéiques pour le Sénior, pour une meilleure évaluation des conséquences d'une malnutrition.

Matériel et méthodes : Le dispositif expérimental combine l'utilisation d'un masticateur in vitro $\left(\mathrm{AM}^{2}\right)$, programmé pour reproduire les troubles de la mastication et de la physiologie orale du Sénior avec l'utilisation d'un digesteur instrumenté (DIDGI) mimant les conditions physiologiques de digestion du Sénior (réduction des concentrations en lipase et pepsine gastrique, en trypsine et chymotrypsine, acidification gastrique réduite, ...). Le modèle d'aliment choisi est une émulsion de type knack pour s'affranchir des effets structure inhérents à la viande. Les cinétiques de relargage des peptides au niveau gastrique sont évaluées par le dosage des peptides entre 30 et 150 minutes (Direct detect). Les peptides sont identifiés et quantifiés en analyses LC/MS (Q Exactive plus Thermo) et Progenesis QI.

Résultats et Analyse statistique : Les spectres peptidiques obtenus en LC/MS sont analysés par le logiciel Progenesis QI. Les intensités cumulées des peptides identifiés correspondant à une protéine (degrés d'hydrolyse de cette protéine) sont ensuite traités par classification hiérarchique ascendante permettant de définir 4 clusters.. La cinétique d'hydrolyse des protéines pour chacun des clusters est analysée par la méthode des kmeans.. Seules les protéines du cluster 1 ( $n=14$ ) ne présentent pas ou peu de différences de cinétiques entre les conditions de digestion Adulte et Senior. En revanche, pour les 54 autres protéines, on observe globalement une diminution, une phase de latence, pour les conditions Sénior... Ces clusters referment des protéines de structure fibreuse, des protéines structurelles du muscle qui sont en quantité majoritaire. Pour la première fois il est établi que la nature de la protéine, ses propriétés, impacte sa cinétique de digestion en conditions digestives du Sénior.

Conclusion : Le couplage des outils de mastication et de digestion in vitro ont permis de mettre en évidence pour la première fois des différences de cinétique de digestion des protéines chez le Sénior. L'impact de la physiologie orale est bien présent mais moindre comparé à la physiologie digestive. Ces approches vont permettre à termes d'évaluer le potentiel nutritionnel des aliments notamment pour des populations spécifiques comme les Séniors.

Remerciements : Remerciements: Les auteurs remercient l'institut Carnot Qualiment pour son soutien financier (Projet QualigrasPhys coordonné par E Guichard)

Conflits d'intérêts : Aucun conflit à déclarer 
P010

Impact de la structure du bol alimentaire des seniors sur la bioaccesibilité des protéines de pois au cours de la digestion in vitro d'une génoise

M. Assad-Bustillos ${ }^{123,{ }^{*}}$, J. Palier ${ }^{1}$, H. Rabesona ${ }^{1}$, Y. Choiset $^{1}$, G. Della-Valle ${ }^{1}$, G. Feron $^{2}$

${ }^{1}$ BIA, INRA, Nantes, ${ }^{2}$ CSGA, INRA, ${ }^{3}$ Cerelab, Dijon, France

Introduction et but de l'étude : L'enrichissement d'aliments céréaliers en protéines de légumineuses constitue une voie prometteuse pour satisfaire les besoins protéiques des seniors. Pour ces personnes, la déstructuration de l'aliment pendant la mastication est une étape du processus digestif d'autant plus importante que leur physiologie orale est fortement altérée. Dans le cas des aliments céréaliers, cette altération impacte les propriétés du bol alimentaire, principalement la viscosité dont la valeur permet de définir un état de déstructuration. Celui-ci pourrait affecter l'accessibilité des protéines durant la digestion. Dans ce contexte, nos objectifs ont été : 1) d'évaluer la bioaccessibilité des protéines de pois au sein d'une génoise enrichie via le protocole de digestion in vitro Infogest ; 2) de déterminer l'impact de la structure du bol alimentaire issu de la mastication par des seniors sur la bioaccesibilité des protéines de pois en utilisant en combinant des approches ex vivo et in vitro.

Matériel et méthodes : Des génoises riches en protéines de pois ont été consommées par 20 seniors ( $>65$ ans) avec physiologie contrastée, $10(6 \mathrm{~F}, 4 \mathrm{H}$ ) avec une bonne dentition (âge=72 \pm 5 , UFP=9 \pm 1 ) et $10(5 \mathrm{~F}, 5 \mathrm{H}$ ) avec une mauvaise dentition (âge=77 \pm 4 , $U F P=3 \pm 1$ ). Leurs bolus ont été récupérés juste avant la déglutition, et leur viscosité apparente a été mesurée par rhéométrie capillaire. Selon ce critère, deux pools ont été établis avec 4 sujets sélectionnés aux extrêmes: faible viscosité (VF) et viscosité élevée (VE), pour des valeurs (à $120 \mathrm{~s}^{-1}$ ) de $124 \pm 18$ Pa.s et $208 \pm 19$ Pa.s, respectivement. Les matrices seules et les deux pools ont subi des digestions in vitro via le protocole standardisé Infogest. Les cinétiques d'hydrolyse quantitative et qualitative ont été déterminées par le dosage de $\mathrm{NH}_{2}$ libérés et par électrophorèse SDS-PAGE, respectivement. Etude approuvée par le CPP NIRB 2016-A00916-45.

Résultats et Analyse statistique : Pour tous les échantillons, les bandes représentatives des protéines légumine, viciline, conviciline sont visibles sur les gels SDS-PAGE à $\mathrm{t}=0$. Celles-ci disparaissent progressivement au cours de la digestion, accompagnées de l'apparition des bandes dont les masses sont inférieurs à $15 \mathrm{kDa}$. De plus, les concentrations de $\mathrm{NH}_{2}$ libérées augmentent significativement avec le temps de digestion $(F=199, p<0,0001)$; cependant, elles ne diffèrent pas entre la génoise et l'isolat de pois $(F=0,05, p=0,81)$. Par ailleurs, le degré de structure du bol alimentaire n'a pas d'effet significatif sur la concentration de $\mathrm{NH}_{2}$ libérée $(\mathrm{F}=0,07, \mathrm{p}=0,62)$.

Conclusion : Les protéines de pois enrichissant une génoise sont bioaccessibles dans des conditions standardisées. Dans ces mêmes conditions, l'état de structure du bol alimentaire issu de la mastication des seniors n'a pas d'influence dans la bioaccessibilité des protéines, du moins dans l'intervalle de viscosité étudié. Il est possible de combiner des méthodes ex vivo et in vitro, en particulier la méthode Infogest, pour suivre la cinétique d’hydrolyse protéique des aliments céréaliers. En perspective, il serait intéressant d'adapter le protocole aux conditions physiologiques digestives des personnes âgées. Projet financé par l'ANR (AlimaSSenS ANR-14-CE20-0003-01).

Conflits d'intérêts : Aucun conflit à déclarer 
Caractérisation du recours aux aliments issus de l'agriculture biologique dans les premiers mois de vie F. Gaudfernau ${ }^{1}$, C. Davisse-Paturet ${ }^{1}$, A. Camier ${ }^{1}$, S. Lioret ${ }^{1}$, S. Nicklaus ${ }^{2}$, M. A. Charles ${ }^{13}$, B. de Lauzon-Guillain ${ }^{4, *}$

${ }^{1}$ CRESS Eq ORCHAD, INSERM, Villejuif, ${ }^{2}$ CSGA, INRA, Dijon, ${ }^{3}$ INED, Paris, ${ }^{4}$ CRESS Eq ORCHAD, INRA, Villejuif, France

Introduction et but de l'étude : Le recours aux aliments issus de l'agriculture biologique et leurs effets sur la santé demeurent insuffisamment étudiés, en particulier chez les enfants. Notre objectif est de caractériser le recours aux aliments issus de l'agriculture biologique $(A B)$ durant les premiers mois de vie en s'intéressant aux caractéristiques familiales associées à cette consommation mais aussi en identifiant les profils alimentaires caractérisés notamment par l'utilisation de ces aliments.

Matériel et méthodes : Notre étude a inclus 8961 enfants issus de la cohorte de naissance ELFE. En complément des entretiens téléphoniques à 2, 12 et 24 mois post-partum, un questionnaire mensuel portant sur le mode d'alimentation lactée et la fréquence de consommation de 28 aliments et boissons, des aliments non mixés et ceux issus de l'AB a été rempli par la mère du $3^{\text {ème }}$ au $10^{\mathrm{èm}}$ mois de l'enfant. L'association entre les caractéristiques familiales et le recours aux aliments issus de l'AB durant la période de diversification alimentaire (jamais, parfois, souvent, toujours) a été analysée à l'aide de régressions logistiques multinomiales. De plus, une analyse en composantes principales (incluant la durée totale d'allaitement, l'âge d'introduction des préparations infantiles, de 13 groupes d'aliments, des morceaux et de la fréquence d'utilisation des aliments bébé et ceux issus de l'AB) a permis d'identifier trois profils de pratiques alimentaires dans la première année de vie : «Introduction tardive des aliments et allaitement plutôt long ", "Recours fréquent aux aliments issus de l'agriculture biologique, allaitement très long et introduction précoce des morceaux » et "Introduction précoce des fruits, légumes et pommes de terre ". L'association entre les caractéristiques familiales et le second profil a été évaluée à l'aide d'une régression linéaire.

Résultats et Analyse statistique : Le recours aux aliments issus de l'AB pour l'enfant était plus fréquent chez les mères plus âgées, avec un niveau d'études élevé, une catégorie socio-professionnelle favorisée, nées à l'étranger, avec un IMC normal, qui avaient cessé de fumer avant la grossesse et qui ont choisi le mode d'alimentation lactée de l'enfant pour des motifs liés à sa santé. Les

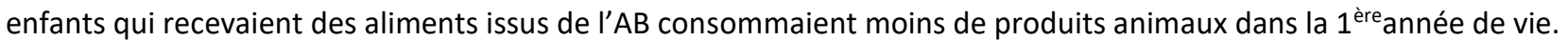

Le profil d'alimentation comprenant l'utilisation fréquente d'aliments issus de l'AB est associé positivement au niveau d'études et au fait que la mère soit née à l'étranger ; mais aussi, contrairement aux résultats précédents, à la parité et à un faible revenu, en lien avec la composante allaitement de ce profil.

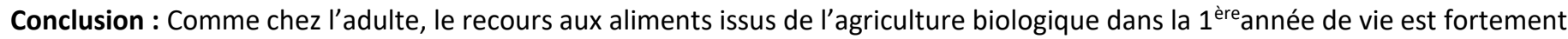
associé aux caractéristiques socioculturelles des familles. Notre étude permet de mettre en évidence qu'il est également associé à des pratiques d'alimentation particulières (allaitement très long) qu'il sera important de prendre en compte pour étudier l'impact de ces produits sur la santé et le développement de l'enfant.

Conflits d'intérêts : Aucun conflit à déclarer 
Effets des graines de lin (Linum usitatissimum) sur les chiffres tensionnels, le statut redox et l'activité de la paraoxonase (PON1), chez des rats rendus hypertendus par l'ouabaïne

S. Berzou ${ }^{1, *}$, D. Krouf ${ }^{1}$, N. Dida-Taleb ${ }^{1}$, A. Guenzet ${ }^{1}$

${ }^{1}$ Laboratoire de Nutrition Clinique et Métabolique, Faculté des Sciences de la Nature et de la Vie. Université d’Oran 1, Oran, Algérie

Introduction et but de l'étude : La graine de lin est en train de devenir l'un des plus important alimentaire fonctionnels en raison de son contenu riche en molécules bioactives qui ont un potentiel effet bénéfique sur la santé et la réduction des maladies cardiovasculaires (MCV). L'objectif de l'étude est de voir les effets des graines de Linum usitatissimum sur les chiffres tensionnels, la peroxydation lipidique et l'activité des enzymes antioxydanteset l'activité de la paraoxonase, chez des rats rendus hypertendus par l'ouabaïne.

Matériel et méthodes : Des rats mâles Wistar $n=24(250 \pm 7 \mathrm{~g})$ sont soumis pendant 21 jours à un régime contenant $20 \%$ de caséine et sont répartis en trois groupes $(n=8)$ : Un groupe témoin $(T)$ reçoit une solution sous-cutanée $(\mathrm{sc})$ de $\mathrm{NaCl}(0,9 \%)$ et les 2 autres groupes reçoivent $10 \mu \mathrm{g} / \mathrm{Kg} / \mathrm{jour}$ d'une solution sc de ouabaïne diluée dans $\mathrm{d} \mathrm{NaCl}(0,9 \%)$ consommant chacun le même régime supplémenté (Oub-Lu) ou non (Oub) avec 1g/100g de graines de lin, pendant 21 jours. Les chiffres tensionnels sont enregistrés chaque semaine (CODA 4, Kent Scientific Corporation, États-Unis).

Résultats et Analyse statistique : Après 21 jours d'expérimentation, les résultats ont montré que le traitement avec Linum usitatissimum diminue significativement les pressions artérielles systolique (PAS) et diastolique (PAD) et améliore la pression artérielle moyenne (PAM) $(P<0,05)$. Les teneurs plasmatiques en cholestérol total, non- HDL cholestérol et en glucose sont réduites de $-26 \%,-38 \%$ et-31\%, respectivement. Au niveau plasmatique et cardiaque, les concentrations des substances réactives à l'acide thiobarbiturique (TBARS) sont significativement diminuées de $-85 \%$ et $-42 \%$, respectivement. Au niveau du cœur, les teneurs en protéines carbonylées sont significativement diminuées $(P<0,05)$. Inversement, les activités enzymatiques de la catalase et de la glutathion peroxydase sont augmentées de $+50 \%$ et de $+54 \%$, respectivement. De même, une augmentation de l'activité de la paraoxonase PON1 (+62\%), est notée.

Conclusion : Ces résultats montrent un effet préventif des graines de lin contre l'hypertension artérielle. De plus, ce traitement induit une diminution de la peroxydation des lipides et des protéines et améliore l'activité de la PON1.

Conflits d'intérêts : Aucun conflit à déclarer 
P013

Évaluation de la qualité bactériologique de la viande rouge dans les boucheries du gouvernorât de Tunis.

S. Ben Youssef ${ }^{1, *}$, G. Dali Temala ${ }^{2}$, I. Sebai ${ }^{3}$, N. Jaziri ${ }^{4}$, S. Ennigrou ${ }^{3}$

${ }^{1}$ Service d'épidémiologie et de médecine communautaire, Hôpital Charles Nicolle de Tunis, Faculté de médecine de Tunis, Université Tunis El-Manar, ${ }^{2}$ Ecole supérieure des sciences et techniques de la santé de Tunis, Université Tunis El-Manar, ${ }^{3}$ Service d'épidémiologie et de médecine communautaire, Hôpital Charles Nicolle de Tunis, Faculté de médecine de Tunis, Université Tunis El-Manar., ${ }^{4}$ Service d'hygiène et de protection de l'environnement, Direction régionale de la santé de Tunis, Tunis, Tunisie

Introduction et but de l'étude : La viande rouge, une denrée périssable, est susceptible de véhiculer de nombreuses maladies chez les humains via la présence éventuelle de nombreux microorganismes pathogènes. Cette contamination est souvent liée aux différentes étapes de l'abattage, au transport, à la conservation et au découpage dans les boucheries. L'objectif de notre étude était d'évaluer la qualité bactériologique de viandes fraîches au sein des boucheries du gouvernorat de Tunis.

Matériel et méthodes : Etude descriptive transversale réalisée durant la période: 20 Mars - 30 Avril 2018. Cinquante boucheries du gouvernorat de Tunis ont été sélectionnées par un sondage systématique parmi une liste disponible de 200 boucheries dans 6 délégations. Les échantillons prélevés ont concerné la viande découpée. Des portions de 100g de l'échantillon ont été prélevées dans des sachets en plastique stériles puis transportées au laboratoire des denrées alimentaires de l'Institut Pasteur de Tun is dans une glacière isotherme munie de plaques réfrigérantes. Au total, nous avons effectué 100 prélèvements ( 2 par boucherie). Les bactéries recherchées étaient les coliformes fécaux, les Staphylococcus aureus, les anaérobies sulfito-réducteurs et salmonella. La qualité bactériologique des viandes prélevées a été évaluée en se référant aux normes l'arrêté Français du 21 décembre 1979 et des normes Tunisiennes.

Résultats et Analyse statistique : Selon les critères microbiologiques requis par l'arrêté Français et les normes Tunisiennes, 53\%, $9 \%$ et $19 \%$ des prélèvements de la viande rouge n'étaient pas conformes aux critères légaux pour les coliformes fécaux, les Staphylococcus aureus et les anaérobies sulfito-réducteurs, respectivement. Les salmonelles étaient identifiées dans 3\% des prélèvements. Un prélèvement sur $8(12 \%)$ était toxique, impropre à la consommation humaine. Seulement le tiers des boucheries (34\%) présentait une viande conforme aux critères légaux pour toutes les bactéries dénombrées.

Conclusion : L'existence de ces microorganismes pathogènes dans la viande constitue un risque potentiel pour le consommateur. D’où la nécessité de mettre en place un programme de lutte efficace contre la contamination des viandes et respecter l'hygiène depuis l'élevage à la ferme, les abattoirs, les étapes de transformation, le transport jusqu'à la vente au consommateur.

Conflits d'intérêts : Aucun conflit à déclarer 
P014

Modifier les apports protéiques pour améliorer l'adéquation nutritionnelle en visant systématiquement ou non davantage de protéines végétales : impacts sur la durabilité

E. De Gavelle ${ }^{1, *}$, P. Leroy ${ }^{2}$, M. Perrimon ${ }^{1}$, J.-F. Huneau ${ }^{1}$, C. Orset ${ }^{3}$, H. Fouillet ${ }^{1}$, L.-G. Soler ${ }^{2}$, F. Mariotti ${ }^{1}$

${ }^{1}$ UMR PNCA, AgroParisTech, INRA, Université Paris Saclay, Paris, ${ }^{2}$ UMR ALISS, INRA, Ivry-sur-Seine, ${ }^{3}$ UMR Economie Publique, AgroParisTech, INRA, Université Paris Saclay, Paris, France

Introduction et but de l'étude : Nos régimes alimentaires s'orientent vers une moindre consommation de protéines animales, en lien avec la durabilité des régimes alimentaires. Etant donné les différences de profil nutritionnel entre sources protéiques, des modifications de consommation, même marginales, posent la question de la qualité nutritionnelle des régimes. Notre objectif était d'identifier les conséquences, sur différents paramètres de durabilité, de modifications des profils de consommation protéique visant à améliorer l'adéquation nutritionnelle, selon qu'on cherche ou pas à augmenter la part des protéines végétales. Matériel et méthodes : A partir des données de consommation de l'étude INCA2 (2006-2007), nous avons simulé, pour chaque individu, toutes les substitutions possibles d'une portion d'un aliment protéique par une portion d'un autre aliment protéique, et sélectionné la substitution augmentant le plus l'adéquation nutritionnelle (estimée selon le score PANDiet). Chaque réduction de taille de portion était ainsi couplée à une augmentation de taille de portion d'un autre aliment déjà consommé par l'individu, ou à l'introduction d'une petite portion d'un aliment consommé par des individus ayant un profil de consommation protéique similaire. Cette étape a été itérée 20 fois pour chaque individu sous deux scénarios différents : en imposant (V) ou non (N) la contrainte d'une augmentation du pourcentage de protéines végétales à chaque itération. Les paramètres de durabilité étudiés étaient le coût, les émissions de gaz à effet de serre, l'exposition aux contaminants et les morts prématurées évitées (estimées avec le modèle PRIME).

Résultats et Analyse statistique : Le pourcentage de protéines végétales (31,1\%) a légèrement diminué dans $\mathrm{N}(30,0 \%)$ et augmenté dans $\mathrm{V}(37,7 \%)$. Les groupes alimentaires dont la contribution à l'apport protéique a le plus augmenté sont les légumineuses $(+225 \%)$, le poisson gras $(+151 \%)$ et le poulet maigre $(+82 \%)$ dans $\mathrm{N}$ et les légumineuses $(+502 \%)$, les pizzas et quiches $(+190 \%)$ et le poisson gras $(+102 \%)$ dans $V$. Le PANDiet a légèrement plus augmenté en $N(+7,5)$ qu'en $V(+6,2)$ du fait de plus fortes probabilités d'adéquation en EPA + DHA, fer, iode, potassium, zinc, riboflavine, vitamine B-6 et B-12 malgré de plus faibles probabilités d'adéquation en fibres et folates. Les émissions de gaz à effets de serre (GES) ont augmenté en $\mathrm{N}$ et diminué en $\mathrm{V}$. Le coût a davantage augmenté en $\mathrm{N}$ qu'en $\mathrm{V}$. Les risques liés à l'exposition à l'arsenic inorganique, aux dioxines et furanes et au nickel ont augmenté en $\mathrm{N}$ et $\mathrm{V}$. Les morts prématurées évitées ont été estimées entre 1500 et 2000/an, le scenario $\mathrm{V}$ permettant d'éviter significativement plus de morts prématurées.

Conclusion : Nous avons identifié des modifications simples et a priori acceptables des apports protéiques de la population qui permettent d'augmenter significativement l'adéquation nutritionnelle des régimes. En contraignant ces modifications dans le sens d'une augmentation systématique de la part des protéines végétales, l'adéquation nutritionnelle augmente moins, mais le prix augmente moins, les émissions de GES diminuent et les morts évitées augmentent.

Conflits d'intérêts : Aucun conflit à déclarer 
UNI-VERT : mise au point de produits alimentaires contenant de la spiruline et répondant aux besoins et attentes des bénéficiaires de l'aide alimentaire.

A.-E. Le Minous ${ }^{1,{ }^{*}}$, S. Harnay ${ }^{1}$, M. Dameron ${ }^{2}$, P. Mahe ${ }^{2}$, C. Deschamp ${ }^{3}$, J. Leuranguer ${ }^{4}$

${ }^{1}$ FPS, ADRIA DÉVELOPPEMENT, Quimper, ${ }^{2}$ Fédération des Paniers de la mer, Ploneour-Lanvern, ${ }^{3}$ BRIT'Inov, Ploufragan, ${ }^{4}$ ALGOSOURCE, Saint Nazaire, France

Introduction et but de l'étude : Les études ABENA concluent que l'état de santé des usagers de l'aide alimentaire demeure préoccupant notamment au regard des troubles liés à la malnutrition : obésité, hypertension, diabète. Des groupes d'aliments sont sous consommés comme les fruits et légumes ou les produits de la mer. La fédération des paniers de la mer s'est donné pour ambition d'améliorer en qualité et quantité l'offre alimentaire en faveur des personnes démunies. La spiruline a montré son intérêt lors d'une étude de faisabilité pour sa qualité nutritionnelle et son potentiel de production en chantier d'insertion. Son aptitude à intégrer des aliments courants et leur acceptabilité restaient à étudier.

Matériel et méthodes : Nous avons étudié l'offre alimentaire dans les structures d'aide alimentaire, interrogé les bénéficiaires et les bénévoles pour qualifier leurs attentes (entretiens qualitatifs). La spiruline produite a été caractérisée pour ses propriétés physico-chimiques et techno-fonctionnelles (ex :activité de l'eau, isothermes de sorption). Différentes matrices alimentaires ont été formulées sur les bases des besoins et attentes détectés, puis testées par un panel de consommateurs $(n=48)$.

Résultats et Analyse statistique : Les usagers s'expriment peu sur leurs attentes, estimant que leur statut de bénéficiaires d'une aide gratuite ne les rend pas légitimes pour formuler des demandes. Ces échanges et observations nous ont permis de construire un cahier des charges de mise au point de produits : aliments courants, contenant des quantités significatives de fruits, légumes et poissons, se conservant à température ambiante, en portion individuelles et/ou familiales, en mettant sous surveillance les teneurs en sodium, sucres ajoutés, matières grasses saturées. La caractérisation de la spiruline a été réalisée $: c$ 'est un ingrédient de très forte hygroscopicité mais aux propriétés texturantes peu stables. Elle possède un très fort pouvoir épaississant et colorant. Les notes d'appréciation globale et l'estimation Nutriscore de ces recettes sont présentées ci-dessous :

\begin{tabular}{|l|c|c|}
\hline & $\begin{array}{c}\text { Approciation } \\
\text { global }\end{array}$ & Nutriscore \\
\hline Soupe haricot vert & 7,3 & B \\
\hline Tartinable sardine & 7,3 & B \\
\hline Tartinable aubergine & 5,0 & A \\
\hline Compote banane & 7,1 & A \\
\hline Compote poire & 7,6 & A \\
\hline
\end{tabular}

Conclusion : Ce projet a permis la création d'un site expérimental de production de spiruline en chantier d'insertion, la conception de produits alimentaire mettant en œuvre cette spiruline et répondant aux manques révélés lors des études d'observation auprès des bénéficiaires. Les notes d'appréciation valident la possibilité de concevoir des aliments qui répondent aux attentes des bénéficiaires de l'aide alimentaire, en respectant les recommandions nutritionnelles.

Références : Grange D, Castetbon K, Guibert G, Vernay M, Escalon H, Delannoy A, Féron V, Vincelet C. Alimentation et état nutritionnel des bénéficiaires de l'aide alimentaire. Etude Abena 2011-2012 et évolutions depuis 2004-2005. Observatoire régional de santé lle-de-France, Institut de veille sanitaire, Institut national de prévention et d'éducation pour la santé ; 2013.186 p.

Remerciements : Les auteurs remercient les régions Bretagne et Pays de la Loire, le département du Finistère et Quimper communauté pour leur soutien au projet.

Conflits d'intérêts : Aucun conflit à déclarer 
Introduction et but de l'étude : Au Niger, la diversité alimentaire des ménages est faible, plus de $40 \%$ des enfants de moins de 5 ans souffraient de retard de croissance en 2016 et seuls $6 \%$ des jeunes enfants de 6-23 mois bénéficient d'un régime alimentaire acceptable tel que défini par l'OMS. La consommation d'aliments de complément (AC) fortifiés pourrait permettre d'améliorer la couverture des besoins en micronutriments des jeunes enfants, à condition que la qualité de ces AC soit adaptée. Ces AC $n$ 'étant pas des matrices inertes, leur qualité nutritionnelle et sanitaire peut être impactée par des conditions de conservation non optimales pouvant induire diverses réactions impliquant des micronutriments. Le but de ce travail était d'étudier les conditions de conservation auxquelles sont soumis au Niger les produits destinés à l'alimentation de complément du jeune enfant, afin de relever d'éventuels facteurs de risque pouvant compromettre la conservation des AC, et formuler des recommandations.

Matériel et méthodes : Les conditions de stockage (température, exposition à la lumière, humidité, durée) d’AC importés ou produits localement ont été observées auprès de 100 commerces (pharmacies, petites boutiques) en zones urbaine et rurale, pour déterminer leur exposition potentielle à divers facteurs de risque. Dans un second temps, 23 enregistreurs de température et d'humidité relative (HR) ont été fixés sur les emballages d'AC produits localement, afin de suivre leurs conditions réelles de stockage depuis la production jusqu'à la consommation, et des mesures d'activité de l'eau ( $\mathrm{a}_{\mathrm{w}}$ ) ont été réalisées à chaque étape sur ces AC. En parallèle, des focus groups ont été réalisés auprès d'une soixantaine de mères de jeunes enfants pour obtenir des précisions sur les pratiques de conservation des AC dans les ménages.

Résultats et Analyse statistique : Parmi les $A C(n=86)$ vendus dans les commerces, ceux produits localement étaient significativement plus récents $(2,3 \pm 0,9$ mois depuis la production) que ceux importés d'Europe $(13,4 \pm 3,4$ mois) ou d'Afrique de l'Ouest (11,8 $\pm 7,2$ mois). La présence de produits périmés a été notée dans $13 \%$ des commerces. Le type de commerce (pharmacie vs petite boutique) $n^{\prime}$ influence pas significativement la température moyenne dans les commerces urbains $\left(35,8 \pm 2,3^{\circ} \mathrm{C}\right)$, mais influence l'amplitude journalière, la température pouvant monter jusqu'à $50^{\circ} \mathrm{C}$ dans les petites boutiques. En zone urbaine, I'HR était significativement plus élevée dans les boutiques $(52,6 \pm 9,8 \%)$ que dans les pharmacies $(34,6 \pm 11,4 \%)$. En zone rurale, des conditions plus extrêmes de températures $\left(60,5^{\circ} \mathrm{C}\right)$ et $\mathrm{HR}(90 \%)$ ont été observées ponctuellement. Une augmentation significative de l'aw des produits a été notée lors du stockage dans les commerces, puis de l'utilisation dans les ménages. Les focus groups ont montré que les femmes développent divers moyens pour conserver au mieux les AC après ouverture.

Conclusion : Le stockage dans les commerces semble être l'étape critique pour la conservation des AC, parfois exposés à des conditions extrêmes, susceptibles d'induire des réactions de dégradation. Il semble nécessaire d'étudier l'effet de telles conditions sur la stabilité des micronutriments au sein des AC fortifiés.

Conflits d'intérêts : Aucun conflit à déclarer 


\section{P017}

"L'arbre en ville " : quand le safou, un Produit Forestier Non Ligneux, régale les consommateurs des villes et des champs, au Cameroun

C. Icard-Vernière ${ }^{1, *}, \mathrm{~S}$. Zoungrana ${ }^{1}, \mathrm{H}$. Womeni ${ }^{2}$, C. Mouquet-Rivier $^{1}$

${ }^{1}$ UMR 204 NUtripass, IRD, Montpellier cedex 5, France, ${ }^{2}$ Department of Biochemistry, University of Dschang, Dschang, Cameroun

Introduction et but de l'étude : Le safou (Dacryodes edulis) est un des Produits Forestiers Non-Ligneux économiquement les plus importants, au Cameroun, et il joue également un rôle prépondérant dans l'économie des ménages. Le safoutier est utilisé comme arbre d'ombrage dans les plantations de café et de cacao, où il est aussi très apprécié pour ses fruits comestibles, les safous. On le trouve dans $80-94 \%$ des exploitations dans certaines régions, mais aussi autour des maisons en zone urbaine. Les safous sont des fruits oléagineux très périssables, saisonniers (juin à octobre) riches en eau (50\%), en lipides (30-35 \%) et micronutriments. Ils sont principalement consommés cuits. Malgré leur abondance, un manque de connaissances sur ces arbres et sur leurs fruits, freine la valorisation du safou, dans un pays où l'augmentation de la population urbaine a dépassé les $70 \%$ entre 2005 et 2015. Aussi, au sein du projet d'agroforesterie ARBOPOLIS, un volet de nutrition a été développé afin de mieux appréhender la place du safou dans l'alimentation des Camerounais, ainsi que le niveau de consommation des plats à base de safou, en zone urbaine comme en zone rurale, en fonction du niveau socio-économique des ménages.

Matériel et méthodes : 12 focus groups (féminins ou masculins) ont été réalisés dans 5 quartiers de Yaoundé et en zone rurale à Loum. Un catalogue photo de plats (7 plats à base de safous, 10 plats d'accompagnements), avec 4 portions par plat, de masses mesurées, a été réalisé, et utilisé dans une enquête de consommation menée auprès de femmes camerounaises (21 à 65 ans) à Yaoundé (31 quartiers, 313 ménages) et en zone rurale autour de Loum et Manjo (24 quartiers, 309 ménages), après obtention d'une clearance éthique. Les premières analyses statistiques ont été réalisées avec le logiciel $R$.

Résultats et Analyse statistique : Les focus groups ont permis d'identifier les principaux plats à base de safou consommés, ainsi que leurs accompagnements. Les consommateurs déclarent s'approvisionner d'abord auprès des arbres autour de leur habitation en zone rurale, mais aussi en zone urbaine, l'achat au marché se faisant secondairement en cas d'indisponibilité des fruits sur ces arbres, ou en l'absence d'accès aux arbres. Les plats sont consommés par tous, exceptés chez les jeunes enfants pour certaines ethnies. Une seule femme sur les 622 enquêtées n'est pas consommatrice de safou. Les plats les plus consommés au cours des 7 jours précédant l'enquête sont les safous braisés, les safous à la cendre et les safous grillés avec respectivement 167 et 117,47 et 129, et 123 et 40 consommatrices en zones urbaine et rurale. La zone d'étude, l'âge de la consommatrice, et le niveau socioéconomique des ménages ont eu un effet significatif sur la consommation du safou, contrairement à l'ethnie. Les fréquences et niveaux de consommation de chaque plat sont en cours d'analyse.

Conclusion : Le safou est très largement consommé au Cameroun, sous forme cuite, avec un accompagnement amylacé. Sa contribution aux apports nutritionnels des Camerounais devrait contribuer à la valorisation des fruits, mais aussi fournir des pistes de réflexion sur la place des arbres « nourriciers » en ville dans un contexte d'urbanisation rapide et de désertification des zones rurales.

Conflits d'intérêts : Aucun conflit à déclarer 
P018

Intérêt pour la truie et sa portée de l'apport en acides gras n-3 dans l'alimentation gestante et allaitante : impact de la durée de distribution

M. Guillevic ${ }^{1, *}$, G. Mairesse ${ }^{1}$, G. Chesneau ${ }^{1}$

${ }^{1}$ VALOREX, Combourtille, France

Introduction et but de l'étude : Dans un contexte d'hyperprolificité des truies où les pertes augmentent, des solutions pour augmenter la vigueur des porcelets sont à rechercher. Parmi celles-ci, des solutions nutritionnelles fonctionnent comme l'incorporation de matières grasses dans les aliments en veillant au profil en acides gras (AG) comme les AG n-3 (Quiniou 2010). L'apport de ces derniers dans les aliments a été testé en station expérimentale dans des conditions maitrisées et a conduit à des effets positifs : hausse des AGn3 du lait \& du plasma (Quelen ; Guillevic 2011) ; croissance porcelet (Boudry 2012) ; profil en AG conduisant à la formation de composés anti-inflammatoires (Mateo 2009). L'objectif de cette étude porte sur la réalisation d'une expérimentation en condition d'élevage de solutions nutritionnelles éprouvées dans les conditions maitrisées.

Matériel et méthodes : L'essai est mené avec des truies nourries avec des rations gestante - allaitante où seule la qualité de la matière grasse changeait. Face au lot TEMOIN, les truies LIN consommaient un aliment à base de graines de lin fournissant $8,2 \mathrm{~g}$ de C18:3/kg pendant toute la gestation et lactation jusqu'à sevrage du porcelet. Le colostrum est prélevé pour en déterminer son profil en $A G$ et sa teneur en immunoglobuline $G(\operatorname{IgG})$. Le sang des porcelets est prélevé pour en déterminer son profil en AG.

Résultats et Analyse statistique : Les performances de reproduction ont été accrues via hausse du nombre de porcelets né vifs (15.5 vs $13.6, p<0.05)$ \& sevrés ( 13.0 vs $11.8, p<0.05)$. La densité nutritionnelle \& immunologique du colostrum est supérieure. La teneur en IgG est plus élevée ( 46.9 vs $88.3 \mathrm{mg} / \mathrm{ml}, \mathrm{p}<0.01$ ). Le profil en AG présente une teneur supérieure en $A G \mathrm{n}-3$ (10.8 vs 3.3 , $\mathrm{p}<0.001$ ) depuis le précurseur jusqu'aux dérivés à longue chaine. Le rapport C20:4/C20:5 est favorable à la voie anti-inflammatoire (1.9 vs 7.0). Le porcelet présente un statut nutritionnel - à l'image de son alimentation lactée - orienté vers un rééquilibrage des AGn6 versus les $n 3$.

Conclusion : Le transfert d'échelles (station/élevage) et de conditions (encadré/terrain) est possible et permet de démontrer l'intérêt nutritionnel de l'apport d'AGn-3 pour la truie et sa portée. Ainsi, il serait pertinent que ces AG essentiels entrent dans les recommandations alimentaires des truies pour ses effets bénéfiques prouvés.

\section{Références}

Quiniou 2010. J. Rech. Porcine, 42, 137-138.

Quelen 2010. J. Rech. Porcine, 42, 139-140.

Guillevic 2011. J. Rech. Porcine, 43, 287-288.

Boudry 2012. J. Rech. Porcine, 44, 191-192.

Mateo 2009. J. Anim. Sci., 87, 948-959.

Conflits d'intérêts : Aucun conflit à déclarer 
Facteurs nutritionnels et non nutritionnels du taux de cholestérol de l'œuf M. Colin ${ }^{1,}{ }^{*}$, C. Riou ${ }^{1}$, J. Delarue ${ }^{2}$, A. Y. Prigent ${ }^{1}$, N. Guriec ${ }^{2}$, M. Van Lissum ${ }^{1}$

${ }^{1}$ COPRI SARL, Ploudalmezeau, ${ }^{2}$ Laboratoire régional de nutrition humaine - FED 4216, Brest, France

Introduction et but de l'étude : L'augmentation du taux de cholestérol sanguin suite à l'ingestion régulière d'œufs constitue un obstacle à la consommation de cet aliment par ailleurs nutritionnellement très intéressant. Ce travail étudie les variations du taux de cholestérol des œufs en fonction de paramètres non nutritionnels ou nutritionnels.

Matériel et méthodes : Cette étude présente une synthèse de 11 essais réalisés par la société COPRI et dans lesquels le taux de cholestérol des œufs a été dosé. Une partie des résultats a déjà été publiée mais sans mention systématique du cholestérol (2, 3 , $4,5,6,7)$.

Résultats et Analyse statistique : Facteurs non nutritionnels: Quatre critères ont été étudiés : poids des œufs, taux de lipides, âge des poules, saison. Le taux de cholestérol de l'œuf n'est lié significativement $(P=0.02)$ qu'au seul taux de lipides. Aucune relation n'existe avec les autres paramètres. Cette observation rejoint différentes études bibliographiques montrant la difficulté de modifier le taux de cholestérol par les seules techniques d'élevage.

Facteurs nutritionnels: Huit paramètres ont été étudiés (Tableau 1).

Tableau 1 : Influence de 7 paramètres nutritionnels sur le taux de cholestérol de l'œuf

\begin{tabular}{|c|c|c|c|c|c|c|c|}
\hline $\begin{array}{l}\text { Paramétre nutritionel étudié } \\
\text { (nb essais) }\end{array}$ & $\begin{array}{l}\text { ALA } \\
(36)\end{array}$ & $\begin{array}{l}\text { DHA } \\
(32)\end{array}$ & $\begin{array}{l}\text { Perfalg } \\
(42)\end{array}$ & $\begin{array}{l}\text { Saponine } \\
(43)\end{array}$ & $\begin{array}{c}\text { Vit E } \\
(4)\end{array}$ & $\begin{array}{c}\text { Vit } D_{3} \\
(3)\end{array}$ & $\begin{array}{c}\text { Choline } \\
(6)\end{array}$ \\
\hline $\begin{array}{l}\text { Cholestérol dans régime témoin } \\
\text { (g/100 g d'oeuf) }\end{array}$ & 0,386 & 0,432 & 0,342 & 0,373 & 0,488 & 0,376 & 0,344 \\
\hline $\begin{array}{l}\text { Cholestérol dans régime } \\
\text { expérimental } \\
\text { (g/100 g d'oeuf) }\end{array}$ & 0,388 & 0,499 & 0,379 & 0,321 & 0,409 & 0,344 & 0,345 \\
\hline $\begin{array}{l}\text { Variation taux de cholestérol } \\
\text { (\% du témoin) }\end{array}$ & 0,31 & 15,52 & 10,82 & $-13,94$ & $-16,19$ & $-8,51$ & 0,29 \\
\hline $\begin{array}{l}\text { Signification statistique / } \\
\text { Cohérence avec la bibliographie }\end{array}$ & NS/OUI & $\mathrm{P}=0,05 / \mathrm{OUI}$ & $P=0,09 / N D$ & $\mathrm{P}=0,03 / \mathrm{OUI}$ & $p=0,10 / O U I$ & $P=0,06 / N D$ & NS/OUI \\
\hline $\begin{array}{l}\text { Effet du paramètre sur le taux } \\
\text { de cholestérol de l'oeuf }\end{array}$ & $\begin{array}{l}\text { Aucun } \\
\text { effet }\end{array}$ & $\begin{array}{l}\text { Légère } \\
\text { augmen- } \\
\text { tation }\end{array}$ & $\begin{array}{l}\text { Tendance à } \\
\text { l'augmen- } \\
\text { tation }\end{array}$ & Diminution & Diminution & Diminution & $\begin{array}{l}\text { Aucun } \\
\text { effet }\end{array}$ \\
\hline
\end{tabular}

En accord avec la bibliographie, l'augmentation des taux d'ALA et de choline de l'aliment n'ont aucun effet sur le contenu en cholestérol de l'œuf. L'accroissement du taux de DHA et l'incorporation de Perfalg $(2,3,4)$ dans la ration tendent à augmenter de 10 à $15 \%$ le cholestérol. Seules les saponines le diminuent significativement d'environ un sixième, observation cohérente avec la bibliographie. Des réductions ont également été observées avec des taux élevés de vitamine $E$ et de vitamine $D$ mais elles portent sur un faible nombre d'observations et ne sont qu'à la limite de la signification statistique. Elles devront donc être confirmées Conclusion : Le taux de cholestérol des œufs apparait difficile à diminuer. Il est peu influencé par les facteurs non nutritionnels et nutritionnels étudiés. D'après nos différents essais, seules les saponines et peut être les vitamines $E$ et $D_{3}$ sont susceptibles de le diminuer.

Références : Roberts, Mac Murry et Connor 1981; (2) Colin et al 2013 ; (3) Guriec et al 2014; (4) Guriec et al 2015 ; (5) Corral et al, 2016 ; (6) Guriec et al 2016; (7) Delarue et al 2017.

Conflits d'intérêts : M. Colin est actionnaire de COPRI SARL, C. Riou: Aucun conflit à déclarer, J. Delarue: Aucun conflit à déclarer, A. Y. Prigent est actionnaire de COPRI SARL, N. Guriec: Aucun conflit à déclarer, M. Van Lissum: Aucun conflit à déclarer 

A. Saint-Eve ${ }^{1, *}$, B. Maurice ${ }^{1}$, J. Delarue ${ }^{2}$, L.-G. Soler ${ }^{3}$, I. Souchon ${ }^{1}$

${ }^{1}$ UMR GMPA, AgroParisTech, INRA, Université Paris-Saclay, 78850, Thiverval-Grignon, ${ }^{2}$ UMR GENIAL, AgroParisTech, INRA, Université Paris-Saclay, 91300, Massy, ${ }^{3}$ ALISS, INRA, 94205, Ivry-Sur-Seine, France

Introduction et but de l'étude : Les consommateurs et citoyens ont aujourd'hui pris conscience que leur alimentation est un facteur clé de leur bien-être, de leur santé et qu'elle impacte l'environnement et le futur de nos sociétés à travers des pratiques plus ou moins durables. Les produits industriels transformés sont pointés du doigt pour être pour partie responsables de maladies métaboliques (Monteiro et al., 2018 ; Moodie et al., 2013). Récemment, une étude sur la cohorte NutriNet a montré des liens entre consommation d'aliments dits « ultra-transformés » (classification NOVA - Monteiro et al., 2018) et risques de cancer (Fiolet et al., 2018).

Il est donc clairement nécessaire de mettre en place des indicateurs fiables et des recommandations pour les consommateurs, les industriels et les pouvoirs publics, afin de construire une offre alimentaire industrielle plus saine et durable. Pour cela, il est nécessaire de comprendre les attentes et les perceptions des consommateurs et d'analyser l'offre alimentaire sous l'angle de critères nutritionnels, technologiques, environnementaux et économiques.

Matériel et méthodes : Notre étude a porté sur les pizzas industrielles qui sont principalement classées dans la catégorie des produits dits " ultra-transformés " selon la classification NOVA, et sont très consommées en France. Par ailleurs, les pizzas offrent des leviers importants de reformulation qu'il sera possible de mettre en œuvre pour tendre vers une alimentation plus saine, plus durable et appréciée.

Afin de réaliser une cartographie complète du marché des pizzas industrielles, la première étape du travail a été de mettre en place un indicateur de process (IDP). Cet indicateur a permis de montrer des différences significatives entre les familles de pizza ainsi qu'entre les secteurs frais et surgelés. Sur la base des critères nutritionnels et technologiques, 16 pizzas représentatives du marché français ont été sélectionnées. Elles ont été évaluées en conditions réelles de consommation (restaurant) par 64 consommateurs selon différents paramètres: perception en bouche, caractère naturel, leur qualité nutritionnelle et leur contribution à la protection de l'environnement.

Résultats et Analyse statistique : Les résultats ont montré que les 16 pizzas présentaient une grande variété de propriétés sensorielles, d'appréciation et de perceptions subjectives. Nous avons notamment observé que la perception de la qualité nutritionnelle était très différente entre les pizzas et pouvait être liée aux attentes spécifiques des consommateurs.

Conclusion : Une pizza de haute qualité nutritionnelle devrait généralement avoir une pâte dure, croustillante, difficile à couper, une garniture généreuse et contenant des ingrédients identifiables, contrairement à une pizza de mauvaise qualité susceptible d'être grasse et molle. Fait intéressant, la naturalité perçue n'était pas liée à ces scores, ce qui montre la complexité de ce paramètre, contrairement à la perception d'une qualité nutritionnelle élevée liée à un faible degré de transformation des aliments.

Références :

Fiolet T et al. 2018. The BMJ 360:k322.

Monteiro CA et al. 2018. Public Health Nutr 21:5-17.

Moodie R et al. 2013. The Lancet 381:670-679.

Conflits d'intérêts : Aucun conflit à déclarer 
P021

Possibilité de couvrir les besoins journaliers de l'Homme en DHA grâce à des menus principalement composés de produits provenant d'animaux terrestres enrichis naturellement

M. Colin ${ }^{1,}{ }^{*}$, L. Caillaud ${ }^{1}$, A. Boutinon ${ }^{1}$, J. Delarue ${ }^{2}$, A. Y. Prigent ${ }^{1}$, M. Van Lissum ${ }^{1}$

${ }^{1}$ COPRI SARL, Ploudalmezeau, ${ }^{2}$ Laboratoire régional de nutrition humaine- FED 4216, Brest, France

Introduction et but de l'étude : Les effets du DHA sur la santé sont abondamment documentés: réduction du risque cardiovasculaire (1), de l'insulinorésistance, du diabète et de l'obésité (2), du syndrome métabolique (3), de la DMLA (5). C'est pourquoi l'ANSES recommande un apport quotidien de $250 \mathrm{mg}$ de DHA/jour/adulte. Actuellement, le DHA est apporté essentiellement par les poissons gras et cette consommation ne permet qu'un apport moyen de $137 \mathrm{mg}$ de $\mathrm{DHA} / \mathrm{jour}$ à la population française; $50 \%$ de la population se situant à un niveau très faible (<100 mg / jour) (6). Or, l'augmentation de la consommation de produits de la mer se heurte à plusieurs problématiques importantes telles que la diminution de la ressource halieutique, l'augmentation des métaux lourds dans le poisson et les difficultés de changement des habitudes alimentaires. Dans ce contexte, l'enrichissement en DHA des produits issus d'animaux terrestres par incorporation de microalgues $(7,8,9)$ dans leur alimentation apparait comme une réelle opportunité permettant d'augmenter la consommation moyenne de DHA de la population tout en évitant les difficultés évoquées précédemment.

Matériel et méthodes : Ce travail consiste à élaborer des menus complets, équilibrés (respectant les recommandations du PNNS) et apportant une quantité de DHA supérieure aux ANC et ce, par consommation de produits issus d'animaux terrestres recevant des microalgues riches en DHA dans leur alimentation. Le poisson n'est utilisé que 2 fois par semaine et les recettes sont des recettes courantes.

Résultats et Analyse statistique : Lorsqu'ils sont réalisés avec des produits animaux naturellement enrichis en DHA, la totalité des menus permettent un apport en DHA supérieur à 2 fois les ANC (Tableau 1).

Tableau 1 : Apport de DHA des menus étudiés réalisés avec des produits standards ou enrichis en DHA

\begin{tabular}{|c|c|c|c|c|c|c|c|}
\hline Jour & 1 & 2 & 3 & 4 & 5 & 6 & 7 \\
\hline $\begin{array}{l}\text { Produits } \\
\text { d'origine } \\
\text { animale sources } \\
\text { de DHA (pour } 3 \\
\text { repas/jour) }\end{array}$ & $\begin{array}{l}\text { Lait, } \\
\text { CEufs, } \\
\text { Yaourt, } \\
\text { Poulet, } \\
\text { Gruyère }\end{array}$ & $\begin{array}{l}\text { Fromage } \\
\text { blanc, } \\
\text { Jambon, } \\
\text { Lapin, } \\
\text { Yaourt, } \\
\text { CEufs }\end{array}$ & $\begin{array}{l}\text { Beurre, Gruyère, } \\
\text { Maquereau, } \\
\text { Porc, Riz au lait, } \\
\text { Saucisse de porc, } \\
\text { Fromage blanc }\end{array}$ & $\begin{array}{l}\text { Lait, Beurre, } \\
\text { CEufs, } \\
\text { Yaourt, } \\
\text { Poulet, } \\
\text { Camembert }\end{array}$ & $\begin{array}{l}\text { Lait, Lapin, } \\
\text { Fromage } \\
\text { blanc, } \\
\text { CFufs, } \\
\text { Semoule au } \\
\text { lait }\end{array}$ & $\begin{array}{c}\text { Beurre, } \\
\text { Fromage } \\
\text { blanc, Lapin, } \\
\text { Yaourt, } \\
\text { đEufs, } \\
\text { Camembert }\end{array}$ & $\begin{array}{l}\text { Lait, CFufs, } \\
\text { Saumon, } \\
\text { Crème } \\
\text { anglaise, } \\
\text { Poulet, } \\
\text { Yaourt }\end{array}$ \\
\hline $\begin{array}{l}\text { DHA produits } \\
\text { standards (mg / } \\
\text { jour) }\end{array}$ & 91 & 41 & 490 & 72 & 62 & 86 & 934 \\
\hline $\begin{array}{l}\text { DHA produits } \\
\text { enrichis ( } \mathrm{mg} / \\
\text { jour) }\end{array}$ & 464 & 429 & 799 & 491 & 371 & 835 & 1215 \\
\hline $\begin{array}{l}\text { Différence (mg / } \\
\text { jour) }\end{array}$ & 373 & 388 & 309 & 419 & 309 & 749 & 280 \\
\hline $\begin{array}{l}\text { DHA produits } \\
\text { enrichis (\% ANC) }\end{array}$ & 186 & 172 & 320 & 196 & 148 & 334 & 486 \\
\hline
\end{tabular}

Conclusion : L'enrichissement de l'alimentation des animaux terrestres en DHA par incorporation de microalgues est l'une des solutions pour couvrir les ANC en cet acide gras essentiel de la population française sans utiliser uniquement des produits provenant de la mer.

Références : (1) Anses (2011); (2) Delarue et al., (2004) ; (3) Delarue et al., (2006), (4) Anses 2011 ; (5) Barnathan (2007) ; (6) Tressou - Cosmao et al., 2015 ; (7) ; Colin et al (2011) ; (8) Colin et al 2013.

Conflits d'intérêts : M. Colin est actionnaire de COPRI SARL, L. Caillaud: Aucun conflit à déclarer, A. Boutinon: Aucun conflit à déclarer, J. Delarue: Aucun conflit à déclarer, A. Y. Prigent: Aucun conflit à déclarer, M. Van Lissum: Aucun conflit à déclarer 
Habitudes alimentaires en matière de consommation du lait et produits laitiers chez la population algérienne M. Bencharif ${ }^{1, *}$, M. Bentaleb ${ }^{2}$

${ }^{1}$ Déparement de Nutrition, Laboratoire de Nutrition et Technologies Alimentaires (LNTA), INATAA, Université Frères Mentouri Constantine 1, Algérie, 'Institut de la Nutrition, de l'Alimentation et des Technologies Agro-Alimentaires (INATAA), Université Frères Mentouri Constantine 1, Algérie, Constantine, Algérie

Introduction et but de l'étude : Le lait et les produits laitiers occupent une place particulière au sein de la consommation de la population algérienne. L'objectif de notre étude était de décrire et d'analyser les habitudes de consommation du lait et dérivés chez un échantillon de population.

Matériel et méthodes : Une étude transversale par questionnaire a été réalisée sur 500 sujets issus de 37 wilayas algériennes, entre le 11 février et le 31 mars 2018. Notre questionnaire était composé de quatre volets : caractéristiques générales, consommation des produits laitiers et leur part dans le revenu, achat des produits laitiers et l'apport calcique quotidien. Le seuil de signification retenu était de 0,05.

Résultats et Analyse statistique : L'enquête a regroupé 466 sujets en milieu urbain ( 351 femmes, 115 hommes) et 34 sujets en milieu rural ( 25 femmes, 9 hommes). Leur moyenne d'âge était de $26,7 \pm 7,9$ ans [ 16,0 ans-82,0 ans]. La part de l'achat des produits

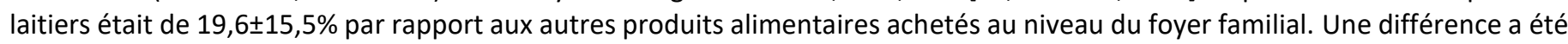
observée par type d'habitat en ce qui concerne la part d'achat des produits laitiers dans le revenu du ménage $(p<0,05)$. Le fromage fondu était le produit laitier le plus consommé avec $92,4 \%$, suivi du lait reconstitué recombiné (70,0\%). Pour la population urbaine, les produits laitiers étaient beaucoup plus consommés durant l'hiver $(34,8 \%)$ et très peu durant l'été $(17,8 \%)$. Deux sujets sur cinq consommaient le lait et dérivés régulièrement. $58,4 \%$ des urbains indiquaient que se sont des consommateurs moyens de produits laitiers, alors que $22,3 \%$ se considéraient comme de grands consommateurs. Les critères d'achat des urbains pour les produits laitiers étaient par rapport au prix, l'hygiène, le goût et la marque. L'apport calcique des sujets $(866,8 \pm 312,4 \mathrm{mg} / \mathrm{jour})$ était inférieur aux recommandations internationales. Aucune différence n'a été remarquée quel que soit le genre et quel que soit le type d'habitat.

Conclusion : Le lait et les produits laitiers entrent très largement dans la consommation de tous les ménages, particulièrement qu'ils sont source de protéines, vitamines et calcium la moins chère. Ils sont à la base des habitudes culinaires et il est donc difficile d'y échapper.

Conflits d'intérêts : Aucun conflit à déclarer 
Enrichissement et préservation de la qualité hygiénique du yaourt par l'extrait aqueux de curcuma, le gel d'aloe vera et l'huile essentielle du romarin

H. Boughellout ${ }^{1, *}$, W. Salmia ${ }^{1}$, F. Adoui ${ }^{2}$

${ }^{1}$ Laboratoire de nutrition et technologie alimentaire (LNTA), équipe transformation et élaboration des aliments (TPEPA) INATAA Université Frères Mentouri Constantine $1,{ }^{2}$ Laboratoire de nutrition et technologie alimentaire (LNTA), équipe transformation et élaboration des aliments (TPEPA) INATAA Université Frères Mentouri Constantine 1, Constantine, Algeria, Constantine, Algérie

Introduction et but de l'étude : La tendance actuelle des consommateurs à chercher une alimentation naturelle sans additifs chimiques a augmenté durant ces derniers décennies. Les extraits de certaines plantes sont douées de plusieurs propriétés biologiques tels que les activités antioxydantes et antimicrobiennes.

Dans ce contexte, le présent travail vise à élaborer un yaourt enrichi avec le gel d'aloe vera (Aloe barbadensis Miller), de l'extrait acqueux du curcuma (Curcuma longa $\mathrm{L}$ ) et de huile essentielle de romarin (Rosmarinus officinalis $L$ ) afin d'augmenter sa valeur alimentaire et diététique et augmenter la qualité hygiénique du yaourt au cours de la conservation.

Matériel et méthodes : L'extraction de l'huile essentielle du romarin a été réalisée par hydrodistillatilon avec un rendement de $0,6 \%$. L'extrait aqueux du curcuma a été obtenu par macération à froid.

Résultats et Analyse statistique : Les résultats obtenus ont montré une activité antibactérienne modérée des trois extraits sur staphylococcus aureus.

Les yaourts obtenus ont présenté des caractéristiques physicochimiques comparables. Les quatre yaourts ont montré une texture ferme, crémeuse, lisse, onctueuse et nappante. Le yaourt enrichi à l'Aloe Vera (AV) a été préféré par $9 / 20$ des dégustateurs.

La charge microbienne des différents yaourts a été différente, un effet antimicrobien du gel de l'aloe vera a été remarqué sur la FTAM et les levures et moisissure mais sans aucun effet sur les bactéries lactiques.

Le suivi de l'évolution des différentes flores au cours de la conservation a montré que la FTAM a été réduite pour les yaourts enrichis avec un taux de 0 UFC après 45 jours de conservation. Les levures et moisissures et la flore lactique n'ont pas été affectées.

Conclusion : A travers ce travail, il a été montré que l'enrichissement du yaourt par ces trois extraits apporte en plus de l'activité antioxydante interessante, une protection de la qualité hygiénique du yaourt au cours de la conservation.

Conflits d'intérêts : Aucun conflit à déclarer 
P024

Effet du jeune du ramadan sur l'alimentation et la corpulence des adultes au niveau de deux régions algériennes (2017) O. Allam ${ }^{1, *}$, H. Oulamara ${ }^{1}$, A. N. Agli ${ }^{1}$

${ }^{1}$ Laboratoire de Nutrition et de Technologie Alimentaire, Université de Constantine, Constantine, Algérie

Introduction et but de l'étude : Durant le mois du ramadan, les musulmans s'abstiennent de manger et de boire depuis le lever jusqu'au coucher du soleil. Le jeune diurne entraine de nombreux changements de mode de vie (alimentation, activités, sommeil, ...), les pratiquants passent d'un mode de vie à un autre du jour au lendemain. La fréquence des prises alimentaires devenant strictement nocturnes et réduites. L'influence du jeune de ramadan sur la quantité et la qualité des prises alimentaires n'étant que rarement aborder. L'objectif est d'étudier l'effet du jeune du ramadan sur l'alimentation, l'activité physique ainsi que la corpulence des adultes algériens.

Matériel et méthodes : Une enquête descriptive transversale a été réalisée sur deux périodes : avant ramadan (du 10 avril au 25 mai 2017) et pendant ramadan (du 19 au 25 juin 2017). La population concerne 400 adultes agés de 18 à 80 ans résidant au niveau de la wilaya de Jijel et la daïra de Ferdjioua. Les mesures anthropométriques concernent le poids et la taille avant et à la fin du ramadan. Le questionnaire recense des informations sur les habitudes alimentaires, l'activité physique et la sédentarité avant et pendant le mois du ramadan. Les apports alimentaires sont déterminés par un rappel de 24 h. L'analyse statistique est réalisée par le logiciel StatView.

Résultats et Analyse statistique : Bien que non significatif, après ramadan, une légère diminution a été trouvée dans le poids et I'IMC moyens des adultes. Un pourcentage de $58 \%$ des sujets ont perdu du poids, $29 \%$ ont pris du poids et $12 \%$ étaient pondéralement stables. Pendant le ramadan, le nombre des repas pris diminue, $76 \%$ prennent deux repas après la rupture du jeune. La fatigue (23\%) et le manque d'appétit (21\%) sont les causes principales de cette diminution. Le repas du lobe est pris quotidiennement par $82 \%$ des sujets. Durant le ramadan, la prise du repas en convivialité augmente et passe de $88 \%$ à $98 \%$ dans les deux régions. Les gâteaux traditionnels riches en sucres simples sont les plus consommés durant les soirées du ramadan (61 $\%)$. Pendant le ramadan, malgré la diminution du nombre des repas, les apports énergétique (1691 $\pm 513,8 \mathrm{vs} 2556 \pm 808,5 \mathrm{kcal} /$ $\mathrm{j} ; \mathrm{p}<0,0001)$, glucidique $(220,1 \pm 83,4$ vs $365,5 \pm 137 ; \mathrm{p}<0,0001)$, protéique $(73,9 \pm 26,5$ vs $93,7 \pm 30,9 ; p<0,0001)$ et lipidique $(57,4 \pm 26$ vs $80,6 \pm 35,1 ; p=0,04)$ augmentent. Aucune corrélation entre les différents apports et l'IMC n'a été trouvée. La pratique du jeune s'est traduite également par une diminution de la durée du sommeil $(8,4 \pm 1,6$ vs $8,1 \pm 2,1 ; p<0,0001)$, de la durée de l'activité physique $(0,2 \pm 0,6$ vs $0,06 \pm 0,3 ; p=0,02)$ ainsi qu'une augmentation des activités sédentaires $(2,5 \pm 1,6$ vs 3,1 $\pm 1,7 ; p<0,0001)$.

Conclusion : Les résultats de cette étude confirment que le jeune du mois du ramadan s'accompagne des changements importants tant dans l'apport énergétique global que dans l'ensemble des apports en nutriments ainsi que le mode de vie général. Les horaires, la fréquence, la qualité et la quantité des prises alimentaires sont les facteurs clés de ces changements. Des études plus approfondies sont nécessaires afin de déterminer la part de chacun de ces facteurs dans les conséquences qu'ils peuvent engendrer surtout vis-à-vis à la corpulence des adultes.

Conflits d'intérêts : Aucun conflit à déclarer 
Traitement cérébral des odeurs subliminales : une étude en IRM fonctionnelle

C. Mignot ${ }^{1, *}$, D. Gounot ${ }^{1}$, S. Chambaron ${ }^{2}$, G. Gaeta ${ }^{3}$, I. Kontaris ${ }^{3}$, L. Marlier ${ }^{1}$

${ }^{1}$ Laboratoire ICube, UMR7357, Centre National de la Recherche Scientifique (CNRS) et Université de Strasbourg, Strasbourg, ${ }^{2}$ Centre des Sciences du Goût et de I'Alimentation, UMR1324, Institut National de la Recherche Agronomique (INRA) et Université de Bourgogne, Dijon, France, ${ }^{3}$ Givaudan UK Ltd, Ashford, Royaume-Uni

Introduction et but de l'étude : Différentes études indiquent que les odeurs ambiantes peuvent influencer les comportements humains, et cela même lorsque les personnes n'en sont pas conscientes. Dans le domaine alimentaire par exemple, il a été montré que des odeurs alimentaires subliminales peuvent guider les choix alimentaires vers des aliments plus sains. Le but de cette étude était de mettre en évidence les réseaux cérébraux impliqués dans le traitement cérébral d'odeurs subliminales.

Matériel et méthodes : Dix participants sains âgés de 19 à 40 ans ont été recrutés sous un prétexte ne faisant pas référence aux odeurs. Lors de l'acquisition d'IRM fonctionnelle (séquence EPI multiband, TR=500 ms), les participants ont été exposés discrètement à deux odeurs (poire, jacinthe) et à un flux d'air non-odorisé (contrôle) en même temps qu'ils réalisaient une tâche visuelle simple. Le paradigme expérimental événementiel est divisé en deux sessions : une première session pendant laquelle les odeurs sont diffusées à une intensité subliminale (IS), suivie par une deuxième session pendant laquelle les odeurs sont diffusées à une intensité supraliminale clairement perceptible (ICP). Les questions posées à la fin de la première session confirment que les odeurs IS n'étaient pas attentivement perçues par les participants. Des contrastes T (SPM) ont été réalisés sur les IS et les ICP pour les deux odeurs. Les réseaux cérébraux impliqués dans le traitement des odeurs ont été explorés en utilisant l'Analyse en Composantes Indépendantes.

Résultats et Analyse statistique : Les premiers résultats en analyse individuelle montrent un traitement sensoriel différentiel entre les odeurs d'IS et d'ICP. L'insula gauche et le cortex cingulaire antérieur gauche s'avèrent par exemple spécifiquement impliqués dans le traitement de l'odeur de poire à intensité subliminale, suggérant qu'un processus de saillance pourrait survenir en olfaction subliminale. De plus, les patterns d'activations cérébrales apparaissent différents entre les odeurs de poire et de jacinthe aux deux intensités considérées.

Conclusion : Ces résultats montrent que des réseaux cérébraux sont activés en présence d'odeurs subliminales. Ils indiquent également que la qualité des odeurs est discriminée par le cerveau même lorsqu'aucune attention n'est portée à ces odeurs. Ce dernier facteur pourrait expliquer l'effet ciblé et spécifique des amorces olfactives alimentaires sur les choix alimentaires.

Remerciements : Nous remercions Thierry Audibert, Paddy Nicholson, Pauline Joussain, et Leo Murison de Givaudan UK Ldt ; Corinne Marrer et Daniel Grucker du laboratoire Icube, France.

Conflits d'intérêts : C. Mignot a reçu une subvention/soutien de Givaudan, D. Gounot: Aucun conflit à déclarer, S. Chambaron: Aucun conflit à déclarer, G. Gaeta: Aucun conflit à déclarer, I. Kontaris: Aucun conflit à déclarer, L. Marlier: Aucun conflit à déclarer 

V. A. Andreeva ${ }^{1,{ }^{*}}$, F. Szabo de Edelenyi ${ }^{1}$, N. Druesne-Pecollo ${ }^{1}$, M. Touvier ${ }^{1}$, S. Hercberg ${ }^{12}$, P. Galan ${ }^{1}$

${ }^{1}$ EREN/Epidémiologie Nutritionnelle, UNIV PARIS 13; INSERM U1153; INRA U1125; CNAM, ${ }^{2}$ Département de Santé Publique, Hôpital Avicenne, Bobigny, France

Introduction et but de l'étude : La migraine est un trouble de la fonction cérébrale dont la prévalence mondiale est estimée à $11,6 \%$. II s'agit de céphalées primaires avec des crises unilatérales et récurrentes ayant des conséquences psychosociales importantes. A ce jour, très peu de données sont disponibles en population générale sur l'alimentation chez les individus souffrant de céphalées. Dans cette étude épidémiologique d'observation constituée d'un échantillon important, les objectifs étaient de déterminer les prévalences actuelles des céphalées (migraines ou céphalées non migraineuses) et de décrire les apports quotidiens en macronutriments chez les adultes français souffrant de céphalées.

Matériel et méthodes : Il s'agit d'une étude ancillaire conduite dans le cadre de la cohorte NutriNet-Santé (lancée en 2009). Cette cohorte est constituée de volontaires de plus de 18 ans, recrutés par des campagnes multimédia grand public et suivis via le site internet https://www.etude-nutrinet-sante.fr. Les céphalées ont été identifiées par un questionnaire d'auto-déclaration en 20132016. Les migraines et les céphalées non migraineuses ont été définies selon des critères déjà publiés. Les apports alimentaires en macronutriments ont été estimés grâce aux enquêtes alimentaires de $24 \mathrm{~h}$. Les apports quotidiens moyens (en g/jour) en glucides (simples, complexes, totaux), protéines et lipides (totaux, acides gras saturés, monoinsaturés, polyinsaturés) étaient les mesures d'exposition. A partir des 37125 individus ayant répondu au questionnaire, un échantillon de 31770 (8 042 hommes et 23728 femmes) pour lesquels l'ensemble des données sociodémographiques et de mode de vie avaient été collectées ont été inclus. Des analyses transversales ont été réalisées à l'aide de modèles ajustés ANOVA stratifiés selon le sexe.

Résultats et Analyse statistique : Au total, 9,2 \% des hommes (âge moyen $=54,3 \pm 13,3$ ans) et 25,7 \% des femmes (âge moyen $=$ $49,6 \pm 12,8$ ans) ont déclaré souffrir de migraine. Dans les modèles ajustés, des apports plus faibles en protéines $(p<0,02)$ et des apports plus élevés en lipides totaux $(p<0,01)$ ont été observés chez les hommes migraineux par rapport aux hommes non migraineux (hommes sans maux de tête ou souffrant de céphalées non migraineuses). En revanche, les apports en lipides totaux $(p<0,0001)$ et en glucides totaux $(p<0,05)$ étaient légèrement plus élevés chez les femmes migraineuses par rapport aux femmes non migraineuses.

Conclusion : Ces résultats permettent d'actualiser les données sur les prévalences des céphalées en France, même si cet échantillon pourrait être sujet d'un biais de sélection. Des associations significatives ont été observées entre les apports en macronutriments et les céphalées, qui différaient selon le sexe. Des recherches longitudinales complèteront ces travaux et serviront à orienter le développement de stratégies de santé publique dans le cadre de la prévention primaire.

Références : Andreeva VA, Szabo de Edelenyi F, Druesne-Pecollo N, et al. Macronutrient intake in relation to migraine and nonmigraine headache. Nutrients. (Soumis)

Remerciements : Les auteurs remercient $\operatorname{Dr}$ Véronique Gourlet pour les analyses statistiques; Pr Tobias Kurth pour le questionnaire i-Share; Dr Françoise Radat pour les informations cliniques sur la migraine.

Conflits d'intérêts : Aucun conflit à déclarer 


\section{P027}

L'acceptabilité d'un repas est affectée par la variation de sa composition par rapport au repas précédent.

L. Boros ${ }^{1}$, F. Gaudfernau ${ }^{1}$, R. Giraudet ${ }^{1}$, S. Raoul ${ }^{1}$, N. Darcel ${ }^{1}$, O. Davidenko ${ }^{1, *}$

${ }^{1}$ UMR PNCA, AgroParisTech, INRA, Université Paris-Saclay, Paris, France

Introduction et but de l'étude : L'acceptabilité d'un repas a été liée à sa composition : un repas varié diminue la sensation de satiété senso-spécifique, ce qui conduit à la consommation énergétique plus élevée comparé à un repas composé d'un seul aliment. Mais l'effet de variété pourrait aussi perdurer d'un repas à l'autre. Cette étude s'est intéressée à l'effet que la variété inter-repas, c'est-à-dire la variation de composition de deux repas consécutifs, pourrait avoir sur leur acceptabilité.

Matériel et méthodes : 345 volontaires ( 237 femmes) qui ont déclaré suivre un régime omnivore ont répondu à un questionnaire en ligne. Le questionnaire comportait 32 questions, chacune présentant deux images de repas. Les volontaires devaient imaginer avoir consommé le premier repas, et indiquer s'ils accepteraient de consommer le second au repas suivant. Chaque repas comportait trois éléments : une source de protéines, un accompagnement et un dessert. La variété inter-repas se mesurait comme le nombre d'éléments différents au sein du couple repas (0 à 3). Ces variations se faisaient soit au sein d'un même groupe alimentaire (ex: saumon et cabillot), soit en changeant de groupe alimentaire (ex: frites et haricots verts). Les volontaires renseignaient par ailleurs leur âge, sexe, IMC, le questionnaire VARSEEK, et leur appréciation pour les différents repas présentés. L'effet de ces facteurs (nombre de variations entre repas, nature d'élément changé, type de variation, caractéristiques du volontaire) sur l'acceptabilité du dîner a été évaluée par régression binomiale.

Résultats et Analyse statistique : La variété inter-repas était positivement associée à la proportion de volontaires qui acceptaient de consommer le dîner $(+31,4 \%$ pour les questions avec 3 variations relativement à 0 variations; $p<0,0001)$. Les changements de groupe alimentaire favorisaient l'acceptabilité du dîner comparé aux variations intra-groupe alimentaire $(p=0,015)$. Les dîners où seul le dessert variait par rapport au déjeuner étaient moins bien acceptés qu'en cas de variation de la source de protéines ou d'accompagnement $(49,9 \%$ d'acceptation contre $62,3 \%$ et $59,7 \%$, respectivement ; $p<0,0001)$. Une meilleure préférence pour le dîner améliorait l'acceptabilité de ce dernier $(p<0,0001)$. Par ailleurs, l'acceptabilité des repas proposés était meilleure chez les hommes (70,9\% contre $57,3 \%$ chez les femmes; $p<0,0001)$ et les consommateurs plus jeunes $(p<0,0001)$.

Conclusion : Les résultats indiquent que la variation de composition entre deux repas consécutifs, quantitative (nombre de variations) et qualitative (changement ou non de groupe alimentaire) pourrait moduler l'acceptabilité d'un repas. Notre méthode pourrait être élargie sur un ensemble de repas plus diversifiés, ou sur une séquence de prises alimentaires plus longue. Par cette étude, nous montrons que la réflexion sur l'acceptabilité des aliments devrait prendre en compte le régime de l'individu au-delà d'un seul repas.

Conflits d'intérêts : Aucun conflit à déclarer 
Le vieillissement et les paramètres physiologiques oraux impactent la libération in vivo d'arômes pendant la consommation d'un aliment

P. Jeltsch ${ }^{1}$, A. Laguerre ${ }^{1}$, C. Septier ${ }^{1}$, H. Labouré ${ }^{1}$, G. Feron ${ }^{1, *}$

${ }^{1}$ Centre des Sciences du Goût et de I'Alimentation, AgroSup Dijon, CNRS, INRA, Univ. Bourgogne Franche-Comté, Dijon, France

Introduction et but de l'étude : Chez les séniors, le vieillissement s'accompagne d'une perte de sensibilité sensorielle et d'une diminution de la prise alimentaire (Schwartz et al. (2018), IFSET, 46:91-106). Pour une population d'âge moyen, des différences interindividuelles de libération d'arômes dans la cavité nasale expliqueraient des régulations de prise alimentaire et de satiété, de par les variations d'intensité et durée de stimulation sensorielle (Ruijshoop et al. (2009), JAFC, 57(21): 9888-9894). Dans ce contexte, l'objectif de cette étude est d'étudier ce mécanisme de libération chez le sujet âgé lors de la consommation d'un aliment modèle.

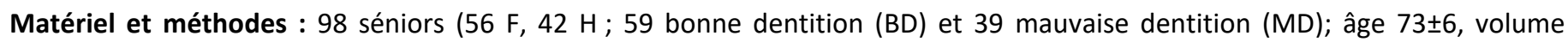
oral $=37,1 \pm 13,8 \mathrm{~mL}$, viscosité salivaire $\mathrm{v}=1,1 \pm 0,5 \mathrm{mPa} . \mathrm{s}$, flux salivaire $\mathrm{FS}=1,7 \pm 1 \mathrm{~mL} / \mathrm{min}$, force langue à déglutition $=46,7 \pm 13,2 \mathrm{kPa}$ ) ont consommé une pastille de menthe selon un protocole (i) libre mesurant le nombre de déglutitions, la quantité d'arôme libérée (menthone) et de pastille consommée (\%pa), (ii) imposé visant à caractériser la libération selon des mouvements oraux fixés (déglutition et mastication). Les mesures de libération ont été faites par analyse des effluves nasales par spectrométrie de masse à ionisation chimique à pression atmosphérique, puis traitées par ANOVA et ANCOVA. Les résultats du protocole imposé ont été

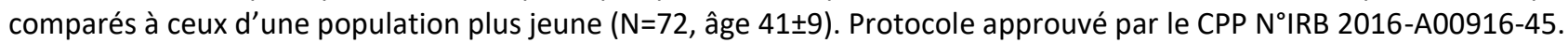

Résultats et Analyse statistique : Le protocole imposé montre que $84 \%$ des séniors libèrent à tous les évènements oraux, contre $54 \%$ chez des adultes d'âge moyen ( $\left.k \mathrm{hi}^{2}=48,25, \mathrm{p}<0,0001\right)$.

Pour le protocole libre, la quantité d'arômes libérée $s^{\prime}$ explique par le \%pa $(F=44,9, p<0,0001)$ et le nombre de déglutition ( $F=19$, $p<0,0001)$. De plus, on observe une interaction entre la dentition et \%pa $\left(F=9,2, p<0,01, \beta_{B D}=0,63\right)$. Le groupe $B D(F S=1,8 \pm 1$ $\mathrm{mL} / \mathrm{min}, \mathrm{v}=1,1 \pm 0,2 \mathrm{mPa} . \mathrm{s})$ libère plus de menthone pour les \%pa élevés que le groupe $\mathrm{MD}(\mathrm{FS}=1,4 \pm 0,9 \mathrm{~mL} / \mathrm{min}, \mathrm{v}=1,3 \pm 0,7 \mathrm{mPa} . \mathrm{s})$ avec FS et $v$ significativement différents entre $B D$ et $M D\left(t_{F s}=1,92, p_{F s}=0,057 ; t_{v}=-2,04, p_{v}=0,043\right)$. Ainsi, un FS élevé et une $v$ faible (groupe $\mathrm{BD}$ ) induiraient plus de dégradation de pastille, donc plus de libération. Une viscosité plus élevé (groupe MD) entrainerait une interaction plus forte de la menthone avec la salive et ainsi moins de libération.

Conclusion : Par rapport à un sujet plus jeune, le sujet âgé libère les arômes à tous les évènements oraux, donc de façon quasicontinue montrant ainsi une difficulté à contrôler, via le velum, le passage des volatils de la cavité orale à la cavité nasale. On peut donc supposer, l'existence d'un mécanisme adaptatif lié à une stimulation sensorielle excessive en condition de consommation. Ceci entrainerait une altération de la sensibilité sensorielle et donc du phénomène de rassasiement sensoriel avec un changement de prise alimentaire. Enfin, cette différence de libération dépend de l'état bucco-dentaire et de la physiologie orale du sujet âgé. La suite de ce travail visera à mesurer la prise alimentaire en la couplant à la libération in vivo d'arômes et en tenant compte de la santé orale.

Remerciements : Projet financé par l'ANR (AlimaSSenS ANR-14-CE20-0003-01)

Conflits d'intérêts : Aucun conflit à déclarer 
Introduction et but de l'étude : Une prise de poids est fréquemment observée chez les patients hospitalisés en psychiatrie, notamment chez ceux atteints de troubles dépressifs ou bipolaires. Le but de cette étude préliminaire est de comprendre les déterminants qui peuvent influencer la prise de poids chez ces patients en s'appuyant sur la population des malades hospitalisés à la clinique du Littoral.

Matériel et méthodes : Deux études ont été réalisées pour comprendre les déterminants de la prise de poids durant l'hospitalisation: une étude rétrospective cas-témoin avec l'analyse des dossiers électroniques des patients dépressifs ou bipolaires ( $n=207$ ) et une étude prospective observationnelle avec l'analyse des dossiers des patients dépressifs et l'administration d'un questionnaire à T0 (entrée) en entretien et d'un questionnaire à T1 (sortie) en auto-administration avec une durée moyenne d'hospitalisation de 24,8 jours $( \pm 6,3)(n=20)$. Une troisième étude transversale a été réalisée pour comprendre les déterminants de la prise de poids des patients dépressifs sous psychotrope accueillis à l'hôpital de jour avec l'étude des dossiers électroniques et l'administration d'un questionnaire en entretien $(n=40)$. Les statistiques descriptives et analytiques (corrélation de rang de Spearman, régression logistique binaire) entre les différents paramètres ont été réalisées sur SPSS statistics 17.0.

Résultats et Analyse statistique : La moyenne d'âge des patients est de 49,3 ans $( \pm 10,7)$. La variation moyenne du poids pendant l'hospitalisation d'après l'étude rétrospective est de $+0,5 \mathrm{~kg}( \pm 1,98)$ [minimum : $-5,4 \mathrm{~kg}$; maximum : $+8,4 \mathrm{~kg}$ ]. La prise de poids moyenne des patients accueillis en hôpital de jour depuis le début de leur traitement psychotrope $($ durée moyenne $=7,8$ ans $\pm 7,8)$ est de $17,6 \mathrm{~kg}( \pm 10,01)$. D’après ces études, les principaux traitements psychotropes associés à la prise de poids appartiennent à la famille des imipraminiques (antidépresseurs tricycliques), des antipsychotiques classiques et des antipsychotiques atypiques. Les psychotropes sont significativement associés à une modification du comportement alimentaire $\left(r_{s}=0,37\right)$ qui est à son tour significativement associée à une prise de poids $\left(r_{s}=0,40\right)$. D'autres facteurs sont également associés significativement à la prise de poids comme le profil socio-économique (difficultés financières, peu de contacts extérieurs, bas niveau d'éducation), la polymédication et l'hygiène de vie (sédentarité, tabac, sommeil). Une personne qui constate une modification de son comportement alimentaire sous psychotrope, qui un a un profil socio-économique défavorisé, qui fume, qui est sédentaire et qui dort peu, a 35 fois plus de risque $(p=0,007)$ de prendre du poids sous psychotrope.

Conclusion : Cette étude préliminaire confirme l'interaction complexe entre certains psychotropes et une modification du comportement alimentaire, en agissant sur des neurorécepteurs régulant la prise alimentaire et l'appétit. Ces résultats ont permis d'entrevoir de nouvelles perspectives comme l'étude de l'effet synergique des différents déterminants et la possibilité de créer un outil de dépistage innovant de la prise de poids des patients dépressifs ou bipolaires.

Conflits d'intérêts : Aucun conflit à déclarer 

L. Thiriet ${ }^{1}$, P. Fernandez ${ }^{1}$, E. Castagna ${ }^{2}$, L. Depezay ${ }^{2, *}$

${ }^{1}$ Centre de recherche, Institut de Tourisme et d'Hôtellerie du Québec, Montréal, Canada, ${ }^{2}$ Fondation Louis Bonduelle, Villeneuve d'Ascq, France

Introduction et but de l'étude : La capacité d'un enfant à cuisiner se traduit globalement par l'acquisition de compétences théoriques, de compétences pratiques, et de compétences transversales. Les compétences s'acquièrent notamment lors d'expériences d'apprentissage actif où l'enfant cuisine lui-même ses aliments comme lors d'un atelier culinaire (Levy \& Auld, 2004). Les enfants développent alors un sentiment de confiance, dit auto-efficacité, en leur capacité de réalisation, déterminant des changements dans leurs attitudes par rapport à la cuisine (Bandura, 1977).

L'objectif de cette étude est de mesurer comment la participation à un atelier culinaire influence à court et à moyen terme, l'acquisition de compétences pratiques (l'utilisation du couteau de cuisine, l'application de méthodes et techniques culinaires et le suivi d'une recette) d'une part et d'autre part les connaissances, le sentiment d'auto-efficacité et les attitudes des enfants par rapport à la cuisine (Cunningham-Sabo \& Lohse, 2014). Aussi, il s'agit de comparer l'acquisition des compétences de deux groupes d'enfants participant à un même atelier dispensé par deux animateurs.

Matériel et méthodes : Deux groupes d'enfants ( $N=27$, âge $12,07 \pm 0,83 ; N=25$, âge $11,0 \pm 0,96)$ ont été sollicités lors d'un atelier culinaire à l'ITHQ (Montréal, Qc). Les trois compétences pratiques ( $1{ }^{\text {ère }}$ partie) et les connaissances, l'auto-efficacité et les attitudes ( $2{ }^{\text {ème }}$ partie) ont été évaluées à travers un même questionnaire auto-administré avant l'atelier (T0), à la fin de l'atelier (T1), et un mois après (T2), respectivement au moyen de questions à choix multiples (1 ${ }^{\text {ère }}$ partie), et d'échelles continues bornées aux extrémités par « pas du tout d'accord » et " extrêmement d'accord " (2 ${ }^{\text {ème }}$ partie).

Résultats et Analyse statistique : L'analyse comparative des résultats pré/post intervention a été effectuée par des tests de Wilcoxon et montre une amélioration des compétences pratiques : suivi d'une recette $(p<0,0001)$, utilisation du couteau $(p=0,003)$ ainsi qu'une amélioration des connaissances $(p<0,0001)$, du sentiment d'auto-efficacité $(p<0,0001)$, et des attitudes $(p<0,0001)$ par rapport à la cuisine.

L'amélioration des compétences est similaire quel que soit le groupe d'enfants, selon le test de Mood appliqué sur les médianes des scores obtenues. La rémanence de l'atelier sur les compétences à moyen terme (T2) sera présentée lors de la conférence.

Conclusion : Pour aller plus loin, de futurs travaux pourraient être envisagés pour valider au moyen d'examens pratiques l'acquisition des compétences sur la base de ce premier travail exploratoire. La finalité de ce projet est de formuler des recommandations sur le contenu d'ateliers culinaires pour optimiser la capacité des enfants à cuisiner.

Références :

Bandura, A. (1977). Social cognitive theory of self-regulation. Organizational Behavior and Human Decision Processes, 50(2), 248287.

Cunningham-Sabo, L., \& Lohse, B. (2014). Impact of a School-Based Cooking Curriculum for Fourth-Grade Students on Attitudes and Behaviors Is Influenced by Gender and Prior Cooking Experience. Journal of Nutrition Education and Behavior, 46(2), 110-120. Levy, J., \& Auld, G. (2004). Cooking Classes Outperform Cooking Demonstrations for College Sophomores. Journal of Nutrition Education and Behavior, 36(4), 197-203.

Conflits d'intérêts : Aucun conflit à déclarer 
P031

Profil de consommation protéique et attitudes vis-à-vis des protéines animales des végétariens, flexitariens et omnivores d'une population représentative française

E. De Gavelle ${ }^{1, *}$, O. Davidenko ${ }^{1}$, H. Fouillet ${ }^{1}$, J. Delarue ${ }^{2}$, N. Darcel ${ }^{1}$, J.-F. Huneau ${ }^{1}$, F. Mariotti $^{1}$

${ }^{1}$ UMR PNCA, AgroParisTech, INRA, Université Paris Saclay, Paris, ${ }^{2}$ UMR GENIAL, AgroParisTech, INRA, Université Paris Saclay, Massy, France

Introduction et but de l'étude : Une transition diététique vers une restructuration de la consommation de sources de protéines a débuté dans les années 2000 dans les pays occidentaux du fait, notamment, de l'émergence de critiques sur les conséquences potentielles des niveaux de consommation actuels de produits carnés sur le bien-être animal, l'environnement et la santé. Plus récemment, cette transition s'est manifestée par une progression des comportements de végétarisme et de flexitarisme. L'objectif de cette étude était de caractériser les différents stades de cette transition diététique, en termes de consommation d'aliments protéiques et d'attitudes vis-à-vis des protéines animales.

Matériel et méthodes : Un questionnaire auto-administré en ligne a été rempli entre avril et mai 2018 par un échantillon représentatif de la population française $(n=2692)$. Les individus ont précisé leur régime alimentaire et rempli un fréquentiel concernant leur consommation alimentaire. Ils ont répondu à des questions sur leurs attitudes et croyances vis-à-vis des protéines animales sur la santé, l'environnement et le bien-être animal (sous forme de scores, un score élevé correspondant à une vision délétère des produits carnés). Nous avons identifié 4 profils de consommateurs en fonction de leur stade dans la transition diététique : les individus se déclarant végétariens ; ceux se déclarant flexitariens ; ceux ayant déclaré envisager de ne consommer de la viande qu'une seule fois par semaine (pro-flexitariens); et ceux sans régime particulier (omnivores).

Résultats et Analyse statistique : Dans la population, 2,0\% des individus se sont déclarés végétariens, 6,5\% flexitariens et 18,7\% pro-flexitariens. La consommation de viande totale diminue en fonction du stade de transition diététique déclaré : $97 \pm 8 \mathrm{~g} / \mathrm{j}$ pour les omnivores, $53 \pm 10 \mathrm{~g} / \mathrm{j}$ pour les pro-flexitariens, $27 \pm 7 \mathrm{~g} / \mathrm{j}$ pour les flexitariens et $8 \pm 2 \mathrm{~g} / \mathrm{j}$ pour les végétariens. Cette gradation est similaire pour tous les types de viande (porc, bœuf et volaille), sauf la charcuterie dont la consommation est similaire entre omnivores et pro-flexitariens et entre flexitariens et végétariens. La consommation d'œufs, de plats préparés, de sandwiches et de fast foods est similaire entre les profils. Les omnivores consomment moins de légumes et légumineuses et plus de pain que les autres profils. Enfin, les flexitariens consomment plus de noix et graines, et moins de pâtes, de lait et de fromage que les autres profils. Les scores liés aux attitudes vis-à-vis des produits carnés sur la santé, l'environnement et le bien-être animal suivent la même tendance que la consommation de viande, avec les scores les plus faibles chez les omnivores puis des scores de plus en plus élevés chez les pro-flexitariens, flexitariens, et végétariens.

Conclusion : La population française présente différents profils de consommation protéique avec, depuis les omnivores jusqu'aux végétariens, une baisse de la consommation de produits carnés et une augmentation de la consommation de légumes et légumineuses. Les attitudes envers la viande en lien avec la santé, l'environnement et le bien-être animal sont associées aux profils de consommation et donc pourraient expliquer le stade des individus au sein de la transition diététique.

Conflits d'intérêts : Aucun conflit à déclarer 
La consommation régulière de snacks de pistaches n'altère ni la régulation pondérale, $\mathrm{ni}$ la composition corporelle M. Fantino ${ }^{1,}{ }^{*}$, F. Bellisle $^{2}$, A. Carughi ${ }^{3}$, M. Feeney ${ }^{4}$

${ }^{1}$ CréaBio Rhône-Alpes, Centre Hospitalier Montgelas, Givors (Lyon), ${ }^{2}$ Nutri Psy Consult, PARIS, France, ${ }^{3}$ APG, FRESNO, Californie, ${ }^{4} A P G$, Los Altos, États-Unis

Introduction et but de l'étude : Le Haut Conseil de la santé publique, dans son avis du 16/02/2014 recommande la consommation quotidienne "d'une petite poignée de fruits à coque ". Cette recommandation s'appuie sur des données scientifiques qui soulignent les bénéfices santé de telles consommations, notamment d'amandes, en terme de qualité du régime alimentaire, gestion pondérale et réduction du risque cardio-métabolique. En revanche, les conséquences de la consommation régulière de pistaches sur le comportement alimentaire, le poids et la composition corporelle ne sont pas clairement établies. L'objectif de cette étude a été d'évaluer les conséquences d'un apport énergétique supplémentaire par la prise ponctuelle ou régulière d'un snack de pistaches sur le comportement alimentaire, le poids, la composition corporelle et les caractéristiques anthropomorphiques.

Matériel et méthodes : La prise alimentaire ad libitum de 60 femmes adultes en bonne santé initialement non consommatrices habituelles de fruits à coque a été mesurée au cours de 4 séances expérimentales de 2 jours consécutifs. Le $1^{\text {er }}$ jour de chaque séance les petits déjeuners et déjeuners pris au laboratoire ont été quantifiés par pesée des aliments ingérés choisis à partir d'un buffet de 49 items. Les aliments du dîner du jour 1 et des repas du jour 2, pris en condition de vie courante, ont été pesés et notés dans un carnet alimentaire. Lors de l'une des 2 premières séances, réalisées à une semaine d'intervalle, les sujets ont reçu chaque jour à $10 \mathrm{~h}$, en cross-over, un snack de $44 \mathrm{~g}$ de pistaches ( $250 \mathrm{kcal})$. Ont été mesurés poids, composition corporelle par DEXA et divers périmètres corporels. Puis les sujets ont été randomisés en un groupe expérimental qui a quotidiennement consommé à $10 \mathrm{~h}$ pendant 12 semaines le même snack de pistaches, et en un groupe contrôle qui a poursuivi ses habitudes alimentaires antérieures sans consommation de fruits à coque. Puis ont été réalisées, à une semaine d'intervalle, les $3^{\text {ème }}$ et $4^{\text {ème }}$ séances de mesures identiques aux séances 1 et 2.

Résultats et Analyse statistique : Aussi bien en début d'étude qu'après la phase de 12 semaines d'habituation, tous les sujets ont compensé le supplément calorique apporté par les snacks par une réduction significative de leur ingéré calorique au cours des repas ad libitum subséquents, associée à une réduction significative de leur motivation alimentaire près la prise du snack. En conséquence tous les sujets avaient en fin d'étude un poids légèrement (mais non significativement) inférieur à leur poids in itial, sans différence significative entre les 2 groupes (contrôles: $-0,37 \pm 0,33 \mathrm{~kg}$, expérimental: $-0,14 \pm 0,26$ ). Les mesures par DEXA n'ont pas mis en évidence de différence de la composition corporelle globale entre le début et la fin de l'étude, ni du contenu en graisse ou de masse maigre des différentes régions anatomiques explorées.

Conclusion : L'apport calorique supplémentaire procuré par la consommation quotidienne régulière pendant 12 semaines d'un snack de pistache $(44 \mathrm{~g}, 250 \mathrm{kcal})$ a été compensé par une réduction équivalente des autres apports énergétiques, sans conséquence délétère sur la régulation pondérale, les caractéristiques anthropométriques ou la composition corporelle.

Conflits d'intérêts : $M$. Fantino a reçu une subvention/soutien de Americano Pistachios Growers (APG), F. Bellisle a reçu une subvention/soutien de APG, A. Carughi est employé(e) de APG, M. Feeney est consultant chez APG 


\section{P033}

Impact des déterminants sensoriels et des informations nutritionnelles sur le comportement alimentaire des consommateurs français à risque de diabète de type 2

A. Saint-Eve ${ }^{1, *}$, E. Boukaïba ${ }^{2}$, B. Maurice ${ }^{1}$, L.-G. Soler ${ }^{3}$, I. Souchon ${ }^{1}$, J. Delarue ${ }^{2}$

${ }^{1}$ UMR GMPA, AgroParisTech, INRA, Université Paris-Saclay, 78850, Thiverval-Grignon, ${ }^{2}$ UMR GENIAL, AgroParisTech, INRA, Université Paris-Saclay, 91300, Massy, ${ }^{3}$ ALISS, INRA, 94205, Ivry-Sur-Seine, France

Introduction et but de l'étude : Le diabète de type 2 (DT2) est responsable d'environ 1,5 million de décès (OMS, 2016) chaque année dans le monde. Le style de vie, et en particulier l'alimentation, est un facteur important impliqué dans cette maladie métabolique chronique. Pour réduire le DT2, il est nécessaire de conduire les consommateurs vers un comportement alimentaire plus sain. Cependant, cela ne peut être réalisé que si les consommateurs, et notamment les populations à risque, sont disposés à se conformer aux recommandations alimentaires. Ainsi, pour rendre les conseils plus efficaces à long terme, nous devons d'abord comprendre le coût hédonique des régimes alimentaires plus sains.

Dans ce contexte, nous avons cherché à évaluer les conséquences du passage à une version plus saine mais moins appréciée d'un produit donné, en mettant l'accent sur les consommateurs à risque de développer le DT2. L'objectif principal de ce projet consistait ainsi à mieux comprendre les arbitrages des consommateurs en situation de repas et l'ajustement des quantités qu'ils consomment. L'impact de recommandations nutritionnelles proposées aux consommateurs lors de ces arbitrages a également été évalué dans ce projet.

Matériel et méthodes : Pour cela, cent vingt volontaires français présentant un risque modéré de développer un DT2 (Findrisc de 1 à 33\%) ont été invités à prendre quatre repas de midi sur une période de deux semaines. Pendant les repas de la première semaine, ils ont été servis avec des pâtes soit classiques (farine de blé blanche), soit complètes (farine complète de blé), puis laissés libres de choisir une sauce tomate, après les avoir goûtées. Deux sauces étaient proposées, l'une étant deux fois plus calorique que l'autre. Les participants ont répété ce scénario au cours de la deuxième semaine, mais des informations nutritionnelles sur les pâtes et la sauce leur ont été fournies. Les participants ont exprimé leur appréciation pour chaque plat du repas et leurs assiettes ont été pesées pour mesurer les quantités de sauce et de pâtes qui ont été consommées dans chaque condition.

Résultats et Analyse statistique : Comme attendu, les résultats montrent que les pâtes complètes étaient légèrement moins appréciées que leurs homologues. Cependant, le type de pâtes n'a pas affecté le choix de la sauce, ni la quantité servie. Paradoxalement, la présence d'informations nutritionnelles a conduit à une plus grande consommation de sauce et de pâtes. De plus, nous avons observé des différences comportementales significatives en fonction du sexe et du niveau de risque du DT2.

Conclusion : Nous avons ainsi pu quantifier le coût hédonique du passage à une version plus saine et mesurer également l'importance du plaisir et des perceptions sensorielles subjectives sur le comportement des consommateurs.

Références : Organisation mondiale de la Santé. (2016). Rapport mondial sur le diabète. Organisation mondiale de la Santé. http://www.who.int/iris/handle/10665/254648.

Conflits d'intérêts : Aucun conflit à déclarer 
Epilepsie et diabète de type 1 : Est- ce que l'hypoglycémie est le seul facteur incriminé ?

N. El Ouarradi ${ }^{1,}{ }^{*}$, G. El Mghari ${ }^{1}$, N. El Ansari ${ }^{1}$

${ }^{1}$ Service d'Endocrinologie diabétologie et maladies métaboliques. Laboratoire PCIM, FMPM, Université Cadi Ayad. CHU Mohamed VI. Marrakech, CHU Errazi, Marrakech, Maroc

Introduction et but de l'étude : L'association diabète de type 1 et de l'épilepsie est connue. Cependant, la physiopathologie de cette association demeure incomprise. L'objectif de cette étude est de décrire l'épilepsie associée au diabète de type 1.

Matériel et méthodes : Nous rapportons le cas de 7 atients diabétiques type 1 épileptiques suivis au service d’Endocrinologie, diabétologie et maladies métaboliques et nutrition de Marrakech.

Résultats et Analyse statistique : Sept patients diabétiques de type 1 ont été étudiés. L'âge de nos patients était compris entre 17 et 21 ans, L'âge de découverte du diabète était compris entre l'âge de 3 ans et de 9 ans. La découverte du diabète précédait la survenue des crises convulsives chez 3 patients .6 patients présentaient une épilepsie généralisée et 1 seul patient présentait une épilepsie partielle. Tous les patients avaient un diabète déséquilibré et présentaient des hypoglycémies fréquentes.

Conclusion : Deux types d'épilepsie sont associés à la survenue d’un diabète de type 1 ; les épilepsies généralisées idiopathiques et les épilepsies temporales. Trois mécanismes peuvent jouer un rôle : l'auto-immunité anti-GAD, l'équilibre glycémique avec la fréquence des hypoglycémies et la génétique. Les auto anticorps anti-GAD retrouvés dans le diabète de type 1 , ont été suggérés pour être le lien entre l'épilepsie et le diabète de type 1. En effet, la GAD est retrouvée dans les cellules béta du pancréas mais c'est aussi une enzyme clef dans la synthèse du GABA qui est le principal neurotransmetteur inhibiteur dans le système nerveux central. Le dysfonctionnement de la GAD cérébrale pourrait perturber la synthèse du GABA et expliquer la plus grande fréquence de l'épilepsie chez les diabétiques.

Conflits d'intérêts : Aucun conflit à déclarer 
Introduction et but de l'étude : L'hyperphagie boulimique est fréquente chez les personnes souffrant d'obésité. En cas de trouble alimentaire, il est nécessaire d'en tenir compte lors du traitement, afin de minimiser le risque de rechute et le phénomène yoyo. L'objectif de notre étude était d'étudier la prévalence des troubles du comportement alimentaire chez un groupe d'obèses tunisiens.

Matériel et méthodes : Il s'agit d'une étude rétrospective menée à l'unité d'obésité de l'institut national de nutrition de Tunis entre Janvier et Mars 2018 à propos de 100 obèses. Le dépistage des troubles du comportement alimentaire s'est basé sur les critères du DSM-IV.

Résultats et Analyse statistique : L'âge moyen de notre population était de $45,08 \pm 13,93$ ans. Nous avons noté une nette prédominance féminine (88\%). L'IMC moyen était à $38,95 \pm 5,72 \mathrm{Kg} / \mathrm{m}^{2}$ avec un tour de taille moyen à $123,88 \pm 13,13 \mathrm{~cm}$. Ils étaient sédentaires dans $60 \%$ des cas. L'apport calorique total moyen était à $3244,25 \pm 1075,08 \mathrm{Kcal} / \mathrm{j}$. Soixante pourcent de nos patients consommaient fréquemment les FastFood. Le grignotage était présent dans $75 \%$ des cas, l'hyperphagie prandiale dans $74 \%$ des cas et les compulsions alimentaires dans $60 \%$ des cas. Quant au Binge syndrome il était présent dans $70 \%$ des cas. Les accès boulimiques étaient notés dans $72 \%$ des cas.

Conclusion : La prévalence des troubles du comportement alimentaire est assez importante parmi nos patients obèses. Psychologues, nutritionnistes et médecins doivent collaborer dans la prévention et le traitement de ces troubles.

Conflits d'intérêts : Aucun conflit à déclarer 


\section{P036}

\section{Etudiants Africains installés en Tunisie, attention à la malbouffe !}

R. Serairi Beji ${ }^{1,}{ }^{*}$, K. Ayed ${ }^{2}$, S. Jameleddine ${ }^{3}$

${ }^{1}$ Département de Nutrition, École supérieure des sciences et techniques de la santé - Laboratoire des plantes aromatiques et médicinales, Technopole Borj Cedia, ${ }^{2}$ Service d'Exploration Fonctionnelle Respiratoire et de Physiothérapie, ${ }^{3}$ Service d'Exploration Fonctionnelle Respiratoire te de Physiothérapie, Hôpital Abderrahmène Mami, Tunis, Tunisie

Introduction et but de l'étude : La Tunisie est un pays qui reçoit beaucoup d'étudiants Africains pour leurs études supérieures. II est certain que quelque soit le pays d'origine, le changement sur le plan alimentaire est très grand. Les légumes, les fruits, la viande et même la volaille sont différents. L'objectif de ce travail est d'approcher le comportement alimentaire d'un groupe de jeunes Africains récemment installés en Tunisie pour leurs études.

Matériel et méthodes : Nous avons préparé un questionnaire comportant des questions fermées et des questions ouvertes. Après l'avoir pré-testé, il a été administré par une nutritionniste qui a interrogé séparément chaque sujet. Le nombre de sujets ayant accepté de participer à l'étude est de 40.

Résultats et Analyse statistique : L'âge moyen de notre population est de $22 \pm 3,8$ ans. La prédominance masculine est nette (69\%). Le BMI moyen est de $29 \pm 3,9 \mathrm{~kg} / \mathrm{m}^{2}$. Les deux tiers des étudiants ont un BMI normal. La ration calorique moyenne est chez les garçons et les filles respectivement de $2629,5 \pm 506,1 \mathrm{kcal} / \mathrm{j}$ et $2322,5 \pm 331,5 \mathrm{kcal} / \mathrm{j}$. L'apport glucidique moyen est de $55,4 \pm 4,4 \%$ de la ration calorique globale avec des extrêmes de $49,6 \%$ et $60,4 \%$. L'apport moyen en saccharose est de $60,5 \pm 27,3$ $\mathrm{g} / \mathrm{j}$ correspondant à $7,9 \pm 3,5 \%$ de l'apport énergétique total. Ceci est conforme aux besoins recommandés chez l'adulte $(5$ à 10 $\%$ AET). Toutefois, $29,7,5 \%$ d'entre eux ont un apport excessif. L'apport lipidique moyen représente 39,8 $\pm 6,1 \%$ de l'apport calorique total et dépasse ainsi les normes recommandées. L'apport moyen en AGS est de $28,5 \pm 11,8 \%$, en AGPI de $23 \pm 11,3 \%$ et en AGMI de 37,6 $\pm 9,7 \%$ de l'apport lipidique quotidien. Les deux tiers des étudiants interrogés ont un apport excessif en AGS. L'apport moyen en AGS ne varie pas en fonction du sexe $(P=0,2)$. Toutefois plus de la moitié des filles ont un apport insuffisant en AGMI (<50\% de l'apport lipidique). L'excès d'apport lipidique intéresse particulièrement les AGS aux dépens des AGPI et des AGMI. L'apport moyen en cholestérol est de $366,8 \pm 188,2 \mathrm{mg} / \mathrm{j}$. L'apport protidique moyen représente 10,6 $\pm 2,3 \%$ de l'apport calorique total. Seuls $19 \%$ des étudiants ont un apport normal. Le rapport protéines animales / protéines végétales est 0,85 $\pm 0,71$ avec un apport excessif en protéines animales chez presque la moitié de la population. L'apport quotidien moyen en fibres alimentaires est de $20,5 \pm 11,7 \mathrm{~g} / \mathrm{j}$.

Conclusion : Notre étude montre que l'alimentation des jeunes étudiants Africains installés en Tunisie est déséquilibrée avec de multiples écarts par rapport aux normes recommandées. Une éducation nutritionnelle ciblée pour cette population est fortement recommandée.

Conflits d'intérêts : Aucun conflit à déclarer 
P038

La composition corporelle prédit la vitesse de course chez plus de $\mathbf{3 0 0 0}$ coureurs à pied

J. Mareschal ${ }^{1,}{ }^{*}$, F. Herrmann ${ }^{2}$, C. Graf ${ }^{3}$, V. Karsegard ${ }^{1}$, N. Achamrah ${ }^{1}$, M. Delsoglio ${ }^{1}$, M. Schindler ${ }^{4}$, C. Pichard $^{1}$, L. Genton ${ }^{1}$

${ }^{1}$ Unité de nutrition, ${ }^{2}$ Service de Gériatrie, ${ }^{3}$ Service de réadaptation médicale, ${ }^{4}$ Service de chirurgie orthopédique et traumatologie de l'appareil locomoteur, Hôpitaux Universitaires de Genève, Genève, Suisse

Introduction et but de l'étude : Le lien entre la composition corporelle et la performance en course à pied n'est pas clair dans la population générale. Cette étude vise à évaluer l'influence de la composition corporelle sur la vitesse de course chez les participants d'une compétition de course à pied.

Matériel et méthodes : Tous les participants d'une compétition de course à pied, ayant lieu en ville de Genève chaque année, qui ont bénéficié d'une première mesure de composition corporelle entre 1999 et 2016 ont été inclus. Les personnes < 16 ans et celles qui n'ont pas terminé la course ont été exclus. La composition corporelle a été mesurée par bio-impédance électrique à 50-kHz avant la course. La masse maigre a été calculée avec la formule de Genève (1) puis soustraite du poids corporel pour déterminer la masse grasse. Les masses grasse et non-grasse ont ensuite été divisées par la taille $(\mathrm{m})$ au carré pour obtenir les indices de masse grasse (FMI) et non-grasse (FFMI). Les distances et temps de course ont été convertis en vitesse (km/h). Les caractéristiques des participants sont exprimées sous forme de moyenne $\pm D S$. Des régressions multiples, ajustées pour l'âge, l'indice de masse corporelle (IMC), l'année de mesure et la distance de course, ont permis de prédire la vitesse de course en fonction des quartiles de FMI et FFMI.

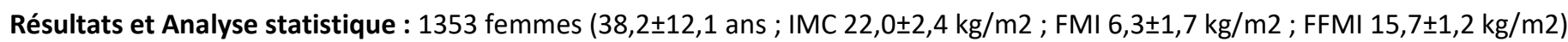

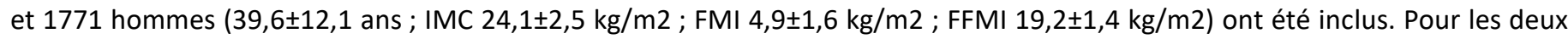
sexes, les régressions multiples ont montré que plus le FMI était élevé, plus la vitesse de course était basse. Chez les femmes, un FFMI dans les quartiles 2 et 3 était associé à une vitesse de course plus élevée en comparaison au quartile 1 . Chez les hommes, un FFMI dans le quartile 4 était associé à une diminution de la vitesse de course.

\begin{tabular}{|c|c|c|c|c|}
\hline & \multicolumn{2}{|c|}{ Femmes $(n=1353)$} & \multicolumn{2}{|c|}{ Hommes $(n=1771)$} \\
\hline & Quartiles & Coefficient (95\%IC), p & Quartiles & Coefficient (95\%IC), p \\
\hline FMI $\left(\mathrm{kg} / \mathrm{m}^{2}\right)$ & $\begin{array}{l}\text { Q1 }:<5,2 \\
\text { Q2 : 5,2-6,1 } \\
\text { Q3 }: 6,2-7,2 \\
\text { Q4 : }>7,2\end{array}$ & $\begin{array}{l}0 \\
-0,81(-1,02 ;-0,60),<0,01 \\
-1,35(-1,56 ;-1,13),<0,01 \\
-2,06(-2,30 ;-1,83),<0,01\end{array}$ & $\begin{array}{l}\text { Q1 }:<3,8 \\
\text { Q2 }: 3,9-4,7 \\
\text { Q3 }: 4,8-5,8 \\
\text { Q4 : }>5.8\end{array}$ & $\begin{array}{l}0 \\
-0,43(-0,66 ;-0,20),<0,01 \\
-1,14(-1,37 ;-0,91),<0,01 \\
-1,93(-2,17 ;-1,68),<0,01\end{array}$ \\
\hline FFMI $\left(\mathrm{kg} / \mathrm{m}^{2}\right)$ & $\begin{array}{l}\text { Q1 }:<15 \\
\text { Q2 : 15-15,6 } \\
\text { Q3 : 15,7-16,4 } \\
\text { Q4 : }>16,4\end{array}$ & $\begin{array}{l}0 \\
0.23(0,01 ; 0,44), 0,04 \\
0.28(0,06 ; 0,50), 0,01 \\
0.19(0,04 ; 0,43), 0,11\end{array}$ & $\begin{array}{l}\text { Q1 : }<18,2 \\
\text { Q2 : 18,2-19,0 } \\
\text { Q3 : 19,1-20,0 } \\
\text { Q4 : >20.0 }\end{array}$ & $\begin{array}{l}0 \\
-0.04(-0,27 ; 0,18), 0,71 \\
-0.21(-0,44 ; 0,02), 0,07 \\
-0.50(-0,74 ;-0,26),<0,01\end{array}$ \\
\hline
\end{tabular}

Conclusion : Le FMI est négativement associé à la vitesse de course chez les deux sexes alors que le FFMI y est positivement associé chez les femmes seulement. Les personnes souhaitant améliorer leur performance en course à pied devraient garder une masse grasse aussi basse que possible.

Références : 1 . Kyle et al, Nutrition, 2001

Conflits d'intérêts : Aucun conflit à déclarer 
Altération du maintien de la masse musculaire et effets immuno-métaboliques induits par un traitement long avec un ligand activateur de PPAR $\beta$

I. Mothe-Satney ${ }^{1}$, V. Guidal ${ }^{2}$, D. Lepousé ${ }^{3}$, S. Le Garf ${ }^{4}$, J. Murdaca ${ }^{1}$, B. Sibille ${ }^{1}$, J. Neels ${ }^{1}$, A.-S. Rousseau ${ }^{15,{ }^{*}}$

${ }^{1}$ UMR/INSERM 1065/C3M, ${ }^{2}$ Université Côte d'Azur, ${ }^{3}$ Université Nice Côte d'Azur, ${ }^{4}$ UMR/INSERM1065/C3M, Université Côte d'Azur, ${ }^{5}$ UMR/INSERM 1065/C3M, Université Nice Côte d'Azur, Nice, France

Introduction et but de l'étude : Les ligands activateurs du peroxisome proliferator activated receptor beta (PPAR $\beta$ ) sont considérés comme des thérapies potentielles du diabète de type 2 du fait de leurs effets " exercice-mimétiques » favorisant la flexibilité du métabolisme vers l'oxydation des acides gras et de leurs effets " anti-inflammatoires ». Toutefois, les effets musculaires et immunitaires en réponse à un traitement long (supérieur à 2 semaines), ainsi que leurs capacités à "mimer" les effets de l'entrainement physique ne sont pas connus. Le but de l'étude a été d'étudier ces effets.

Matériel et méthodes : Nous avons réalisé un protocole de traitement de 6 semaines avec un agoniste de PPAR 3 (GW0742, $n=12$ ) ou son véhicule (DMSO, $n=12$ ) administrés dans la nourriture, chez des souris C57BI/6J. La moitié des souris était entrainée sur tapis roulant. Des analyses par qPCR, western-blots et cytométrie (FACS) ont été réalisées sur le muscle, les organes lymphoïdes et le sang.

Résultats et Analyse statistique : Une ANOVA à deux facteurs (entrainement, traitement) ou des tests non-paramétriques (Kruskall-Wallis) ont été réalisés. L'expression de CPT1a, principal gène-cible de PPAR $\beta$, est augmentée significativement dans le muscle squelettique, les organes lymphoïdes secondaires et le sang. Indépendamment des effets de l'entrainement, le traitement avec le GW0742 diminue d'environ $40 \%$ la masse du tissu adipeux viscéral $(p<0,005)$ mais aussi de $20 \%$ celle du vastus lateralis $(p<0,05)$. La force musculaire maximale (grip-test) est également significativement diminuée $(p<0,0005)$. Ces derniers effets, potentiellement indésirables, nous amènent à rechercher les mécanismes moléculaires sous-jacents. Nos résultats dans le muscle vastus lateralis montrent une altération de la synthèse protéique en réponse au traitement GW0742 caractérisée par une diminution significative de la quantité de protéines substrats de mTOR (S6 et 4EBP1). De plus, la régulation négative de l'activité de mTOR semble être favorisée (augmentation de la phosphorylation de l'AMPK, diminution d'Akt). Le système ubiquitineprotéasome (dégradation protéique) ne semble pas être affecté de manière significative. En revanche, l'expression des gènes impliqués dans la régulation de l'autophagie (BNIP-3, LC3, Gabarap) et de l'apoptose (Bax, Bcl2) est diminuée ( $p<0,005)$. Un changement de profil des cellules T matures $(C D 4+, C D 8+, \alpha \beta$ et $\gamma \delta T)$ n'est pas détectable dans le sang. Le pourcentage de cellules $T$ régulatrices est augmenté significativement avec l'entrainement. Toutefois, seul le traitement GW0742 chez les souris entrainées augmente de manière significative le pourcentage de cellules Foxp3+.

Conclusion : Pris dans leur globalité, nos résultats semblent montrer des effets potentiellement délétères du traitement GW0742 chez la souris normo-pondérée, affectant sa composition corporelle et sa capacité immuno-régulatrice. Mieux caractériser les mécanismes impliqués est nécessaire car les répercussions physiopathologiques des effets du GW0742 à long terme pourraient être importantes.

Remerciements : Agence Française de Lutte contre le Dopage

Conflits d'intérêts : Aucun conflit à déclarer 
Cancer du sein métastatique et masse musculaire : rôle de l’activité physique dans la prévention de la sarcopénie et des toxicités L. Delrieu ${ }^{12,{ }^{*}, \text { A. Martin }}{ }^{3}$, B. Fervers ${ }^{1}$, M. Morelle ${ }^{1}$, O. Febvey-Combes ${ }^{1}$, O. Pérol ${ }^{1}$, D. Freyssenet ${ }^{3}$, P. Bachmann $^{1}$, O. Trédan $^{1}$, F. Pilleul ${ }^{1}$, M. Touillaud ${ }^{1}$, V. Pialoux ${ }^{2}$

${ }^{1}$ Centre Léon Berard, Lyon, ${ }^{2}$ Université Claude Bernard Lyon 1, Université de Lyon, Villeurbanne, ${ }^{3}$ Université Claude Bernard Lyon 1, Université de Lyon, Université Jean Monnet Saint-Etienne, Saint-Etienne, France

Introduction et but de l'étude : La sarcopénie correspond à une diminution du volume et du nombre de fibres musculaires associée à une baisse de la force musculaire et touche entre $11 \%$ et $74 \%$ des patients atteints de cancer. La pratique de l'activité physique (AP) augmenterait la masse maigre et stabiliserait voire corrigerait la sarcopénie. La sarcopénie a aussi été associée à une toxicité sévère de la chimiothérapie. La prise en charge précoce de la sarcopénie est un enjeu clinique important pour limiter la détérioration de l'état de santé des patientes. L'objectif de l'étude était d'évaluer le rôle d'une intervention en AP sur l'évolution de la masse musculaire chez des patientes atteintes d'un cancer du sein métastatique et de tester les associations entre la sarcopénie et les performances physiques, la qualité de vie, la fatigue et la toxicité des traitements.

Matériel et méthodes : Une cohorte de 49 patientes atteintes d'un cancer du sein métastatique a été suivie entre octobre 2016 et janvier 2018. L'intervention de 6 mois était non supervisée et personnalisée en AP, basée sur un objectif de nombre de pas à atteindre. A l'inclusion et à 6 mois, des évaluations anthropométriques, des tests fonctionnels (test de marche de 6 min et force d'extension du quadriceps), et des questionnaires (AP et qualité de vie) ont été réalisés. Les coupes transversales de scanner au niveau de la troisième vertèbre lombaire ont été analysées à l'inclusion, 3 mois et 6 mois pour évaluer la sarcopénie (indice de masse musculaire $<40 \mathrm{~cm}^{2} / \mathrm{m}^{2}$ ) et la qualité musculaire (mauvaise $\mathrm{si}<37,8$ Hounsfield Unit).

Résultats et Analyse statistique : L'évolution des variables a été testée par des analyses de la variance à mesure répétées, les associations par des tests de Mann-Whitney ou de Khi-deux et les corrélations par le coefficient de Spearman. L'âge moyen des patientes était de 55 ans (10) avec un indice de masse corporelle moyen de $26,1 \mathrm{~kg} / \mathrm{m}^{2}(5,8)$ et $96 \%$ des patientes étaient compliantes à l'intervention. A l'inclusion, $51 \%$ des patientes étaient sarcopéniques et $71 \%$ avaient une mauvaise qualité musculaire. Aucune différence n'a été trouvée entre les patientes sarcopéniques et non sarcopéniques concernant les performances aux tests fonctionnels, la fatigue et la qualité de vie mesurés à l'inclusion et à la fin de l'étude. Les patientes ayant une force d'extension du quadriceps élevée à l'inclusion avaient significativement moins de risque d'être sarcopéniques durant l'étude $(p=0,05)$. Le fait d'être sarcopénique à l'inclusion était associé à un risque plus élevé de développer des toxicités sévères $(p=0,02)$.

Conclusion : Cette étude suggère un effet bénéfique de l'AP comme facteur protecteur de risque de sarcopénie et de toxicité. Cependant il est nécessaire de mener des études contrôlées randomisées auprès d'un plus grand effectif pour confirmer ces résultats et déterminer un seuil d'AP qui permettrait de prévenir une sarcopénie.

Remerciements : Ligue contre le cancer, le Cancéropôle Auvergne Rhône-Alpes, Odyssea et Activ'Ra.

Conflits d'intérêts : Aucun conflit à déclarer 
Effets du jeûne du ramadan sur le profil nutritionnel chez des footballeurs amateurs

F. Chiha ${ }^{1}$, H. Djemai ${ }^{23,{ }^{*}}$, P. Noirez ${ }^{23}$, F. Desgorces ${ }^{2}$, Y. Benkara ${ }^{1}$

${ }^{1}$ Laboratoire d'expertise et d'analyse de la performance sportive (LEAPS), Institut STAPS, Constantine 2, Constantine, Algérie,

${ }^{2}$ EA7329 IRMES, INSEP, ${ }^{3}$ UFR STAPS, Université Paris Descartes, Paris, France

Introduction et but de l'étude : Le jeûne du Ramadan représente une période d'absence de tout apport alimentaire et hydrique durant la journée et pendant un mois. Il serait responsable de modifications métaboliques transitoires et de perturbations du cycle sommeil-veille. Les modifications des habitudes alimentaires dans le milieu sportif et en particulier chez les footballeurs posent un grand problème sur la gestion de l'entraînement et le dosage de la charge de l'entraînement. Notre objectif est d'évaluer les effets du jeûne intermittent du Ramadan sur les apports nutritionnels chez des footballeurs amateurs.

Matériel et méthodes : 12 footballeurs masculins amateurs (S) âgés de 19,8 $\pm 2,3$ ans et 9 sujets non sportifs (NS) âgés de 22,8 \pm 5,3 ans en bonne santé ont participé dans cette étude. Deux périodes d'évaluation nutritionnelle ont été effectuées avec un suivi anthropométrique. La masse grasse et masse maigre ont été déterminés par la méthode des plis cutanés. Un questionnaire a été établi afin d'évaluer les apports nutritionnels tant quantitatif que qualitatif sur une semaine. La première évaluation (T1) a eu lieu toute au long de la semaine qui précédait le mois du Ramadan. La seconde (T2) était durant toute la quatrième semaine du mois. Pour cette seconde semaine, le questionnaire a été adapté aux exigences du jeûne du Ramadan (nombre et horaire des prises alimentaires). L'estimation de l'apport énergétique et de sa composition a été réalisée en utilisant le programme Bilnut (version 2.0, 1990).

Résultats et Analyse statistique : Nos résultats montrent que le poids corporel et la masse grasse ont significativement diminué au cours du Ramadan chez les sujets $S$ et NS ( $p<0,01, p<0,05$; respectivement). En revanche, la masse maigre n'est pas modifiée. L'apport nutritionnel a été modifié durant la période du jeûne. Une augmentation significative du taux des lipides dans l'apport alimentaire (\%AET) chez les deux groupes $(p<0,05)$, accompagnée d'une diminution du taux protéique chez les $S(p<0,05)$. Une diminution d'apport calorique a été observée chez les NS ( $2875 \pm 358$ vs. $2483 \pm 420 \mathrm{kcal} ; p<0,001)$. Comparés aux NS, les $\mathrm{S}$ avaient un apport journalier en protéine plus bas à T1 (S:69,35 $\pm 9,38$ vs. NS: 80,21 $\pm 13,68 \mathrm{~g} ; p<0,05)$ et qui a diminué significativement entre T1 et T2 chez ces sujets NS (T1 : 80,21 $\pm 13,68$ vs.T2 : 70,05 $\pm 9,42 \mathrm{~g} ; p<0,01)$. Cette diminution en protéine chez les NS était accompagnée d'une diminution de l'apport journalier en glucide $(435,48 \pm 58,31$ vs. $379,36 \pm 71,35 \mathrm{~g} ; p<0,01)$, ainsi d'une réduction en apport hydrique $(p<0,001)$.

Conclusion : Nos résultats montrent que la pratique du jeûne du Ramadan a un effet sur la composition corporelle par une diminution de la masse grasse et du poids corporel. Le jeûne intermittent affecte les habitudes nutritionnelles des footballeurs mais d'une façons moins importante que les NS et leur alimentation est orientée vers des apports plus gras.

Conflits d'intérêts : Aucun conflit à déclarer 
${ }^{1}$ Laboratoire AME2P, Université Clermont Auvergne, Aubière, ${ }^{2}$ INRA, UMR1019, UNH, Université Clermont Auvergne, ClermontFerrand, France

Introduction et but de l'étude : L'activité de pédalage aquatique est souvent mise en avant pour ses effets positifs concernant la perte de poids. Cependant, aucune évidence scientifique ne justifie encore cette affirmation. Quelques travaux se sont intéressés aux réponses cardio-ventilatoires de cette activité (Bréchat 1999, Garzon 2015) mais aucune ne s'est intéressée aux aspects énergétiques. De plus, nombreux sont les travaux ayant souligné l'importance d'étudier à la fois le versant de la dépense énergétique liée à l'activité mais également la compensation alimentaire qu'elle peut engendrer (Blundell et al 2015). Ainsi, l'objectif de notre étude était de comparer les réponses énergétiques (apports et dépenses) lors d'un exercice de pédalage réalisé en milieu aquatique avec ou sans jets hydromassants.

Matériel et méthodes : Huit femmes entre 21 et 30 ans ont réalisé 3 visites expérimentales : i) une session contrôle (sans exercice) (CONT) ; ii) une session de pédalage aquatique de 40 minutes à $75 \%$ de la fréquence cardiaque maximale sans jets hydromassants (JETS-); iii) une session de pédalage aquatique de 40 minutes à $75 \%$ de la fréquence cardiaque maximale avec jets hydromassants (JETS+). Chaque session s'est déroulée sur une matinée avec un petit déjeuner calibré, un exercice de pédalage (ou repos) trois heures après le petit déjeuner, un buffet ad libitum une demie heure après la session expérimentale. Lors de la session contrôle (CONT), les participantes sont restées assises au calme pendant 40 minutes. La dépense énergétique durant les 40 minutes de repos et les deux exercices de pédalage a été évaluée par calorimétrie indirecte. La composition corporelle (BIA) ainsi que le niveau d'activité physique (IPAQ) des participantes ont été évalués.

Résultats et Analyse statistique : La dépense énergétique lors de la session CONT était significativement inférieure à celle des sessions d'exercice $(p<0.05)$. L'utilisation de jets hydromassants latéraux au cours d'une session de pédalage augmente significativement la dépense énergétique pour une même intensité de travail cardiaque $(p<0.05)$. La prise énergétique relative au repas ad libitum est significativement inférieure suite aux sessions d'exercice en comparaison à la session contrôle $(p<0.05)$.

Conclusion : Les résultats de cette étude montrent que le pédalage en milieu aquatique est propice pour favoriser le déficit énergétique. L'utilisation de jets hydromassants semble être un paramètre favorisant d'autant plus ce déficit. Ces résultats confirment l'intérêt, de mieux identifier les modalités d'activité physique permettant d'agir sur les deux versants de la balance énergétique.

Références : Bréchat, P.H., Wolf, J.P., Simon-Rigaud, M.L., Brechat, N., Kantelip, J.P., Berthelay, S. and Regnard, J. (1999) Influence of immersion on respiratory requirements during 30-min cycling exercise. European Respiratory Journal 13, 860-866.

Blundell, J. E., Gibbons, C., Caudwell, P., Finlayson, G., \& Hopkins, M. (2015). Appetite control and energy balance: impact of exercise. Obes Rev, 16 Suppl 1, 67-76.

Conflits d'intérêts : Aucun conflit à déclarer 
L'activité physique et le comportement sédentaire chez une population représentative de la ville Casablanca, Maroc : étude GPAQ

R. Msaad ${ }^{1}$, K. Mohtadi ${ }^{1}$, N. Benalioua ${ }^{1}$, Y. Elkardi ${ }^{1}$, R. Essadik ${ }^{1}$, H. Lebrazi ${ }^{1}$, A. Jafri ${ }^{2}$, A. Derouiche ${ }^{1}$, A. Kettani $^{1,}{ }^{*}$, H. Taki $^{1}$, R. Saile ${ }^{1}$

${ }^{1}$ Laboratoire Biologie et Santé (URAC 34), Faculté des Sciences Ben M'Sik, Université Hassan II de Casablanca, ${ }^{2}$ Université Mohammed VI des Sciences de la Santé, Faculté des Sciences et Techniques de Santé, Casablanca, Maroc

Introduction et but de l'étude : Il existe des preuves solides que l'activité physique (AP) est associée à un certain nombre de maladies chroniques comme le cancer et les maladies cardiovasculaires. De ce fait, l'évaluation du niveau d'activité physique et du comportement sédentaire permet d'indiquer l'état de santé. Le but de notre étude est de décrire la fréquence l'AP et du comportement sédentaire auprès d'une population casablancaise représentative.

Matériel et méthodes : Une enquête transversale a été menée au niveau de la ville de Casablanca en 2017. Un échantillonnage en grappes a été utilisé en se basant sur le recensement national de 2014 pour sélectionner les participants. Les données sur l'activité physique ont été recueillies à l'aide du questionnaire GPAQ qui contient 16 questions sur la fréquence (jours) et la durée (minutes/heures) d'intensité modérée et vigoureuse de l'AP dans trois domaines (travail, transport et loisirs) et sur la sédentarité; les questions sont posées en termes de comportement dans une semaine typique ou habituelle. L'analyse a été effectuée à l'aide du logiciel statistique SPSS 20.

Résultats et Analyse statistique : L'étude a concerné 730 sujets dont 379 hommes et 461 femmes. L'âge moyen est de $39,07 \pm$ 15,46 ans. La fréquence du niveau d'activité physique élevée était de $34,24 \%$ (femmes $38,8 \%$, hommes $61,2 \%, p<0,001$ ) et elle diminue avec l'âge $(p<0,001)$, alors que la fréquence du niveau d'activité physique modérée était de $40,54 \%$. La fréquence du niveau d'activité physique faible était de $25,20 \%$ (femmes $59,8 \%$, hommes $40,2 \%, p<0,001$ ). Le pourcentage des sujets ne pratiquant aucune activité physique dans leur travail, pour leurs loisirs ou pour se déplacer respectivement était $53,28 \%, 64,93 \%$ et $24,24 \%$. La durée médiane de l'activité physique totale quotidienne était de 72,85 minutes (femmes 45,71 min, hommes 94,28 $\mathrm{min}$ ). La durée moyenne du comportement sédentaire quotidien était 227,11 $\pm 157,9$ (femmes 235,9 $\pm 156,05$ min, hommes $219,09 \pm 159,51 \mathrm{~min})$.

Conclusion : Notre étude a révélé une inactivité physique très répandue chez notre population, ainsi qu'elle est influencée par le sexe et l'âge, cependant son suivi est indispensable pour l'élaboration de politiques publiques dans le domaine de la santé et de la prévention des maladies chroniques.

Conflits d'intérêts : Aucun conflit à déclarer 


\section{Dénutrition hospitalière - CLAN - Nutrition à domicile}

P044

Nutrition enterale : une bonne transition à la norme enfit ?

C. Chabut ${ }^{1,}{ }^{*}$, R. Gervais ${ }^{1}$, G. Foy ${ }^{1}$, X. Deviot ${ }^{1}$

${ }^{1}$ Pharmacie, Centre hospitalier Delafontaine, Saint Denis, France

Introduction et but de l'étude : L'erreur d'administration par injection parentérale au lieu de la voie orale ou entérale fait partie des 12 " never events", évènements indésirables pouvant être évités. Après une longue période de transition au sein de notre établissement, la mise en place de la norme ENFit (ISO 80369-3) touche à sa fin. La multiplication des références et adaptateurs ayant perturbé les pratiques en nutrition entérale (NE), nous avons donc souhaité établir un état des lieux de ces pratiques et des connaissances des services en NE.

Matériel et méthodes : Nous avons réalisé un audit dans 14 services consommateurs de dispositifs médicaux (DM) de NE (4 services pédiatrique, 2 services gériatriques, 8 services de médecine aiguë) à l'aide d'un questionnaire à 15 items concernant leurs connaissances, leurs pratiques de montage et observation des DM en stock dans les services de soins (SS).

Résultats et Analyse statistique : Dans les 14 services audités, un questionnaire a été complété par chaque équipe. Nous avons constaté une non-connaissance globale de la norme ENFit. Dans 9 services les agents n'était pas informés de sa mise en application expliquant une incompréhension quant au changement des références ainsi qu'une mauvaise connaissance des recommandations de NE, exposant ainsi le patient à des risques d'erreurs d'administration. Nous avons observé un mélange d'une dizaine de références avec des connectiques incompatibles (non normées et ENFit) dans 10 services entrainant des problèmes de connexion entre tubulure et sonde et des montages complexifiés avec l'ajout d'adaptateurs. Dans les services de pédiatrie, un autre type de connectique sécurisée incompatible est utilisé. Une note de service a été rédigée pour que les équipes soignantes prennent connaissance des DM à disposition, afin de supprimer des références et d'harmoniser leurs dotations. Nous avons recensé des mésusages dans 9 services. Notamment 6 utilisaient des sondes de Salem pour la nutrition, principalement en réanimation et en neurologie. Egalement, la vérification de la pose des sondes par radio n'étaient pas réalisée dans 6 services, principalement en gériatrie (qui se trouve dans une structure différente) et en neurologie (vérification au stéthoscope). Cela s'explique aussi par du matériel inadapté, une mauvaise gestion des dotations et de la transmission de l'information entre les services. Suite à cet audit, nous avons réalisé un support de formation adapté que nous avons présenté dans chaque SS. Nous avons défini la norme ENFit, présenté les DM ENFIT disponibles, rappelé les recommandations et bonnes pratiques en NE ainsi que les conséquences des différents mésusages des DM de NE.

Conclusion : Cet audit met en évidence l'importance de la connaissance du matériel dans la pratique ainsi que la bonne communication entre les services de soins et la pharmacie. La formation s'est avérée nécessaire pour sensibiliser les soignants aux risques qu'entrainent un usage détourné des DM de NE et à l'importance d'un montage sécurisé avec le matériel adapté. Suite à cela, nous avons fait remonter ces informations au CLAN (Comité de Liaison Alimentation Nutrition), afin de sensibiliser le personnel médical et de trouver des solutions pour améliorer les pratiques sur le long terme.

Conflits d'intérêts : Aucun conflit à déclarer 


\section{P045}

Le changement d'offre du petit déjeuner améliore la satisfaction du patient

C. Meyer ${ }^{1, *}$, L. Joly ${ }^{1}$, A. Gaude ${ }^{2}$, S. Langlais ${ }^{2}$, C. Lirot ${ }^{2}$, E. Fontaine ${ }^{12}$

${ }^{1}$ Comité de Liaison Alimentation Nutrition, ${ }^{2}$ Equipe Mobile de Pesage, CHU Grenoble Alpes, Grenoble cedex 9, France

Introduction et but de l'étude : Le petit déjeuner semble être un repas particulièrement bien consommé à l'hôpital. En 2010, l'audit conduit dans notre établissement le confirmait : $79 \%$ des patients interrogés consommaient entièrement leur petit déjeuner. Néanmoins en 2008, les quantités servies à ce repas étaient jugées insuffisantes par $18 \%$ des patients, et près de $80 \%$ d'entre eux suggéraient la présence d'une composante supplémentaire. Ces constats ont mis en évidence le besoin d'améliorer ce repas, dans un contexte connu de difficultés de couverture du besoin nutritionnel des patients hospitalisés.

Matériel et méthodes : L'offre alimentaire du petit déjeuner a été améliorée en janvier 2015 avec l'ajout d'un yaourt au lait entier et d'un jus de fruits amenant les apports de ce repas à $500 \mathrm{kcal}$ et $10 \mathrm{~g}$ de protéines. Une campagne d'information pour les patients a été mise en œuvre conjointement : les correspondants CLAN ont été informés et une affiche a été distribuée dans tous les services de soins pour mise en place dans chaque chambre. En 2018, un nouvel audit a été réalisé par le CLAN afin d'évaluer l'impact des actions réalisées sur l'information du patient de la nouvelle offre ainsi que sa satisfaction, et de dégager de nouvelles pistes d'amélioration. Les patients ont été interrogés à l'aide d'un questionnaire entre janvier et mai 2018.

Résultats et Analyse statistique : 93\% des patients interrogés consomment leur petit déjeuner $(n=319)$.

Comparaison audits 2008 et 2018 :

\begin{tabular}{|l|c|c|c|c|c|c|}
\hline $\begin{array}{l}\text { Population : } \\
\text { patients } \\
\text { adultes MCO }\end{array}$ & $\begin{array}{l}\text { Echantillon } \\
\text { total }(\mathrm{N})\end{array}$ & $\begin{array}{l}\text { Echantillon exploité pour la } \\
\text { question de la satisfaction vis- } \\
\text { à-vis des quantités servies }(\mathrm{n})\end{array}$ & $\begin{array}{l}\text { \% de patients } \\
\text { jugeant les } \\
\text { quantités } \\
\text { satisfaisantes }\end{array}$ & $\begin{array}{l}\text { \% de patients } \\
\text { jugeant les } \\
\text { quantités } \\
\text { insatisfaisantes }\end{array}$ & $\begin{array}{l}\% \text { de patients } \\
\text { jugeant les } \\
\text { quantités } \\
\text { excessives }\end{array}$ & $\begin{array}{l}\% \text { de } \\
\text { patients } \\
\text { sans } \\
\text { opinion }\end{array}$ \\
\hline Audit 2008 & $\mathrm{N}=173$ & $\mathrm{n}=127$ & $76 \%$ & $18 \%$ & $5 \%$ & $1 \%$ \\
\hline Audit 2018 & $\mathrm{N}=319$ & $\mathrm{n}=292$ & $90 \%$ & $4 \%$ & $1 \%$ & $5 \%$ \\
\hline
\end{tabular}

$90 \%$ des patients jugent les quantités satisfaisantes en 2018 contre $76 \%$ en 2008 (différence significative, $p<0.001$, test du khi ${ }^{2}$ ). L'affiche est présente dans seulement $31 \%$ des chambres, et est citée à $8 \%$ comme mode d'information de l'offre du petit déjeuner. Le vecteur principal de l'information reste le personnel soignant (cité par $77 \%$ des patients interrogés sur la question). Conclusion : L'amélioration de la satisfaction des patients concernant le petit déjeuner semble concorder d'une part avec l'offre d'un petit déjeuner complet et d'autre part, avec la campagne d'information mise en œuvre. L'information du patient sur cette nouvelle offre reste cependant perfectible (diffusion de l'information sur TV, nouvelle campagne de sensibilisation et formation du personnel). Des actions sont également à envisager du côté de la gestion des stocks des produits de petit déjeuner (ruptures rencontrées). Enfin, ajouter de nouveaux aliments à haute densité énergétique (aliments cités par les patients) reste une piste à explorer pour améliorer encore les apports caloriques et protéiques des patients hospitalisés.

On peut regretter l'impossibilité de comparer les apports nutritionnels du petit déjeuner entre 2008 et 2018 , ces données n'ayant pas été recueillies au départ. Une étude de ce type semblerait intéressante à mener dans le cadre d'une nouvelle amélioration de l'offre du petit déjeuner.

Conflits d'intérêts : Aucun conflit à déclarer 
P046

Prévalence de la dénutrition en consultation plaies et cicatrisation au CHU de Montpellier

C. Goruc ${ }^{1, *}$, L. Teot ${ }^{2}$, S. Pujol ${ }^{2}$ et UTN / Consultation plaies et cicatrisation

${ }^{1}$ Unité Transversale Nutrition (UTN), ${ }^{2}$ Consultation plaies et cicatrisation, CHU Montpellier, Montpellier, France

Introduction et but de l'étude : L'unité de "Consultation Plaies et Cicatrisation" du Dr TEOT du CHU de Montpellier accueille des patients présentant des plaies complexes et diverses avec des retards/difficultés de cicatrisation. La cicatrisation est un processus complexe au cours duquel les facteurs nutritionnels tiennent une place importante. L'évaluation de l'état nutritionnel reste cependant difficile chez ces patients suivis en ambulatoire avec peu d'études sur la prévalence de la dénutrition. Ce constat a motivé la mobilisation de l'Unité Transversale de Nutrition (UTN) afin d'évaluer la prévalence de la dénutrition chez ces patients suivis pour plaies chroniques et proposer des outils adaptés tant sur le dépistage que sur les prises en charges nutritionnelles.

Matériel et méthodes : Etude observationnelle prospective quantitative réalisée du 11/2017 au 12/2017 auprès des patients venant en consultation "plaies et cicatrisation". Les paramètres étudiés pour dépister la dénutrition et le risque de dénutrition sont ceux définis par I'HAS/ANSES et l'ESPEN : taille, poids, perte de poids à $1 / 6$ mois, Mini Nutritional Assessment, Nutritional Risk Screening, Echelle de Prise Alimentaire. Le grade nutritionnel péri-opératoire (GN) a également été pris en compte. L'évaluation a été réalisée par un diététicien de I'UTN et l'infirmière impliquée lors de la consultation.

Résultats et Analyse statistique : 102 patients évalués : $30 \%$ des patients sont dénutris et $23 \%$ sont à risque de dénutrition ce qui devrait impliquer une prise en charge nutritionnelle (PEC) pour 53\% des patients consultant dans l'unité de plaies et cicatrisation. $87 \%$ des patients proviennent du domicile ce qui invite à une réflexion sur le développement d'un maillage Ville/Hôpital (réseau, télémédecine, dossier partagé...) impliquant tous les acteurs du dépistage, de la prise en charge et du suivi nutritionnel (chirurgiens, médecins, IDE libéraux et diététiciens).

Spécificités des patients nécessitant un recours chirurgical post-consultation : $20 \%$ des patients sont orientés vers une chirurgie (greffe de peau artificielle et autologue, parage de plaie, VAC, lambeau, pontage...). Il est nécessaire lors de chirurgie programmée d'évaluer le GN qui tient compte de l'état nutritionnel, des facteurs de risque de dénutrition péri-opératoire (dont le type d'acte chirurgical en lui-même). $86 \%$ des patients nécessitant un recours chirurgical sont GN2/GN3 ce qui nécessiteraient une PEC nutritionnelle adaptée en pré-opératoire et post-opératoire. L'évaluation nutritionnelle semble indispensable. L'objectif étant d'optimiser la cicatrisation postopératoire et d'en prévenir les complications.

Etat nutritionnel par type de plaies : les patients présentant une escarre sont ceux présentant le plus fort taux de dénutrition (47\%). Cette population est la plus dénutrie mais également la plus difficile à évaluer. Il semble important de la cibler précocement.

Conclusion : Cette étude confirme la forte prévalence de la dénutrition chez les patients suivi en ambulatoire en consultation "Plaies et cicatrisation". Il apparaît ainsi indispensable d'intégrer au parcours de soins de ces patients une stratégie de dépistage précoce et de prise en charge nutritionnelle de la dénutrition.

Conflits d'intérêts : C. Gourc: Aucun conflit à déclarer, L. Teot est instructeur remunéré par ACELITY ET URGO, S. Pujol: Aucun conflit à déclarer 
${ }^{1}$ Departement de biologie laboratoire de chimie analytique et electrochimie, Faculté Abou Bekr Belkaid Tlemcen, Sba, ${ }^{2}$ Departement de biologie laboratoire de chimie analytique et electrochimie, Faculté Abou Bekr Belkaid Tlemcen, ${ }^{3}$ Cardiologie, CHU Tlemcen, Tlemcen, Algérie

\section{Introduction et but de l'étude :}

Selon le Club francophone gériatrie et nutrition ; la polymédication, est l'un des signes d'alerte d'une éventuelle dénutrition chez une personne âgée. Pour cela, il est donc particulièrement intéressant de guetter le risque de dénutrition chez les patients hypertendus. Ce volet reste très peu exploré et les études manquent. On se propose dans cette modeste contribution de préciser l'influence de la polymédication sur la dénutrition de personnes âgées hypertendues.

Matériel et méthodes : II s'agit d'une étude descriptive transversale étalée sur l'année 2017. Le recrutement des patients ( 956 patients) a été fait auprès de médecins spécialistes en cardiologie et en médecine interne du CHU Docteur Benzerdjeb Tlemcen, conformément aux critères d'inclusions. Tous les patients avaient bénéficié d'une évaluation de l'état nutritionnel à l'aide du Mini Nutritional Assessement (MNA) test. Une analyse de la variance (ANOVA) était effectuée afin de vérifier que la polymédication constitue un risque de dénutrition chez les hypertendus.

Résultats et Analyse statistique : Les personnes âgées hypertendues n'étaient pas exposées au même risque de dénutrition. Les moyennes des scores (risque de dénutrition) par modalités de polymédication ne sont pas égales $(P=0.000)$. Chez les patients polymediqués, quelque soit le sexe $(P>0.714)$. Le risque de dénutrition était différent selon l'IMC $(P=0.010)$. Cette tendance est inversée chez les patients non polymediqués. En effet, a IMC égale ( $P>0.05)$, les hommes et les femmes de ce groupe étaient exposés a un risque différent de dénutrition. $(P=0.001)$. Chez les patients non polymediqués, les moyennes des scores par modalités des classes d'antihypertenseurs ne sont pas différentes $(P>0.05)$. Ces derniers ne semblent pas avoir un effet direct sur le risque de dénutrition. Par ailleurs, dans le groupe des patients hypertendus qui prennent plus de trois médicaments, les moyennes des scores (risque de dénutrition) par modalités des classes de traitement sont pratiquement différentes ( $P=0.056)$. Bien que les co-morbidités conduisent souvent à orienter le choix, les ARA2 utilisés en monothérapie sont significativement corrélés au risque de dénutrition. Les IEC, les diurétiques, les bétabloquants et les inhibiteurs calciques n’ont que peu ou pas d'effet, et ce quelque soit la stratégie thérapeutique utilisée (mono ou bithérapie) ( $P>0.05$ )

Conclusion : Notre étude suggère que la polymédication est une cause importante de la dénutrition chez hypertendus .II doit y avoir désormais une prise de conscience concernant le suivi des patients sous ARA2 dans les populations de l'extrême ouest Algérie.

Conflits d'intérêts : Aucun conflit à déclarer 


\section{P048}

La Dyslipidémie, et risque de dénutrition chez les patients âgés hypertendus.

Y. Kachekouche ${ }^{1}$, M. Dali-Sahi ${ }^{1}{ }^{*}$, N. Medjati-Dennouni ${ }^{1}$, H. N. Merad Boudia ${ }^{1}$, A. Meziane ${ }^{2}$

${ }^{1}$ Département de biologie laboratoire de chimie analytique et d'électrochimie, Université de Tlemcen, ${ }^{2}$ Service de cardiologie, CHU de Tlemcen, Tlemcen, Algérie

Introduction et but de l'étude : La dyslipidémie et les antiyhpertenseurs sont connus comme facteurs qui majorent le risque de dénutrition.

La dénutrition augmente la durée moyenne de séjour à l’hôpital, le nombre de complications, la mortalité, et le coût global de la prise en charge.

Le but de notre travail est de déterminer la part de la dyslipidémie dans la survenue de la dénutrition chez les sujets âgés hypertendus.

Matériel et méthodes : Il s'agit d'une étude descriptive étalée sur 04 mois de l'année 2018 intéressant des patients hypertendus présentant une dyslipidémie (263) et des patients hypertendus non dyslipidémiques (429) hospitalisés ou consultant au service de cardiologie du CHU Docteur Benzerdjeb Tlemcen. Le questionnaire Mini Nutritional Assessement (MNA) et les analyses en composantes principales (ACP) sont utilisées pour déterminer les liaisons entre le risque de dénutrition et les différentes classes des traitements chez les deux groupes de patients.

Résultats et Analyse statistique : Pour les mêmes seuils d'IMC ( $P>0,05)$, les moyennes des scores par modalités de sexe n'étaient pas égales, en effet le risque de dénutrition chez les hypertendus dyslipidémiques était différent chez les hommes par rapport aux femmes $(P=0,000)$. L'analyse en composante principale montre aussi que le risque de dénutrition peut aussi être lié aux antihypertenseurs utilisés notamment les ARA2. Cependant nous ne soulignons aucunes liaisons avec les autres types de classes antihypertenseurs utilisés chez les hypertendus dyslipidémiques (Les bétabloquants, les inhibiteurs de l'enzyme de conversion, les inhibiteurs calciques et les diurétiques).

Cependant chez les hypertendus indemnes de dyslipidémie, l'effet sexe n'est pas retenu $(P>0,05)$. Cependant nos résultats indiquent que les moyennes des score par modalités $d^{\prime} I M C$ sont différentes $(P=0,000)$. L'obésité est associée à un mauvais pronostic de dénutrition chez les individus hypertendus de notre étude.

Conclusion : Une prise en charge adéquate et correcte du traitement antihypertensif permet d'améliorer le pronostic de ces patients et prévient de la dénutrition chez le sujet hypertendus dyslipidémiques. Enfin il est impératif de prendre en charge les hypertendus dénutris en situation d'obésité afin d'améliorer leur statut fonctionnel.

Conflits d'intérêts : Aucun conflit à déclarer 
Introduction et but de l'étude : En institution comme à domicile, certains patients peuvent avoir des difficultés à se mobiliser, se déshabiller ou refuser de se mettre en sous-vêtements. II est donc probable que peser les patients habillés est une pratique fréquente. La surestimation du poids corporel pourrait induire une erreur d'évaluation du statut nutritionnel. L'objectif était de déterminer dans quelle mesure peser les patients adultes habillés versus en sous-vêtements pouvait modifier leur poids et leur statut nutritionnel.

Matériel et méthodes : Tous les patients adultes vus, entre juin et septembre 2017, en consultation externe dans I'Unité de Nutrition du CHU de Limoges étaient inclus, quelle que soit la pathologie. Quatre poids étaient recherchés: habillé avec chaussures, en sous-vêtements, poids des vêtements et poids des chaussures. L'indice de masse corporelle (IMC) était calculé. Les données étaient recueillies pour quatre conditions de température différentes: temps froid, pluvieux, chaud et très chaud. L'analyse statistique utilisait l'ANOVA, le test de Fisher, la régression simple.

Résultats et Analyse statistique : 51 patients étaient inclus ( 38 femmes, 13 hommes). La pesée habillée surestimait le poids réel de $1,6 \mathrm{~kg}(80,2$ versus $78,6 \mathrm{~kg}, \mathrm{p}<0,0001)$, et induisait une surestimation de classification du statut nutritionnel dans $13,7 \%$ des cas. Le poids des vêtements était plus élevé pour le temps froid $(p=0,02)$. Les poids des vêtements et des chaussures étaient plus élevés chez les hommes ( $p=0,03$ et $p=0,002)$. Il n'y avait pas de différence significative en fonction de l'âge.

Conclusion : Il existe bien une surestimation significative du poids et du statut nutritionnel si l'on pèse les patients habillés versus en sous-vêtements, majorée si la température extérieure est froide. Une seule étude sur le sujet chez l'adulte a été retrouvée, réalisée aux États-Unis, qui suggérait que le poids corporel en sous-vêtements pourrait être obtenu en soustrayant du poids habillé environ $0,8 \mathrm{~kg}$ chez les femmes et $1,2 \mathrm{~kg}$ chez les hommes. Nos résultats vont dans le sens de la recommandation française, à savoir de peser les patients adultes en sous-vêtements et non pas habillés.

\section{Références :}

Whigham LD, Schoeller DA, Johnson LK, Atkinson RL. Effect of clothing weight on body weight. Int J Obesity 2013;37:160-1.

Censi L, Spinelli A, Roccaldo R, Bevilacqua N, Lamberti A, Angelini V, et al. Dressed or undressed? How to measure children's body weight in overweight surveillance? Public Health Nutr 2014;17:2715-20.

Tuan T, Marsh DR, Ha TT, Schroeder DG, Thach TD, Dung VM, et al. Weighing Vietnamese children: how accurate are child weights adjusted for estimates of clothing weight? Food Nutr Bull 2002;23:48-52.

Melchior JC, Anachi M, Hankard R. Méthodes d'évaluation et du risque nutritionnel. In: Traité de nutrition clinique. Paris: SFNEP; 2016. p. 647-68.

https://fr.climate-data.org/location/342/\#climate-graph [Accéder le 7 mai 2018].

World Health Organization. Physical status: the use and interpretation of anthropometry. Report of a WHO Expert Committee. WHO Technical Report Series; 1995. p. 854.

Cole TJ, Bellizzi MC, Flegal KM, et al. Establishing a standard definition for child overweight and obesity worldwide: international survey. BMJ 2000;320:1240L 1243.

Conflits d'intérêts : Aucun conflit à déclarer 
Vers une amélioration des prescriptions médicamenteuses chez les patients ayant des troubles de la déglutition et/ou une sonde nasogastrique au $\mathrm{CHI}$ de Villeneuve-Saint-Georges

J. Potier ${ }^{1}$, S. Fondaneche ${ }^{2}$, N. Robquin ${ }^{2}$, G. Goujon ${ }^{3}$, C. Martin ${ }^{1}$, J. Naturel ${ }^{4}$, F. Chaumel ${ }^{4}$, C. Burnat ${ }^{5}$, E. Barsam $^{2, *}$

${ }^{1}$ Pharmacie, ${ }^{2}$ Réanimation, ${ }^{3}$ Chirurgie CMF-ORL, ${ }^{4}$ Gériatrie aiguë, ${ }^{5}$ Biologie, CHI de Villeneuve-Saint-Georges, Villeneuve-SaintGeorges, France

Introduction et but de l'étude : L'écrasement et l'ouverture des médiacaments (MDT) peuvent être une source d'iatrogènie (incompatibilité, inefficacité, toxicité). C'est pourquoi le CLAN et la Pharmacie du CHIV ont réalisé une EPP (Evaluation des Pratiques Professionnelles) afin d'effectuer un état des lieux et de prendre des mesures correctives selon les recommandations.

Matériel et méthodes : L'EPP a été réalisée dans trois services : réanimation, chirurgie et gériatrie.

Un questionnaire a été adressé aux infirmières (IDE) concernant les modalités de préparation des MDT en cas de troubles de la déglutition ou de sonde naso-gastrique (SNG), sur l'adéquation de la prescription médicale et sur le bon usage des MDT. Par ailleurs, un suivi sur la pratique a été réalisé durant une semaine dans les trois services avec un questionnaire en rapport.

Résultats et Analyse statistique : 41 IDE ont répondu aux 12 items.

On constate un manque de connaissances concernant la possibilité d'écrasement selon la forme galénique et une absence de prescription médicale pour $77 \%$ des IDE.

En pratique, $71 \%$ des MDT sont écrasés dans la chambre, sans gants (47\%) et sans masque (94\%) et pour $29 \%$ dans le couloir. $88 \%$ sont donnés avec de l'eau et $12 \%$ avec du yaourt.

Nous avons donc rédigé une procédure institutionnelle rappelant la nécessité légale d'une prescription médicale de l'écrasement /ouverture des MDT.

Nous avons répertorié tous les MDT disponibles sur le CHIV et renseigné leur écrasabilité et les alternatives. Ce tableau a été adapté à chaque service afin de l'afficher dans les postes de soins sous forme de poster.

Sur le plan informatique, nous avons renseigné dans notre logiciel d'aide à la prescription la possibilité d'écraser/ouvrir les MDT afin de faciliter la prescription médicale.

Enfin, nous avons acheté 50 broyeurs mécaniques qui permettent de broyer individuellement dans des sachets plastique les MDT et ainsi de réduire les contaminations croisées et l'exposition des soignants.

Conclusion : Au total, cette EPP nous a permis de voir qu'il n'y avait pas d'outils permettant une optimisation de la prise des MDT chez les patients atteints de troubles de la déglutition ou avec une SNG.

La diffusion d'outils (informatique et poster) ainsi que la mise en place de broyeurs mécaniques sera complémentée par une formation des personnels soignants et médicaux.

Il serait par ailleurs intéressant de refaire une EPP afin de vérifier l'impact de ces mesures.

Conflits d'intérêts : Aucun conflit à déclarer 
P051

Évaluation de la consommation des plateaux repas et de la couverture des objectifs caloriques et protidiques au sein de plusieurs unités du CHU de Strasbourg

E. Merkt ${ }^{1, *}$, L. Parizet ${ }^{1}$, A. Botz ${ }^{1}$, E. Riehl ${ }^{1}$, S. Dekaj ${ }^{1}$, L. Trasrieux $^{1}$, A. Pradignac ${ }^{1}$

${ }^{1}$ CLAN, Hôpitaux Universitaires de Strasbourg, Strasbourg, France

Introduction et but de l'étude : L'hôpital est un lieu où malheureusement les patients se dénutrissent. Cela peut-être dû aux pathologies sous-jacentes mais également à une offre de restauration qui peut-être inadaptée et souvent décriée par les patients hospitalisés. Le but de cette étude a été d'évaluer si les plateaux repas proposés aux patients permettent de couvrir les objectifs d'apports caloriques et protidiques.

Matériel et méthodes : Nous avons recueilli de façon prospective 1680 fiches patients-repas concernant les 56 plats proposés dans le cadre du menu normal salé. Pour chaque plat, nous avons évalué la quantité consommée à l'aide d'une échelle analogique visuelle en 10 points. Les consommations caloriques et protidiques de chaque plateau repas et au sein d'une journée complète ont pu ainsi être calculées et comparées aux objectifs caloriques et protidiques fixés par le CLAN (respectivement $960 \mathrm{kcal}$ et $36 \mathrm{~g}$ de protéine par plateau repas). Cette étude a été menée dans différentes UF de notre CHU (pneumologie, cardiologie, médecine et ophtalmologie) pour tenir compte d'une certaine diversité de pathologies rencontrées chez les patients hospitalisés. Nous nous sommes limités à l'analyse de couverture des objectifs caloriques et protidiques, les besoins energétiques ne pouvant être calculés. Les comparaisons de moyennes ont été réalisées à l'aide du test $t$ ou de l'ANOVA selon les cas.

Résultats et Analyse statistique : D'une manière générale, les femmes hospitalisées présentent des consommations caloriques et protidiques plus faibles que celles des hommes (respectivement $630 \pm 291$ vs $706 \pm 308 \mathrm{kcal} p<0,05$ et $28 \pm 14$ vs $73 \pm 32 \mathrm{~g}$ de protéines $p<0,05)$, avec pour corollaire une moins bonne couverture des objectifs caloriques $(65 \% p<0,05)$ ou protéiques $(79 \%$ $p<0,05)$ que leurs congénères masculins. Quand on s'interesse aux secteurs de soins, c'est en pneumologie que les pourcentages de couverture caloriques et protidiques sont les plus faibles $(66 \% \pm 32$ et $79 \% \pm 42 p<0.001)$. Lorsqu'on conjugue les deux états, on retrouve les valeurs les plus basses observées chez les femmes hospitalisées en pneumologie (63\% de couverture calorique et $76 \%$ de couverture protéique) alors que les valeurs les plus élevées observées sont chez les hommes des UF d'ophtalmologie (90\% de couverture calorique et $99 \%$ de couverture protéique $p<0,001$ ).

Conclusion : Cette enquête démontre une inadéquation entre les repas produits par le service de restauration et la couverture des objectifs caloriques et protidiques des patients fixés par le CLAN. Ces résultats mettent en évidence une grande hétérogénéité de couverture des objectifs qui dépend à la fois du sexe, du contexte pathologique et du repas servi. Ils suggèrent également qu'il serait sans doute nécessaire d'adapter la composition du plateau repas (plat, périphériques) à la typologie des patients hospitalisés ce qui n'est habituellement pas fait en pratique clinique.

Conflits d'intérêts : Aucun conflit à déclarer 
Projet de téléconsultation diététique dans le suivi de la personne âgée dénutrie à domicile N. Masseboeuf ${ }^{1,}{ }^{*}$, F. Diebold ${ }^{2}$, V. Attalin ${ }^{3}$

${ }^{1}$ Dietique, ${ }^{2}$ Geriatrie, CHI Frejus Saint Raphael, Frejus, ${ }^{3}$ Nutrition, CHU Montpellier, Montpellier, France

Introduction et but de l'étude : Pour le collectif de lutte contre la dénutrition, $100 \%$ des patients dénutris suivis au domicile après l'hospitalisation, doivent bénéficier de la mise en place d'un parcours de soin nutritionnel coordonné ville-hôpital. Ce parcours se heurte actuellement à des difficultés, notamment l'absence de consultation diététique en ville. Pour optimiser cette prise en charge, le recours à la télémédecine (TLM) diététique, pourrait être une piste d'amélioration, avec de nombreux bénéfices attendus, médicaux, sociaux, économiques. Le but est de concevoir un projet de téléconsultation (TLC) diététique chez la personne âgée (PA) dénutrie après l'hospitalisation, organisée avec un professionnel de santé (médecin et/ou infirmière) associé ou non à un aidant, sur un territoire de 110000 habitants, dont 38\% sont retraités.

Matériel et méthodes : 1) Bibliographie : aspect médico-économique de la dénutrition, impact de l'intervention à domicile d'une diététicienne, résultats d'expériences de télésanté en nutrition

2) Etude du parcours de soin diététique chez les PA dénutries dans l'établissement

3) Pistes sur les moyens technologiques, humains et financiers, garantissant le projet, ainsi que son évaluation

4) Proposition d'un protocole de coopération permettant à la diététicienne de réaliser légalement un acte TLM avec la délégation d'un médecin référent en nutrition (non soumis à l'ARS)

Résultats et Analyse statistique : 1) Des études ont montré un meilleur état nutrtionnel, une diminution de nombre de réhospitalisations, de moindres coûts avec l'intervention d'une diététicienne à domicile. La TLC est aussi efficace qu'en présentiel. Le suivi téléphonique diététique a un impact positif sur la qualité de vie chez la PA dénutrie.

2) Diagramme de flux 2017 : 28400 séjours au total (41\% >= 70 ans). 6013 en Méd./Chir. (hors Réa.), dont 78\% du domicile via les urgences. 2402 prises en charge par une diététicienne, dont 1628 codages E43 et E44 (=27\% des séjours Méd./Chir., 85\% >= 70 ans). Parmi ces derniers, $65 \%$ retournent à domicile dont $29 \%$ via le SSR, ce qui conforte le besoin d'un suivi à domicile, ce que permet la TLC.

3) Pilotage du projet avec le président du CLAN, gériatre référent en nutrition. La création d'un poste de diététicienne permettrait d'inclure 200 patients/année dédié à la TLC. Trois outils technologiques envisagés : dossier informatisé du patient, portail régional de santé ou carnet de santé en nutrition déployé dans l'obésité au sein d'une UTN, adaptable et accessible à partir d'un smartphone. Ils sont sécurisés, interfaçables et disposent de la TLC. Les indicateurs de suivi : état nutritionnel, réhospitalisations, fréquence d'utilisation, satisfaction des utilisateurs, évènements indésirables.

4) Protocole de coopération : "Dépistage, prévention et prise en charge diététique de la dénutrition chez l'adulte via la TLM". II vise à déléguer à la diététicienne l'acte de TLC grâce à ses compétences en diététique, relationnelles et transversales.

Conclusion : La TLC diététique serait une opportunité, faute de disponibilité, de sensibilisation et de formation des professionnels à domicile. Elle pourrait faciliter une plus grande coopération avec la ville, une meilleure qualité des soins avec une rémunération liée au basculement récent des actes de TLC dans un financement de droit commun.

Conflits d'intérêts : Aucun conflit à déclarer 
Evaluation de la qualité et de la pertinence des prescriptions de nutrition parentérale en oncologie médicale au sein du centre de lutte contre le cancer de Nantes au cours des années 2015 et 2016

C. Saintes ${ }^{1}$, F. Dayot ${ }^{1}$, S. Dauffy ${ }^{1}$, S. Folliard ${ }^{1}$, H. Lusson ${ }^{1}$, E. Perrien ${ }^{1}$, H. Senellart ${ }^{1}$, D. Vansteene ${ }^{1, *}$

${ }^{1}$ Institut de Cancérologie de l'Ouest, Nantes, France

Introduction et but de l'étude : La nutrition parentérale (NP) nécessite un encadrement strict, du fait de ses possibles complications. La SNFEP a proposé en 2012 une grille d'évaluation des pratiques professionnelles à ce sujet.

L'objectif de l'étude est d'évaluer la pertinence et la qualité des prescriptions de nutrition parentérale en hospitalisation d'oncologie médicale à l'Institut de Cancérologie de l'Ouest - site René Gauducheau (CRG) à Nantes.

Matériel et méthodes : L'ensemble des prescriptions de nutrition parentérale réalisées entre le 01/01/15 et le 31/12/16 en hospitalisation d'oncologie médicale a été analysé rétrospectivement. L'évaluation portait sur l'indication, le bilan préthérapeutique, les modalités d'administration et la surveillance du traitement.

Résultats et Analyse statistique : Parmi les quatre-vingt-deux dossiers analysés (78 patients), 40 étaient des hommes (48,8\%) et 42 des femmes (51,2\%). Leur âge médian était de 62,1 ans (36-85 ans). Les localisations primitives du cancer les plus représentées sont ovaire (17 patients, 20,7\%), estomac et œsophage (16 patients, 19,5\%), digestif bas (10 patients, 12,2\%) et pancréas, poumon et sein (7 patients pour chaque localisation, 8,5\%). Le Performans status de l'OMS était à 0 ou 1 pour 22 patients (26,8\%), à 2 pour 28 patients $(34,1 \%)$, à 3 pour 23 patients $(28,1 \%)$ et à 4 pour 4 patients $(5 \%)$. L'IMC moyen était de 21.7 et médian de 21,6 (amplitude de 13.7 à 34.1).

Parmi ces 82 patients, la prise en charge palliative active concernait 75 patients $(91,4 \%)$, et les problématiques digestives représentaient la première cause d'hospitalisation $(53,7 \%)$.

Le tube digestif était fonctionnel chez 11 patients (13,4\%), la nutrition entérale a été testée dans un premier temps pour 8 d'entre eux, mais relayée par une nutrition parentérale du fait d'une complication. L'indication était pertinente pour $96,3 \%$ des patients. La durée entre la prescription initiale et la date de décès était de 66 jours en médiane (amplitude 7 à 491 jours). Le décès survenait dans les 90 jours après la prescription pour 49 patients (59,8\%). La NP a duré moins de 7 jours pour 10 patients. La durée médiane de NP était de 18 jours. Le motif d'arrêt le plus fréquent était l'arrêt des soins.

L'évaluation nutritionnelle était complète (IMC, poids à 1 et 6 mois) pour $68,3 \%$ des patients, et le bilan pré thérapeutique pour $50 \%$. Les apports étaient conformes aux recommandations (1,2 à 1,5g/kg/jour de protéines et 30 à $35 \mathrm{kcal} / \mathrm{kg} / \mathrm{j})$ pour seulement 3 des 41 patients en NP exclusive.

La surveillance clinico-biologique était insuffisante pour 90,1\% des patients, principalement du fait d'une fréquence insuffisante des bilans biologiques et du poids. Aucun des patients en NP n'a bénéficié d'une consultation avec un médecin nutritionniste alors que 5 patients ont reçu plus de 3 mois de NP.

Conclusion : Nos résultats, meilleurs qu'au CRG en 2009 et que dans d'autres centres, restent insuffisants, en particulier concernant le suivi clinico-biologique. La mise en place d'une consultation de surveillance dédiée semble indispensable.

Conflits d'intérêts : Aucun conflit à déclarer 
P054

Le supplément nutritionnel oral : impact en terme de compliance du patient et en terme financier lorsque celui-ci est prescrit via une prescription médicale informatisée.

M. Lardinois ${ }^{1}$, M. D'Alu ${ }^{2}$, C. Michel ${ }^{1}$, G. Remy ${ }^{3}$, V. Vanesse ${ }^{2}$, D. Noël ${ }^{1}$, D. Lacrosse ${ }^{1, *}$

${ }^{1}$ UTN, ${ }^{2}$ Service alimentation et diététique, ${ }^{3}$ Pharmacie, CHU UCL Namur, Yvoir, Belgique

Introduction et but de l'étude : La dénutrition a de multiples conséquences sur l'évolution de l'état de santé des patients. Sa prévention et sa prise en charge sont donc essentiels. La prescription de suppléments nutritionnels oraux (SNO) est souvent la première étape de cette prise en charge, encore faut-il que les patients les consomment de façon régulière. Pour cela, il est essentiel de trouver des solutions pour augmenter leur compliance par rapport à leur traitement nutritionnel. De plus, ces SNO non consommés représentent un gaspillage et donc un coût important pour les hôpitaux puisque à charge de celui-ci.

Matériel et méthodes : Nous avons cherché à déterminer si la compliance augmentait et la gaspillage diminuait lorsque les compléments étaient prescrits via la PMI (prescription médicale informatisée) et apportés par les infirmières, plutôt que via les plateaux repas, comme c'est habituellement le cas. Nous avons réalisé une étude interventionnelle prospective au sein du CHU UCL Namur sur le site Godinne, dans les unités de chirurgies digestives (unité d'intervention) et de gastroentérologie (unité témoin). La diététicienne en charge des deux unités prescrivait les SNO via la PMI dans l'unité d'intervention ; les SNO étaient alors délivrés par l'infirmière. Pour l'unité témoin, le processus de délivrance classique via le plateau repas est resté en place. Les données suivantes étaient récoltées par l'équipe investigatrice : caractéristiques générales et état nutritionnel du patient, type de SNO prescrit, délai d'administration du SNO, quantité consommée quotidiennement, raison éventuelle d'un refus. L'analyse statistique était descriptive (Excel).

Résultats et Analyse statistique : Septante-six patients ont été inclus : 26 dans le groupe témoin et 50 dans le groupe d'intervention. Les patients non dénutris de l'unité témoin présentaient une compliance de $74,8 \%$ contre $82,9 \%$ à l'unité d'intervention. Nous avons objectivé une compliance de $83,3 \%$ pour les patients dénutris de l'unité témoin et $70,4 \%$ pour ceux de l'unité d'intervention. Pour les patients dénutris sévères, la compliance à l'unité témoin était de $98 \%$ contre $79,2 \%$ à l'unité d'intervention. Le coût des SNO non consommés était de 6.10 euros pour l'unité témoin et 22.39 euros pour l'unité d'intervention. Le délai d'administration du SNO aux patients dénutris était inférieure de quasi deux jours dans le groupe intervention.

Conclusion : Nous n'avons pu objectiver ni une compliance supérieure ni des coûts inférieurs dans le groupe intervention. On peut discuter l'influence de certains biais: pathologies différentes, biais lié au passage de l'équipe investigatrice pouvant influencer les comportements tant des patients que de l'équipe soignante. L'amélioration du délai entre la prescription et l'administration du SNO constitue un point positif pour une prise en charge nutritionnelle plus précoce. Enfin, cette nouvelle méthode de prescription de SNO permet une meilleure visibilité du traitement nutritionnel pour l'ensemble du personnel soignant. Une évaluation à plus grande échelle au sein de la totalité de l'hôpital pourrait être la prochaine étape.

Conflits d'intérêts : Aucun conflit à déclarer 

120 patients de soins intensifs

A. Martinez ${ }^{1,{ }^{*}}$, E. Favre ${ }^{1}$, T. Kelevina $^{1}$, G. Bagnoud ${ }^{1}$, M. Charrière ${ }^{1}$, D. Favre ${ }^{1}$, O. Pantet $^{1}$, M. Berger $^{1}$, P. $^{\text {Eckert }}{ }^{1}$

${ }^{1}$ Service de Médecine Intensive Adulte, Centre Hospitalier Universitaire Vaudois (Suisse), Lausanne, Suisse

Introduction et but de l'étude : Les patients nécessitant des traitements de soins intensifs prolongés sont en augmentation (1). Un programme dédié a été créé dans l'unité de soins intensifs (USI). Un de ses objectifs est d'optimiser leur apport nutritionnel. Le suivi nutritionnel de cette population représente un défi en termes de tolérance digestive et de continuité des soins. Les infirmières exercent un rôle pivot dans la gestion de l'administration de l'alimentation et des surveillances en lien.

Matériel et méthodes : Etude descriptive rétrospective monocentrique dans une USI polyvalente universitaire. Les données ont été récoltées entre février 2017 et juillet 2018. Unique critère d'inclusion : un séjour en USI $\geq 14$ jours. Sont exclues : les admissions pour brûlure ou neuro-réanimation. Les variables d'intérêt: démographie, diagnostic, ventilation mécanique (VM), durée de séjour, déficit en énergie (couperet $-500 \mathrm{kcal} / \mathrm{j}$ ou cumulé $-6000 \mathrm{kcal}$ ) et protéines (couperet $-300 \mathrm{~g}$ ). Données extraites du système $\mathrm{d}^{\prime}$ information clinique (MetaVision ${ }^{\circledR}$ ), durant les 60 premiers jours. Les recommandations nutritionnelles du service sont diffusées via un protocole interne. Données : médianes, Interquartiles 25-75.

Résultats et Analyse statistique : Au total, 120 patients ont été inclus dans le programme. Leurs caractéristiques: âge 60,5 ans [IQR 19], SAPSII 54 [IQR 27,5], mortalité 18,5\% (sur 1900 patients USI/an, âge 65 ans, mortalité 13\%), durée de séjour (DUSI) de 32 jours [IQR 23] et durée de VM 17,3 jours [IQR 11,7]. Les patients ont reçu $23 \mathrm{kcal} / \mathrm{kg} / \mathrm{j}$ [IQR 4,3] ce qui est l'énergie recommandée et $0,9 \mathrm{~g} / \mathrm{kg} / \mathrm{j}$ [IQR 0,32$]$ de protéines, soit moins que la recommandation. De $\mathrm{J} 1$ à $\mathrm{J} 7,47 \%$ des journées sont caractérisées par un déficit kcal >-500 par rapport à cible prescrite. Avec la progression de la nutrition, le bilan d'énergie s'équilibre entre J1 et J7. La variabilité des apports inter- et intra-individu est maximale durant les premiers 14 jours.

Une association significative est relevée entre le déficit en protéines entre J1-J14 et la durée de séjour totale, $p=0,0044$. Un déficit cumulé >-6000 kcal de J1 à J7, est associé à une prolongation de la durée de VM, p=0,046. Des escarres sont présentes chez $97 / 120$ patients (médiane 3 ) et sont associées avec un déficit en protéines $(p=0.06)$.

Conclusion : La surveillance quotidienne des apports a montré la grande variabilité de l'attribution calorique et protéinique pendant la première semaine avec une amélioration progressive. Les observations relevées questionnent sur la pertinence à débuter le programme avant $\mathrm{J} 14$, car 2 indicateurs semblent sensibles à un apport nutritionnel ciblé durant les 2 premières semaines en USI, soit précédant leur inclusion dans le programme. Alors que l'apport en énergie est acceptable, le faible apport en protéines semble associé au développement d'escarres. Ces observations soulignent le besoin d'un soutien de l'évaluation infirmière quotidienne pour assurer la continuité du soin nutritionnel, pour mettre en place des stratégies d'application du protocole nutritionnel de I'USI, en adéquation avec la situation clinique rencontrée.

Références : 1. Nelson JE, Cox CE, Hope AA, Carson SS. Chronic critical illness. Am J Resp Crit Care Med. 2010;182(4):446-54.

Conflits d'intérêts : Aucun conflit à déclarer 
P056

Second audit de prescription, d'administration et de suivi des alimentations parentérales au Centre Hospitalier Universitaire de Mont-Godinne. Intérêt de l'implication d'une diététicienne.

G. Dauw ${ }^{1}$, D. Noël ${ }^{1}$, D. Lacrosse ${ }^{1}$, V. Vanesse ${ }^{2}$, J.-D. Hecq ${ }^{3}$, L. Soumoy ${ }^{3}$, C. Michel $^{13,{ }^{*}}$

${ }^{1}$ UTN, ${ }^{2}$ service alimentation et diététique, ${ }^{3}$ Pharmacie, CHU UCL Namur, Yvoir, Belgique

Introduction et but de l'étude : La dénutrition est associée à une augmentation de la durée de séjour, de la mortalité et des coûts. Il est donc important de la prévenir et de la prendre en charge. Divers supports nutritionnels peuvent être mis en place. Les connaissances du personnel soignant sur le bon usage des alimentations parentérales (AP) sont souvent limitées alors qu'un mésusage peut mener à une augmentation des complications et des coûts. Plusieurs stratégies d'amélioration des pratiques dont " un tour multidisciplinaire des AP » ont été mises en place après le premier audit.

Matériel et méthodes : Ce second audit de 2 mois réalisé au CHU UCL Namur Site Godinne avait pour but d'analyser prospectivement les pratiques de prescription, d'administration et de suivi des AP et de confronter ces résultats à l'audit de 2011. Nous avons également évalué le coût direct engendré par les jours inadéquats d'AP et analysé l'apport de l'implication d'une diététicienne investigatrice. Une série de critères qualitatifs et de paramètres ont été récoltés lors de la prescription et pendant la durée de l'AP en lien avec les recommandations internes. L'analyse statistique est descriptive.

Résultats et Analyse statistique : Nous avons inclus 51 patients et analysé 623 jours d'AP. Le dépistage nutritionnel a été réalisé chez $70,6 \%$ patients $(44,6 \%$ en 2011$)$. Le poids a été objectivé dans les 7 jours avant AP pour $80,4 \%$ des patients (61,7\% en 2011). L'indication était inadéquate pour $6 \%$ (27,7\% en 2011). Parmi les patients à risque de SRI, $21,4 \%$ n'ont pas eu de dépistage nutritionnel (64,7\% en 2011). Les AP de 2 à 6 jours ont réduites de moitié. Un bilan calorique a été calculé pour $88.6 \%$ des jours avec apports per os (46.5\% en 2011). L'apport calorique total était adéquat pour $51,6 \%$ des jours analysés, $61.9 \%$ pour le protéique total. Une administration de médicament en Y avec l'AP a été observé pour 22,4\% (avec un risque d'incompatibilité pour $16 \%$ ) des jours avec observation de la voie veineuse. Le suivi biologique et celui du poids respectaient mieux les recommandations que lors du premier audit : 35.3\% des patients pour les triglycérides (6.1\% en 2011), 72.5\% pour le magnésium et phosphore (61.5\% en 2011 ), $76.5 \%$ pour le poids ( $25.5 \%$ en 2011$) ; 43.5 \%$ des hypophosphorémies étaient traitées (17.7\% en 2011). Le surcoût occasionné par le nombre de jours inappropriés d'AP a été évalué à 2272,2€ sur 2 mois (7813,12€ sur 3 mois en 2011)

Conclusion : L'intégration d'une diététicienne dans cet audit a permis d'obtenir plus de données sur les bilans calorico-protéiques. Elle souligne l'importance de la réalisation de ces bilans, de leur communication aux équipes soignantes afin de limiter les risques d'overfeeding et de surcoût. Le travail de I'Unité Transversale de Nutrition (UTN) semble permettre une amélioration des pratiques de prescription et de suivi des AP même s'il reste encore des points d'amélioration. Cet audit souligne la richesse et l'importance d'une collaboration multidisciplinaire au sein l'UTN et des unités de soins. La sensibilisation, la formation des équipes seront poursuivies et de nouvelles stratégies seront proposées pour poursuivre l'amélioration.

Conflits d'intérêts : Aucun conflit à déclarer 


\section{P057}

Impact de la dénutrition sur la survenue des infections nosocomiales au sein de deux pôles du CHU de Strasbourg. C. Dos Reis ${ }^{1,}{ }^{*}$, T. Lavigne ${ }^{1}$, D. Mutter ${ }^{1}$, D. Christmann ${ }^{1}$, A. Pradignac ${ }^{1}$

${ }^{1} \mathrm{CLAN}$ CHU, Strasbourg, France

Introduction et but de l'étude : La dénutrition est un véritable problème de santé publique engendrant d'importantes complications parmi lesquelles les infections nosocomiales (IN). Néanmoins, la dénutrition ne fait classiquement pas partie des facteurs de risque reconnus d'IN. L'objectif de cette étude a donc été d'évaluer l'impact de la dénutrition sur la survenue d'IN, et notamment d'estimer dans quelles proportions la dénutrition pouvait être considérée comme un facteur de risque indépendant d'IN.

Matériel et méthodes : L'étude était prospective, monocentrique, composée de patients adultes hospitalisés dans 2 pôles médicochirurgicaux du CHU de Strasbourg. L'enquête s'est déroulée sur cinq semaines successives, de février à mars 2017. Les patients ont bénéficié d'une évaluation de l'état nutritionnel par une diététicienne selon les recommandations HAS 2003-2007, avec le recueil de I'IMC, la perte de poids, l'albuminémie, l'EVA des ingesta et calcul du NRI. La survenue d'une IN a été ensuite recherchée puis validée par un médecin expert de l'Equipe Opérationnelle d'Hygiène du CHU selon les références de l'Enquête Nationale de Prévalence des IN. Sur le plan statistique, les comparaisons de moyennes ont été réalisées à l'aide d'un test t ou d'une ANOVA selon les cas, les comparaisons des distributions des variables qualitatives par un test du Chi ${ }^{2}$ et une régression logistique utilisée pour déterminer les variables prédictives d'une IN.

Résultats et Analyse statistique : Parmi les 609 patients éligibles, 339 ont bénéficié d'une évaluation nutritionnelle complète. $49.9 \%$ des patients étaient dénutris avec $35.4 \%$ et $14.5 \%$ respectivement modérément et sévèrement dénutris. 57 patients ont présenté une IN, soit une incidence de $16.8 \%$. II existe une plus forte incidence des IN en fonction de la gravité de la dénutrition ( $5.9 \%$ non dénutris vs $23.3 \%$ modérément dénutris vs $38.8 \%$ sévèrement dénutris ; $p<0.0001$ ). Les Odds Ratio (OR) de développer une IN chez les patients dénutris modérés et sévères sont respectivement de $4.9(p<0.0001)$ et 10.1 ( $p<0.0001)$. Les OR sont également majorés en fonction de la durée d'hospitalisation de 7-14 jours ou $\geq 14$ jours avec respectivement des valeurs de de 7.1 et de 33.3 ( $p<0.0001)$. L'analyse multivariée démontre que la dénutrition est un des facteurs de risque prépondérant au développement d'une IN et proportionnel à sa sévérité (OR $2.3(p=0.063)$ pour les dénutris modérés; OR 5.3 ( $p<0.001)$ pour les dénutris sévères). Les autres facteurs retenus sont la présence d'une durée d'hospitalisation prolongée (OR 7.0 ( $p<0.0001)$ pour 7-14jours; OR $17.4(p<0.0001)$ pour $\geq 14$ jours), la présence d'un cathéter $(K T)$ central (OR 4.7 ( $p<0.0001)$ ) ou d'une chirurgie récente (OR $2.6(p=0.012)$ ). Plus, la durée d'hospitalisation se prolonge moins bon est l'état nutritionnel $(p<0.001)$.

Conclusion : Au vu de nos résultats, la dénutrition semble être un facteur de risque indépendant et important de développement d'une IN au même titre qu'une durée d'hospitalisation prolongée ou la présence d'un KT central. L'impact de la dénutrition sur la survenue d'une IN est amplifié par une durée d'hospitalisation qui se prolonge, ces deux éléments étant souvent intriqués en pratique clinique courante, avec l'aggravation de l'état nutritionnel des patients dont l'hospitalisation se prolonge.

Conflits d'intérêts : Aucun conflit à déclarer 


\section{P058}

La Prévalence de la dénutrition chez les patientes atteintes le cancer du sein

T. SALMI ${ }^{1}$, M. Dali-Sahi ${ }^{1,}{ }^{*}$, Y. Kachkouche ${ }^{1}$, N. Medjati-Dennouni ${ }^{1}$

${ }^{1}$ Laboratoire d'électrochimie et de chimie analytique département de biologie, Université de Tlemcen, Tlemcen, Algérie

Introduction et but de l'étude : La pathologie cancéreuse entraîne des troubles nutritionnels majorés par les traitements oncologiques. Les apports alimentaires des patients peuvent être aléatoires et irréguliers. Par ailleurs, certains traitements favorisent la prise de poids, en particulier l'hormonothérapie pour le cancer du sein

L'objectif de la présente étude est d'évaluer les risques liés à la dénutrition chez des patients atteints du cancer du sein.

Matériel et méthodes : Il s'agit d'une étude analytique de 211 patientes atteintes du cancer du sein au centre pierre et Marie curie d'Alger. Le questionnaire Mini Nutritional Assessement (MNA), les tests de Khi deux et le test Kruskal- Wallis ainsi que les corrélations ont été utilisés pour déterminer d'abord les liens de la dénutrition avec le cancer du sein

Résultats et Analyse statistique : La prévalence de la dénutrition chez la population atteinte du cancer du sein est de $52.6 \%$ avant le traitement.

Le test Khi deux démontre que la dénutrition est liée fortement au cancer du sein avec une $p$ significative $\left(p^{* * *}=0.0004 ;\right.$ Khi deux $=126.61$ ).

Il existe aussi une faible corrélation entre l'état nutritionnel des patientes atteintes du cancer du sein et l'IMC ( $r=20.7 ; p=0.0025)$

.Par ailleurs on note une corrélation inverse avec l'âge de survenue du cancer du sein et le score de nutrition ( $r=-35.5 ; p=0.0001)$.

Conclusion : Les patients atteints de cancer du sein représentent une population à un risque accru de dénutrition.

Conflits d'intérêts : Aucun conflit à déclarer 

sponsoriée par l'ESICM et ESPEN

${ }^{1}$ Unité de Nutrition, Hôpitaux Universitaires de Genève, Genève, Suisse

Introduction et but de l'étude : Les calorimètres indirects $(\mathrm{Cl})$ actuellement sur le marché sont incapables de mesurer les patients ventilés mécaniquement à des niveaux de $\mathrm{FiO}_{2}$ supérieurs à $60 \%$. Cette étude visait à valider in vitro la précision du nouveau $\mathrm{Cl}$ développé pour l'étude ICALIC* (Q-NRG, Cosmed, Italie) dans des conditions de ventilation mécanique avec $\mathrm{FiO}_{2} \geq 60 \%$, en utilisant un spectromètre de masse (SM, Extrel, USA) comme référence.

Matériel et méthodes : Des cycles respiratoires à des $\mathrm{FiO}_{2} 60,70$ et $80 \%$ ont été simulés dans un poumon artificiel au moyen d'un ventilateur mécanique (Dräger, Allemagne). Des mélanges de gaz à des concentrations de $\mathrm{CO}_{2}$ égales à la $\mathrm{FiO} 2(60,70,80 \%$, balance $\mathrm{N}_{2}$ ) ont été injectés dans le circuit du poumon artificiel à l'aide d'un contrôleur de débit massique de précision (Bronkhorst, Allemagne) pour simuler une consommation d' $\mathrm{O}_{2}\left(\mathrm{VO}_{2}\right)$ et une production de $\mathrm{CO}_{2}\left(\mathrm{VCO}_{2}\right)$ de $250 \mathrm{ml} / \mathrm{min}$. Les mesures de Q-NRG ont été comparées aux mesures simultanées du SM, en utilisant la technique de la chambre de mélange.

Résultats et Analyse statistique : Le tableau présente des moyennes de 12 mesures simultanées avec le Q- $\mathrm{NRG}_{(\mathrm{VO}}$, VCO $2 \mathrm{Q}-$ $\mathrm{NRG}$ ) et le $\mathrm{SM}$ de référence ( $\mathrm{VO}_{2}, \mathrm{VCO}_{2} \mathrm{MS}$ ). Le Q-NRG a mesuré avec moins de $2.6 \%$ de différence par rapport à la mesure du SM $\left(\mathrm{VO}_{2}, \mathrm{VCO}_{2} \_\right.$Diff) jusqu'à une $\mathrm{FiO}_{2}$ de $70 \%$. Au delà, les mesures du Q-NRG étaient moins précises avec des différences $>10 \%$ à une $\mathrm{FiO}_{2}$ de $80 \%$.

\begin{tabular}{|c|c|c|c|c|c|c|}
\hline $\mathrm{FiO}_{2}(\%)$ & $\begin{array}{c}\mathrm{VO}_{2} \_\mathrm{Q}-\mathrm{NRG} \\
(\mathrm{ml} / \mathrm{min})\end{array}$ & $\begin{array}{c}\mathrm{VO}_{2} \mathrm{SM}_{(\mathrm{m} / \mathrm{min})} \\
(\mathrm{ml}\end{array}$ & $\begin{array}{c}\mathrm{VO}_{2} \text { Diff } \\
(\%)\end{array}$ & $\begin{array}{c}\mathrm{VCO}_{2} \mathrm{Q}_{-} \mathrm{NRG} \\
(\mathrm{ml} / \mathrm{min})\end{array}$ & $\begin{array}{c}\mathrm{VCO} \\
(\mathrm{ml} / \mathrm{min})\end{array}$ & $\begin{array}{c}\mathrm{VCO}_{2} \text { Diff } \\
(\%)\end{array}$ \\
\hline 60 & 252 & 249 & +1.3 & 248 & 245 & +1.5 \\
\hline 70 & 223 & 217 & +2.6 & 225 & 225 & +0.5 \\
\hline 80 & 257 & 216 & +19.3 & 213 & 241 & -11.4 \\
\hline
\end{tabular}

Conclusion : Une excellente concordance entre les mesures du Q-NRG du SM dans ces expériences de simulations in vitro avec des $\mathrm{FIO}_{2}$ jusqu'à 70\% confirme que le Q-NRG est actuellement le dispositif le plus efficace pour effectuer une calorimétrie indirecte chez les patients dans des conditions de ventilation mécanique extrême.

Références : *Etude multicentrique internationale pour le développement et la validation d'un nouveau calorimètre indirect, sponsorisée par les Sociétés Européennes de Nutrition Clinique et Métabolisme (ESPEN) et de Soins Intensifs (ESICM).

Conflits d'intérêts : Y. Dupertuis: Aucun conflit à déclarer, T. Oshima a reçu une subvention/soutien de Bourse de voyage de Cosmed, M. Delsoglio: Aucun conflit à déclarer, N. Achamrah: Aucun conflit à déclarer, C.-P. Heidegger: Aucun conflit à déclarer, C. Pichard a reçu une subvention/soutien de Fresenius, Abbot, Nestlé, Nutricia, Cosmed 
Validation in vitro d'un nouveau calorimètre indirect pour des niveaux de consommation d'O2 et de production de CO2 très faibles

Y. M. Dupertuis ${ }^{1,{ }^{*}}$, T. Oshima ${ }^{1}$, M. Delsoglio ${ }^{1}$, N. Achamrah ${ }^{1}$, C.-P. Heidegger ${ }^{1}$, C. Pichard ${ }^{1}$ et Etude multicentrique ICALIC sponsoriée par l'ESICM et ESPEN

${ }^{1}$ Unité de Nutrition, Hôpitaux Universitaires de Genève, Genève, Suisse

Introduction et but de l'étude : Les méthodes d'évaluation objective de la dépense énergétique chez les patients ventilés mécaniquement avec une consommation d' $\mathrm{O}_{2}\left(\mathrm{VO}_{2}\right)$ et une production de $\mathrm{CO}_{2}\left(\mathrm{VCO}_{2}\right)$ très faibles sont limitées. Cette étude visait à évaluer in vitro si le nouveau calorimètre indirect $(\mathrm{CI})$ développé pour l'étude ICALIC* (Q-NRG, Cosmed, Italie) est capable de mesurer avec précision des valeurs $\mathrm{VO}_{2}$ et $\mathrm{VCO}_{2}$ inférieures à $150 \mathrm{ml} / \mathrm{min}$ par rapport à un spectromètre de masse (SM; Extrel, États-Unis) comme mesure de référence.

Matériel et méthodes : Des cycles respiratoires à $\mathrm{FiO}_{2}$ de 21,40 et $60 \%$ ont été simulés dans un poumon artificiel au moyen d'un ventilateur mécanique (Dräger, Allemagne). Des mélanges de gaz à des concentrations de $\mathrm{CO}_{2}$ égales à la $\mathrm{FiO}_{2}(21,40,60 \%$, balance $\mathrm{N}_{2}$ ) ont été injectés dans le circuit du poumon artificiel à l'aide d'un contrôleur de débit massique de précision (Bronkhorst, Allemagne) pour simuler une consommation d' $\mathrm{O}_{2}\left(\mathrm{VO}_{2}\right)$ et de $\mathrm{CO}_{2}$ de 75 et $100 \mathrm{ml} / \mathrm{min}$. Les mesures du Q-NRG ont été comparées aux mesures simultanées du SM, en utilisant la technique de la chambre de mélange.

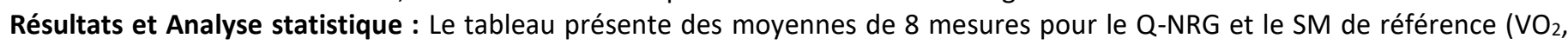
$\mathrm{VCO}_{2}$ ). Le Q-NRG a mesuré avec moins de 3,5\% de différence par rapport à la mesure du SM $\left(\mathrm{VO}_{2}, \mathrm{VCO}_{2} \mathrm{Diff}\right)$. À des $\mathrm{FIO} 2 \mathrm{plus}$ élevées $(\geq 70 \%)$, la mesure de $\mathrm{VCO}_{2}$ est restée très précise, tandis que la mesure du $\mathrm{VO}_{2}$ est devenue moins précise.

\begin{tabular}{|c|c|c|c|c|}
\hline $\mathrm{FiO}_{2}(\%)$ & $\begin{array}{c}\mathrm{VO}_{2}(\mathrm{ml} / \mathrm{min}) \\
\text { Q-NRG }-\mathrm{SM}\end{array}$ & $\begin{array}{c}\mathrm{VO}_{2} \text { _Diff } \\
(\%)\end{array}$ & $\begin{array}{c}\mathrm{VCO}_{2}(\mathrm{ml} / \mathrm{min}) \\
\text { Q-NRG - SM }\end{array}$ & $\begin{array}{c}\mathrm{VCO}_{2} \text { Diff } \\
(\%)\end{array}$ \\
\hline 21 & $68-71$ & -3.5 & $68-68$ & -0.7 \\
\hline 60 & $68-71$ & -3.5 & $68-68$ & -0.7 \\
\hline 21 & $91-93$ & -1.8 & $89-91$ & -2.0 \\
\hline 60 & $91-93$ & -1.8 & $89-91$ & -2.0 \\
\hline
\end{tabular}

Conclusion : Une excellente concordance entre les mesures du Q-NRG et du SM dans ces expériences de simulations in vitro suggère que des mesures de calorimétrie indirecte à de très faibles $\mathrm{VO}_{2}$ et $\mathrm{VCO}_{2}$ sont possibles avec le Q-NRG. D'autres expériences sont nécessaires pour confirmer la validité du Q-NRG pour évaluer une dépense énergétique très faible comme observée chez les patients anorexiques ou pédiatriques.

Références : *Etude multicentrique internationale pour le développement et la validation d'un nouveau calorimètre indirect, sponsorisée par les Sociétés Européennces de Nutrition Clinique et Métabolisme (ESPEN) et des Soins Intensifs (ESICM).

Conflits d'intérêts : Y. Dupertuis: Aucun conflit à déclarer, T. Oshima a reçu une subvention/soutien de Bourse de voyage de Cosmed, M. Delsoglio: Aucun conflit à déclarer, N. Achamrah: Aucun conflit à déclarer, C.-P. Heidegger: Aucun conflit à déclarer, C. Pichard a reçu une subvention/soutien de Fresenius, Abbot, Nutricia, Nestlé, Bourse de voyage de Cosmed 
P061

Evaluation des pratiques professionnelles : prise en charge nutritionnelle des patients atteints de cancer des voies aérodigestives supérieures traités par radiothérapie ou radio-chimiothérapie dans un Centre français de Lutte contre le Cancer

D. Vansteene ${ }^{1, *}$, M. Pons ${ }^{2}$, P. Bachmann ${ }^{3}$, B. Raynard ${ }^{2}$ et InterCLAN Unicancer

${ }^{1}$ Institut de Cancérologie de I'Ouest, Nantes, ${ }^{2}$ Gustave Roussy, Villejuif, ${ }^{3}$ Centre Léon Bérard, Lyon, France

Introduction et but de l'étude : Selon l'étude Nutricancer 2012, 39\% des patients atteints de cancer sont dénutris. Ce taux monte à $42 \%$ chez les patients atteints de cancer des voies aérodigestives supérieures (VADS). La SFNEP en 2012, a écrit des recommandations pour la prise en charge nutritionnelle des patients adultes atteints de cancer. Ces recommandations se déclinent en 10 plans personnalisés de soins (PPS) dont le 4ème porte spécifiquement sur les patients ayant un cancer des VADS traité par radiothérapie (RT) ou radio-chimiothérapie.

La SFNEP a ensuite publié en 2013 un guide d'évaluation des pratiques professionnelles (EPP) en nutrition clinique pour les cancers des VADS.

L'InterCLAN des Centres Régionaux de Lutte contre le Cancer (CRLCC) a décidé de participer à un programme d'EPP, afin de déterminer si ces recommandations sont appliquées. Ensuite, une démarche d'amélioration des pratiques professionnelles pourra être entreprise dans chaque centre.

Matériel et méthodes : Dans chaque centre de lutte contre le cancer, 1 ou 2 auditeurs (médecin ou diététicien) ont audité 10 dossiers des derniers patients ayant terminé une RT pour un cancer des VADS au plus tard le 30 juin 2017. L'ensemble des données a été analysé ensuite par une seule personne.

Résultats et Analyse statistique : Vingt-huit personnes réparties dans 17 des 20 CRLCC ont audités 253 dossiers de patients (pt) (201 hommes, 52 femmes), d'âge médian 62 ans (27-93). La localisation primitive est : oropharynx (73), cavité buccale (66), larynx (33), sinus (26), hypopharynx (21) et autres (34). Les stades III et IV représentent 76,7\% des pt. La moitié des pt ont été opérés. Lors de la consultation initiale, le poids, l'IMC, le poids à 1 et 6 mois, l'albuminémie et l'EPA sont notés respectivement pour $92,9 \%$, $85,4 \%, 61,3 \%, 40,3 \%, 29,2 \%$ et $13,8 \%$ des pt. Le diagnostic nutritionnel est évalué pour $37,5 \%$ des pt, il est correct chez $51,6 \%$ d'entre eux. Parmi les pt évaluables, 20,3\% d'entre eux sont dénutris. Des conseils nutritionnels, des compléments nutritionnels oraux (CNO), une nutrition entérale (NE) ou parentérale (NP) sont évoqués respectivement chez $47,4 \%, 26,1 \%, 30 \%$ et $0 \%$ des pt. A chaque venue en RT et en chimiothérapie, les pt sont pesés respectivement en médiane 6 (0-42) et 4 (0-49) fois.

Pendant la RT, les apports caloriques sont d'au moins $30 \mathrm{kcal} / \mathrm{kg} / \mathrm{J}$ chez 22,1\% des pt (non renseignés pour $61,3 \%$ ).

La prise en charge nutritionnelle a consisté en des CNO, une NE ou une NP chez respectivement 62,5\%, 43,9\% et 2,8\% des pt. Lorsqu'une NE a été prescrite, elle a été proposée au pt par un médecin dans $79,1 \%$ des cas.

Un mois après la fin de la RT, le poids est noté dans 70,8\% des dossiers. Environ 2 et 6 mois après la fin de la RT, 51,5\% et 34,9\% des pt évaluables sont dénutris.

Conclusion : Cette EPP souligne la nécessité de sensibiliser les radiothérapeutes et les oncologues médicaux à l'importance de la prise en charge nutritionnelle en cours de radiothérapie des cancers des VADS.

Alors que le taux de dénutrition est faible au début de la prise en charge, il augmente considérablement à distance des traitements.

Conflits d'intérêts : Aucun conflit à déclarer 

hospitalisés dans une unité de Nutrition Clinique-Trouble du Comportement Alimentaire (TCA)

M. Guinhut ${ }^{1, *}$, J.-C. Melchior ${ }^{12}$, N. Godart ${ }^{23}$, M. Hanachi ${ }^{12}$

${ }^{1}$ Unité de nutrition clinique, Hôpital Raymond Poincaré, Garches, ${ }^{2}$ UVSQ, Versailles, ${ }^{3}$ Fondation Santé des Etudiants de France, Paris, France

Introduction et but de l'étude : L'unité de nutrition clinique de l'hôpital Raymond Poincaré est spécialisée dans la prise en charge de la dénutrition sévère et de ses complications, chez les patients atteints d'AM. Centre de référence reconnu par l'ARS, elle est une unité de recours national pour des patients atteints de formes extrêmes d'AM, souvent chronicisées. Le but de l'étude est de caractériser le profil clinique de cette population de patients hospitalisés le plus souvent pour dénutrition extrême et d'identifier les complications médicales qu'ils développent.

Matériel et méthodes : Les données démographiques, cliniques et paracliniques de 386 patients (21 H et $365 \mathrm{~F}$ ) atteints d'AM, lors de leur première hospitalisation dans l'unité de nutrition clinique de l'hôpital Raymond Poincaré, entre novembre 1997 et janvier 2014, ont été recueillies grâce aux dossiers médicaux.

Résultats et Analyse statistique : L'âge moyen était 29,4 ans $\pm 12 ; 89$ patients (23\%) étaient en couple ; 56 (14,5\%) avaient un ou plusieurs enfants, $97(25,1 \%)$ avaient un niveau de formation bac+4 ou plus, $123(32,5 \%)$ avaient interrompu leur activité professionnelle ou étudiante depuis plus de 6 mois.

L'âge de début du TCA était 19 ans $\pm 8 ; 181$ patients (47\%) avaient une AM restrictive pure avec un IMC moyen à l'admission de $12,7 \pm 2,2 ; 301$ patients $(78,2 \%)$ avaient déjà été hospitalisés dans d'autres services hospitaliers auparavant.

Sur le plan des conduites addictives, 117 patients (30,3\%) avaient un tabagisme actif, 24 (6,2\%) une consommation d'alcool pathologique, $24(6,2 \%)$ un antécédent de toxicomanie. La répartition des comorbidités psychiatriques était : $21,8 \%$ ( $n=84$ ) de trouble de la personnalité, 8,3\% $(n=32)$ de TOC, 47,1 \% ( $n=182)$ d'antécédent de trouble de l'humeur ou de trouble anxieux, $21,2 \%$ ( $n=82$ ) d'antécédents de tentative de suicide mentionnés dans le dossier médical. Le(s) comorbidité(s) somatique(s) associées concernaient 88 patients $(22,8 \%)$.

L'évolution clinique au cours de l'hospitalisation était marquée par une prise de poids de $3,8 \mathrm{~kg} \pm 4$ pour une durée d'hospitalisation de 35,2 jours $\pm 30,2$ sous nutrition entérale administrée chez $82 \%$ des patients ( $n=317$ ), ce qui correspondait à une prise de poids moyenne de $0,8 \mathrm{~kg}$ par semaine.

Les principales complications médicales identifiées en cours d'hospitalisation étaient : l'ostéoporose ( $n=132 ; 45,8 \%$ ), les complications infectieuses $(n=93 ; 24,1 \%)$, la transformation gélatineuse de la moelle $(n=24 ; 6,22 \%)$, la cytolyse hépatique $>10 \mathrm{~N}$ (de dénutrition et/ou de renutrition) ( $n=22 ; 5,7 \%)$, la dysfonction cardiaque ( $n=50 ; 12,9 \%$ ), l'hypokaliémie et/ou l'hypophosphorémie ( $\mathrm{n}=194 ; 50,2 \%)$.

Pendant leur hospitalisation 99 patients $(25,6 \%)$ ont fait un séjour en réanimation.

Le taux de mortalité brut à 5,7 ans était de $11,5 \%$ ( $n=44$ décès).

Vingt-quatre patients $(6,2 \%)$ étaient hospitalisés sous contrainte.

Conclusion : Les patients atteints d'AM hospitalisés pour dénutrition sévère en secteur somatique présentent des complications médicales graves et fréquentes avec un taux de mortalité élevé. Les comorbidités psychiatriques, fréquentes, compliquent la prise en charge. Une prise en charge spécialisée et multidisciplinaire de ces patients est donc indispensable.

Conflits d'intérêts : Aucun conflit à déclarer 
Réalisation d'un e-learning sur le branchement - débranchement de la nutrition parentérale dans un centre hospitalier A. Monribot ${ }^{1,}{ }^{*}$, J. Bataille ${ }^{1}$, S. Peyronnet ${ }^{2}$, V. Bonal ${ }^{2}$, S. Nérome ${ }^{3}$, L. Billiauws ${ }^{2}$, F. Joly ${ }^{2}$, J. Le Grand ${ }^{1}$

${ }^{1}$ Pharmacie à usage intérieur, ${ }^{2}$ Gastro-entérologie et assistance nutritive, ${ }^{3}$ Unité d'Hygiène et de Lutte Contre les Infections Nosocomiales, Hôpital Beaujon, Clichy, France

Introduction et but de l'étude : La nutrition parentérale (NP) et/ou hydratation sur voie veineuse centrale (VVC) est un acte technique à haut risque de complications. Suite à une complication létale sur une erreur de manipulation d'une VVC, une réflexion dans un centre hospitalo-universitaire a mené à la proposition de formations pour le personnel infirmier. L'objectif de ce travail est d'élaborer un e-learning sur la manipulation des VVC chez des patients sous NP-hydratation cyclisée.

Matériel et méthodes : Un groupe pluridisciplinaire (GP) a été créé afin d'identifier les étapes de manipulation et d'entretien des VVC chez des patients sous NP-hydratation cyclisée. Les notions fondamentales à maitriser ont été définies et une recherche des recommandations, référentiels a été réalisée. La population cible étant les infirmiers titulaires et étudiants du service. Afin de favoriser l'apprentissage et de motiver l'apprenant, le GP souhaitait réaliser le e-learning sous forme d'illustrations, vidéos, textes avec un questionnaire avant et après chaque thème.

Résultats et Analyse statistique : Le GP constitué de médecins, cadres, infirmiers, hygiénistes, pharmaciens a élaboré un elearning sur les étapes de branchement, débranchement simple et débranchement complet avec réfection d'un pansement et changement de valve, d'une NP-hydratation cyclisée. Chacune des étapes précédemment citées sont abordées sous forme de vidéos dans 3 chapitres. Le GP a souhaité expliciter chaque soin dans son intégralité. L'apprenant visualise la technicité de chaque geste et intègre la chronologie des étapes. Les notions fondamentales sur les caractéristiques techniques et fonctionnelles des cathéters et des valves, les règles d'hygiène, les complications liées à la manipulation des VVC sont détaillées dans 4 chapitres sous forme de textes, vidéos, illustrations. Les étapes préalables au soin : préparation du chariot de soin et du patient font l'objet d'un chapitre. Dans le dernier chapitre, une chambre des erreurs permet d'insister sur les dérives. Afin de maintenir un niveau d'attention élevé, les vidéos durent en moyenne 3 minutes. Les 5 QCM avant et après chaque chapitre testent l'apprenant sur les notions fondamentales à acquérir. Ils lui permettent de vérifier ses connaissances, d'évaluer sa pratique. La correction des QCM aura lieu avec une personne référente. Le e-learning sera réalisé lors des semaines de formation des étudiants ou lors de sessions dédiées pour les infirmiers titulaires en poste. Afin de maintenir la concentration de l'apprenant, chaque chapitre fera l'objet de sessions de maximum $1 \mathrm{~h}$.

Conclusion : Lors de la réalisation des vidéos, des écarts de pratiques ont été identifiés. Ils ont immédiatement fait l'objet de corrections. Ce e-learning permet d'uniformiser et de sécuriser des pratiques spécifiques, à risque, quotidiennes du service. Un élargissement de celui-ci aux chambres implantables est à réaliser. Il serait intéressant de réaliser une évaluation des pratiques professionnelles avant et après la mise en place du e-learning afin d'évaluer la qualité de l'apprentissage et l'impact du e-learning sur les pratiques notamment sur la fréquence de survenue des complications et évènements indésirables.

Conflits d'intérêts : Aucun conflit à déclarer 
Introduction et but de l'étude : La dénutrition en France, touche environ 10\% des enfants hospitalisés avec pour conséquence une augmentation du taux de morbi-mortalité, de la durée d'hospitalisation et donc son coût. Le but de cette étude est de comparer la valeur prédictive de 4 scores (SRNP, STRONGkids, STAMP et PYMS) afin d'évaluer le risque de dénutrition.

Matériel et méthodes : Cette étude monocentrique inclue 130 enfants âgés de 2 à 17 ans. Pour chaque enfant, l'état nutritionnel a été apprécié par l'examen clinique, le z-score d'IMC, l'indice de Waterlow et les 4 scores de risque nutritionnel ont été calculés. Les données ont ensuite été analysées grâce au logiciel $R^{\circledR}$.

Résultats et Analyse statistique : Parmi les 130 enfants, 12 enfants (9,8\%) ont été dépistés comme dénutris après le calcul de I'IMC, tandis que 6 (4.9\%) l'ont été avec l'indice de Waterlow. Les 4 scores sont corrélés entre eux. Les scores PYMS et STAMP sont corrélés négativement au z-score d'IMC à l'entrée à l'hôpital. Les scores SRNP et STAMP sont corrélés négativement à la perte de poids pendant l'hospitalisation.

\begin{tabular}{|l|c|c|c|c|}
\hline & SRNP & STRONGkids & STAMP & PYMS \\
\hline z-score IMC au début de I'hospitalisation & $r=-0,09$ & $r=-0,16$ & $r=-0,45$ & $r=-0,38$ \\
& $p=0,33 N S$ & $p=0,08$ NS & $p=2,53 \times 10^{-7}$ & $p=1,64 \times 10^{5}$ \\
\hline Perte de poids pendant I'hospitalisation & $t=-3,4$ & $t=-1,2$ & $t=-5,1$ & $t=-1,2$ \\
& $p<0,001^{*}$ & $p=0,2$ (NS) & $p$-value $<0,001^{*}$ & $p=0,3$ (NS) \\
\hline $\begin{array}{l}\text { Malnutrition (BMI } \leq 3^{\text {rd }} \text { percentile) à la fin de } \\
\text { I'hospitalisation }\end{array}$ & $t=7,8$ & $t=7,9$ & $t=7,7$ & $t=7,9$ \\
& $p<0,001^{*}$ & $p<0,001^{*}$ & $p<0,001^{*}$ & $p<0,001^{*}$ \\
\hline
\end{tabular}

Conclusion : Les scores PYMS/STAMP prédisent le mieux la dénutrition existante à l'entrée de l'hospitalisation, tandis que les scores SRNP et STAMP prédisent le mieux l'installation d'une dénutrition au cours de l'hospitalisation.

Conflits d'intérêts : Aucun conflit à déclarer 
Evaluation de l'état nutritionnel chez l'enfant polyhandicapé (étude Polynut)

O. Zaghet ${ }^{1, *}$, R. Enaud ${ }^{1}$, H. Clouzeau ${ }^{1}$, L. Rebouissoux ${ }^{1}$, T. Lamireau ${ }^{1}$

${ }^{1}$ Gastroentérologie et Nutrition Pédiatriques, CHU Bordeaux, Bordeaux, France

Introduction et but de l'étude : On estime actuellement à 15000 le nombre de patients polyhandicapés âgés de moins de 20 ans en France. Les progrès dans la prise en charge globale ont permis une augmentation de l'espérance de vie, souvent au prix de complications orthopédiques, neurologiques ou respiratoires, qui altèrent leur qualité de vie. Les difficultés alimentaires sont habituelles, expliquant la fréquence de la dénutrition qui touche 15 et $80 \%$ des enfants atteints de polyhandicap. L'étude a pour but d'évaluer l'aspect nutritionnel, faire le recueil des apports nutritionnels afin d'apporter des solutions d'amélioration de la prise en charge nutritionnelle des enfants polyhandicapés.

\section{Matériel et méthodes :}

PolyNut est une étude transversale et multicentrique menée dans les différents centres d'accueil d'enfants polyhandicapés sur la région Nouvelle-Aquitaine. Deux questionnaires, un médical et un autre alimentaire avec le recueil des apports nutritionnels sur 3 jours, ont été remplis. Les indices nutritionnels tels que l'indice de masse corporelle (IMC), l'indice de Waterlow, le rapport Taille pour l'Age, le périmètre brachial (PB) ont été relevés et exprimés en z-scores et percentiles. Les apports nutritionnels en énergie et macronutriments ont été calculés à partir d'un relevé des ingestas en s'aidant de la table de Composition Ciqual. L'analyse statistique réalisée avec le logiciel $R^{\circledR}$ comprenait des tests de corrélation et des analyses univariées.

Résultats et Analyse statistique : Onze centres (67\%) ont accepté de participer. L'étude comprend 71 enfants polyhandicapés (29 garçons et 42 filles) âgés de 10.9 ans en moyenne ( \pm 4.8$)$. Le pourcentage d'enfants dénutris était de $26.8 \%$ (IMC $\leq 3^{\mathrm{e}}$ percentile) et $39,7 \%$ (PB $\leq 10^{\mathrm{e}}$ percentile). Plus de $80 \%$ des enfants ont des apports caloriques insuffisants, et la quasi-totalité des enfants ont des apports hydriques insuffisants (<80\% des ANR). Les différents variables (âge à l'entrée dans le centre et lors de l'étude, apport protéique, nombre de classes médicamenteuses utilisées, durée moyenne des repas) n'étaient pas corrélés à l'état nutritionnel.

Conclusion : Un bilan évaluant l'état nutritionnel et l'adéquation des apports alimentaires est nécessaire à l'entrée et régulièrement au cours de tout séjour dans les établissements pour enfant polyhandicapé.

Conflits d'intérêts : Aucun conflit à déclarer 
P066

L'échelle des prises alimentaires $\left(\mathrm{EPA}^{\circledR}\right)$ permet elle en consultation diététique ambulatoire de cancérologie d'apprécier le niveau d'apport calorique et de prédire l'échec de prise en charge diététique orale ?

J. Rieubon ${ }^{1}$, P. Roux ${ }^{1}$, I. Fiorletta ${ }^{1,}{ }^{*}$, C. Fingal ${ }^{1}$, B. Poirée ${ }^{2}$, C. Occhipinti ${ }^{3}$, V. Garabige ${ }^{4}$, S. Buzzo ${ }^{5}$, M. Pons ${ }^{6}$, P. Senesse ${ }^{7}$, B. Raynard ${ }^{6}$, P. Bachmann ${ }^{1}$ et Interclan CRLCC - Unicancer

${ }^{1}$ Unité de nutrition et diététique, CRLCC L BERARD, Lyon Cedex 08, ${ }^{2}$ Service diététique, Centre François Baclesse, Caen, ${ }^{3}$ Service diététique, Institut Curie - Hôpital René Huguenin, Saint Cloud, ${ }^{4}$ Service diététique, Institut Curie, Paris, ${ }^{5}$ Service diététique, Centre Antoine Lacassagne, Nice, ${ }^{6}$ Unité de nutrition, Institut Gustave Roussy, Villejuif, ${ }^{7}$ Unité de nutrition, CRLCC Val d'Aurelle, Montpellier, France

Introduction et but de l'étude : En cancérologie, le dépistage d'une insuffisance des prises alimentaires est recommandé. Les recommandations SFNEP 2012 préconisent l'utilisation de l'échelle des prises alimentaires (EPA ${ }^{\circledR}$ ). II existe une bonne corrélation de l'EPA avec l'apport calorique et le risque de dénutrition chez l'hospitalisé principalement (Thibault R, et al, 2009, Clin Nutr 28;134-40)( Raynard B et al, 2014 https://doi.org/10.1016/S0985-0562(14)70597-7). II n'y a pas d'étude en consultation de cancérologie (CS) ambulatoire validant l'outil. Enfin, une EPA $<7$ pourrait faire suspecter un échec de prise en charge diététique et/ou par compléments nutritionnels oraux (CNO) (Guex E et al, NCM, 2013, 27 (3) ; 139-47). L'objectif de l'étude est de confirmer en CS ambulatoire de diététique, la valeur de l'EPA dans la prédiction de l'insuffisance des PA, du risque de dénutrition, d'échec de prise en charge orale.

Matériel et méthodes : L'étude multicentrique porte sur les données d'une première CS (CS1) diététique ambulatoire et/ou d'hôpital de jour chez des adultes non sévèrement dénutris. Le receuil porte sur la pathologie et son traitement, I'EPA, le poids, la perte de poids, les ingesta, les symptômes en rapport avec la réduction des PA, le score performance status PS OMS, le type de prise en charge nutritionnelle actuelle et préconisée. Lors de la CS de suivi (CS2), les données sont comparées à celles de la CS1. Les critères HAS définissent le niveau de dénutrition. Les résultats préliminaires sont exprimés en moyenne $+/$ - écart-type.

Résultats et Analyse statistique : Du 14/3 au 27/8/2018, 101 dossiers avec CS2 ( 6 centres) sont analysés. A la CS1, 59 femmes, 42 hommes, de $69 \pm 14$ ans, sont atteints principalement de cancer ORL (25,5\%), gynécologique (22,5\%) ou digestif (20,6 \%), métastatique 1 fois sur $2 ; 27$ ont été opérés, 40 non encore reçus ni chimio-, ni radio-thérapie. L'IMC est de $23,5 \pm 4.4$ et 80 patients ont perdu du poids ( 27 fois $\geq 10 \%$ en 6 mois ; 34 de 5 à $10 \% ; 21$ sont stables ou ont pris du poids), 72 ont un PS 0 ou 1 ; la principale cause de réduction des PA est l'anorexie (40 cas), les nausées-vomissements (12), la dysphagie (10); 52 patients sont dénutris. L'EPA est $<7$ dans 53 cas : Selon que l'EPA est $<7$ ou $\geq 7,66 \%$ des patients sont dénutris et les apports oraux sont de $16,8 \pm 6,7 \mathrm{Kcal} / \mathrm{Kg}$ de poids, versus $35 \%$ et $24,2 \pm 6,2$. Lors de la CS2, 39 patients ont perdu du poids depuis la CS1 (21 avec EPA à $\mathrm{CS} 1<7,18$ avec une EPA $\geq 7$ ) et la couverture des besoins estimés à $30 \mathrm{Kcal} / \mathrm{kg}$ de poids (CNO inclus) est assurée chez 8 patients avec EPA à $\mathrm{CS} 1<7$ versus 11 avec EPA $\geq 7$.

Conclusion : Ces résultats préliminaires en ambulatoire semblent confirmer les données sur l'utilité en hospitalisation de l'EPA dans le dépistage d'une insuffisance d'apport ou d'une dénutrition. L'analyse en cours devrait permettre de déterminer les raisons ou symptômes expliquant la probable incapacité de l'EPA à prédire les chances de succès d'une prise en charge diététique ambulatoire.

Conflits d'intérêts : Aucun conflit à déclarer 


\section{DIABETE}

\section{P067}

Evaluation du risque de survenue de la dénutrition chez des sujets diabétiques hypertendus

M. Dali-Sahi ${ }^{1,}{ }^{*}$, N. Dennouni-Medjati ${ }^{1}$, Y. Kachkouche ${ }^{1}$, H. N. Merad Boudia ${ }^{1}$

${ }^{1}$ Biologie Molèculaire, université de Tlemcen, Tlemcen, Algérie

Introduction et but de l'étude : L'aspect nutritionnel est central dans la prise en charge des sujets âgés diabétiques hypertendus. Ils sont à plus haut risque de dénutrition. Cependant la dénutrition est fréquemment sous-estimée chez les sujets âgés diabétiques hypertendus, car ses manifestations cliniques sont non spécifiques.

Le "Mini Nutritionnal Assessement» (MNA) est un outil visant le dépistage de la dénutrition qui peut être utilisé au moment du diagnostic de diabète chez les hypertendus et dans le suivi du traitement. L'objectif de la présente étude est d'évaluer les risques liés à la dénutrition chez cette catégorie de patients recrutés au CHU de Tlemcen.

Matériel et méthodes : Une étude descriptive est réalisée sur un échantillon de 692 individus, 366 diabétiques hypertendus, et 326 hypertendus normo glycémique âgés de 50 ans et plus, recrutés au CHU de Tlemcen durant trois mois de l'année 2018: Le questionnaire MNA et une régression linaire nous ont permit de proposer un modèle prédictif du risque de dénutrition chez les diabétiques hypertendus

Résultats et Analyse statistique : Notre modèle retient le sexe comme facteur, indépendant de l'âge, $(p=0,001), L^{\prime} I M C(p=0,000)$, le traitement par les ARA2 $(p=0.009)$, les IEC $(p=0.026)$, et l'association ARA2/Diurétique $(p=0.013)$.

Le modèle rejette l'effet âge et la survenue du diabète de type2.

Conclusion : La population diabétique hypertendue est une population à un risque accru de dénutrition. Cette dernière est particulièrement liée à la thérapie utilisée.

Conflits d'intérêts : Aucun conflit à déclarer 
Introduction et but de l'étude : Le diabète sucré expose aux morbidités cardio-vasculaires.

Des stratégies de divers types sont mises en place pour contenir cette pathologie; des mesures hygièno-diététiques (MHD) participent à ce programme de lutte contre cette'épidémie.

Nous avons voulu faire un état des lieux, dans une région de l'intérieur de l'Algérie, concernant l'éducation thérapeutique et les MHD qui devraient accompagner le schéma thérapeutique.

Matériel et méthodes : Il s'agit d'une étude observationnelle transversales descriptive effectuée entre octobre 2017 et mars 2018. 402 diabétiques de 3 municipalités (région de Chlef située à environ 200 kms à l'ouest d'Alger)) ont participé à l'étude.

L'examen comportait une interrogatoire (questionnaire de 73 items), un examen clinique, des mesures anthropométriques et une bio-impédancemétrie. Une deuxième visite est nécessaire pour revoir les bilans biologiques. La deuxième consultation comprend des conseils nutritionnels.

Les données de l'étude ont été saisies à l'aide d'un logiciel de calcul informatique (SPSS 14.0). L'analyse statistique comprenait des moyennes et des écarts-types pour les données qualitatives et des pourcentages pour les données quantitatives.

Résultats et Analyse statistique : Le taux de prévalence du diabète était de $17,3 \%$, dont $57 \%$ d'hommes et $43 \%$ de femmes et un âge moyen de 68 ans pour une population global de 26000 habitants.

$7 \%$ seulement des patients ont répondu correctement aux questions sur les différents aspects du diabète.

$70 \%$ des patients ont des croyances erronées sur le lien entre les aliments et le diabète.

$60 \%$ des sujets interrogés n'ont jamais consulté chez un diabétologue.

La pratique du sport ou d'une activité physique soutenue et régulière concerne $12 \%$ des patients de l'étude.

Conclusion : La promotion chez les diabétiques d'un meilleur changement de mode de vie pour améliorer le contrôle métabolique de la maladie et son évolution, en respectant les multiples dimensions du mode de vie: culturelles, sociales et familiales, est primordiale.

Pour cette raison, la prescription doit être personnalisée et négociée en tenant compte des particularités de chaque diabétique et de ses possibilités financières en particulier.

L'instauration des MHD doit être précédée de l'évaluation du profil alimentaire initial par le prescripteur.

Références : - Eves ND, Plotnikoff RC, Resistance training and type 2 diabetes: Considerations for implementation at the population level, Diabetes Care, 2006; 29: 1933-41

- Knowler WC, Barrett-Connor E, Fowler SE, Hamman RF, Lachin JM, Walker EA. et al. « Reduction in the incidence of type 2 diabetes with lifestyle intervention or metformin » [archive] N Engl J Med. 2002;346: 393-403.

- Lagger G, Pataky Z, Golay A. Efficacy of therapeutic patient education in chronic diseases and obesity. Patient Educ Couns. 2010 Jun;79 (3): 283-6. Epub 2010 Apr 21.

Remerciements : Nous tenons à remercier le personnel administratif et le personnel de santé pour la collaboration active et l'aide fournie ayant permis la réalisation de l'étude.

Conflits d'intérêts : Aucun conflit à déclarer 
Effet de l'entraînement en résistance chez les femmes post-ménopausées, obèses et saines sur la sensibilité à l'insuline P. Noirez ${ }^{1,}{ }^{*}$, S. Berhaiem ${ }^{1}$, A. D. Karelis ${ }^{2}$, M. Aubertin-Leheudre ${ }^{2}$, J.-F. Brun ${ }^{3}$

${ }^{1}$ ufr staps - IRMES, EA7329, UNIVERSITE PARIS DESCARTES, paris, France, ${ }^{2}$ Département des sciences de l'activité physique, UQAM, Montréal, Canada, ${ }^{3} \mathrm{CHU}$, Université de Montpellier, Montpellier, France

Introduction et but de l'étude : L'obésité est devenue un grave problème de santé publique, elle atteint maintenant tous les âges et en particulier les personnes âgées. Elle cause plusieurs problèmes métaboliques, l'un d'eux la résistance à l'insuline. L'objectif de l'étude est de déterminer l'effet de l'entrainement en résistance sur la sensibilité à l'insuline.

Matériel et méthodes : La population de cette étude se compose de 31 femmes sédentaires, post-ménopausées, obèses et saines (âge : 63,81 $\pm 3,12$; IMC : 34,17 $\pm 3,65$ ). Dix-neuf d'entre elles (Groupe ER) ont réalisé un entrainement de renforcement musculaire en résistance pendant 16 semaines et 12 ont servi de groupe témoin (Groupe $\mathrm{C}$ ). Un test d'hyperglycémie provoquée par voie orale (HGPO) a été réalisé pour mesurer la sensibilité à l'insuline. Ensuite en utilisant les équations de l'oral minimal model, on a pu calculer l'insulino sensibilité (SI) et classer le groupe ER en 2 sous-groupes : Insulino-Résistant [ER-IR, SI < 6,3 min $\left.{ }^{1} /(\mu \mathrm{U} / \mathrm{ml}) \cdot 10^{-4}\right)$ et Insulino-Sensible (ER-IS, SI $>6,3 \mathrm{~min}^{-1} /(\mu \mathrm{U} / \mathrm{ml}) .10^{-4}$ ]. Des mesures de la composition corporelle (masse maigre, masse grasse, masse osseuse, masse grasse viscéral), des caractéristiques métaboliques (pression artérielle, glycémie, insulinémie, HOMA-IR, triglycérides, HDL, LDL, ApoB, CRP, cholestérol total) et caractéristiques physiques (force de préhension, force musculaire (1RM) et $\left.\mathrm{VO}_{2} \mathrm{max}\right)$ ont été effectuées.

Résultats et Analyse statistique : L'intervention en renforcement musculaire de 16 semaines n'a induit aucun effet sur la composition corporelle, la pression artérielle, la glycémie, l'insulinémie, les triglycérides, HDL, LDL, ApoB, CRP et le cholestérol total. Après cette intervention on a observé chez le groupe ER une baisse de l'indice simplifié de la résistance à l'insuline (HOMA$I R, P<0,05)$, une augmentation du VO $\mathrm{V}_{2} \max (P<0,01)$ ainsi que la force musculaire $1 \mathrm{RM}(\mathrm{P}<0,01)$. Lorsqu'on a divisé le groupe en fonction de leur insulino résistance, on constate l'augmentation du VO2max que chez les $E R-I R(P<0,01)$. Une baisse de la glycémie à jeun et insulinémie à jeun ont été observé chez les $E R-I R(P<0,05)$ et non pas chez les ER-IS.

Conclusion : Ce protocole d'entrainement en résistance améliore la force musculaire et plusieurs indices de glycorégulation chez des femmes âgées, obèses et insulino-résistantes.

Références : Caumo A, Bergman RN, Cobelli C. Insulin sensitivity from meal tolerance tests in normal subjects: a minimal model index. J Clin Endocrinol Metab. 2000 Nov;85(11):4396-402

Conflits d'intérêts : Aucun conflit à déclarer 
Analyse du statut en viamine $\mathrm{E}$ chez les diabétiques de type 1.

F. Z. Meziane ${ }^{1,{ }^{*}}$, A. Bahar ${ }^{1}$, M. Dali-Sahi ${ }^{1}$, N. Medjati-Dennouni ${ }^{1}$, Y. Benslama $^{1}$

${ }^{1}$ Le laboratoire de chimie analytique et d'électrochimie, Université Abou Bekr Belkaid, Tlemcen, Algérie

Introduction et but de l'étude : La vitamine E est le principal composant liposoluble dans le système de défense antioxydant cellulaire et provient exclusivement de l'alimentation. Il a de nombreux rôles importants notamment dans la régulation du système immunitaire.

Chez les diabétiques, I'hyperglycémie induit un stress oxydatif qui peut expliquer la pathogenèse de toutes les complications diabétiques : rétinopathie, la néphropathie et l'athérosclérose.

Des études suggèrent que la vitamine $E$ réduit cette charge oxydative car elle est proposé comme étant l'antioxydant majeur qui a la capacité de rompre les chaînes solubles des lipides et protège les membranes biologiques de la peroxydation des lipides.

L'objectif de notre étude est de mesurer la prévalence de la carence en vitamine E chez les diabétiques de type1, afin qu'une stratégie populationnelle plus appropriée soit mise en œuvre, selon l'anamnèse, d'avoir une carence en vitamine $E$.

Matériel et méthodes : Il s'agit d'une étude descriptive (cas-témoins) portant sur un échantillon total de 134individus dont 79 diabétiques avec un âge moyen de $\mathbf{9 , 8 \pm 3 , 6 9}$ ans et $\mathbf{5 5}$ témoins dont l'âge moyen est de $\mathbf{9 . 9 9} \pm \mathbf{2 . 9 5}$ ans avec un sexe ration de 0,9 et des extrêmes allant de 2 ans et 4 mois à 17 ans. L'analyse statistique a été réalisé par le logiciel Minitab /version 16 pour le test d'indépendance khi deux et le test $T$ de Student afin de déterminer si la carence en vitamine $E$ est significatif et le test de corrélation afin de quantifier la relation linéaire entre les variables $\mathrm{HbA} 1 \mathrm{C}$ et la vitamine $\mathrm{E}$.

Les données de consommation alimentaire ont été collectées via la méthode du rappel de consommation alimentaire de 24 heures. L'apport en vit $E$ a été calculé grâce à un fichier « CIQUAL, 2017».

Résultats et Analyse statistique : L'apport journalier moyen en viatmine E est nettement au-dessous de l'apport conseillé chez 75 diabétiques soit $(94,94 \%)$.

Le test d'indépendance khi deux révèle une relation significative entre cas et témoins ayant une carence en vitamine $E$ avec un $p$ value égale à $p=0,011$ donc le diabète est lié à une carence en vitamine $E(p=0,011$ et khi deux=6,438)

Le test $T$ de Student révèle une relation hautement significative pour le taux de la vitamine $E$ des deux groupes ( $p=0.000)$. Cela confirme le résultat du khi-deux donc les cas sont plus carencé en vit $E$ par apport aux témoins.

Aucune corrélation significative n'a été retrouvée entre la Vitamine $E$ et l'HbA1c $(p=0,476)$ ni entre la Vitamine $E$ et la glycémie à jeun (p 0,706).

Conclusion : On conclusion, notre étude préliminaire démontre que le régime alimentaire imposé aux diabétiques de type 1 doit être revu puisqu'il présente une carence significative en vitamine $E$.

Cependant aucune corrélation n'a été retrouvée entre la vitamine E et l'équilibre glycémique

Conflits d'intérêts : Aucun conflit à déclarer 
Apport alimentaire du sélénium chez des diabètiques de type 1 de la ville de Tlemcen (extrême ouest Algérien)

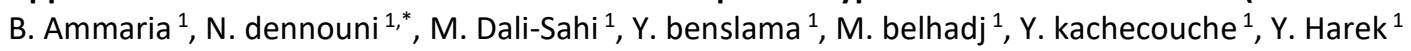

${ }^{1}$ Laboratoire de chimie analytique et d'électrochimie, Université de tlemcen Algérie, Tlemcen, Algérie

Introduction et but de l'étude : Le sélénium (Se) est un micronutriment d'une grande importance nutritionnelle jouant un rôle fondamental dans de nombreuses fonctions biologiques notamment contre le stress oxydatif et les maladies autoimmunes.

L'objectif de cette étude est d'estimer l'apport alimentaire quotidien en Se chez des diabétique de type 1 dans la ville de Tlemcen.

Matériel et méthodes : II s'agit d'une étude analytique (cas témoins) portant sur un échantillon total de 100 individus dont 45 diabétiques avec un age moyen de $10.17 \pm 3.84$ et 55 témoins avec un age moyen de $9.88 \pm 2.98$. Les données de consommation alimentaire ont été collectées via la méthode du rappel de consommation alimentaire de 24 heures. L'apport en Se a été calculé grâce à un fichier « CIQUAL, 2017» et les tests statistiques ont été réalisés par le logiciel Minitab /version 16.

Résultats et Analyse statistique : Le test $t$ de student révèle une relation hautement significative entre l'apport alimentaire en "Se » et la glycémie pour les diabétiques de type $1(p=0.00)$.

On observe que le plus grand nombre des sujets ont un apport alimentaire normal en sélénium, mais la polpulation diabétique présente un nombre de sujets carencés en « Se » plus élevé par rapport aux sujets témoins dont $25 \%$ des cas sont carencés. Ce résultat est confirmé par le test d'indépendance du khi deux $(p=0.003)$.

Une régression logistique binaire montre que la carence en « Se » indique un risque de DT1 1.68 fois plus élevé.

Le test de corrélation bi-variée entre $\mathrm{Hba1c}$; I'IMC, l'âge et la glycémie a montré une corrélation positive avec l'apport en Se avec un degré de significativité $p=0.94 ; p=0.962 ; p=0.07, p=0.5$ respectivement. Néanmoins des régressions multiples ont montré une association du taux de " Se " avec la fréquence et la quantité de consommation de quelques aliments riche en « Se » tel que les légunineuses, le pain et les oeufs.

Conclusion : L'apport journalier moyen en « Se » était nettement au-dessous de l'apport conseillé chez $25 \%$ des patients, cette carence pourrait aggraver l'état de santé des diabétiques de type 1. C'est pour cela qu' une correction dans leurs régime alimentaire surtout dans la fréquence et la quantité de certains aliments tel que les légumineuses est recommandée.

Enfin, II convient d'élargir la compréhension du lien entre le statut en sélénium et le diabète qui représente encore une des premières causes de mortalité dans le monde.

Conflits d'intérêts : Aucun conflit à déclarer 
Apport nutritionnel en Magnésium des diabétiques de type 1 dans la population de Tlemcen (extrême ouest Algérien). Y. Benslama ${ }^{1}$, A. Behar ${ }^{1}$, N. Dennouni ${ }^{1,},{ }^{*}$, M. Dali-Sahi ${ }^{1}$, Y. Harek $^{1}$

${ }^{1}$ Laboratoire de chimie analytique et d'électrochimie, Université Aboubekr Belkaid Tlemcen Algérie, Tlemcen, Algérie

Introduction et but de l'étude : Le Magnésium (Mg) est un micronutriment important qui provient exclusivement de l'alimentation. C'est le $4^{\text {ème }}$ minérale le plus répandu dans l'organisme jouant le rôle d'un co-facteur pour de nombreuses réactions enzymatiques.

Il participe également au métabolisme des lipides, la synthèse de protéines, la production de l'énergie par dégradation du glucose et à l'activité de certaines hormones, telle que l'insuline.

Des études récentes suggèrent l'intérêt d'un apport suffisant en magnésium dans la prévention de certaines complications liées au diabète de type 1.

L'objectif de notre étude vise à évaluer le statut magnésique chez les diabétiques de type 1 et étudier ses corrélations avec l'équilibre glycémique

Matériel et méthodes: II s'agit d'une étude transversale ayant inclus 39 diabétiques de type 1. Chaque patient a bénéficié d'une enquête alimentaire (rappel des 24 heures) et d'un dosage de la glycémie à jeun et de l'hémoglobine glyquée (HbA1c).

L'apport en Mg a été calculé grâce au fichier « CIQUAL, 2017 » et les tests statistiques ont été réalisés par le logiciel Minitab /version 16.

Résultats et Analyse statistique : L'âge moyen de nos patients était de 10.092 \pm 3.71 ans. L'apport journalier moyen en magnésium était nettement au-dessous de l'apport conseillé chez 79.48\% des patients avec une moyenne de $167.12 \mathrm{mg}$.

Le test de corrélation bi-variée entre $\mathrm{Hba1c}$; I'IMC, l'âge et la glycémie a montré une corrélation positive avec l'apport en Mg avec un degré de significativité $p=0.182 ; p=0.007 ; p=0.310, p=0.113$ respectivement.

Conclusion : Les résultats obtenus ont démontré que notre population diabétique est carencée en Mg. On pense qu'un apport correct de magnésium améliore l'effet de l'insuline injectée, et pourrait prévenir les complications liées au diabète de type1.

Conflits d'intérêts : Aucun conflit à déclarer 
Effet à 3 ans sur le poids et l'équilibre glycémique de l'exercice en endurance ciblé sur l'oxydation des lipides (LIPOXmax) chez des diabétiques de type 2 comparés à des non-diabétiques.

J.-M. Nguyen ${ }^{1}$, M. Richou ${ }^{1}$, F. Bughin ${ }^{1}$, J. Mercier ${ }^{1}$, E. Raynaud de Mauverger ${ }^{1}$, J.-F. Brun ${ }^{1,{ }^{*}}$

${ }^{1}$ Physiologie Clinique, INSERM U1046, Montpellier, France

Introduction et but de l'étude : L'activité physique, puissant insulino-sensibilisateur, est désormais reconnue comme l'un des outils thérapeutiques du diabète. Le réentrainement en endurance de faible intensité ciblé au LIPOXmax a montré son efficacité sur l'équilibre glycémique notamment dans l'essai randomisé "LIPOXmax Réunion". On sait par ailleurs qu'il permet une perte de poids prolongée dans l'obésité. La perte de poids est également un objectif important dans le diabète de type 2 (DT2) et est souvent particulièrement difficile à obtenir. Nous avons donc suivi un groupe de DT2 sur 36 mois pour évaluer l'efficacité sur le poids de cette démarche sur 3 ans.

Matériel et méthodes : 16 DT2 traités par le traitement routinier actuel (ADO, GLP1 analogues et analogues d'insuline) âgés de de 40-71 ans comparés à 287 témoins appariés pour la corpulence l'âge et le sexe. Après calorimétrie d'effort les sujets réalisent 8 séances d'induction de réentraînement en milieu hospitalier pour mettre en place dans leur vie quotidienne une activité ciblée au LIPOXmax, 3x45 minutes par semaine. Ils sont suivis en consultation au long cours.

Résultats et Analyse statistique : Les deux courbes de perte de poids sont superposées jusqu'à 6 mois puis se séparent de plus en plus $(p<0.01)$, amorçant une remontée dans le DT2 qui contraste avec la poursuite de l'amaigrissement chez les non diabétiques. Entre 30 et 36 mois le poids des DT2 se maintient à $-0,82 \pm 0,30 \%$ du poids initial tandis que celui des témoins se maintient à $-7,45$ $\pm 0,78 \%(p<0.001)$. L'HbA1c baisse au cours de la période considérée $(p<0.01)$ et se maintient en moyenne à 3 ans à $-0,65 \pm 0,34$. La baisse d'HbA1c n'est pas corrélée à celle du poids. Les sujets non traités par sulfonylurées et/ou insuline ( $n=9$ ) perdent davantage de poids $(p<0.001)$ que ceux qui reçoivent ces traitements $(n=7)$ mais ils présentent tout de même ce profil d'échappement après 10 mois. Les sujets traités par sulfonylurées et/ou insuline présentaient une moindre aptitude à oxyder les lipides à l'exercice $(p<0.01)$.

Conclusion : Ces résultats confirment qu'il reste plus difficile de faire maigrir des DT2 que des non diabétiques avec l'exercice physique, même avec une démarche douce et facile à mettre en œuvre comme le LIPOXmax, et ce d'autant plus que les patients sont traités par insulinothérapie et/ou sulfonylurées (ce qui s'accompagne d'une moindre utilisation oxydative des lipides à l'exercice). La baisse d'HbA1c de $0.6 \%$ est évidemment plurifactorielle dans cette étude de suivi de "vraie vie " mais concorde avec les données d'études interventionnelles démontrant une efficacité intéressante de l'exercice en endurance de faible intensité sur l'équilibre glycémique.

Conflits d'intérêts : Aucun conflit à déclarer 
Les grignons d'olive diminuent la glycémie et améliorent l'activité antioxydante tissulaire, chez le rat rendu diabétique par injection à la streptozotocine.

H. Cherrad ${ }^{1, *}$, S. Bouderbala ${ }^{1}$

${ }^{1}$ Laboratoire de Nutrition clinique et métabolique LNCM, Faculté des Sciences de la Nature et de la Vie. Université Oran 1, Algérie, Oran, Algérie

Introduction et but de l'étude : Le diabète est un important problème de santé. Les remèdes naturels constituent un antique et un nouvel recours utilisés dans le traitement et l'amélioration de cette pathologie. L'intérêt de ce travail est de voir l'effet des grignons d'olive (GO) sur la glycémie et le statut oxydatif chez des rats rendus diabétique par injection à la streptozotocine.

Matériel et méthodes : Des rats mâles Wistar pesant $260 \pm 20 \mathrm{~g}$, soumis à un régime contenant $20 \%$ de caséine, sont rendus diabétiques par une seule injection intra péritonéale de streptozotocine $(55 \mathrm{mg} / \mathrm{kg} \mathrm{PC})$. Ils sont divisés en 2 groupes consommant chacun, pendant 28 jours, le même régime traité (DGO) ou non (D) avec 7,5\% de grignons d'olive.

Résultats et Analyse statistique : Les rats diabétiques soumis au régime supplémentés avec les GO (D-GO) sont comparés au groupe non traité D. Les teneurs en glucose et I'HBA1c sont respectivement, 1,7- et 1,5-fois plus faibles, alors que le taux d'insuline reste inchangé. Au niveau du foie, du cœur, du rein et du muscle, une augmentation de l'activité de la superoxyde dismutase (SOD) (1,4-, 1,52-, 1,2- et 1,4-fois, respectivement), de la glutathion peroxydase (GSH-Px) (2,2-, 4-, 1,9- et 1,7-fois) et de la catalase CAT (1,3-, 1,6-, 2,2- et 1,8-fois) est noté. Pour ces mêmes organes, les teneurs en glutathion réduit sont respectivement 2,5, 1,4-, 1,3-et 1,3-fois plus élevées. Au niveau sérique, les valeurs de l'acide urique et de la vitamine C sont 1,4-et 2,7-fois plus augmentées chez le groupe traité comparé au groupe non traité.

Conclusion : Chez le rat rendu diabétique, la consommation du régime supplémenté par les grignons d'olive induit a une diminution de la glycémie. Il semblerait aussi que ce régime stimule la défense antioxydante tissulaire, en augmentant l'activité enzymatique endogène en particulier, de la SOD, CAT et GPX-Px et non enzymatique tel que l'acide urique et la vitamine C. Mots Clés : Rat - Diabète - Grignons d'olive - Glycémie - HbA1C- SOD - GSH-Px- CAT - GSSH-red -Acide urique - Vitamine C.

Conflits d'intérêts : Aucun conflit à déclarer 

LCAT chez les rats rats rendus diabétiques par la streptozotocine

F. Z. Alachaher ${ }^{1, *}$

${ }^{1}$ Faculté des sciences, Oran, Algérie

Introduction et but de l'étude : Linum usitatissimum est l'un des nombreux remèdes traditionnels utilisés dans le traitement du diabète dans les déserts de l'Algérie. Le but de cette étude est d'étudier les effets de cette plante sur l'hyperglycémie, les indices de sensibilité à l'insuline (QUICKI, HOMA-IR, HOMA-B), les profils lipidiques, les activités lécithine: cholestérol acyltransférase (LCAT) et paraoxonase chez le rat diabétique.

Matériel et méthodes : Le diabète est induit par voie intrapéritonéale par une injection unique de streptozotocine (STZ) (60 mg / $\mathrm{kg}$ pc). Vingt rats diabétiques, pesant $263 \pm 5 \mathrm{~g}$, sont répartis en deux groupes nourris avec un régime de caséine additionné ou non avec l'extrait de $L u$ ( $1 \mathrm{~g} / \mathrm{kg}$ de poids corporel) pendant 8 semaines.

Résultats et Analyse statistique : Les analyses phytochimiques de $L u$ révélent la présence des alcaloïdes et des flavonoïdes. À J56, la glycémie, le cholestérol total (CT), les triacylglycérols (TG), les phospholipides (PL) et les taux de HOMA-IR sériques diminuent de manière significative $(p<0,05)$, chez le groupe diabétique traités par Lu vs non traités. L'effet hypolipidémiant induit par l'extrait de $L u$ est dû à la réduction du cholestérol total (CT) dans les LDL-HDL1 (-64\%) et les TG dans les VLDL (-65\%). Le traitement avec l'extrait $L u$ améliore les activités de la PON1 et la LCAT de $+38 \%$ et $+43 \%$ respectivement. Les taux de TBARS dans le sérum, LDLHDL1 et HDL3 sont 2,1, 1,7 et 2 fois plus faibles.

Conclusion : Les résultats de cette étude indiquent que les propriétés thérapeutiques de l'extrait de Linum usitatissimum pourraient prévenir de nombreuses complications diabétiques en améliorant la dyslipidémie diabétique et les dommages oxydatifs.

Références : Wilbert S.A. (2014). Treatment of Dyslipidemia in Diabetics. Int J Clin Cardiol. 1:1.

Zheng T., Shu G., Yang Z., Mo S., Zhao Y. \& Mei Z. (2012). Antidiabetic effect of total saponins from Entada phaseoloides (L.) Merr. in type 2 diabetic rats. J of Ethnopharmacol. 139: 814-821.

Zheng X., Zhang L., Wang W., Wu Y., Zhang Q. \& Feng W. (2011). Anti-diabetic activity and potential mechanism of total flavonoids of Selaginella tamariscina (Beauv.) Spring in rats induced by high fat diet and low dose STZ. J Ethnopharmacol. 137: 662- 668.

Conflits d'intérêts : F. Z. Alachaher est consultant chez SAN 
Effet des grignons d'olive sur la peroxydation lipidique sérique et tissulaire, chez le rat rendu diabétique H. Cherrad ${ }^{1, *}$, S. Bouderbala ${ }^{2}$, M. Bekhtaoui ${ }^{2}$

${ }^{1}$ Département de Biologie, Faculté des Sciences de la Nature et de la Vie. Université Oran1, ${ }^{2}$ Département de Biologie, Faculté des Sciences de la Nature et de la Vie. Université Oran 1, Oran, Algérie

Introduction et but de l'étude : De nombreuses études se sont intéressées aux produits végétaux ainsi que leurs effets sur la prévention et/ou le traitement de certaines maladies. L'effet des grignons d'olive (GO) est étudié sur la peroxydation lipidique des tissus et du sérum, chez des rats rendu diabétiques.

Matériel et méthodes : Des rats mâles de souche Wistar $(n=20)$ pesant $260 \pm 20 \mathrm{~g}$ sont soumis pendant 4 jours à un régime contenant $20 \%$ de caséine combinée à $5 \%$ d'huile de tournesol. Après cette phase d'adaptation, les rats sont rendu diabétiques (D) par injection de streptozotocine et sont divisés en 2 groupes consommant pendant 28 jours le même régime de caséine supplémenté ou non avec les grignons d'olive à 7,5\% (D-GO et D, respectivement).

Résultats et Analyse statistique : Les rats diabétiques soumis au régime supplémentés avec les GO (D-GO) sont comparés au groupe D. Le poids corporel et la nourriture ingérés sont similaires chez les deux groupes. Au niveau hépatique les teneurs en lipides totaux sont 1,36-fois plus augmentées, cependant les teneurs en esters de cholestérol sont 2-fois plus diminués. Le contenu en cholestérol total, cholestérol libre et triglycérides du foie ne représente aucune différence significative. Les teneurs en cholestérol total, cholestérol libre et esters de cholestérol du sérum sont similaires. Les concentrations sériques en triglycérides sont 1,24-fois plus faibles. Au niveau du sérum, foie, reins, cerveau, cœur et muscle, les teneurs en substances réactives à l'acide thiobarbiturique sont respectivement, 2,7-, 1,5-, 1,3-, 1,6- et 3- et 3-fois plus faibles.

Conclusion : Chez le rat rendu diabétique, la supplémentation du régime avec les grignons d'olive semble avoir un effet hypolépimiant. De plus, il apparait que les grignons d'olive protègent contre l'attaque radicalaire en diminuant la peroxydation lipidique au niveau sérique et tissulaire.

Conflits d'intérêts : Aucun conflit à déclarer 
P078

Les protéines de sardine atténuent l'hyperglycémie, l'insulinorésistance ainsi que l'inflammation, au cours du diabète de type 2, chez le rat Wistar

N. Benaicheta ${ }^{11}$, F. O. Boukortt ${ }^{1, *}$

${ }^{1}$ Biologie, Université, Oran, Algérie

Introduction et but de l'étude : Notre objectif est d'examiner l'effet des protéines de sardine sur les marqueurs du diabète et de l'inflammation chez des rats présentant un diabète de type 2 (DT2).

Matériel et méthodes : Le DT2 est induit par l'administration d'un régime hyperlipidique (RH) (30\% de lipides dont $67 \%$ d'acides gras saturés), pendant 5 semaines. Au bout de cette période, l'injection intrapéritonéale d'une faible dose de streptozotocine est administrée aux rats. Les rats diabétiques sont répartis en 4 lots $(n=6)$ et consomment durant un mois $20 \%$ de caséine (CAS) ou protéines de sardine (PS) combinées à 30\% (RH-CAS ou RH-PS) ou 5\% de lipides (CAS ou PS). La glycémie, l’hémoglobine glyquée (HbA1c), I'insuline, la leptine et la resistine sont déterminées. Les indices de l'insulinorésistance (HOMA-IR) et de la fonction des cellules $\beta$ (HOMA- $\beta$ ) sont calculés.

Résultats et Analyse statistique : Les résultats indiquent que la glycémie diminue tout au long de l'expérimentation avec PS combinées au régime hyperlipidique ou non comparé à la CAS. De même, les PS vs la CAS diminuent l'HbA1c et l'indice HOMA-IR quel que soit le régime consommé. La teneur en insuline sérique diminue de $51 \%$ chez le groupe PS vs CAS. L'indice HOMA- $\beta$ augmente chez les rats consommant les PS associées au régime hyperlipidique ou au régime normolipidique. Par ailleurs, les teneurs en resistine augmentent chez le groupe RH-CAS vs CAS. En revanche, ces teneurs diminuent avec PS combinées à $5 \%$ ou $30 \%$ de lipides. Les teneurs en leptine sont similaires chez le groupe PS vs CAS et le groupe RH-PS vs RH-CAS.

Conclusion : En conclusion, les protéines de sardine atténuent la glycémie, l'insulinorsistance et améliorent les marqueurs de I'inflammation.

Conflits d'intérêts : Aucun conflit à déclarer 
P079

La consommation alimentaire et l'activité physique chez les diabétiques de la région Oujda Angad. Eating habits, physical activity and among diabetes patients in Oujda Angad.

A. Hsaini ${ }^{1, *}$, Y. Aboussaleh ${ }^{1}$

${ }^{1}$ Laboratoire de nutrition, Université ibn tofail, Kenitra, Maroc

Introduction et but de l'étude : L'association entre les habitudes alimentaires, l'activité physique et le risque de diabète sucré est très importante, mais la littérature au Maroc est rare dans ce domaine.

Objectif : L'objectif de cette étude était d'examiner prospectivement les associations entre la fréquence alimentaire, l'activité physique et le risque de diabète sucré

Matériel et méthodes : L'étude a été réalisée dans le cadre d'une enquête transversale du 1er décembre 2016 au 30 mars 2017 , centre de référence de la diabétologie à Oujda au Maroc. Un questionnaire structuré a été utilisé pour recueillir des informations sur les données sociodémographiques, les habitudes alimentaires, l'activité physique. Les pratiques alimentaires ont été évaluées à l'aide d'un questionnaire semi-quantitatif sur la consommation alimentaire. Le poids et la taille ont été mesurés selon les normes internationales. Hémoglobine A1C (HBA1C), la pression artérielle de chaque patient a été mesurée directement après l'entretien. Les données ont été analysées par le logiciel SPSS version 20.0

Résultats et Analyse statistique : 40,4\% des patients diabétiques étaient en surpoids (IMC> RC = $25 \mathrm{~kg} / \mathrm{m} 2$ ), 27,5\% étaient obèses $(\mathrm{IMC}>\mathrm{ou}=30 \mathrm{~kg} / \mathrm{m} 2)$. Les bonnes habitudes alimentaires, le score d'activité physique étaient associés à un risque réduit. Une association significative entre une activité physique insuffisante, les comportements alimentaires et le diabète a été observée. La consommation de pain / féculents et la consommation de viandes sont des comportements systématiquement associés au taux élevé d'hémoglobine.

Conclusion : À la lumière de ces résultats, il semble concevable que le risque de développer une maladie diabétique puisse être réduit en modifiant les habitudes alimentaires et le mode de vie.

Conflits d'intérêts : Aucun conflit à déclarer 
Les habitudes alimentaires chez les diabétiques de type 1 au cours du mois de Ramadan :

N. El Ouarradi ${ }^{1,}$, G. El Mghari ${ }^{1}$, N. El Ansari ${ }^{1}$

${ }^{1}$ Service d'Endocrinologie diabétologie et maladies métaboliques. Laboratoire PCIM, FMPM, Université Cadi Ayad. CHU Mohamed VI. Marrakech, CHU Errazi, Marrakech, Maroc

Introduction et but de l'étude : Le diabète type 1 est l'une des pathologies qui exempte le musulman de jeûner du fait des risques encourus de complications et de décompensation. Mais malgré les recommandations médicales, 43 \%des patientsdiabétiques de type 1 jeûnent pendant le mois du Ramadan selon l'étude Epidiar. L'objectif de notre étude est d'évaluer les habitudes alimentaire des patients diabétiques type 1 au cours du Ramadan.

Matériel et méthodes : Il s'agit d'une étude observationnelle transversale à visée descriptive menée au cours du mois de ramadan 2016 et ayant inclus les patients diabétiques de type 1 suivis au sein du service d'endocrinologie et de diabétologie du CHU Mohamed VI.

Résultats et Analyse statistique : Sur les 20 patients inclus dans l'étude, 4 d'entre eux ont tenté de jeuner le Ramadan. La moyenne d'âge des participants était de 22 ans avec des extrêmes allant de 14 ans à 33 ans. Le nombre moyen de repas était de 3.5 et 25 $\%$ des patients prenait de façon régulière le Sohour. L'apport calorique total journalier était plus élevé au cours du mois de Ramadan (1765 kcal/j contre $1715 \mathrm{kcal} / \mathrm{j})$. La plupart de nos patients avaient une activité physique réduite au cours du Ramadan, avec une augmentation de la ration glucidique : L'apport en glucides représentait $55 \%$ de l'apport calorique total au cours de ramadan contre $49 \%$ en dehors de ramadan.

Conclusion : L'étude des effets métaboliques du jeune au cours du Ramadan a fait l'objet de nombreux travaux, par contre, l'influence du Ramadan sur les habitudes alimentaires chez les non jeuneurs n'a été que rarement abordée. Les résultats de notre étude étaient en faveur d'une augmentation de l'apport glucidique pendant le Ramadan. La gestion du patient diabétique durant ce mois revêt une importance particulière et l'éducation du patient diabétique type 1 , encore plus durant ce mois, est l'un des piliers essentiels de la prise en charge de la maladie et de la prévention de ses complications.

Conflits d'intérêts : Aucun conflit à déclarer 
P081

Impact du "manger-mains" chez le résident institutionnalisé ne mangeant pas seul, en raison de troubles cognitifs et/ou physiques, à travers l'interdisciplinarité soignants/cuisiniers d'une recherche en EHPAD.

S. Verbrugghe ${ }^{1,{ }^{*}}$, E. Coulon-Bidet ${ }^{2}$, J. $^{\text {Dimet }}{ }^{3}$

${ }^{1}$ Unité de Recherche Clinique, ${ }^{2}$ Service Restauration, CHD Vendée, La Roche Sur Yon, ${ }^{3}$ Centre de Recherche Clinique, $\mathrm{CH}, \mathrm{Mont}$ de Marsan, France

Introduction et but de l'étude : La dénutrition et la prévention de son risque chez les personnes âgées institutionnalisées constituent un enjeu de santé publique majeur. L'alimentation y est une problématique car souvent inadaptée aux capacités de préhension et aux besoins nutritionnels. Une offre alimentaire «manger-mains» (MM), mets saisis et consommés avec les doigts, pourrait être une solution. Cette approche n'est pas nouvelle mais les études sur le sujet manquent dans la littérature. Nous avons réalisé une étude dont l'objectif principal évaluait les apports caloriques à six mois. Parmi les objectifs secondaires, l'autonomie et le ressenti des soignants ont été évalués.

Matériel et méthodes : Etude pilote, prospective, randomisée, réalisée sur 5 Etablissements d'Hébergement pour Personnes Agées Dépendantes (EHPAD) du CH départemental de Vendée. Cette étude s'adressait aux résidents avec troubles cognitifs et/ou physiques ne s'alimentant pas seuls. Les résidents recevaient une alimentation classique ou MM par tirage au sort. L'offre MM était fabriquée à partir du menu habituel mixé, elle concernait les 2 repas principaux. La mesure calorique des ingesta a été réalisée mensuellement, sur 3 jours consécutifs, par une diététicienne en analysant les photos des plateaux repas avant et après consommation. Le ressenti des soignants a été évalué avec un questionnaire.

Résultats et Analyse statistique : L'étude s'est déroulée sur 7 mois. Sur les 269 résidents institutionnalisés, 47 ont été inclus (25 dans le groupe contrôle / 22 dans le groupe intervention MM). L'âge moyen des résidents était de 83,7 ans +/- 8,2, dont 72\% de femmes. Parmi eux, $64 \%$ étaient à risque de dénutrition, $36 \%$ dénutris et $81 \%$ présentaient une démence. Durant l'étude, 18 résidents du bras $M M$ sont sortis prématurément ( 3 décès, 1 retrait de consentement, 1 détérioration de l'état général et 13 perte d'appétit avec refus de tout ou partie de la prestation $\mathrm{MM}$ ). En analysant les variations d'apports caloriques résident par résident, jusqu'à 3 mois, dans le groupe MM, la plupart perdent moins d'apports caloriques que dans le bras standard et l'autonomie autour du repas est augmentée ; puis cette tendance s'inverse. Ceci s'explique certainement par la nouveauté de la prestation proposée aux résidents et l'aspect pratique de manger avec les doigts. A 3 mois, nous avons 9 sorties d'étude ou perte d'appétit avec refus de tout ou partie de la prestation MM, expliquées par la lassitude des résidents, en regard des questionnaires agents renseignés. Les soignants sont $69 \%$ à être satisfaits de ce concept et respectivement $56 \%$ et $83 \%$ à juger qu'il permet d'améliorer la quantité de mets consommés et l'autonomie.

Conclusion : Le «manger-mains» semble être une piste intéressante, pour améliorer les apports caloriques et l'autonomie des résidents d'EHPAD. Nouveauté et aspect pratique à consommer avec les doigts ont été des facteurs favorisant l'acte alimentaire. L'étude a permis d'identifier des pistes de travail pour améliorer la nutrition des résidents en manque d'autonomie, avec une optimisation sur la durée et le rythme de l'offre $\mathrm{MM}$; axes qui seront à évaluer par d'autres études.

Conflits d'intérêts : Aucun conflit à déclarer 


\section{P082}

Lors d'une inflammation intestinale à bas bruit, $S$. Thermophilus CRZ170 permet de limiter les pertes de masse maigre chez l'âgé

I. Savary-Auzeloux ${ }^{1}$, M. Jarzaguet ${ }^{1}$, J. David ${ }^{1}$, M. De Avezedo ${ }^{2}$, J.-M. Chatel ${ }^{2}$, D. Dardevet ${ }^{1, *}$

${ }^{1}$ Unité de Nutrition Humaine, INRA, CEYRAT, ${ }^{2}$ Unité Micalis, INRA, Jouy en Josas, France

Introduction et but de l'étude : Le vieillissement se traduit, au niveau systémique, par le développement d'une inflammation à bas bruit qui est un des éléments explicatifs du développement de plusieurs pathologies dont la sarcopénie. Au niveau du tube digestif (TD), une dégradation de son intégrité ainsi qu'une altération de son métabolisme et de sa flore endogène (Lynch et al. 2015) ont été démontrés, notamment une augmentation de la perméabilité intestinale et la génération d'une micro inflammation locale puis généralisée. Ces altérations physiologiques et métaboliques de l'intestin se traduisent par une compétition pour l'utilisation des nutriments entre le TD enflammé et les tissus périphériques comme le muscle. L'objectif de notre étude est donc de tester in vivo au cours du vieillissement, l'effet protecteur d'une souche bactérienne avec des propriétés anti-inflammatoires in vitro, sur une inflammation intestinale bas bruit et son impact la préservation de la masse maigre et le muscle

Matériel et méthodes : Des rats âgés $(18 \mathrm{~m})$ ont été traités au DSS $(4 \% \mathrm{p} / \mathrm{v})$ dans l'eau de boisson pendant $28 \mathrm{j}$ avec ou pas quotidiennement une dose de $S$. Thermophilus CRZ170 $\left(10^{9} \mathrm{CFU} / \mathrm{j}\right)$ présentant un potentiel anti-inflammatoire in vitro (Junjua.2016[d1] ). Ils ont été comparés à des rats témoins recevant la même quantité de nourriture (PF). Le poids corporel et l'ingéré ont été suivi quotidiennement, la masse maigre et la masse grasse mesurées par EchoMRI à J0, J14 et J28 et les poids de muscles et colon mesurés à $\mathrm{J} 28$ à l'abattage. L'analyse statistique a été faite par ANOVA et la significativité par test posthoc Fisher ( $p<0.05)$

Résultats et Analyse statistique : Pour les DSS, la perte de poids corporelle, de masse maigre, de masse grasse à J28 est significativement plus importante que celle des témoins PF (-110 vs $-86 \mathrm{~g} ;-51$ vs $-36 \mathrm{~g}$ et -65 vs $-47 \mathrm{~g}$, respectivement, $p<0.05)$. De même, les muscles gastrocnémien et le tibialis sont significativement plus petits de $12 \%$ et $10 \%$ vs $P F(p<0.05$ ). En revanche, le poids du colon est augmenté significativement de $13 \%$ avec le DSS. Le traitement avec CRZ170 a permis sous DSS de maintenir normal le poids du colon ( 2.09 vs $2.14 \mathrm{~g}$ ), de limiter la perte de masse maigre (-38 vs $-36 \mathrm{~g}$ ) et de limiter à 4 et $5 \%$ la perte de masse musculaire (CRZ vs PF et DSS non significatif).

Conclusion : Chez l'âgé, la perte de masse maigre et musculaire associée à une inflammation bas bruit d'origine intestinale peut être diminuée par l'ingestion de $S$. Thermophilus qui possèdent des propriétés anti-inflammatoires sur les entérocytes. II pourrait donc présenter une stratégie probiotique intéressante de lutte contre la fragilité au cours du vieillissement.

Conflits d'intérêts : Aucun conflit à déclarer 
Effets combinés d'une supplémentation en citrulline et d'un entraînement par intervalles de haute intensité sur le profil corporel, musculaire et fonctionnel de personnes âgées : Influence de l'apport protéique initial.

M. Aubertin-Leheudre ${ }^{12,{ }^{*}}$, L. P. Carvalho ${ }^{1}$, M. C. Dulac ${ }^{1}$, G. El Hajj Boutros ${ }^{1}$, P. Noirez ${ }^{3}$, J. A. Morais ${ }^{4}$, V. Marcangeli $^{1}$

${ }^{1}$ Faculté des Sciences, UQAM, ${ }^{2}$ CRIUGM, Montreal, Canada, ${ }^{3}$ Université Paris Descartes, Paris, France, ${ }^{4}$ Mc Gill University, Montreal, Canada

Introduction et but de l'étude : Le vieillissement normal est lié à des détériorations corporelles et fonctionnelles. L'entrainement par intervalles de haute intensité (EIHI) et la citrulline (CIT) semble être des options intéressantes pour contrer ces changements même si leur effet combiné est méconnu. De plus, les adaptations liées à ces 2 interventions semblent être médiées par la quantité de protéines consommée. Ainsi l'objet de ce projet est de vérifier chez des personnes âgées obèses 1) si, pour un même apport de protéines, la supplémentation en CIT induit de meilleures adaptations musculaires que sous placebo (PLA) lorsque combiné à un EIHI, et; 2) si, l'apport initial de protéines influence les adaptations suite à un EIHI combiné à une supplémentation en CIT.

Matériel et méthodes : Devis a-posterioriissu d'une ECR. Soixante-treize personnes âgées (>60ans) obèses (\% masse grasse $(\mathrm{MG})>27(\mathrm{H})$ et $35(\mathrm{~F})$ ) ont complété aléatoirement et à double insu 12 semaines d'une supplémentation de CIT ou PLA (10gr/j) combinée à un EIHI sur elliptique (3sessions/sem;30min/session). Elles ont été divisées en 4 groupes selon leur supplémentation et leur apport initial en protéines (<ou >1g/kgPC/j): $1-\mathrm{CIT}(n=19)$ vs. $1-P L A(n=14)$ vs. $1+C I T(n=21)$ vs. $1+P L A(n=19)$.

La composition corporelle (masses maigre (MM) et grasse totales, jambes, gynoïdes; DXAC), les habitudes de vie (apport alimentaire (3j; Nutrifit@) et nombre de pas/j (7j; armband sensewear (C), la force de préhension (FP/poids corporel; Lafayette @ ), la force (FMI; cellule de force) et puissance (PMI; Leg power rigC) des membres inférieurs, les capacités fonctionnelles (SPPB) et aérobie ( $6 \mathrm{~min}$ marche) ont été évaluées pré et post-intervention.

Résultats et Analyse statistique : Une $2 * 2$ ANOVA à mesures répétées (SPSS 22.0 C) a été utilisée pour répondre à nos objectifs $(P<0,05$ : significatif). Aucune différence n'a été observée avant l'intervention entre nos groupes sauf pour l'apport en protéines (par devis). L'EIHI améliore tous les paramètres corporels, musculaires et fonctionnels chez des personnes âgées obèses. Néanmoins, nous notons que le groupe 1-CIT améliore significativement plus la FP, les MG et MM des jambes et la MG gynoïde que les groupes 1-PLA ou 1+CIT. De plus, le groupe 1+CIT améliore significativement plus sa FMI que le groupe 1+PLA.En revanche, aucune différence significative entre les groupes 1-PLA et 1+PLA est observée.

Conclusion : La quantité de protéine initiale ne semble pas médier les adaptations suite à un EIHI. Par contre, la CIT lorsque combinée à un EIHI induit de meilleures améliorations et surtout chez ceux ingérant initialement peu de protéines. De futures études devront confirmer nos résultats prometteurs.

Remerciements : Nous remercions tous les participants et la technicienne Roy $\mathrm{C}$ pour son support. Cette étude a été financé par les agences suivantes RQRV, FRQS-UQAM, FCI, GRAPA. La supplémentation a été offerte gracieusement par Citrage(c). MAL et MD sont supportées par le fond de recherche du québec en santé (FRQS).

Conflits d'intérêts : Aucun conflit à déclarer 
P084

État nutritionnel des patients âgés d'un Établissement d'Hébergement pour Personnes Âgées Dépendantes (EHPAD) et liens entre la dénutrition, la consommation de compléments oraux et le service à l'assiette

F. Desbordes ${ }^{1, *}$, S. Jouveau ${ }^{2}$, A. Broussaudier ${ }^{2}$, V. Noailletas ${ }^{2}$, P. Fayemendy ${ }^{13}{ }^{4}$, B. Morin ${ }^{1}$, B. Misset ${ }^{1}$, F. Rebière ${ }^{1}$, J. C. Desport ${ }^{134}, \mathrm{P}$. Jésus ${ }^{134}$

${ }^{1}$ Unité de nutrition, CHU Limoges, ${ }^{2}$ Service diététique, Hôpital Chastaingt, ${ }^{3}$ Inserm UMR 1094 NET, Faculté de médecine, ${ }^{4}$ Réseau Limousin Nutrition (LINUT), Limoges, France

Introduction et but de l'étude : En Établissement d'Hébergement pour Personnes Âgées Dépendantes (EHPAD), la dénutrition est fréquente, avec des conséquences sévères. Paradoxalement, peu d'études en France ont déterminé le statut nutritionnel des résidents d'EHPAD et/ou l'influence de la consommation de compléments nutritionnels oraux (CNO) et de la pratique d'un service à l'assiette (SAA) sur l'état nutritionnel des résidents. Les objectifs de l'étude étaient de déterminer i) l'état nutritionnel de résidents dans un EHPAD de grande taille et ii) le lien éventuel entre la consommation de CNO, la pratique d'un SAA et la présence d'une dénutrition.

Matériel et méthodes : L'étude était descriptive rétrospective monocentrique. Les données étaient issues de l'ensemble des dossiers médicaux consultés entre juillet 2017 et janvier 2018. Le statut nutritionnel était déterminé selon les critères français pour les adultes et les personnes âgées. Les résultats sont présentés en médiane [IQ25-75] pour les variables quantitatives, et pourcentage pour les variables qualitatives. Des analyses univariées et une analyse multivariée étaient réalisées afin d'établir une association entre la présence d'une dénutrition et les variables d'intérêt enregistrées.

Résultats et Analyse statistique : Le nombre de lits de l'EHPAD était de 258. La population d'étude était constituée de 248 patients (âge : 88,0 [83,0-93,0] ans), dont 78,2\% de femmes, le poids était de 62,8 [52,9-74,4] kg, l'IMC de 25,7 [21,9-29,4] kg/m2, l'albuminémie de 35,8 [31,6-37,8] g/l. La CRP (médiane : 5 [2-11] mg/l) était prise en compte pour évaluer le statut nutritionnel. Au total, 39,5\% des résidents étaient dénutris, en cohérence avec les données usuelles (1). En analyse univariée, les patients dénutris étaient plus âgés, consommaient plus souvent des CNO et avaient moins souvent un service à l'assiette que les patients non dénutris (respectivement $p=0,002, p<0,0001, p=0,01$ ). En analyse multivariée, il existait une association négative entre la présence d'une dénutrition et le niveau d'IMC, l'albuminémie et la concentration en CRP. La prise de CNO et le SAA n'étaient pas associés à la dénutrition.

Conclusion : L'EHPAD étudié, bien que de grande taille, semblait représentatif des établissements publics français pour le nombre de lits, la répartition par âge et par genre des résidents. Près de $40 \%$ des patients étaient dénutris. L'analyse multivariée suggère que l'utilisation des CNO et la mise en œuvre du SAA dans cet EHPAD doivent être précisées.

Références : (1) Pauly L et al. Z Für Gerontol Geriatr. 2007;40:3-12

Conflits d'intérêts : Aucun conflit à déclarer 
Le blocage du système endocannabinoïde stimule la synthèse protéique via un mécanisme mTOR et calcium dépendant dans un modèle de myotubes en culture.

O. Le Bacquer ${ }^{1, *}$, K. Lanchais ${ }^{1}$, Y. Boirie ${ }^{12}$, S. Walrand ${ }^{1}$

${ }^{1}$ UMR1019, Unité de Nutrition Humaine, INRA/Université Clermont Auvergne, ${ }^{2}$ Service de Nutrition Clinique, CHU ClermontFerrand, Clermont-Ferrand, France

Introduction et but de l'étude : Les endocannabinoïdes (EC), dérivés des acides gras polyinsaturés synthétisés par l'organisme, sont des ligands des récepteurs cannabinoïdes. Ils exercent une action autocrine et paracrine en se liant à leurs récepteurs et contrôlent de nombreux processus métaboliques au sein des différents tissus de l'organisme. Au niveau musculaire, des études ont montré que les EC participent au contrôle de la signalisation de l'insuline et de la captation du glucose. Les EC régulent également la différenciation musculaire et modulent les capacités oxydatives du muscle, probablement via la biogenèse mitochondriale, comme précédemment observé dans les cellules neuronales. Dans une étude précédente, nous avons observé qu'un traitement au rimonabant (agoniste inverse du récepteur $\mathrm{CB} 1$ ) stimulait la synthèse protéique de myotubes C2C12 de façon dose- et temps-dépendant. L'objectif de cette étude était d'identifier les mécanismes responsables de la stimulation de la synthèse protéique musculaire par le rimonabant.

Matériel et méthodes : Après différenciation, des cellules $\mathrm{C} 2 \mathrm{C} 12$ (modèle de myotubes en culture) ont été incubées en présence de rimonabant. La synthèse protéique a été mesurée par Western Blot en utilisant la technique SUnSET (incorporation de puromycine dans les protéines naissantes). L'implication des voies Akt, ERK, mTOR a été évaluée par l'utilisation d'inhibiteurs spécifiques. L'implication du système calcique a été étudiée par l'utilisation de chélateurs de calcium ou de ryanodine/2-APB. Enfin le rôle de CB1 a été étudié en utilisant divers agonistes de ce récepteur. Les résultats ont été analysés par ANOVA à une voie et exprimés en moyenne \pm sem.

Résultats et Analyse statistique : En présence de $10 \mu \mathrm{M}$ de rimonabant, la synthèse protéique est accrue de $90 \%$ après $1 \mathrm{~h}$ de traitement $(p<0.05)$, et de $150 \%$ après $6 \mathrm{~h}(p<0.01)$. Cet effet n'est pas modifié par l'utilisation de wortmannine ou d'U0126 (respectivement inhibiteurs de la PI3K et de ERK), mais est diminué de $40 \%$ en présence de rapamycine (inhibiteur de mTOR, $\mathrm{p}<0.05$ ) et de façon dose-dépendante en présence de BAPTA-AM (chélateur intracellulaire de calcium, $p<0.05$ ). L'utilisation de ryanodine ou de 2-APB ne modifie pas l'effet du rimonabant sur la synthèse protéique, ce qui suggère que le relargage du calcium par le réticulum endoplasmique n'intervient pas dans ce processus. Enfin, différents agonistes de CB1 (AEA, MetAEA, ACEA ou 2AG) seuls ne modifient pas la synthèse protéique dans ce modèle de myotubes en culture, et ne modifie pas l'effet du rimonabant.

Conclusion : Dans des cellules $\mathrm{C} 2 \mathrm{C} 12$, une incubation courte en présence de rimonabant induit une stimulation de la synthèse protéique en faisant intervenir la voie mTOR et le calcium intracellulaire. Cet effet ne semble pas dépendre du calcium du réticulum endoplasmique et semble indépendant du récepteur CB1.

Conflits d'intérêts : Aucun conflit à déclarer 
Auton'Al 60 : Programme de Prévention de la perte d'autonomie des seniors de l'Oise

L. Lavefve ${ }^{1}$, J. Branchu ${ }^{1}$, B. Breil ${ }^{1}$, F. Depeint ${ }^{1}$, P. Pouillart ${ }^{1}$, C.-N. Niamba ${ }^{1}$, H. Younes ${ }^{1}$, C. Buche-Foissy ${ }^{1}$, A.-K. Illner ${ }^{1,}{ }^{*}$

${ }^{1}$ PANASH (Pôle d'Activité en Nutrition, Alimentation et Santé Humaine), Institut Polytechnique UniLaSalle, Beauvais, France

Introduction et but de l'étude : La perte d'autonomie varie selon les individus: elle n'est pas uniquement fonction de l'état de santé, mais aussi de l'environnement social et psychologique. Les facteurs de risque sont multiples, incluant le manque d'accès à une alimentation équilibrée, une activité physique ou du lien social. La prévention doit donc se fonder sur une approche multidimensionnelle, souvent peu étudiée par rapport à sa pertinence préventive.

Matériel et méthodes : Le projet Auton'Al 60 vise à prévenir la perte d'autonomie liée à l'alimentation, à l'activité physique et au bien-être mental auprès de bénéficiaires, âgés de 60 à 89 ans, avec un GIR 5 ou 6 dans l'Oise. Entre février et juillet 2018,7 ateliers de prévention ont été proposés dans 5 zones géographiques (urbaines et rurales). Un blog et une newsletter mensuelle ont été créés afin de garder un contact avec les bénéficiaires entre chaque atelier. L'évaluation s'est basée sur un entretien approfondi à To sur le niveau d'autonomie, les habitudes alimentaires et l'état de santé ainsi que sur 2 questionnaires auto-administrés à miparcours et à la fin du projet. L'analyse statistique a été réalisée à l'aide de SPSS 17.

Résultats et Analyse statistique : 69 ateliers ont été réalisés en 5 mois. Au total, 91 séniors ( $27,8 \%$ hommes - 71,9\% femmes) ont tous participé à au moins un atelier, avec un âge moyen de 69,1 ans $( \pm 6,3)$. Ils ont été $72,6 \%$ à être présents à 6 ou 7 ateliers, et $49,5 \%$ à réaliser l'ensemble des 7 ateliers. 6,7\% déclarent avoir un degré d'autonomie avec des aides ponctuelles ou être en perte d'autonomie. $32,6 \%$ de la population ont déclaré une absence de maladie chronique et $43,8 \%$ ont un IMC $\left(\mathrm{kg} / \mathrm{m}^{2}\right)$ normal. La prévalence de l'obésité est de 15,7\%.

$52,9 \%$ des participants interrogés en fin de projet ont indiqué avoir diminué leur consommation de produits riches en matières grasses, 60,9\% leur consommation de sucre, et 52,9\% affirment consommer moins de sel. $80 \%$ pratiquent du sport régulièrement à la fin du projet, contre $60 \%$ au début. Enfin, 23,2 \% des bénéficiaires ont démarré une nouvelle activité associative durant le projet, dans des associations sportives (5,5\%), culturelles (6,8\%), loisirs (2,7\%) ou sociales (8,2 \%). Cependant, la population âgée de 66 à 69 ans a été identifiée comme plus fragilisée : leur état de santé est perçu comme moins bon, ils pratiquent moins d’activité physique et ont un IMC ainsi qu'une prévalence de personnes en surpoids (61,9\%) plus importante que pour les autres tranches d'âge.

Le rythme auquel ont été proposés les ateliers a plu à tous les bénéficiaires, et le nombre d'ateliers a été jugé satisfaisant par 88,6 $\%$ des participants. Concernant les outils annexes, la newsletter est un outil qui semble plus pertinent et plus apprécié que le blog pour cette population.

Conclusion : Le projet Auton'Al 60 confirme l'hétérogénéité des personnes âgées par rapport à leur degré d'autonomie, mais aussi à leurs comportements vis-à-vis de leur santé. L'approche multidimensionnelle a montré une pertinence préventive satisfaisante. Des stratégies restent à définir pour proposer un outil de prévention plus adapté aux personnes isolées et en situation de précarité. Remerciements : Nous remercions tous les participants ainsi que la Conférence des financeurs du département de l'Oise.

Conflits d'intérêts : Aucun conflit à déclarer 
R. Mizouri ${ }^{1{ }^{*}}{ }^{*}$, I. Rezgani ${ }^{1}$, F. Boukhayatia ${ }^{1}$, K. Ben Naceur ${ }^{1}$, H. Zahra ${ }^{1}$, M. Khiari ${ }^{1}$, Y. Hatira ${ }^{1}$, A. Temssek ${ }^{1}$, B. M. Faika ${ }^{1}$

${ }^{1}$ Service $C$ de Nutrition, Institut National de Nutrition de Tunis, Tunis, Tunisie

Introduction et but de l'étude : Le sujet âgé diabétique est particulièrement fragile puisqu'il cumule les effets du vieillissement et de la maladie. Le diabète constitue la sixième cause de mortalité à cet âge compte tenu de la morbidité vasculaire, dont il est responsable.

L'objectif de cette étude était d'étudier le risque cardio-métabolique que représente le sujet âgé diabétique.

Matériel et méthodes : Il s'agissait d'une étude descriptive réalisée chez 100 diabétiques âgés de type 2 hospitalisés dans notre service pour diabète déséquilibré. Chaque patient a bénéficié d'un examen clinique complet, d'un bilan biologique avec un bilan de retentissement complet.

Résultats et Analyse statistique : L'âge moyen des patients est de 69,36 ans avec une prédominance de la tranche d'âge allant de 65 ans à 70 ans (56\%). La durée moyenne d'évolution de diabète était de 15 ans et dont 54\% étaient sous insulinothérapie. La majorité de ces patients avaient un diabète déséquilibré (78\%) avec une $\mathrm{HbA} 1 \mathrm{c}$ moyenne de 10,2\%. Chez ces patients les facteurs de risques cardiovasculaires soulevés étaient: la sédentarité (29\%), une obésité androïde (38,5\%), le tabagisme (28,6 \%), l'hypertension artérielle $(61,47 \pm 4,40 \%)$, une microalbuminémie (48\%), une hypertriglycéridémie (33\%) et une hypo-HDL cholestérolémie $(27,4 \%)$. L'atteinte cardiovasculaire était représentée essentiellement par une cardiopathie ischémique chez $13,11 \pm 3,05 \%$ de la population, l'artériopathie oblitérante des membres inférieurs chez 9,83 $\pm 2,69 \%$ et un accident vasculaire cérébrale chez $0,81 \pm 0,21 \%$ des cas. Sur le plan thérapeutique une statine a été reçue chez $49 \%$ des cas et des antiagrégants plaquettaires dans $36,6 \%$ des cas.

Conclusion : Le risque cardio-métabolique augmente parallèlement avec l'âge. La stratégie préventive et thérapeutique nécessite une bonne prise en charge du diabète et des différents facteurs de risque associés.

Conflits d'intérêts : Aucun conflit à déclarer 


\section{METABOLISME DES MACRO- ET MICRONUTRIMENTS}

\section{P088}

Impact d'un régime hyperlipidique sur la biochimie et l'ultrastructure cardiaque de modelé prepubere femelle

D. Sibouakaz ${ }^{1}$, K. Othmani-Mecif ${ }^{1,}{ }^{*}$, A. Fernane ${ }^{1}$, A. Taghlit ${ }^{1}$, S. Ait-Benali ${ }^{1}$, Y. Benazzoug ${ }^{1}$

${ }^{1}$ Faculté des Sciences Biologiques, Université des Sciences Biologiques H.B, Bab Ezzouar Alger, Alger, Algérie

Introduction et but de l'étude : Les effets délétères des régimes hyperlipidiques (HL) sur le métabolisme sont bien connus, et leur consommation s'accompagne souvent d'un surpoids et d'une atteinte cardiaque. Le but de l'étude est d'évaluer l'impact du régime enrichi en lipides sur les paramètres biochimiques et l'ultrastructure du ventricule cardiaque gauche du lapin femelle prépubère.

Matériel et méthodes : L'étude concerne 12 lapins pré-pubères femelles, un lot témoin (FT) et un lot soumis au régime hyperlipidique pendant 3 mois $(F R H L)(n=6)$ (régime standard $+1,5 \mathrm{~g}$ de graisse animale et végétale). Un suivi du poids corporel est réalisé et le ratio poids cœur/poids corporel des lapins est calculé. Nous évaluons la CK-Mb au niveau plasmatique et les teneurs en protéines totales et en calcium au niveau cardiaque par des méthodes enzymatiques. Les lipides totaux (LT) et les phospholipides (PL) cardiaques extraits selon la méthode de Folch et al. (1957) sont analysés par chromatographie sur couche mince (CCM). L'ultrastructure cardiaque est observée par microscopie électronique à transmission (MET). Une analyse comparative des différents résultats est réalisée entre le lot $\mathrm{RHL}$ et le lot témoin.

Résultats et Analyse statistique : Sous l'effet du RHL, une augmentation significative du poids corporel est constatée comparativement au lot FT $(p<0,0001)$. Le rapport poids du cœur / poids corporel est faible chez FRHL vs FT, indiquant que l'évolution corporelle dépasse l'évolution cardiaque $(p<0,01)$. Nous constatons que le régime $\mathrm{RHL}$ réduit légèrement la teneur protéique au niveau cardiaque chez les FRHL vs FT $(p<0,05)$. Pour le taux de calcium, au niveau du ventricule gauche du lot FRHL nous enregistrons une augmentation significative de sa valeur comparativement au lot $\mathrm{FT}(p<0,0001)$. II est noté au niveau du plasma du lot FRHL une augmentation de l'activité CK-MB $(p<0,05)$. L'analyse des classes lipidiques, après séparation par CCM, révèle une augmentation du taux des LT et des PL chez le groupe RHL, notamment pour la sphingomyéline et la lysophsphatidyl choline.

L'analyse du tissu cardiaque en microscopie électronique à transmission montre une diminution des myofibrilles qui apparaissent en dégénérescence avec une désorganisation du sarcomère, une abondance de mitochondries dispersées, des complexes jonctionnels endommagés et de multiples gouttelettes lipidiques. Les mitochondries altérées apparaissent gonflées, allongées avec une variation de leur taille, désorganisation de leurs crêtes et quelques figures de duplication.

Conclusion : La pathogénicité du régime hyperlipidique se traduit par un surpoids, une altération de la biochimie cardiaque de l'organisme animal pré-pubère femelle, avec de remarquables anomalies ultrastructurales au niveau du ventricule gauche.

Conflits d'intérêts : Aucun conflit à déclarer 
Introduction et but de l'étude : Le microbiote est considéré comme un organe à part entière dont les déséquilibres sont associés à la survenue de pathologies chroniques métaboliques, mais également prolifératives. La composition du microbiote est influencée par de nombreux facteurs, notamment alimentaires. Notre objectif est d'évaluer la relation entre les principaux composants alimentaires et la composition du microbiote fécal.

Matériel et méthodes : L'analyse d'enquêtes alimentaires sur 3j, (Nutrilog 3.11) a été mise en lien avec la composition du microbiote fécal, chez 30 femmes en bonne santé. Les analyses du microbiote ont été menées par séquençage de la région V3-V4 de l'ADNr 16S (MiSeq, Illumina ${ }^{\odot}$ ). Richesse, diversité microbienne, abondances et \% des phyla microbiens sont comparés entre le $1^{\text {er }}$ quartile (Q1) et le $4^{\text {ème }}$ quartile (Q4) d'apport des principaux macronutriments (test de Wilcoxon, SAS v 9.4): apport en glucides (simples et complexes), en protéines (végétales et animales), en lipides (AGS, AGMI, AGPI, $\Omega 3, \Omega 6$, LA, ALA, DHA et EPA) et alcool (résultats exprimés en médiane [Q1 vs Q4].

Résultats et Analyse statistique : La diversité (indice Shannon) et la richesse microbienne (indice Chao1) ne sont pas impactées par les variations d'apport de macronutriments. L'apport quotidien en alcool est associé à l'abondance bactérienne (Log bact. totales : $11,29[11.24-11,37]$ vs $11,46[11,39-11,57], p=0,0128)$, et l'apport en protéines animales à des différences d'abondance relative en Bacteroidetes $(p=0,032)$.

Les abondances relatives des principaux phyla sont significativement différents selon l'apport lipidique total, avec notamment une abondance relative plus importante en Firmicutes $(58,54 \%[52,44-60,60]$ vs $51,27 \%[45,51-54,49]$, $p=0,04)$ et plus faible en Bacteroidetes $(33,69 \%$ [29,48-36,39] vs 41,89\% [34,26-45,11], $p=0,04)$ pour les forts consommateurs. Un apport accru en acides gras saturés est associé de façon significative à une diminution de l'abondance relative du phylum Proteobacteria (3,94\% [2,79$4,62]$ vs $1,79 \%[1,28-3,23], p=0,04)$ et une diminution du ratio Firmicutes/Bacteroidetes $(2,05[0,94-2,48]$ vs 0,40 [0,27-0,72], $p=0,009)$. De la même façon, les abondances relatives des phyla bactériens diffèrent significativement selon l'apport en AGMI et AGPI, notamment les AGPI $\Omega 6$ avec une abondance relative augmentée du phylum Verrucomicrobia associée à un apport accru en LA $(0,88 \%[0,12-1,38]$ vs 2,9\% [1,92-4,27], $p=0,003)$. Les AGPI 23 , tels que EPA et le DHA, sont associés à une diminution de l'abondance relative de certains phyla en particulier Proteobacteria $(p=0,005$ et $p=0,01)$, respectivement.

Conclusion : L'analyse de la composition du microbiote fécal en lien avec les apports nutritionnels de femmes en bonne santé met en évidence la relation entre les apports journaliers en alcool, protéines animales et végétales et l'abondance relative des principaux phyla. L'apport lipidique apparait étroitement lié à la composition de la flore, selon le type d'acides gras considéré. Cette étude préliminaire suggère qu'une modulation du microbiote pourrait être testée à travers des apports nutritionnels, notamment lipidiques, adaptés.

Conflits d'intérêts : Aucun conflit à déclarer 
P090

Relations entre nutrition avant la grossesse, polymorphisme du gène FADS et statut en acides gras polyinsaturés pendant la grossesse dans la cohorte EDEN.

E. Petit ${ }^{1}$, M. Armand ${ }^{2}$, M. De Agostini ${ }^{1}$, A. Forhan ${ }^{1}$, M.-A. Charles ${ }^{1}$, B. Heude ${ }^{1}$, J. Y. Bernard ${ }^{1, *}$

${ }^{1}$ Centre de recherche en Epidemiologie et Statistique Sorbonne Paris Cité, Inserm, Villejuif, ${ }^{2}$ Centre de Résonance Magnétique Biologique et Médicale, Université Aix-Marseille, CNRS, Marseille, France

Introduction et but de l'étude : Les acides gras polyinsaturés à longue chaîne (AGPI-LC), acides arachidonique (AA, $n-6)$ et docosahexaénoïque (DHA, n-3), jouent un rôle clé dans le bon développement du fœtus. Ils sont à la fois apportés par l'alimentation et biosynthétisés à partir des acides gras essentiels précurseurs, les acides linoléique (LA, n-6) et alpha-linolénique (ALA, n-3) sous le contrôle notamment des gènes FADS (Fatty Acid Desaturase). Notre objectif était d'examiner les associations entre la nutrition maternelle, un polymorphisme nucléotidique (SNP) des gènes FADS et le statut en AGPI pendant la grossesse.

Matériel et méthodes : L'étude EDEN est une cohorte mère-enfant française de 2002 femmes enceintes recrutées entre 2003 et 2006 dans les maternités de Poitiers et Nancy. Des échantillons de sang maternel (à 24-28 semaines de grossesse) et du cordon ombilical (à l'accouchement) ont été prélevés. Le profil en AGPI n-6 et n-3 des membranes des érythrocytes (en \% des acides gras totaux) a été analysé par chromatographie en phase gazeuse. Un questionnaire de fréquence alimentaire, couplé à une table de composition des aliments, a permis d'estimer les quantités d'AGPI consommés (en \% des acides gras totaux) avant la grossesse. Le SNP rs174546 (C/T) sur le gène FADS1 a été génotypé à partir de l'ADN maternel. Les associations entre apport nutritionnel d'un AGPI donné et sa teneur dans les érythrocytes ont été étudiées avec des coefficients de corrélation partielle de Spearman. Les associations entre le statut en AGPI et le variant rs174546, ainsi que les interactions entre rs174546 et l'apport nutritionnel, ont été étudiées par régression linéaire.

Résultats et Analyse statistique : Au total, 1595 femmes disposaient de données sur la nutrition et le statut en AGPI ; 1416 femmes disposaient aussi du génotypage de rs174546 parmi lesquelles 49,4\% était homozygotes pour l'allèle majeur (CC) et 9,9 \% l'était pour l'allèle mineur (TT). Les teneurs moyennes ( \pm écart-type) de LA, d'AA et de DHA dans les érythrocytes maternels étaient respectivement de $7,1 \%( \pm 0,8), 12,9 \%( \pm 0,8)$ et $5,3 \%( \pm 0,9)$. L'apport alimentaire en DHA avant la grossesse était corrélé positivement avec sa teneur dans le sang maternel (DHA : $r=0,34$ ) tandis que les AGPI $n-6$ ne l'étaient pas ou peu (LA : $r=0,11 ; A A$ : $r=0.01)$. Les femmes CC avaient plus de DHA et de AA que les femmes TT $(0,40$ [IC $95 \%: 0,23 ; 0,56]$ et $0,94[0,80 ; 1,08])$, mais moins de LA et de $\operatorname{ALA}(-0,74[-0,88 ;-0,60]$ et $-0,019[-0,025 ;-0,013])$. Le variant rs 174546 expliquait une plus forte part de la variance des teneurs des érythrocytes en AGPI n-6 (LA : 8,9\% de la variance; AA : 14,9\%) qu'en AGPI n-3 (ALA : 3,5\% ; DHA : 1,6\%). Aucunes interactions entre le variant rs174546 et l'apport nutritionnel en AGPI n'ont été observées.

Conclusion : L'étude EDEN confirme l'existence d'une relation entre l'apport nutritionnel et le statut en DHA pendant la grossesse, ainsi qu'un rôle modeste de FADS. Le statut en AGPI n- 6 des érythrocytes semble en revanche plus fortement déterminé par le facteur génétique que nutritionnel, et ce de manière indépendante l'un de l'autre.

Remerciements : Prix IDF/FRM pour les Sciences de I'Alimentation 2015.

Conflits d'intérêts : Aucun conflit à déclarer 
Analyse des relations entre la consommation de produits laitiers et le syndrome métabolique chez l'homme par métabolomique F. Capel ${ }^{1,}{ }^{*}$, V. Bongard ${ }^{2}$, C. Malpuech-Brugère ${ }^{1}$, E. Karoly ${ }^{3}$, G. Michelotti ${ }^{3}$, J.-P. Rigaudière ${ }^{4}$, C. Jouve ${ }^{4}$, J. Ferrieres ${ }^{2}$, C. Marmonier ${ }^{5}$, J.-L. Sebedio ${ }^{1}$

${ }^{1}$ Unité de Nutrition Humaine, Université Clermont Auvergne, INRA, CRNH Auvergne, Clermont Fd, ${ }^{2}$ Epidémiologie, Economie de la Santé et Santé Publique, INSERM-CHU, Toulouse, France, ${ }^{3}$ Metabolon, Durham, États-Unis, ${ }^{4}$ Unité de Nutrition Humaine, INRA, Clermont Fd, ${ }^{5}$ Service Recherche Nutrition-Santé, CNIEL, Paris, France

Introduction et but de l'étude : Bien que des études récentes ont démontré que la consommation de produits laitiers peut protéger du syndrome métabolique (MetS), les mécanismes restent méconnus. Dans cette étude observationnelle, nous avons analysé comment la consommation de produits laitiers pouvait modifier les perturbations du métabolome plasmatique induites par le MetS.

Matériel et méthodes : Une analyse du métabolome par HPLC-MS/MS et du profil en acides gras par chromatographie gazeuse a été effectuée dans le plasma d'une population de 298 participants (61 avec MetS) de l'étude française MONALISA. L'échantillon a été stratifié en 6 groupes $(n=48-53)$ en fonction du niveau de consommation de produits laitiiers exprimé en portions par jour $(<1.5$, entre 1.5 et 2.15 , entre 2.15 et 2.75 , entre 2.75 et 3.5 , entre 3.5 et $4.3,>4.3$. Les comparaisons entre les sujets sains et atteints de MetS ont été faites à l'aide du test du Chi2 pour les variables catégorielles et un test de Student pour les variables continues. Les analyses statistiques des données de métabolomiques ont été faites par des ANOVA à 1 ou 2 voies selon la comparaison avec une prise en compte du taux de faux positif, FDR<0.1. Des corrélations entre les concentrations plasmatiques des différents métabolites et les paramètres biologiques ont été faites en utilisant la méthode de Pearson en considérant un FDR<0.1.

Résultats et Analyse statistique : Des altérations majeures de nombreuses voies métaboliques ont été observées chez les sujets atteints de MetS. Des différences significatives dans les taux plasmatiques d'acides aminés à chaîne ramifiée, de di-peptides comprenant un gamma-glutamate et de métabolites de l'arginine et de la proline ont été observées entre les sujets témoins et les sujets Mets. Les concentrations plasmatiques de nombreuses espèces lipidiques étaient également augmentées avec le MetS (mono- et diacylglycérols, eicosanoïdes, lysophospholipides et lysoplasmalogènes), avec une diminution des acides gras à chaîne courte et des plasmalogènes. La composition plasmatique en acides gras est fortement perturbée par le MetS. Certaines perturbations du métabolome liées au MetS sont modulées avec la consommation de produits laitiers, plus spécifiquement ceux à faible teneur en lipides (lait et produits laitiers frais). Elles concernent les dipeptides issus d'altérations du métabolisme du glutathion et d'une protéolyse partielle de protéines. En outre, nous confirmons que la consommation de produits laitiers augmente la concentration plasmatique en C15:0.

Conclusion : La consommation de produits laitiers, notamment le lait et les produits laitiers frais à teneur réduite en lipides, pourrait atténuer certaines altérations métaboliques liées au MetS en réduisant l'inflammation et le stress oxydatif.

Conflits d'intérêts : F. Capel: Aucun conflit à déclarer, V. Bongard: Aucun conflit à déclarer, C. Malpuech-Brugère: Aucun conflit à déclarer, E. Karoly: Aucun conflit à déclarer, G. Michelotti: Aucun conflit à déclarer, J.-P. Rigaudière: Aucun conflit à déclarer, C. Jouve: Aucun conflit à déclarer, J. Ferrieres: Aucun conflit à déclarer, C. Marmonier est employé(e) de CNIEL, J.-L. Sebedio: Aucun conflit à déclarer 
Un apport élevé d'Amidon Lentement Digestible diminue les réponses glycémique et insulinémique de façon équivalente dans la population caucasienne et asiatique

A. Goux ${ }^{1, *}$, L. Neufcourt ${ }^{1}$, O. Brack ${ }^{2}$, F. Atkinson ${ }^{3}$, S. Vinoy ${ }^{1}$

${ }^{1}$ Nutrition Research, Mondelez International, Saclay, ${ }^{2}$ SOCIETE K.S.I.C., Esches, France, ${ }^{3}$ Sydney University's Glycemic Index Research Service (SUGiRS) , The University of Sydney, Sydney, Australie

Introduction et but de l'étude : L'intolérance au glucose ainsi que le diabète de type 2 sont des pathologies de plus en plus répandues dans le monde. De nombreux travaux de recherche ont montré que les sujets de phénotype asiatique auraient une moins bonne tolérance au glucose que les caucasiens. Par ailleurs, il a été démontré dans la population caucasienne que la consommation de produits présentant un contenu élevé en amidon lentement digestible (Slowly Digestible Starch, SDS) diminue la réponse glycémique postprandiale significativement par rapport à des produits amylacés pauvre en SDS. Le but de cette étude est d'évaluer les réponses glycémique et insulinémique postprandiales de sujets asiatiques et de sujets caucasiens consommant des produits présentant des profils de digestibilité de l'amidon différents.

Matériel et méthodes : Cinq produits céréaliers présentant des profils de digestibilité de l'amidon différents (détermination par la méthode SDS) et une solution de glucose ont été testés. Une étude randomisée contrôlée, et croisée mesurant les réponses et indexes glycémiques (IG) et insulinémiques (II) des produits sélectionnés pendant deux heures postprandiales a été réalisée à l'université de Sydney. 12 sujets caucasiens et 12 sujets asiatiques ont été inclus dans l'étude et ont consommé $50 \mathrm{~g}$ de glucides disponibles de chaque produit, selon la norme ISO-26642(2010). Les analyses statistiques ont été réalisées avec Jump-12-Pro.

Résultats et Analyse statistique : Les sujets inclus avaient un âge et un indice de masse corporel moyen de 28,0 $\pm 2,6$ ans et de $21,4 \pm 0,34 \mathrm{~kg} / \mathrm{m}^{2}$ pour les asiatiques et $26,0 \pm 1,1$ ans et $22,4 \pm 0,52 \mathrm{~kg} / \mathrm{m}^{2}$ pour les caucasiens. Parmi les produits céréaliers testés, 3 produits présentaient un contenu élevé en SDS (entre 26 et $28 \mathrm{~g}$ de SDS $/ 100 \mathrm{~g}$ de produit) et deux contenaient peu de SDS (de 0 à $2 \mathrm{~g}$ de SDS/100g). Les IG des produits riches en SDS étaient bas et variaient entre 40 et 48 pour les sujets caucasiens et entre 44 et 54 pour les sujets asiatiques. Les produits pauvres en SDS présentaient des IG moyens ou élevés, avec des valeurs de 60 et 79 pour les sujets caucasiens et 64 et 90 pour les sujets asiatiques. L'étude de différents paramètres des réponses glycémique et insulinémique (IG ou II, aire incrémentale sous la courbe iAUC, valeur de pic) par des modèles statistiques incluant les effets produits, ethnie, session et l'interaction produit*ethnie démontre que l'effet de l'ethnie n'explique pas les réponses physiologiques observées. Seul l'effet produit est significatif et permet de différencier les groupes de produits en fonction de leur contenu en SDS. De plus, les produits avec un contenu élevé en SDS diminuent la valeur du pic de glycémie d'environ 1 mM, que ce soit chez des asiatiques ou des caucasiens. En ce qui concerne la réponse insulinémique, les produits riches en SDS induisent une demande en insuline plus faible comparé aux produits riches en SDS, de $29 \%$ chez les asiatiques et de $32 \%$ chez les caucasiens. Conclusion : Notre étude démontre que la consommation de produits riches en SDS permet de diminuer la réponse glycémique et insulinémique, et ce quelle que soit l'ethnie des sujets. Cette diminution peut avoir un intérêt à long terme dans la prévention des maladies métaboliques.

Conflits d'intérêts : A. Goux est employé(e) de Mondelez International, L. Neufcourt a un conflit d'intérêt avec Ancien Stagiaire chez Mondelez International, O. Brack est consultant chez Mondelez International pour ce travail, F. Atkinson: Aucun conflit à déclarer,

S. Vinoy est employé(e) de Mondelez International 
P093

Pharmacocinétique d'une nouvelle forme galénique de vitamine D3 100000 UI en capsule molle

P. Fardellone ${ }^{1, *}$, R. Mentaverri ${ }^{2}$, G. Brami ${ }^{3}$, J. C. Souberbielle ${ }^{4}$

${ }^{1}$ Rhumatologie, ${ }^{2}$ Pharmacie, CHU Amiens, Amiens, ${ }^{3}$ Laboratoires IPRAD, ${ }^{4}$ Exploration fonctionnelle, APHP Necker, Paris, France

Introduction et but de l'étude : Dans le contexte d'une carence fréquente en vitamine $D$ et d'une supplémentation nécessaire, il est souhaitable de mettre à disposition des patients et des professionnels de la santé une gamme plus large de médicaments à base de vitamine D3.

Matériel et méthodes : Une étude de bioéquivalence en ouvert, randomisée, groupes parallèles a comparé une dose unique d'une nouvelle forme galénique en capsule molle contenant $100000 \mathrm{UI}$ de vitamine D3 (Groupe 1) et la vitamine D3 100000 UI solution buvable en ampoule (Groupe 2) chez des sujets sains (EudraCT N²013-002041-10). Les sujets ont été suivis pendant une période de 4 mois. Le critère principal de l'étude était l'aire sous la courbe (ASC) des concentrations sériques de 25-hydroxyvitamine-D (25(OH)D) au Jour 112.

Résultats et Analyse statistique : Cette étude a été menée dans le nord de la France entre février et mai 2014 chez 53 volontaires sains, âgés en moyenne de 26,9 ans. La population de sujets inclus dans les 2 groupes était homogène, sans aucune différence entre les groupes. De faibles concentrations sériques de 25(OH)D ont été observées dans les deux groupes (10,6 ng/ml dans le Groupe 1 et 9,0 $\mathrm{ng} / \mathrm{ml}$ dans le Groupe 2).

En fin d'étude à J112, I'ASC des concentrations sériques de 25(OH)D était de 2499,4 $\pm 463,8 \mathrm{nmol} / \mathrm{ml}(7,8 \pm 0,2$ pour LogAUC) pour le Groupe 1 et $2152,3 \pm 479,8 \mathrm{nmol} / \mathrm{ml}(7,6 \pm 0,2$ pour LogAUC) pour le Groupe 2 . L'intervalle de confiance à $90 \%$ de la différence [$0,26 ;-0,06]$ n'était pas compris dans les limites d'équivalence $[-0,223 ; 0,223]$. Par conséquent, la bioéquivalence des deux traitements n'a pas été démontrée. En revanche, le test de supériorité a conclu à une supériorité de la vitamine D3 en capsule molle par rapport à la vitamine D3 en ampoule en utilisant un test non paramétrique de Wilcoxon. Cette supériorité a été observée avec $p=0,029$ pour l'ASC et $p=0,03$ pour LogAUC. Le modèle ANCOVA a également conclu à une différence significative ( $p=0,0093)$ pour LogAUC entre les deux groupes.

Le profil de la concentration sérique de $25(\mathrm{OH}) \mathrm{D}$ a montré une différence statistiquement significative en faveur du Groupe 1 à $\mathrm{J} 1, \mathrm{J3}, \mathrm{J} 7, \mathrm{~J} 14$ et J90. La concentration sérique moyenne de 25(OH)D dans le Groupe 1 était comprise entre 20 et $30 \mathrm{ng} / \mathrm{ml}$ pendant les 4 mois de suivi, alors qu'elle était inférieure à $20 \mathrm{ng} / \mathrm{ml}$ durant toute l'étude dans le Groupe 2, hormis à J112.

La Cmax moyenne dans le Groupe $1(28,5 \pm 5,0 \mathrm{ng} / \mathrm{ml})$ était significativement plus élevée $(p=0,002)$ que la Cmax moyenne dans le groupe $2(23,9 \pm 4,3 \mathrm{ng} / \mathrm{ml})$. Le Tmax a été atteint en seulement 14 jours dans plus de la moitié des sujets ayant reçu une capsule molle de 100000 UI de vitamine D3 alors qu'il a été atteint en 45 jours dans le Groupe 2.

Les deux traitements ont été bien tolérés. Aucun effet indésirable grave ou lié au traitement n'a été rapporté au cours de l'étude. Conclusion : Le profil pharmacocinétique de la nouvelle forme galénique de vitamine D3 100000 Ul en capsule molle était supérieur à celui de la solution buvable en ampoule. Il a été démontré qu'une dose unique de 100000 UI de vitamine D3 en capsule molle pouvait augmenter le taux sérique de $25(\mathrm{OH}) \mathrm{D}$ au-dessus de $20 \mathrm{ng} / \mathrm{ml}$ et le maintenir entre 20 et $30 \mathrm{ng} / \mathrm{ml}$ pendant 4 mois en période d’hiver. La nouvelle formulation de vitamine D3 était bien tolérée.

Conflits d'intérêts : P. Fardellone est consultant chez AMGEN, Expanscience, JANSEN-CILAG, Roche, Lilly, MSD, Nordic-Pharma, TEVA, UCB, IPRAD, Abbvie, R. Mentaverri: Aucun conflit à déclarer, G. Brami est employé(e) de IPRAD, J. C. Souberbielle est consultant chez DiaSorin, Siemens, Roche Diagnostics, Rottapharm/Mylan, Effik, Lilly, Amgen 
Introduction et but de l'étude : La qualité des sources alimentaires de protéines est devenue une question particulièrement sensible dans le cadre des débats actuels sur un rééquilibrage entre les sources alimentaires animales et végétales. Les protéines sont un composant alimentaire indispensable à la survie, fournissant de l'azote et des acides aminés (AA), et en particulier les neuf acides aminés indispensables (AAI). En outre, parmi les AAl, la lysine et la thréonine sont dits strictement indispensables car ne participant pas aux processus de transamination. La capacité d'une protéine à couvrir les besoins nutritionnels en AAl est à la base de l'évaluation de la qualité des sources alimentaires de protéines. L'objectif de cette étude était de caractériser l'impact de régimes à base de gluten déficients en lysine et en thréonine sur le métabolisme de rats en croissance et d'identifier des biomarqueurs de ces régimes. L'hypothèse est qu'un déficit en un AAl entraine une activation des systèmes enzymatiques de catabolisme des autres AA, produisant des métabolites spécifiques de ces dégradations détectables dans les fluides biologiques et susceptibles de représenter des biomarqueurs de tels régimes.

Matériel et méthodes : Des rats en croissance ont été nourris pendant 3 semaines avec un régime à base de gluten supplémenté en thréonine et déficient à $70 \%$ en lysine, un régime à base de gluten supplémenté en lysine et déficient à $47 \%$ en thréonine, un régime à base de gluten supplémenté en lysine et en thréonine couvrant $100 \%$ des besoins en tous les $A A$, et un régime témoin à base de protéine de lait couvrant $100 \%$ des besoins en tous les AA (APAFiS \#5497). Le poids et la prise alimentaire ont été mesurés quotidiennement. A la fin de l'expérience, les tissus et fluides biologiques ont été prélevés. La composition corporelle a été analysée, des mesures d'expression de gènes impliqués dans le métabolisme protéique et lipidique ont été réalisées et le métabolome urinaire a été analysé par LC-MS. L'analyse statistique a été faite par analyse de variance et l'analyse des métabolomes par analyse discriminante des composants indépendants (IC-DA).

Résultats et Analyse statistique : La déficience en ces AAI ne modifie pas la prise alimentaire. La déficience en lysine induit une diminution du gain de poids corporel, et de la masse maigre, en lien avec une augmentation de la protéolyse et une diminution de la protéosynthèse, une diminution de la densité minérale osseuse, et aucun effet sur le métabolisme lipidique. La déficience en thréonine, induit une diminution du gain de poids corporel, et du poids du foie et de la peau, sans modification du métabolisme protéique, de la densité minérale osseuse et métabolisme lipidique. Après validation du modèle de déficience, l'analyse métabolomique réalisée sur des échantillons d’urine a mis en évidence la présence de molécules discriminantes spécifiques des régimes et des types de protéines.

Conclusion : La déficience en un AAI a un impact sur la croissance et le métabolisme osseux et protéique de rats en croissance. Ces états de déficience se sont traduits par des profils de métabolomes différents qui pourraient conduire à l'identification de biomarqueurs spécifiques des sources de protéines et des déficiences associées en AAI.

Conflits d'intérêts : Aucun conflit à déclarer 
${ }^{1}$ Laboratoire de Recherche en Surveillance et Epidémiologie Nutritionnelles en Tunisie, Institut National de Nutrition et de Technologie Alimentaire, ${ }^{2}$ Laboratoire de toxicologie, Unité de toxicologie, recherche et environnement, Centre d'assistance médicale urgente, Tunis, Tunisie, ${ }^{3}$ UMR NUTRIPASS, IRD-Université de Montpellier-Montpellier Supagro, Montpellier, France

Introduction et but de l'étude : L'obésité constitue un problème de santé publique mondial notamment du fait de sa forte progression dans les pays du sud dont particulièrement la région Afrique du Nord et Moyen Orient. Aussi, nombre de ces pays ont instauré des programmes d'iodation universelle du sel (IUS) pour réduire la carence en iode. Mais des processus d'iodation mal contrôlés conduisent souvent à la commercialisation de sel excessivement iodé. Cela augmente le risque d'apports excessifs en iode, avec, dans ces contextes de transition nutritionnelle, un effet cumulatif potentiel dû à la consommation d'aliments gras, sucrés et salés (également facteurs du surpoids et des maladies chroniques associées). Nous évaluons la cooccurrence intra-sujet du surpoids et d'un statut en iode inadéquat

Matériel et méthodes : Une enquête nationale a été réalisée en Tunisie en 2012 chez les enfants de 6-12 ans ( $n=1560)$. Le surpoids était IMC (Indice de Masse Corporelle) pour l'âge $\geq+1$ z-score (OMS, 2007). Les seuils de concentration de l'iode urinaire (CIU), étaient $\mathrm{CIU}<100 \mu \mathrm{g} / \mathrm{l}$ (carence) et $\mathrm{CIU} \geq 200 \mu \mathrm{g} / \mathrm{l}$ (excès) selon OMS, 2007. Nous avons étudié les double charges intra-sujet " surpoids - carence en iode » (Sp-Cl) et « surpoids - excès d'iode " (Sp-EI). Les associations brutes et ajustées avec un ensemble de facteurs sociodémographiques ont été estimées par des modèles de régression logistique.

Résultats et Analyse statistique : Les prévalences de surpoids, de carence en iode et d'excès d'iode étaient respectivement de $18,4 \%$ (95 \% Cl:[15,5-21,7]), 11,4 \% (95 \% Cl:[8,6-14,9]) et 52,2\% (95\% Cl:[50,1-62,2]). La prévalence de Sp-Cl était de 2,7 \% (95\% $\mathrm{Cl}:[1,4-5,3])$ alors que celle de Sp-El était de 9,8\% (95\% Cl:[7,7-12,3]). La carence ou l'excès d'iode étaient indépendants du surpoids ( $P=0,48$ et $P=0,23)$. La prévalence de $S p$-El semblait augmenter avec l'âge $(\mathrm{OR}=1,7 ; 95 \% \mathrm{Cl}:[1,1-2,7])$, alors qu'un niveau $d^{\prime}$ instruction bas chez la mère constituait un facteur de protection $(\mathrm{OR}=0,6 ; 95 \% \mathrm{Cl}:[0,4-0,9])$.

Conclusion : Dans ce contexte, chez les enfants d'âge scolaire, la cooccurrence du surpoids et du statut inadéquat en iode touchait seulement un enfant sur dix et les deux problèmes nutritionnels semblaient indépendants. Des recherches supplémentaires sont néanmoins nécessaires pour confirmer ces résultats notamment chez les adultes. Toutefois, dans ces contextes, il est possible que la prévalence de Sp-El augmente au cours des prochaines années dans la mesure où : - le sel est une molécule obésogène, la surconsommation de sel prédispose à l'excès d'apport en iode (d'autant plus si le sel est excessivement iodé), - un apport inadéquat en iode peut être la cause d'hypothyroïdisme, désordre métabolique souvent associé à un gain de poids. Les programmes d'IUS devront donc être cordonnés avec les stratégies de lutte contre l'obésité et des maladies chroniques (dont la réduction de la consommation de sel), pour avoir l'effet escompté de ces différentes interventions en population.

Conflits d'intérêts : Aucun conflit à déclarer 
Introduction et but de l'étude : La demande mondiale en protéines connait une forte croissance et il parait nécessaire de proposer de nouvelles alternatives durables et de bonne qualité nutritionnelle aux protéines d'origine animale. Les légumineuses sont de bons candidats en raison de leur profil d'acides aminés indispensables (AAI) relativement équilibré. Notre objectif est d'évaluer à travers différents indices la qualité nutritionnelle d'un isolat de protéine de pois (NUTRALYS ${ }^{\circledR}$ pea protein) seul ou complémenté en méthionine (Met). Pour cela, nous avons évalué chez le rat le «Protein Efficiency Ratio » (PER), le « Digestible Indispensable Amino Acid Score " (DIAAS) et le bilan azoté.

Matériel et méthodes : Etude $1: 40$ rats Wistar mâles de $50 \mathrm{~g}$ ont été nourris ad libitum pendant 28 jours avec un régime contenant $10 \%$ de protéines dont seule la source de protéine varie. 5 groupes $(n=8)$ ont été constitués: pois, caséine, gluten de blé, combinaison pois-gluten (indice chimique 100), pois supplémenté en Met (à concentration équivalente à la caséine). La prise alimentaire et le poids corporel ont été relevés quotidiennement pour le calcul du PER, obtenu par le rapport entre le gain de poids corporel et l'apport protéique sur toute la période.

Etude $2: 45$ rats Wistar mâles de $250 \mathrm{~g}$ ont été placés en cage métabolique pendant 2 jours et nourris avec un régime contenant $14 \%$ de protéines de différentes sources. 5 groupes $(n=9)$ ont été constitués : pois, caséine, gluten, pois supplémenté en Met, régime exempt de protéine. La prise alimentaire a été mesurée quotidiennement. L'azote contenu dans les régimes, les fèces et les urines a été dosé par analyseur élémentaire pour établir un bilan azoté (azote ingéré - azote perdu). Les rats ont ensuite reçu un repas calibré contenant un marqueur indigestible (oxyde de chrome) et ont été euthanasiés 6h après l'ingestion. Les contenus de l'estomac, de l'intestin grêle, de l'iléon, du cæcum et du côlon ont été collectés. Le DIAAS a été calculé selon la formule suivante : mg AAl digestible dans $1 \mathrm{~g}$ de protéine testée*100/mg de cet AAl dans $1 \mathrm{~g}$ de protéine de référence. Les AAl du contenu iléal ont été dosés par HPLC par la méthode AccQtag ultra, et le chrome a été dosé par spectrométrie d'absorption atomique. Les pertes endogènes ont été estimées grâce au groupe protéiprive.

Résultats et Analyse statistique : Les résultats sont présentés sous la forme moyenne $\pm E T$. L'effet régime a été testé par une ANOVA avec ajustement de Bonferroni.

\begin{tabular}{|l|l|l|}
\hline Protéine & PER $\mathbf{( g / g )}$ & Bilan azoté $(\mathbf{m g})$ \\
\hline Pois & $1,14 \pm 0,27 a$ & $230,8 \pm 57,4 a$ \\
\hline Caséine & $2,55 \pm 0,27 b$ & $315,1 \pm 43,0 b$ \\
\hline Gluten & $0,47 \pm 0,19 c$ & $235,1 \pm 86,7 a$ \\
\hline Pois+gluten & $1,60 \pm 0,16 d$ & $/$ \\
\hline Pois+ Met & $2,52 \pm 0,33 b$ & $311,6 \pm 72,5 b$ \\
\hline
\end{tabular}

La supplémentation du pois en Met permet d'obtenir un PER identique à la caséine. Les bilans azotés sont tous positifs et supérieurs pour les groupes caséine et pois supplémenté en Met. Les analyses d'AA sont en cours pour le calcul du DIAAS.

Conclusion : Chez le rat en croissance dont les besoins en Met sont élevés, le déficit en Met du pois n'est pas compensé par un apport de gluten. Le pois permet cependant une meilleure croissance que le gluten et sa supplémentation en Met permet d'atteindre les performances de la caséine.

Conflits d'intérêts : F. Guillin est employé(e) de Roquette, J. Calvez: Aucun conflit à déclarer, L. Guérin-Deremaux est employé(e) de Roquette, C. Lefranc-Millot est employé(e) de Roquette, D. Azzout-Marniche: Aucun conflit à déclarer, N. Khodorova: Aucun conflit à déclarer, C. Gaudichon: Aucun conflit à déclarer 
${ }^{1}$ Nutrition, Institut Pasteur de Lille, ${ }^{2} \mathrm{CHRU}$ de Lille, Lille, France

Introduction et but de l'étude : L'hypertriglycéridémie majeure (triglycérides $>10 \mathrm{~g} / \mathrm{l}$ ) est une situation métabolique relativement rare, le but de cette étude est d'en analyser les caractéristiques cliniques et génétiques.

Matériel et méthodes : 100 patients reçus à la consultation de lipidologie du CHRU de Lille et ayant présenté au moins une fois une triglycéridémie $>10 \mathrm{~g} / \mathrm{l}$ à jeun, ont eu un bilan clinique, alimentaire, biologique et génétique. Le screening a été réalisé sur les gènes majeurs d'intérêt diagnostique (Lipoprotéine lipase (LPL), APOA5, APOE) après consentement éclairé des sujets. Ils ont été classés selon leur génotype en trois groupes: pathogène (une ou plusieurs mutations majeures) (P), composite (variants fonctionnels mineurs) (C), sauvage (absence de mutation ou de variant fonctionnel validé) (S). La cohorte comportait $83 \%$ d'hommes.

Résultats et Analyse statistique : Clinique: Age moyen lors du diagnostic : 39 ans - IMC moyen $29 \pm 4,6 \mathrm{~kg} / \mathrm{m}^{2}-\mathrm{Syndrome}$ métabolique $83 \%$ - HTA $47 \%$ - Diabète de type $240 \%$ - Fumeurs $32 \%$ - Stéatose hépatique $44 \%$ - Pancréatite aigüe $13 \%$ Maladies cardiovasculaires (42\%) dont cardiopathies ischémiques (22\%) - Douleurs abdominales inexpliquées $28 \%$ - Alimentation non équilibrée $62 \%$ - Activité physique insuffisante $73 \%$ - Consommation d'alcool régulière $77 \%$ - Antécédents familiaux d'hypertriglycéridémie $34 \%$ - Xanthomatose éruptive $4 \%$.

Sur le plan biologique : Valeur maximale des triglycérides $24 \pm 17,5 \mathrm{~g} / \mathrm{l}-\mathrm{CHDL} 0,34 \pm 0,1 \mathrm{~g} / \mathrm{l}$ - C non HDL 2,14 g/l - Glycémie 1,20 $\mathrm{g} / \mathrm{l}$ - Ferritinémie $306 \pm 329 \mathrm{ng} / \mathrm{ml}$ - CRPus 2,9 mg/l - Homocystéinémie $16 \pm 13 \mu \mathrm{mol} / \mathrm{l}$ - Lipidogramme (94 patients) type III 3, type IV 67, type V 13, ND 6.

Génétique (89 patients) : Pathogène $20 \%$ - Composite $64 \%$ - Sauvage $16 \%$.

Le nombre d'allèles à risque était beaucoup plus élevé dans le génotype $C$. Il existe une association significative $(p<0,05)$ entre le nombre d'allèles à risque du gène de l'APOA5 et le génotype $(C>P>S)$.

Réponse au traitement (triglycérides): Favorable (baisse) avec la diététique seule $18 \%$ - Favorable avec le traitement médicamenteux et diététique $30 \%$ - Variable (fluctuant) $30 \%$ - Inconnu $22 \%$.

Données en fonction du génotype : Le génotype Composite était plus souvent associé à une hyperlipidémie de type IV (44\%) versus $11 \%(P)$ ou $12 \%(S)$.

Le génotype $P$ répondait plus (triglycérides) au traitement (- $43 \%)$ que le génotype $C$ (- $16 \%$ ) ou $S$ (- $35 \%)$.

Le génotype $P$ était associé à une valeur maximale de triglycérides plus élevée que le génotype $C(35 \mathrm{~g} / \mathrm{l} v \mathrm{v} 20 \mathrm{~g} / \mathrm{l}) \mathrm{p}<0,05$. Le génotype $P$ avait une ancienneté de la maladie plus grande que le génotype $S$ (18 ans vs 8 ans) $p<0,05$. Le génotype $S$ était plus souvent associé à une pancréatite aigüe (36\%) que le génotype $P(17 \%)$ et le génotype $C(7 \%) p=0,031$.

Conclusion : L'hypertriglycéridémie majeure est une affection sévère par ses complications cardio-vasculaires fréquentes (42 \%) souvent dans un contexte de cumul de facteurs de risques majeurs. La pancréatite aiguë touche $13 \%$ des sujets. Dans $84 \%$ des cas il existe des anomalies sur 3 gènes majeurs de prédisposition. Le plus souvent il s'agit d'un génotype Composite (variants fonctionnels mineurs). Certains traits cliniques sont associés au génotype. Une analyse élargie de gènes d'intérêt par séquençage génomique (NGS) est programmée pour compléter l'étude.

Conflits d'intérêts : Aucun conflit à déclarer 
Apports alimentaires et statut biologique en magnésium dans la population de diabétiques de type 2 H. Zahra ${ }^{1,}{ }^{*}$, O. Berriche ${ }^{1}$, R. Mizouri ${ }^{1}$, M. Khiari ${ }^{1}$, F. Boukhayatia ${ }^{1}$, R. Ben Othman ${ }^{1}$, F. Mahjoub ${ }^{1}$, H. Jamoussi $^{1}$

${ }^{1}$ Service A, Institut National de Nutrition de Tunis, Tunis, Tunisie

Introduction et but de l'étude : Le magnésium est un minéral participant dans plus de 300 réactions métaboliques dans le corps et qui pourrait interagir avec les paramètres de l'homéostasie glucidique. Il a été démontré qu'une consommation plus élevée en magnésium semble améliorer l'homéostasie du glucose et de l'insuline.

L'objectif de notre étude était d'évaluer le statut en magnésium et sa corrélation avec l'apport alimentaire en magnésium.

Matériel et méthodes : Nous avons mené une étude descriptive transversale concernant 101 diabétiques de type 2 sur une période d'un an entre le mois de Septembre 2016 et le mois d'Août 2017 suivis à l'Institut National de Nutrition. Les patients ont bénéficié d'une enquête alimentaire et d'un bilan biologique comportant une glycémie à jeun, une HbA1c, une magnésémie, une magnésurie de $24 \mathrm{~h}$.

Résultats et Analyse statistique : L'âge moyen de nos patients était de 56 $\pm 7,9$ ans avec des extrêmes allant de 39 à 76 ans. Une prédominance féminine était notée avec une sex-ratio égale à 0,60 . La moyenne de l'HbA1c était de $8,56 \pm 1,91 \%$. Un déficit en magnésium était noté chez $12,9 \%$ des patients. L'apport alimentaire quotidien moyen en magnésium de nos patients était de $391,61 \mathrm{mg} / \mathrm{j}$ chez les hommes et de $287,78 \mathrm{mg} / \mathrm{j}$ chez les femmes. Dans notre étude, $65,4 \%$ des hommes et $78,7 \%$ des femmes avaient des apports alimentaires inférieurs aux recommandations. Dans notre étude, la magnésémie augmentait avec l'augmentation de l'apport en magnésium, mais cette relation n'était pas statistiquement significative $(p=0,09)$. La magnésémie était comparable chez les patients ayant un apport suffisant en magnésium et chez ceux ayant un apport insuffisant ( $p=0,4)$. Nous n'avons pas trouvé de corrélation significative entre le taux de la magnésémie et l'apport alimentaire en magnésium ( $r=0,17$; $p=0,09)$. Dans notre étude, L'apport moyen en magnésium était plus faible chez les patients ayant un mauvais contrôle glycémique par rapport à ceux ayant un bon équilibre glycémique mais la différence n'était pas statistiquement significative ( $p=0,2)$. Ainsi, l'HbA1c diminuait avec l'apport alimantaire en magnésium mais cette relation n'était pas significative $(p=0,2)$. L'HbA1c diminuait avec l'augmentation de l'apport alimentaire en magnésium mais cette relation n'était pas significative $(r=-0,15 ; p=0,2)$.

Conclusion : L'apport moyen en magnésium était associé à un mauvais contrôle glycémique soulignant ainsi l'effet bénéfique de l'apport en magnésium sur l'équilibre glycémique. Un dépistage du défaut d'apport en ce minéral et sa correction par une prescription diététique adaptée devrait être mise en oeuvre.

Références : 1-Song Y, Manson JE, Buring JE, Liu S. Dietary magnesium intake in relation to plasma insulin levels and risk of type 2 diabetes in women. Diabetes Care. 2004;27(1):59-65.

2- Van Dam RM, Hu FB, Rosenberg L, Krishnan S, Palmer JR. Dietary calcium and magnesium, major food sources, and risk of type 2 diabetes in US black women. Diabetes Care. 2006;29(10):2238-43.

Conflits d'intérêts : Aucun conflit à déclarer 
${ }^{1}$ UMR PNCA, Agroparistech, Paris, ${ }^{2}$ RMes, INSERM UMR 1229, Nantes, France

Introduction et but de l'étude : Le but de ce travail est d'étudier l'effet du monosodium glutamate (MSG), un exhausteur de goût, sur la physiologie osseuse en situation de restriction protéique. Nous avons développé au laboratoire un modèle murin qui, lorsqu'il est soumis à un régime modérément carencé en protéines ( $6 \%$ de l'énergie totale sous forme de protéine de soja) présente une perte de densité minérale osseuse, une réduction de la longueur des fémurs et une altération de la microarchitecture osseuse par rapport aux valeurs mesurées lorsque le régime inclut $20 \%$ de protéine de soja ou $6 \%$ de caséine. Ce modèle induit une ostéopénie par inhibition des ostéoblastes. Dans la mesure où le glutamate semble activer les ostéoblastes, nous avons choisi le modèle de restriction protéique pour tester les effets de cet acide aminé sous sa forme monosodique sur la perte de densité osseuse liée à une restriction protéique.

Matériel et méthodes : Des souris Balb/c femelles ont été mises sous régime modérément carencé en protéines de soja pendant 12 semaines. L'étude incluait 8 groupes de 12 animaux : un groupe témoin ingérant un régime normoprotéique (NP) et 7 groupes ingérant un régime hypoprotéique (LP) qui incluait différentes concentration de MSG $(0,05,0,1,0,5,1$ ou $2 \%)$ ou de la PTH utilisée comme témoin positif et un groupe sans MSG recevait un apport isosodé et isoazoté d'alanine. Les animaux sont placés en cages individuelles afin de pouvoir mesurer leur consommation alimentaire et réaliser les expériences en " pair-feeding ». L'effet du complément MSG a été évalué par la mesure de la densité minérale osseuse par absorptiométrie biphotonique à rayons $\mathrm{X}$, et par l'évaluation de marqueurs du remodelage osseux qui permettent de rendre compte des activités de résorption et de formation osseuse, assurées respectivement par les ostéoclastes et les ostéoblastes. Nous avons également évalué la macroarchitecture de l'os cortical et la microarchitecture de l'os trabéculaire.

Résultats et Analyse statistique : L'ingestion du régime LP arrête la croissance des souris au cours des 12 semaines de l'étude, et nous observons pendant cette période une réduction de gain de masse osseuse. Le MSG dans le régime n'a pas d'effet sur la croissance des souris, mais nous observons un gain de masse osseuse similaire à celui observé lorsque les animaux ingèrent le régime NP. La préservation de la densité minérale osseuse est corrélée à une augmentation des concentrations plasmatiques des marqueurs de l'activité des ostéoblastes, sans modification des marqueurs de l'activité des ostéoclastes. Le MSG semble restaurer la densité minérale osseuse de souris sous régime modérément carencé en protéine en stimulant l'activité des ostéoblastes. L'évaluation de la microarchitecture nous a permis de constater que l'os cortical était moins sensible à la présence de MSG que l'os trabéculaire. Le MSG stimule également la synthèse du collagène de type I, constituant protéique majeur de la matrice osseuse.

Conclusion : Cette étude a permis de montrer que le MSG a un effet positif sur les paramètres osseux de souris sous restriction protéique. Une complémentation en MSG pourrait donc être bénéfique pour maintenir la qualité des os en particulier chez les personnes dénutries à risque d'ostéoporose.

Conflits d'intérêts : Aucun conflit à déclarer 
Évaluation du statut en calcium chez des enfants Marocains par l'utilisation de l'excrétion urinaire de 24-heures

A. Bouziani ${ }^{1,}{ }^{*}$, N. Saeid ${ }^{1}$, M. Idrissi ${ }^{1}$, A. Rami ${ }^{1}$, A. El Hamdouchi ${ }^{1}$, Y. Taboz ${ }^{1}$, M. El Mzibri ${ }^{1}$, K. El Kari ${ }^{1}$, H. Aguenaou ${ }^{1}$, H. Benkirane ${ }^{1}$

${ }^{1}$ Unité Mixte de la recherche en Nutrition et Alimentation, Faculté des sciences-Université Ibn Tofail/ Laboratoire de Nutrition et Alimentation, Kenitra, Maroc

Introduction et but de l'étude : Selon les rapports de l'organisation mondiale de la santé, la carence en calcium est l'une des carences les plus importantes de tous les micronutriments présentant ainsi un problème majeur de santé. La carence en calcium est responsable de nombreuses maladies fonctionnelles, notamment l'ostéoporose et les fractures. Au niveau de la population marocaine, les études sur le calcium restent limitées. Le dosage urinaire du calcium est l'une des méthodes utilisées pour étudier la carence en calcium et déterminer l'équilibre corporel en ce minéral. L'objectif de la présente étude est de déterminer le statut en calcium par l'utilisation de l'excrétion urinaire de 24-heures, et par la suite l'évaluation du niveau de carences chez un échantillon d'enfants marocains.

Matériel et méthodes : Au total, 280 enfants âgés de 6 à 18 ans ont été recrutés au niveau des écoles publiques dans la région Rabat-Kenitra pour participer à l'étude. Seulement 131 enfants ont pu provenir une collecte urinaire de 24heures complète. Le statut socioéconomique et le statut de morbidité ont été évalués pour chaque participant et les paramètres anthropométriques ont été aussi mesurés. Le dosage du calcium urinaire a été évalué par spectrométrie de masse ICP-MS.

Résultats et Analyse statistique : La moyenne totale du calcium urinaire est de $72,48 \mathrm{mg} /$ jour. Environ $73 \%$ des enfants présentent une carence en calcium confirmée par le ratio $\mathrm{Ca} / \mathrm{Cr}$, qui fournit un bon indicateur de la carence en calcium urinaire et qui est estimé par une valeur de référence de 0,2. Selon le sexe, aucune différence significative n'a été détectée pour l'excrétion urinaire calcique et sa corrélation avec l'état nutritionnel s'est trouvée négative.

Conclusion : les résultats de notre étude montrent que le statut en calcium chez les enfants étudiés est très faible ce qui les expose aux carences. Ceci peut être du au niveau de consommation de produits riches en calcium ou à une déficience en vitamine $D$, ce qui reste à confirmer par d'autres analyses.

Conflits d'intérêts : Aucun conflit à déclarer 


\section{NUTRITION EN PATHOLOGIE : CANCER, INSUFFISANCE D'ORGANES, REANIMATION, PATHOLOGIES}

DIGESTIVES...

\section{P101}

Évaluation de l'état nutritionnel des hémodialysés chroniques

M. Fadli ${ }^{1}{ }^{*}$, N. Kennab ${ }^{1}$, A. Ghouini ${ }^{2}$, K. Khelfat ${ }^{1}$, M. Boulghiti, N. Ayad, N. Kassa

${ }^{1}$ Physiologie Métabolique et Nutrition, CHU Hussein Dey, Alger, ${ }^{2}$ Laboratoire de Physiologie, Faculté de Médecine, Blida, Algérie

Introduction et but de l'étude : L'insuffisance rénale chronique est souvent associée à un état de dénutrition protéino-énergétique (DPE). La DPE constitue un facteur important de morbi-mortalité. L'objectif de cette étude est de préciser la prévalence de la DPE chez les patients hémodialysés chroniques à l'unité d’hémodialyse de l'EPH d'EL MENIAA.

Matériel et méthodes : C'est une étude transversale descriptive monocentrique, portant sur 41 patients suivis pour une insuffisance rénale chronique traités par hémodialyse au sein de l'unité d'hémodialyse de l'établissement publique hospitalier d'El Méniaa du 17 décembre 2017 au 5 janvier 2018. L'évaluation de l'état nutritionnel s'est basée sur l'enquête alimentaire, les mesures anthropométriques, la composition corporelle et le bilan biologique. Le diagnostic de dénutrition a été posé en se référant aux critères de I'ISRNM (International Society of Renal Nutrition and Metabolism).

Résultats et Analyse statistique : L'âge moyen de nos patients est de 44,21 $\pm 3,65$ ans, avec une prédominance masculine. L'apport calorique moyen est de $22.49 \pm 3.16 \mathrm{kcal} / \mathrm{kg} / \mathrm{j}$, la moitié des patients de notre série avaient un apport énergétique $<20 \mathrm{kcal} / \mathrm{kg} / \mathrm{j}$ tandis que seuls $12 \%$ des patients avaient un apport adéquat $>35 \mathrm{kcal} / \mathrm{kg} / \mathrm{j}$. L'apport protéique moyen est de $1.05 \pm 1,09 \mathrm{~g} / \mathrm{kg} / \mathrm{j}$, uniquement $17 \%$ avaient un apport protéique optimal $>1.2 \mathrm{~g} / \mathrm{kg} / \mathrm{j}$. La moyenne du poids de base est de 65,68 $\pm 4,05 \mathrm{~kg}$. L'IMC moyen est de $24,34 \pm 2.28 \mathrm{~kg} / \mathrm{m}^{2}$, un $\mathrm{IMC}<21 \mathrm{~kg} / \mathrm{m}^{2}$ est retrouvé chez $29.3 \%$ des patients. La quasi-totalité des hommes avaient un rapport TT/TH optimal $(<1)$, alors que $90 \%$ des femmes présentaient un rapport TT/TH élevé $(>0.85)$. La circonférence moyenne brachiale $(\mathrm{CMB})$ était normale $(>21 \mathrm{~cm})$ chez $3 / 4$ des femmes, cependant, seuls $19 \%$ des hommes avaient une CMB normale $(>26$ $\mathrm{cm})$. La valeur moyenne de la masse grasse est de $29,34 \pm 3,19 \%$, plus des $3 / 4(78 \%)$ des patients avaient un excès de masse grasse. La valeur moyenne de la masse maigre est de $68,43 \pm 3,73 \%$, à noter que $80.4 \%$ des patients présentaient un pourcentage en masse maigre inférieur aux valeurs normales. Plus de la moitié des patient hémodialysés présentaient un $3^{\text {ème }}$ secteur, lequel était très important ( $>1$ litre) pour $1 / 3$ des patients. La moyenne du nPNA est de $1,84 \pm 0,8 \mathrm{~g} / \mathrm{kg} / \mathrm{j}$. La majorité des patients hémodialysés avaient un nPNA normal (>1g/ $\mathrm{kg} / \mathrm{j})$. La moyenne d'albuminémie est de $35.63 \pm 2,40 \mathrm{~g} / \mathrm{l}$. Une albuminémie inférieure à $35 \mathrm{~g} / \mathrm{l}$ était retrouvée chez $41.2 \%$ des patients. La cholestérolémie moyenne est de $1.46 \pm 0,58 \mathrm{~g} / \mathrm{l}$ et $94.3 \%$ des patients présentaient une cholestérolémie $>1 \mathrm{~g} / \mathrm{l}$. L'évaluation de l'état nutritionnel des patients selon les recommandations de l'ISRNM a retrouvé une DPE chez 32\% des patients, parmi eux $22 \%$ présentaient une dénutrition modérée et $10 \%$ une dénutrition sévère.

Conclusion : La valeur pronostique de la DPE chez les patients hémodialysés chroniques incite à une reconnaissance et une prise en charge précoce de l'ensemble des perturbations nutritionnelles chez ces patients. Une prise en charge multidisciplinaire par le néphrologue, le médecin nutritionniste et la diététicienne est impérative.

Références :

- N.CANO, K.MOREAU, C.LASSEUR, et al. Nutrition et maladie rénale chronique. Traité de Nutrition Clinique. s.I.: Sosciété Froncophone de Nutrition Clinique et Métabolisme, 2016.

- N.CANO. Nutrition de l'hémodialysé chronique. Nutrition clinique et métabolisme. 2004, Vols. 7-10.

Conflits d'intérêts : Aucun conflit à déclarer 
P102

Intérêt du rapport Créatinine/Cystatine $C$ et de l'impédancemétrie bioélectrique (BIA) dans l'évaluation de la composition corporelle chez le patient atteint de cancer.

G. Ulmann ${ }^{12,{ }^{*}}$, J. Kai ${ }^{1}$, J. P. Durand ${ }^{23}$, N. Neveux ${ }^{12}$, A. Jouinot ${ }^{3}$, J. P. De Bandt ${ }^{12}$, F. Goldwasser ${ }^{23}$, L. Cynober $^{12}$

${ }^{1}$ Biochimie, APHP-HUPC-HÔPITAL COCHIN, ${ }^{2}$ EA 4466, Faculté de pharmacie - USPC, ${ }^{3}$ Oncologie médicale, APHP-HUPC-HÔPITAL COCHIN, Paris, France

Introduction et but de l'étude : Une sarcopénie est présente avant traitement chez environ 40\% des patients atteints de cancer et représente un risque accru de morbi-mortalité1. En pratique, elle est évaluée par le scanner en L3 ou l'impédancemétrie (BIA). Le rapport créatinine/cystatine $C$ a été récemment proposé pour évaluer la masse musculaire, la créatinine reflétant la masse musculaire et la fonction rénale et la cystatine $C$ plasmatique la seule fonction de filtration glomérulaire. Mais ce dernier paramètre n'a pas été étudié chez le patient attient de cancer. Le but de cette étude est de comparer l'utilisation de ces trois méthodes dans l'évaluation de la composition corporelle chez le patient atteint de cancer.

Matériel et méthodes : Entre octobre 2017 et avril 2018, tous les patients ayant eu un scanner abdominal au maximum 1,5 mois avant leur évaluation pré-thérapeutique en hôpital de jour de cancérologie ont été inclus dans l'étude. Une mesure de la composition corporelle par BIA (Bodystat Quadscan $4000^{\circledR}$ ) et le dosage de la créatinine (Roche, Cobas $8000^{\circledR}$ ) et de la cystatine $\mathrm{C}$ (Siemens, BNII ${ }^{\circledR}$ ) plasmatiques ont été réalisés à cette occasion. Les analyses statistiques (corrélation de Pearson, Bland-Altman, Passing-Bablok et courbes ROC) ont été faites avec RStudio (v. 1.1.442).

Résultats et Analyse statistique : Nous avons inclus 44 patients (14 femmes) d'âge médian 65 ans (36 à 93 ans), tous cancers confondus (17 localisations dont poumon : 27\%, prostate : 15\%). Les corrélations entre les différentes méthodes sont bonnes:

\begin{tabular}{|l|l|l|}
\hline \multicolumn{3}{|c|}{ coefficient de corrélation ( $p$-value) } \\
\hline & Scanner & BIA \\
\hline BIA & $0,763\left(1,7.10^{-9}\right)$ & $x$ \\
\hline Créatinine/Cystatine C & $0,65\left(2,0.10^{-6}\right)$ & $0,490\left(7,3.10^{-4}\right)$ \\
\hline
\end{tabular}

La BIA tend à surestimer la masse maigre $(\mathrm{MM})$ d'environ $3 \mathrm{~kg}$ (test de Bland-Altman). Sur la base des critères de sarcopénie au scanner $^{2}$ (seuil : 19,1 kg/m² $(\mathrm{H}), 15,76 \mathrm{~kg} / \mathrm{m}^{2}(\mathrm{~F})$ ), la sensibilité de la BIA pour diagnostiquer une MM faible est de $75 \%$ et la spécificité de $67 \%$ chez l'homme et de $38 \%$ et $100 \%$ respectivement chez la femme. Selon les mêmes critères, le seuil de rapport créatinine/cystatine C pour une masse maigre faible est de 95,01 $\mu \mathrm{mol} / \mathrm{g}$ chez l'homme et de 65,3 $\mu \mathrm{mol} / \mathrm{g}$ chez la femme avec une sensibilité et une spécificité respectivement $96 \%$ et de $50 \%$ chez l'homme et de $38 \%$ et $100 \%$ chez la femme.

Conclusion : La corrélation entre la MM déterminée par le scanner en L3 et par la BIA ainsi qu'avec le rapport créatinine/cystatine $\mathrm{C}$ est bonne. En revanche, la concordance entre le scanner et la BIA est médiocre. La BIA et le rapport Créatinine/Cystatine C peuvent aider au diagnostic de masse maigre faible, en particulier chez les hommes. Ce rapport est un marqueur peu couteux et facile à mesurer qui pourrait entrer dans l'évaluation de l'état nutritionnel du patient atteint de cancer.

Références : ${ }^{1}$ Pamoukdjian $\mathrm{F}$ et al. Clin Nutr 2018;37:1101-1113

${ }^{2}$ Mourtzakis M et al. Appl Physiol Nutr Metab 2008;33:997-1006

Conflits d'intérêts : Aucun conflit à déclarer 
Retrouver le plaisir de manger après un cancer ORL

M. Burgevin ${ }^{1, *}$

${ }^{1}$ Diététique, CHU Toulouse, Toulouse, France

Introduction et but de l'étude : Cette étude fait suite à une constatation récurrente lors des consultations diététiques : les patients ayant eu un cancer oto-rhino-laryngé présentent une perte de plaisir à l'alimentation provoquant une diminution des prises per os et une perte de poids. On estime que $72 \%$ de ces patients sont dénutris.

On observe dans les suites du cancer ORL des modifications sensorielles et, parfois, une nécessité de changement de texture entraînant un bouleversement du rapport à l'alimentation. Le mangeur ne reconnaît plus ce qu'il mange, il a du mal à identifier ses aliments et à se les approprier. Perte de repères et d'identité rendent difficile la décision de consommer. L'alimentation peut devenir un acte contraint, dénué de tout plaisir.

Cette étude, réalisée au Centre de Rééducation Intensive des Laryngectomisés du CHU de Toulouse, avait comme question de départ "Comment redonner l'envie, le plaisir de manger à des personnes dont le rapport à la nourriture a été profondément perturbé par la maladie?".

Matériel et méthodes : La méthodologie appliquée repose sur une démarche qualitative.

La phase exploratoire, constituée d'entretiens d'experts, de focus groups avec les patients aura permis de déterminer les trois hypothèses de travail :

- la redécouverte du plaisir passe par la reconstruction d'une expérience positive par rapport au repas mixé,

- la cuisine autrement : un levier pour retrouver le plaisir,

- l'importance de l'implication de l'entourage et de la commensalité.

Par la suite, la phase de terrain, composée d'un questionnaire qualitatif, a mis à jour les comportements de mangeurs, les attentes et les perceptions autour du repas mixé.

Résultats et Analyse statistique : L'âge moyen des personnes interrogées est de 56 ans, 53\% d'hommes et $47 \%$ de femmes. La majorité d'entre elles mangent mixé depuis plus d'un an (60\%). Globalement, l'alimentation mixée est vécue comme une prescription médicale nécessaire à leur bonne santé.

L'analyse du questionnaire a contribué à confirmer les hypothèses.

La majorité des personnes interrogées affirment aimer beaucoup la cuisine (64\%).

La moitié des enquêtés estiment n'être pas complètement compris par leurs proches. Ce sentiment, associé à la gêne sociale ressentie, affecte les prises alimentaires à l'extérieur.

Manger différemment est source d'exclusion. Pourtant la moitié d'entre eux, aimeraient pouvoir retourner au restaurant. La commensalité a donc un rôle prépondérant dans la qualité des prises alimentaires.

Conclusion : Cette étude a permis de mettre en évidence que le changement de texture n'est pas un acte anodin. L'alimentation représente bien plus qu'un simple acte biologique visant à l'ingestion de nutriments.

Le passage à un repas mixé s'accompagne d'une difficulté d'identification des produits et donc d'incorporation, d'une modification du rapport à l'alimentation et des relations avec ses commensaux. Toutes ces perturbations peuvent se traduire par une diminution du plaisir de manger.

Il est donc indispensable que le mangeur crée une expérience positive pour accepter ce nouveau mode d’alimentation.

Ce travail a conduit à mettre en place des actions concrètes afin de lui redonner le goût de manger :

- une carte décrivant la texture modifiée à présenter aux restaurateurs,

- un listing des restaurants proposant des plats mixés,

- des ateliers d'éducation sur la prévention de la dénutrition et l'utilisation des compléments,

- un classeur de recettes mixées susceptible de devenir un livre.

Conflits d'intérêts : Aucun conflit à déclarer 
Excès de méthionine et altération de la structure rénale chez le rat des sables, Psammomys obesus

B. Chaouad ${ }^{12}$, A. Ghoul ${ }^{1}$, F. Zerrouk ${ }^{1}$, A. Moulahoum ${ }^{1}$, K. Othmani-Mecif ${ }^{1,}{ }^{*}$, B. Yasmina ${ }^{1}$

${ }^{1}$ Faculté des Sciences Biologiques, Université des Sciences \& de la Technologie Houari Boumediene USTHB., ALGER, ${ }^{2}$ Faculté des Sciences de la nature et de la vie et des sciences de la terre, Université Djillali Bounaama, Khemis Miliana, Algérie

Introduction et but de l'étude : De nombreux travaux ont montré qu'un régime alimentaire riche en méthionine est à l'origine d'une hyperhomocystéinémie, facteur de risque de plusieurs pathologies telles que les maladies cardiovasculaires et l'insuffisance rénale chronique. L'objectif de notre étude est de déterminer l'impact d'une l'hyperhomocystéinémie sur la structure histologique et la morphométrie rénale du rat des sables, Psammomys obesus.

Matériel et méthodes : Pour ce faire, 10 Psammomys obesus sont répartis équitablement en 2 lots : un lot témoin nourri exclusivement de plantes halophiles (régime naturel) et un lot expérimenté recevant le même régime alimentaire et soumis à une administration chronique, par voie intra péritonéale, de méthionine à raison de $150 \mathrm{mg} / \mathrm{kg}$ de poids corporel/jour pendant 6 mois. Les répercussions de cet excès de méthionine sur la structure cellulaire et matricielle rénale ont été analysées par une étude histologique (coloration au trichrome de masson) et histochimique (coloration à l'acide périodique-Schiff). Une étude morphométrique nous a permis de compléter cette analyse.

Résultats et Analyse statistique : L'excès de méthionine administré aux rats des sables, est à l'origine d'une hyperhomocystéinémie. Nos résultats histomorphométriques montrent que l'administration chronique de la méthionine provoque une importante accumulation des collagènes fibrillaires caractérisant l'installation d'une fibrose interstitielle. Celle-ci est observée au niveau des espaces intertubulaires (environ $110 \%$ vs témoins), dans les glomérules (environ 78\% vs témoins) caractérisant une glomérulosclérose et autour des artères (fibrose périvasculaire). L'accumulation des éléments de la matrice extracellulaire au niveau des lames basales entourant les corpuscules et les tubules rénaux provoque un épaississement de ces structures. Nous avons également observé, chez les animaux expérimentés, un rétrécissement de la chambre urinaire (d'environ $28 \%$ ) associé à une augmentation de la surface glomérulaire (d'environ $20 \%$ ) et la présence de nombreux glomérules totalement détruits ce qui indique l'installation d'une insuffisance rénale. Une importante infiltration leucocytaire, en particulier au niveau interstitiel et périvasculaire, mais également au niveau glomérulaire et tubulaire est observée.

Conclusion : Ces résultats montrent que l'hyperhomocystéinémie engendrée par un excès de méthionine est à l'origine d'altérations de la structure rénale chez le rat des sables et par conséquent d'altérations fonctionnelles.

Conflits d'intérêts : Aucun conflit à déclarer 
Corrélations entre les paramètres biologiques et la composition corporelle dans la sclérose latérale amyotrophique. P. Jésus ${ }^{12} 3^{3}$, , F. Patin ${ }^{45}$, S. E. Bakkouche ${ }^{6}$, S. Beltran ${ }^{46}$, C. R. Andrès ${ }^{45}$, P. Vourc'h ${ }^{45}$, H. Blasco ${ }^{45}$, P. Corcia ${ }^{46}$

${ }^{1}$ Institut de Neuroépidémiologie et de Neurologie Tropicale, CNRS FR 3503 GEIST, Université de Limoges, ${ }^{2}$ INSERM, UMR 1094, Neuroépidémiologie Tropicale, Faculté de Médecine de Limoges, ${ }^{3}$ Unité de Nutrition, CHU Limoges, Limoges, ${ }^{4}$ INSERM UMR 1253 , Imagerie et Cerveaux, Université François-Rabelais, ${ }^{5}$ Laboratoire de Biochimie et de Biologie Moléculaire, ${ }^{6}$ Centre SLA, service de Neurologie, CHU Tours, Tours, France

Introduction et but de l'étude : Au cours de la sclérose latérale amyotrophique (SLA) la composition corporelle par impédancemétrie bioélectrique (BIA) ainsi que des paramètres biochimiques tels que la ferritine ou les marqueurs du bilan lipidique peuvent être associés à l'évolution et à la survie. Le but de notre travail était de déterminer la variation des paramètres biologiques, de BIA et cliniques au cours de la maladie, ainsi que le lien des données biologiques avec la BIA et les données cliniques.

Matériel et méthodes : Les données biologiques (albumine, lipides sériques, ferritine), la composition corporelle par BIA (masse maigre $[\mathrm{MM}]$, eau corporelle totale $[E C T]$, eau intracellulaire [EIC]) et les données cliniques (poids, indice de masse corporelle [IMC], le score de l'ALS functional rating scale revised [ALSFRS-R] et la capacité vitale forcée [CVF]) des patients SLA ont été recueillies tous les 3 mois au cours des 12 mois (T1 à T4) suivant le diagnostic. Le test de Freidman a été utilisée pour étudier l'évolution des données au cours des quatre évaluations. Le test de Wilcoxon a été effectués pour comparer l'évolution des données entre la première évaluation (T1) et la dernière évaluation (T4). Le test de Spearman a été réalisés pour rechercher des corrélations des données biologiques avec la BIA et les données cliniques.

Résultats et Analyse statistique : 42 patients atteints de SLA certaine ou probable selon les critères de l'El Escorial révisés, ont été inclus dans cette étude. L'âge médian était de 70,7 ans (62.7 - 78.8) au moment du diagnostic. Le sex H/F ratio était de 1 et le site de début bulbaire dans $26,8 \%$ des cas. Pendant le suivi, seul l'IMC a évolué au cours des quatre évaluations ( $p=0,009)$. L'IMC, le score de l'ALSFRS-R et de la CVF ont diminué de manière significative $(p=0,01, p=0,006, p<0,0001$ et $p<0,0001$, respectivement) entre T1 à T4. La composition corporelle et les paramètres biologiques n'ont pas évolué pas au cours du suivi. Une corrélation positive entre les variations de l'albumine et de l'ALSFRS-R était observée $(r=0,48, p=0,04)$. Plusieurs corrélations positives entre les variations du LDL-cholestérol et du poids, de la MM, de l'ECT et de l'EIC ont été observées $(r=0,46, p=0,007 ; r=0,53, p=$ 0,$002 ; r=0,51, p=0,003$ et $r=0,60, p=0,004$, respectivement). Des corrélations négatives ont aussi été retrouvées entre les variations de ferritine et de poids, de la MM, de l'ECT et de I’EIC $(r=-0,49, p=0,002 ; r=-0,58, p=0,0002 ; r=-0,51, p=0,001$ et $r=-0,53 . p=0,008$, respectivement).

Conclusion : Cette première étude permet d'objectiver des relations entre les biomarqueurs biochimiques, la composition corporelle et les données cliniques sur cette maladie. L'albumine pourrait permettre de suivre l'évolution fonctionnelle des patients atteints de SLA. La BIA peut donc constituer un outil précieux associé au bilan biologique pour la prise en charge nutritionnelle des patients atteints de SLA. Une confirmation sur une plus grande cohorte est nécessaire pour la confirmation de ces résultats.

Conflits d'intérêts : Aucun conflit à déclarer 
Caractéristiques nutritionnelles des patients séjournant plus de 2 semaines en réanimation : identification de facteurs de risque liés au pronostic chez 120 patients

G. E. Bagnoud ${ }^{1,}{ }^{*}$, A. Martinez ${ }^{1}$, E. Favre ${ }^{1}$, M. Charrière ${ }^{2}$, D. Favre ${ }^{2}$, T. Kelevina ${ }^{1}$, O. Pantet ${ }^{1}$, P. Eckert ${ }^{1}$, M. M. Berger $^{1}$

${ }^{1}$ Service de médecine intensive adulte et brûlés, ${ }^{2}$ Nutrition Clinique-EDM, Centre Hospitalier Universitaire Vaudois, CHUV, Lausanne, Suisse

Introduction et but de l'étude : Les patients de soins intensifs (USI) nécessitant des traitements prolongés de plusieurs semaines sont en augmentation. Les données nutritionnelles disponibles sont rares alors que l'on sait qu'une nutrition adaptée peut faire la différence entre un retour à domicile ou une institutionnalisation (1). Puisque la prolongation du séjour engendre des coûts humains et économiques importants, un programme de suivi a été implanté : cette étude a pour objectif d'identifier les éventuels risques et particularités du traitement nutritionnel de ces patients.

Matériel et méthodes : Etude observationnelle rétrospective mono-centrique dans une USI multidisciplinaire universitaire, conduite entre février 2017 et juillet 2018. Critères d'inclusion: $>18$ ans, séjour en USI >14 jours (J). Critère d'exclusion: admission pour brûlure ou neuro-réanimation. Variables saisies: démographie, SAPSII, NRS, nutrition (énergie : couperet -500 kcal/j, cumulé $-6000 \mathrm{kcal}$; protéines couperet -300 g), épuration extrarénale (ERRC présence), ventilation mécanique (VM), escarres, durée de séjour (DUSI), force musculaire (score MRC). Observation jusqu'à J60. Données extraites du Système d'information clinique (MetaVision). Statistiques descriptives. Données en médianes [IQR 25-75].

Résultats et Analyse statistique : Au total, 120 patients ont été inclus dans le programme âgés de 60,5 ans [IQR 19], NRS à 5 [IQR 2], SAPSII 54 [IQR 27,5], mortalité 18,5\%, durée de séjour 32 jours [IQR 23] et durée VM de 17,3 jours [IQR 11,7]; 50\% sont admis pour sepsis, $50 \%$ ont requis ERRC. La cible énergétique prescrite évolue, passant de $1675 \mathrm{kcal} / \mathrm{j}$ la $1^{\text {ère }}$ semaine $(21,8 \mathrm{kcal} / \mathrm{kg})$ à $1750 \mathrm{kcal} / \mathrm{j}$ la $2^{\mathrm{ème}}$. L'énergie délivrée est inférieure à la cible pendant la phase de progression de la nutrition avec une stabilisation dès la $2^{\text {ème }}$ semaine vers $23 \mathrm{kcal} / \mathrm{kg} / \mathrm{j}$ : le bilan d'énergie cumulé s'améliore, passant de $-3732 \mathrm{kcal}$ la $1^{\text {ère }}$ semaine à -917 kcal durant la $2^{\text {ème }}$ semaine (-256 kcal sur le séjour total). L'apport de protéines n'est que de $0,9 \mathrm{~g} / \mathrm{kg} / \mathrm{j}[\mathrm{IQR} 0,32]$ malgré une recommandation interne de 1,1-1,3 g/kg/j. La survenue d'escarres chez 97/120 patients est associée au déficit en protéines ( $p=0.06$ ). La force musculaire à la sortie (MRC 34 [IQR 19]) est faible sans lien avec l'énergie ou protéines fournies. Le NRS d'admission est significativement lié à la mortalité (OR 1,34, $p=0,048$ ), alors que le SAPSII ne l'est pas. On note des associations significatives entre le déficit en protéines J1-J14 et la durée USI totale $(p=0,0044)$; ainsi qu'entre la durée de VM et le déficit calorique J1-J7 >-6000 kcal ( $p=0,0462)$.

Conclusion : Ces données originales montrent une grande variabilité des apports (calories/protéines). L'état nutritionnel altéré à l'entrée (NRS) est un facteur de risque d'évolution défavorable, tout comme le déficit en protéines qui prime sur celui en énergie. Ces données montrent l'importance d'évaluer le patient à l'entrée, de rester attentif aux NRS >5 et d'un contrôle quotidien de l'atteinte de la cible protéino-énergétique prescrite.

Références : 1. Yeh DD, et al. Adequate Nutrition May Get You Home. JPEN 2016;40:37-44.

Conflits d'intérêts : Aucun conflit à déclarer 
Le régime pauvre en FODMAPs, sans consultation diététique, dans la prise en charge des patients souffrant du syndrome de l'intestin irritable

P. Van Ouytsel ${ }^{1,},{ }^{*}$ A. Szalai ${ }^{1}$, M. Arvanitakis ${ }^{1}$, A. Van Gossum ${ }^{1}$, J.-C. Preiser ${ }^{1}$, H. Louis $^{1}$

${ }^{1}$ Hopital Erasme, Bruxelles, Belgique

Introduction et but de l'étude : Le régime pauvre en glucides fermentescibles à chaine courte (Oligo-, Di-, Mono-saccharides et Polyols) (FODMAPs), a été démontré efficace dans le traitement des patients souffrant du syndrome de l'intestin irritable (SII). L'intervention d'un(e) diététicien(ne) n'est pas toujours possible lorsque les patients sont vus en consultation médicale. Le but de l'étude a été d'évaluer la prise en charge de patients souffrant de SII à qui un régime pauvre en FODMAPs est proposé directement par le gastroentérologue.

Matériel et méthodes : Une étude prospective a été menée chez des patients souffrant de SII. Des explications sommaires sur le régime pauvre en FODMAPs sont données par le gastroentérologue à l'aide d'une fiche explicative. Un premier questionnaire comportant des questions sociodémographiques a été soumis au patient lors la consultation. Six semaines plus tard, un deuxième questionnaire a évalué la compréhension des explications données, l'impact social et l'adhérence au régime, l'évolution des symptômes liés au SII à l'aide d'une échelle de Likert (0 à 10), et enfin le souhait d'une prise en charge diététique spécifique. L'analyse statistique a été réalisée au moyen de tests pairés de Student avec un seuil de p-valeur fixé à $5 \%$.

Résultats et Analyse statistique : Trente-cinq patients ont été inclus (69\% de femmes; âge moyen $45 \pm 15$ ans). Septante quatre pour cent des patients pensent que la nourriture est impliquée dans la genèse de leurs symptômes, $97 \%$ se disant prêts à suivre un régime pour contrôler leurs symptômes. Dans le questionnaire de suivi après régime, 91\% des patients ont bien compris les fiches qui leur ont été remises. Concernant l'adhérence au régime, $52 \%$ affirment avoir suivi le régime de manière régulière, $28 \%$ l'ont parfois suivi et $20 \%$ ne l'ont jamais ou rarement suivi. Les facteurs de non-adhérence comprennent principalement la complexité du régime, le manque de temps, l'oubli ou un dégoût pour les aliments proposés. Plusieurs facteurs tendent à être reliés à une meilleure adhérence au régime (niveau d'études plus élevé, identifier l'alimentation comme facteur impliqué dans l'apparition des symptômes, vivre seul, le sexe féminin) (NS). Pour un tiers des patients, suivre le régime a eu un impact négatif significatif sur leur vie sociale. Tous les symptômes, sauf la constipation, ont diminué d'intensité après instauration du régime. Finalement, $77 \%$ des patients sont satisfaits de leur prise en charge et la plupart d'entre eux (69\%) sont demandeurs d'une consultation diététique spécifique.

Conclusion : Proposer un régime pauvre en FODMAPs sans l'intervention d'un(e) diététicien(ne) est possible, mais seulement un patient sur deux va adhérer au régime. Un suivi par une diététicienne spécialisée est souhaité par une majorité de patients.

Conflits d'intérêts : Aucun conflit à déclarer 
P108

L'évaluation de la sarcopénie avant greffe hépatique pourrait contribuer à déterminer le pronostic post-greffe des patients en ACLF 2 et 3

S. Testu ${ }^{1, *}$

${ }^{1}$ Hépatologie, Hôpital de La Croix rousse, Lyon, France

Introduction et but de l'étude : La décompensation aiguë de cirrhose associée à une défaillance multi-viscerale, ou Acute On Chronic Liver Failure (ACLF), est associée à un risque de décès majeur en l'absence de transplantation hépatique (TH). Une étude récente a montré que la TH était une alternative thérapeutique efficace pour les patients les plus sévères (avec trois défaillances d'organe (ACLF3)) malgré un nombre de complications et une durée d'hospitalisation majorés. Plusieurs auteurs ont montré l'intérêt de l'évaluation de la sarcopénie pour prédire la mortalité sur liste d'attente de TH ou après TH. L'intérêt de l'évaluation de la sarcopénie en pré -TH n'a pas encore été étudié dans la population spécifique des patients en ACLF, pour lesquels l'evolution de la pathologie hépatique est accélérée en comparaison aux autres patients cirrhotiques.

Matériel et méthodes : Les patients en ACLF 2 (2 défaillances d'organe) ou 3, transplantés hépatiques dans notre centre entre 2016 et 2017, ont été inclus rétrospectivement. La surface musculaire au niveau de la 3ème vertèbre lombaire (L3) a été mesurée sur des scanners injectés datant de moins de 6 mois avant la greffe. La surface des muscles psoas (PMA) et la surface musculaire totale rapportée à la taille ${ }^{2}$ (SMI) ont été rapportées pour chaque patient. Le critère de jugement principal était la durée d'hospitalisation en réanimation et les critères de jugement secondaires étaient la durée d'hospitalisation totale et la mortalité à 1 an.

Résultats et Analyse statistique : Parmi les 15 patients inclus, 9 étaient en ACLF 3 et 6 en ACLF 2. Le MELD médian était 28 (27,5 ; 40,5). Parmi les femmes $(\mathrm{n}=6)$, la médiane du SMI et du PMA étaient respectivement de $3776 \mathrm{~mm} / \mathrm{m}^{2}(3288 ; 4466)$ et $1743 \mathrm{~mm}{ }^{2}$ $(1114 ; 1965)$. Chez les hommes $(n=9)$, ces valeurs étaient respectivement de $4657 \mathrm{~mm} / \mathrm{m}^{2}(3609-4997)$ et $1886 \mathrm{~mm} \mathrm{~m}^{2}(1589 ; 2213)$. Selon le SMI et la définition de Tandon_et al._et Van Vugt_et al_., 67\% des patients inclus étaient sarcopéniques (_vs_40\% parmi l'ensemble des patients en attente de TH selon la littérature).

II n'y avait pas de différence en fonction du statut ACLF 2 ou 3. La durée d'hospitalisation en réanimation et la durée d'hospitalisation totale étaient plus importantes pour les patients sarcopéniques selon le SMI (respectivement, médiane 29 jours $(10,8 ; 49)$ et 75.5 jours $(28.5-122.3)$ pour les patients sarcopéniques_vs_ 12 jours $(10 ; 26,5)$ et 51 jours $(36 ; 77.5)$ pour les patients non sarcopéniques). Deux patients sont décédés lors de la première année post-greffe, soit une mortalité à 1 an de $13 \%$, similaire à celle rapportée dans la littérature. On ne retrouvait pas de différence selon la surface musculaire mesurée en L3.

Conclusion : La mesure de la sarcopénie par imagerie en pré-TH chez les patients ACLF 2 et 3 pourrait présenter un intérêt pour estimer le pronostic post-greffe de ces patients très sévères et sélectionner les patients pouvant bénéficier de cette stratégie thérapeutique. Cette observation doit être confirmée dans une plus large cohorte en associant l'étude de la mortalité post-TH et le nombre de complications.

Conflits d'intérêts : Aucun conflit à déclarer 
Valeur prédictive de la sarcopénie et de la lymphopénie préopératoires sur la survie sans récidive dans l'adénocarcinome pancréatique opérable

J. Raillat ${ }^{1}$, J. Grillot ${ }^{1, *}$, A. Vienot ${ }^{2}$, D. Vernerey ${ }^{3}$, F. Fein ${ }^{1}$, C. Turco ${ }^{4}$, B. Heyd ${ }^{4}$, S. Koch ${ }^{1}$, L. Vuitton ${ }^{1}$, C. D'Engremont ${ }^{1}$, C. Borg ${ }^{2}$

${ }^{1}$ Gastroentérologie et Nutrition, ${ }^{2}$ Oncologie médicale, ${ }^{3}$ Unité méthodologie et qualité de vie en cancérologie, ${ }^{4}$ Service de chirurgie viscérale, CHRU BESANCON, BESANCON CEDEX, France

Introduction et but de l'étude : L'adénocarcinome pancréatique est un enjeu majeur de santé publique. Lorsque la maladie est découverte à un stade localisé, la chirurgie carcinologique associée à la chimiothérapie adjuvante est le meilleur traitement susceptible de conduire à une survie prolongée. Malgré des progrès incontestables, la chirurgie pancréatique est grevée d'une morbidité élevée (40 à 50\%) justifiant une meilleure sélection des patients. Le but de cette étude était de déterminer les facteurs pronostiques préopératoires, notamment biologiques et nutritionnels, sur la survie sans récidive des patients atteints d'un adénocarcinome pancréatique résécable.

Matériel et méthodes : 146 patients atteints d'un adénocarcinome pancréatique et opérés au CHRU de Besançon entre 2006 et 2014 ont été rétrospectivement inclus. Les paramètres clinico-biologiques et nutritionnels ont été recueillis au diagnostic et un mois après la chirurgie. La sarcopénie radiologique était définie par une valeur de l'index musculaire squelettique inférieure à $52,4 \mathrm{~cm}^{2} / \mathrm{m}^{2}$ chez l'homme et inférieure à $38,5 \mathrm{~cm}^{2} / \mathrm{m}^{2}$ chez la femme. Les analyses statistiques ont permis de développer un score pronostique.

Résultats et Analyse statistique : 58,5 \% des 94 patients inclus dans l'analyse finale étaient sarcopéniques en préopératoire et $19,1 \%$ présentaient une lymphopénie.

En analyse univariée, les paramètres suivants étaient corrélés à la survie sans récidive (SSR): la taille tumorale, le taux sérique de Ca 19.9, la lymphopénie et la sarcopénie.

En analyse multivariée, seules la lymphopénie et la sarcopénie étaient indépendamment corrélées à la $S S R$ ( $H R=4,6 ; p<0,0001$ et $\mathrm{HR}=1,8 ; \mathrm{p}=0,0047$ respectivement).

La constitution d'un score incluant ces 2 paramètres a permis d'identifier 3 groupes de patients avec un profil pronostique différent à savoir : le groupe sans lymphopénie ni sarcopénie, le groupe sarcopénie sans lymphopénie et le groupe lymphopénie sans sarcopénie (SSR respectives: 21,2; 11,5 et 5,6 mois $p<0,0001$ ). La SSR était par ailleurs conditionnée par le degré de sévérité de la sarcopénie préopératoire.

Conclusion : La sarcopénie apparait en plus de la lymphopénie comme un marqueur pronostique préopératoire dans l'adénocarcinome pancréatique localisé et devrait être évaluée en pratique courante pour discuter de l'orientation thérapeutique des malades.

Conflits d'intérêts : Aucun conflit à déclarer 
Evaluation des paramètres nutritionnels préopératoires dans une cohorte d'adénocarcinome pancréatique résécable J. Grillot ${ }^{1,}{ }^{*}$, A. Vienot ${ }^{2}$, J. Raillat ${ }^{1}$, D. Cazaux ${ }^{1}$, C. Prothe ${ }^{1}$, C. Turco ${ }^{3}$, S. Borot ${ }^{4}$, C. D'Engremont ${ }^{1}$

${ }^{1}$ Gastroentérologie et Nutrition, ${ }^{2}$ Oncologie Médicale, ${ }^{3}$ Chirurgie Viscérale, ${ }^{4}$ Endocrinologie, Diabétologie et Nutrition, CHRU Besancon, Besancon, France

Introduction et but de l'étude : La chirurgie permet d'augmenter la survie à long terme dans l'adénocarcinome pancréatique (ADKP) localisé, cependant la morbidité reste élevée justifiant une meilleure sélection des patients.

Les données actuelles démontrent que dans I'ADKP, la perte de poids, un IMC bas et la perte de masse maigre sont corrélés à une majoration du risque de complications post opératoires.

Il est donc recommandé de réaliser un dépistage nutritionnel systématique lors du diagnostic et la prise en charge nutritionnelle est depuis les dernières recommandations de la Société Française d’Anesthésie et Réanimation (SFAR) et de la Société Française de Nutrition Clinique et Métabolisme (SFNCM) bien codifiée.

Le but de ce travail était d'évaluer la prise en charge nutritionnelle préopératoire des patients présentant un ADKP résécable et de comparer l'évolution des patients suivant leur grade nutritionnel et statut sarcopénique préopératoire.

Matériel et méthodes : 87 patients atteints d'un ADKP et opérés dans un centre universitaire entre 2010 et 2014 ont été rétrospectivement inclus. Les paramètres clinico-biologiques et nutritionnels ont été recueillis au diagnostic et un mois après la chirurgie. La dénutrition était un critère composite, et devait comporter un des trois paramètres suivant: une perte de poids $\geq 10$ $\%$ en 6 mois, un IMC $\leq 18.5 \mathrm{~kg} / \mathrm{m}^{2}$ ou $<21 \mathrm{~kg} / \mathrm{m}^{2}$ en cas d'âge $>70$ ans, une albuminémie $<30 \mathrm{~g} / \mathrm{dL}$. La sarcopénie radiologique était définie par une valeur de l'index musculaire squelettique inférieure à $52,4 \mathrm{~cm}^{2} / \mathrm{m}^{2}$ chez l'homme et inférieure à $38,5 \mathrm{~cm}^{2} / \mathrm{m}^{2} \mathrm{chez}^{\prime}$ la femme.

Résultats et Analyse statistique : $63.4 \%$ des patients étaient dénutris et $60 \%$ sarcopéniques. $13 \%$ des patients avaient eu une évaluation nutritionnelle en préopératoire et $42 \%$ avaient reçu une immunonutrition. Moins de $5 \%$ des malades dénutris avaient eu une nutrition artificielle comme recommandée en préopératoire.

Les marges de résection était significativement meilleure (RO) chez les patients non dénutris $(96.2 \%$ vs $75 \%$, $p=0.0251)$. Les patients du groupe dénutris tendaient à présenter plus de complications chirurgicales (33.3\% vs $15.4 \%, p=0,0998)$.

La sarcopénie était cependant le seul marqueur nutritionnel associé à la survie globale (SG) avec une SG de 35 mois pour les non sarcopéniques contre 17,4 mois pour les patients sarcopéniques ( $p=0,0274)$.

Conclusion : La sarcopénie est un marqueur nutritionnel préopératoire prometteur qu'il faudra intégrer à notre pratique courante, toutefois, il conviendra dans un premier temps de veiller à faire appliquer les recommandations de prise en charge nutritionnelle périopératoire

Conflits d'intérêts : Aucun conflit à déclarer 


\section{P111}

Etude de la nature des allergies alimentaires en consultation au Laboratoire National de référence de Casablanca-Maroc L. Slimani ${ }^{1, *}$, S. Souat ${ }^{1}$, J. El Bakkouri ${ }^{2}$, A. Jafri ${ }^{1}$, F. Dehbi ${ }^{1}$

${ }^{1}$ Faculté des Sciences et techniques de santé, Université Mohammed VI des Sciences de la Santé, ${ }^{2}$ LNR, Hôpital Cheikh Khalifa, Casablanca, Maroc

Introduction et but de l'étude : L'allergie alimentaire est une hypersensibilité, de mécanisme immunologique connu, à un ou plusieurs allergènes alimentaires. Elle représente aujourdh'hui un sujet de préoccupation majeur au Maroc, compte tenu de sa prévalence et de sa gravité potentielle.

Le présent travail a pour objectif de faire le point sur la nature des allergies alimentaires et d'étudier la fréquence des différents groupes alimentaires.

Matériel et méthodes : II s'agit d'une étude rétrospective effectuée au niveau du Laboratoire National de Référence (LNR) de Casablanca (Maroc) au sein de l'Hôpital Cheikh Khalifa du 12/01/2017 au 09/05/2018. Cette étude a concerné 118 sujets consultant le LNR. L'analyse des IgE spécifique a été effectuée sur des échantillons sanguins par la technique immunoblot sur l'automate PLEX MAT et nous a permis d'évaluer la fréquence des différentes allergies aux groupes alimentaires.

Résultats et Analyse statistique : Nos résultats montrent que les deux groupes de fruits et légumes et de poissons et fruits de mer prennent place dans la première ligne des fréquences de sensibilité alimentaire avec un taux de $51.16 \%$ et $34.88 \%$ respectivement pour les enfants et de $40.30 \%, 50,75 \%$ respectivement pour les adultes. Pour les nourissons, la proportion du groupe du lait et produits laitiers est de $57.17 \%$ suivie du groupe fruits et légumes de $42.85 \%$.

Conclusion : Les données obtenues dans cette étude nous permettent d'avoir une estimation de la fréquence des différents types d'allergènes alimentaires qui arrivent au niveau du LNR.Une analyse plus approfondie pourrait mettre en évidence les facteurs de risques impliqués dans l'incidence de ces allergies et améliorer leur prise en charge.

Conflits d'intérêts : Aucun conflit à déclarer 
P112

Complications cardiométaboliques et déficits cognitifs chez les survivants de la leucémie lymphoblastique aiguë pédiatrique : associations avec l'inflammation périphérique ?

P. Léveillé ${ }^{123,{ }^{*}}$, A. Boulet-Craig ${ }^{12}$, J. Laniel ${ }^{12}$, A. Franco ${ }^{13}$, S. Morel ${ }^{134}$, S. Drouin ${ }^{1}$, F. Rodier ${ }^{56}$, M. Krajinovic $^{17}$, C. Laverdière ${ }^{7}$, D. Sinnett ${ }^{17}$, P. Robaey ${ }^{17}$, S. Sultan ${ }^{17}$, E. Levy ${ }^{134}$, V. Marcil ${ }^{134}$, S. Lippé ${ }^{12}$

${ }^{1}$ Centre de Recherche du CHU Sainte-Justine, ${ }^{2}$ Département de Psychologie, Université de Montréal, Montréal, ${ }^{3}$ Institut sur la nutrition et les aliments fonctionnels, Québec, ${ }^{4}$ Département de Nutrition, ${ }^{5} \mathrm{CRCHUM}$ et Institut du cancer de Montréal, ${ }^{6}$ Département de Radiologie, radio-oncologie et médecine nucléaire, ${ }^{7}$ Département de Pédiatrie, Université de Montréal, Montréal, Canada

Introduction et but de l'étude : Les traitements de la leucémie lymphoblastique aiguë (LLA) pédiatrique laissent des séquelles importantes entre autres sur le plan cardiaque, métabolique et neurocognitif. Les survivants de la LLA présentent un risque accru d'obésité, de résistance à l'insuline, et de dyslipidémie ${ }^{1}$. L'excès de tissu adipeux qui accompagne l'obésité favorise entre autres la libération d'adipokines inflammatoires favorisant le développement d'un état inflammatoire systémique. Le cerveau est vulnérable aux changements du métabolisme périphérique. Toutefois, le lien entre les marqueurs de l'inflammation et les fonctions cognitives demeure peu exploré dans cette population. Le but de l'étude est d'étudier les associations entre les biomarqueurs de l'inflammation et les déficits cognitifs.

Matériel et méthodes : Les analyses ont été effectuées sur la cohorte PETALE regroupant 246 survivants de la LLA ${ }^{2}$. Les biomarqueurs de l'inflammation ont été mesuré par V-Plex. Un score validé (DIVERGT) ciblant des domaines cognitifs sensibles aux traitements a été déterminé ${ }^{3}$. Des corrélations de Spearman ont été réalisées entre les biomarqueurs et le score DIVERGT. Les biomarqueurs les plus pertinents ont été catégorisés en tertiles et des comparaisons entre les groupes ont été faites à l'aide du test de Kruskal-Wallis.

Résultats et Analyse statistique : Les analyses préliminaires ont montré que les niveaux plasmatiques de TNF- $\alpha$ sont inversement corrélés au score du DIVERGT ( $r=0,189 ; P=0,003)$. Lorsque les niveaux de TNF- $\alpha$ sont catégorisés en tertiles, le score DIVERGT était significativement différent $(P=0,003)$. Les comparaisons post-hoc révèlent que le score au DIVERGT des participants du tertile 3 $(M=7,98 E C=2,41)$ était différent du tertile $1(M=8,87 E C=2,08 ; P=0,018)$ et du tertile $2(M=9,00 E C=2,23 ; P=0,005)$.

Conclusion : Les niveaux de TNF- $\alpha$, un marqueur d'inflammation systémique, sont associés à des déficits cognitifs chez les survivants de la LLA pédiatrique. Il est possible que l'identification de biomarqueurs inflammatoires mène à l'adoption de nouvelles stratégies thérapeutiques permettant de prévenir les troubles métaboliques et cognitifs suite au traitement du cancer de l'enfant.

Références : ${ }^{1}$ Levy E. et al. 2017.Sci Rep.

${ }^{2}$ Marcoux S. et al. 2017.Pediatr Blood Cancer.

${ }^{3}$ Boulet-Craig A. et al. 2018.Pediatr Blood Cancer.

Remerciements : Réseau Cardiométabolisme Diabète et Obésité

Instituts de recherche en santé du Canada

Société de recherche sur le cancer

Centre de cancérologie familiale Garron de l'Hôpital pour enfants malades

Fondation de l'Hôpital Sainte-Justine

Fonds de Recherche en santé du Québec

Groupes d'oncologie pédiatrique de l'Ontario

Conflits d'intérêts : Aucun conflit à déclarer 


\section{P113}

DHA des spermatozoïdes et infertilité : revue de la littérature et implications physiopathologiques

F. Saur ${ }^{1}$, C. Ingueneau ${ }^{1}$, L. Bujan ${ }^{2}$, S. Caspar-Bauguil ${ }^{1, *}$, S. Hamdi ${ }^{1}$

${ }^{1}$ Laboratoire de biochimie, Hôpital Purpan, Place Dr Baylac, ${ }^{2}$ Médecine de la reproduction, CHU Toulouse, Toulouse, France

Introduction et but de l'étude : L'infertilité touche 10 à $15 \%$ des couples, l'origine étant masculine dans $50 \%$ des cas. La moitié des étiologies de l'infertilité masculine ne sont pas identifiées. Le lien entre les facteurs nutritionnels et la qualité des spermatozoïdes (spz) a émergé comme une piste de recherche. Des études humaines ou animales pointent en particulier le rôle des acides gras poly-insaturés (AGPI) et notamment des oméga-3 (en particulier le DHA) dont l'implication n'a pas été clairement établie. L'objectif de ce travail est de faire le point par une analyse poussée de la littérature sur l'implication du DHA dans la qualité du sperme.

Matériel et méthodes : L'exploration de la base PubMed sur le thème lipides et spz humains a permis d'identifier 120 articles. Ils ont été triés en fonction des critères suivants : présence d'un groupe de patients normozoospermes, analyse lipidique des spz en chromatographie phase gazeuse et détection à ionisation de flamme (GC-FID), composition en acides gras (AG) numériquement exploitable et conversion possible en \%mol. Un AG a été sélectionné lorsque trois études au moins en exprimaient le résultat. Pour chaque $A G$, une moyenne pondérée a été calculée.

Résultats et Analyse statistique : L'analyse de la littérature a permis d'identifier neuf articles entre 1998 et 2012 totalisant 312 sujets normozoospermes. L'AGPI majoritaire est le DHA confirmant une composition membranaire unique. Cet enrichissement en DHA serait impliqué dans la fluidité membranaire et donc la mobilité des spz mais protégerait également l'ADN germinal du stress oxydant. Cinq études observationnelles ont montré que la proportion membranaire en DHA était significativement diminuée chez les hommes présentant une infertilité liée à des anomalies du spermogramme et en particulier une asthénozoospermie (mobilité anormale). Cinq études observationnelles réalisées à partir d'un questionnaire de fréquence alimentaire, ont montré une association inverse entre l'apport exogène d'oméga-3 et le niveau d'asthénozoospermie. Enfin, seules trois études interventionnelles sur six ont montré un effet bénéfique de l’apport en oméga-3 sur la qualité spermatique (mobilité, statut antioxydant).

Conclusion : Les données de la littérature font apparaitre que les spz font partie des types cellulaires les plus enrichis en DHA. Une question fondamentale émerge : quel est le rôle et le mécanisme de cet enrichissement ? Le testicule apparait comme ayant un métabolisme lipidique propre (avec élongations et désaturations), indispensable à la spermatogenèse. Les souris KO pour LPAAT3, impliqué dans la synthèse de phospholipides contenant du DHA au cours de la spermatogénèse, ou bien KO pour la désaturase FADS2, impliquée dans la synthèse du DHA à partir de l'acide alpha-linolénique, présentent une infertilité sévère associée à une baisse drastique du DHA dans les spz. II est aussi probable que d'autres compartiments interviennent (épididyme et plasma séminal). Le rôle de l'alimentation qui contient peu de DHA est controversé car il est possible que le compartiment génital mâle soit autonome dans sa synthèse. Des études complémentaires sont indispensables pour identifier l'implication des AGPI dans la physiopathologie de l'infertilité masculine et poser le rationnel des futurs essais interventionnels.

Conflits d'intérêts : Aucun conflit à déclarer 
Introduction et but de l'étude : Les carences nutritionnelles au cours de la grossesse sont des facteurs de comorbidité pendant la grossesse et au décours, pour l'enfant. L'objectif est de comparer le statut nutritionnel en vitamines et fer chez des patientes enceintes opérées d'une chirurgie bariatrique (CB) ou au cours de grossesses à risques (T) reçues dans une maternité de niveau 3. Matériel et méthodes : Cette étude concerne 87 patientes opérées (CB : 30 sleeve, 50 bypass, 7 autres chirurgies) et 73 femmes enceintes $(T)$ au cours du suivi d'une grossesse à risque (diabète, HTA, syndrome d'apnée obstructif du sommeil, obésité grade 2/3 non opérées, autres pathologies) vues en consultation de second trimestre (25,5 semaines d'aménorrhée (SA) [24,1 26.9]) entre 2015 et 2017. L'âge moyen est de 32 ans. Le délai moyen entre la chirurgie bariatrique et la grossesse actuelle est de 4,5 ans [3,9-5,2]. La parité et le geste sont comparables pour les 2 groupes ainsi que le terme [38,1-38,9 SA]. Les données biologiques du bilan hématologique et micronutritionnel (vitamines B9, B12, B6 (pyridoxal phosphate), C, A, E et D sériques, B1 (thiamine pyrophosphate) sang total, et ferritinémie) ont été recueillies rétrospectivement.

Les seuils définis ont été discutés par le Groupe d'Etude Français sur les grossesses après chirurgie bariatrique (BARIA-MAT) dans le cadre de la rédaction des recommandations françaises pour la pratique clinique en 2018.

Résultats et Analyse statistique : Les carences en vitamines B1, B6, B9, D et C sont plus fréquentes chez les femmes avec grossesse à risque $(T)$ que chez les femmes enceintes opérées $(C B)$.

\begin{tabular}{|l|c|c|c|c|}
\hline Paramètres : seuils & CB & T & $\mathbf{X}^{\mathbf{2}}$ & p \\
\hline B9 : $5 \mathrm{ng} / \mathrm{mL}$ & $80 / \mathbf{1 1 \%}$ & $73 / \mathbf{4 9 \%}$ & 26.64 & $* * *$ \\
\hline B6 : $10 \mathrm{nmol} / \mathrm{L}$ & $92 / \mathbf{1 8 \%}$ & $83 / \mathbf{3 4 \%}$ & 5.31 & $*$ \\
\hline B1 : $91 \mathrm{nmol} / \mathrm{L}$ & $91 / \mathbf{2 6 \%}$ & $71 / \mathbf{4 4 \%}$ & 5.32 & $*$ \\
\hline D $/ 30 \mathrm{ng} / \mathrm{mL}$ & $81 / \mathbf{4 2 \%}$ & $58 / \mathbf{7 2 \%}$ & 12.64 & $* * *$ \\
\hline C $/ 6 \mathrm{mg} / \mathrm{L}$ & $78 / \mathbf{1 7 \%}$ & $49 / \mathbf{3 7 \%}$ & 6.57 & $*$ \\
\hline
\end{tabular}

Données exprimées en effectif / \% au-dessous du seuil ; Test $X^{2}$ comparant les femmes CB vs $T$ à partir des seuils définis : $p<0.05$ $*, p<0.01^{* *}, p<0.001 * * *$. test non significatif pour les vitamines B12, A et $E$, l'hémoglobine et la ferritinémie.

Pour chacun de ces paramètres, dans les deux groupes, l'absence de supplémentation majore la prévalence de la carence.

Conclusion : Chez les femmes suivies pour une grossesse à risque, la prévalence des carences en vitamines est élevée. Cette prévalence est plus importante chez les femmes enceintes non opérées que chez les femmes enceintes opérées d'une chirurgie bariatrique, pourtant elles-mêmes considérées comme à haut risque de carence. La supplémentation en vitamines réduit la prévalence de ces carences.

Ces résultats nous incitent à organiser une politique de dépistage et de prise en charge des carences micronutritionnelles mises en évidence dans cette étude, au cours du suivi des grossesses pathologiques.

Conflits d'intérêts : Aucun conflit à déclarer 
Evaluation nutritionnelle des patients sous traitement anti néoplasique en Algérie M. A. Melzi ${ }^{12, *}$, Z. Derbouz ${ }^{2}$, Z. Bachiri ${ }^{2}$, A. Bounedjar ${ }^{12}$

${ }^{1}$ Faculté de Médecine, Université de Blida 1, ${ }^{2}$ Oncologie Médicale, Centre Hospitalo-Universitaire Frantz Fanon de Blida, Blida, Algérie

Introduction et but de l'étude : Le cancer est un problème de santé publique en Algérie, avec plus de 48000 cas de cancers en 2013. Une dénutrition est associée dans 30 à $40 \%$ des cas. C'est une cause majeure de morbidité, qui peut conduire à l'interruption de la stratégie thérapeutique. Son étiologie est complexe faisant intervenir plusieurs facteurs liée au patient, au siège de la tumeur et aux traitements. Les cancers de l'estomac, de l'œsophage, du pancréas et de la tête est du cou sont le plus souvent associés à une dénutrition.

Nous avons effectué ce travail pour évaluer l'état nutritionnel des patients sous antinéoplasiques.

Matériel et méthodes : Nous avons réalisé une étude prospective sur les patients recevant un traitement anti néoplasique pour leur maladie cancéreuse au niveau du service d'oncologie médicale du centre hospitalo-universitaire de Blida. L'objectif de ce travail est l'évaluation clinique de l'état nutritionnel des patients avant le début du traitement cytostatique, et son évolution sous traitement. L'indice de masse corporelle (IMC) est utilisé comme indicateur de l'état nutritionnel, calculé sur la base du poids initial du patient avant traitement, puis sur la base du poids mesuré après des séances de traitement antinéoplasique (évaluation) . Les résultats sont analysés selon le sexe et le siège de la tumeur primitive (test exact de Fisher).

Résultats et Analyse statistique : Nous avons colligé 232 patients évaluables. Les hommes représentent $56 \%$ des cas. L'âge moyen est de 57,71 $\pm 0,88$ ans. Les cancers de l'estomac, du sein et le colon représentent 19,8\%, 19,8\% et 19,4\% respectivement. L'évaluation de l'état initial des patients (avant le traitement anti néoplasique) montre un IMC moyen est de 23,37 $\pm 0,3$ (22,03 chez les hommes et 25,07 chez les femmes). Un sous poids est retrouvé chez $15,08 \%$ des patients (7,8\% chez les femmes et $20,7 \%$ chez les hommes; $p=0,0089)$. L'obésité est observée chez $7,32 \%$ des patients $(11,7 \%$ chez les femmes et $3,8 \%$ chez les hommes ; $p=0.039)$.

L'évaluation en cours du traitement cytostatique retrouve un IMC moyen de 23,33 $\pm 0,30$ (hommes 21,96 $\pm 0,37$; femmes 25,07 \pm 0.45 ), avec une variation moyenne de $1,43 \pm 0,11$ (hommes $1,61 \pm 0,13$; femmes $1,21 \pm 0,18$ ). La perte moyenne du poids est estimée à $5,9 \% \pm 0,45$ (hommes $6,8 \pm 0,5 \%$; femmes $4,7 \pm 0,7 \%$ ). Une perte du poids de plus de $30 \%$ est retrouvée chez $1.7 \%$ des patients $(2,9 \%$ chez les femmes et $0,7 \%$ chez les hommes ; $p=0,32)$. Une perte de poids entre 10 et $30 \%$ est observée chez $12 \%$ des patients $(5,8 \%$ chez les femmes et $16,9 \%$ chez les hommes; $p=0,01)$. Un gain de poids est observé chez $6,9 \%$ des patients $(13,7 \%$ chez les femmes et $1,5 \%$ chez les hommes ; $p=0,0003)$.

Conclusion : L'altération de l'état nutritionnel est observée chez $15 \%$ des patients au moment du diagnostic de leur cancer. Les hommes semblent être plus susceptibles à avoir un état nutritionnel initial altéré, et à dégrader leur état sous traitement anti néoplasique, par rapport aux femmes, qui ont plus tendance à améliorer leur statut.

Conflits d'intérêts : Aucun conflit à déclarer 
Effets de la $\mathrm{N}$-carbamoyl-putrescine sur le métabolisme protéique musculaire du rat âgé dénutri.

P. Jegatheesan ${ }^{1,{ }^{*}}$, N. Akir ${ }^{1}$, N. El-Hafaia ${ }^{1}$, R. Ramassamy ${ }^{1}$, M. Aboubacar ${ }^{1}$, S. Nakib ${ }^{2}$, N. Neveux $^{2}$, C. Loï $^{3}$, L. Cynober $^{12}{ }^{2}$ J. P. De Bandt ${ }^{12}$

${ }^{1}$ EA4466 PRETRAM, Faculté de Pharmacie - UNIVERSITÉ PARIS DESCARTES - Sorbonne Paris Cité, ${ }^{2}$ Service de Biochimie, Hôpital Cochin, Hôpitaux Universitaires Paris Centre, APHP, Paris, ${ }^{3}$ Citrage, Créteil, France

Introduction et but de l'étude : Une prévalence accrue des situations de dépendance liées à la sarcopénie est constatée avec l'augmentation de la population des personnes âgées. L'efficacité limitée des différents moyens de prise en charge disponibles en font un problème préoccupant. Nous avons montré chez le jeune rat qu'une molécule métaboliquement apparentée aux polyamines et dérivée de la citrulline, la N-carbamoyl-putrescine (NCP), augmentait le contenu protéique du muscle soleus. Dans ce contexte, l'objectif de cette étude était d'évaluer les effets de la NCP sur le métabolisme protéique musculaire au cours de la renutrition de rats âgés dénutris et d'en explorer les mécanismes sous-jacents, notamment l'évolution de la balance anabolisme/catabolisme protéique.

Matériel et méthodes : Des rats (18 mois) ont été dénutris par 6 semaines d'une alimentation à $50 \%$ de leurs apports spontanés puis répartis en 4 groupes : dénutris (DEN), dénutris puis renourris pendant 4 jours avec un régime standard à $100 \%$ de leurs apports spontanés, seul (REN) ou avec 1 ou $10 \mathrm{mg}$ de NCP/kg/j (NCP1, NCP10). Nous avons mesuré en particulier les acides aminés (AA) plasmatiques et musculaires, l'homéostasie azotée (bilan d'azote), l'équilibre entre catabolisme (3 méthylhistidine (3MH)) et synthèse protéique musculaires (SPM, mesurée par la méthode SunSet dans l'EDL, le soleus (Sol) et le tibialis (Tib)) et l'expression/l'activation des protéines de signalisation de l'anabolisme (4EBP1, S6K) et du catabolisme (MuRF1). Statistiques : ANOVA et PLSD de Fisher.

Résultats et Analyse statistique : Le gain et la vitesse de prise du poids au cours de la renutrition étaient semblables. La renutrition était associée à une augmentation de la triglycéridémie $(p<0,05)$ et, dans les groupes $N C P$, de la glycémie $(p<0,05)$. Quelle que soit la dose, la NCP favorisait un gain de masse du Tib ( $p<0,05$ vs DEN) mais pas de l'EDL ni du Sol et augmentait le contenu protéique de l'EDL (ANOVA $p=0.07$, NCP10 vs DEN, $p=0,05$ ) sans effet au niveau des autres muscles. La phosphorylation de 4EBP1 augmentait dans les groupes REN $(p<0,05)$, NCP1 $(p=0,06)$ et NCP10 $(p<0,05)$ par rapport au groupe DEN dans l'EDL. II en était de même dans le groupe REN vs NCP1 $(p=0,07)$ dans le Tib. Celle de S6K1 augmentait dans le Tib (NCP vs REN $(p=0,007))$ et l'EDL (+24 \% NCP vs REN et DEN (NS)). Toutefois, la vitesse de SPM n'était pas modifiée. La NCP diminuait l'expression de MuRF1 dans le Tib (NCP vs REN $(p<0,05)$ ) mais pas dans le Sol et l'EDL. La NCP ne modifiait pas le profil des AA plasmatiques, le bilan azoté, le rapport Phe/Tyr plasmatique, l'élimination urinaire de $3 \mathrm{MH}$, de créatinine et d'urée.

Conclusion : Au cours de la renutrition, l'apport de NCP maintient la masse maigre en agissant sur la balance protéique. Cet effet varie selon le type de muscle. Les effets de la NCP semblent limités soit par les effets immédiats de la renutrition et l'insulinorésistance associée soit par la disponibilité en AA pour la SPM.

Conflits d'intérêts : P. Jegatheesan: Aucun conflit à déclarer, N. Akir: Aucun conflit à déclarer, N. El-Hafaia: Aucun conflit à déclarer, R. Ramassamy: Aucun conflit à déclarer, M. Aboubacar: Aucun conflit à déclarer, S. Nakib: Aucun conflit à déclarer, N. Neveux: Aucun conflit à déclarer, C. Loï est employé(e) de Citrage, L. Cynober est actionnaire de Citrage, J. P. De Bandt est actionnaire de Citrage 
La spasticité est-elle un facteur prédictif de l'état nutritionnel des patients en état de conscience altérée ?

M. Fadeur ${ }^{1,}{ }^{*}$, C. Ippoliti ${ }^{2}$, C. Malherbe ${ }^{3}$, A.-M. Verbrugge ${ }^{3}$, O. Gosseries ${ }^{2}$, J. De Flines ${ }^{1}$, A. Thibaut ${ }^{2}$, S. Laureys ${ }^{2}$, N. Paquot ${ }^{1}$

${ }^{1}$ Diabétologie, Nutrition et Maladies métaboliques, ${ }^{2}$ Coma Science Group, ${ }^{3}$ Diététique, CHU Liège, Liège, Belgique

Introduction et but de l'étude : La littérature scientifique concernant l'état nutritionnel des patients en état de conscience altérée (ECA) est à l'heure actuelle très pauvre. Le but de cette étude prospective observationnelle est d'investiguer la relation entre le profil nutritionnel de ces patients et leur état de conscience.

Matériel et méthodes : Nous avons collecté et analysé les données cliniques (état de conscience, spasticité) et nutritionnelles (indice de masse corporelle (IMC), apports nutritionnels, biologie) de 80 patients en état d'éveil non répondant (ENR), en état de conscience minimale (ECM) ou en état de conscience minimale émergeant (EECM). Les patients ont été classés selon le résultat de l'analyse nutrtitionnelle: bien nourri, à risque de dénutrition et dénutri.

Résultats et Analyse statistique : De nos 80 patients ( $43 \pm 15$ ans), 19 étaient en ENR (24\%), 47 en ECM (59\%) et 14 en EECM (17\%). Parmi eux, 7 (9\%) étaient à risque de dénutrition et les 73 autres (91\%) étaient bien nourris.

$L^{\prime} I M C$ de ces sujets n'est statistiquement pas lié à leur niveau d'ECA $(p=0,09)$, au délai après l'accident $(p=0,12)$ ou à l'étiologie de leur état neurologique $(p=0,51)$.

Une différence entre l'apport énergétique réellement administré à ces patients et celui qu'ils devraient théoriquement recevoir sur base des recommandations ( $30 \mathrm{kcal} / \mathrm{kg}$ de poids de calcul) est observée. Les sujets obèses $\left(\geq 30 \mathrm{~kg} / \mathrm{m}^{2}\right.$ ) recevaient $631 \pm 328$ $\mathrm{kcal}$ en moins/jour par rapport aux recommandations et ceux en surpoids $\left(25-29,9 \mathrm{~kg} / \mathrm{m}^{2}\right), 415 \pm 390 \mathrm{kcal} / \mathrm{j}$ en moins également. En revanche, les patients avec un IMC faible $\left(<18,5 \mathrm{~kg} / \mathrm{m}^{2}\right)$ recevaient $106 \mathrm{kcal} \pm 299 \mathrm{kcal} / \mathrm{j}$ en plus.

La comparaison du niveau de spasticité en fonction de l'IMC révèle une corrélation significative $(r=-0,30, p=0,009)$ et montre que les patients les moins spastiques au niveau des membres inférieurs sont plus à risque d'avoir un surpoids (IMC $=27,7 \pm 7,5$ $\left.\mathrm{kg} / \mathrm{m}^{2}\right)$. Au contraire, les plus spastiques à ce niveau sont plus à risque d'avoir un IMC plus faible $\left(22,1 \pm 3,8 \mathrm{~kg} / \mathrm{m}^{2}\right)$. Parmi les 9 sujets avec un $I M C<18,5 \mathrm{~kg} / \mathrm{m}^{2}, 7$ présentent une spasticité sévère et 2 une spasticité modérée au niveau des membres inférieurs. L'étude de la différence d'apport énergétique par rapport à la spasticité montre que les patients non spastiques recevaient $297 \pm$ $318 \mathrm{kcal} / \mathrm{j}$ en moins par rapport aux recommandations, contre $222 \pm 430 \mathrm{kcal} / \mathrm{j}$ pour une spasticité moyenne et $41 \pm 406 \mathrm{kcal} / \mathrm{j}$ pour des niveaux de spasticité plus élevés.

Conclusion : Nos résultats indiquent que la majorité des patients ECA ont un état nutritionnel satisfaisant. Cet état nutritionnel ne semble pas lié au niveau de conscience, au délai ou à la cause de leur accident. Les patients les moins spastiques présentent des critères de surpoids malgré un apport énergétique considérablement réduit par rapport aux recommandations nutritionnelles. En revanche, un niveau élevé de spasticité est associé à un sous poids $\left(\mathrm{IMC}<18,5 \mathrm{~kg} / \mathrm{m}^{2}\right)$ en dépit d'un apport énergétique supérieur à l'objectif préconisé par ces recommandations. Des analyses complémentaires sont en cours (calorimétrie indirecte, impédancemétrie, étude de la spasticité et imagerie métabolique) afin de mieux comprendre les mécanismes sous-jacents.

Conflits d'intérêts : Aucun conflit à déclarer 
Introduction et but de l'étude : Chez le patient de réanimation la mise en place de sonde gastrique pour nutrition entérale peut être difficile, en raison de l'absence de collaboration et de l'abolition fréquente du réflexe de toux. L'incidence rapportée de placement dans les voies respiratoires est de l'ordre de $2 \%$ et est associée à un taux de pneumothorax et de décès de $10 \%$ et de $1,5 \%$ des patients dont la sonde a été mal placée (1). En cas d'échec répété ou de mauvais placement d'une sonde gastrique,

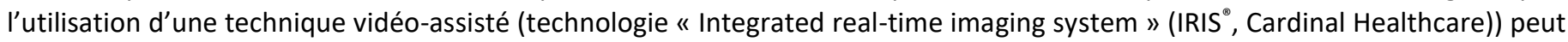
représenter un progrès substantiel et améliorer la sécurité des patients $(2,3)$. Cette étude a évalué la procédure de mise en place vidéo-assisté de sonde gastrique par le personnel infirmier de réanimation.

Matériel et méthodes : Après une séance de formation, une équipe d'infirmiers-référents a été constituée et disponible en cas de placement difficile ou infructueux de sonde gastrique, défini par deux échecs successifs de placement à l'aveugle chez des patients adultes nécessitant un accès pour nutrition entérale. La mise en place de sondes Kangaroo ${ }^{\star}$ en polyuréthane d'un calibre de 12 French d'une longueur de $91 \mathrm{~cm}$ équipé de la technologie IRIS ${ }^{\circledR}$ a été réalisée par l'infirmier en charge du patient sous la supervision d'un infirmier-référent. Une feuille d'évaluation a été remplie pour chaque placement vidéo-assisté.

Résultats et Analyse statistique : Dix-huit patients ont été inclus. Les sondes ont été placées par 8 infirmiers différents. Soixanteet-un pourcent des patients étaient conscients et $67 \%$ étaient intubés ou trachéotomisé au moment du placement vidéo-assisté. Lors du placement, les cordes vocales et la trachée ont été visualisées chez $22 \%$ des patients, le passage dans une bronche chez $11 \%$, l'œsophage a été visualisé chez $67 \%$, la paroi gastrique chez $94 \%$ et le pylore chez $11 \%$. Le placement final en site gastrique a été confirmé chez $100 \%$ des patients.

Conclusion : Chez le patient de réanimation, le placement vidéo-assisté de sonde naso-gastrique par l'équipe infirmière constitue une solution de recours en cas d'échec de placement à l'aveugle et permet d'assurer le positionnement correct de la sonde, même si la visualisation de l'ensemble des structures anatomiques n'est pas fréquente. La formation par les collègues référents facilite probablement la technique de placement. Une évaluation coût-bénéfice de cette technique est requise.

Références : 1- Sparks DA et al :JPEN $35: 625$ (2011)

2- $\quad$ Mizzi A et al : Nutrition $37: 48$ (2017)

3- Wischmeyer $P$ et al : doi: 10.1002/jpen.1313 JPEN 2018 (sous presse)

Conflits d'intérêts : G. Crohin: Aucun conflit à déclarer, S. Waeytens: Aucun conflit à déclarer, R. Sand: Aucun conflit à déclarer, N. Bailly: Aucun conflit à déclarer, M. De Mahieu: Aucun conflit à déclarer, J.-C. Preiser est consultant chez Cardinal Healthcare 
Introduction et but de l'étude : La dysphagie est un symptôme fréquemment retrouvé chez les patients hospitalisés aux soins intensifs (USI) après intubation trachéale, trachéotomie ou présence d'une sonde naso-gastrique. Les conséquences de la dysphagie comportent une incidence accrue de fausses routes et de pneumonies acquises en USI. L'objectif principal de l'étude est de déterminer si la mise en place d'un dépistage systématique de la dysphagie diminue (1) l'incidence des pneumonies acquises, (2) la durée de séjour en USI et à l'hôpital et (3) la mortalité à l'USI et à l'hôpital.

Matériel et méthodes : Les données de patients hospitalisés plus de 48 heures à l'USI, âgés de plus de 18 ans, et présentant au moins un facteur de risque de dysphagie ont été comparées avant (groupe " pré » - novembre 2015) et après instauration d'un dépistage systématique de la déglutition (groupe " post » - avril à juin 2017). Le risque de dysphagie a été établie à l'aide d'un bilan fonctionnel basé sur dix critères, réalisé par le kinésithérapeute et confirmé (ou infirmé) par un(e) orthophoniste et/ou par une naso-fibroscopie réalisée par le médecin ORL. Le Clinical Pulmonary Infection Score (CPIS), a été utilisé pour déterminer si les patients avaient acquis une pneumonie à I'USI. Un score CPIS $\geq 6$ était associé à une forte probabilité de pneumonie. Les données ont été comparées par tests-t, le test $U$ de Mann-Whitney, le test Chi-Carré ou le test exact de Fisher.

Résultats et Analyse statistique : Après consentement éclairé, 27 patients ont été inclus dans le groupe pré (16 hommes ; admis pour motif médical $(n=18)$, chirurgical $(n=9)$ ) et 35 dans le groupe post ( 26 hommes; admis pour motif médical ( $n=31)$, chirurgical $(n=4))$. Trois patients du groupe pré $(11,1 \%)$ ont été diagnostiqués dysphagiques lors du séjour à l'USI versus 27 patients dans le groupe post $(77,1 \%)(p<0,001)$. Cinq nouveaux cas de dysphagie ont été diagnostiqués dans le groupe pré après la sortie de I'USI $(18,5 \%)$ vs 0 nouveau cas de dysphagie dans le groupe post $(p=0,012)$. Dans le groupe pré, 5 patients $(18,5 \%)$ ont acquis une pneumonie à l'USI et 6 patients $(17,1 \%)$ dans le groupe post. La durée de séjour à l'USI et à l'hôpital des patients du groupe post sont plus longues que celles du groupe pré (14 [8-32] vs 8 [5-13], $p=0,006$ et 58 [31-77] vs 23 [15-38], p $<0,001$ respectivement). II n'y a pas de différence statistiquement significative pour la mortalité à l'USI ou à l'hôpital entre les deux groupes (5/27 vs 4/35 et $7 / 27$ vs $5 / 35$, respectivement).

Conclusion : La dysphagie recherchée systématiquement était très fréquente et associée à une incidence élevée de pneumonies. Par comparaison avec une période sans dépistage systématique de dysphagie, la fréquence des pneumonies était similaire, mais les troubles de déglutition étaient beaucoup plus rarement diagnostiqués à l'USI, et plus fréquemment observés après la sortie du patient. Ces données plaident en faveur de la poursuite du dépistage systématique de la déglutition à l'USI.

Conflits d'intérêts : Aucun conflit à déclarer 
Le cancer et la chimiothérapie affecte la fonction mitochondriale hépatique dans un modèle murin de cancer colique. B. Martin ${ }^{1,}{ }^{*}$, F. Larmarche ${ }^{1}$, E. Fontaine ${ }^{1}$, C. Moinard $^{1}$, C. Breuillard ${ }^{1}$

${ }^{1}$ INSERM U1055, LBFA, Grenoble, France

Introduction et but de l'étude : Il a été proposé qu'un dysfonctionnement de la fonction mitochondriale pourrait contribuer au développement de la cachexie (1). Cependant, à ce jour, il n'existe pas de données confirmant ce phénomène dans les cancers extra-hépatiques. Notre objectif était d'évaluer le métabolisme mitochondrial hépatique dans un modèle de rat cancéreux sous chimiothérapie (2).

Matériel et méthodes : Nous avons utilisé des rats femelles de 10 semaines qui ont été randomisé en deux groupes ( $n=6)$. Un groupe témoin d'animaux sains (C), et un groupe ayant une implantation tumorale sous cutanée au niveau du flan (tumeur du côlon Ward) et une chimiothérapie (K). Après l'implantation du cancer, et une phase de croissance tumorale, la chimiothérapie, incluant deux cycles (CPT-11 et 5-FU) à une semaine d'intervalle, est injectée.

A l'euthanasie, un jour après la dernière chimiothérapie, le foie est rapidement prélevé et les mitochondries sont isolées. Puis la respiration mitochondriale lors de la synthèse d'ATP et la respiration sans synthèse d'ATP en fonction du potentiel membranaire (fuite de protons et/ou découplage des pompes à proton) ont été mesurées tel que décrit (3). Les résultats sont exprimés en moyenne \pm SEM.

Le protocole a été validé par le comité d'éthique (APAFIS\#11492-2017112715178992).

Résultats et Analyse statistique : La respiration sans synthèse d'ATP en fonction du potentiel membranaire n'a pas été affectées par le cancer et la chimiothérapie. Par contre, la respiration lors de la synthèse d'ATP a été fortement réduite de $32 \%$ ( $C=222 \pm 21$ $\mathrm{nmol} \mathrm{O} / \mathrm{min} / \mathrm{mg}$ de protéine, vs $\mathrm{K}=153 \pm 29 \mathrm{nmol} \mathrm{O} / \mathrm{min} / \mathrm{mg}$ de protéine, test-t unilatéral, $\mathrm{p}=0,03$ ).

Conclusion : Nos résultats préliminaires suggèrent une altération du métabolisme mitochondrial hépatique lié au cancer et à la chimiothérapie. La contribution de cette altération mitochondriale dans la cachexie cancéreuse reste à confirmer.

Références : 1. Dumas et al., J.Hepatol., 2011

2. Xue et al., Br J Nutr., 2009

3. Nicholls et al., Methods Mol Biol., 2012

Conflits d'intérêts : Aucun conflit à déclarer 
P121

Effets d'un régime à haute teneur en protéines dans un contexte de surnutrition lipidique et calorique sur la synthèse protéique musculaire et l'infiltration lipidique chez les rats adultes et âgés.

E. Poggiogalle ${ }^{1, *}$, A. Carayon ${ }^{1}$, J. Salles ${ }^{1}$, C. Giraudet ${ }^{1}$, J.-P. Rigaudière ${ }^{1}$, S. de Saint-Vincent ${ }^{1}$, P. Sanchez ${ }^{1}$, O. LeBacquer $^{1}$, F. Capel ${ }^{1}$, S. Walrand ${ }^{1}$, Y. Boirie $^{1}$, C. Guillet $^{1}$

${ }^{1}$ Centre de Recherche en Nutrition Humaine, Université de Clermont Ferrand, Clermont Ferrand, France

Introduction et but de l'étude : L'infiltration lipidique ectopique peut influencer négativement l'efficacité anabolique de l'apport protéique au niveau musculaire surtout au cours du vieillissement. L'objectif de l'étude était de déterminer les effets d'un apport protéique élevé sur les altérations du métabolisme musculaire, chez les rats adultes et âgés en situation de surnutrition lipidique et calorique.

Matériel et méthodes : Deux groupes de soixante rats Wistar adultes et âgés ont été répartis aléatoirement en quatre groupes consommant quatre régimes différents pendant dix semaines: un régime conventionnel isocalorique (12\% protéines, $14 \%$ lipides, STD12); un régime isocalorique riche en protéines (25\% protéines, $14 \%$ lipides, STD25); un régime hypercalorique, hyperlipidique et isoprotéique (12\% protéines, $45 \%$ lipides, HFD12); et un régime hypercalorique, hyperlipidique à haute teneur en protéines (25\% protéines, 45\% lipides, HFD25). À partir de l'enrichissement en [13C]-valine mesuré par spectrométrie de masse, la vitesse de synthèse fractionnaire (FSR) et la vitesse de synthèse absolue (ASR) des protéines musculaires totales ont été mesurées au sein du muscle tibial antérieur (TA). Le contenu lipidique intramusculaire a été évalué par chromatographie.

Résultats et Analyse statistique : À cause de l'autolimitation de la prise alimentaire chez les rats dans les groupes hypercaloriques et hyperlipidiques, l'apport énergétique n'était pas différent parmi le quatre groupes. Au-delà des types de régime, le poids du muscle TA était inférieur chez les rats âgés comparés aux groupes des rats adultes $(p<0.01)$. La vitesse de synthèse protéique fractionnaire était inférieure chez les rats âgés dans le groupe HFD25 comparé au groupe STD12 (effet du régime: $p=0.02$ ), mais la vitesse de synthèse fractionnaire dans les groupes âgés était plus élevé que dans les groupes adultes (effet de l'âge, $p<0.05$ ). Par contre la vitesse absolue de synthèse protéique n'était pas différente parmi les quatre groups. Le niveau de triglycérides intramusculaires dans le muscle TA était plus élevé dans le groupe de rats âgés qui avaient reçu le régime hyperlipidique et isoprotéique (HFD12) (effet de l'âge: $p=0.02$; effet du régime: HFD12 vs. STD12: $2.04 \pm 1.74$ vs. 0.83 $\pm 0.49 u g / g, p=0.02$ ).

Conclusion : Chez les rats âgés le poids musculaire était diminué même si la vitesse de synthèse protéique fractionnaire était augmentée; ces observations suggèrent des troubles spécifiques de la régulation nutritionnelle du renouvellement protéique musculaire. Dans un contexte isocalorique, un haut teneur en protéines interfère avec l'infiltration lipidique intramusculaire, sans améliorer la résistance anabolique chez les rats âgés consommant un régime hyperlipidique.

Conflits d'intérêts : Aucun conflit à déclarer 
Intérêt du hand grip test dans la prise en charge diététique du patient cirrhotique compliqué de décompensation oedématoascitique

B. Sainton ${ }^{1, *}$, A. Paillard ${ }^{1}$, J. Capelle ${ }^{1}$, R. Piquemal ${ }^{1}$

${ }^{1}$ Unité transversale diététique et nutrition, Centre Hospitalier Simone Veil de Blois, Blois, France

Introduction et but de l'étude : L'évaluation de l'état nutritionnel du patient cirrhotique compliqué de décompensation oedémato-ascitique est difficile puisque les critères de dépistage usuels peuvent être faussés : le delta poids et l'IMC sont surévalués en cas de rétention hydrique, l'albumine et la pré-albumine sont diminuées par le biais de l'insuffisance hépatocellulaire. Notre étude a pour objectif de déterminer l'intérêt du grip test comme outil de dépistage de la dénutrition chez le patient cirrhotique décompensé avec ascite.

Matériel et méthodes : Il s'agit d'une étude interventionnelle prospective comparative. Les patients inclus sont cirrhotiques, avec décompensation oedémato ascitique ayant été ponctionnée. Les critères d'exclusion sont les patients avec carcinome hépatocellulaire et/ou encéphalopathie permanente. Les critères de dénutrition recueillis sont I'IMC, la circonférence brachiale (CP) et circonférence musculaire brachiale (CMB), le hand grip test (HGT), l'évaluation de la prise alimentaire (EPA), l'évaluation subjective globale (SGA) et les apports énergétiques et protéiques $\left(I M C<24 \mathrm{~kg} / \mathrm{m}^{2}\right.$, ingesta en calories $<2 / 3$ des besoins estimés, ingesta en protéines $<2 / 3$ des besoins estimés, $E P A<7, C B<24 \mathrm{~cm}, C M B<19 \mathrm{~cm})$. Le HGT était mesuré à 3 reprises sur le bras non dominant. Le seuil retenu caractérisant la dénutrition sur le HGT est $<30 \mathrm{~kg}$ chez l'homme et $<17 \mathrm{~kg}$ chez la femme. La significativité de la corrélation de ces données était évaluée par le test de Pearson par le logiciel Statistica.

Résultats et Analyse statistique : 7 patients étaient inclus dans cette étude (4 hommes et 3 femmes). L'âge moyen était de 57,9 +/- 6,2 ans. A l'entrée, 5 patients avaient un score Child-Pugh B et 2 patients un score Child-Pugh C. Le score de MELD était en moyenne de $14+/-4,5$. Parmi les 7 patients inclus :

- 1 patiente présentait 6 des 7 critères de dénutrition à l'entrée. Cette patiente était classée dénutrie sévère par le SGA. Seul le HGT ne semblait pas conclure à une dénutrition.

- 2 patients présentaient 4 des 7 critères de dénutrition à l'entrée. Les 2 patients étaient classés comme modérément dénutri avec le SGA. Seul I'un des deux patients présentait une dénutrition sur le HGT.

- 4 patients présentaient 3 des 7 critères de dénutrition à l'entrée. Deux patients étaient classés comme modérément dénutri par le SGA dont un présentant une dénutrition sur le HGT.

- 2 patientes présentant un score MELD>15 étaient modérément et sévèrement dénutries.

L'IMC, EPA, CB, CMB, SGA n'étaient pas corrélés de manière significative à l'HGT hormis le score de MELD corrélé négativement au HGT mesurée à l'entrée $(r=-0.94, p=0.016)$. La prise en charge diététique a permis une augmentation significative de l'EPA, du HGT, de la $C B$ et de la CMB soit 4 des 7 critères de dénutrition mesurés entre la consultation diététique initiale et le suivi diététique.

Conclusion : Dans notre étude, le HGT ne s'avère pas être un outil fiable de diagnostic de la dénutrition au regard de l'absence de corrélation avec une $\mathrm{CMB}<5^{\text {ème }}$ percentile et le SGA. Néanmoins, la corrélation négative entre le MELD score et le HGT nous permet de suggérer une association entre la gravité de la cirrhose et l'altération de la force musculaire. D'autre part, le HGT apparaît comme un moyen de mesure de l'efficacité de la prise en charge nutritionnelle.

Conflits d'intérêts : Aucun conflit à déclarer 
Analyse longitudinale du support nutritionnel chez des patients primo traités pour un cancer des voies aérodigestives supérieures (VADS) au Centre Antoine Lacassagne

C. Hebert ${ }^{1, *}$, C. Michel $^{2}$, J. Sicurani ${ }^{1}$, Y. Chateau ${ }^{3}$, J. Viotti ${ }^{4}$, M.-N. Falewee ${ }^{1}$

${ }^{1}$ Nutrition, ${ }^{2}$ activités scientifiques, ${ }^{3}$ Data Management, ${ }^{4}$ Statistiques, Centre Antoine Lacassagne, Nice, France

Introduction et but de l'étude : Le support nutritionnel est jugé nécessaire et est intégré suivant les traitements (tt) proposés dans les recommandations nationales pour les patients (pts) atteints de cancer.

Matériel et méthodes : L'unité de nutrition du centre a évalué l'efficacité de sa prise en charge dans une étude longitudinale observationnelle tout tt confondu sur les 2 critères suivants : compliance aux recommandations, pourcentage (\%) de couverture des besoins théoriques.

Résultats et Analyse statistique : Sur 2 ans, 135 pts ont été inclus et suivis avant tt, puis 1, 2, 3, 6, 9, 12, et 18 mois après le début $\mathrm{du}$ tt initial. Sept ont eu un suivi diététique sans complémentation (groupe $1 ; 5,2 \%), 37$ ont nécessité uniquement des compléments nutritionnels oraux (CNO) (groupe $2 ; 27,4 \%$ ), et 91 une nutrition entérale (NE) (groupe $3 ; 67,4 \%$ ).

Dans le groupe 1, 4 pts ont bénéficié d'une chimiothérapie d'induction suivie de radiochimiothérapie sans retentissement sur leur statut nutritionnel.

Dans le groupe 2, les recommandations nutritionnelles ont été respectées malgré l'hétérogénéité des tt.

Le pourcentage des besoins théoriques couvert par les CNO était en moyenne de 41,1\% [36,7]. Pendant les 6 premiers mois, les CNO couvraient $40,5 \%$ à $49,5 \%$ des besoins. Plus de la moitié des pts continuaient à prendre des CNO au-delà de 9 mois après l'initiation du $\mathrm{tt}$, avec une couverture des besoins théoriques de $20 \%$.

Dans le groupe 3, les 91 pts ont été analysés en 3 groupes.

La NE transitoire exclusive a été nécessaire chez 19 pts sur une durée cumulée moyenne de 65,5 [60,9] jours, dont 14 du fait d'une chirurgie. Pour les 5 autres pts, la NE a été instaurée du fait d'une dénutrition sévère ou dysphagie totale. Seize \% des pts avaient encore une NE au-delà de 9 mois après le début de tt.

La NE a été associée à des CNO chez 37 pts avec une durée cumulée moyenne de 119,3 [114,6] jours. La prise en charge nutritionnelle a été conforme aux recommandations.

Durant le tt, $70 \%$ des pts avaient leur besoins théoriques couverts par la NE à hauteur de $88 \%$. Six mois après tt, seul un quart des pts nécessitait une NE. La NE a été nécessaire à 9 mois après le début de tt pour $21,6 \%$ des pts avec une couverture des besoins par la NE $>60 \%$.

Enfin, 35 pts ont reçu en alternance une $\mathrm{NE}$, des $\mathrm{CNO}+$ /- associés à une alimentation orale. Dans ce groupe, les recommandations n'ont pas été respectées du fait du patient ou des médecins. La durée cumulée moyenne de NE était de 113,3 [128,8] jours. Cinq pts avaient encore des CNO 18 mois après le début de tt.

Conclusion : Notre étude a montré l'importance du suivi nutritionnel tout traitement confondu chez les patients porteurs d'un cancer des VADS et sur une longue période. Une prescription de support nutritionnel permettant la couverture des besoins théoriques a été nécessaire chez $95 \%$ des patients (dont $71 \%$ de NE).

Le suivi nutritionnel par une équipe médicale spécialisée permet d'optimiser la prise en charge de chaque patient en expliquant et en adaptant les référentiels pendant et après le traitement. Un suivi à long terme est justifié car plus d'un tiers des patients reçoit encore un support nutritionnel 9 mois après le début de traitement.

Conflits d'intérêts : Aucun conflit à déclarer 
${ }^{1}$ néphrologie, Hôpital Marie Curie, Lodelinsart, Belgique

\section{Introduction et but de l'étude :}

La prévalence de malnutrition est évaluée à plus de $30 \%$ dans les populations de patients hémodialysés. Paradoxalement et contrairement à la population générale, les patients arrivés en dialyse avec un BMI > 30 présentent une mortalité moindre. La masse musculaire interfère avec de nombreux facteurs de morbi/mortalité (résistance aux agents anabolisants, inflammation cryptogénique, stress oxydatif, traumatismes et chutes, etc...) La cascade de la dénutrition est aussi influencée par l'urémie, l'inflammation gastrique, l'anémie, la fatigue, la dépression et l'insuffisance cardiaque. Des modèles animaux de sarcopénie ont démontré un effet spectaculaire d'une supplémentation en AAE et éllagitannins contenus dans des extraits de grenade sur le syndrome inflammation / malnutrition et la masse musculaire.

Le but de cette étude est de mesurer les effets d'une supplémentation orale de ce type à notre population de patients hémodialysés durant 6 mois.

Matériel et méthodes : 26 patients en hémodialyse chronique à raison de $3 \times 4$ heures/semaine ont été sélectionnés au hasard et appariés avec 26 autres hémodialysés (groupe témoin). Chaque patient traité a absorbé pendant $10 \mathrm{mois}$ un flacon de $125 \mathrm{ml} / \mathrm{jour}$ de Myogran Drink ${ }^{\circledR}$. Le Kt/V a fait l'objet d'une surveillance étroite et chaque participant a été observé longitudinalement tant pour ses paramètres biologiques que pour les données anthropomorphiques et la bio-impédance. La composition du Myogran Drink ${ }^{\circledR}$ est reprise au tableau (1).

Résultats et Analyse statistique : Sur 26 patients traités (T0), 20 ont terminé l'étude(T6). Les 6 autres patients furent soit transplantés ( $\mathrm{N}=2$ ) soit décédés. $48 \%$ de femmes et $52 \%$ d'hommes ont été inclus avec un âge moyen de 69.2 ans.

Après 10 mois de cette étude, l'ALB augmente de manière significative (graphique 1). La CRP et les paramètres lipidiques s'améliorent nettement (graphiques 2 et 3 ) tandis que la masse maigre se stabilise contrairement au groupe témoin. Notons aussi qu'un paramètre important tel que la résistance à l'EPO s'améliore sous traitement.

Conclusion : Après 10 mois de prise de suppléments oraux à base d'AAE, de collagène hydrolysé et d'extraits de grenade riches en éllagitannins, les paramètres biologiques, inflammatoires et lipidiques sont favorablement influencés chez des patients dialysés.

II nous semble que l'amélioration de la synthèse protéique par les AAE et les effets antioxydants des éllagitannins, déjà bien étayés dans la littérature, peuvent exercer un effet favorable chez le patient dialysé chronique.

Références :

Malnutrition-inflammation complex syndrome in dialysis patients: causes and consequences. Kalantar-Zadeh $\mathrm{K}$ et al. :. Am $\mathrm{J}$ Kidney Dis. 2003 Nov;42(5):864-81.

Réactivité de polyphénols du vin sous conditions oxydantes : hémisynthèse des mongolicaïnes, et d'adduits entre polyphénols et thiols odorants. Emilie Petit Université Sciences et Technologies - Bordeaux I, 2013. Français. NNT : $2013 B O R 14915$

Urolithin B, a newly identified regulator of skeletal muscle mass. Julie Rodriguez et al : J Cachexia Sarcopenia Muscle. 2017 Aug; 8(4): 583-597. Published online 2017 Mar 1. doi: 10.1002/jcsm.12190

Conflits d'intérêts : Aucun conflit à déclarer 
Prévalence de la dénutrition à l’admission et impact sur la mortalité en réanimation, expérience dans un service de réanimation polyvalente.

P.-A. Rogghe ${ }^{1,{ }^{*}}$, V. Pages ${ }^{1}$, F. Rousseau ${ }^{1}$, T. Vanderlinden ${ }^{1}$

${ }^{1}$ Réanimation, GHICL, Lomme-Les-Lille, France

Introduction et but de l'étude : La dénutrition en réanimation est une préoccupation démontrée par son impact sur la morbidité et le pronostic. Sa prévalence est importante et probablement sous-estimée ; les données actuelles semblent cependant assez variables selon l'outil de dépistage utilisé. L'objectif de ce travail est d'évaluer la prévalence de la dénutrition à l'admission dans notre service de réanimation polyvalente, via l'utilisation de l'algorithme algorea du PNNS, ainsi que son impact sur la mortalité et les méthodes de suppléance vitale en réanimation (épuration extra-rénale, ventilation mécanique).

Matériel et méthodes : Etude rétrospective, monocentrique, dans un service de réanimation polyvalente de 12 lits, sur une période de 20 mois. L'ensemble des dossiers a été analysé à l'exception des patients admis pour surveillance programmée de chirurgie. Le bilan nutritionnel est basé sur l'algorithme algorea, effectué endéans les $48 \mathrm{~h}$ après admission, permettant une répartition en 2 sous groupes : non dénutris « ND » et dénutris « $D$ » (modérés et sévères). Cet outil de dépistage est basé sur: l'index de masse corporelle, la perte de poids, l'albumine corrigée ainsi que le "nutrition risk index". L' « outcome » primaire est la mortalité en réanimation, les " outcomes " secondaires sont la durée de séjour en réanimation, le recours à la ventilation mécanique et à l'épuration extra-rénale. Comparaison des sous-groupes via âge, sexe, score IGS 2 (indice de gravité simplifié), score Omega (indice de charge thérapeutique). Les analyses statistiques " chi-deux » et " test T de Student » ont été réalisées à l'aide du logiciel SPSS Statistics (version 20).

Résultats et Analyse statistique : 556 dossiers ont été examinés et 476 ont pu être analysés, 80 non inclus pour données manquantes. $77 \%$ des patients sont dénutris. $58 \%$ sont des hommes pour $42 \%$ de femmes. Les deux sous-groupes sont comparables et il n'existe pas de différence de gravité initiale. L'âge (ND 66,2 $\pm 16,5$ vs D 65,5 $\pm 15,1 ; p$ 0,733), l'IGS2 (ND 47,5 \pm 17,8 vs D 51,2 $\pm 19,9 ;$ p 0,149), l'Omega (ND 330,6 $\pm 384,2$ vs D 379,11 $\pm 435 ; p$ 0,310) ne montrent pas de différence significative. La mortalité en réanimation est supérieure dans le groupe dénutri (ND : 25,9\%, D : 38,3\%, p <0,001), ainsi que le recours à la ventilation mécanique (ND : 69,4\%, D : 74,7\%, $p<0,001$ ) et à l'épuration extra-rénale (ND : 13\%, D : 20,1\%, $p<0,001$ ). Nous n'observons par contre pas de différence en terme de durée de séjour (ND : 15,9 $\pm 16,4$ vs $D: 17,2 \pm 17 ; p: 0,529$ ). La « ProtéineC-réactive » est nettement supérieure dans le groupe dénutri (ND : 34,4 $\pm 46,2$ vs $D: 150,1 \pm 108,8 ; p<0,001$ ).

Conclusion : Nos données confirment la prévalence importante de la dénutrition à l'admission en réanimation et son influence significative sur la mortalité et le recours aux techniques de suppléance (ventilation mécanique, épuration extra-rénale). Le diagnostic rapide et la prise en charge optimale de ce déficit apparaissent donc fondamentaux dans la prise en charge globale du patient en réanimation.

Conflits d'intérêts : Aucun conflit à déclarer 
Protection contre le développement futur de l'athérosclérose via l'administration périnatale d'un extrait liquide de spiruline chez la souris APO E -/-

M. Coué ${ }^{12,{ }^{*}, \text { B. Castellano }}{ }^{2}$, J. Falewée ${ }^{1}, A$. Aguesse $^{2}$, O. Lépine ${ }^{3}$, K. Ouguerram $^{12}$

${ }^{1}$ Université de Nantes, ${ }^{2}$ UMR PhAN, INRA, ${ }^{3}$ AlgoSource Group, Nantes, France

Introduction et but de l'étude : L'athérosclérose représente la première cause de mortalité dans les pays industrialisés. Une étude récemment publiée du laboratoire a démontré qu'une hypercholestérolémie maternelle augmente le développement des lésions athéromateuses dans la descendance chez la souris APO E-/-. II est connu que l'hypercholestérolémie est associée au stress oxydant (SO). Le but de ce travail est d'étudier l'effet d'une supplémentation maternelle avec un extrait liquide de spiruline (ELS), microalgue très riche en antioxydants $(\mathrm{AO})$, sur le développement de plaque dans la descendance.

Matériel et méthodes : Nous avons croisé des souris APO E-/- et les femelles gestantes ont reçu de l'eau (contrôles) ou de l'ELS (80 mg de AO/souris/jour) pendant la gestation (ELS G) ou pendant la gestation et la lactation (ELS G+L). Après sevrage, toute la descendance a suivi un régime contrôle. Les descendants mâles et femelles ont été étudiés séparément et ont été mis à mort à 25 semaines d'âge. L'aire des plaques d'athérosclérose a été quantifiée après coloration à l'huile rouge de la racine aortique. Les tests statistiques ont été réalisés sur $n=6-12$ souris/groupe via un test ANOVA.

Résultats et Analyse statistique : Il n'y a pas de différence concernant le poids corporel des descendants à 25 semaines d'âge. A $600 \mathrm{~nm}$ du cœur, nous avons vu une diminution significative de la surface des plaques d'athérosclérose chez les descendants ELS G F, ELS G+L F et ELS G M $(-39 \%$; $-39 \%$ et $-52 \%$ respectivement, $p<0,05)$. A 16 semaines d'âge, les souris $E L S$ G $+\mathrm{L} F$ ont significativement moins de choline $(-23 \%, p<0,05)$ et de TMAO $(-35 \%, p<0,05)$ plasmatiques alors que les mâles ELS $G$ présentent moins de carnitine $(-24 \%, p<0,05)$. Les souris ELS $G$ ont une tendance à avoir une augmentation plasmatique de l'apolipoprotéine A1 $(p=0,08)$.

Conclusion : Dans cette étude, nous démontrons qu'un ELS administré durant la période fœtale protège du développement futur des plaques d'athérosclérose chez la souris APO E KO. D’autres analyses sont nécessaires pour mettre en évidence les mécanismes d'action.

Conflits d'intérêts : M. Coué: Aucun conflit à déclarer, B. Castellano: Aucun conflit à déclarer, J. Falewée: Aucun conflit à déclarer, A. Aguesse: Aucun conflit à déclarer, O. Lépine a un conflit d'intérêt avec Directeur d'AlgoSource et financeur du projet de recherche., K. Ouguerram: Aucun conflit à déclarer 
L'activité physique spontanée en condition d'obésité ralentit la croissance tumorale en modulant les signaux hormonaux tissulaires

D. Le Guennec ${ }^{1,{ }^{*}}$, V. Hatte ${ }^{1}$, M. Goepp ${ }^{1}$, M.-C. Farges ${ }^{1}$, S. Rougé ${ }^{1}$, F. Caldefie-Chezet ${ }^{2}$, M.-P. Vasson ${ }^{1}$, A. Rossary $^{1}$

${ }^{1}$ Laboratoire de biochimie, biologie moléculaire et nutrition; Equipe ECREIN ; UMR1019 UNH, ${ }^{2}$ Laboratoire de sciences végétales et fongiques ; Equipe ECREIN ; UMR1019 UNH, Université Clermont Auvergne - INRA, Clermont-Ferrand, France

Introduction et but de l'étude : En situation d'obésité, les dérégulations du tissu adipeux induisent la sécrétion d'œstradiol et de leptine (pro-inflammatoire) aux dépens d'adiponectine (anti-inflammatoire), une augmentation du stress oxydant associée à une baisse des capacités anti-oxydantes favorisant un microenvironnement pro-carcinogène. L'activité physique (AP), facteur de prévention de l'obésité et des cancers, favorise une inflammation aigüe modérée, la réponse anti-oxydante et la sécrétion d'adipokines anti-inflammatoires. Les mécanismes de protection de l'AP dans le cancer mammaire sont mal connus. Le but est de caractériser le dialogue inter-organe induit par l'AP spontanée et l'impact sur la croissance tumorale dans un modèle de carcinogenèse mammaire murin en situation d'obésité.

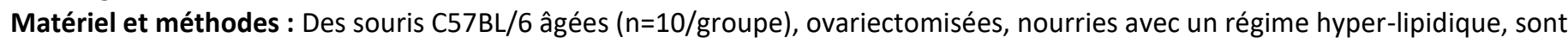
hébergées en environnement standard (ES) ou en environnement enrichi (EE) favorisant l'AP spontanée. Après 4 semaines, des cellules tumorales syngéniques E0771 sont implantées par fat-pad. Au sacrifice, l'exploration des adipokines et des cytokines a été conduite dans les organes d'intérêt (tumeur, tissu adipeux inguinal, gastrocnémien, plasma) ainsi que la caractérisation du statut oxydatif et des voies de signalisation (JNK, AKT, NFKB, p38, S6K1) de la tumeur.

Résultats et Analyse statistique : L'EE entraîne une augmentation de l'AP sans modification des masses maigres et adipeuses, ni des métabolites énergétiques (triglycérides, glucose). L'analyse factorielle multiple montre que les variables expliquant les différences entre individus sont les masses adipeuses (totale, viscérale et inguinale), l'adiponectine plasmatique, les taux de leptine des tissus d'intérêt, les capacités anti-oxydantes et les voies de signalisation intra-tumorales. Les adipokines plasmatiques sont corrélées aux masses adipeuses et aux voies de signalisation tumorales. Le taux de leptine dans le gastrocnémien est corrélé avec celui de la tumeur $\left(r^{2}=0,57 p<0,05\right)$ et montre une forte diminution chez les animaux EE vs ES (tumeur: 0,30 $\pm 0,07$ vs $0,60 \pm$ $0,10 \mathrm{pg} / \mathrm{mg} ; \mathrm{p}<0,05$; gastrocnémien : $0,09 \pm 0,03$ vs $0,45 \pm 0,11 \mathrm{pg} / \mathrm{mg} ; \mathrm{p}<0,05)$. L'IL6 plasmatique est diminuée et associée à une réduction du stress oxydant tumoral (thiols protéiques, GST, thiorédoxine) pour les souris EE vs ES. Une baisse de l'activation des voies tumorales de signalisation est observée pour les souris EE vs ES, corrélée positivement avec les masses musculaires et négativement avec les masses adipeuses.

Conclusion : Ces résultats montrent que l'activité physique spontanée en condition d'obésité ralentie la croissance tumorale, en modifiant le dialogue inter-organe, conduisant à une réduction de l'inflammation et du stress oxydatif du microenvironnement tumoral. Une consommation de la leptine est observée dans la tumeur et le gastrocnémien alors que la production au niveau du tissu adipeux et la distribution plasmatique ne semblent pas affectées. Ainsi, l'activité physique module les signaux hormonaux tissulaires conduisant à une moindre stimulation des voies de signalisation nécessaires à la croissance tumorale.

Conflits d'intérêts : Aucun conflit à déclarer 
Signature postprandiale des microARN circulants et association à l'hypertriglycéridémie

R. Daclat ${ }^{12, *}$, P. Giral ${ }^{123}$, J.-E. Salem ${ }^{124}$, M. Guérin ${ }^{12}$, J.-M. Lacorte ${ }^{125}$

${ }^{1}$ Sorbonne Université, ${ }^{2}$ UMRS1166, INSERM, Institut de recherche des maladies cardiovasculaires, Métabolisme et Nutrition,

${ }^{3}$ Endocrinologie-Métabolisme, ${ }^{4} \mathrm{CIC},{ }^{5}$ Biochimie Endocrinienne et Oncologique, APHP Hôpital Pitié-Salpêtrière, Paris, France

Introduction et but de l'étude : Les microARN (miR) sont de petits ARN non codants, de 18 à 25 nucléotides dont certains sont retrouvés dans la circulattion. Ils régulent l'expression des gènes cibles à un niveau post-transcriptionnel et ont été impliqués dans la régulation de nombreux processus biologiques, y compris le métabolisme glucido-lipique. La physiopathologie de I'hypertriglycéridémie postprandiale (PP), qui est un facteur de risque cardiovasculaire, n'est pas complètement élucidée. Notre étude, vise à comparer l'expression relative des miRs circulants entre des sujets normolipidémiques (NLS) et les patients hypertriglycéridémiques (HTG) avant et après l'ingestion d'un repas standardisé en faisant les hypothèses suivantes 1) les miRs peuvent varier en PP 2) la variation PP des miRs est associée aux troubles métaboliques.

Matériel et méthodes : Après extractions des mIRs circulants, nous avons utilisé les cartes microfluidiques Taqman Low Density Array (TLDA, ThermoFisher). Nous avons analysé le profil d'expression de 754 miRs chez 5 sujets NLS avant et 2 heures après l'ingestion d'un repas standardisé pour identifier les miRs qui varient en PP. Les miR-candidats ont été quantifiés par RT-qPCR durant la phase PP $(0,2 h, 4 h$ et $6 h$ après la prise du repas) dans 2 populations plus larges : NLS ( $n=15)$ et HTG ( $n=29)$. L'analyse statistique a été réalisée avce le logiciel "R".

Résultats et Analyse statistique : Dans une première étape, nous avons identifié, chez 5 sujets NLS, 10 miRs dont l'expression variait significativement 2 heures après la prise de repas. La variation des miRs a été confirmée chez 15 NLS, montrant une augmentation significative $(p<0,005)$ de leur expression relative au cours de la phase $P P$, allant de 1,8 à 4,4 fois par rapport à leur expression à jeun. En revanche, cette variation PP n'a pas été détectée pour l'ensemble des 10 miRs chez les HTG (n=29). Une analyse en composante principale de la variation PP des miRs a permis de mettre en évidence d'une part une signature PP spécifique associée au groupe HTG et d'autre part de distinguer 2 sous-groupes au sein des HTG (HTG1 et HTG 2). L'analyse des paramètres biologiques montre que les sujets HTG se différencient par le niveau d'insulino-résistance évalué par le HOMA ( $p$ $<0,05)$. Une analyse multivariée comparant les sous-groupes HTG 1 et HTG 2 permet d'identifier 9 variables qui contribuent pour près de $80 \%$ à la différence entre les sous-groupes. Parmi ces variables, on distingue en plus de l'insulino-résistance, 6 miRs ainsi que la concentration en LDL et HDL.

Conclusion : Ces observations originales mettent en évidence pour la première fois une variation post-prandiale des miRs circulants établissant une signature dépendante du contexte métabolique. Dans la littérature, ces miR-candidats ont été associés soit au métabolisme lipidique soit à des troubles métaboliques. II s'agit maintenant d'établir si ces variations sont des biomarqueurs associés aux troubles métaboliques ou s'ils contribuent à l'apparition et au développement d'un dysmétabolisme.

Conflits d'intérêts : Aucun conflit à déclarer 
Introduction et but de l'étude : Le niveau d'apport protéique en cancérologie est un sujet de controverse. En effet un apport trop élevé en protéine pourrait favoriser la croissance tumorale, et au contraire des apports hypo protéiques favoriseraient la dénutrition et l'état de cachexie, ce dernier étant un facteur de morbi-mortalité chez les patients. A ce jour il n'existe pas d'étude clinique ou expérimentale permettant de trancher la question.

L'objectif est donc d'évaluer l'influence de différents apports protéiques dans un modèle de rats cancéreux sous chimiothérapie. Matériel et méthodes : Soixante-dix-huit rats femelles (Fisher 344) ont été divisés en 6 groupes : un groupe témoin (C) sans cancer ni chimiothérapie $(n=8)$ et avec un régime standard ( $100 \%$ des besoins en protéines) et 5 groupes avec cancer (tumeur du colon "Ward » injectée en sous cutané) et deux cycles de chimiothérapie (CPT-11 et 5-FU) (K), avec des régimes différents ( $n=14$ ) et des quantités différentes de protéines (50\%, $75 \%, 100 \%, 150 \%$ et $200 \%$ des besoins en protéines). La prise alimentaire, le poids des animaux ainsi que la taille de la tumeur sont mesurés quotidiennement. Lors de l'euthanasie, les organes sont pesés et la synthèse protéique est évaluée dans les muscles et la tumeur.

Le protocole a été validé par le comité d'éthique (APAFIS\#11492-2017112715178992).

Résultats et Analyse statistique : Le cancer se traduit par une perte de poids des animaux ( $C=171.5 \pm 2.6$ vs $\mathrm{K}=150.8 \pm 2.4)$. De même au niveau tissulaire, le cancer induit une atrophie du foie $(p<0,005)$, du thymus $(p<0,005)$, de la masse maigre $(p<0,005)$ et de la masse grasse $(p<0,005)$. De façon étonnante, ces altérations de la composition corporelle ne sont pas affectées ni par les apports hyper protéiques ni par les apports hypo protéiques. Par ailleurs, la synthèse protéique n'a pas montré de différence entre les groupes. Concernant la croissance tumorale, celle ci n'est pas affectée par le niveau d'apport protéique.

Conclusion : Nos premières données suggèrent que ni le statut nutritionnel ni la croissance tumorale ne semble modulée par le niveau d'apport protéique.

Conflits d'intérêts : Aucun conflit à déclarer 
Profil alimentaire sélénié des cardiopathes de la population de Tlemcen (extrême Ouest Algérien)

M. Belhadj ${ }^{1}$, N. Dennouni ${ }^{1,}{ }^{*}$, A. Behar ${ }^{1}$, M. Dali-Sahi ${ }^{1}$, Y. Harek ${ }^{1}$

${ }^{1}$ Laboratoire de chimie analytique et d'électrochimie, Université Abou Bekr Belkaid Tleamcen Algérie, Tleamcen, Algérie

Introduction et but de l'étude : Le sélénium "Se" est un micronutriment d’une grande importance en nutrition humaine. II exerce ses fonctions biologiques grâce à des protéines auxquelles il est associé "sélénoprotéines". La plupart des sélénoprotéines, dont les fonctions sont connues, interviennent dans les systèmes redox et les voies de signalisation. Une carence modérée en sélénium, semble accroître la sensibilité à diverses maladies dans lesquelles le stress oxydant est impliqué : maladies cardiovasculaires, maladies inflammatoires, infections virales, maladies neurodégénératives et cancers.

L'objectif de cette étude est d'estimer l'apport alimentaire en Se chez une population de cardiopathes et, d'évaluer son éventuelle association à la maladie chez la population étudiée.

Matériel et méthodes : Il s'agit d'une étude descriptive analytique de type cas-témoins réalisée sur un échantillon de 100 sujets dont 39 cardiopathes et 61 témoins. Les données de consommation alimentaire ont été collectées via la méthode du rappel de consommation alimentaire de 24 heures. L'apport en Se a été calculé grâce à un fichier "CIQUAL, 2017» et les tests statistiques ont été réalisés par le logiciel Minitab /version 16.

Résultats et Analyse statistique : La comparaison entre les moyennes des apports des cas 37,1 $\pm 29,2 \mu \mathrm{g} / \mathrm{J}$ et les témoins $101,2 \pm$ $34,9 \mu \mathrm{g} / \mathrm{J}$ par le test de Student a révélé une différence significative avec $\mathrm{p}=0,000$.

Le test d'ANOVA a montré une différence significative entre l'apport alimentaire du Se et la fréquence de consommation de certains aliments: pattes $(p=0,002)$, viande $(p=0,004)$, fruits $(p=0,007)$, pain $(p=0,042)$, lait et produits laitiers $(p=0,048)$.

Le test de corrélation a montré que les valeurs de l'apport sont positivement corrélées avec les valeurs du: triglycéride $(p=0,05)$ et l'âge ( $p=0,239)$; et inversement corrélées avec: I'indice de la masse corporelle (IMC) $(p=0,026)$ et cholestérol $(p=0,221)$.

Conclusion : D'après ces résultats on peut dire qu'une carence en Se est un facteur de risque pouvant aggraver l'état de sujets atteints de maladies cardiovasculaires ou bien prédisposer les sujets sains à la mise en place d'une cardiopathie.

Conflits d'intérêts : Aucun conflit à déclarer 
Traitement des tumeurs des voies aérodigestives supérieures : faut-il mettre une sonde de gastrostomie prophylactique ? M. M. Van Gossum ${ }^{1}$, A. Yanni ${ }^{2,}{ }^{*}$, D. Dequanter ${ }^{2}$, A. Barik ${ }^{1}$, V. Pierart ${ }^{3}$, P. Eisendrath $^{1}$

${ }^{1}$ Gastroenterlogie, ${ }^{2}$ Stomatologie, ${ }^{3}$ Diététique, CHU Saint-Pierre, Bruxelles, Belgique

Introduction et but de l'étude : : Les patients atteints d'un cancer des voies aérodigestives supérieures (VADS) sont souvent dénutris avant et pendant leur traitement. La sonde de gastrostomie prophylactique s'est avérée être un outil efficace pour assurer une alimentation suffisante. Cependant, il n'existe pas d'algorithme capable d'identifier les patients à haut risque de dénutrition. Le but de cette étude a été de décrire la prise en charge nutritionnelle, d'évaluer l'impact de la mise en place prophylactique d'une gastrostomie et d'identifier des facteurs prédictifs de dénutrition.

Matériel et méthodes : Cette étude rétrospective inclus 152 patients atteints de cancer des VADS traités par chirurgie,

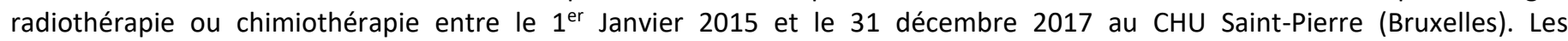
caractéristiques nutritionnelles, tumorales et thérapeutiques ont été rapportées et évaluées avant et après traitement. Des tests statistiques ont été réalisés dont notamment une régression logistique pour identifier les facteurs associés à la dénutrition.

Résultats et Analyse statistique : La mise en place d'une sonde de gastrostomie prophylactique a été effectuée chez 41 patients sur 152 en raison d'un index de masse corporelle bas, d'une dénutrition sévère et d'un trouble de l'ingestion orale, sans prendre en considération la localisation tumorale, le stade tumoral et le plan thérapeutique.

La comparaison du groupe avec sonde prophylactique et du groupe sans sonde prophylactique montre une différence significative en terme de réhospitalisation ( $p=0,042)$, perte de poids relative $(p<0,0001)$, dysphagie, dénutrition sévère et dégradation de l'état général $(p=0,001)$. Aucune différence significative n'a été observée quant aux complications infectieuses et/ou fistules postopératoires.

Dans le groupe des patients sans gastrostomie prophylactique, en analyse univariée, une atteinte ganglionnaire, des troubles de l'ingestion orale, un traitement par radiochimiothérapie et une localisation hypopharyngée ou nasopharyngée étaient des facteurs prédictifs significatifs de dénutrition, avec un seuil de perte de poids relatif de $5 \%$.

Conclusion : Notre étude a démontré les avantages d'une gastrostomie endoscopique percutanée prophylactique en terme de réhospitalisation, perte de poids, dysphagie, dénutrition sévère et dégradation de l'état général. Elle précise les indications de mise en place sur base des facteurs prédictifs de dénutrition.

Conflits d'intérêts : Aucun conflit à déclarer 
La prévalence de la boulimie chez les adolescents au Maroc : Région de Fès

M. Makdad ${ }^{1,}{ }^{,}$, K. Harraqui ${ }^{1}$, Z. Hannoun ${ }^{1}$, A. Bour ${ }^{2}$

${ }^{1}$ Doctorante, ${ }^{2}$ Professeur, Université Ibn Tofail, faculté des Sciences, kénitra, Maroc

Introduction et but de l'étude : La boulimie est un trouble de comportements alimentaires qui touche principalement les adolsecents. L'objectif de notre étude était de déterminer la prévalence de la boulimie chez les adolescents de la ville de Fès, Maroc.

Matériel et méthodes : Etude transversale portant sur un échantillon de 367 élèves des collèges et des lycées privés et publiques. Un auto-questionnaire sur les caractères sociodémographiques et sur les habitudes de vie et alimentaires a été administré. Le dépistage de la boulimie s'est fait grâce à une échelle spécifique Eating Attitudes Test (EAT-26). Les troubles anxieux et dépressifs ont été évalués grâce à l'échelle Hospital Anxiety and Depression (HAD). Des mesures anthropométriques ont été réalisées de manière standard chez tous les adolescents.

Résultats et Analyse statistique : L'âge moyen était de 15,58 $\pm 2,01$ avec l'étendue de [12-19] ans. L'indice de masse corporelle (IMC) moyen était de 19,99 $\pm 2,47 \mathrm{~kg} / \mathrm{m}^{2}$. 51,5\% étaient des filles. La prévalence de la boulimie était de 9,6 \% (IC 95 \% [6,6-12,5]). La boulimie était significativement plus fréquente chez les adolescents de sexe féminin ( $p=0,007$ ), ayant un état dépressif $(p=0,002)$ et un IMC normal $(p=0,000)$. Le régime alimentaire était le moyen de contrôle du poids le plus utilisé chez les adolescents souffrant de la boulimie $(40,8 \%)$.

Conclusion : La boulimie est un problème de santé fréquent dans notre contexte, d'où la nécessité de prendre des mesures préventives pour cette pathologie.

Conflits d'intérêts : Aucun conflit à déclarer 
${ }^{1}$ Hépato-gastro-entérologie et Nutrition, ${ }^{2}$ Chirurgie Digestive et Générale, CHU Caen Normandie, Caen, France

Introduction et but de l'étude : La chirurgie carcinologique sus-mésocolique est considérée comme à risque de syndrome de renutrition inappropriée (SRI). Bien que des supplémentations ioniques soient prescrites de manière systématique, ce risque n'est pas spécifiquement identifié en préopératoire. Le but de cette étude était d'évaluer la survenue du SRI dans notre population. Les objectifs secondaires étaient d'évaluer en préopératoire l'état nutritionnel, le risque de SRI et leur impact sur la survenue de troubles ioniques post-opératoires.

Matériel et méthodes : Etude observationnelle, rétrospective, monocentrique, incluant consécutivement tous les patients ayant eu une chirurgie sus-mésocolique carcinologique programmée du 29/01/2018 au 29/06/2018 et ayant reçu une nutrition artificielle post-opératoire. Les données recueillies étaient: dénutrition (critères HAS/SFNEP/SFAR ou perte de poids $>10 \%$ sans délai), ingesta, compléments oraux, hydratation, nutrition artificielle en préopératoire et en post-opératoire, risque de SRI (critères du National Institute for Health and Care Excellence, NICE), biologie ( $K, P, M g$ ) et la présence d'évènements intercurrents. Un SRI était défini par un trouble ionique associé à une symptomatologie clinique compatible.

Statistiques : test $\mathrm{t}$ de Student, $\mathrm{Chi}^{2}$ Yates ou test exact de Fisher, seuil de significativité fixé à $p<0.05$.

Résultats et Analyse statistique : Sur la période, 22 patients ont bénéficié d'une chirurgie majeure sus-mésocolique carcinologique (hors hépatectomie), 1 a été exclu par manque de données. Age médian 67 ans [51 - 85] et 76\% d'hommes. Le geste était : duodénopancréatectomie céphalique (57\%), œsophagectomie (24\%), gastrectomie totale (9,5\%) et gastrectomie partielle (9,5\%). L'évaluation préopératoire montrait $38 \%$ de patients dénutris. Les apports oraux préopératoires (compléments nutritionnels inclus) étaient supérieurs chez les patients non dénutris par rapport aux patients dénutris $(2509 \mathrm{kcal}$ vs $1734 \mathrm{kcal}-p=0,02)$. Le risque de SRI était présent chez $11 \%$ des patients. De J0 à J10 post-opératoire, $45 \%$ des patients ont présenté une hypophosphorémie $<0,80 \mathrm{mmol} / \mathrm{l}, 20 \%$ une hypomagnésémie $<0,75 \mathrm{mmol} / \mathrm{l}$ et $14 \%$ une hypokaliémie $<3,6 \mathrm{mmol} / \mathrm{l}$. Le nombre d'hypophosphorémies $<0,60 \mathrm{mmol} / \mathrm{I}$ de $\mathrm{J} 0$ à $\mathrm{J} 10$ post-opératoire était significativement différent entre les patients non dénutris et dénutris ( 0 vs $57 \% p=0,007$ ). Celles-ci survenaient de J0 à J4 malgré des apports en énergie et phosphore non significativement différents. L'appartenance à la population à haut risque ou la présence d'au moins un facteur de risque de SRI selon NICE n'était pas prédictif d'une hypophosphorémie $<0,60 \mathrm{mmol} / \mathrm{I}$. Un patient dénutri a développé un SRI (avec troubles neurologiques et admission en réanimation), bien qu'il n'ait pas été identifié à risque de SRI.

Conclusion : Le SRI est rare mais grave. Dans ce travail, la dénutrition était un meilleur facteur prédictif de SRI que les critères du NICE. Il apparait important de mesurer les phosphorémies, magnésémies et kaliémies en période post-opératoire, afin de mettre en place, le plus rapidement possible les supplémentations adaptées, surtout chez les patients dénutris.

Conflits d'intérêts : Aucun conflit à déclarer 
Hyperadiponectinémie chez les patients diabétiques dénutris hospitalisés pour traitement de lésions du pied R. Charchour ${ }^{1,}{ }^{*}$, F. Phan ${ }^{2}$, S. Fourati ${ }^{1}$, E. Ben Salah ${ }^{3}$, F. Mestari ${ }^{4}$, O. Bourron ${ }^{2}$, J.-M. Lacorte ${ }^{156}$

${ }^{1}$ Biochimie Endocrinienne et Oncologique, ${ }^{2}$ Diabétologie, ${ }^{3}$ Département d'Immunologie, ${ }^{4}$ Biochimie Métabolique, HU Pitié Salpêtrière-Charles Foix, ${ }^{5}$ UMRS 1166, INSERM, ${ }^{6}$ Sorbonne Université, Paris, France

Introduction et but de l'étude : Une dénutrition se caractérise entre autre sur le plan biologique, par la diminution de la préalbumine sérique (P-Alb) qui s'observe également au cours des processus inflammatoires. Dans ces conditions, il est difficile d'interpréter l'état nutritionnel par exemple chez des patients diabétiques lorsque apparaissent des lésions infectieuses. L'adiponectine (ADPN), une adipokine produite par le tissu adipeux, est paradoxalement augmentée chez les patients dialysés dénutris et chez les insuffisants cardiaques cachectiques. Nous avons fait l'hypothèse que l'ADPN pouvait être un marqueur de dénutrition chez les sujets diabétiques ayant des lésions ulcéreuses du pied.

Matériel et méthodes : II s'agit d'une étude prospective chez 82 hommes, hospitalisés dans le service de diabétologie aux HU Pitié-Salpêtrière-Charles Foix pour la prise en charge d'une ulcération infectée du pied chez des patients diabétiques. Un bilan biologique de dénutrition et d'inflammation est réalisé à l'entrée (T0) puis une semaine après la prise en charge thérapeutique (T1). Les patients ont été répartis en 3 groupes selon la concentration de $P$-Alb: groupe dénutri (DN), P-Alb <110 mg/L ( $n=13)$; groupe indéterminé (I) $110 \mathrm{mg} / \mathrm{L}<\mathrm{P}-\mathrm{Alb}<200 \mathrm{mg} / \mathrm{L}$ ( $\mathrm{n}=34$ ); groupe non-dénutri (NDN) P-Alb >200 mg/L ( $\mathrm{n}=34$ ). L'ADPN totale a été dosée par technique Elisa (Bulhmann, Suisse), la P-Alb et la CRP par immunoturbidimétrie et I'IL-6 par chimiluminescence. Le traitement statistique des données a été réalisé avec le logiciel Prism version 7.

Résultats et Analyse statistique : Lors de l'admission à TO, nous trouvons une corrélation inverse entre les concentrations d'ADPN et de P-Alb $(p<0.0001)$ mais pas de corrélation avec les marqueurs de l'inflammation tels que la CRP et IL-6. En comparant les groupes DN et NDN, il y a une augmentation significative des concentrations de CRP ( $p<0,0001)$ et d'IL-6 ( $<<0,0001)$ dans le groupe DN mais pas de différence pour l'ADPN. Entre T0 et T1, nous observons dans le groupe DN une augmentation significative de I'ADPN ( $p<0.05)$, en même temps qu'une diminution de la CRP $(p<0.01)$ et de l'IL-6 $(p<0.05)$ alors que nous ne constatons aucune différence pour l'ADPN, la CRP et l'IL-6 dans le groupe NDN. Enfin, à T1 il existe une différence significative entre le groupe DN et NDN pour la P-Alb $(p<0,001)$, l'IL-6 $(p<0,01)$ et l'ADPN $(p<0,0001)$ mais pas pour la CRP.

Conclusion : Lors de la prise en charge de lésions infectieuses du pied chez le patient diabétique, l'adiponectine ne permet pas de distinguer les groupes dénutris et non dénutris. Cependant, après diminution de l'inflammation, il apparait une augmentation des concentrations circulantes d'ADPN uniquement dans le groupe dénutri. Ces résultats sont cohérents avec l'effet inhibiteur de l'IL6 sur l'adiponectine déjà rapporté dans la littérature. Dans ces conditions, nous proposons d'évaluer la combinaison de plusieurs marqueurs biologiques pour mieux suivre l'état nutritionnel des patients en particulier au cours du traitement du pied diabétique.

Conflits d'intérêts : Aucun conflit à déclarer 
${ }^{1}$ Oncologie/Hematologie/Secteur Protegé, Centre Hospitalier de la Cote Basque, Bayonne, ${ }^{2}$ utnc chu Caen, chu caen, caen , France

\section{Introduction et but de l'étude :}

Malgré les recommandations indiquant qu'il est primordial d'utiliser la nutrition entérale (NE) lorsque le tube digestif est viable, l'utilisation de la nutrition parentérale (NP) reste la méthode la plus utilisée en hématologie. Le but était d'évaluer la faisabilité et l'impact de la nutrition entérale chez des patients admis pour autogreffe de moelle.

\section{Matériel et méthodes :}

Etude observationnelle menée dans un $\mathrm{CH}$ dont les données ont été recueillies prospectivement. La promotion de la NE a été décidée en équipe, mais la proposition à chaque patient était laissée au libre choix de l'hématologue référent qui demandait l'accord du patient. Les objectifs nutritionnels étaient de 30-35 kcal/kg/j. Les données cliniques (poids, appétit par EPA, échelle visuelle analogique de bien-être) et biologiques (albumine, prealbumine, CRP) étaient recueillies entre J-1 et J+1 de la greffe, puis à la sortie d'aplasie. Un questionnaire était donné au personnel du service pour évaluer sa perception de la NE. Les résultats étaient exprimés en médiane [extrêmes] comparées par un test de Mann-Whitney.

Résultats et Analyse statistique : Entre décembre 2017 et mai 2018, 18 patients (9H/9F, âge 60,5 ans [36-70] ont été traités par autogreffe pour myélome $(n=11)$, lymphome $(n=6)$ ou Waldenström $(n=1)$. Une patiente décédée précocement $n$ 'a pas été inclue. Le support nutritionnel était assuré par NE $(n=5)$, NP $(n=9)$ ou sans support $(n=4)$. Sur les 13 patients $n$ 'ayant pas eu de NE, 7 avaient refusé la NE, et c'était le choix du médecin pour 6 . Les caractéristiques initiales des patients étaient identiques quelque soit le support nutritionnel reçu, sauf le poids initial plus élevé dans le groupe sans support (97 kg vs 65 kg pour NE et 74 kg pour $N P ; p=0,03)$. L'évolution après versus avant greffe est indiquée dans le tableau

\begin{tabular}{|l|c|c|c|c|}
\hline Variation après vs avant greffe & NE $(n=5)$ & NP $(n=9)$ & Oral seul $(n=4)$ & $\begin{array}{c}\text { NE versus NP } \\
p\end{array}$ \\
\hline Poids $(\mathrm{kg})$ & $-2(-13$ à +3$)$ & $-1,8(-8$ à $-0,8)$ & $-2,45(-4,7$ à 1,5$)$ & $N S$ \\
\hline Albumine $(\mathrm{g} / \mathrm{l})$ & $-1,4(-7$ à 14$)$ & $-8(-14$ à -1$)$ & $-9,7(-20$ à -1$)$ & 0,03 \\
\hline Préalbumine $(\mathrm{g} / \mathrm{l})$ & $-0,01(-0,11$ à 1$)$ & $-0,07(-0,13$ à $-0,02)$ & $-0,085(-0,13$ à $-0,05)$ & 0,05 \\
\hline EPA (0-10) & $0(0$ à 5$)$ & $-2(-4$ à 0$)$ & $-1(-4$ à 0$)$ & 0,006 \\
\hline EVA bien être & $1(0$ à 5$)$ & $-2(-4$ à -1$)$ & $-2(-3$ à -1$)$ & 0,003 \\
\hline
\end{tabular}

Les questionnaires ont été renseignés par 16 professionnels du service (5 médecins, 11 IDE/AS), 62\% exprimaient une image négative de la NE et $69 \%$ s'estimaient mal à l'aise avec cette prise en charge.

Conclusion : Malgré une difficulté de mise en place de la NE chez les patients autogreffés en lien avec une réserve des patients et des équipes soignantes, I'utilisation de la NE améliore le statut nutritionnel et devrait être privilégiée.

Remerciements : Remerciements aux medecins, infirmieres et AS du service qui ont permis de réaliser cette etude. Remerciements à Marie Astrid Piquet pour son aide et son soutien.

Conflits d'intérêts : Aucun conflit à déclarer 
Les paramètres de composition corporelle (CC) sont-ils associés au devenir clinique de patients atteints d'un cancer du pancréas avancé traités par chimiothérapie à base de fluoropyrimidine ?

M. Som ${ }^{12, *}$, C. Brac de la Perrière ${ }^{23}$, J. Viaud ${ }^{23}$, B. Campillo-Gimenez ${ }^{23}$, M. Thomas ${ }^{2}$, L. Lacaze ${ }^{2}$, J. Edeline ${ }^{23}$, R. Thibault $^{12}$

${ }^{1}$ Unité de nutrition- CHU Rennes, ${ }^{2}$ Institut Nutrition Métabolisme et Cancer, NuMeCan, INRA, INSERM, Université de Rennes, ${ }^{3}$ Centre Eugène Marquis, Rennes, France

Introduction et but de l'étude : Le cancer du pancréas (CP) est l'un des cancers les plus agressifs avec près de $80 \%$ des patients présentant une maladie métastatique au diagnostic. La chimiothérapie à base de fluoropyrimidine (Folfirinox) est l'un des traitements standards de première ligne métastatique. Ce protocole est le plus efficace mais également le plus toxique. L'impact de la CC du patient incluant la sarcopénie, spécifiquement dans le CP avancé reste débattu. L'objectif de notre étude était donc d'évaluer l'impact de différents paramètres de CC sur le pronostic du CP et la toxicité du Folfirinox (FOX).

Matériel et méthodes : Tous les patients traités par FOX en première ligne d'un CP avancé entre 2007 et 2015 dans notre Centre de Lutte contre le Cancer ont été rétrospectivement analysés. La CC a été évaluée par coupe scanner au niveau de la troisième vertèbre lombaire. Les images ont été analysées avec le logiciel ImageJ (NIH, USA). La sarcopénie était définie par l'index musculaire squelettique (IMS) $\left(<52.4 \mathrm{~cm}^{2} / \mathrm{m}^{2}\right.$ pour les hommes et $38.5 \mathrm{~cm}^{2} / \mathrm{m}^{2}$ pour les femmes). Les index du muscle psoas (IMP), du tissu adipeux viscéral (IAV), sous-cutané (IASC) et intramusculaire étaient évalués. Satistiques : Kaplan Meier et Cox pour comparer les effets des paramètres de CC sur les survies globale et sans progression. Tests du Chi-2 et Fisher Exact pour la toxicité de la chimiothérapie.

Résultats et Analyse statistique : Les données de 137 patients ont été extraites : 91 avaient un scanner abdominal disponible et ont été inclus. L'âge moyen ( \pm SD) était de 60.7 ( \pm 9.2 ) ans. La maladie était localisée dans $42 \%$ des cas et métastatique dans $58 \%$. IMS moyen : $H, 48.63( \pm 7.03) \mathrm{cm}^{2} / \mathrm{m}^{2}, F, 36.92( \pm 6.00) \mathrm{cm}^{2} / \mathrm{m}^{2}$. La prévalence de la sarcopénie était de $64 \%(n=58)$. En analyse multivariée, ni la sarcopénie ni les autres paramètres de CC (hormis l'index de tissu adipeux intrapsoas) n'étaient significativement associés à la survie globale ou à la survie sans progression. En revanche, le performans status (HR=0.42 [0.20;0.85], $p=0.015)$, la chirurgie du primitif $(H R=0.55$ [0.32;0.93], $p=0.03)$, la présence de métastases hépatiques $(H R=2.62[1.59 ; 4.32], p<0.001)$ ainsi que l'index de tissu adipeux intrapsoas ( $H R=0.52$ [0.29;0.95], $p=0.03$ ) étaient des facteurs indépendants prédictifs de la mortalité. Les patients ayant un IMS faible $\left(<37.3 \mathrm{~cm}^{2} / \mathrm{m}^{2}, \mathrm{n}=22\right)$ avaient plus de vomissement que ceux ayant un IMS haut (54\% vs $24 \%$, $\mathrm{p}=0.016)$. Les patients avec un IASC et IAV élevé $\left(>24.6 \mathrm{~cm}^{2} / \mathrm{m}^{2} \mathrm{n}=63,>21.8 \mathrm{~cm}^{2} / \mathrm{m}^{2} \mathrm{n}=62\right.$, respectivement) avaient significativement plus de toxicité hématologique $\geq$ grade 3 ( $35 \%$ vs $5 \%, p=0.009 ; 34 \%$ vs $9 \%, p=0.028$, respectivement).

Conclusion : Cette étude ne met pas en évidence de lien entre les paramètres de CC et la survie chez les patients avec CP localement avancé ou métastatique. En revanche, un IMS bas, un IASC ou IAV haut est associé à une toxicité de la chimiothérapie plus sévère. Cela mérite des études complémentaires, notamment pour savoir si une intervention nutritionnelle ciblée pourrait améliorer la tolérance de la chimiothérapie de ces patients.

Conflits d'intérêts : Aucun conflit à déclarer 
Introduction et but de l'étude : En réanimation, la gestion de la nutrition entérale (NE) est complexe car de nombreux facteurs contribuent souvent à l'instauration d'un déficit énergétique et protéique tout au long du séjour des patients.L'objectif de l'étude a été de confronter nos pratiques professionnelles au protocole de nutrition entérale en vigueur en réanimation adulte et de comparer les apports en énergie et en protéines réellement administrés avec les recommandations des sociétés savantes.

Matériel et méthodes : Etude prospective, observationnelle, menée dans le service de réanimation de l'établissement durant 6 mois. Population étudiée: 39 patients ( $\mathrm{F}, \mathrm{n} \mathrm{H}$, âge moyen IMC moyen) sur un total de 215 patients admis, pris en charge par la diététicienne dans les 24 premières heures et pour lesquels une NE est prescrite ou susceptible de l'être au cours séjour, en complément ou non d'une autre nutrition. Les besoins énergétiques et protéiques de chaque patient sont calculés à partir du référentiel de nutrition artificielle en réanimation.

Résultats et Analyse statistique : - 79\% des patients reçoivent une NE au cours des 24 heures suivant leur admission. II existe un écart quotidien entre ce qui est prescrit et réellement administré au patient. Si le $1^{\text {er }}$ jour, seule $36 \%$ de la quantité prescrite est administrée, cette différence s'estompe au fils du séjour bien qu'elle reste de l'ordre de $10 \%$ en moyenne.

- La comparaison entre les apports moyens quotidiens par patient en énergie et en protéines avec les apports recommandés montre qu'un apport supplémentaire de $630 \mathrm{kcal} /$ jour et $46 \mathrm{~g}$ de protéines est nécessaire pour couvrir les besoins patients. Le bilan cumulé en énergie et protéines illustre l'évolution des déficits et met en exergue des déficits croissants dès le $3^{\text {ème }}$ jour et qui doublent presque entre J3 et J15.

Conclusion : Cette étude nous a permis de mettre en évidence des différences importantes entre les apports protéinoénergétiques et la cible recommandée, ceci dès le début de la prise en charge. A partir de ces constats des mesures d'amélioration ont été mises en place, en particulier l'individualisation des apports nutritionnels, la nécessité de faire évoluer notre protocole de NE de réanimation pour atteindre les cibles sous 48 heures ainsi que de viser la fourchette haute de la cible nutritionnelle recommandée.

Références : Fontaine $E$, Massanet $P$, Schneider S. Prévalence et conséquences des troubles de l'état nutritionnel en réanimation. Annales Françaises d'Anesthésie et de Réanimation. 33 (2014) 202-218

Hurel J-Y. Lefrant N.J. Cano C. Ichaï J-C. Preiser F. Tamion. Guidelines for Nutrition Support in Critically III Patient 23 (2014) $332-$ 350

Remerciements : Au service de Diététique du CH de Fréjus Saint Raphaël

Au service de réanimation du CH de Fréjus Saint Raphaël

Au Pr Schneider

Conflits d'intérêts : Aucun conflit à déclarer 
Impact de l'immunonutrition orale sur la morbidité postopératoire en chirurgie oncologique digestive: une étude de cohorte à l'échelle nationale

C. Rives-Lange ${ }^{12, *}$, A. Challine ${ }^{2}$, D. Danoussou ${ }^{3}$, S. Katsahian ${ }^{45}$, A. Ait Boudaoud ${ }^{12}$, S. Gaujoux ${ }^{16}$, B. Dousset $^{17}$, C. Carette ${ }^{2}$, A. Lazzati ${ }^{8}$, S. Czernichow ${ }^{2}$

${ }^{1}$ Université Paris Descartes, ${ }^{2}$ Nutrition, HEGP, ${ }^{3}$ INSERM, UMR_S 1138, Université Paris Descartes, Centre de Recherche des Cordeliers,, ${ }^{4}$ INSERM, UMR_S 1138, Université Paris Descartes, Centre de Recherche des Cordeliers, ${ }^{5}$ Unité d'Épidémiologie et de Recherche Clinique, HEGP, ${ }^{6}$ Service de chirurgie digestive, Hopital Cochin, 27, rue du Faubourg Saint Jacques, 75014, 7 Service de chirurgie digestive, Hopital Cochin, 27, rue du Faubourg Saint Jacques, 75014, Paris, ${ }^{8}$ service de chirurgie générale, Centre hospitalier intercommunal de Créteil, Créteil, France

Introduction et but de l'étude : L'immunonutrition se défini par l'ajout de nutriments spécifiques en quantité supérieure à la normale pour moduler l'immunité, dans le cadre d'une assistance nutritionnelle. Les recommandations nationales indiquent qu'elle devrait être prescrite avant toute chirurgie digestive oncologique majeure, dans le but de réduire la morbidité postopératoire. Néanmoins, cette pratique demeure controversée. L'objectif de notre étude est d'évaluer son effet en préopératoire sur un échantillon national représentatif.

Matériel et méthodes : Nous avons utilisé la base de données administrative nationale " échantillon généraliste des bénéficiaires ". Les sujets ont été sélectionnés à partir des codes cancers CIM-10 croisés avec les actes de chirurgie digestive de 2012 à 2016. Deux groupes ont été identifiés, l'un avec remboursement de l'immunonutrition 45 jours avant la chirurgie et l'autre sans ce remboursement. Le critère de jugement principal était la morbidité post-opératoire à 90 jours, les critères secondaires, la durée de séjours et la mortalité.

Résultats et Analyse statistique : Une régression logistique et une analyse de survie ajustées sur la pondération inverse du score de propension (IPW)) ont été réalisées. 1771 patients ont été inclus, $72 \%(n=1279)$ atteints de cancers colorectaux, 14\% ( $n=256)$ de cancers hépato-pancréato-biliaires et $12 \%(n=218)$ de cancers oeso-gastriques et instestinaux. L'immunonutrition avait été remboursée pour $34 \%$ des patients $(n=606)$. Les patients du groupe immunonutrition étaient significativement plus jeunes, avec plus de syndrome d'apnée du sommeil, plus d'antécédents de chimiothérapie et utilisaient plus de compléments nutritionnels oraux. II n'y avait pas de différence significative entre les deux groupes concernant la morbidité à 90 jours en post opératoire (OR 0.95 (IC $95 \%: 0.87 ; 1.04)$ ) ni sur la survie. Cependant, le nombre de séjour long était plus faible dans le groupe immunonutrition (OR : 0.77 IC $95 \%: 0.63-0.94)$.

Conclusion : A l'échelle nationale, l'utilisation préopératoire de l'immunonutrition avant chirurgie digestive oncologique majeure n'était pas associée à une réduction de la morbidité post opératoire. Cependant, elle était associée à une réduction de la durée de séjour.

Conflits d'intérêts : Aucun conflit à déclarer 

France

Introduction et but de l'étude : La prévalence de la dénutrition dans le cancer du poumon est de 30 à $80 \%$ selon les études [1]. Le dépistage et le diagnostic font partie intégrante de la prise en charge d'un patient cancéreux [2]. La dénutrition est associée à un risque accru de morbi-mortalité ainsi qu'à une moins bonne tolérance des chimiothérapies obligeant parfois à une réduction de dose [3]. Le NIVOLUMAB est préconisé en deuxième ligne de traitement dans les cancers bronchiques en stade avancé [4]. Nous avons voulu étudier l'impact du statut nutritionnel sur l'efficacité et la tolérance du NIVOLUMAB dans les cancers bronchopulmonaires.

Matériel et méthodes : Nous avons réalisé un suivi rétrospectif des patients ayant reçu du NIVOLUMAB pour un cancer bronchopulmonaire. Chez chacun des patients, des données nutritionnelles et des données oncologiques ont été recueillies concomitamment et rapportées dans le temps. Le recueil de ces données a été fait avant le traitement par immunothérapie, ainsi que lors de l'introduction du NIVOLUMAB (M0) et à 2 et 4 mois du début de l'immunothérapie (respectivement M2 et M4). L'influence du statut nutritionnel à $M 0$ a été évalué sur la réponse et la toxicité à $M 2$ à $M 4$, ainsi que sur la survie. Le rôle du suivi diététique ainsi que la variation du poids au cours du temps ont été évalués sur la survie.

Résultats et Analyse statistique : 127 patients ont été inclus avec un âge moyen de 67 ans. Le NIVOLUMAB était la deuxième ligne de traitement pour 84 patients (64,1\%). A M0, 64 patients (51\%) avaient bénéficié d'au moins une consultation diététique. Le statut nutritionnel a été évalué selon les critères HAS à M0 pour 120 patients : 80 n'étaient pas dénutris (67\%), 24 présentaient une dénutrition modérée (20\%) et 16 une dénutrition sévère (13\%). II n'y a pas de lien statistiquement significatif entre le statut nutritionnel à $\mathrm{M} 0$ et la réponse au traitement à $\mathrm{M} 2$ et $\mathrm{M} 4$. On ne met pas en évidence de variation significative du poids dans le temps, ni de différence d'évolution de la maladie selon que le patient était ou pas pris en charge par un diététicien à l'instauration du NIVOLUMAB $(p=0.9)$. En analyse multivariée, la dénutrition sévère à M0 avait un H.R (Hazard Ratio) = 2,32 - 95\% C.I : 1,13$4,75(p=0,02)$; et une diminution mensuelle de $1 \%$ du poids par rapport au poids à MO avait un H.R = 1,17 - 95\% C.I : 1,13-1,21 $(\mathrm{p}<0,0001)$.

Conclusion : La dénutrition sévère et la diminution du poids dans le temps sont des facteurs indépendants associés à une moins bonne survie chez les patients atteints de cancer pulmonaire pour lesquels un traitements par NIVOLUMAB est instauré. Des études intégrant la détection précoce de la sarcopénie associée à un suivi nutritionnel rapproché pourraient mettre en évidence un bénéfice sur la survie.

Références : [1] M. Pressoir et al, Prevalence, risk factors and clinical implications of malnutrition in French comprehensive cancer centres, Br J Cancer, 102 (2010)

[2] Khan et al, Détection et évaluation de la dénutrition en oncologie : quels sont les outils, pour quel type de cancer et dans quels buts?, Bulletin du cancer, 2016

[3] Capuano $\mathrm{G}$ et al, 2008, Head \& Neck, 30 ; 503-8

[4] Novello et al, Metastatic non-small-cell lung cancer: ESMO Clinical Practice Guidelines for diagnosis, treatment and follow-up, Annals of Oncology, 2016

Conflits d'intérêts : Aucun conflit à déclarer 
P140

Effet de la 20-hydroxyecdysone sur l'obésité viscérale induite par un stress nutritionnel chez un modèle du syndrome métabolique "Gerbillus tarabuli" : Implication du cortisol

Z. Bellahreche ${ }^{1,{ }^{*}}$, N. Semiane ${ }^{1}$, A. Mallek ${ }^{1}$, Y. Dahmani ${ }^{1}$

${ }^{1}$ Laboratoire de Physiologie et Biologie des Organismes - Nutrition et Métabolisme, Université Des Sciences et de Technologie Houari-Boumediene - Faculté des Sciences Biologiques (USTHB-FSB), Alger, Algérie

Introduction et but de l'étude : L'obésité résulte d'un déséquilibre entre apport et dépense énergétique. L’OMS l'a considéré comme maladie chronique associée à de nombreuses complications : la résistance à l'insuline, le syndrome plurimétabolique et le diabète.

Le but de notre étude est de tester l'effet de la 20-hydroxyecdysone (20 E) sur l'histophysiologie du tissu adipeux chez Gerbillus tarabuli.

Matériel et méthodes : Dans notre expérimentation qui a duré 5 mois nous avons utilisé 10 gerbilles de sexe mâle reparties en 2 groupes : Groupe RH : Les animaux sont soumis à un régime riche en hydrates de carbone ( $70 \%$ dattes $+30 \%$ orge). Groupe $20 \mathrm{E}$ : Les gerbilles recevaient le même régime que le groupe $\mathrm{RH}$ supplémenté en 20 -hydroxyecdysone (50mg/kg du poids corporel). Les animaux sont pesés chaque semaine afin d'évaluer le poids corporel. Après 5 mois, les animaux ont été sacrifiés et les tissus adipeux viscéraux ont été prélevés et pesés. Une fraction de chaque tissu est fixée pour une étude morphométrique et le reste est broyé pour le dosage tissulaire des triglycérides et du cortisol. Les plasmas ont été recueillis pour le dosage de certains paramètres biochimiques et hormonaux.

Résultats et Analyse statistique : Nos résultats montrent une hypertriglycéridémie chez le groupe RH par rapport aux valeurs initiales et l'étude morphométrique a révélé une hypertrophie adipocytaire accompagnée des taux élevés du cortisol et triglycérides in situ. En revanche, l'administration de la $20 \mathrm{E}$ améliore le taux des triglycérides plasmatiques et tissulaires comparé au groupe RH. En plus, nous avons remarqué une diminution de la taille des adipocytes chez le groupe $20 \mathrm{E}$ peut être liée à la diminution du cortisol plasmatique ou à une inhibition des enzymes de régénération locale du cortisol dans l'adipocyte.

Conclusion : Ces résultats montrent que la diète hyperglucidique a entrainé une obésité viscérale chez la gerbille aggravée par une hypercortisolémie. Cependant, l'administration de la $20 \mathrm{E}$ semblait exercer des effets bénéfiques sur le tissu adipeux via la diminution du taux du cortisol à l'état obèse.

Conflits d'intérêts : Aucun conflit à déclarer 
P141

Prise en charge et dépistage de l’obésité infantile : Pratiques et attentes des Médecins Généralistes et des Pédiatres libéraux du Limousin.

H. Guibert ${ }^{1}$, A. Lienhardt-Roussie ${ }^{2}$, P. Fayemendy ${ }^{345}$, B. Morin ${ }^{3}$, A. Voisin ${ }^{2}$, H. Thibault ${ }^{678}$, J. C. Desport ${ }^{1345}$, P. Jésus $^{1345,{ }^{*}}$ ${ }^{1}$ Centre Spécialisé de I'Obésité du Limousin, ${ }^{2}$ Service de Pédiatrie, ${ }^{3}$ Unité de Nutrition, CHU de Limoges, ${ }^{4}$ INSERM UMR 1094, Neuroépidémiologie Tropicale, Faculté de Médecine de Limoges, ${ }^{5}$ nnstitut de Neuroépidémiologie et Neurologie Tropicale, CNRS FR 3503 Geist, Université de Limoges, Limoges, ${ }^{6}$ Centre Spécialisé de l'Obésité de l'Aquitaine Nord, ${ }^{7}$ Service de Pédiatrie, ${ }^{8}$ Réseau de Prévention et de Prise en charge de l'Obésité Pédiatrique, $\mathrm{CHU}$ de Bordeaux, Bordeaux, France

Introduction et but de l'étude : L'obésité chez l'enfant est un problème de santé publique (3,5 à $4 \%$ en France). Devant les difficultés rencontrées par les professionnels de santé, les Réseaux de Prévention et de Prise en charge de l'Obésité Pédiatrique (RéPPOP)sont d'une aide importante. Le but de notre travail était de comprendre et d'évaluer les besoins et pratiques des Médecins Généralistes (MG) et des Pédiatres libéraux du Limousin.

Matériel et méthodes : Un questionnaire avec les données sociodémographiques des médecins, leurs pratiques concernant le dépistage et la prise en charge (PEC) de l'obésité pédiatrique, ainsi que leurs difficultés et suggestions d'amélioration, a été envoyé par courrier à tous les MG $(n=777)$ et Pédiatres Libéraux $(n=18)$ du Limousin en Avril 2016. Un rappel par voie postale a aussi été réalisé. L'analyse statistique comprenait les tests $t$ de Student, d'ANOVA et du Chi2.

Résultats et Analyse statistique : 238 questionnaires ont été inclus dans l'analyse (230 pour les MG et 8 pour les Pédiatres). Les MG déclaraient avoir 23,2 $\pm 18,1 \%$ d'enfants de moins de 16 ans dans leur patientèle. Les MG déclaraient avoir en moyenne dans leur patientèle 3,5\% $\pm 4,8 \%$ d'enfants obèses alors que les Pédiatres libéraux déclaraient en avoir $4,7 \% \pm 3,7 \%$. 22\% des $M G$ déclaraient avoir suivi une formation complémentaire sur la thématique de l'obésité chez l'enfant dans les 5 dernières années. Les MG calculaient systématiquement l'indice de masse corporelle dans 39,3\% des cas, le reportaient sur les courbes de corpulence dans $31,8 \%$ des cas et traçaient les courbes dans 26,6\% des cas. Les Médecins formés à l'obésité réalisaient ces 3 étapes de façon significativement différente des Médecins non formés ( $p=0,005, p=0,03, p=0,05$, respectivement). Les principales difficultés étaient la motivation des familles $(80,7 \%)$, les difficultés socio-économiques $(51,2 \%)$ et les échecs fréquents $(45,6 \%)$. Les suggestions d'aide étaient le remboursement des consultations diététiques (63,0\%), la création d'un RéPPOP (53,5\%) et une

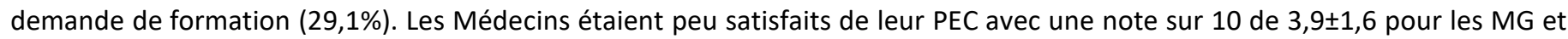
de 5,1 $\pm 1,9$ pour les Pédiatres. Trente MG (13\%) étaient intéressés par une formation réalisée en Limousin par le RéPOPP Aquitaine.

Conclusion : Bien que non exhaustive, notre enquête a permis de faire la mise au point sur les pratiques, difficultés et attentes des MG et Pédiatres du Limousin face à l'obésité infantile. A la suite de cette enquête une formation a été réalisé par le RéPOPP Aquitaine aux MG du Limousin.

Conflits d'intérêts : Aucun conflit à déclarer 
${ }^{1}$ Laboratoire de Nutrition et de Technologie Alimentaire, Université de Constantine, Constantine, Algérie

Introduction et but de l'étude : Le goût est une modalité sensorielle qui permet d'apprécier les saveurs d'une substance ingérée grâce aux récepteurs gustatifs situés dans la cavité buccale. Chez l'homme, désirer manger un aliment peut conditionner le choix alimentaire. Les graisses contribuent au plaisir de l'acte de « manger » en raison des propriétés sensorielles qu'ils apportent ce qui pourrait favoriser leur surconsommation et de ce fait influencer la corpulence par une prise du poids.

L'objectif de notre travail est de déterminer le niveau de sensibilité gustative aux aliments apportant le goût gras à travers l'évaluation des préférences alimentaires et d'étudier le possible lien avec la surcharge pondérale.

Matériel et méthodes : L'étude porte sur 210 jeunes adultes ( 157 femmes et 53 hommes) agés de 18 à 30 ans au niveau du Laboratoire de Nutrition et Technologie Alimentaire (LNTA). Les mesures anthropométriques concernent le poids et la taille. La surcharge pondérale est définie par un $I M C \geq 25$. Le questionnaire comporte une partie sur l'évaluation du degré de préférence envers des aliments apportant le goût gras (aliments hyper gras et gras rajouté) sur une échelle de 4 points (DEGLAIRE et coll, 2012) et une autre sur les préférences alimentaires en relation avec ce goût. Le niveau de sensibilité est estimé à partir des scores moyens de préférence attribués à chaque groupe d'aliments apportant le goût étudié (gars-sucré, gras-salé et gras rajouté). L'analyse statistique est réalisée avec le logiciel StatView

Résultats et Analyse statistique : Le taux de surpoids est de $45.7 \%$ dont $20 \%$ d'obésité. Le pourcentage du surpoids chez les femmes est plus élevé que celui des hommes (49.68\% vs $33.96 \% ; p=0.04)$. Les sujets en surpoids ont un score moyen de préférence au gras plus élevé que les normo pondéraux ( $2.18 \pm 0.95$ vs $1.72 \pm 1.03 ; p=0.001)$. L'IMC augmente avec le score de préférence du beurre $(p=0.04)$ et de l'huile d'olive $(p=0.002)$. Une relation significative a été trouvée entre la sensibilité au gras rajouté (beurre et huile d'olive) et l'état pondéral des adultes, ceux en surpoids sont les moins sensibles par rapport au gras rajouté (83.33\% vs $60.53 \% ; p=0.0003$ ). Aucune différence significative n'a été trouvée entre le niveau de sensibilité pour les aliments gras-sucré ou gras-salé et l'état pondéral. Cependant, les sujets les moins sensibles au gras-sucré sont plus nombreux à déclarer préférer des pâtisseries et du chocolat $(30.21 \%$ vs $0 \%, p=0.01 ; 27.66 \%$ vs $0 \%, p=0.02)$.

Conclusion : Ces résultats suggèrent l'existence d'une relation entre le niveau de préférence envers les aliments apportant le goût gras et la corpulece des adultes. Il est possible que c'est la préférence du gras en lui-même (gras rajouté) qui peut influencer le choix alimentaire des adultes par une consommation excessive des aliments palatables riches en gras liée à une faible sensibilité gustative à ces derniers ce qui peut entrainer une prise de poids, voir une obésité à long terme.

Références : ALLAM O., TFYECHE M., OULAMARA H et AGLI AN. Préférence et sensibilité aux aliments apportant les goûts gras, sucré, salé et amer et état pondéral. Nutr. Santé, 2017, Vol.06 N02: 45-51. DOI: 10.30952/ns.6.2.1

DEGLAIRE A., MEJEAN C., CASTETBON., KESSE-GUYOT., URBANO C et coll. Development of a questionnaire to assay recalled liking for salt, sweet and fat. Food Quality and Preference, 2012, $23: 110-124$

Conflits d'intérêts : Aucun conflit à déclarer 
${ }^{1}$ Faculté des sciences biologiques, USTHB (université des sciences et de la technologie Houari Boumediene), Alger, Algérie

Introduction et but de l'étude : L'inflammation chronique de bas grade caractérisée par l'expression des cytokines inflammatoires est associée à l'insulinorésistance. Cette dernière joue un rôle crucial dans le développement de la stéatose et constitue un puissant prédicateur des affections hépatiques appelées NAFLD.

Nous avons évalué le potentiel bénéfique de l'extrait aqueux d'une molécule antioxydante, la spiruline, dans la résorption des altérations métaboliques et morphologiques hépatiques chez le rat des sables, Psammomys obesus, modèle nutritionnel d'insulinorésistance et de Diabète de type 2.

Matériel et méthodes : Notre étude a porté sur 3 groupes expérimentaux : témoins soumis au régime naturel du biotope (PN), placebo (PI), et traités (PS) à raison de $100 \mathrm{mg} /$ jour de spiruline durant 12 semaines. Les Psammomys $\mathrm{PI}$ et $\mathrm{PS}$ reçoivent le régime hypercalorique. L'effet de la spiruline a été évalué à travers certains paramètres biochimiques plasmatiques, et sur la structure du foie.

Résultats et Analyse statistique : Au terme de l'expérimentation, les animaux placebo présentent un gain pondéral significatif de $42 \%$ avec une adiposité viscérale importante. Comparativement aux témoins, le lot placebo ou insulinorésistant affiche une hyperinsulinémie spectaculaire $(+279 \%)$ associée à une hypertriglycéridémie (1.75 vs $0.62 \mathrm{~g} / \mathrm{L})$, une hausse significative des transaminases, le TNF alpha a doublé et le NO subit 38\% d'augmentation. L'examen morphologique révèle la présence d'une stéatose hépatique et de quelques infiltrats inflammatoires lobulaires.

Grâce à ses composants actifs (phycocyanine, $\beta$ carotène) aux vertus insulino sensibilisantes et anti-inflammatoires, la spiruline prévient significativement les troubles métaboliques et améliore grandement l'inflammation en inhibant l'activité des macrophages pro-inflammatoires M1.

En effet, chez les animaux traités une stabilisation de la masse corporelle est observée vers la dernière semaine, et les taux des paramètres biochimiques étudiés sont proches de ceux des animaux témoins, hormis le cholestérol total qui est peu corrigé (0.94 $\mathrm{g} / \mathrm{L}$ vs $0.63 \mathrm{~g} / \mathrm{L}$ ). Par ailleurs, on note l'absence de stéatose et des infiltrats immunitaires au niveau du parenchyme hépatique.

Conclusion : Ces résultats suggèrent qu'un traitement préventif par la spiruline permet de contrer les effets délétères du régime hypercalorique, empêchant ainsi l'installation de l'insulinorésistance et de l'inflammation chronique et leurs répercussions sur la structure hépatique.

Conflits d'intérêts : Aucun conflit à déclarer 
${ }^{1}$ UMR 0866 DMEM, INRA, ${ }^{2}$ Phycobiotech, Montpellier, France

Introduction et but de l'étude : La prévalence de l'obésité et des désordres métaboliques associés, tels la stéatose hépatique non alcoolique, l'inflammation et la résistance à l'insuline, augmente rapidement et devient un problème majeur de santé publique. Le silicium ( $\mathrm{Si}$ ) a des effets bénéfiques marqués sur la physiopathologie osseuse et cardiovasculaire et l'incorporation de Si dans la spiruline augmente la biodisponibilité de cet élément. L'objectif de ce projet est d'étudier les effets de la spiruline enrichie en silicium sur différentes composantes du syndrome métabolique chez le rat Zucker.

Matériel et méthodes : Trente rats mâles obèses Zucker répartis en trois groupes $(n=10)$ ont reçu un placebo, un comprimé de spiruline ou un comprimé de spiruline enrichie en Si. Un test oral de tolérance au glucose (OGTT) a été réalisé à 6 et 12 semaines de régimes. Les rats ont ensuite été sacrifiés puis l'insuline et la leptine sériques ainsi que les lipides sériques et hépatiques ont été quantifiés. L'activité de la citrate synthase et des complexes respiratoires de la chaîne mitochondriale du foie, différents marqueurs de l'inflammation et du stress oxydant ont également été déterminés.

Résultats et Analyse statistique : La consommation de spiruline a diminué la consommation alimentaire des rats Zucker, sans effet cependant sur leur poids corporel. De plus, elle a amélioré la tolérance au glucose et diminué l'activité hépatique de la NADPH oxydase, une enzyme impliquée dans la production de radicaux libres. Par rapport à la spiruline seule, la consommation de spiruline enrichie en Si n'a pas eu d'effet significatif sur les caractéristiques des animaux ni sur les paramètres métaboliques sanguins et hépatiques explorés.

Conclusion : Alors que la spiruline seule améliore la tolérance au glucose et le stress oxydant des rats Zucker, la spiruline enrichie en Si n'a pas d'effet bénéfique supplémentaire sur les paramètres mesurés. La spiruline enrichie en Si pourrait avoir des effets plutôt au niveau osseux et contre le développement de l'athérosclérose, comme cela a déjà été montré chez le hamster. Des études supplémentaires dans d'autres modèles animaux et chez l'Homme sont nécessaires pour clarifier les effets de la spiruline enrichie en Si.

Références : Vidé J et al. Dietary silicon-enriched spirulina improves early atherosclerosis markers in hamsters on a high-fat diet. Nutrition. 2015 Sep;31(9):1148-54.

Remerciements : Ces résultats font partie du projet Spirulicium Cardio cofinancé par la région Occitanie et par le Fonds européen de développement régional

Conflits d'intérêts : J. Vidé: Aucun conflit à déclarer, B. Bonafos: Aucun conflit à déclarer, G. Fouret: Aucun conflit à déclarer, N. Jouy est consultant chez Phycobiotech, C. Coudray: Aucun conflit à déclarer, S. Gaillet: Aucun conflit à déclarer, C. Feillet-Coudray: Aucun conflit à déclarer 
Effets de la spiruline enrichie en silicium sur les désordres métaboliques associés à l'obésité chez le rat.

J. Vidé ${ }^{1}$, B. Bonafos ${ }^{1}$, G. Fouret ${ }^{1}$, N. Jouy ${ }^{2}$, C. Feillet-Coudray ${ }^{1,}{ }^{*}$, S. Gaillet ${ }^{1}$, C. Coudray $^{1}$

${ }^{1}$ UMR 0866 DMEM, INRA, ${ }^{2}$ Phycobiotech, Montpellier, France

Introduction et but de l'étude : La prévalence de l'obésité et des désordres métaboliques associés, tels la stéatose hépatique non alcoolique, l'inflammation et la résistance à l'insuline, augmente rapidement et devient un problème majeur de santé publique. Le silicium (Si) a des effets bénéfiques marqués sur la physiopathologie osseuse et cardiovasculaire et l'incorporation de Si dans la spiruline augmente la biodisponibilité de cet élément. L'objectif de ce projet est d'étudier si la mitochondrie est impliquée dans les mécanismes préventifs de la spiruline enrichie en Si contre le stress oxydant et l'inflammation préalablement démontré chez le hamster (1).

Matériel et méthodes : Des rats Wistar mâles ont reçu un régime témoin contenant $5 \%$ d'huile de soja ( $n=10$ ) ou un régime obésogène contenant $30 \%$ de saindoux $(n=30)$. Ces derniers ont été répartis dans 3 groupes $(n=10)$ et ont reçu un placebo, un comprimé de spiruline ou un comprimé de spiruline enrichie en Si. Après 12 semaines, les rats ont été sacrifiés et l'ALAT, l'insuline, la leptine et l'adiponectine sériques ainsi que les lipides sériques et hépatiques ont été quantifiés. L'activité de la citrate synthase et des complexes respiratoires de la chaîne mitochondriale du foie, différents marqueurs de l'inflammation et du stress oxydant ont également été déterminés.

Résultats et Analyse statistique : Le régime riche en graisses induit une obésité, une stéatose hépatique (augmentation de l'ALAT plasmatique et du taux de triglycérides hépatiques) et un stress oxydant (diminution des groupements -SH et du GSH et augmentation de l'activité NADPH oxydase hépatique). La spiruline et la spiruline enrichie en Si modulent de façon modérée quelques paramètres métaboliques (intolérance au glucose et activité NADPH oxydase), sans effet spécifique de la spiruline enrichie en Si.

Conclusion : Contrairement à ce qui a été observé chez le hamster, la spiruline enrichie en Si chez le rat n'induit pas d'effet bénéfique supplémentaire par rapport à la spiruline seule. Des études supplémentaires dans d'autres modèles animaux et chez l'Homme sont nécessaires pour clarifier les effets de la spiruline enrichie en Si sur les désordres métaboliques associés à l'obésité. Références : Vidé J et al. Dietary silicon-enriched spirulina improves early atherosclerosis markers in hamsters on a high-fat diet. Nutrition. 2015 Sep;31(9):1148-54.

Remerciements : Ces résultats font partie du projet Spirulicium Cardio cofinancé par la région Occitanie et par le Fonds européen de développement régional.

Conflits d'intérêts : J. Vidé: Aucun conflit à déclarer, B. Bonafos: Aucun conflit à déclarer, G. Fouret: Aucun conflit à déclarer, N. Jouy est consultant chez Phycobiotech, C. Feillet-Coudray: Aucun conflit à déclarer, S. Gaillet: Aucun conflit à déclarer, C. Coudray: Aucun conflit à déclarer 
Inflammation et stress oxydant au cours du développement de l'intolérance au glucose et de la stéatose hépatique chez des rats nourris avec un régime obésogène

C. Feillet-Coudray ${ }^{1,}{ }^{*}$, G. Fouret ${ }^{1}$, C. Vigor ${ }^{2}$, B. Bonafos ${ }^{1}$, B. Jover ${ }^{3}$, A. Blachnio-Zabielska4 ${ }^{4}$, J. Rieusset ${ }^{5}$, S. Gaillet $^{1}$, T. Durand ${ }^{2}$, C. Coudray ${ }^{1}$

${ }^{1}$ UMR 0866 DMEM, INRA, ${ }^{2}$ IBMM, ${ }^{3}$ U1046, INSERM, Montpellier, France, ${ }^{4}$ Hygiene, Epidemiology Metabolic Disorders Department, Medical University of Bialystok, Bialystok, Pologne, ${ }^{5}$ Unité 1060 CarMen/ Univ.Lyon1 / INRA 1235, INSERM/ Univ.Lyon1 / INRA 1235, Lyon, France

Introduction et but de l'étude : La prévalence de l'obésité, de la stéatose hépatique non alcoolique et de la résistance à l'insuline augmente rapidement et devient un problème majeur de santé publique. L'obésité et ses complications s'accompagnent souvent d'une inflammation et d'un stress oxydant, lesquels joueraient un rôle important dans ces désordres métaboliques. Cette étude vise à déterminer l'évolution à long terme de l'inflammation et du stress oxydant au cours de l'installation de l'intolérance au glucose et de la stéatose hépatique sous régime obésogène.

Matériel et méthodes : Soixante-quinze rats Wistar mâles ont été divisés en deux lots : un lot recevant un régime témoin et un lot recevant un régime riche en graisses et en sucre (HFHFr). Un groupe de chaque lot ( $n=7 / 8)$ a été sacrifié à $4,8,12,16$ et 20 semaines de régime. Les principaux marqueurs de l'intolérance au glucose et de l'insulinorésistance, du métabolisme lipidique, de l'inflammation et du stress oxydant ont été mesurés.

Résultats et Analyse statistique : Le régime HFHFr induit un surpoids chez les rats, une intolérance au glucose et une stéatose hépatique. II augmente les teneurs en diacylglycérols et en céramides hépatiques, l'expression du gène hépatique de l'IL-6 mais sans modification d'autres paramètres de l'inflammation. Il augmente l'activité de la NADPH oxydase hépatique (enzyme qui produit des radicaux libres), mais pas la production de ROS mitochondriale. De plus, les taux plasmatiques et/ou hépatiques des isoprostanoides et des TBARS (lipides oxydés) sont diminués. Enfin, le régime HFHFr induit une diminution de la teneur en GSH (antioxydant cellulaire) et de l'activité des enzymes antioxydantes (SOD, catalase et GPX).

Conclusion : Bien que le régime $\mathrm{HFHFr}$ induise une intolérance au glucose et une accumulation de lipides hépatiques, l'inflammation et le stress oxydant ne sont pas ou peu modifiés. Un stress oxydant de faible intensité pourrait donc être le résultat et non la cause de l'accumulation des lipides hépatiques et de l'intolérance au glucose observée. Plus d'études sont nécessaires pour confirmer ces résultats, en particulier chez l'Homme.

Conflits d'intérêts : Aucun conflit à déclarer 
Introduction et but de l'étude : Malgré les recommandations de l'ANSES de 2010, la prise en charge de l'obésité reste trop centrée sur le contenu de l'assiette. Les approches ciblant la régulation sur les signaux alimentaires internes et le renforcement des compétences psycho-sociales semblent plus pertinentes que le " bien manger-bien bouger ". Peu d'études à ce jour ont évalué les bénéfices d'un programme éducatif orienté sur ces approches. Nous avons analysé l'impact d'un programme d'éducation thérapeutique court soutenant la réflexivité des sujets atteints d'obésité quant aux déterminants de leur excès de poids et aux changements nécessaires pour perdre du poids durablement.

Matériel et méthodes : Analyse des questionnaires de qualité de vie (QDV) des sujets atteint d'obésité (IMC > 30) ayant participé au programme d'éducation thérapeutique ambulatoire "Comprendre mon poids pour agir » de 2014 à 2017. Ce programme consiste en 5 ateliers collectifs dont les objectifs sont de favoriser la prise de conscience des éléments à l'origine du fait de manger au-delà des besoins du corps, et d'aider à trouver des solutions personnalisées et durables, sans demande de modifications du profil et des quantités alimentaires. Le même questionnaire a été rempli au début et 3 mois après la fin du programme, soit un intervalle de 4 mois et demi. Il comporte des données anthropométriques (poids, taille, évolution pondérale récente) et un mixte des questionnaires EQVOD ${ }^{1}$ et SF-36 $6^{2}$ (55 questions sur la QDV). Les résultats sont exprimés en moyenne +/- écart-type ; Utilisation du test de Student ; $p<0.05$ considéré comme significatif.

Résultats et Analyse statistique : 103 personnes ont participé au programme. 46 participants (âge : 50,4 +/- 12,0 ans ; femmes : 93,5\% ; IMC : 40,1 +/- 6,7) ont rempli les deux questionnaires. Les 69 autres (âge : 47+/- 14,9 ans ; femmes : 95,7 \% ; IMC : 38,9 $+/-6,7)$ n'ont rempli que le questionnaire initial ; $\neq$ non significative entre les 2 groupes. Les 46 répondeurs ont présenté une perte de poids de $3,0+/-4,4 \mathrm{~kg}\left(\mathrm{p}<10^{-4}\right)$ et une baisse de I'IMC de $1,1+/-1,7\left(\mathrm{p}<10^{-4}\right)$. Une évolution pondérale à la hausse était présente chez $39 \%$ des sujets au début du programme et chez $17 \%$ à l'issue. L'analyse des $\neq$ dimensions de la QDV est en faveur d'une amélioration des compétences psychosociales (notamment de l'estime de soi), d'une meilleure perception de leur santé et d'une diminution de la valeur hédonique de l'alimentation sans perte de la satisfaction liée au fait de manger.

Conclusion : Ces résultats sont en faveur de l'efficacité d'un programme éducatif court centré sur la réflexivité de la relation à l'alimentation et ses déterminants, et soutenant l'auto-détermination dans la conduite de changements.

Références : ${ }^{1}$ Ziegler O, Filipecki J, Girod I, Guillemin F. Development and validation of a French obesity-specific quality of life questionnaire: Quality Of Life, Obesity and Dietetics (QOLOD) rating scale. Diabetes Metab 2005, 31: 273-83

${ }^{2}$ Leplège A, Ecosse E, Verdier A, Perneger T. The French SF-36 Health Survey : translation, cultural adaptation and preliminary psychometric evaluation. J Clin Epidemiol 1998, $51: 1013-1023$

Conflits d'intérêts : Aucun conflit à déclarer 
Association prospective entre les comportements alimentaires durables évalués par l'index générique de durabilité des régimes (SDI), la prise de masse corporelle et le risque d'obésité dans la cohorte NutriNet-Santé

L. Seconda ${ }^{1, *}$, J. Baudry ${ }^{1}$, M. Egnell ${ }^{1}$, C. Julia ${ }^{1}$, S. Hercberg ${ }^{1}$, P. Pointereau ${ }^{2}$, D. Lairon ${ }^{3}$, B. Allès ${ }^{1}$, E. Kesse-Guyot ${ }^{1}$

${ }^{1}$ EREN, université Paris 13 , Bobigny, ${ }^{2}$ Agriculture, Solagro, Toulouse, ${ }^{3}$ Nort, Université Aix-Marseille, Marseille, France

Introduction et but de l'étude : La non-durabilité des systèmes alimentaires est à l'origine d'enjeux environnementaux et de santé publique. Depuis quelques années nous remarquons un intérêt croissant pour l'identification de comportements alimentaires plus durables. Notre objectif est d'étudier les associations prospectives entre la durabilité des régimes alimentaires, évaluée par un indice holistique, le SDI (Sustainable Diet Index), et le risque d'obésité ou d'augmentation de l'indice de masse corporelle (IMC) au sein d'une population d'adultes français sur un temps de suivi d'environ 3 ans.

Matériel et méthodes : Le SDI a été mesuré chez 26594 participants de l'étude NutriNet-Santé grâce aux bases de données développées dans le cadre du projet BioNutrinet. Pour ces participants, le poids et la taille ont été collectés tous les ans entre 2014 et 2018. Des modèles mixtes nous ont permis d'étudier d'éventuelles différences de corpulence entre les participants au sein des quintiles sexe-spécifiques de SDI. Le risque d'obésité a été évalué par un modèle de Cox après la prise en compte de multiples facteurs d'ajustements.

Résultats et Analyse statistique : Au début de l'étude, des différences importantes de corpulence sont observées selon les quintiles de SDI. Les personnes ayant un IMC supérieur ou égal à $30 \mathrm{~kg} / \mathrm{m}^{2}$ étaient plus nombreuses dans le quintile le moins durable. De plus, le modèle mixte principal montre une augmentation d'IMC de $0,14 \mathrm{~kg} / \mathrm{m}^{2}$ par an dans ce dernier quintile alors que cette augmentation est significativement plus basse dans les trois quintiles les plus durables. Aussi, le risque d’obésité était significativement plus bas dans le quintile le plus durable par rapport au moins durable, après de multiples ajustements $\mathrm{HR}_{05}$ vs. Q1 $=0.21($ IC95\%=0.15-0.29).

Conclusion : Cette étude montre ainsi une forte association entre l'adoption d'un régime durable et le risque d'obésité ou de prise de poids. Cette association peut s'expliquer par des différences importantes des quantités consommées par groupes alimentaires, ainsi qu'une consommation plus forte d'aliments issus de l'agriculture biologique, et une plus faible de produits préparés industriellement réduisant l'exposition à différents composés. Ces derniers pouvant être impliqués dans les mécanismes d'augmentation de la masse corporelle. Finalement, les résultats mettent en évidence l'importance pour la protection de l'environnement et des ressources ainsi que pour la santé publique de promouvoir une alimentation plus durable et de la rendre accessible à tous.

Conflits d'intérêts : Aucun conflit à déclarer 
Indicateurs anthropométriques et risque cardiovasculaire dans la population adulte Oranaise - Etude ISOR

L. Houti ${ }^{1,}{ }^{*}$, S. Mediene-Benchekor ${ }^{23}$, H. Ouhaibi-Djellouli ${ }^{23}$, S. Lardjam-Hetraf ${ }^{3}$, I. Hamani-Medjaoui ${ }^{4}$

${ }^{1}$ LABSIS, Faculté de Médecine, ${ }^{2}$ Département de Biotechnologie, Faculté des Sciences, Université Oran $1,{ }^{3}$ LGMC, Université USTO$\mathrm{MB},{ }^{4} \mathrm{CSORVAT}, \mathrm{CNAS}$, Oran, Algérie

Introduction et but de l'étude : L'augmentation des valeurs anthropométriques, mesurées par le tour de taille, le rapport taillehanche ou l'indice de masse corporelle, est associée classiquement à l'élévation du risque de développer une maladie cardiovasculaire. Dans ce travail, il s'agit de déterminer lequel de ces indicateurs est le plus pertinent selon le sexe, dans la population oranaise.

Matériel et méthodes : Une enquête en population a été réalisée, en 2007-2009 dans la population oranaise (30 à 64 ans). Les sujets tirés au sort dans la liste des assurés sociaux de la ville d'Oran, ont participé volontairement à un bilan clinique (PAS, PAD), anthropométrique (poids, taille, tour de taille) et biologique (glycémie à jeun, triglycérides, Cholestérol-HDL). Au total, 787 sujets (Ratio $\mathrm{M} / \mathrm{F}=0,94$ ) ont participé à l'enquête. Trois niveaux de tour de taille, rapport taille-hanche et indice de masse corporelle (normal, intermédiaire et élevé) ont été définis dans les deux sexes, selon les critères IDF et NCEP-ATPIII. Le syndrome métabolique a été défini selon les critères NCEP-ATPIII.

Résultats et Analyse statistique : Dans les deux sexes, le tour de taille élevé est plus fortement associé à l'obésité (82,4\% chez les hommes et $93,2 \%$ chez les femmes) que le rapport taille-hanche élevé (26,5\% chez les hommes et $53,4 \%$ chez les femmes). Chez les femmes, le risque cardiovasculaire (diabète, hypertension artérielle et syndrome métabolique) augmente significativement dans les niveaux élevés des trois indicateurs d'obésité corporelle et abdominale, comparé aux niveaux normaux et intermédiaires et varie de $42 \%$ à $94 \%$. C'est dans le groupe tour de taille élevé que le risque est maximum ( $73 \%$ à $94 \%, p<0,001)$. Chez les hommes, les résultats sont plus singuliers. 80 à $85 \%$ des diabétiques et des hypertendus présentent des niveaux de tour de taille, de rapport taille-hanche et d'indice de masse corporelle, normaux ou intermédiaires. En revanche, le syndrome métabolique est plus fréquent dans le groupe tour de taille élevé $(49 \%, p<0,001)$.

Conclusion : Dans notre population, le risque cardiovasculaire est maximum chez les femmes dans les niveaux élevés des trois indicateurs pendant qu'il est présent chez les hommes quelques soient les niveaux d'obésité abdominale et corporelle. Chez les hommes, d'autres facteurs de risque comme le tabac ou l'activité physique restent à explorer.

Conflits d'intérêts : Aucun conflit à déclarer 

S. Le Garf ${ }^{1,}{ }^{*}$, J. Murdaca ${ }^{2}$, I. Mothe-Satney ${ }^{2}$, G. Le Menn ${ }^{2}$, B. Sibille ${ }^{2}$, J. Neels ${ }^{2}$, A.-S. Rousseau ${ }^{2}$

${ }^{1}$ UMR/INSERM1065/C3M, ${ }^{2}$ UMR/INSERM 1065/C3M, Université Côte d'Azur, Nice, France

Introduction et but de l'étude : L'inflammation liée à l'obésité est sinequanone au développement des comorbidités. Il existe une perturbation de la balance lymphocytaire pro-inflammatoire et anti-inflammatoire avec une diminution de cellules $\mathrm{T}$ régulatrices (LTreg). Les LTreg contrôlent l'inflammation systémique et possèdent un métabolisme oxydo-lipidique. L'activation de la voie du peroxisome proliferator activated receptor beta (PPARb) augmente le métabolisme oxydo-lipidique des lymphocytes T. Les agonistes de PPARb sont des traitements potentiels des maladies métaboliques. II convient d'en distinguer leurs effets propres de ceux induits par des stratégies non-médicamenteuses (alimentation et activité physique) recommandées dans la réduction de l'obésité. Le but de l'étude a été de caractériser les effets de ces différentes stratégies sur les changements immunométaboliques, l'intolérance au glucose et la perte de poids chez la souris rendue obèse puis soumise à un régime normal.

Matériel et méthodes : 24 souris femelles C57BL/6J de 7 semaines ont été rendues obèses et intolérantes au glucose par un régime riche en gras (HFD) administré pendant 12 semaines. A l'issue des 12 semaines (TO), les souris sont mises sous un régime normal et aléatoirement réparties en différents sous-groupes $(n=6)$ : traitées pendant 8 semaines avec un agoniste de PPARb (GW0742) vs véhicule (DMSO) et/ou entraînées sur tapis roulant (3 sessions/semaine). Nous avons utilisé comme groupe contrôle des souris soumises à un régime normal et entrainées ( $n=6 ; N D-E x)$. Des tests de tolérance au glucose (GTT), l'expression des gènes cibles de PPARb (qPCR) et le profil des LTreg dans les organes lymphoïdes (FACS, marquage FoxP3+ et CD25+CD127-) ont été réalisés. Selon les conditions des ANOVAs ou des tests non-paramétriques ont été effectués.

Résultats et Analyse statistique : A l'issue des 8 semaines les souris traitées (GW0742 et/ou exercice) ont une masse adipeuse viscérale et une tolérance au glucose non différente de celles du groupe contrôle (ND-Ex). Toutefois la masse adipeuse viscérale reste significativement plus élevée dans le groupe sans traitement ( $p<0,01 ; v s$ les autres groupes). Les différents traitements $n$ 'ont pas d'effet sur le pourcentage de LT CD4+ dans les ganglions. En revanche, le pourcentage de Treg CD25+CD127- est variable selon le traitement. Le traitement GW0742 lorsque combiné à l'exercice conduit à une augmentation du pourcentage de LT CD25+CD127- comparativement au groupe traité seulement avec du GW0742 ( $<<0,05)$. Le pourcentage de cellules Foxp3+ est significativement plus élevé en réponse à la combinaison du traitement GW0742 et de l'entrainement (GW0742+Ex) que celui observé dans le groupe contrôle (ND-Ex).

Conclusion : L'ensemble de nos résultats suggère des effets d'une activation de la voie PPAR $\beta$ redondants à ceux de l'entrainement sur la masse adipeuse viscérale mais complémentaires sur le pourcentage de cellules Treg. Cette combinaison pourrait être favorable dans un contexte de réduction de l'obésité. Il semble toutefois important de considérer la plasticité des cellules T et leur fonction qui pourraient-être changées selon ces conditions, et ce, indépendamment de leur nombre.

Conflits d'intérêts : Aucun conflit à déclarer 
Peut-on utiliser le Quotient de Satiété comme prédicteur de la prise alimentaire chez l'adolescent en situation d'obésité ?

A. Fillon ${ }^{1}$, M. Miguet ${ }^{1}$, M. Khammassi ${ }^{1}$, J. Masurier ${ }^{2}$, M. Duclos ${ }^{1}$, Y. Boirie ${ }^{1}$, A. Tremblay ${ }^{3}$, V. Drapeau $^{3}$, M. $^{-E .}$ Mathieu $^{4}$, D. Thivel ${ }^{1, *}$

${ }^{1}$ Université Clermont Auvergne, ${ }^{2}$ SSR UGECAM, clermont-ferrand, France, ${ }^{3}$ Université Laval, Quebec city, ${ }^{4}$ Université de Montreal, Montreal, Canada

Introduction et but de l'étude : L'évaluation des sensations d'appétit est une méthode précise pour mesurer la motivation à manger avant et en réponse à un repas (Flint et al., 2000). L'évolution de ces sensations d'appétit en réponse à une prise énergétique permet aussi de renseigner la capacité satiétogène de cette dernière, à travers le calcul du Quotient de Satiété (QS). Si ce QS est couramment utilisé chez l'adulte sain et en situation d'obésité, notamment comme un prédicteur fiable de la prise alimentaire et de la perte de poids, son application reste incertaine en pédiatrie. L'objectif est ici d'évaluer la reproductibilité de ce QS chez l'adolescent obèse et de questionner sa capacité à prédire sa prise alimentaire subséquente.

Matériel et méthodes : Trente-neuf enfants (13,2 $\pm 0,9$ ans, 13 garçons) présentant une obésité (IMC $>97^{\text {eme }}$ percentile) ont réalisés deux sessions expérimentales dans un ordre aléatoire : i) une condition lors de laquelle ils ont reçu un Petit Déjeuner Fixe uniquement (PDF); ii) une condition composée du même PDF mais au cours de laquelle leur prise alimentaire ad libitum a été évaluée sur la journée (PDF+adlib). Lors de la condition PDF+adlib, leur prise alimentaire au repas de midi et du soir a été pesée et évaluée. Leurs sensations alimentaires (faim, satiété, désir de manger, propension à manger) ont été évaluées à l'aide d'échelles visuelles analogiques (EVA) (Flint et al., 2001) à intervalles réguliers au cours des deux journées. Le QS pour chaque sensation a été calculé à partir du petit déjeuner tel que : $S Q(\mathrm{~mm} / \mathrm{kcal})=[($ Sensation pré-repas $(\mathrm{mm}))-($ moyenne des sensations sur les 60 minutes post repas $(\mathrm{mm})$ ) / valeur calorique du repas (kcal)] *100 (Green et al., 1997) (CPP Sud Est VI).

Résultats et Analyse statistique : Les quotients de satiété (QS) relatifs à la sensation de faim, de désire de manger et de propension à manger, ainsi que les sensations alimentaires à jeun, ne sont pas significativement différents entre les deux conditions. Des analyses graphiques de Bland \& Altman soulignent la concordance entre les QS mesurés lors des deux conditions. Le QS de faim est corrélé avec la prise alimentaire au repas du soir $(p<0,05, r=0,353)$ et la prise alimentaire totale sur la journée ( $p<0,05, r=0,379)$. En revanche, les QS ne sont pas corrélés avec les sensations alimentaires pré et post-déjeuner.

Conclusion : Ces résultats préliminaires suggèrent le QS comme étant une méthode reproductible d'évaluation de la réponse satiétogène chez les adolescents en situation d'obésité. Cependant, des analyses supplémentaires sont nécessaires afin de déterminer si cette méthode peut être utilisée comme prédicteur de la prise alimentaire et de la perte de poids dans cette population.

Conflits d'intérêts : Aucun conflit à déclarer 
Sentiment de satisfaction après chirurgie bariatrique : quels prédicteurs à un an post-opératoire ?

B. Gaudrat ${ }^{12,{ }^{*}, \text { S. Andrieux }}{ }^{1}$, V. Florent ${ }^{1}$, A. Rousseau ${ }^{2}$

${ }^{1}$ Nutrition, CH Arras, Arras, ${ }^{2}$ PSITEC EA 4072, Université Lille, Lille, France

Introduction et but de l'étude : Le sentiment de satisfaction a été mis en évidence comme un facteur favorisant le maintien des comportements de santé et notamment dans le domaine de la perte pondérale. En effet, les patients maintenant une perte de poids sur le long terme rapportent un sentiment de satisfaction supérieur aux patients reprenant du poids. La satisfaction du patient après chirurgie bariatrique $(C B)$ a été peu étudiée malgré le rôle que celle-ci pourrait jouer dans le maintien de la perte de poids à long terme via la pérennisation des comportements de santé associés à la CB. Plusieurs facteurs semblent entrer en jeu dans la formation de la satisfaction : les attentes par rapport au résultat, l'attribution causale (interne versus externe) et le sentiment d'équité ( $c$ 'est à dire le fait de percevoir comme "juste » le résultat obtenu comparativement à ce que les autres obtiennent dans les mêmes circonstances). Cette étude a pour objectif d'évaluer la satisfaction après CB et d'identifier les facteurs influençant le développement de la satisfaction post-opératoire.

Matériel et méthodes : 53 patients opérés d'une CB au CSO du CH d'Arras (âge moyen=37,69 $\pm 11,10$, IMC moyen avant $\mathrm{CB}=45,50 \pm 5,85$, IMC moyen 1 an après $\mathrm{CB}=31,77 \pm 5,80$, femmes $=65,3 \%$ ) ont complété lors du 1er contact avec le service et à 1 an post-opératoire différentes mesures visant à évaluer leurs attentes quantitatives (en terme de poids chiffré) et qualitatives (santé/mobilité, image corporelle, rapports interpersonnels, améliorations psychologiques). Un an après CB, ont été évalués : la satisfaction (quantitative et qualitative), le sentiment d'équité et l'attribution causale (interne vs externe). Lors des deux temps de mesure (pré et post-opératoire) différentes variables psychologiques ont été mesurées.

Résultats et Analyse statistique : Les scores de satisfaction un an après CB sont élevés (>4/5). Les régressions identifient le sentiment d'équité comme principal prédicteur de la satisfaction du patient par rapport au résultat pondéral (expliquant $37 \%$ de la variance). Cette variable est également un prédicteur important d'autres mesures de satisfaction (centrée sur l'image corporelle [23\% de la variance], centrée sur les améliorations interpersonnelles [ $28 \%$ de la variance] et centrée sur les améliorations psychologiques [ $10 \%$ de la variance]. Concernant la satisfaction centrée sur la santé/mobilité, c'est la qualité de vie physique 1 an après CB qui apparait comme facteur prédicteur dominant ( $42 \%$ de la variance).

Conclusion : Cette étude met en évidence des scores élevés de satisfaction un an après $\mathrm{CB}$. Par ailleurs, cette satisfaction ne semble ni liée au poids perdu ni aux attentes préopératoires du patient mais c'est le sentiment d'équité qui apparait comme un facteur prédictif majeur de la satisfaction post-opératoire. Ces résultats semblent indiquer que majorer la satisfaction du patient après $C B$ nécessite sans doute plus d'amener celui-ci à percevoir sa perte de poids comme " juste " par rapport aux résultats obtenus par les autres patients plutôt que de tenter d'infléchir sa courbe pondérale. Ainsi, le sentiment d'équité mériterait d'être pris en considération dans nos accompagnements pré et post-opératoire afin d'améliorer la satisfaction post-opératoire du patient en permettant l'optimisation du maintien des modifications comportementales contemporaines à la CB.

Conflits d'intérêts : Aucun conflit à déclarer 
Un nouvel effet secondaire de la chirurgie bariatrique : la rhinorrhée post-prandiale

M. Pouget ${ }^{1,}{ }^{*}$, C. Lemaire ${ }^{1}$, L.-A. Gendre ${ }^{1}$, M. Riquelme ${ }^{2}$, E. Gentes ${ }^{1}$, N. Farigon ${ }^{1}$, C. Palmier-Forestier ${ }^{1}$, M. Miolanne $^{1}$, K. Slim ${ }^{3}$, Y. Boirie ${ }^{1}$

${ }^{1}$ Nutrition Clinique, ${ }^{2}$ Délégation Recherche Clinique \& Innovation, ${ }^{3}$ Chirurgie digestive, CHU de Clermont-Ferrand, ClermontFerrand, France

Introduction et but de l'étude : Multifactorielle, l'obésité peut être à l'origine de nombreuses comorbidités diminuant l'espérance et la qualité de vie des patients. La chirurgie bariatrique permet une réduction de la morbi-mortalité mais comporte des risques nutritionnels. Un symptôme aujourd'hui non rapporté dans la littérature a été exploré : la rhinorrhée post-prandiale après chirurgie bariatrique.

Matériel et méthodes : Cette étude rétrospective a inclus 82 patients obèses, ayant eu une chirurgie bariatrique et suivis lors des visites post-opératoires à 3, 6, 12, 18 et 24 mois. La présence ou l'absence de rhinorrhée a été recueillie lors de l'interrogatoire médical. Le but de cette étude etait de déterminer la prévalence de cette rhinorrhée et dans un second temps, regarder si elle est associée aux changements morphologiques, biologiques ou clinique (dumping syndrome, ...).

Les comparaisons entre groupes de patients ont été réalisées avec les tests statistiques usuels : test de Student ou le test de MannWhitney si conditions du t-test non respectées (normalité, homoscédasticité étudiée par le test de Fisher-Snedecor) pour les variables quantitatives. S'agissant des données répétées longitudinales, des modèles mixtes comme l'ANOVA en données répétées ont été mis en œuvre afin de prendre en compte la variabilité inter et intra patient (considéré comme effet aléatoire d’étudier les effets groupe (rhinorrhée), temps après la chirurgie et leurs interactions.

Résultats et Analyse statistique : Parmi les 82 patients (âge $43 \pm 12$ ans, $78 \%$ femmes, $76 \%$ sleeve gastrectomie vs $24 \%$ by-pass, IMC $47 \pm 7 \mathrm{~kg} / \mathrm{m}^{2}$ ), 28 patients (34\%) ont développé une rhinorrhée post-prandiale après chirurgie bariatrique. Au sein de la population, les patients ayant une rhinorrhée $(R+)$ sont comparables aux patients sans rhinorrhée ( $R$-) sur le plan morphologique, biologique ou encore clinique avant la chirurgie. Cependant, la rhinorrhée est associée à l'intensité de la perte de poids (effet temps : $p<0,001$; effet rhinorrhée : $p=0,056$; ANOVA). Dans le groupe $\mathrm{R}+$, la perte de poids est de 21, 33, 44, 40, 41 kg à 3, 6, 12, 18, 24 mois respectivement, alors que dans le groupe $\mathrm{R}-$, la perte de poids est de 20, 28, 34, 31, $30 \mathrm{~kg}$.

La glycémie semble plus faible chez les patients $R+(p=0,09)$ et le dumping syndrome plus fréquent $(47 \%$ chez les $R+$ et $8 \%$ chez les R- à 12 mois; $p=0,005)$. Cependant, aucun lien n'est constaté avec les allergies saisonnières, le type de chirurgie ou le tabagisme. La qualité de vie des patients avec une rhinorrhée n'est pas détériorée selon le score de santé générale du SF-36 $(p=0,10)$.

Conclusion : Cette étude révèle une prévalence significative de rhinorrhée post-prandiale après chirurgie bariatrique en lien avec l'intensité de la perte de poids et le dumping syndrome. Son mécanisme physiopathologique reste encore à élucider.

Conflits d'intérêts : Aucun conflit à déclarer 
Introduction et but de l'étude : La chirurgie bariatrique représente à l'heure actuelle la méthode thérapeutique la plus efficace pour induire une perte de poids chez les patients obèses morbides. Néanmoins, les résultats pondéraux post-opératoire demeurent pour certains décevants à moyen terme. Le suivi de programme d'éducation thérapeutique concernant la diététique et l'activité physique, notamment lors d'un séjour en SSR spécialisé, est souvent préconisé afin d'améliorer la perte de poids postopératoire. Le but de cette étude a été d'évaluer l'impact d'un séjour en SSR spécialisé sur l'évolution pondérale et la survenue de complications post-chirurgie bariatrique chez des patients obèses.

Matériel et méthodes : Nous avons réalisé une étude rétrospective portant sur 672 patients opérés d'une chirurgie bariatrique entre 2014 et 2017. Nous avons comparé deux groupes en fonction d'un passage en SSR ( $n=112 ; 17 \%$ ) ou non ( $n=560 ; 83 \%$ ), sur l'évolution pondérale et la survenue de complications médicales, psychiatriques ou chirurgicales en post opératoire en tenant compte d'éventuels facteurs confondants tels que le sexe, un antécédent de chirurgie bariatrique et le moment du séjour en SSR (supérieur ou inférieur à 12 mois avant ou après chirurgie). L'évolution pondérale post-opératoire a été évaluée par l'IMC, le pourcentage de perte de poids (PPdP) et par l'Excess Weight Loss (EWL), à 3 mois, 6 mois, 1 an et 2 ans. Les comparaisons de moyennes ont été réalisées avec un test t ou une analyse de covariance selon les cas.

Résultats et Analyse statistique : Les résultats concernent 672 patients, 526 (78,3\%) femmes et 146 (21,7\%) hommes, d'âge moyen de 42,2 ans, l'IMC opératoire étant de $44,4 \mathrm{~kg} / \mathrm{m}^{2}$. La perte de poids post-opératoire a été significativement plus importante tout au long du suivi, chez les patients n'ayant pas bénéficié de SSR (à 2 ans, PPdp : 36\% vs 33\%, p=0,04; EWL : 76\% vs 65\%, $p=0,009)$, la différence étant essentiellement liée à l'évolution pondérale des femmes. Ce résultat, en apparence paradoxal, semble s'expliquer par l'influence du délai entre le séjour en SSR et la chirurgie. Les patients qui ont bénéficié d'un SSR dans l'année péri opératoire objectivaient une perte de poids équivalente à 2 ans (PPdp de 36\%) et rattrapaient ainsi leur retard sur I'IMC de départ ( 30 vs $28 \mathrm{~kg} / \mathrm{m}^{2}, p=0,043$ ) d'autant qu'ils présentaient un profil clinique plus défavorable : IMC plus important (48 vs $44 \mathrm{~kg} / \mathrm{m}^{2}, \mathrm{p}<0,001$ ), plus de comorbidités (diabète, hypertension artérielle, stéatose, SAOS, complications ostéo articulaires) que les patients qui n'en avaient pas bénéficié. Nous n'avons pas observé de différence d'incidence des complications médicales, psychiatriques ou chirurgicales entre les différents groupes.

Conclusion : Cette étude met en évidence que les patients adressés en SSR en pratique clinique courante sont souvent plus " compliqués " avec une surcharge pondérale plus importante et des comorbidités plus nombreuses. Le bénéfice d’un séjour en SSR pour ces patients semble plus conséquent s'il a lieu dans l'année du geste de chirurgie bariatrique avec une l'évolution pondérale post opératoire plus favorable sur le moyen terme.

Conflits d'intérêts : Aucun conflit à déclarer 
Prévalence de l'obésité et du surpoids chez des écolières et écoliers de Casablanca : Maroc

H. Makhlouki ${ }^{1}{ }^{*}$, S. Lougda ${ }^{1}$, A. Jafri ${ }^{2}$, F. Achibane ${ }^{1}$, M. El Arbaoui ${ }^{1}$, Y. Elkardi ${ }^{1}$, H. Taki ${ }^{1}$, R. Saile ${ }^{1}$, A. Derouiche ${ }^{1}$

${ }^{1}$ Groupe de Recherche Nutrition Humaine Laboratoire Biologie et Santé, URAC34. Faculté des Sciences Ben M'sik. Université Hassan II de Casablanca, Maroc, ${ }^{2}$ Faculté des sciences de la santé, Université Mohammed VI, Casablanca, Maroc

Introduction et but de l'étude : De 1975 à 2016 L'obésité juvénile est passée de 1\% à 6\% chez les filles et $8 \%$ chez les garçons âgés de 5 ans à 19 ans.

Des études épidémiologiques et des recherches indiquent que l'obésité juvénile surpassera l'insuffisance pondérale d'ici 2022 et qui serai due en particulier à l'existence d'une forte association entre les troubles du comportement alimentaire et l'obésité.

L'objectif de ce travail est d'évaluer la prévalence de l'obésité et surpoids juvénile selon les références OMS chez des écoliers âgés de moins de 12 ans.

Matériel et méthodes : L'échantillonnage a concerné 80 enfants choisis au hasard âgés de 8 ans à 12 ans de la région de Médiouna du grand Casablanca Maroc. Les mesures de la taille ont été réalisées par un stadiomètre doté d'une toise verticale, le poids et I'IMC à l'aide d'un impédencemètre (Karada scan) selon les recommandations de l'OMS et la surcharge pondérale a été évaluée en comparant l'IMC aux normes de références de l'OMS 2007.

Résultats et Analyse statistique : La moyenne d'âge de notre échantillon était de $9,8 \pm 1,4$ ans et le sexe ratio filles/garçons était de 1,4 . Dans notre étude la prévalence du surpoids a été estimée à $13,28 \%$ (15,2\% des filles et $8,8 \%$ des garçons) dont 5,1\% était obèse $(6,5 \%$ des filles et $8,8 \%$ des garçons).

Conclusion : Ces résultats mettent en évidence l'existence d'une prévalence très élevée de surpoids et d'obésité dans les différentes tranches d'âges des enfants dans ladite région. Les données concernant le surpoids et l'obésité sont variable dans la littérature, avec des prévalences au Maroc allant respectivement de $8 \%$ et 3\% (M. Sebbani et all 2012). D'où l'intérêt de mettre en place un plan d'action pour une prise en charge précoce afin d'agir contre la progression de ce problème de santé.

Remerciements : Nous remercions tous les enfants qui ont participé à cette étude.

Conflits d'intérêts : Aucun conflit à déclarer 
La prévalence de la surcharge pondérale et les facteurs sociodémographiques associés à Casablanca, Maroc

K. Mohtadi ${ }^{1}$, R. Msaad ${ }^{1}$, N. Benalioua ${ }^{1}$, Y. Elkardi ${ }^{1}$, R. Essadik ${ }^{1}$, H. Lebrazi ${ }^{1}$, A. Jafri ${ }^{2}$, A. Derouiche ${ }^{1}$, E. H. Tahri $^{3}$, A. Kettani $^{1}$, H. Taki ${ }^{1}$, R. Saile ${ }^{1, *}$

${ }^{1}$ Laboratoire de Biologie et Santé (URAC 34), Faculté des Sciences Ben M'Sik, Université Hassan II de Casablanca, ${ }^{2}$ Université Mohammed VI des Sciences de la Santé, Faculté des Sciences et Techniques de Santé, Casablanca, Maroc, ${ }^{3}$ Laboratoire de Biologie Moléculaire et Physiopathologie, Faculté des Sciences Ben M’Sik, Université Hassan II de Casablanca, Casablanca, Maroc

Introduction et but de l'étude : L'obésité constitue un problème majeur de santé publique à l'échelle mondiale. Elle favorise la survenue de plusieurs pathologies affectant le pronostic vital des individus, principalement les maladies cardio-vasculaires. L'objectif de notre étude était d'évaluer la prévalence de la surcharge pondérale chez la population adulte de Casablanca et les facteurs sociodémographiques associés à son occurrence.

Matériel et méthodes : Une enquête transversale a été menée au niveau de la ville de Casablanca en 2017. Un échantillonnage en grappes a été utilisé en se basant sur le recensement national de 2014 pour sélectionner les participants. Les données sociodémographiques (âge, niveau d'instruction et état matrimonial ...) ont été collectées à l'aide d'un questionnaire et les paramètres anthropométriques ont été mesurés selon les recommandations de l'OMS. L'obésité était définie par l'indice de masse corporelle (IMC) $(\geq 30 \mathrm{~kg} / \mathrm{m} 2)$. La régression logistique a été utilisée pour prédire les principaux facteurs associés à l'obésité.

Résultats et Analyse statistique : L'étude a concerné 730 sujets dont 379 hommes et 461 femmes. L'âge moyen était de $39,07 \pm$ 15,46 ans. Concernant le niveau d'étude, 19,2\% étaient analphabètes et 17,9\% avaient un niveau supérieur. Parmi les sujets de l'étude, $55,3 \%$ étaient actifs et $22 \%$ étaient des femmes au foyer. Les sujets mariés et célibataires représentaient respectivement $52,7 \%$ et $39,4 \%$ des cas. La prévalence de l'obésité était de $21,6 \%$ (8,7 et 35,6\% pour les hommes et les femmes, respectivement). La prévalence du surpoids était de $28,2 \%$ ( $28,3 \%$ chez les femmes et $28,2 \%$ chez les hommes). Le sexe, la zone de naissance, l'état matrimonial, la profession et les groupe d'âge sont significativement associés à l'obésité dans notre population ( $p<0,001$ ). Chez les femmes, l'âge, l'état matrimonial, le niveau de scolarité et la profession étaient significativement associés à l'obésité, alors que chez les hommes, seuls l'âge et l'état matrimonial étaient significativement associés à l'obésité $(p<0,05)$.

Conclusion : Cette étude montre que la prévalence de l'obésité est élevée dans la population étudiée. Ainsi que les facteurs sociodémographiques étudiés étaient associés au risque d'obésité mais d'une manière différente selon le sexe. Et que cette pathologie nécessite d'être prise en compte dans les interventions de santé publique.

Conflits d'intérêts : Aucun conflit à déclarer 
Un régime à teneur faible en protéine et en méthionine induit une augmentation de la prise alimentaire, de la dépense énergétique et de FGF21 et une baisse de l'IGF-1 et affecte peu l'adiposité chez les souris femelle BalbC

P. Even ${ }^{1, *}$, C. Chaumontet ${ }^{1}$, D. Azzout-Marniche ${ }^{1}$, A. Blais ${ }^{1}$, J. Piedcoq ${ }^{1}$, D. Tomé ${ }^{1}$, C. Gaudichon $^{1}$

${ }^{1}$ UMR PNCA, AgroParisTech, INRA, Université Paris-Saclay, Paris, France

Introduction et but de l'étude : Les régimes faibles en protéine sont soupçonnés d'augmenter la prise alimentaire et le gain de masse grasse, une réponse qui pourrait être exacerbée selon la qualité des protéines, comme les protéines de soja avec une teneur réduite en méthionine. Le but de cette étude était de comparer, chez des souris femelles BalbC recevant un régime à teneur réduite en protéine et en méthionine, la prise alimentaire, la composition corporelle, la dépense énergétique, la sensibilité à l'insuline et les taux plasmatiques de FGF21 et d'IGF-1.

Matériel et méthodes : 56 souris femelles BalbC de 7 semaines logées en cages individuelles ont été soumises pendant 8 semaines à 7 régimes dont la teneur en protéine a été modifiée en quantité $(20 \%, 6 \%, 3 \%)$ et en qualité (caséine, soja, soja complémenté en méthionine) ( $\mathrm{N}^{\circ} \mathrm{d}^{\prime}$ agrément du comité national d'éthique Cométhéa 13-012). Le poids et la prise alimentaire ont été mesurés 3 fois par semaine. Un test de tolérance au glucose a été effectué après 4 semaines de régime. La dépense énergétique et l'activité motrice ont été mesurées entre 4 et 8 semaines de régime. A la fin de l'étude les souris ont été euthanasiées, le sang prélevé par ponction cardiaque pour le dosage de l'insuline, FGF21 et IGF-1. La composition corporelle a été déterminée par pesée des tissus. L'hypothalamus et des morceaux de foie, muscle, tissu adipeux blanc et brun ont été rapidement congelés dans l'azote liquide pour la mesure de l'expression des gènes. L'analyse statistique a été faite par analyse de variance sous RC.

Résultats et Analyse statistique : Une baisse de la teneur en protéine du régime de $20 \%$ à $6 \%$ a induit une augmentation de la prise alimentaire, n'a pas eu d'effet sur le poids total mais a induit une augmentation légère de l'adiposité, davantage chez les souris recevant les protéines de soja qu'avec la caséine. La dépense énergétique a augmenté proportionnellement à la prise alimentaire expliquant les effets limités sur le poids et la masse grasse. Les taux plasmatiques d'IGF-1 ont diminué et ceux de FGF21 ont augmenté avec la baisse de la teneur en protéine du régime. La teneur en méthionine des régimes a affecté les variations des taux de plasmatique de FGF21 mais pas celles d'IGF-1. Au niveau hypothalamique, des variations significatives de l'expression des neurotransmetteurs ont été observées pour les souris soumises à un régime contenant 3\% de protéine, avec en particulier une augmentation de l'expression de NPY et une baisse de celle de POMC, reflétant que, chez ces souris, l'augmentation de la prise alimentaire était insuffisante pour atteindre les besoins en protéine et assurer une croissance équivalente à celle observée dans les autres groupes.

Conclusion : Une baisse de l'apport protéique induit chez la souris une augmentation de la prise alimentaire qui est compensée par une augmentation de la dépense énergétique ce qui se traduit par un impact limité sur la masse grasse. Ces effets semblent médiés par une augmentation de FGF21 qui est sensible la quantité et la qualité des protéines et une baisse de IGF-1 qui ne semble sensible qu'à la quantité des protéines.

Conflits d'intérêts : Aucun conflit à déclarer 
Accompagnement familial à domicile et de PROXimité de l’OBésité infanto-juvénile PROXOB : étude pilote de faisabilité en recherche interventionnelle en santé.

R. Rigondet ${ }^{1}$, A. Rigal $^{1}$, C. Desblès ${ }^{1}$, Q. Lesaichot ${ }^{1}$, J. Masurier ${ }^{2}$, C. Cardenoux ${ }^{3}$, D. Thivel ${ }^{4}$, B. Pereira ${ }^{5}$, C. Lambert $^{5}$, Y. Boirie $^{16, *}$, M. Miolanne ${ }^{1}$

${ }^{1}$ Nutrition, CHU Gabriel Montpied, ${ }^{2} \mathrm{SSR}$ Nutrition Obésité, ${ }^{3} \mathrm{CMI},{ }^{4} \mathrm{AME} 2 \mathrm{P}$, Université Clermont Auvergne, ${ }^{5} \mathrm{DRCl}$, CHU Gabriel Montpied, ${ }^{6}$ UNH-UMR 1019, Université Clermont Auvergne, Clermont-Ferrand, France

Introduction et but de l'étude : Les principes de la prise en charge de l'obésité infanto-juvénile reposent sur un accompagnement multidisciplinaire de longue durée incluant l'environnement familial. Le projet-pilote PROXOB (accompagnement familial de PROXimité de l'OBésité infanto-juvénile) a pour buts la prévention primaire et l'initiation précoce ou le renforcement de la prise en charge de l'obésité infantile par l'accompagnement à domicile de familles concernées par l'obésité. Il s'agit également de lutter contre les inégalités sociales de santé et contre la discrimination en permettant l'accès aux soins de proximité des populations notamment précaires. Le but de l'étude est de démontrer la faisabilité, l'acceptabilité et la transférabilité de cette approche innovante, personnalisée, interdisciplinaire, familiale et à domicile.

Matériel et méthodes : Cette recherche interventionnelle en santé a comporté 3 phases (PROXOB I, II et III) avec évaluation continue et systématique du protocole en vue de son amélioration. L'accompagnement consiste en 18 séances à domicile sur une période de 6 mois pour toute la famille : 3 bilans éducatifs partagés à domicile, 5 ateliers diététiques, 5 d'activité physique adaptée et 5 autour de la parentalité. Le bilan médical de la famille est réalisé en centre de soin avant (M0), à la fin des 6 mois d'intervention (M6) puis 6 mois plus tard (M12).

Résultats et Analyse statistique : 62 familles ( $n=235$ personnes) ont participé aux deux premières vagues 2015-2016 et 20162017. 95\% des familles sont satisfaites, disent avoir changé leurs habitudes et recommandent PROXOB à d'autres familles. L'adhésion est de $95 \%$ en milieu rural et de $73 \%$ en milieu urbain. Les professionnels relatent un réel bénéfice d'une prise en charge interdisciplinaire de l'ensemble de la famille à domicile. Le protocole a été optimisé pour favoriser le recrutement, l'adhésion, l'organisation, l'évaluation, la pérennisation et le déploiement de PROXOB. La troisième vague en cours voit une adhésion de $100 \%$ en milieu urbain comme rural.

Conclusion : PROXOB est devenu un protocole opérationnel faisable et accepté par le public cible et les professionnels. II est transférable et reproductible, l'expérience acquise pouvant être exploitée dans tous les territoires, régionaux ou nationaux. L'évaluation des résultats permettra de conforter la position de PROXOB au sein du parcours de prévention et de soin de l'obésité pédiatrique.

Conflits d'intérêts : Aucun conflit à déclarer 
Étude pilote chez 45 patients obèses : évaluation d'un Traitement innovant Ambulatoire Pluridisciplinaire d'aide à la Gestion de I'Impulsivité Alimentaire.

H. Lelandais ${ }^{1, *}$, T. Saillard ${ }^{1}$, E. Diologent ${ }^{1}$, M. Riguet ${ }^{1}$, C. Meret $^{12}$, V. Risselin ${ }^{1}$, C. Lemiere ${ }^{1}$, M. Valentino ${ }^{1}$, D. Hazard ${ }^{1}$, P. Prié ${ }^{1}$, G. Le Foll ${ }^{1}$, A. Raimbault ${ }^{1}$, J. Dijoux ${ }^{1}$, P. Dechelotte ${ }^{13}$, S. Grigioni ${ }^{13}$, V. Folope ${ }^{123}$

${ }^{1}$ Nutrition, ${ }^{2}$ Centre Spécialisé Obésité Haute Normandie, CHU de Rouen, ${ }^{3}$ INSERM UMR 1073, Normandie Université, ROUEN, France

Introduction et but de l'étude : Dans la prise en charge classique médicale ou médico-chirurgicale de l'obésité, la ré-ascension pondérale est fréquemment liée à la survenue ou à la réapparition de compulsions alimentaires. Afin de prendre en compte cette problématique, nous avons proposé un Traitement Ambulatoire Pluridisciplinaire (TAP) qui associe un protocole de thérapie cognitivo-comportementale (TCC) innovant appelé " Méditation basée sur la gestion de l'Impulsivité Alimentaire » (MBGIA) à un protocole d'hypnose médicale créé à cette intention.

Matériel et méthodes : Les patients obèses compulsifs, inclus dans ce TAP entre Février et Juin 2018, ont bénéficié de 13 séances de 3 modules chacune, dont 8 modules de TCC MBGIA, 4 modules d'hypnose, 6 modules de diététiques, 2 modules culinaires, 5 modules d'activité physique adaptée, 1 module de socio-esthétique et 13 modules infirmiers avec revue des tâches à domicile. Ils ont rempli des questionnaires avant et après le TAP pour évaluer leurs poids, taille, comportement alimentaire (EDI-2), anxiétédépression (HAD) et activité physique (Ricci et Gagnon).

Résultats et Analyse statistique : 45 patients compulsifs (88,9\% de femmes) d'âge moyen 46,1 $\pm 15,5$ ans et d'IMC moyen $36,8 \pm 9,4$ $\mathrm{kg} / \mathrm{m}^{2}$ ont été inclus ; ils ont réalisé en moyenne $9,2 \pm 3,4$ séances. Les scores de comportement alimentaire global et notamment d'impulsivité alimentaire, d'anxiété, de dépression et de pratique d'activité physique ont été significativement améliorés (cf. tableau 1).

Tableau 1: Evolution des scores EDI total (comportement alimentaire global), d'impulsivité alimentaire (EDI composante impulsivité), d'anxiété (HAD-A), de dépression (HAD-D) ainsi que du score d'activité physique au cours du TAP; résultats exprimés en moyennestécart-type.

* $: p<0.05$ avec le test de Kruskal-Wallis

\begin{tabular}{|l|c|c|c|}
\hline & Score avant le TAP & Score après le TAP & p \\
\hline EDI total & $91,3 \pm 30,4(n=29)$ & $71,7 \pm 24,8(n=29)$ & 0,00 \\
\hline EDI composante impulsivité & $6 \pm 3,7(n=29)$ & $3 \pm 4(n=29)$ & 0,00 \\
\hline HAD-A & $10,3 \pm 3(n=29)$ & $9,3 \pm 3,5(n=29)$ & 0,042 \\
\hline HAD-D & $9,5 \pm 3,6(n=29)$ & $7,5 \pm 4,1(n=29))$ & 0,005 \\
\hline Ricci et Gagnon & $19,4 \pm 7,7(n=28)$ & $24,7 \pm 7,2(n=28)$ & 0,00 \\
\hline
\end{tabular}

Conclusion : Ce traitement intégratif alliant TCC et hypnose améliore la composante compulsive et le bien être global des patients obèses au décours immédiat du programme : il offre de nouvelles perspectives dans la prise en charge de l'obésité et nous incite à confirmer ces résultats par une étude contrôlée.

Conflits d'intérêts : Aucun conflit à déclarer 
${ }^{1}$ Centre des Sciences du Goût et de I'Alimentation, AgroSup Dijon, CNRS, INRA, Univ. Bourgogne Franche-Comté, ${ }^{2}$ Service de Diabétologie et Nutrition Clinique, CHU Dijon, Dijon, France

Introduction et but de l'étude : Des études suggèrent que les biais attentionnels (BA) envers les aliments (tendance des stimuli alimentaires à attirer automatiquement l'attention) jouent un rôle dans le maintien/développement de l'obésité. Notre objectif est de caractériser ces BA chez des adultes normo-pondéraux (NP), en surpoids (S) et obèses (O), ainsi que la manière dont l'environnement olfactif peut les influencer afin de mieux comprendre des choix alimentaires délétères pour la santé.

Matériel et méthodes : Une mesure d'orientation de l'attention visuelle envers les stimuli alimentaires a été développée. Les participants (36 NP, 26 S, 24 O) ont réalisé une tâche informatique (Food Adapted Visual Probe Task) couplée à un paradigme d'amorçage olfactif, nous permettant d'évaluer l'influence d'odeurs alimentaires non-attentivement perçues (séance implicite) vs. attentivement perçues (séance explicite) sur les biais attentionnels. L'exposition à des odeurs signalant des aliments à haute densité énergétique (HDE) (odeur de quatre quarts) vs. faible densité énergétique (LDE) (odeur de poire) nous permet de mesurer la différence d'impact de signaux sensoriels sur ces individus de statuts pondéraux différents.

Résultats et Analyse statistique : Les résultats ont été analysés en utilisant un modèle linéaire mixte estimé à l'aide du package nlme du logiciel R (v. 3.4.3.). Les résultats indiquent un BA envers l'alimentation, quel que soit le statut pondéral des individus $(p<0,0001)$. Ce BA est exacerbé lorsque les aliments sont à HDE $(p<0,0001)$. Un effet d'amorçage significatif est observé uniquement lorsque les odeurs sont non-attentivement perçues (séance implicite). Dans cette condition implicite, les $O$ et NP ne réagissent pas de la même façon aux odeurs alimentaires $(p=0,02)$. En effet, les $O$ ont une orientation plus prononcée de l'attention envers les stimuli alimentaires lorsqu'ils sont non attentivement exposés à une odeur HDE (quatre-quarts) comparé à l'odeur LDE (poire $(p=0,02)$.

Conclusion : Finalement, nos résultats confirment l'intérêt d'étudier des facteurs cognitifs (les BA), et soulignent la pertinence d'utiliser l'amorçage pour impacter ces derniers, afin de mieux comprendre les liens entre l'environnement et le comportement alimentaire. Des études à venir chercheront à confirmer ces résultats en utilisant des techniques EEG et tenteront de relier la présence de tels biais à des choix alimentaires délétères pour la santé.

Conflits d'intérêts : Aucun conflit à déclarer 
${ }^{1}$ Laboratoire AME2P, Université Clermont Auvergne, Aubière, ${ }^{2}$ INRA UMR1019, UNH, Université Clermont Auvergne, ClermontFerrand, France

Introduction et but de l'étude : Les mécanismes expliquant le gain de poids ont été très étudiés ces dernières années. Les stéroïdes et notamment les œstrogènes ont été montrés comme jouant un rôle important dans les processus de régulation du stockage et déstockage lipidique. Différentes stratégies sont reconnues comme permettant le gain ou la perte de poids mais il existe une variabilité interindividuelle de réponse à ces stratégies. Nous avons émis l'hypothèse que l'expression des récepteurs aux œstrogènes (ER $\alpha$ et $E R \beta)$ serait dépendante de cette sensibilité interindividuelle à répondre aux stratégies de modulation pondérale.

Objectif: Le but de cette étude était d'une part d'évaluer l'effet de deux régimes alimentaires (chow vs HFHS) sur la sensibilité à prendre du poids, l'expression des ERs et la tolérance au glucose, d'autre part d'évaluer l'effet de stratégies de perte de poids (adaptation nutritionnelle vs activité physique) sur la sensibilité à perdre du poids, l'expression des ERs et la tolérance au glucose. Matériel et méthodes : Dans la première phase de ce projet 15 rats mâles Wistar ont été soumis à 16 semaines de régime HFHS et 15 rats ont suivi un régime standard. Les rats ont été analysés en fonction de leur capacité à prendre du poids (ob-prone vs obrésistant). Dans la deuxième phase de l'étude, 45 rats rendus obèses suite à un régime HFHS ont été soumis à différentes stratégies de perte de poids (adaptation nutritionnelle vs activité physique) pendant 8 semaines. Les rats ont été analysés en fonction de leur capacité à perdre du poids (répondeurs vs non-répondeurs).

Tous les rats ont été soumis à un test oral de tolérance au glucose avant sacrifice ainsi qu'à une analyse de la composition corporelle par DXA. L'expression d'ER $\alpha$ et ER $\beta$ a été évalué dans différents tissus adipeux et dans le gastrocnemius par western blot.

Résultats et Analyse statistique : Phase 1, les rats « ob-prone » avaient une AUC pour l'insuline supérieure au groupe « obrésistant ». L'expression d'ERß était également augmentée dans le tissu adipeux péri-rénal des rats "ob-prone ». Dans la deuxième phase de notre étude l'expression d'ERß était augmentée chez les « répondeurs » aux différentes stratégies de perte de poids.

Conclusion : Notre équipe a déjà démontré que l'expression d'ER $\alpha$ était impactée par les modifications de régime nutritionnel, cette nouvelle étude montre que l'expression d'ER $\beta$ dans le tissu adipeux est associée à la variabilité interindividuelle de prendre ou perdre du poids.

Références : Ponnusamy S, Tran QT, Harvey I, Smallwood HS, Thiyagarajan T, Banerjee S, Johnson DL, Dalton JT, Sullivan RD, Miller DD et al: Pharmacologic activation of estrogen receptor beta increases mitochondrial function, energy expenditure, and brown adipose tissue. FASEB J 2017, 31(1):266-281.

King NA, Hopkins M, Caudwell P, Stubbs RJ, Blundell JE: Individual variability following 12 weeks of supervised exercise: identification and characterization of compensation for exercise-induced weight loss. Int J Obes (Lond) 2008, 32(1):177-184.

Pagliassotti MJ, Knobel SM, Shahrokhi KA, Manzo AM, Hill JO: Time course of adaptation to a high-fat diet in obesity-resistant and obesity-prone rats. Am J Physiol 1994, 267(3 Pt 2):R659-664.

Conflits d'intérêts : Aucun conflit à déclarer 
L'addiction alimentaire diagnostiquée par la Yale Food Addiction Scale version 2.0 est fréquente chez les patients obèses mais n'est pas associée aux complications de l'obésité.

M. Som ${ }^{12, *}$, D. Val-Laillet ${ }^{23}$, A. Constant ${ }^{24}$, R. Moirand ${ }^{25}$, R. Thibault ${ }^{12}$

${ }^{1}$ Unité de nutrition- CHU Rennes, ${ }^{2}$ Institut Nutrition Métabolismes et Cancer, NuMeCan, INRA, INSERM, Université de Rennes, ${ }^{3}$ INRA, ${ }^{4}$ EHESP, ${ }^{5}$ Service des Maladies du Foie - CHU Rennes, Rennes, France

Introduction et but de l'étude : L'obésité est une pandémie responsable de nombreuses complications. L'addiction alimentaire (AA) est un concept récent pouvant expliquer en partie l'échec de la prise en charge de l'obésité. L'objectif de notre étude était donc de déterminer la prévalence de l'AA dans une population de patients obèses éligibles à la chirurgie de l'obésité, et le phénotype clinique du patient obèse ayant une AA.

Matériel et méthodes : Le Yale Food Addiction Scale (YFAS) 2.0 est le questionnaire validé qui a été utilisé pour le diagnostic d'AA. Il était rempli par chaque nouveau patient consultant dans l'unité de nutrition pour la prise en charge d'une obésité.

Les données sociodémographiques et cliniques étaient collectées rétrospectivement à partir de patients obèses vus en consultation de nutrition de décembre 2016 à mars 2018. Tous les patients ayant rempli le questionnaire YFAS ont été inclus dans l'analyse. Les données qualitatives étaient analysées à l'aide du test du Khi2 ou Fisher exact et les données quantitatives par l'analyse de variance à un facteur (ANOVA).

Résultats et Analyse statistique : Deux-cent-vingt-six patients obèses ont rempli le questionnaire YFAS 2.0. Parmi ces patients, 44 (19\%) ont été exclus de l'analyse car les questionnaires étaient mal remplis. Quatre-vingt pour cent étaient des femmes. L'âge moyen était de 43,3 ans (+/-13,7). L'indice de masse corporelle (IMC) moyen était de $42,9 \mathrm{~kg} / \mathrm{m}^{2}(+/-6.6)$. La prévalence d'AA était de $33 \%(n=60 / 182)$. Parmi ceux-ci, $57 \%$ avait une AA sévère, $35 \%$ une modérée et $8 \%$ une légère. Parmi les patients éligibles à une chirurgie de l'obésité $39 \%$ ( $n=34 / 88$ ) avaient une AA. L'AA touchait davantage les femmes et cela de manière significative : $37 \%$ des femmes et $17 \%$ des hommes $(p=0,02)$. Les données sociodémographiques telles que l'âge, la profession, le statut marital, la parentalité, le lieu de résidence et l'IMC ne différaient significativement pas entre AA et non AA. Il en était de même pour la prévalence des complications médicales (diabète, hypertension artérielle, infarctus du myocarde, syndrome métabolique, dyslipidémie, stéatose hépatique, syndrome d'apnées obstructives du sommeil, dyspnée).

Conclusion : Près de $40 \%$ des patients obèses éligibles à la chirurgie de l'obésité et adressés dans notre centre de référence rapportaient une AA. Cependant, l'AA n'était pas associée aux complications de l'obésité. II reste maintenant à déterminer si la présence d'une AA est ou non prédictive du succès de la prise en charge médico-chirurgicale de l'obésité, et si une prise en charge addictologique personnalisée dédiée permettrait le maintien de la perte de poids à long terme.

Conflits d'intérêts : Aucun conflit à déclarer 
Effets bénéfiques d'une prise en charge pluridisciplinaire sur l'inflammation systémique : Impact de l'endurance cardiorespiratoire chez des adolescents obèses présentant des troubles du sommeil

J. Roche ${ }^{123}$, L. Isacco ${ }^{12}$, D. Thivel ${ }^{4,}{ }^{*}$, F. Perret ${ }^{5}$, G. Dumoulin ${ }^{26}$, V. Gillet ${ }^{3}$, F. Mougin $^{12}$

${ }^{1}$ UPFR des Sports, Université de Bourgogne Franche-Comté, ${ }^{2} E A 3920$ - Marqueurs Pronostiques et Facteurs de Régulation des Pathologies Cardiaques et Vasculaires, Besancon, ${ }^{3}$ Centre Médical Santé-Sommeil Ellipse, Franois, ${ }^{4}$ EA 3533, Laboratoire des Adaptations Métaboliques à l'Exercice en conditions Physiologiques et Pathologiques (AME2P), Clermont-Ferrand, ${ }^{5}$ Centre de soins de suites et de réadaptation La Beline, UGECAM, Salins-les-Bains, ${ }^{6}$ Département de biochimie, CHU Jean Minjoz, Besancon, France

Introduction et but de l'étude : Une prise en charge (PEC) associant modification des habitudes alimentaires et réentrainement à l'exercice est efficace pour traiter le syndrome d'apnées obstructives du sommeil (SAOS) du jeune obèse, mais ses effets sur l'inflammation ne sont pas totalement élucidés. Les objectifs de cette étude ont été d'évaluer, chez des adolescents obèses sévères, la relation entre l'inflammation et le SAOS et de déterminer les facteurs expliquant une baisse de l'inflammation à l'issue d'une PEC pluridisciplinaire de 9 mois.

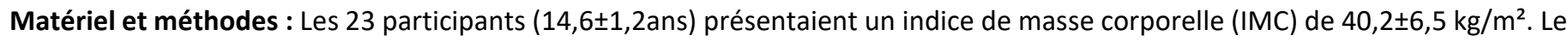
sommeil, les paramètres anthropométriques, le profil inflammatoire et l'endurance cardiorespiratoire ont été mesurés en pré et post PEC. La $V O_{2 \text { pic }}$ a été rapportée au poids $\left(V_{2} O_{2 \text { pic }} ; \mathrm{ml} / \mathrm{min} / \mathrm{kg}\right)$ et à la masse maigre $\left(V^{\prime} O_{2 \text { picmM }} ; \mathrm{ml} / \mathrm{min} / \mathrm{kg} \mathrm{Mm}\right)$. Les relations entre concentrations de protéine C-réactive (CRP) et IMC, sexe, index de désaturation en oxygène (IDO), index de $\mu$-éveils, temps de sommeil total (TST) et $V^{\prime} O_{2 p e a k}$ ont été étudiées par ANCOVA. Le SAOS a été déterminé selon un index d'apnées-hypopnées obstructives (IAHO) $\geq 2$ pour la constitution de groupes "SAOS » et « Non SAOS ».

Résultats et Analyse statistique : A l'admission, les sujets SAOS ( $n=13)$ présentaient des concentrations de CRP plus élevées que les sujets Non-SAOS. L'IMC, ajusté pour le sexe, l'index de $\mu$-éveils, l'IDO, le TST et la $V O_{2}{ }_{2}$ icPC, était associé aux taux de CRP ( $r^{2}$ ajusté $=0,32, p<0,05)$. En post PEC le SAOS a été normalisé chez 6 sujets (46\%). Les taux de CRP et de leptine ont significativement diminué et ceux d'adiponectine ont augmenté dans le groupe SAOS et dans la population totale. La diminution des concentrations de CRP a été expliquée par une amélioration de la $V^{`} O_{2 p i c m m}$ ajustée pour le sexe, la perte de poids et la modification des paramètres du sommeil étudiés ( $r^{2}$ ajusté $\left.=0,75, p<0,05\right)$.

Conclusion : Malgré des niveaux supérieurs de CRP dans le groupe SAOS comparés à ceux du groupe Non-SAOS, l'obésité prédomine sur les effets pro-inflammatoires induits par les troubles du sommeil et la faible endurance cardiorespiratoire, à l'admission. Bien que le programme n'ait pas permis de normaliser le SAOS chez tous les sujets, les taux de CRP et des adipokines se sont améliorés, mais restent cependant alarmants. La baisse de l'inflammation systémique est expliquée par l'amélioration de l'endurance cardiorespiratoire indépendamment du sexe, de la perte de poids et des paramètres du sommeil.

Nos résultats confirment que le SAOS et l'obésité ont bien un impact sur la santé cardio-métabolique. L'exercice représente une alternative thérapeutique majeure capable de réduire l'inflammation systémique déjà très élevée chez des jeunes obèses avec des troubles du sommeil associés.

Conflits d'intérêts : Aucun conflit à déclarer 
Évaluation de la santé sexuelle des patientes obèses par les médecins généralistes nutritionnistes M. Lavenat ${ }^{1,}{ }^{*}$, A.-M. Guedj ${ }^{2}$, D. Costa ${ }^{3}$, P. Mares ${ }^{4}$, V. Taillard ${ }^{2}$

${ }^{1} \mathrm{CHU}$ Nîmes, Nîmes, France, ${ }^{2}$ Endocrinologie, CHU Nîmes, Nîmes, ${ }^{3}$ Médecine générale, Faculté de médecine Montpellier-Nimes, Montpellier, ${ }^{4}$ Gynécologie-obstétrique, CHU Nîmes, Nîmes, France

Introduction et but de l'étude : L'obésité est devenue un réel enjeu de santé publique, de par sa prévalence croissante et ses comorbidités associées. Tout comme la dimension nutritionnelle, la santé sexuelle est un élément essentiel de la qualité de vie. La sexualité peut jouer un rôle d'initiateur ou de facteur d'entretien de l'obésité et/ou en être une des complications. L'objectif de notre étude est d'évaluer l'intérêt porté par les médecins généralistes nutritionnistes sur la sexualité de leurs patientes obèses lors de consultations dédiées à la prise en charge nutritionnelle.

Matériel et méthodes : Etude descriptive observationnelle par questionnaire anonyme adressé à 133 médecins généralistes de France métropolitaine ayant une compétence en nutrition du 8 janvier au 1 mars 2018. Le critère de jugement principal était le nombre de médecin abordant la sexualité avec leurs patientes. Les critères de jugements secondaires étaient l'abord de la contraception et de la programmation de la grossesse ainsi que les raisons et les freins à aborder la sexualité avec leurs patientes obèses.

Résultats et Analyse statistique : 80 médecins (60,2\%) ont répondu au questionnaire. 35 médecins (44\% (IC95\% [32,6;55,3])) abordaient le sujet de la sexualité avec leurs patientes obèses lors de consultations dédiées à la prise en charge de l’obésité alors que 67 médecins (84\% (IC 95\% [73,81; 91,05])) parlaient de la contraception et que 63 médecins (79\% (IC 95\% [68,17; 87,1])) parlaient des risques encourus lors de la grossesse et l'intérêt de sa programmation.

Les principales raisons qui poussaient les médecins à aborder la sexualité étaient que la sexualité était importante dans la prise en charge globale de la patiente (57\%), qu'ils l'incluaient dans l'anamnése (43\%), dans la prise en charge de la contraception (31\%) ou à la recherche de complications (57\%) et que la sexualité faisait partie de leur interrogatoire systématique (29\%) ou des attentes de la patiente (40\%).

Les principaux freins à aborder la sexualité étaient que les patientes n'étaient pas en attente (78\%), que la sexualité n'était pas le problème prioritaire de la prise en charge (60\%), que les médecins n'avaient pas les compètences nécessaires (49\%) ou par manque de temps (44\%) et que la sexualité ne s'incluait pas dans la prise en charge de l'obésité (38\%).

Conclusion : La sexualité reste un sujet encore insuffisamment abordé au cours de consultations de prise en charge de l'obésité alors que celle-ci peut être altérée par de nombreux facteurs intriqués et doit par conséquent, si nécessaire, s’intégrer dans la prise en charge globale.

Conflits d'intérêts : Aucun conflit à déclarer 
Coût matériel d'une ration journalière d'un obèse : proposition d'une ration équilibrée à moindre coût R. Serairi Beji ${ }^{1,}{ }^{*}$, J. Boumaiza ${ }^{2}$, S. Ennaifer ${ }^{3}$, K. Ayed $^{4}$, R. Ksouri ${ }^{5}$, S. Jameleddine ${ }^{4}$

${ }^{1}$ Département de Nutrition, École supérieure des sciences et techniques de la santé - Laboratoire des plantes aromatiques et médicinales, Technopole Borj Cedia, ${ }^{2}$ Département de Nutrition, Université Mahmoud El Matri, ${ }^{3}$ Département de Nutrition, Ecole Supérieure des Sciences et Techniques de la Santé de Tunis, ${ }^{4}$ Exploration Fonctionnelle Respiratoire, Hôpital Abderrahmene Mami, ${ }^{5}$ Laboratoie des Plantes Aromatiques et Médicinales, Technopole Borj Cedria, Tunis, Tunisie

Introduction et but de l'étude : En Tunisie, $57 \%$ de la population est en surpoids ou obèse, d'après l'institut National de la Consommation. Le phénomène est dû à une alimentation très industrielle, mais aussi au manque d'activité physique et à la consommation excessive de sucres rapides, de pain et de pâtes alimentaires. Se nourrir convenablement n'est forcément pas synonyme de coût élevé. D'où l'objectif de ce travail qui est d'accompagner des sujets obèses dans la prise en charge de leur excès pondéral en leur proposant un régime adapté à leur physiopathologie et à moindre coût que celui auquel il était soumis.

Matériel et méthodes : Une centaine d'adultes volontaires, obèses (IMC > 30kg /m2 ), âgés de 20 à 60 ans ont été recrutés sur une période de quatre mois allant de décembre 2017 à mars 2018. Une approche anthropométrique et une histoire alimentaire sur 7 jours leur ont été réalisées. Après détermination de la composition des plats consommés en utilisant le livre des recettes Tunisiennes « LA SOFRA », nous avons calculé le prix de revient de chacun de leur plat en utilisant la "Calculette gratuite du prix de revient d'un plat » offerte par LGPST solutions.

Résultats et Analyse statistique : Les principaux résultats montrent que les apports spontanés de la population étudiée sont excédentaires par rapport aux besoins (respectivement $3026.87 \pm 515.3$ et $1800-2000 \mathrm{kcal} / \mathrm{j}$ ). Ce déséquilibre concerne également et surtout les différents types de lipides avec $14.92 \pm 2.11 \%$ pour les acides gras saturés, $12.57 \pm 3.2 \%$ pour les acides gras monoinsaturés et $17.21 \pm 3.3 \%$ pour les acides gras polyinsaturés, en pourcentage des apports caloriques globaux. II en est de même pour le cholestérol dont les apports sont de $353.8 \pm 219.1$ alors que les besoins ne dépassent pas 300mg. Sur le plan comportement alimentaire, beaucoup de déviations ont été notées à savoir les fringales pour les boissons sucrées, le night eating syndrome, la consommation d'alcool surtout sous forme de bière et les tentatives, non sans échec, de régimes hyperprotéinés. Le coût moyen du repas des sujets enquêtés était de 10,307 \pm 2.400 dinars tunisiens. Nous avons fourni à chacun des participants une prise en charge nutritionnelle adaptée à son état. Nous avons insisté sur la correction du comportement alimentaire et nous leur avons fourni un semainier, à moindre coût que le leur, pour qu'ils puissent l'adopter dans leur démarche de perte de poids.

Conclusion : Dans la prise en charge de ce groupe de sujets obèses, nous leur avons expliqué que se nourrir convenablement suivant sa physiopathologie est possible à un coût intéressant.

Conflits d'intérêts : Aucun conflit à déclarer 
Une nouvelle méthode pour étudier les déterminants gustatifs chez la souris : étude de l'impact d'une obésité nutritionnelle sur la perception du sucré.

A. Dastugue ${ }^{1, *}$, J.-F. Merlin ${ }^{1}$, G. Maquart ${ }^{1}$, A. Bernard ${ }^{1}$, P. Besnard ${ }^{1}$

${ }^{1}$ NUTox, UMR 1231 INSERM/AgroSup Dijon/UBFC, Dijon, France

Introduction et but de l'étude : Des études récentes suggèrent l'existence d'un lien entre obésité, chute de la sensibilité gustative et consommation préférentielle d'aliments dense en énergie chez le rongeur et chez l'Homme. Cependant les mécanismes à l'origine de cette association potentiellement délétère restent mal connus notamment en raison de la complexité des changements de perception gustative. En effet, l'influence du goût sur le choix alimentaire est étroitement liée aux composantes psychologiques du processus de récompense qui sont selon Berridge (Physiol Behav. 2009 97:537-50) la réponse hédonique déclenchée par l'aliment («liking»), la motivation ou non de le consommer («wanting») et la mémorisation du plaisir ou du déplaisir ressenti («learning»). Nous décrivons ici un nouveau concept de gustomètre, appelé FRM8 (brevet $n^{\circ}$ FR3 062 783), produisant des informations substantielles sur ces relations goût-récompense. Puisqu'une obésité nutritionnelle altère la perception gustative du sucré chez le rat (Shin et al, 2011, Am J Physiol 2011, 301 : R1267-80), nous avons choisi comme preuve de concept d'analyser la perception gustative du sucré au moyen du FRM8 chez des souris rendues obèses par un régime riche en graisses saturées.

Matériel et méthodes : Le FRM8 a une structure octogonale, les 8 faces de l'appareil équipées d'un biberon avec un système de détection (lickomètre) permettant de mesurer en temps réel le nombre de lapées données sur la tétine. Devant chaque biberon est placée une trappe guillotine permettant de réguler son accès. Un programme informatique enregistre en continu le nombre de lapées/biberon et contrôle le fonctionnement des trappes (ordre et durée d'ouverture). Après deux sessions d'entrainement avec 8 biberons d'eau pour habituer les souris au contexte expérimental, les animaux (témoins minces et obèses) ont été confrontés à une gamme de 7 concentrations de saccharose, le $8^{\text {ème }}$ biberon contenant de l'eau (solution témoin). Le design original du FRM8 exigeant le déplacement de la souris vers le biberon accessible au temps $t$, ce système permet une exploration simultanée du comportement de lapée («liking») et de la motivation à consommer (« wanting»).

Résultats et Analyse statistique : Comme escompté, l'obésité nutritionnelle s'accompagne d'un changement significatif de la perception du sucré qui se traduit par $\left.1^{\circ}\right)$ une chute du nombre total de lapées/30min $(358,15$ vs 816,93 chez les controles, $\mathrm{P}<0,001) 2^{\circ}$ ) un seuil de réponse au saccharose augmenté indiquant une chute de la sensibilité gustative au sucré $(0,32$ vs $0,19 \mathrm{M}$ chez les controles, $\left.\mathrm{P}<0,05), 3^{\circ}\right)$ une diminution de la motivation à boire $(2,54$ vs 3,93 blocks* chez les controles, $\mathrm{P}<0,001)$. Ces données ont été systématiquement reproduites au cours de 3 études supplémentaires indépendantes.

$\left(^{*}\right)$ Block = Quand une souris a lapé les 8 biberons, elle a réalisé un " block ».

Conclusion : Ces résultats, originaux pour la souris, démontrent l'intérêt de notre dispositif pour l'étude du lien entre gustation et comportement alimentaire. Une meilleure compréhension des mécanismes moléculaires responsables des défauts sensoriels identifiés pourrait ouvrir de nouvelles perspectives pharmacologiques et/ou nutritionnelles permettant de corriger des comportements alimentaires à risques.

Conflits d'intérêts : Aucun conflit à déclarer 
Explorations fonctionnelles digestives en post chirurgie bariatrique.

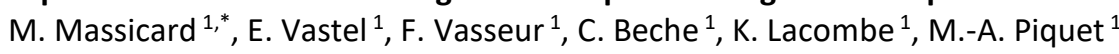

${ }^{1}$ Hépato-gastro-entérologie et nutrition, CHU DE CAEN, Cqen, France

Introduction et but de l'étude : La place des explorations fonctionnelles digestives (EFD) dans le bilan de troubles digestifs en post chirurgie bariatrique n'est pas établie. L'objectif de cette étude est de déterminer l'intérêt des EFD dans la prise en charge des patients en post chirurgie bariatrique.

Matériel et méthodes : Etude exploratoire observationnelle, rétrospective sur des patients opérés d'une chirurgie bariatrique et ayant été explorés pour des troubles fonctionnels digestifs au CHU de 2007 à 2017. Les données recueillies sur dossiers étaient : type et nombre de chirurgie, IMC avant et après la chirurgie, présence de trouble digestif, résultats de pH-métrie (Ohmega MMS), manométrie œsophagienne (manométrie à diffusion Solar GI MMS), breath test au glucose (GASTRO+TM Gastrolyser) ainsi que la conduite tenue après ces tests (introduction ou changement d'une thérapeutique) et leur éventuel bénéfice clinique. Résultats exprimés en moyenne \pm écart-type, comparaisons par test de chi- 2 .

Résultats et Analyse statistique : Sur 1832 patients opérés au CHU en chirurgie bariatrique sur la période, 40 patients (2\%) ont

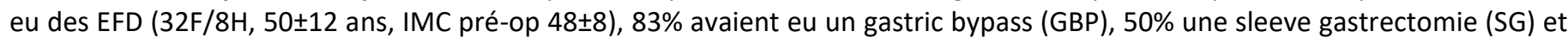
$10 \%$ un anneau gastrique (AGA), $40 \%$ des patients avaient eu plusieurs chirurgies bariatriques. Avant chirurgie, $20 \%$ présentaient un trouble fonctionnel digestif dont la quasi-totalité était représentée par un pyrosis. Après la 1ère intervention les symptômes étaient : pyrosis (49\%), épigastralgies (36\%), douleurs abdominales (31\%) et diarrhées (23\%). Les symptômes digestifs hauts (pyrosis, épigastralgies, vomissements, dysphagies et régurgitations) étaient plus fréquents après SG et/ou AGA alors que les symptômes digestifs bas (douleurs abdominales, diarrhées, ballonnements, dumping) étaient plus fréquents après $G B P(p<0,001)$. Après conversion d'un AGA ou SG en BP, les symptômes étaient : dumping (37,5\%), épigastralgies (25\%), diarrhées (19\%) et pyrosis (19\%). Seize pH-métries ont été réalisées pour pyrosis et/ou épigastralgies et mettaient en évidence un RGO chez $75 \%$ des patients (dont $75 \%$ déjà traités par IPP et $25 \%$ ont eu un IPP prescrit après le test). Chez les patients qui avaient un IPP préalable, $22 \%$ ont poursuivi leur traitement et $78 \%$ ont été converti en GBP (amélioration clinique pour 60\%). Dix manométries, réalisées pour régurgitations et/ou dysphagies, étaient normales sauf une (achalasie traitée par dilatation). Treize breath tests ont été réalisés pour l'exploration de douleurs abdominales, diarrhées et ballonnements, $62 \%$ retrouvaient une pullulation bactérienne. Tous ces patients en reçus des antibiotiques (25\% améliorés, contre $75 \%$ non améliorés cliniquement). Chez les patients non améliorés, 2 patients ont subi un démontage chirurgical pour une dénutrition et une malabsorption (amélioration clinique pour les 2).

Conclusion : Les EFD sont peu utilisées après chirurgie bariatrique. La pH-métrie semble être une aide à la décision de conversion en GBP chez les patients présentant un RGO post SG ou AGA. La fréquente normalité de la manométrie suggère une mauvaise discrimination de la dysphagie à l'interrogatoire des patients opérés. Les breath tests mettent en évidence des pullulations bactériennes mais celles-ci ne sont souvent pas améliorées par l'antibiothérapie, et leur utilité reste à établir.

Conflits d'intérêts : Aucun conflit à déclarer 
Le Yucatan Minipig comme futur modèle d'études précliniques en dénutrition et obésité sarcopénique L. Lacaze ${ }^{1,}{ }^{*}$, K. Boudjema $^{1}$, J. Georges ${ }^{2}$, J. Delamarre ${ }^{2}$, F. Le-Gouevec ${ }^{2}$, A. Chauvin ${ }^{2}$, D. Val-Laillet ${ }^{3}$, R. Thibault ${ }^{4}$

${ }^{1}$ chirurgie hépatobiliaire et digestive, CHU Pontchaillou, rennes, ${ }^{2}$ INRA, UEPR, ${ }^{3}$ Univ Rennes, Nutrition Métabolismes et Cancer, NuMeCan, INRA, INSERM, SaintGilles, ${ }^{4}$ Unité de Nutrition, CHU Pontchaillou, rennes, France

Introduction et but de l'étude : La physiopathologie de la dénutrition chez les patients obèses est mal connue, justifiant le développement de modèles animaux de dénutrition et d'obésité sarcopénique. Les miniporcs présentent des profils métaboliques et nutritionnels proches de ceux de l'homme. Il s'agit d'une étude de faisabilité sur un modèle miniporc d'obésité sarcopénique. Matériel et méthodes : Deux types de régime ont été testés : hypoprotidique et protéiprive, en comparaison à un régime standard. Le régime pauvre en protéines (7,8 MJ / kg d'énergie nette) était isocalorique par rapport au régime standard (7,3 MJ / kg d'énergie nette), alors que le régime protéiprive était plus énergétique (13,7 MJ / kg d'énergie nette). Les régimes contenaient $10,9 \%$ (standard) , 6,8\% (hypoprotidique), et $0 \%$ (protéiprive) de protéines digestibles.

Résultats et Analyse statistique : Sept miniporcs obèses du Yucatan ( 3 mâles et 4 femelles) ont été utilisés. Dans la première partie de l'étude, nous avons montré chez trois animaux que le régime protéiprive était le seul régime associé à une perte de poids à un mois. Les pertes pondérales étaient respectivement pour chaque animal de $14,0 \%, 14,4 \%$ et $11,0 \%$. Dans la deuxième partie de l'étude, nous avons confirmé la perte de poids à un mois avec le régime protéiprive sur trois autres miniporcs obèses du Yucatan. La perte de poids était de $9,0 \%, 11,9 \%$ et $12,0 \%$. Avec le régime protéiprive, une perte de poids moyenne de $12,3 \% \pm 2,2$ ont été observés chez les 6 animaux. Un animal a refusé de s'alimenter avec le régime proteiprive et a été euthanasié avant la fin de l'expérience.

Conclusion : Cette étude préliminaire a révélé qu'un régime alimentaire protéiprive pouvait induire une perte de poids corporel moyenne de $12 \%$ chez les miniporcs obèses, contrairement au régime hypoprotidique qui n'entrainait pas de perte pondérale. Ces données ont confirmé la faisabilité d'un nouveau modèle de dénutrition chez les miniporcs. Une étude approfondie est prévue avec des analyses de la composition corporelle par scanner pour mieux phénotyper la dénutrition

Conflits d'intérêts : Aucun conflit à déclarer 
${ }^{1}$ UTDN, Centre Hospitaliser de Blois, Blois, France

Introduction et but de l'étude : La chirurgie bariatrique est le traitement le plus efficace de l'obésité sévère et modérée associée à des comorbidités. L'objectif de notre étude est d'évaluer l'efficacité de la chirurgie bariatrique au centre hospitalier de Blois, depuis la création de la filière datant de 2015.

Matériel et méthodes : Il s'agit d'une étude rétrospective, observationnelle, monocentrique. Tous les patients ayant bénéficié d'une chirurgie bariatrique au centre hospitalier de Blois de novembre 2015 à Décembre 2017 étaient inclus. Les patients opérés répondaient aux critères de l'HAS et étaient suivis exclusivement en ambulatoire sous forme de consultations. Les données anthropométriques (pourcentage de perte d'excès de poids (PEP), IMC et pourcentage de perte de poids) étaient recueillies à 3 , 6,12 et 24 mois ainsi que les comorbidités à 12 mois.

Résultats et Analyse statistique : 75 patients (63 femmes et 12 hommes) étaient opérés dans notre cohorte: 67 patients d'une sleeve gastrectomie (89\%), 8 d'un bypass gastrique (11\%). La durée moyenne de préparation à la chirurgie était de 11,7 mois Le suivi moyen post-opératoire était de 12,4 mois. L'âge moyen était de 44,29 ans (+/-10,40). Avant la chirurgie, l'IMC moyen était de 43,62 (+/- 5,65) et l'excès de poids moyen de 49,42 kg (+/-13,19). A 12 mois, le PEP moyen était de 72,7 \%(+/-27,2), I'IMC de $30,54(+/-5,49)$, et le pourcentage de perte de poids 43,5\% (+/-19,08). A 24 mois, le PEP moyen était de 69,3\%(+/-22,9), I'IMC de $29,95(+/-3,87)$, et le pourcentage de perte de poids $33 \% \%(+/-22,04)$. La perte de poids selon les différents paramètres anthropométriques était significative $(p<0,001)$. A 12 mois de la chirurgie, $16 \%$ patients présentaient une HTA contre $44 \%$ en préopératoire, $2,6 \%$ un diabète contre $21,3 \%$ en préopératoire, $1,78 \%$ une stéatose hépatique contre $60 \%$ en préopératoire, $3,6 \%$ une dyslipidémie contre $37,3 \%$ en préopératoire $(p<0,001), 21,4 \%$ un SAS contre $68 \%$ en préopératoire $(p=0,16)$. 4 patients ne sont pas présentés aux rendez vous de consultations (entre 3 et 12 mois).

Conclusion : Notre travail montre d'excellents résultats en terme de perte de poids et de réduction des comorbidités. Ce travail valide la qualité de l'organisation de la filière bariatrique au $\mathrm{CH}$ de Blois. La prise en charge ambulatoire pluridisciplinaire permet un suivi personnalisé. Ceci pourrait permettre de prévenir les risques de reprise pondérale et de perdus de vue sur le long terme.

Conflits d'intérêts : Aucun conflit à déclarer 
P170

Exposition périnatale à un régime de quantité et de qualité variables en protéines chez le rat- croissance, préférences alimentaires et risque de surpoids chez la descendance femelle adulte

G. Carlin ${ }^{1,}{ }^{*}$, C. Chaumontet ${ }^{1}$, C. Delteil ${ }^{1}$, N. Darcel ${ }^{1}$, B. Van de Heijning ${ }^{2}$, A. Kodde ${ }^{2}$, D. Tome ${ }^{1}$, A.-M. Davila ${ }^{1}$

${ }^{1}$ UMR PNCA, AgroParisTech INRA Université Paris-Saclay, PARIS, France, ${ }^{2}$ Danone Nutricia Research, Utrecht, Pays-bas

Introduction et but de l'étude : La manipulation du niveau de protéines dans le régime maternel impacte la santé de la descendance. La restriction en protéines au cours de la gestation augmente le risque de surpoids chez la descendance adulte. A ce jour, l'impact de l'excès de protéines et de leurs sources durant la période périnatale est moins investigué. Ces travaux étudient chez le rat les effets d'un régime protéique maternel variant en quantité et en qualité durant la gestation et l'allaitement sur: la croissance, les préférences alimentaires et le risque de surpoids chez la descendance femelle adulte dans un modèle de choix alimentaires multiples (Dietary Self-Selection, DSS).

Matériel et méthodes : 6 groupes de mères ont été soumis pendant la gestation à un des deux types de régimes isocaloriques : hyperprotéique (HP, 47\% de protéines) ou normoprotéique (NP, témoin, 19\% de protéines). Les sources protéiques composant les régimes étaient des protéines de lait total (LT), de pois (P) ou de dinde (D). Durant l'allaitement, les mères ont reçu le régime NP de source identique à celle de gestation. Au sevrage et jusqu'au jour post-natal (JPN) 70, les ratons issus des 6 groupes de mères ont été exposés au DSS et un groupe témoin de ratons (sans DSS), issus des groupes de mères LT, a été soumis au régime complet NPLT (8 groupes de ratons où $n=8$ /groupe). Le DSS était composé de 5 gamelles distinctes: HPLT, HPP, HPD, glucides ou lipides. Les profils des régimes en acides aminés et en acides gras variaient dû à la composition des différentes sources protéiques. Le poids, la prise alimentaire ont été relevés quotidiennement; la composition corporelle et la leptinémie à jeun ont été déterminées à la fin de l'étude.

Résultats et Analyse statistique : Le régime de gestation n'a pas eu d'influence sur le poids de naissance. Pendant l'allaitement, le gain de poids des ratons était plus faible dans les groupes $\mathbf{P}$ et $\mathbf{D}$ comparativement aux groupes $\mathbf{L T}(P<0,0001)$ ainsi que pendant la période post-sevrage dans les groupes $D$ uniquement $(P<0,0001)$. Le DSS a entrainé une augmentation de la prise énergétique totale (PET) par rapport aux groupes témoins recevant le NPLT $(P<0,0001)$. Les groupes DSS ont consommé plus de protéines $(7-$ $11 \%$ PET) et de lipides (8-11\% PET) et moins de glucides (17-22 \% PET) par rapport aux ratons nourris avec le NPLT $(P<0,0001)$. La source protéique maternelle n'a pas induit de préférences pour celle-ci après le sevrage dans les groupes DSS. Dans tous les groupes HP gestation, les tissus adipeux total et sous-cutané ont augmenté (respectivement, $P=0,06 \& P<0,01$ ). Cette augmentation d'adiposité était corrélée positivement à la leptinémie à jeun $(P<0,05)$.

Conclusion : La qualité et la quantité de protéines maternelles consommées impactent la croissance de la descendance. Après le sevrage, les ratons sous DSS ont spontanément augmenté leurs apports en protéines et en lipides indépendamment du régime maternel. En revanche, quelle que soit la source maternelle de protéines, le régime HP gestationnel a programmé l'adiposité et augmenté le risque de surpoids chez la descendance adulte.

Remerciements : Merci à tous les membres de l'UMR PNCA et les collaborateurs DNR pour leur investissement.

Conflits d'intérêts : G. Carlin: Aucun conflit à déclarer, C. Chaumontet: Aucun conflit à déclarer, C. Delteil: Aucun conflit à déclarer, N. Darcel: Aucun conflit à déclarer, B. Van de Heijning est employé(e) de Danone Nutricia Research, A. Kodde est employé(e) de Danone Nutricia Research, D. Tome: Aucun conflit à déclarer, A.-M. Davila: Aucun conflit à déclarer 
Nouvelles courbes de croissance $\&$ diagnostic de l'état nutritionnel chez l'enfant, quels impacts ?

V. Harivel ${ }^{1,}{ }^{*}$, D. Grey ${ }^{1}$, R. Gandy ${ }^{1}$ et Unité Transversale de Nutrition (UTN)

${ }^{1}$ Unité Transversale Nutrition, CHU Montpellier, Montpellier, France

Introduction et but de l'étude : Depuis avril 2018, de nouvelles courbes de croissance (NCC) de référence sont diffusées à l'ensemble des professionnels de santé. Notre objectif est d'étudier l'impact de ces NCC sur le diagnostic de l'état nutritionnel des enfants par rapport aux anciennes courbes de croissances (ACC).

Matériel et méthodes : Etude réalisée sur des données recueillies de mars 2017 à mai 2018, au CHU de Montpellier. Les critères $d^{\prime}$ inclusion sont : enfant âgé de 1 mois à 18 ans hospitalisé depuis plus de $24 \mathrm{~h}$ dans les services de pédiatrie. Les critères d'exclusion sont : enfant non mesurable, refusant d'être mesuré ou pesé, ou présentant un diagnostic de déshydratation.

Pour chaque enfant a été répertorié puis comparé la taille moyenne attendue pour l'âge (T/A), le poids moyen attendu pour la taille $(\mathrm{P} / \mathrm{T})$, le poids moyen attendu pour l'âge $(\mathrm{P} / \mathrm{A})$ et l'IMC (enfant +2 ans), avec les ACC puis les NCC. Le diagnostic de dénutrition modérée et sévère a été établi via l'indice de Waterloo, conformément aux recommandations françaises du PNNS et de la SFNEP. Résultats et Analyse statistique : $\mathbf{3 7 1}$ enfants ont été inclus. Nous avons comparé le diagnostic nutritionnel posé pour chaque enfant avec les ACC vs les NCC. Principaux résultats : $+5.4 \%(n=20)$ enfants dénutris, $+16.3 \%$ ( $n=29)$ enfants en dénutrition chronique, $+3.9 \%(n=9)$ enfants en dénutrition sévère.

Les NCC semblent rapporter plus de cas de dénutrition sur cet échantillon (+ 20 enfants), avec une augmentation notable de la dénutrition chronique. L'IMC ne semble pas être impacté tout comme les diagnostics de surpoids et d'obésité.

\begin{tabular}{|l|c|c|c|c|}
\hline & \multicolumn{2}{|c|}{ Anciennes courbes $\mathbf{n = 3 7 1}$} & \multicolumn{2}{c|}{ Nouvelles courbes $\mathbf{n = 3 7 1}$} \\
\hline Dénutrition (total) & $\mathrm{n}=115$ & $\mathbf{3 1}$ & $\mathrm{n}=135$ & $\mathbf{3 6 . 4}$ \\
\hline Dénutrition Modérée & $\mathrm{n}=93$ & 80.9 & $\mathrm{n}=104$ & 77 \\
\hline Dénutrition Sévère & $\mathrm{n}=\mathbf{2 2}$ & 19.1 & $\mathrm{n}=31$ & $\mathbf{2 3}$ \\
\hline Dénutrition Aigüe & $\mathrm{n}=75$ & $\mathbf{6 5 . 2}$ & $\mathrm{n}=66$ & 48.9 \\
\hline Dénutrition Chronique & $\mathrm{n}=40$ & 34.8 & $\mathrm{n}=69$ & $\mathbf{5 1 . 1}$ \\
\hline
\end{tabular}

Concernant les résultats en fonction de la spécialité médicale : il semble y avoir une augmentation du nombre d'enfant dénutris en chirurgie viscérale/urologie (+10\% soit 4 enfants), médecine générale/infectieuse (+8\% soit 5 enfants), et la neuro-pédiatrie (+8.7\% soit 4 enfants). Les autres spécialités ont peu ou pas de changement du nombre d'enfants diagnostiqués dénutris. Cependant, il nous semble nécessaire d'avoir une cohorte plus importante afin de vérifier si les spécificités de diagnostic sont bien en lien avec la spécialité médicale.

Conclusion : Les NCC semblent engendrer un dépistage plus précoce de la dénutrition des enfants, leur utilisation permettrait donc une optimisation de la prise en charge nutritionnelle.

Conflits d'intérêts : Aucun conflit à déclarer 
Changement de sources protéiques des formules infantiles : degré d'hydrolyse et bioaccessibilité des acides aminés L. Le Roux ${ }^{1,}{ }^{*}$, F. Nau ${ }^{2}$, P. Schuck ${ }^{2}$, R. Chacon ${ }^{3}$, R. Jeantet ${ }^{2}$, A. Deglaire ${ }^{2}$, D. Dupont ${ }^{2}$

${ }^{1}$ R\&D, Sill Dairy International, ${ }^{2}$ INRA, Agrocampus Ouest, UMR1253 Sciences et Technologie du Lait et de l'CEuf, Rennes, ${ }^{3} R \& D$, Sill, Plouvien, France

Introduction et but de l'étude : Les protéines généralement utilisées dans les formules infantiles proviennent du lait de vache et sont majoritairement des protéines de lactosérum afin de se rapprocher de la composition du lait maternel. Cependant, certaines protéines végétales offrent également de bonnes propriétés nutritionnelles et pourraient constituer d'intéressants substituts dans un contexte de compétition d'usage des protéines de lactosérum. Le but de cette étude était de comprendre l'impact de la substitution des protéines de lactosérum par des protéines végétales sur le degré d'hydrolyse et sur la bioaccessibilité des acides aminés.

Matériel et méthodes : Quatre protéines végétales ont été sélectionnées (PV1 à PV4) en raison de leur composition en acides aminés essentiels adaptée aux besoins du nourrisson. Elles ont été utilisées pour développer des formules infantiles de composition identique à la référence $(R)$, à ceci près que les protéines de lactosérum étaient remplacées par une des quatre protéines végétales. Les cinq formules infantiles ont ensuite été soumises à un modèle de digestion in vitro gastro-intestinale en conditions statiques (Ménard et al. 2018). Le degré d'hydrolyse des protéines était mesuré par la méthode OPA (Ophtaldialdehyde). Les acides aminés libres ont été quantifiés par chromatographie.

Résultats et Analyse statistique : Tableau 1. Teneur en acides aminés libres et degré d'hydrolyse en point final de digestion de cinq formules infantiles

\begin{tabular}{|l|c|c|c|c|c|c|c|}
\hline $\begin{array}{l}\text { Formules } \\
\text { infantiles }\end{array}$ & $\begin{array}{c}\text { Thréonine } \\
\mathbf{( \% )}\end{array}$ & $\begin{array}{c}\text { Isoleucine } \\
\mathbf{( \% )}\end{array}$ & $\begin{array}{c}\text { Leucine } \\
\mathbf{( \% )}\end{array}$ & $\begin{array}{c}\text { Lysine } \\
\mathbf{( \% )}\end{array}$ & $\begin{array}{c}\text { AAS } \\
\mathbf{( \% )}\end{array}$ & $\begin{array}{c}\text { AAA } \\
(\%)\end{array}$ & $\begin{array}{c}\text { Degré d'hydrolyse } \\
\text { (\%) }\end{array}$ \\
\hline R & $12,6 \pm 0,7$ & $19,1 \pm 0,6$ & $47,9 \pm 2,9$ & $71,1 \pm 3,2$ & $23,6 \pm 2,6$ & $79,6 \pm 3,7$ & $53,0 \pm 6,1$ \\
\hline PV1 & $5,8 \pm 0,1^{* * *}$ & $1,9 \pm 0,2^{* * *}$ & $12,1 \pm 0,7^{* * *}$ & $16,9 \pm 0,9 * * *$ & $9,1 \pm 1,2 * * *$ & $21,1 \pm 0,9 * * *$ & $29,0 \pm 4,1^{* * *}$ \\
\hline PV2 & $15,7 \pm 1,2^{*}$ & $20,9 \pm 0,7$ & $51,4 \pm 1,6$ & $72,6 \pm 1,1$ & $22,0 \pm 2,3$ & $74,8 \pm 5,2$ & $65,6 \pm 1,2$ \\
\hline PV3 & $14,9 \pm 4,0$ & $17,2 \pm 1,1$ & $49,7 \pm 4,9$ & $73,1 \pm 4,4$ & $24,1 \pm 4,8$ & $75,5 \pm 5,3$ & $45,4 \pm 4,4$ \\
\hline PV4 & $11,8 \pm 2,3$ & $16,4 \pm 2,8^{*}$ & $45,6 \pm 5,1^{*}$ & $68,7 \pm 6,6$ & $25,8 \pm 6,8$ & $71,1 \pm 8,2$ & $40,2 \pm 2,7 *$ \\
\hline
\end{tabular}

Les différences entre les valeurs moyennes des formules PV1 à 4 par rapport à $R$ sont considérées significatives lorsque $p<0,05$ ( $t$ Test : $\left.{ }^{*} 0,05 ;{ }^{* *} 0,01 ;{ }^{* *} 0,001\right)$. AAS (acides aminés soufrés); AAA (acides aminés aromatiques)

Les formules PV2 et PV3 libèrent autant d'acides aminés que la référence $(R)$ et présentent un degré d'hydrolyse équivalent $(p>0,05)$. En revanche, la formule PV4 se distingue de $R$ par une moindre libération d'isoleucine et de leucine et présente également un degré d'hydrolyse plus faible. Enfin, la formule PV1 conduit à des valeurs très inférieures à celles obtenues avec $R$ pour toutes les variables étudiées.

Conclusion : Il est établi que la cinétique de digestion des protéines dans une matrice et par conséquence, la libération des acides aminés et leur absorption peuvent être influencées notamment par la composition en protéines (Le Feunteun et al., 2014). De manière concordante, nous montrons ici que la nature des protéines des formules infantiles aurait un effet sur la protéolyse. Ainsi, parmi les quatre formules "végétalisées " testées, seules PV2 et PV3 sont nutritionnellement comparables à la référence. Des digestions in vitro en conditions dynamiques (plus proches des conditions physiologiques) seront réalisées prochainement afin de compléter ces résultats quant à la qualité nutritionnelle de ces formules infantiles innovantes.

Conflits d'intérêts : Aucun conflit à déclarer 

S. Krinitzki ${ }^{1}$, S. Lioret $^{1}$, M. A. Charles ${ }^{12}$, B. De Lauzon-Guillain ${ }^{3, *}$

${ }^{1}$ CRESS Eq ORCHAD, INSERM, Villejuif, ${ }^{2}$ INED, Paris, ${ }^{3}$ CRESS Eq ORCHAD, INRA, Villejuif, France

Introduction et but de l'étude : Bien que l'influence des caractéristiques maternelles sur le poids de naissance (PN) soit bien connue, il existe, à ce jour, très peu d'études sur le potentiel rôle des caractéristiques paternelles (alimentaires, sociodémographiques). L'objectif est d'étudier dans quelle mesure l'alimentation du père et sa consommation d'alcool avant la conception sont associées au poids de naissance de l'enfant.

Matériel et méthodes : Un questionnaire de fréquences alimentaires, portant sur les mois précédant le début de la grossesse, a été rempli par 983 pères au cours de la dernière vague d'inclusion de la cohorte ELFE (Etude Longitudinale Française depuis l'Enfance). Une analyse en composantes principales a été réalisée afin de caractériser les profils alimentaires des pères. Après avoir étudié les associations entre les caractéristiques sociodémographiques des pères et leur alimentation, nous avons analysé la relation entre les profils alimentaires paternels ainsi que leur consommation d'alcool et le poids de naissance de l'enfant (zscore tenant compte de l'âge gestationnel, en continu ou en 3 catégories), respectivement par le biais de régressions linéaires et logistiques multinomiales, ajustées sur 1) les facteurs sociodémographiques, anthropométriques et le tabagisme maternel 2) sur l'alimentation de la mère.

Résultats et Analyse statistique : Quatre profils alimentaires paternels ont pu être identifiés : « Energie » (associé positivement à la fréquence de consommation de tous les groupes d'aliments), "Equilibré » (associé positivement aux fruits, légumes et huile d'olive et négativement aux sodas et frites), "Alcool » ( associé positivement à l'alcool et négativement aux produits laitiers) et "Grignotage » (associé positivement aux gâteaux, chips et chocolat et négativement à la consommation de viande). Les profils alimentaires « Equilibré » $(p=0,03)$ et "Grignotage » $(p=0,001)$ étaient associés positivement au poids de naissance ( $z$-score en continu), alors que la consommation d'alcool n'y était pas associée linéairement. L'association avec le profil « Equilibré » était toutefois atténuée quand l'alimentation maternelle était prise en compte dans le modèle. Par ailleurs, le profil « Grignotage » était associé négativement avec le risque de petit poids de naissance (OR $z$-score <25ème percentile vs 25 -75ème percentile $=0,79[0,66 ; 0,94]$ ) alors que le profil « Alcool » était associé positivement avec le risque de gros poids de naissance (OR $z$-score $>25$ ème percentile vs 25 -75ème percentile $=1,23[1,03 ; 1,47])$. Des résultats similaires ont été retrouvés en ajustant sur l'alimentation maternelle.

Conclusion : En accord avec les données d'expérimentation animale, l'alimentation du père avant la conception semble être associée au PN de son enfant en population générale humaine.

Conflits d'intérêts : Aucun conflit à déclarer 
Teneur en matières grasses et profil en acides gras des laits de suite commercialises en officine de pharmacie à Abidjan K.-A. Kouassi ${ }^{1}$, A. N'bra ${ }^{2}$, J. S. Yao ${ }^{2}$, S. Dibi ${ }^{3}$, M. Aké $^{12, *}$

${ }^{1}$ Département de Chimie Analytique, Bromatologie, Chimie Générale et Minérale, UFR Sciences Pharmaceutiques et Biologiques, Université Félix Houphouët-Boigny, ${ }^{2}$ Laboratoire de Nutrition, Institut National de Santé Publique, ${ }^{3}$ Laboratoire de ChimieToxicologie, Direction du Laboratoire Central de la Police, Abidjan, Côte d'Ivoire

Introduction et but de l'étude : De nos jours, les substituts du lait maternel représentent une alternative à l'allaitement pour les nourrissons. En vue de garantir la santé des nourrissons, il importe de s'assurer de leur qualité en particulier au niveau nutritionnel. La présente étude avait pour objectifs de : i) énumérer les caractéristiques des laits de suite vendus en officine de pharmacie à Abidjan ; ii) déterminer la teneur en matières grasses de ces laits; iii) établir le profil en acides gras; iv) comparer les résultats obtenus à ceux affichés sur les conditionnements.

Matériel et méthodes : L'étude s'est déroulée en deux étapes : une enquête en officines de pharmacie afin de relever les caractéristiques commerciales des laits de suite. L'étape analytique a été réalisée sur cinq (5) échantillons de toutes les marques des laits de suite, commercialisées en officine de pharmacie. La détermination de la teneur en matières grasses a été réalisée par gravimétrie après leur extraction à froid. Le profil en acide gras a été établi par chromatographie gazeuse couplée à la spectrométrie de masse.

Résultats et Analyse statistique : Il ressort de cette étude que neuf (9) marques de lait de suite étaient commercialisés et disponibles en officine de pharmacie. La dénomination de ces marques est suivie du chiffre " 2 ", indiquant la tranche d'âge concernée de 6 à 12 mois. Plusieurs autres mentions ont été systématiquement retrouvées sur les conditionnements telles que le type de lait, le mode de reconstitution du lait, les différentes informations nutritionnelles... Des teneurs en matières grasses variant entre 5,01 à 21,32 g/100 g soit 0,67 à 3,044 g/100 ml ont été objectivées. Ces valeurs étaient proches des teneurs indiquées sur les conditionnements des différentes marques analysées. Le profil en acides gras a permis de relever la présence de sept (7) acides gras dont un seul acide gras essentiel (acide linoléique) tandis que les conditionnements indiquaient la présence d'acide alphalinolénique. L'acide gras, en proportion la plus importante dans les laits analysés, est l'acide oléique.

Conclusion : L'étude réalisée a permis de relever les caractéristiques des laits de suite commercialisés en officine de pharmacie. L'étape analytique a objectivé des teneurs suffisantes en matières grasses et un profil en acides gras variés, dont la présence revêt un intérêt nutritionnel pour le nourrisson de 6 à 12 mois.

Conflits d'intérêts : Aucun conflit à déclarer 
P175

Introduction des aliments texturés dans l'alimentation des enfants de 4 à 36 mois : descriptions des pratiques en France et facteurs associés

C. Tournier ${ }^{1,}{ }^{*}$, L. Demonteil ${ }^{12}$, E. Ksiazek ${ }^{1}$, H. Weenen ${ }^{3}$, M. Dusoulier ${ }^{2}$, A. Marduel $^{2}$, S. Nicklaus ${ }^{1}$

${ }^{1}$ UMR CGSA, INRA, Dijon, ${ }^{2}$ Blédina SA, Limonest, France, ${ }^{3}$ Danone Nutricia Research, Utrecht, Pays-bas

Introduction et but de l'étude : Les expériences alimentaires de la petite enfance jouent un rôle déterminant dans la construction des préférences alimentaires futures. Il est actuellement suggéré que les enfants apprennent à manger, et à aimer les morceaux, s'ils sont exposés à des aliments de textures variées pendant la diversification alimentaire. Selon les nouvelles recommandations du Comité Nutrition de I'ESPGHAN (2017), l'alimentation sous forme de purée devrait faire place aux premiers morceaux fondants entre 8 à 10 mois au plus tard. En France, l'introduction des textures est actuellement peu abordée dans les recommandations nationales (PNNS) et les pratiques parentales sont peu connues. Cette étude décrit en détails quels types de textures sont proposés aux enfants de 4 à 36 mois en France et identifie les facteurs associés à ces pratiques.

Matériel et méthodes : Nous avons développé un questionnaire en ligne destiné aux parents d'enfant de 4 à 36 mois. Ce questionnaire comprend une liste de 188 couples aliment-texture pour lesquels les parents doivent mentionner s'ils les ont déjà proposés à leur enfant. Ces couples aliment-texture ont été classés en 3 niveaux de texture : purées lisses et granuleuses (T1), petits morceaux fondants (T2), et morceaux gros et/ou durs et doubles textures (T3). L'exposition d'un enfant à la texture des aliments a été objectivée par le calcul de scores d'exposition : global (188 couples aliment-texture) et par niveau de texture (T1, $\mathrm{T} 2$ et T3). L'association entre le score d'exposition global et les caractéristiques de la mère, de l'enfant et les pratiques d'alimentation a ensuite été explorée par régressions linéaires multivariées, par classe d'âge $(n=14)$.

Résultats et Analyse statistique : Les données de 2999 mères, vivant en France, majoritairement primipares et ayant atteint le niveau universitaire, ont été collectées. Les analyses montrent que la consommation de purées augmente significativement au cours de la première année ( $n=8.0 \pm 4.7$ à $4-5$ mois à $27.2 \pm 5.9$ à 12 mois) puis reste relativement constante jusqu'à 36 mois. Les petits morceaux mous sont très progressivement introduits entre 6 et 22 mois ( $n=0.7 \pm 3.2$ à 30.4 \pm 5.1 ) alors que les morceaux plus gros ou plus durs sont surtout introduits après $13-15$ mois (de $n=25.0 \pm 17.3$ à $74.9 \pm 18.4$ à $30-36$ mois). Différents facteurs expliquent ces pratiques d'introduction de textures. Les enfants diversifiés plus tôt, ayant plus de dents, et étant davantage capables de se nourrir seul avec leurs mains ou une fourchette sont généralement davantage exposés aux textures. Par ailleurs, pour quelques classes d'âge étudiées $(n=5)$, le score d'exposition est plus bas chez les enfants consommant uniquement des produits commerciaux pour bébés et plus haut chez les enfants exposés uniquement à une alimentation fait maison et/ou des aliments du commerce non destinés aux bébés.

Conclusion : Notre étude montre qu'avant 12 mois, la majorité des enfants sont essentiellement nourris avec des purées et donc peu exposés aux morceaux. Ces résultats ont permis de mettre en évidence les facteurs influençant le plus l'exposition aux textures, et suggèrent la nécessité de communiquer sur l'introduction de la texture auprès des parents en France.

Conflits d'intérêts : C. Tournier a reçu une subvention/soutien de Travaux réalisés dans le cadre de la thèse CIFRE de L Demonteil financée par Blédina et l'ANRT, L. Demonteil a reçu une subvention/soutien de Travaux réalisés dans le cadre de la thèse CIFRE de L Demonteil financée par Blédina et l'ANRT, E. Ksiazek: Aucun conflit à déclarer, H. Weenen est employé(e) de Danone Nutricia Research, M. Dusoulier est employé(e) de Blédina SA, A. Marduel est employé(e) de Blédina SA, S. Nicklaus a reçu une subvention/soutien de Travaux réalisés dans le cadre de la thèse CIFRE de L Demonteil financée par Blédina et l'ANRT 
Les nourrissons dont l'alimentation est exclusivement lactée sont-ils capables de compenser des modifications de la densité énergétique du lait lors du repas suivant ? (Prix SFN 2015)

C. Schwartz ${ }^{1, *}$, P. Brugaillères ${ }^{1}$, S. Issanchou ${ }^{1}$, S. Nicklaus ${ }^{1}$

${ }^{1}$ CSGA, INRA, Dijon, France

Introduction et but de l'étude : Un gain de poids trop rapide dans les premiers mois de vie est un facteur de risque de développement de surpoids et d'obésité (Stettler \& lotova, 2010). Comprendre les facteurs permettant le maintien de l'équilibre de la balance énergétique chez les nourrissons est donc crucial. Etre capable d'ajuster les quantités consommées en fonction de la densité énergétique (DE) des aliments est une façon d'équilibrer la balance énergétique, à dépense énergétique constante. Les travaux de Fomon (1975) et Timby (2014) attestent que les nourrissons dont l'alimentation est exclusivement lactée sont capables, après un apprentissage, d'apprendre à ajuster les quantités de lait en réponse à une modification de la DE. Dans quelle mesure sont-ils capables d'un tel ajustement d'un repas à l'autre ? Aucune étude expérimentale permettant de répondre à cette question n'a été publiée à ce jour.

Matériel et méthodes : Pour répondre à cette question, une mesure de réactivité à la DE a été développée sur la base du paradigme de compensation calorique classiquement utilisé chez l'enfant plus âgé ou l'adulte. Ce paradigme permet d'évaluer dans quelle mesure le sujet ajuste sa prise alimentaire lors d'un repas ad libitum, suite à l'ingestion d'un aliment de DE plus ou moins élevée. Ainsi, environ 15 jours avant le démarrage de la diversification alimentaire (date décidée par les parents), les parents et leur nourrisson sont venus deux fois au laboratoire. Chaque jour de suivi, les nourrissons ont reçu une première prise alimentaire à domicile : un biberon de lait dont la DE était modifiée de + ou - 20\% par rapport à la DE de leur lait habituel (selon un ordre contrebalancé). La prise alimentaire suivante (du même jour) avait lieu au laboratoire où leur était proposé un biberon de lait d'une DE habituelle (moyenne $=67 \mathrm{Kcal} / 100 \mathrm{~mL}$ ), à consommer en ad libitum. Le relevé des quantités consommées à domicile et au laboratoire a permis de calculer un score COMPX. Ce score traduit la capacité du nourrisson à ajuster sa prise alimentaire en réponse à l'apport calorique du repas précédent. Lorsque l'ajustement est parfait, le score COMPX=100 \%. Si ce score est $<100 \%$, cela traduit une sous-compensation. Des questionnaires ont permis de collecter certaines caractéristiques maternelles et enfantines. L'étude a reçu un avis favorable du CPP Est-I (2015-A00014-45).

Résultats et Analyse statistique : Un score COMPX a pu être calculé pour 27 nourrissons (15 filles / 12 garçons) à 4,4 $\pm 0,7$ mois. Les nourrissons sous-compensent avec un COMPX moyen significativement inférieur à $100 \%$ : COMPX=30 $\pm 56 \%$ (N=27; $t=-6.49$; p<.0001), comme déjà observé chez des enfants plus âgés ou des adultes.

Conclusion : A court terme, les nourrissons en alimentation lactée exclusive n'ajustent que partiellement leur prise énergétique après la modification de l'énergie apportée (DE $\pm 20 \%$ ) lors de la prise lactée précédente. En termes de perspectives, cette mesure de compensation calorique à court terme sera mise en lien avec des données sur l'historique d'alimentation lactée et du tempérament alimentaire.

Remerciements : Elodie Trioulet, Valérie Feyen et Emilie Szleper pour leur aide dans la collecte de données. Prix SFN 2015, ANR PUNCH (ANR-15-CE21-0014). Conflits d'intérêts : Aucun conflit à déclarer

Conflits d'intérêts : Aucun conflit à déclarer 
Dégradation de $\mathbf{N}$-acétyle-cystéine en N,N-diacétyle-cystine dans les Nutritions Parentérales Pédiatriques

I. Sommer ${ }^{12,{ }^{*}, \text { H. Schwebel }}{ }^{3}$, V. Adamo ${ }^{3}$, L. Bouchoud ${ }^{4}$, P. Bonnabry ${ }^{4}$, F. Sadeghipour ${ }^{1}$

${ }^{1}$ Pharmacie, Centre Hospitalier Universitaire Vaudois CHUV, Lausanne, ${ }^{2}$ Section des sciences pharmaceutiques (EPGL), Université de Genève, Université de Lausanne, Genève, Lausanne, ${ }^{3}$ Pharmaceutical Development, B.Braun Medical AG, Crissier, ${ }^{4}$ Pharmacie , Hôpitaux Universitaires de Genève HUG, Genève, Suisse

Introduction et but de l'étude : Les guidelines de I'ESPGHAN/ESPEN/ESPR sur la nutrition parentérale pédiatrique (NPP) recommandent l'administration de la cystéine aux enfants prématurés. Le besoin de cet acide aminé (AA) semi-essentiel est dû à l'immaturité biochimique de ces patients qui résulte dans l'incapacité de synthétiser de la cystéine endogène depuis la méthionine et la sérine. Le précurseur soluble N-acétyle-cystéine (NAC) permet de supplémenter la NPP avec de la cystéine car il est facilement fractionné en sa forme biodisponible. Mais il peut aussi s'oxyder en son dimer N,N-diacétyle-cystine (DAC) durant le processus de fabrication.

Les buts de cette étude sont la compréhension du processus de dégradation de la NAC en DAC et ses déclencheurs et l'évaluation de la criticité et la concentration limite de la DAC dans des NPP.

Matériel et méthodes : Des solutions d'AA contenant la NAC ont été passées à l'autoclave après avoir injecté différents volumes d'air $(2,8,16 \mathrm{~mL})$ dans l'espace de tête primaire. Une partie des échantillons contenaient des absorbeurs d'oxygène dans l'emballage secondaire. Les concentrations d'oxygène ont été mesurées dans la solution et dans les espaces de tête des emballages primaires et secondaires à des intervalles de temps différents $(0,7,13,45$ jours). Aux mêmes intervalles, les concentrations de DAC ont été analysées par HPLC.

Résultats et Analyse statistique : Les bilans molaires démontrent que la NAC se dégrade exclusivement en DAC.

Les concentrations de DAC augmentent considérablement dans les échantillons sans absorbeur d'oxygène. Dans ce cas, après 45 jours aucune stabilisation de la dégradation de NAC n'a pu être observée car le taux d'oxygène est saturé aux trois points de mesure (solution et espaces de tête primaire et secondaire).

Dans les échantillons avec absorbeurs, les taux d'oxygène sont drastiquement réduits aux trois points de mesure. La durée nécessaire pour absorber tout l'oxygène dépend du volume d'air initialement présent. Les concentrations de DAC augmentent en fonction des taux d'oxygène dans la solution et l'espace de tête primaire.

Conclusion : Tout d'abord, il faut noter qu'à notre connaissance, il n'y a aucune publication dans la littérature ni sur les mécanismes de cette dégradation, ni sur la toxicité de DAC dans les NPP.

Les analyses réalisées confirment une corrélation entre la concentration d'oxygène et la dégradation de NAC en DAC en fonction du temps permettant ainsi d'établir des cinétiques de formation de la DAC. Il est nécessaire de limiter la quantité d'oxygène en contact avec la NAC dans le cas d'emballages semi perméables. L'utilisation d'absorbeurs d'oxygène est un moyen efficace de réduire le taux de l'oxygène dans la solution et les espaces de tête des emballages primaires et secondaires.

II n'y a pas d'évidence de non-sécurité et/ou toxicité de la DAC dans les NPP. D'autres recherches sur les effets de la DAC par voie veineuse sur l'être humain sont nécessaires.

Conflits d'intérêts : Aucun conflit à déclarer 
Pancréatite aigue secondaire à une hypertriglycéridémie chez l'enfant : une cause rare à évoquer

J. Lemale ${ }^{1,}{ }^{*}$, S. Keloua ${ }^{1}$, B. dubern ${ }^{1}$, J.-P. Girardet ${ }^{1}$, P. Tounian ${ }^{1}$

${ }^{1}$ Nutrition et gastroentérologie pédiatriques, hopital Trousseau, Paris, France

Introduction et but de l'étude : A l'inverse de l'adulte, l'hypertriglycéridémie est une cause rare de pancréatite aiguë chez l'enfant, seuls quelques cas isolés sont décrits dans la littérature. L'objectif de cette étude multicentrique était de décrire les caractéristiques cliniques, biologiques et évolutives de 14 enfants hospitalisés pour une pancréatite aiguë secondaire à une hypertriglycéridémie.

Matériel et méthodes : Quatorze enfants hospitalisés entre août 2005 et novembre 2017 dans 8 services de pédiatrie pour une pancréatite aiguë (lipasémie supérieure à 3 fois la normale) associée à une hypertriglycéridémie supérieure à $10 \mathrm{~g} / \mathrm{l}$ ont été inclus. Les autres causes de pancréatite aiguë, biliaires, malformatives, virales, médicamenteuses ou traumatiques ont été éliminées. Les antécédents familiaux, l'âge de survenue de la pancréatite et son évolution ont été analysés. L'exploration de l'hypertriglycéridémie comportait un dosage des triglycérides, du cholestérol total, et HDL, une électrophorèse des lipoprotéines et une analyse des gènes connus pour coder des protéines impliquées dans le métabolisme des triglycérides: lipoprotéine-lipase, ApoCII, ApoCIII, ApoA5, ApoE, LMF1, GPIHBP1.

Résultats et Analyse statistique : L'âge médian de survenue de la pancréatite aiguë était de 7 ans (extrêmes : 6 semaines-14 ans), la triglycéridémie moyenne était de $22 \mathrm{~g} / \mathrm{l}$ (extrêmes : $10,8 \mathrm{~g} / \mathrm{l}-82 \mathrm{~g} / \mathrm{l}$ ) avec une hyperchylomicronémie. L'évolution de la pancréatite a été favorable dans tous les cas après la baisse du taux des triglycérides. La durée d'hospitalisation moyenne était de 13 jours (extrêmes : 8 jours-47 jours). Au cours du suivi, d'une durée médiane de 4 ans (extrêmes : 9 mois-12 ans) cinq enfants ont eu une récidive de pancréatite aiguë. Le dernier taux médian de triglycérides sous traitement diététique hypolipidique était de $8,2 \mathrm{~g} / \mathrm{l}$ (extrêmes : 0,5g/l-24,3g/l). Une mutation homozygote majeure du gène codant pour la lipoprotéine lipase a été retrouvée dans sept cas. Chez deux enfants, des polymorphismes du gène de la lipoprotéine lipase ou de l'Apo A5 ont été mis en évidence de façon isolée. Chez cinq enfants, aucune anomalie génétique n'a été retrouvée en dehors d'un génotype E3/E4 ou E2/E3 de l'Apo E. Aucune anomalie n'a été identifiée sur les gènes codant pour l'ApoCII, l'ApoCIII, LMF1 et la protéine GPIHBP1.

Conclusion : L'hypertriglycéridémie est une cause de pancréatite aiguë qu'il faut savoir rechercher chez l'enfant. Les anomalies génétiques (mutations ou polymorphismes) responsables d'hypertriglycéridémie majeure sont diverses et leurs expressions phénotypiques variables. Pour certaines hypertriglycéridémies, aucune étiologie n'est identifiée. Du fait de leur rareté, des études génétiques systématiques permettront de trouver de nouvelles mutations. L'évolution de la pancréatite aiguë est favorable mais des récidives sont possibles en cas d’hypertriglycéridémie mal contrôlée malgré les mesures diététiques.

Remerciements : Dr P Benlian, Pr J Sarles, Pr A lachaux, Pr F Gottrand, Dr Cremillieux

Conflits d'intérêts : Aucun conflit à déclarer 
V. Raoult ${ }^{1}$, L. Bouvart ${ }^{1}$, D. Guimber ${ }^{2}$, N. Peretti ${ }^{3}$, H. Piloquet ${ }^{4}$, R. Hankard ${ }^{567}$, A. De Luca ${ }^{56,{ }^{*}}$ et The Pediatric Nutritional Assessment Network (www.epinut.fr)

${ }^{1}$ Pédiatrie, CHRU Tours, Tours, ${ }^{2}$ Pédiatrie, CHRU Lille, Lille, ${ }^{3}$ Nutrition, HCL, Lyon, ${ }^{4}$ Pédiatrie, CHU Nantes, Nantes, ${ }^{5}$ Nutrition, CHRU Tours, ${ }^{6}$ Inserm UMR 1069, ${ }^{7}$ Université F Rabelais, Tours, France

Introduction et but de l'étude : Le diagnostic de dénutrition comprend l'analyse de la courbe de croissance. L'objectif principal de notre étude était d'analyser les données de croissance antérieures chez l'enfant hospitalisé.

Matériel et méthodes : Cette étude observationnelle a inclus les enfants hospitalisés du 12 au 23 mars 2018 dans les centres participants. Les enfants âgés de 0 à 18 ans ont été inclus, en dehors des prématurés. La date, le poids et la taille du dernier point connu du carnet de santé était notée, une vitesse de perte d'1ET en 3 mois était compatible avec une dénutrition. L'état nutritionnel était évalué à l'entrée et la sortie d'hospitalisation. Les données ont été saisies sur l'outil internet e-Pinut (www.epinut.fr), permettant le calcul des index selon les normes françaises.

Résultats et Analyse statistique : Parmi les 1385 enfants inclus, 671 enfants soit 48\% avaient une donnée antérieure de croissance documentée. La fréquence d'un index de Waterlow <-2 ET était de 6\% au dernier point connu, $11 \%$ à l'admission. Un suivi récent $<3$ mois pour les $<2$ ans, $<6$ mois pour les $2-5$ ans, $<1$ an pour les $\geq 6$ ans était présent respectivement dans $87 \%, 69 \%$ et $64 \%$ des cas $\left(\mathrm{p}<10^{-4}\right.$ ) (global : 76\%). Les enfants $<2$ ans et $\geq 6$ ans porteurs de maladies chroniques avaient plus fréquemment un suivi récent que les enfants sans maladie chronique ( $90 \%$ vs $81 \%, p<0,05 ; 77 \%$ vs $50 \%, p<10^{-4}$ ) mais pas les $2-5$ ans. Les enfants avec une vitesse de perte d'1ET en 3 mois n'avaient pas une DMS différente des autres enfants $(4,0 \pm 4,0 \mathrm{j}$ vs $4,0 \pm 4,3 \mathrm{j}$, NS), ni une fréquence de traitement nutritionnel différente à l'entrée (14\% vs $16 \%)$.

Conclusion : La fréquence d'un index de Waterlow <-2ET est plus importante à l'entrée qu'au dernier point du carnet de santé suggérant un impact aigu de la pathologie amenant à l'hospitalisation. Lors d'une hospitalisation, des données antérieures de croissance sont disponibles pour seulement un enfant sur deux, et récentes dans $3 / 4$ des cas. L'évaluation de la dynamique de croissance, élément majeur de l'état nutritionnel, est insuffisamment disponible chez l'enfant hospitalisé, notamment chez les $\geq 6$ ans sans pathologie chronique.

Conflits d'intérêts : V. Raoult: Aucun conflit à déclarer, L. Bouvart: Aucun conflit à déclarer, D. Guimber: Aucun conflit à déclarer, N. Peretti: Aucun conflit à déclarer, H. Piloquet: Aucun conflit à déclarer, R. Hankard: Aucun conflit à déclarer, A. De Luca a reçu une subvention/soutien de Nutricia Nutrition Clinique 
P180

Influence des apports nutritionnels durant les premières semaines de vie du prématuré sur la croissance et le développement psychomoteur jusqu'à l'âge de 2 ans

P. Poidevin ${ }^{1}$, A. LEKE ${ }^{1, *}$

${ }^{1}$ Néonatologie, CHU Amiens, Amiens, France

Introduction et but de l'étude : La nutrition optimale du prématuré est une problématique majeure en néonatologie.

L'objectif principal était d'étudier le lien entre nutrition initiale du prématuré et trajectoire de croissance jusque 2 ans. L'objectif secondaire était d'étudier le lien entre nutrition et développement psychomoteur jusque 2 ans

Matériel et méthodes : Il s'agit d'une étude monocentrique, rétrospective, descriptive, incluant des prématurés < 32 semaines d'aménorrhée (SA) nés entre 2005 et 2015 hospitalisés dans les unités de néonatologie et suivis par le même observateur en consultation du CHU Amiens-Picardie.

Résultats et Analyse statistique : 40 prématurés de terme moyen de naissance de $28.9 \pm 2.1 \mathrm{SA}$ et de poids moyen de naissance moyen de $1190 \pm 350 \mathrm{~g}$ ont été inclus. $20 \%$ des enfants présentaient un retard de croissance intra-utérin (RCIU), $47.5 \%$ un retard de croissance à la sortie du service dont $17.5 \%$ de prématurés avec RCIU donc $30 \%$ d'enfants eutrophes à la naissance ayant présenté un retard de croissance extra-utérin (RCEU). Il a été mis en évidence un déficit d'apports nutritionnels importants au cours des 2 premières semaines d'hospitalisation. Le retard de croissance pondéral, statural et du périmètre crânien était retrouvé respectivement chez $32 \%, 26 \%$ et $19 \%$ des enfants à 1 an, et $18 \%, 18 \%$ et $6 \%$ des enfants à 2 ans. A 2 ans, l'IMC était insuffisant pour $24 \%$ des enfants et le rapport PB/PC déficitaire pour $14 \%$. Les apports énergétiques durant la $2^{\text {ème }}$ semaine de vie étaient associés avec une meilleure croissance pondérale jusqu'à l'âge de 2 ans. A 2 ans, un retard psychomoteur était observé chez $26 \%$ des enfants évalués, dont 6\% présentaient un poids inférieur à -2 DS et taille inférieure à -2 DS. Nous n'avons pas observé de lien entre apports nutritionnels durant l'hospitalisation et développement psychomoteur.

Conclusion : Une nutrition optimale dès les premiers jours de vie des grands prématurés est nécessaire pour prévenir un RCEU pouvant avoir des conséquences sur la santé à long terme.

Conflits d'intérêts : Aucun conflit à déclarer 
Evaluation des pratiques du dépistage de la dénutrition chez l'enfant au cabinet du médecin généraliste. V. Bertrand ${ }^{1,}{ }^{*}$, B. Pichot ${ }^{1}$, P. Déchelotte ${ }^{2}$

${ }^{1}$ Pédiatrie, Hopital Jacques Monod, Le Havre, Montivilliers, ${ }^{2}$ Département de Nutrition, CHU de Rouen et INSERM U1073, Université de Rouen, IRIB, Rouen, France

Introduction et but de l'étude : La malnutrition reste un problème de santé publique en Europe, non seulement en gériatrie et dans les maladies chroniques de l'adulte, mais aussi en pédiatrie. La prévalence de la dénutrition en pédiatrie est estimée à 5 à 10 $\%$ en milieu hospitalier. Les pratiques de dépistage de la dénutrition chez l'enfant au cabinet du médecin généraliste sont mal connues. Ce travail a eu pour objectif de dresser un état des lieux du dépistage de la dénutrition chez l'enfant en médecine de ville.

Matériel et méthodes : Un questionnaire anonymisé a été envoyé par mail et courrier aux médecins généralistes libéraux de Seine-Maritime. Le questionnaire comprenait 12 questions à choix simple ou multiples et 1 question ouverte. Les données étudiées concernaient les médecins (âge, sexe, \% de patientèle pédiatrique), leurs connaissances (critères cliniques utilisés pour évaluer la dénutrition, conseils ou bilans nutritionnels), leurs pratiques (prise de poids taille IMC, lecture des courbes de croissance), leurs outils (IMC calculé sur disque ou logiciel informatique, type de courbe de croissance papier ou numérique), et leurs attentes en terme de formation.

Résultats et Analyse statistique : 102 médecins ont répondus au questionnaire (sex ratio 1,16, majorité de plus de 50 ans 57,8\%), ayant une patientèle pédiatrique comprise entre 5 à $15 \%$ pour $55,9 \%$ d'entre eux. Les critères cliniques utilisés pour évaluer la dénutrition étaient l'examen clinique $92 \%$, le poids $79 \%$, l'IMC 97\%, le PB/PC 6\%, l'indice de Waterlow 1\%. $82 \%$ ne connaissaient pas l'indice de Waterlow et $72 \%$ ne connaissaient pas le $\mathrm{PB} / \mathrm{PC}$. $79,4 \%$ des médecins mesuraient systématiquement à chaque consultation le poids, 33,3\% mesuraient systématiquement la taille et l'IMC. $72.2 \%$ avaient un logiciel qui calcule l'IMC, 5.5\% n'avaient que le disque d'IMC pédiatrique, 16,7\% les deux, et 5.5\% n'avaient ni disque d'IMC pédiatrique ni logiciel. Parmi les $43.6 \%$ qui regardaient systématiquement les courbes de croissance à chaque consultation, une majorité $(77,5 \%)$ utilisaient les courbes de croissance du carnet de santé, et $20.6 \%$ les courbes du logiciel informatique. $79.2 \%$ reportaient systématiquement les poids/taille dans le carnet de santé, la raison principale de ne pas le faire étant l'oubli du carnet de santé par les familles. $71 \%$ se sentaient à l'aise pour donner des conseils diététiques et $31 \%$ pour faire un bilan nutritionnel. 13,7\% prescrivaient parfois des compléments alimentaires chez l'enfant. Seuls $9,8 \%$ des médecins ont participé à des réunions d'information sur le dépistage de la dénutrition chez l'enfant et $89 \%$ souhaiteraient plus de formation à ce sujet dans le département.

Conclusion : La mesure de la taille et la détermination de l'IMC restent insuffisamment réalisées, malgré la diffusion croissante des logiciels. Les courbes de croissance ne sont pas remplies et regardées systématiquement, ce qui expose à un retard de dépistage, alors que le non remplissage du carnet de santé expose à une perte d'information. Il existe une attente des médecins en termes de formation. La mise en œuvre du dossier médical partagé devrait permettre l'harmonisation des supports de recueil des indicateurs nutritionnels.

Conflits d'intérêts : Aucun conflit à déclarer 
Introduction et but de l'étude : L'exposition précoce aux acides gras polyinsaturés (AGPI) oméga 6 (acides linoléique LA et arachidonique $A A, n-6$ ) et oméga 3 (acides eicosapentaénoïque EPA et docosahexaénoïque DHA, n-3) pourrait programmer le système cardio-métabolique (1-3). Le but était d'examiner les relations entre le statut en AGPI, maternel et à la naissance, et des paramètres cardio-métaboliques ultérieurs des enfants.

Matériel et méthodes : L'étude EDEN est une cohorte mère-enfant française de 2002 femmes enceintes recrutées entre 2003 et 2006 dans les maternités de Poitiers et Nancy. Le profil en acides gras des membranes des érythrocytes du sang maternel (prélevé à 24-28 semaines de grossesse) et du cordon ombilical (prélevé à l'accouchement) a été analysé par chromatographie en phase gazeuse. Les relations, entre les taux d'AGPI et des paramètres cardio-métaboliques (cholestérol total, cholestérol-HDL et LDL, triglycéridémie, leptine, insuline, glycémie) des enfants à 5 ans, ont été examinées par régression linéaire avec ajustement sur des facteurs de confusion potentiels.

Résultats et Analyse statistique : Le profil en AGPI et en acétal diméthylique (DMA, indicateur des plasmalogènes) a été obtenu chez 1876 mères et 1402 nouveau-nés (filles: 46\%) (moyennes en \% des acides gras totaux \pm écart-type).

\begin{tabular}{|l|c|c|c|c|c|c|c|}
\hline & AGPI $\mathbf{n - 6}$ & LA & AA & AGPI $\mathbf{n - 3}$ & EPA & DHA & DMA \\
\hline Mères & $25,6 \pm 1,4$ & $7,2 \pm 0,8$ & $12,9 \pm 0,8$ & $7,8 \pm 1,2$ & $0,44 \pm 0,17$ & $5,3 \pm 0,9$ & $6,3 \pm 0,6$ \\
\hline Cordon des Filles (n= 637) & $26,4 \pm 1,1$ & $2,9 \pm 0,5$ & $15,1 \pm 0,9$ & $7,2 \pm 1,1$ & $0,17 \pm 0,11$ & $6,3 \pm 1,0$ & $5,6 \pm 0,5$ \\
\hline Cordon des Garçons (n= 739) & $26,1 \pm 1,1$ & $2,9 \pm 0,5$ & $15,1 \pm 0,9$ & $7,0 \pm 1,0$ & $0,17 \pm 0,12$ & $6,1 \pm 0,9$ & $5,7 \pm 0,5$ \\
\hline
\end{tabular}

Le statut en DHA et en AGPI n-3 des mères était associé positivement à la glycémie $(0,08$ [95\% IC: 0,$02 ; 0,13] \mathrm{mmol} / \mathrm{L}$ et 0,07 $[0,01 ; 0,12]$ pour une variation d'un écart type du taux de l'AGPI) chez les filles à 5 ans, alors que le statut maternel en DMA et le rapport n-6/n-3 l'étaient négativement $(-0,05[-0,09 ;-0,00]$ et $-0,06[-0,12 ;-0,00])$. Le cholestérol-HDL était positivement associé aux AGPI n-6 maternels chez les filles $(0,04[0,00 ; 0,09])$, alors qu'il était associé négativement à l'AA $(-0,03[-0,07 ; 0,00])$ et positivement à I'EPA $(0,04[0,01 ; 0,08)]$ maternels chez les garçons. Le statut à la naissance révélait des associations à 5 ans uniquement chez les filles. Ainsi, le DMA du sang du cordon était associé positivement au cholestérol total $(0,15$ [0,04;0,26]), cholestérol-HDL $(0,05[-0,00 ; 0,09])$, et cholestérol-LDL $(0,11[0,02 ; 0,20])$, et négativement à l'insuline $(-0,14[-0,24 ;-0,04])$. Le DHA et les AGPI n-3 étaient positivement associés à la glycémie $(0,07[-0,00 ; 0,14])$. Les AGPI n-3 et le DHA $(0,03[0,00 ; 0,06])$, ainsi que I'EPA $(0,03[0,01 ; 0,06])$ étaient associés positivement à la triglycéridémie, et le rapport $n-6 / n-3$ l'était négativement $(-0,03[-$ $0,06 ; 0,00])$.

Conclusion : L'exposition prénatale aux AGPI oméga 6, oméga 3, et aux plasmalogènes était associé à la glycémie et à la lipémie de l'enfant à 5 ans de façon différente chez les filles et les garçons. Ces résultats princeps de la cohorte EDEN suggèrent que ces lipides pourraient programmer la santé cardio-métabolique.

Références : 1- Jochems SH et al. PLEFA 2015, 100:21-7.

2- Vidakovic AJ et al. Nutr Metab Cardiovasc Dis 2017, 27:78-85.

3- Voortman T et al. Clin Nutr 2018, 37:984-92.

Remerciements : Prix IDF/FRM pour les Sciences de I'Alimentation 2015.

Conflits d'intérêts : Aucun conflit à déclarer 


\section{P183}

Les suspicions d'allergies au lait de vache non IgE-médiée sont rarement confirmées

A. Lemoine ${ }^{1,}{ }^{*}$, J. Aroulandom ${ }^{2}$, C. O'Neill $^{1}$, J. Lemale ${ }^{1}$, P. Tounian ${ }^{1}$

${ }^{1}$ Nutrition et gastroentérologie pédiatrique, ${ }^{2}$ Urgences pédiatriques, Hôpital Trousseau - APHP, Paris, France

Introduction et but de l'étude : Trop de nourrissons suspects d'allergies aux protéines du lait de vache (APLV) suivent des régimes prolongés sans PLV non justifiés. Le but de cette étude était de montrer que la majorité des suspicions d'APLV ne sont pas confirmées et qu'elles guérissent rapidement.

Matériel et méthodes : Soixante-trois nourrissons âgés de 21 jours en moyenne et suspects d'APLV non IgE-médiée devant des symptômes disparaissant après exclusion des PLV (hors syndrome d'entérocolite induite par les protéines alimentaires) ont été inclus. Un test de provocation orale diagnostique était réalisé 4 semaines après l'exclusion des PLV, puis tous les 2 mois pour tester l'acquisition de la tolérance lorsque le diagnostic était confirmé.

Résultats et Analyse statistique : Les symptômes faisant suspecter l'APLV étaient des rectorragies ( $n=43 ; 68,3 \%$ ), des vomissements ( $n=14 ; 22,2 \%$ ) et/ou une diarrhée ( $n=14 ; 22,2 \%$ ). L'APLV était confirmée chez seulement 20 patients (31,7 \%). L'âge médian d'acquisition de la tolérance des PLV était de 8,9 mois (min-max : 3,5-13,6 mois).

Conclusion : Dans les formes non IgE-médiées d'APLV, le diagnostic est confirmé dans moins d'un tiers des cas par le test d'éviction-réintroduction. Une fois l'APLV confirmée, la moitié des nourrissons guérit avant l'âge de 9 mois. Ce travail souligne l'importance de systématiquement confirmer précocement les suspicions d'APLV, mais aussi de tester dès l'âge de 4 mois l'acquisition de la tolérance au lait de vache.

Conflits d'intérêts : A. Lemoine a un conflit d'intérêt avec Modilac, Picot, J. Aroulandom: Aucun conflit à déclarer, C. O'Neill: Aucun conflit à déclarer, J. Lemale: Aucun conflit à déclarer, P. Tounian a un conflit d'intérêt avec Danone/Blédina, Mead-Johnson, Nestlé/Guigoz, Novalac/Ménarini, Nutricia, Picot, Sodilac 
P184

Vélocité de croissance des nouveau-nés prématurés : comparaison du modèle exponentiel de Patel avec les variations de Zscore de poids

L. Simon ${ }^{1, *}$, M. Hanf ${ }^{2}$, A. Frandas-Chauty ${ }^{3}$, D. Darmaun ${ }^{4}$, G. Gascoin ${ }^{5}$, C. Flamant ${ }^{1}$, J.-C. Rozé ${ }^{1}$

${ }^{1}$ Néonatologie, CHU de Nantes, ${ }^{2}$ INSERM, CIC, Université de Nantes, ${ }^{3}$ Néonatologie, CHU Nantes, ${ }^{4}$ INRA, UMR 1280, Université de Nantes, Nantes, ${ }^{5}$ Néonatologie, CHU d'Angers, Angers, France

Introduction et but de l'étude : De nombreuses méthodes sont utilisées pour évaluer la croissance des nouveau-nés prématurés pendant l'hospitalisation. L'objectif principal était de comparer deux méthodes pour évaluer la vélocité de croissance pondérale: le modèle exponentiel décrit par Patel ${ }^{1}$ en $\mathrm{g} / \mathrm{kg} / \mathrm{j}$ et la variation de Z-score de poids selon les courbes de Fenton ${ }^{2}$. L'objectif secondaire était de déterminer les facteurs expliquant un défaut de concordance entre les deux méthodes.

Matériel et méthodes : Les prématurés nés avant 33 SA entre 2003 et 2015 étaient inclus. Un modèle de régression linéaire a été construit pour prédire ce que serait la vélocité de croissance par modèle exponentiel en utilisant la vélocité de croissance par Zscore. La vélocité de croissance par modèle exponentiel et la vélocité de croissance par modèle exponentiel prédite par le Zscore ont été utilisées pour évaluer la concordance entre les deux méthodes. Nous considérons une limite de $+/-2 \mathrm{~g} / \mathrm{kg} / \mathrm{j}$ comme une concordance acceptable, $+/-4 \mathrm{~g} / \mathrm{kg} / \mathrm{j}$ comme une concordance pauvre et au-dessus de $4 \mathrm{~g} / \mathrm{kg} / \mathrm{j}$ une mauvaise concordance.

Résultats et Analyse statistique : Parmi les 3954 enfants inclus, 2471 enfants (62,5\%) ont une différence de moins de $2 \mathrm{~g} / \mathrm{kg} / \mathrm{j}$ entre les deux méthodes de vélocités de croissance, 1278 enfants (32,3\%) ont une différence entre 2 et $4 \mathrm{~g} / \mathrm{kg} / \mathrm{j}$ et 205 enfants $(5,2 \%)$ une différence supérieure à $4 \mathrm{~g} / \mathrm{kg} / \mathrm{j}$. Dans une analyse multivariée, le Z-score de poids de naissance et l'âge gestationnel expliquent respectivement $31 \%$ et $25 \%$ de la variance de ces différences. Les autres facteurs réunis expliquent moins de $1 \%$ de la variance.

Conclusion : Chez plus d'un tiers des enfants inclus, les deux méthodes de mesure de la vélocité de croissance avaient une mauvaise concordance. Les variations de Z-score de poids prennent en considération l'âge gestationnel et le sexe et semblent plus adaptées pour analyser la vélocité de croissance d'une population avec des âges gestationnels variés.

Références : 1. Patel AL, Engstrom JL, Meier PP, Kimura RE. Accuracy of methods for calculating postnatal growth velocity for extremely low birth weight infants. Pediatrics. 2005 Dec;116(6):1466-73.

2. Fenton TR, Nasser R, Eliasziw M, Kim JH, Bilan D, Sauve R. Validating the weight gain of preterm infants between the reference growth curve of the fetus and the term infant. BMC Pediatr. 2013 Jun 11;13:92.

Remerciements : Nous remercions Madame le Dr Fenton pour le partage des LMS des courbes de croissance publiées en 2013.

Conflits d'intérêts : Aucun conflit à déclarer 
La matière grasse laitière et le probiotique L. fermentum incorporés dans des préparations pour nourrissons modulent leur digestion ainsi que la composition du microbiote et les propriétés barrière de l'intestin chez le mini-porc

M. Lemaire ${ }^{12}$, G. Boudry ${ }^{1}$, O. Ménard ${ }^{3}$, P. Le Ruyet ${ }^{2}$, C. Baudry ${ }^{2}$, P. Gérard ${ }^{4}$, D. Dupont ${ }^{3}$, A. Deglaire $^{3}$, I. Le Huërou-Luron ${ }^{1, *}$, S. Blat ${ }^{1}$

${ }^{1}$ NUMECAN, INRA, INSERM, Univ Rennes, Rennes, ${ }^{2}$ Lactalis R\&D, Retiers, ${ }^{3}$ STLO, INRA, Agrocampus Ouest, Rennes, ${ }^{4}$ MICALIS, INRA, AgroParisTech, Univ Paris-Saclay, Jouy-en-Josas, France

Introduction et but de l'étude : Rapprocher les préparations pour nourrissons (PPN) du lait maternel, d'un point de vue fonctionnel, constitue un enjeu majeur pour optimiser la santé des nourrissons qui ne sont pas allaités par leur mère. La structure et la composition très particulières de la matrice lipidique du lait maternel et la présence d'un microbiote laitier pourraient en partie expliquer les différences physiologiques et métaboliques observées entre enfants allaités au sein et ceux recevant des PPN. L'objectif de cette étude était d'évaluer les effets de l'ajout dans les PPN de matière grasse laitière (MGL) bovine associée ou non à une souche probiotique (L. fermentum CECT5716, Lf) sur la digestion, le microbiote et la fonction barrière de l'intestin du nourrisson à l'issue de la période d'allaitement.

Matériel et méthodes : Vingt-six miniporcs Yucatan ont été allaités avec une PPN classique à base de matières grasses végétales (MGV), ou une PPN à base d'un mélange de MGV et de MGL supplémentée ou non en Lf (MGL et MGL+Lf respectivement). La digestion des PPN (protéolyse, lipolyse), la composition et l'activité du microbiote rectal et la fonction barrière de l'intestin ont été évaluées à l'âge de 28 jours.

Résultats et Analyse statistique : L'ajout de MGL associée ou non au probiotique Lf a induit une protéolyse gastrique plus importante et a augmenté la diversité de peptides bioactifs et potentiellement antimicrobiens présents dans l'iléon et le côlon. Une lipolyse intestinale plus importante a également été observée avec la MGL. L'association de la MGL et de Lf a de plus renforcé les défenses intestinales non spécifiques de l'hôte, via une augmentation de la densité des cellules à mucus dans le jéjunum, ainsi qu'une plus forte expression des ARN codant les protéines des jonctions serrées (ZO-1 et occludine) et une diminution de la perméabilité transcellulaire dans le côlon. Ces effets pourraient en partie être médiés par la modulation de la composition et du métabolisme du microbiote intestinal observée dans le groupe MLG+Lf avec notamment une sur-abondance de genres bactériens tels que Lachnospiraceae UCG-001 et Coprococcus 3, et une augmentation de la production de métabolites bactériens tels que le butyrate, l'isobutyrate et le glutamate.

Conclusion : La MGL et le probiotique Lf ont modifié la digestion des PPN, l'implantation du microbiote et la fonction barrière intestinale du nourrisson. Ces résultats soulignent l'importance de la composition des FI sur la santé digestive du nourrisson. Une étude à plus long terme nous a permis de mettre en évidence des conséquences bénéfiques de ces modifications précoces sur la physiologie intestinale du jeune adulte.

Conflits d'intérêts : M. Lemaire a reçu une subvention/soutien de Lactalis, est employé(e) de Lactalis, G. Boudry: Aucun conflit à déclarer, O. Ménard: Aucun conflit à déclarer, P. Le Ruyet est employé(e) de Lactalis, C. Baudry est employé(e) de Lactalis, P. Gérard: Aucun conflit à déclarer, D. Dupont: Aucun conflit à déclarer, A. Deglaire: Aucun conflit à déclarer, I. Le Huërou-Luron a reçu une subvention/soutien de Lactalis, S. Blat a reçu une subvention/soutien de Lactalis 
P186

Fréquence de la malnutrition (dénutrition, surpoids/obésité) et prévalence de la carence martiale chez les nourrissons de l'Ouest algérien

K. Bouziane-Nedjadi ${ }^{1,}{ }^{*}$, A. Hadj Kadi ${ }^{2}$, A. Hamous ${ }^{2}$, S. Benadouda ${ }^{2}$, N. Dida ${ }^{2}$, I. Boureghda ${ }^{3}$, M. Bouchenak ${ }^{2}$

${ }^{1}$ Pédiatrie C , CHU Oran, Oran, ${ }^{2}$ Laboratoire de Nutrition Clinique et Métabolique, Faculté des Sciences de la Nature et de la Vie. Université d'Oran1 Ahmed Ben Bella, Oran, ${ }^{3}$ Association Algérienne pour la Promotion de la Nutrition de l'Enfant et de la Mère AAPEM, Alger, Algérie

Introduction et but de l'étude : Les enfants présentant un retard de croissance lors de la petite enfance sont à risque de surpoids et d'obésité à l'adolescence et à l'âge adulte, d'où l'intérêt de dépister et d'identifier précocement le retard de croissance chez les enfants de moins de 1 ans. Par ailleurs, la carence martiale (CM) est l'un des désordres nutritionnels les plus fréquents que ce soit dans les pays en développement ou les pays développés, constituant ainsi un véritable problème de santé publique.

Le but de cette étude était d'évaluer la fréquence de la malnutrition (dénutrition, surpoids et obésité) et de la prévalence de la carence martiale parmi une population de nourrissons de l'Ouest algérien.

Matériel et méthodes : De janvier à juin 2016, 130 nourrissons sains (72 mâles), moyenne d'âge 21,2 $\pm 3,9$ mois, avaient été prospectivement enrôlés lors de leur présentation dans 3 centres de vaccination à Oran. Après consentement écris, les paramètres anthropométriques (poids/âge, taille/âge, poids/taille, IMC, exprimés en déviations standard (DS) selon les standards de l'OMS, avaient été déterminés. Les apports alimentaires avaient été évalués par méthode de rappel des 24 heures, suivie par un enregistrement prospectif sur 3 jours.

Les dosages de l'hémoglobine, de la férritinémie, et des récepteurs solubles de la transferrine (sTfR) ont été réalisés pour déterminer le statut martial. Les valeurs continues ont été exprimées en moyenne \pm déviations standard, et celle discontinues en pourcentages.

Résultats et Analyse statistique : La détermination des poids et tailles chez 112 nourrissons, avait retrouvé un poids/âge moyen

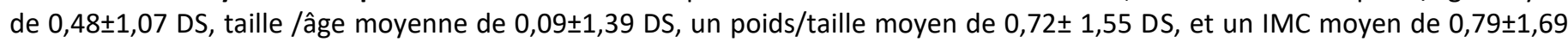
DS. Une émaciation (poids/taille <-2 DS) a été retrouvé dans 5,5 \% ( $n=6)$, retard de croissance (taille/âge < $-2 D S)$ dans $6,2 \%(n=7)$, surpoids dans $14,3 \%(n=16)$, et une obésité dans $6,3 \%(n=7)$.

L'anémie était présente chez $46,9 \%$ des nourrissons (61/130) et la carence martiale (férritine $<11 \mathrm{mg} / \mathrm{dl}$ et/ou sTfR élévés) chez $59,6 \%$ (71/119). L'enquête alimentaire avait retrouvé un déficit d'apport en micronutriments ; fer (84 \%), zinc (65\%), iode (48\%) et vitamine $\mathrm{D}(95 \%)$.

Conclusion : Notre étude a montré qu'environ $10 \%$ des nourrissons présentaient une dénutrition (émaciation, retard de croissance), et $18,7 \%$ une surnutrition. De plus, l'anémie et la carence martiale étaient particulièrement prévalentes. Ceci démontre l'impact de la dénutrition et de la « faim cachée » dans notre contexte.

Conflits d'intérêts : Aucun conflit à déclarer 


\section{P187}

Impact des lipides polaires laitiers sur le gain de poids, le microbiote et des marqueurs de la barrière intestinale in vivo et rôle de la sphingomyéline in vitro

M. Milard ${ }^{1, *}$, A. Penhoat ${ }^{1}$, V. Sauvinet ${ }^{2}$, A. Durand ${ }^{1}$, E. Meugnier ${ }^{1}$, E. Loizon ${ }^{1}$, C. Buisson ${ }^{1}$, L. Bindels ${ }^{3}$, A. Neyrinck $^{3}$, N. Delzenne ${ }^{3}$, F. Laugerette ${ }^{1}$, M.-C. Michalski ${ }^{1}$

${ }^{1}$ Laboratoire CarMeN, INSERM U1060, INRA1397, Univ-Lyon1, Lyon, ${ }^{2}$ CRNH Rhône-Alpes, Univ-Lyon1, HCL, Pierre Bénite, France, ${ }^{3}$ LDRI, MNut Research group, Université Catholique de Louvain, Bruxelles, Belgique

Introduction et but de l'étude : L'obésité est associée à des altérations du microbiote et de la barrière intestinale et à l'établissement d'une inflammation à bas bruit. Dans ce contexte, les lipides polaires laitiers (LP) ont montré des effets bénéfiques sur le métabolisme lipidique chez le rongeur, pouvant être liés à leur teneur en sphingomyéline (SM, 25\% de ces LP) qui aurait un impact sur le microbiote intestinal. Nos objectifs sont de montrer (i) in vivo, qu'un régime hyperlipidique supplémenté en LP laitiers induit une modulation du microbiote et de la barrière intestinale (incluant les protéines de jonctions serrées) et (ii) in vitro, que la SM participe à la modulation de l'expression des protéines de jonctions serrées.

Matériel et méthodes : Des souris C57BI/6 mâles adultes ont été nourries pendant 8 semaines avec un régime hyperlipidique (high-fat, HF ; $21 \%$ d'huile de palme dans des croquettes d'entretien) dépourvu de LP ou supplémenté avec 1.1\% (HF-LP1) ou 1.6\% (HF-LP2) de LP laitiers. L'impact des régimes a été observé sur le gain de poids, le poids du tissu adipeux épididymal, l'histomorphologie de l'intestin (profondeur des cryptes et expression des protéines de jonctions serrées), le microbiote caecal et les acides gras fécaux. Pour vérifier l'impact spécifique de la SM sur les jonctions serrées in vitro, des cellules Caco-2/TC7 ont été incubées avec des micelles mixtes lipidiques supplémentées avec $0,0,2$ ou $0,4 \mathrm{mM}$ de SM laitière pure ou dans un mélange de LP totaux.

Résultats et Analyse statistique : Après 8 semaines de régime, le groupe HF-LP2 présente un gain de poids moindre ( $P<0.01$ vs $\mathrm{HF}$ ) corrélé à moins de tissu adipeux épididymal sans différence de prise alimentaire. Les souris HF-LP1 présentent une augmentation de la teneur en Bifidobacterium animalis ( $P<0.05$ vs HF). Le groupe HF-LP2 présente une diminution de Lactobacillus reuteri $(P<0.05)$ corrélée à la perte fécale en acides gras à longue chaîne spécifiques de la SM laitière $(C 23: 0, C 24: 0$ et $C 24: 1)$. La profondeur des cryptes coliques est augmentée dans ce groupe. Parmi les protéines de jonctions serrées, seule l'expression de ZO-1 tend à être augmentée dans le duodénum. In vitro, une augmentation significative de l'expression génique des protéines de jonctions serrées (ZO-1, occludine, JAM-1, claudine-1) est observée lorsque les cellules Caco-2/TC7 sont incubées avec des micelles supplémentées en SM pure ( $P<0.05$ vs micelles dépourvues de SM), cet effet n'étant pas observé avec les LP laitiers totaux.

Conclusion : Nos résultats montrent qu'un régime hyperlipidique enrichi en LP laitiers limite le gain de poids, module l'abondance de bactéries intestinales bénéfiques et module des marqueurs de la barrière intestinale. Les pertes fécales en acides gras et les effets obtenus in vitro laissent suggérer que les impacts des LP laitiers seraient en partie dus à la SM.

Remerciements : Nous remercions F. Joffre et K. Bertrand (ITERG) pour la préparation et l'analyse lipidiques des régimes, Corman pour la fourniture de l'ingrédient et $\mathrm{C}$. Louche-Pélissier (CRNH-RA) pour son aide dans l'analyse des lipides fécaux. Cette étude a été financée par le CNIEL.

Conflits d'intérêts : M. Milard a reçu une subvention/soutien de CNIEL, A. Penhoat: Aucun conflit à déclarer, V. Sauvinet: Aucun conflit à déclarer, A. Durand: Aucun conflit à déclarer, E. Meugnier: Aucun conflit à déclarer, E. Loizon: Aucun conflit à déclarer, C. Buisson: Aucun conflit à déclarer, L. Bindels: Aucun conflit à déclarer, A. Neyrinck: Aucun conflit à déclarer, N. Delzenne: Aucun conflit à déclarer, F. Laugerette: Aucun conflit à déclarer, M.-C. Michalski a reçu une subvention/soutien de CNIEL, est consultant chez CNIEL 
L'inflammation intestinale de rats carencés en folate et vitamine B12 et exposés au DSS produit une stéatohépatite nonalcoolique (NASH) liée à l'augmentation de MCP-1 et IL-1beta

Z. Harb ${ }^{1,}{ }^{*}$, L. Lagrost ${ }^{2}$, A. Marchal Bressenot ${ }^{3}$, J. Raso ${ }^{1}$, J.-M. Alberto ${ }^{1}$, R. Umoret ${ }^{1}$, J.-P. Bronowicki ${ }^{4}$, J.-L. Guéant ${ }^{5}$

${ }^{1}$ Inserm - U1256, Nancy, ${ }^{2}$ Inserm - U866 Faculté de Médecine, Dijon, ${ }^{3}$ Hôpital Robert Debré, Reims, ${ }^{4}$ Hépato-Gastro-Entérologie, CHRU Nancy, ${ }^{5}$ Laboratoire de biochimie et de biologie moléculaire, CHRU Nancy,Inserm U - 1256, Nancy, France

Introduction et but de l'étude : La stéatohépatite non-alcoolique (NASH) est une forme sévère de maladie non-alcoolique du foie (NAFLD) dont le risque de survenue est augmenté chez les patients atteints d'une maladie inflammatoire chronique de l'intestin ( $\mathrm{MICl}$ ). La carence en donneurs de méthyle (CDM) combinée à l'exposition au sulfate de dextran (DSS) dans l'eau de boisson est un modèle expérimental de forme sévère de $\mathrm{MICl}$ qui fragilise la barrière intestinale. L'augmentation de la perméabilité intestinale et la translocation des endotoxines bactériennes (LPS) sont deux mécanismes des MICl qui participent aux manifestations inflammatoires systémiques des $\mathrm{MICl}$. Nous avons d'étudié si l'inflammation intestinale sévère et la translocation des endotoxines bactériennes (LPS) contribuent à l'augmentation du risque de NASH dans le modèle CDM/DSS.

Matériel et méthodes : Des rats Wistar mâles âgés de deux mois ont été soumis à un régime CDM et ingestion de DSS et comparés à des rats témoins. Les concentrations de LPS et de cytokines plasmatiques ont été mesurées. Des coupes histologiques du foie ont été effectuées. L'expression hépatique et intestinale des différents cytokines et chimiokines été étudié par western blot. L'inflammasome et l'immunité innée liée à l'activation du Toll-like receptor 4 (TLR4) par le LPS ont également été étudiées au niveau de foie.

Résultats et Analyse statistique : Les rats du groupe DSS présentaient une stéatose, une inflammation sans fibrose et un taux normal de I'LPS. Les concentrations plasmatiques et l'expression hépatique et intestinale de la chimiokine CCL2, également denomée monocyte chemoattractant protein $1(\mathrm{MCP}-1)$ et d'IL-1ß étaient augmentées. La voie NFkB était activée au niveau hépatique demontré par une expression accrue de ces differents modulateurs. L'activation du complexe IKK (IKK $\alpha$ et IKKß) était associée à la phosphorylation d'IkB $\alpha$ et la translocation nucléaire de NFkB. Cette inflammation n'était pas médiée par le LPS. La voie TLR4 n'était pas activée dans le foie. L'analyse statistique des résultats a été faite par T-test et ANOVA.

Conclusion : Nos résultats suggèrent que la NASH produite dans le modèle CDM/DSS n'est pas secondaire à un mécanisme médié par l'activation de TLR4 par le LPS. L'inflammation sévère intestinale du modèle CDM/DSS augmentent le risque de NASH via l'augmentation d'IL-1ß et MCP-1. Ces résultats suggèrent d'évaluer le rôle d'IL-1ß et de MCP-1 dans les manifestations systémiques des $\mathrm{MICl}$.

Références : Min Chen et al.2011. "Methyl deficient diet aggravates experimental colitis in rats". J. Cell. Mol.Med.Vol 15, No 11, pp. 2486-2497

Johannie du Plessis et al. "Pro-inflammatory cytokines but not Endotoxin-related parameters associate with disease severity in patients with NAFLD". Plos One.DOI :10.1371

Wendy L. et al. 2009. "Inflammatory cytokines stimulate the chemokines CCL2/MCP-1 and CCL2/MCP-7 through NFkB and MAPK dependent pathways in rat astrocytes. Brain resh.doi:10.1016

Remerciements : Ce travail a été financé par un contrat doctoral du MESRI et par le FHU ARRIMAGE.

Conflits d'intérêts : Aucun conflit à déclarer 
L'effet antiprolifératif du cholest-4-en-3-one, un métabolite du microbiote intestinal, est associé à une diminution de la lipogenèse et à une désorganisation des radeaux lipidiques dans deux modèles de cellules cancéreuses mammaires

J. Elia ${ }^{1,{ }^{*}}$, D. Carbonnelle ${ }^{1}$, C. Gaudin ${ }^{1}$, J.-M. Huvelin ${ }^{1}$, A. Burghelea ${ }^{1}$ au nom de Technicienne, M. Tannoury ${ }^{2}$, M. Diab-Assaf $^{2}$, K. Petit ${ }^{1}$, E.-H. Nazih ${ }^{1}$

${ }^{1}$ Université de Nantes, Nantes, France, ${ }^{2}$ Université Libanaise, Fanar, Liban

Introduction et but de l'étude : Le cholest-4-en-3-one est un métabolite du cholestérol résultant notamment de son oxydation dans le tractus gastro-intestinal, en particulier par le microbiote intestinal. Il a été identifié dans les algues marines vertes (Ulva armoricana) et rouges (Solieria chordalis, Laurencia obtusa), et par notre équipe "Mer, Molécules, Santé » dans un extrait de Laurencia papillosa collectée de la côte Libanaise. Récemment, il a été démontré que le cholest-4-en-3-one possède des activités biologiques intéressantes telles que des activités antitumorales et anti-obésité. Il est également considéré comme un composé thérapeutique potentiel pour la prévention et / ou le traitement de la maladie d'Alzheimer. Plusieurs études ont montré une exacerbation du métabolisme lipidique dans les cellules tumorales. L'objectif de nos travaux consiste à étudier les effets biologiques du cholest-4-en-3-one sur les cellules tumorales mammaires (MCF-7 et MDA-MB-231) en étudiant la cytotoxicité et la migration cellulaire, ainsi que l'expression de la Flotillin-2 au niveau des radeaux lipidiques, plateformes de signalisation membranaires. Parallèlement, nous évaluons l'influence du cholest-4-en-3-one sur le métabolisme lipidique des cellules tumorales mammaires en étudiant l'expression des enzymes lipogéniques.

Matériel et méthodes : La cytotoxicité du cholest-4-en-3-one sur les cellules tumorales mammaires a été évaluée par le test MTT et la migration cellulaire par le test wound-healing. Les radeaux lipidiques ont été isolés par ultracentrifugation sur gradient de densité et l'expression de la Flotillin-2 a été étudiée par western blot sur les fractions récoltées. Des RT-qPCR ont également été réalisées pour déterminer l'expression des gènes et des enzymes impliquées dans la lipogenèse ainsi que dans la synthèse du cholestérol (acétyl-CoA carboxylase (ACC), acide gras synthase (FASN) et hydroxyméthylglutaryl-CoA réductase (HMGCR), respectivement).

Résultats et Analyse statistique : Le cholest-4-en-3-one a présenté une activité cytotoxique sur les cellules tumorales mammaires et a réduit la migration cellulaire. De plus, ce composé a altéré les radeaux lipidiques, ce qui est démontré par une diminution de l'expression de la Flotillin-2, impliquée dans plusieurs voies de signalisation dans les cellules tumorales. Le cholest-4-en-3-one a également diminué l'expression de l'ACC et de la FASN réduisant ainsi la lipogenèse. En outre, il a réduit l'expression de HMGCR, l'enzyme clé dans la synthèse du cholestérol.

Conclusion : Nos résultats révèlent que le cholest-4-en-3-one pourrait avoir une activité antiproliférative sur les cellules tumorales mammaires via l'inhibition de la lipogenèse et de la synthèse du cholestérol. En conséquence, l'apport nutritionnel en cholest-4en-3-one ou l'induction de sa production via le microbiote intestinal pourrait être une stratégie prometteuse pour le traitement et / ou la prévention du cancer du sein.

Conflits d'intérêts : Aucun conflit à déclarer 
La taurine prévient la peroxydation lipidique chez les souris sensibilisées à la $\boldsymbol{\beta}$-Lactoglobuline

S. Ainad Tabet ${ }^{1,},{ }^{*}$ A. Haddi ${ }^{1}$, H. Negaoui ${ }^{1}$, O. Kheroua ${ }^{1}$, D. Saidi ${ }^{1}$

${ }^{1}$ Laboratoire de la Physiologie de la Nutrition et de la Sécurité Alimentaire. Faculté des Sciences de la Nature et de la Vie, Université d'Oran 1 Ahmed Benbella, Algérie, Oran, Algérie

Introduction et but de l'étude : L'augmentation du stress oxydant via la production d'espèces réactives de l'oxygène (ROS) par les cellules immunitaires entraîne des effets délétères sur l'architecture villositaire ainsi qu'un dysfonctionnement de la fonction intestinale. Il a été montré sur des modèles animaux d'inflammation intestinale que la taurine (acide 2-aminoéthanesulfonique) pouvait exercer certains effets protecteurs antioxydants.

L'objectif de cette étude est d'évaluer l'effet de la taurine sur la peroxydation lipidique intestinale chez des souris sensibilisées à la $\beta$-Lactoglobuline $(\beta$-Lg), allergène majeur du lait bovin.

Matériel et méthodes : Pour le besoin de cette étude, 18 souris Balb/c sont réparties en 3 lots de 6 souris chacun :

-Le groupe témoin négatif ne recevant aucun traitement (T-);

-Le groupe expérimental (Tau) recevant $3 \mathrm{mmol} / \mathrm{kg} / \mathrm{jour}$ de taurine par gavage pendant 15 jours puis immunisé à la $\beta$-Lg par voie intrapéritonéale.

-Le groupe témoin positif immunisé à la $\beta$-Lg par voie intrapéritonéale $(T+)$. Chaque souris reçoit une dose de $100 \mu l$ d'une solution de PBS, pH 7,4 contenant $10 \mu \mathrm{g}$ de $\beta$ - $\mathrm{Lg}$ mélangée à $2 \mathrm{mg} \mathrm{d}^{\prime} \mathrm{Al}(\mathrm{OH})_{3}$. Les injections ont lieu à Jo puis, sous forme de rappels et dans les mêmes conditions au $14^{\text {ème }}, 21^{\text {ème }}$ et $28^{\text {ème }}$ jour du protocole.

Le degré de sensibilisation des souris à la $\beta$-Lg est évalué par un dosage ELISA des IgG sériques spécifiques. La peroxydation lipidique au niveau jéjunal est mesurée par la détermination des substances réactives à l'acide thiobarbiturique (TBARS).

Résultats et Analyse statistique : Le niveau de sensibilisation à la $\beta$-Lg atteint un titre très élevé de $1 / 10000000^{\mathrm{ème}}$ chez les souris $(T+)$ et $n^{\prime}$ est que de $1 / 516^{\text {ème }}$ chez les souris (Tau) $(p<0,001)$.

Les teneurs en TBARS au niveau intestinal des souris $(T+)$ sont significativement plus élevées que celles des souris témoins ( $T$-) (4 \pm 0.008 vs $5.2 \pm 0.015) \mu \mathrm{mol} / \mathrm{mL}(\mathrm{p}<0,01)$.

L'administration préventive de la taurine à $3 \mathrm{mmol} / \mathrm{kg} / \mathrm{j}$ chez les souris (Tau) réduit significativement la peroxydation lipidique par rapport à celle du groupe sensibilisé à la $\beta-\operatorname{Lg}(T+)(4.8 \pm 0.006 \pm 5.2 \pm 0.015) \mu \mathrm{mol} / \mathrm{mL}(p<0,03)$.

Conclusion : Nos résultats montrent clairement que l'effet de la sensibilisation des souris par la $\beta$-Lg se traduit par l'induction d'un stress oxydant au niveau intestinal. La taurine, par des mécanismes qui restent à élucider, semble diminuer significativement cet effet.

Mots clés: Taurine, $\beta$-Lactoglobuline, Sensibilisation, Peroxydation lipidique, Souris Balb/c.

Conflits d'intérêts : Aucun conflit à déclarer 


\section{P191}

Mise en place d'une hyperphagie associée à une adaptation jéjunale et colique dans un modèle de grêle court chez le rat A. Le Moigne ${ }^{1}$, A.-C. Jarry ${ }^{1}$, L. Ribeiro-Parenti ${ }^{1}$, M. Le Gall ${ }^{1}$, A. Bado ${ }^{1}$, F. Joly ${ }^{1}$, J. Le Beyec - Le Bihan ${ }^{1,{ }^{*}}$

${ }^{1}$ U1149, INSERM, Paris, France

Introduction et but de l'étude : Le syndrome de grêle court (SGC) résulte d'une résection étendue de l'intestin et se caractérise par une insuffisance intestinale chronique. Des adaptations spontanées (hyperphagie, microbiote dysbiotique, adaptation morpho-fonctionnelle) sont observées chez les patients SGC. Nous avons validé un modèle murin de SGC et montré que dès 7 jours après résection une adaptation intestinale se met en place avec hyperplasie colique et augmentation de l'expression et de la sécrétion de nombreuses hormones gastro-intestinales (GLP-2, GLP-1, PPY mais aussi de la ghréline). Parallèlement, une forte augmentation de l'expression des neuropeptides orexigènes hypothalamiques (AgRP et NPY) a été observée (Gillard et al. 2016). L'objectif de cette étude était d'analyser l'adaptation à long terme (21 jours post-op.) de ce modèle murin de résection étendue de l'intestin avec anastomose jéjuno-colique (IRJC).

Matériel et méthodes : Des rats Wistar ont subi une résection de 75\% d'intestin grêle, de la valve iléo-caecale et une colectomie partielle (IR) ou une transsection intestinale (sham). La perte de poids et la prise alimentaire ont été mesurées quotidiennement pendant 21 jours. Le jour du sacrifice, du sang a été prélevé (dosages d'albumine, d'entérhormones) ainsi que l'hypothalamus, l'intestin grêle et le colon restants. Les fragments intestinaux et coliques ont été fixés pour des analyses morphométriques ou congelés pour analyse par RT-qPCR des ARNm du proglucagon, PYY, Pept1. Les ARNm codant pour les neuropeptides hypothalamiques (AgRP, NPY...) ont également été quantifiés par RT-qPCR. Les résultats sont exprimés en moyenne \pm esm et analysés par des tests non paramétriques.

Résultats et Analyse statistique : Sept jours après la résection les rats IRJC avaient perdu 20\% de leur poids initial, comme précédemment décrit (Gillard et al. 2016) et leur prise alimentaire était similaire à celle des rats sham. A 14 jours post-opératoires certains rats IRJC ont repris du poids, et à J21 les rats IRJC ne présentaient plus que $10 \%$ de perte de poids par rapport au poids initial. La prise alimentaire des rats IRJC a augmenté à J8 post-opératoire, s'est stabilisée entre J14 et J21 et était significativement plus importante que celle des rats sham ( $65 \% \mathrm{P}<0.001)$. Cette hyperphagie est associée à l'augmentation de l'expression des neuropeptides hypothalamiques orexigènes, qui a débuté à $J 6$ et est encore observée à J21 (AgRP : x3.8, $P<0.01$ vs sham et NPY $x 2, P<0.01$ vs sham). A J21, l'hyperplasie de la muqueuse colique restante s'accompagne d'une hyperplasie de la muqueuse jéjunale. Une adaptation fonctionnelle avec une augmentation de l'expression de transporteurs de nutriments (Pept1...) et des taux d'ARNm d'entérohormones du tractus gastro-intestinal est aussi rapportée.

Conclusion : Ces résultats indiquent que l'adaptation se poursuit avec mise en place d'une hyperphagie probablement secondaire à l'augmentation des neuropeptides orexigènes. L'augmentation de la surface d'échange participe également à l'amélioration de la fonction d'absorption. Ce modèle murin de SGC nous permet maintenant de développer des approches thérapeutiques pour augmenter ces adaptations.

Références : L. Gillard et al. Sci.Rep. 2016;6:28345

Conflits d'intérêts : Aucun conflit à déclarer 
Evaluation de la flore bactérienne intestinale chez le rat wistar malnutris : impact de la réalimentation par un blé fermenté type Hamoum.

D. Yssaad ${ }^{1, *}$, B. M. Benakriche ${ }^{2}$, D. Saidi ${ }^{1}$, O. Kheroua ${ }^{1}$

${ }^{1}$ Laboratoire de Physiologie de la Nutrition et de Sécurité Alimentaire. LPNSA, Université d'Oran1- Faculté des Sciences de la Vie et de la Nature, Oran, ${ }^{2}$ Université de Mostaganem, Mostaganem, Algérie

Introduction et but de l'étude : : La malnutrition protéique entraine non seulement de lourdes déficiences sur la croissance mais affecte également les structures digestives et la flore bactérienne intestinale. L'objectif de travail est de vérifier si le protocole de réalimentation avec un régime à base de blé fermenté Hamoum (BFH), riche en bactéries lactiques, en nutriments essentiels a un impact sur le rétablissement de la flore bactérienne intestinale chez le rat malnutris en période de sevrage.

Matériel et méthodes : Nous avons utilisé 48 rats Wistar mâle âgés de 4 semaines, répartis en cinq groupes. Le premier groupe constitue le groupe témoin a reçu un régime standard équilibré avec un apport protéique à $20 \%$ caséine, le deuxième groupe constitue le groupe malnutris a reçu un régime carencé en protéines pendant 28 jours ( $2 \%$ de caséine). Le troisième, quatrième et cinquième groupe constituant les groupes malnutris et réalimentés respectivement avec le BFH, le régime à base de blé non fermenté (BNF) et le régime standard (STD) pendant une période de 28 jours. Nous avons évalué la croissance pondérale des rats, le taux des bactéries lactiques, de coliformes totaux et d'E.Coli au niveau de l'estomac et l'intestin grêle.

Résultats et Analyse statistique : Les résultats obtenus montrent que la malnutrition protéique induit une perte très significative du poids total des rats par rapport aux témoins $(p<0.0001)$. En revanche, les résultats de la phase de réalimentation montrent une reprise significative du poids dans le groupe malnutris et réalimenté avec le BFH. Nos résultats montrent que le taux des bactéries lactiques au sein de la flore bactérienne intestinal est très faible chez le groupe malnutris par rapport au témoin, alors que le nombre des coliformes totaux et des E.coli était plus élevé. Après la réalimentation par le BFH, nous avons constaté une diminution des coliformes totaux et des E.coli et une augmentation du nombre des bactéries lactique dans les différents compartiments intestinaux.

Conclusion : Le blé fermenté traditionnel type Hamoum renferme des microorganismes ayant des vertus nutritionnelles et diététiques très appréciés dans le rétablissement du microbiote intestinale dans les situations de malnutrition protéique sévère.

Conflits d'intérêts : Aucun conflit à déclarer 
Etude du métabolisme central et périphérique du tryptophane et de la kynurénine dans un modèle de dénutrition secondaire à une inflammation aigue

H. Hounkponou ${ }^{1}$, P. Tirelle ${ }^{1}$, E. Salameh ${ }^{1}$, C. L'Huillier ${ }^{1}$, W. Bahlouli ${ }^{1}$, C. Guérin ${ }^{1}$, C. Bôle-Feysot ${ }^{1}$, J. C. do Rego ${ }^{2}$, A. Goichon ${ }^{1}$, P. Déchelotte ${ }^{13}$, R. Marion-Letellier $^{1}, \mathrm{~N}$. Achamrah ${ }^{13}$, M. Coëffier $^{13,{ }^{*}}$

${ }^{1}$ INSERM UMR1073, ${ }^{2}$ Plateforme d'analyse du comportement SCAC, Université de Rouen Normandie, ${ }^{3}$ Département de Nutrition, $\mathrm{CHU}$, Rouen, France

Introduction et but de l'étude : L'inflammation est une cause de dénutrition majeure, elle-même fréquemment associée à des troubles de l'humeur. La régulation de l'humeur est en partie liée au métabolisme du tryptophane (TRP), précurseur de la sérotonine, mais également de la kynurénine (KYN), de l'acide kynurénique (KYNA) et de l'acide quinolinique (QA). Au niveau central, KYNA joue un rôle neuroprotecteur alors que QA a des propriétés neurotoxiques. Or, l'inflammation régule ces différentes voies métaboliques du TRP. Le but de ce travail a été d'étudier, chez la souris, le métabolisme périphérique et central du TRP et de la KYN dans un modèle de dénutrition secondaire à une inflammation.

Matériel et méthodes : Des souris mâles C57BI/6 ( $n=8-10$ / groupe) ont été réparties en 3 groupes : contrôle ad libitum (CT), LPS et contrôle pair-fed (vs LPS noté PF). Les souris ont reçu à deux temps (J1 et J4) des doses croissantes de LPS ( 1 et $3 \mathrm{mg} / \mathrm{kg}$ en i.p.) ou du PBS. A J5, un test comportemental à deux compartiments " éclairé-obscur » a été réalisé pour évaluer le comportement anxio-dépressif. Les taux des ARNm codant pour les enzymes impliquées dans le métabolisme du TRP et de la KYN ont été mesurés dans différents tissus périphériques et centraux, ainsi que des marqueurs inflammatoires (CXCL1 plasmatique et calprotectine fécale).

Résultats et Analyse statistique : Les concentrations de CXCL1 plasmatique et de calprotectine fécale étaient significativement augmentées chez les souris LPS à $J 2$ mais plus à J5. La perte de poids était de $9,6 \pm 2,2 \%$ à J5 dans le groupe LPS ( $p<0,05$ vs CT) contre $14,7 \pm 0,8 \%$ dans le groupe $P F(p<0,05$ vs CT et LPS). Concernant le métabolisme périphérique TRP-KYN, les souris $P F$ présentaient une diminution du taux d'ARNm codant pour IDO2 au niveau hépatique, associée à une diminution de la concentration plasmatique de son produit, la $\operatorname{KYN}(1,22 \pm 0,07$ vs $1,70 \pm 0,18, p<0,05)$, sans modification au niveau central. Au contraire, les souris LPS présentaient une diminution des taux d'ARNm codant pour la TRP hydroxylase-2 au niveau de l'hypothalamus et de l'amygdale ( $p<0,05$ vs $C T$ ). Seules les souris LPS présentaient une réponse comportementale modifiée avec une diminution des activités totales horizontale et verticale par rapport aux souris $C T$ et $P F(p<0,05)$, une diminution du nombre de changements de compartiments (15 \pm 3 chgts) et une augmentation du temps d'immobilité dans le compartiment éclairé (76,9 $\pm 3,3 \mathrm{sec})$ par rapport aux souris CT ( $27 \pm 3$ chgts ; 62,2 $\pm 2,4 \mathrm{sec}, \mathrm{p}<0,05)$ et PF $(25 \pm 1$ chgts ; 67,4 $\pm 1,7 \mathrm{sec}, p<0,05)$.

Conclusion : La dénutrition persistante après un épisode $d^{\prime}$ inflammation aigue est associée à des troubles anxio-dépressifs et des modifications centrales du métabolisme du TRP, modifications centrales non observées dans un modèle de dénutrition par carence d'apports. Ce travail incite à explorer les flux des métabolites du TRP et de la KYN dans différentes situations de dénutrition.

Remerciements : Ce travail a reçu le soutien financier de la Nutricia Research Foundation et de la société Européenne de Nutrition Clinique et Métabolisme (ESPEN)

Conflits d'intérêts : Aucun conflit à déclarer 


\section{P194}

La supplémentation en glutamine restaure la fonction de barrière intestinale dans un modèle murin d'anorexie.

C. L'huillier ${ }^{12}{ }^{*}$, M. Jarbeau ${ }^{12}$, L. Belmonte ${ }^{12} 3$, S. Nobis ${ }^{12}$, A. Goichon ${ }^{12}$, É. Salameh ${ }^{12}$, W. Bahlouli ${ }^{12}$, J.-C. do Rego ${ }^{24}$, P. Déchelotte ${ }^{123}, M$. Coëffier ${ }^{123}$

${ }^{1}$ INSERM UMR 1073, Normandie Université, '2Institut pour la Recherche et l'Innovation Biomédicale (IRIB), UNIROUEN, ${ }^{3}$ Département de Nutrition, CHU de Rouen, ${ }^{4}$ Service Commun d'Analyse du Comportement, SCAC, Rouen, France

Introduction et but de l'étude : L'axe microbiote-intestin-cerveau est impliqué dans la régulation du comportement alimentaire. Au cours de l'anorexie mentale, la dénutrition est fréquemment associée à des troubles de l'humeur et des troubles fonctionnels digestifs. Dans le modèle murin activity-based anorexia ( $A B A)$, la dénutrition s'accompagne d'une augmentation de la perméabilité intestinale. Nous avions donc pour objectif ici d'évaluer les effets de la glutamine (GIn), substrat entérocytaire préférentiel et facteur de trophicité intestinale, sur la fonction de barrière intestinale chez des souris ABA.

Matériel et méthodes : Quarante-huit souris mâles C57BL/6 ont été placées dans des cages individuelles et réparties entre groupes Contrôle (CTRL; $n=16)$ et $A B A(n=32)$. Les souris $A B A$ avaient un accès libre à une roue d'activité. Après 5 jours d'acclimatation, l'accès à la nourriture a été progressivement réduit pour les souris ABA de 6h/jour au jour 6 à $3 \mathrm{~h} / \mathrm{jour}$ au jour 9 et jusqu'au jour 17. À partir de J11, les souris $A B A$ ont reçu une supplémentation en Gln à $1 \%(A B A-G ; n=16)$ ou non $(A B A-C$; $\mathrm{n}=16)$. Cette supplémentation a été administrée via l'eau de boisson pour atteindre l'objectif de $2 \mathrm{~g} / \mathrm{kg} / \mathrm{jour}$. La solution de $\mathrm{Gln}$ a été renouvelée chaque jour. Une injection intrapéritonéale de puromycine $(0,04 \mu \mathrm{mol} / \mathrm{g})$ était réalisée 20 minutes avant la mise à mort en vue d'évaluer le taux de synthèse protéique. La perméabilité intestinale a été mesurée ex vivo en chambres de Ussing sur des segments coliques. Les résultats ont été analysés en ANOVA une-voie avec un seuil de significativité à 0,05.

Résultats et Analyse statistique : Chez les souris $A B A$, la prise alimentaire et le poids étaient significativement diminués $(p<0,05$ vs CTRL), avec une diminution de la masse maigre et de la masse grasse. La Gln n'a pas eu d'effet sur la prise alimentaire, le poids, la composition corporelle et sur l'activité physique. Au niveau hypothalamique, les taux d'ARNm codant pour les neuropeptides orexigénique NPY et anorexigénique POMC n'étaient pas modifiés par la Gln. La perméabilité colique était augmentée dans le groupe ABA-C ( $+59 \%$ vs CTRL; $p<0,05)$ mais pas dans le groupe ABA-G $(-3 \%$ vs CTRL). Le taux d'ARNm codant pour la mucine- 2 , composant majeur du mucus, était augmenté dans le groupe $A B A-G(p<0,05$ vs $A B A-C)$. Enfin, la synthèse protéique de la muqueuse colique était diminuée dans le groupe $A B A-C(50 \%$ vs $C T R L ; p<0,05)$ mais pas dans le groupe $A B A-G(94 \%$ vs $C T R L)$.

Conclusion : En conclusion, la glutamine restaure la fonction de barrière colique dans le modèle murin $A B A$ sans effets à court terme sur le poids ou la composition corporelle. Ces résultats incitent à évaluer les effets de la glutamine sur les troubles intestinaux fréquemment observés au cours de l'anorexie.

Remerciements : Cette étude a été financée par un don de la société Nutriset à la Fondation Charles Nicolle.

Conflits d'intérêts : Aucun conflit à déclarer 
Effet d'un régime riche en sel sur la fibrose intestinale dans un modèle murin de colite chronique.

A. Amamou ${ }^{12, *}$, M. Rouland ${ }^{12}$, E. Salameh ${ }^{12}$, L. Yaker ${ }^{12}$, M. Jarbeau ${ }^{12}$, C. Guérin ${ }^{12}$, A. Goichon ${ }^{12}$, G. Savoye ${ }^{12} 3$, R. Marion-Letellier ${ }^{12}$

${ }^{1}$ INSERM UMR 1073, Université Rouen Normandie, ${ }^{2}$ Institut de Recherche et d'Innovation Biomédicale (IRIB), Rouen université, ${ }^{3}$ Département de gastroentérologie, CHU Charles Nicolle, Rouen, France

Introduction et but de l'étude : La fibrose intestinale est une complication fréquente chez les patients atteints de maladies inflammatoires chroniques de l'intestin ( $\mathrm{MICl}$ ). Les $\mathrm{MICl}$ se développent chez des personnes avec une susceptibilité génétique sous l'influence de facteurs environnementaux comme l'alimentation. Une alimentation riche en sel est délétère dans les fibroses extraintestinales et dans le développement de colites expérimentales. Le but de ce travail a été d'étudier l'effet d'une alimentation riche en sel sur la fibrose intestinale dans un modèle de colite chimio-induite chronique chez le Rat.

Matériel et méthodes : La colite chronique a été induite de façon hebdomadaire par une injection rectale de 2,4,6 trinitrobenzene sulfonic acid (TNBS) à doses croissantes $(15,30,45 \mathrm{mg})$ chez des rats Sprague-Dawley pendant 3 semaines. Les animaux ont reçu une alimentation standard pour les groupes contrôles $(n=10)$ et TNBS $(n=6)$ ou une alimentation enrichie à $4 \%$ de $\mathrm{NaCl}$ pour le groupe TNBS+sel $(n=9)$ pendant 4 semaines. Le poids et la composition corporelle ont été mesurés. Différents marqueurs de l'inflammation (longueur/poids du colon) et de la fibrose intestinale ( $\alpha$-SMA, COL1A1, CTGF) ont été évalués par Western Blot ou par qPCR. L'activité des métalloprotéinases matricielles 2 et 9 (MMP2 et MMP9) a été évaluée par zymographie.

Résultats et Analyse statistique : La colite chronique induit une diminution significative de la prise de poids $(-8,7 \% ; p<0,001)$ sans modification significative de la composition corporelle. La colite chronique induit une augmentation du ratio taille/poids du colon, marqueur inflammatoire (CT 0,08 $\pm 0,01$ vs TNBS $0,23 \pm 0,04 ; \mathrm{p}<0,001)$. L'alimentation riche en sel diminue significativement la prise de poids des animaux colitiques $(-10 \% ; p<0,01)$ ainsi que leurs masses maigre $(-17 \% ; p<0,001)$ et grasse $(-31 \% ; p<0,05)$. L'alimentation riche en sel augmente significativement l'activité de la MMP-9 colique $(p<0,001)$.

Conclusion : Cette étude montre qu'une alimentation riche en sel a des effets délétères sur les animaux avec une colite chronique : perte de poids plus marquée et augmentation de marqueurs de fibrose. Le sel pourrait être un facteur environnemental qui contribue au développement de la fibrose intestinale.

Remerciements : Asma Amamou est allocataire d'une bourse de thèse financée par l'INSERM à 100\%.

Conflits d'intérêts : Aucun conflit à déclarer 
Etude de l'interaction entre des dérivés du tryptophane et le récepteur aryl hydrocarbone dans un modèle in vitro de fibrose intestinale

A. Amamou ${ }^{12, *}$, L. Yaker ${ }^{12}$, C. Bôle-Feysot ${ }^{12}$, G. Savoye ${ }^{123}$, R. Marion-Letellier ${ }^{12}$

${ }^{1}$ INSERM UMR 1073, Université Rouen Normandie, ${ }^{2}$ Institut de Recherche et d'Innovation Biomédicale (IRIB), Université de Rouen, ${ }^{3}$ Département de gastroentérologie, CHU Charles Nicolle, Rouen, France

Introduction et but de l'étude : La fibrose intestinale est une complication fréquente chez les patients atteints de maladies inflammatoires chroniques de l'intestin. Elle se caractérise par un dépôt excessif de protéines de la matrice extracellulaire produites par les myofibroblastes activés. Dans des modèles expérimentaux de fibrose extra-intestinale, il a été montré que les dérivés du tryptophane tel que le 6-formylindolo[3,2-b]carbazole (FICZ), la kynurénine et le (1'H-indole-3'-carbonyl)thiazole-4acide carboxylique methyl ester (ITE) exercent des propriétés anti-fibrotiques via le récepteur aryl hydrocarbone (AhR). Le but de ce travail a été d'étudier l'effet des dérivés du tryptophane dans un modèle de fibrose intestinale in vitro et leurs effets sur le récepteur AhR.

Matériel et méthodes : La lignée cellulaire de fibroblastes humains coliques CCD-18Co a été induite par 10ng/ml de TGF $\beta$ pendant $24 \mathrm{~h}$ pour reproduire un modèle in vitro de fibrose intestinale. Les cellules ont ensuite été incubées 24h avec le FICZ (10-100-1000 $n M)$, la kynurénine (0,1-1-10 $\mu \mathrm{M})$ ou l'ITE (1-10-100 $\mu \mathrm{M}) ;(\mathrm{n}=4$ expériences indépendantes). La modulation de l'AhR et des marqueurs de fibrose tels que $\alpha$-SMA, CTGF, COL1A1 et COL3A1 ont été étudiés par Western Blot ou RT-qPCR. L'activité des métalloprotéinases matricielles 2 et 9 a été étudiée par zymographie.

Résultats et Analyse statistique : Le TGF $\beta$ augmente l'expression des ARNm codant pour les marqueurs de fibrose CTGF, COL1A1 et COL3A1 (TGF $\beta$ vs CT; $p=0,0286$ ) et augmente sans atteindre le seuil de significativité l'expression protéique de l' $\alpha$-SMA $(p=0,0571)$. Aucune différence significative de l'expression de l'AhR ou des marqueurs fibrotiques n'a été mise en évidence après traitement des cellules par le FICZ, la kynurénine ou I'ITE.

Conclusion : Le traitement par le TGF $\beta$ induit l'expression de marqueurs de fibrose dans la lignée CCD-18Co. Cependant, les dérivés du tryptophane testés n'ont pas modifié de façon significative les marqueurs de fibrose intestinale dans les conditions testées.

Remerciements : Asma Amamou est allocataire d'une bourse de thèse financée par I'INSERM à 100\%.

Conflits d'intérêts : Aucun conflit à déclarer 
Identification de la protéine ClpB (caseinolytic peptidase B), mimétique du neuropeptide anorexigène $\alpha$-MSH ( $\alpha$-melanocytestimulating hormone) chez la souche Hafnia alvei 4597 par technique LC-MS/MS DIA

N. Lucas ${ }^{1}$, M. Dominique ${ }^{123}$, C. Deroissart ${ }^{1}$, J. Vowinckel ${ }^{4}$, K. Novy $^{4}$, T. Treiber $^{4}$, P. Déchelotte ${ }^{1235}$, G. Lambert $^{1}$, R. Legrand $^{1, *}$ ${ }^{1}$ TargEDys SA, Université de Rouen, ${ }^{2}$ Inserm UMR1073 - nutrition, inflammation et dysfonction de l'axe intestin-cerveau, ${ }^{3}$ Institute for Research and Innovation in Biomedicine (IRIB), Normandie Université, Rouen, France, ${ }^{4}$ Biognosys AG, Wagistrasse 21, Schlieren, Suisse, ${ }^{5}$ Centre Hospitalier Universitaire Charles Nicolle, Normandie Université, Rouen, France

Introduction et but de l'étude : De nombreuses altérations du microbiote intestinal (ou dysbioses) ont été recensées chez des patients atteints d'obésité ou d'anorexie. En effet, il a été démontré une augmentation de la population appartenant à l'ordre des Enterobacteriaceae et notamment de la souche Escherichia coli dans l'anorexie. Cette augmentation était inversement corrélée à l'indice de masse corporelle. Ainsi, les entérobactéries pourraient jouer un rôle clé dans le développement et/ou le maintien des troubles du comportement alimentaire et de l'obésité. De récentes études ont mis en évidence que la souche Escherichia coli K12 (E.coli) appartenant aux entérobactéries est capable de produire une protéine possédant un mimétisme avec le neuropeptide anorexigène $\alpha$-MSH ( $\alpha$-melanocyte-stimulating hormone) : la ClpB (caseinolytic protease $B$ ). La présente étude a pour but de confirmer l'expression de la ClpB dans une autre souche d'entérobactérie, Hafnia alvei 4597 par une approche protéomique LCMS/MS dite de "data independent acquisition" (DIA).

Matériel et méthodes : Pour cette étude, les protéines issues de trois cultures de H.alvei 4597 et E.coli K12 ont été utilisées. Afin de ne détecter que les protéines ayant des séquences similaires avec l' $\alpha-M S H$, une immunoprécipitation avec un anticorps anti$\alpha-M S H$ a été effectuée puis une technique LC-MS/MS DIA a été réalisée. Les données obtenues ont été analysées grâce aux logiciel Spectronaut Pulsar 11 (Biognosys) et les résultats ont été croisés avec les bases de données d’E.coli (UniProt TrEMBL, 83333) et d'H.alvei (UniProt TrEMBL, 13337) afin d'identifier les protéines. Seules les protéines ayant une $Q$-value $<0,001$ et un log2 fold change $>0,4$ ont été considérées dans cette étude.

Résultats et Analyse statistique : Grâce à cette technique LC-MS/MS DIA, 112 protéines ont été identifiées chez H.alvei 4597 contre 116 chez E.coli K12. La ClpB figurait parmi les 18 protéines communes aux deux souches. De plus, la quantification relative a permis de mettre en évidence que la ClpB était la protéine mimétique $d^{\prime} \alpha-M S H$ la plus abondante après immunoprécipitation dans la souche H.alvei 4597 et qu'elle était significativement augmentée par rapport à E.coli $K 12\left(1,55 \pm 0,08 \times 10^{7} \mathrm{UA}\right.$ versus 0,22 $\pm 0,09 \times 10^{7} \cup A ; p<0,001$ test $t$ de Student). L'alignement des séquences des protéines identifiées a finalement permis de confirmer le mimétisme de la ClpB issue d'H.alvei 4597 pour l'a-MSH sur la séquence pharmacophore du neuropeptide.

Conclusion : Cette étude a permis de démontrer que la souche H.alvei 4597 est capable de produire la ClpB et, ce, en quantité plus importante que la souche E.coli K12. De plus, la ClpB de cette souche présente le même mimétisme avec l' $\alpha$-MSH que la ClpB d'E.coli K12. Ces données sont donc favorables à l'utilisation de la souche H.alvei 4597 comme probiotique visant à lutter contre le surpoids en renforçant les mécanismes naturels de satiété grâce à un effet similaire à celui de l'a-MSH.

Conflits d'intérêts : N. Lucas est employé(e) de TargEDys SA, M. Dominique: Aucun conflit à déclarer, C. Deroissart est employé(e) de TargEDys SA, J. Vowinckel : Aucun conflit à déclarer, K. Novy: Aucun conflit à déclarer, T. Treiber: Aucun conflit à déclarer, P. Déchelotte est consultant chez TargEDys SA, G. Lambert est employé(e) de TargEDys SA, R. Legrand est employé(e) de TargEDys SA 
Introduction et but de l'étude : La chirurgie de l'obésité, incluant la procédure de Roux-en-Y by-pass gastrique (RYGB), est actuellement le seul traitement permettant d'obtenir une perte de poids significative et durable ainsi qu'une amélioration des paramètres métaboliques chez le patient obèse lorsque d'autres méthodes ont échoué. Cependant, les mécanismes sous-jacents sont encore mal connus et les données disponibles sur les adaptations gastro-intestinales restent fragmentaires et parfois contradictoires.

Matériel et méthodes : Afin d'explorer les modifications induites par un RYGB au niveau de la morphologie et des fonctions endocrines gastro-intestinales, nous avons comparé dans cette étude 8 miniporcs Yucatan obèses soumis à un RYGB avec restriction alimentaire et 8 miniporcs ayant subi une chirurgie fantôme (SHAM) et dont la ration alimentaire a été étalonnée sur la consommation des animaux RYGB.

Résultats et Analyse statistique : Trois mois après chirurgie, la concentration plasmatique de la ghréline a augmenté dans le groupe RYGB ( $385 \pm 88$ vs. $234 \pm 75 \mathrm{pg} / \mathrm{ml}, \mathrm{P}<0,05)$ comme dans le groupe SHAM $(428 \pm 108$ vs. $253 \pm 76 \mathrm{pg} / \mathrm{ml}, \mathrm{P}<0,05)$, tandis que celle de la leptine a diminué (RYGB : $11,5 \pm 4,1$ vs. $26,7 \pm 14,7 \mathrm{ng} / \mathrm{ml}$; SHAM : 10,6 $\pm 4,6 \mathrm{vs.} 22,2 \pm 9,0 \mathrm{ng} / \mathrm{ml}, \mathrm{P}<0,05)$. A quatre mois, une perte de poids significative $(P<0,05)$ de $17 \%$ a été observée chez les RYGB (de $94,4 \pm 2,8$ à $76,8 \pm 3,2 \mathrm{~kg}$ ), et de $11 \% \mathrm{chez}$ les SHAM (de $95,2 \pm 1,9$ à $84,1 \pm 2,2 \mathrm{~kg}$ ). Nous avons démontré une atrophie cryptique au niveau de l'estomac exclu (115 $\pm 48 \mu \mathrm{m} v s$. $162 \pm 34 \mu \mathrm{m}$ dans l'estomac normal), mais une augmentation de la taille des cryptes dans la poche gastrique des animaux RYGB $(219 \pm 45 \mu \mathrm{m})$, ainsi qu'une augmentation de la profondeur des glandes $(206 \pm 49 \mu \mathrm{m}$ dans la poche gastrique $v s$. $173 \pm 30 \mu \mathrm{m}$ pour l'estomac normal) $(\mathrm{P}<0,05)$. Au niveau intestinal, la profondeur des cryptes a diminué dans l'anse alimentaire $(284 \pm 45 \mu \mathrm{m} v$. $319 \pm 38 \mu \mathrm{m}$ dans le jéjunum proximal SHAM), tandis que la longueur des villosités a diminué dans l'anse biliaire ( $324 \pm 58 \mu \mathrm{m})$ et l'anse commune $(397 \pm 60 \mu \mathrm{m})$ chez les RYGB en comparaison du jéjunum proximal préopératoire ( $545 \pm 83 \mu \mathrm{m})$ ou du jéjunum distal SHAM $(445 \pm 17 \mu \mathrm{m})(P<0,05)$. Si le nombre de cellules endocrines n'a pas été affecté par le RYGB, en revanche le nombre de cellules à GLP-1 a augmenté dans l'anse alimentaire $\left(2,8^{-6} \pm 9,3^{-7}\right.$ vs. $1,7^{-6} \pm 7,2^{-7}$ cellules/mm2 dans le jéjunum proximal préopératoire, $\mathrm{P}<0,05)$.

Conclusion : Ces résultats montrent l'ampleur des adaptations gastro-intestinales induites par un RYGB, incluant des modifications morphologiques et fonctionnelles dans les parties n'étant plus en contact avec le chyme, une adaptation de la muqueuse gastrique pour compenser la perte de surface, ainsi qu'une modulation de la sécrétion des hormones gastro-intestinales.

Remerciements : Cette étude a été financée par l'Action Prioritaire Hed-O-Shift du département AlimH de l'INRA, pilotée par David Val-Laillet, ainsi que par une bourse de recherche obtenue par Damien Bergeat auprès de l'ESPEN. Yentl Gautier a reçu une bourse de thèse de l'INRA et de la Région Bretagne. Les auteurs remercient les personnels techniques de l'INRA de St Gilles pour leur participation aux soins aux animaux et aux analyses de laboratoire.

Conflits d'intérêts : Aucun conflit à déclarer 
Evaluation du tissu adipeux viscéral et des paramètres glycémiques en post chirurgie bariatrique.

N. C. Dagher ${ }^{1, *}$, R. Damade ${ }^{2}$, A. Monier ${ }^{1}$, C. de Brauer ${ }^{3}$, S. Antoun ${ }^{4}$

${ }^{1}$ Diabétologie nutrition, ${ }^{2}$ Medecine interne, CH Chatres, Chartres, ${ }^{3}$ Santé publique, CHU Avicenne, Bobigny, ${ }^{4}$ Urgences, IGR, Villejuif, France

Introduction et but de l'étude : Les données concernant l'évolution du tissu adipeux viscéral (TAV) lors de la perte de poids sont rares. La perte de poids rapide chez des patients obèses ou en surpoids suivis pour un diabète ou une hyperglycémie à jeun se voit souvent accompagnée d'une amélioration des paramètres glycémiques. L'objectif principal de ce travail était recherche d'un lien entre l'amélioration glycémique et la perte de TAV. Nous avons aussi cherché quel type de tissu adipeux (TAV vs tissu adipeux sous cutané TASC) était le plus impacté par la perte de poids et si la dépense énergétique de repos (DER) était altérée avec le perte de poids.

Matériel et méthodes : La mesure du TA (corps entier) a été faite par absoptiométrie biphotonique à rayons $X$ (DEXA) muni du logiciel coreScan pour l'estimation du TAV, chez 33 sujets ( 27 femmes, 6 hommes) obèses candidats à une chirurgie bariatrique (by pass et sleeve). Les variations de poids, d' indice de masse corporelle (IMC), de masse (MG), la DER, ont été évalué en préopératoire et en post opératoire dans un délai moyen de 12 mois. La glycémie et l' hémoglobine glycquée ont également été mesurés. Nous avons cherché les paramètres associés à la diminution du TAV en fonction des paramètres initiaux des patients. Les test de Wilcoxon a été utilisé pour les variable quantitatives ou continues, de comparaison de moyenne en raison de nombre petit échantillon. Le test de Mac Nemar a servi à faire des comparaisons de pourcentage pour nos varibales qualitatives.

Résultats et Analyse statistique : Avant la chirurgie, la quantité de TAV est d'autant plus élevée que le poids l'est ( $<<0.0002$ ). En préopératoire,nous n'avons pas noté de corélation entre la quantité de TAV et la présence d'une hyperglycémie modérée à jeun ou d'un diabète. Les patients ayant des IMC élevés ont aussi une masse musculaire (MM) plus élevée. La DER est plus importante chez les patients ayant les IMC les plus élevé, avec une corrélation avec la quantité de MM. La mobilisation du TAV est corrélée à l'importance de la perte de poids totale (coeff $0.63, p=0.0003$ ). II n'y a pas de lien entre la perte de MG ni la perte de TAV et la régression des paramètres glycémiques. En moyenne, avant le chirurgie, le TAV représente $3.4 \%$ de la MG totale contre $3.1 \%$ après la chirurgie. Le TASC a diminué de $36.7 \%$ tandis que le TAV a regressé de $45.4 \%(p<0.0001)$. La perte de poids est corrélée en post opératoire à une diminution de la DER.

Conclusion : Cette étude n'a pas montré le lien entre l'importance du TAV et la glycémique avant la chirurgie bariatrique chez le sujet obèse, ni de corrélation entre mobilisation du TAV et amélioration de la glycémie. Ceci est probablement du à un manque de puissance. En outre, nous avons noté une diminution proportionnellement plus importante pour le TAV que pour le TASC avec la perte de poids. Il serait intéressant d'identifier des éléments prédictif de l'amélioration des paramètres glycémiques en préopératoire d'une chirurgie bariatrique.

Remerciements : Mille mercis aux Drs Monier, Damade et Antoun, des thérapeutes précieux et d'excellents mentors. Au Dr C. de Brauer, fine stasticienne, toujours intéressée par la clinique.

Conflits d'intérêts : Aucun conflit à déclarer 
Effet d'un mois de sevrage en hormone de croissance (GH) chez des patients déficitaires en GH : étude du rôle de la GH dans la régulation du métabolisme des lipoprotéines riches en triglycérides (LRT)

S. Béliard ${ }^{12}$, R. Valéro ${ }^{12}$, J. Dupont-Roussel ${ }^{2}$, A. Calabrese ${ }^{2}$, M. Maraninchi $^{2,}{ }^{*}$

${ }^{1}$ Service de Nutrition, Maladies métaboliques et Endocrinologie, APHM, ${ }^{2}$ UMR C2VN, Aix Marseille Univ, INSERM, INRA, Marseille, France

Introduction et but de l'étude : Le déficit en hormone de croissance (GH) est associé à une surmortalité cardiovasculaire et les patients déficitaires en $\mathrm{GH}$ présentent un état d'insulino-résistance et de nombreuses perturbations du profil lipidique: hypertriglycéridémie, baisse du HDL-cholestérol, augmentation des LDL petites et denses et hyperlipidémie postprandiale. L'objectif principal de cette étude est de préciser le rôle spécifique de la GH dans la régulation du métabolisme des lipoprotéines riches en triglycérides (LRT).

Matériel et méthodes : Etude des profils lipidiques et apolipoprotéiques à jeun et en phase postprandiale dans les fractions plasmatiques et LRT lors d'un repas-test réalisé avant (M0) et après un mois de sevrage en GH (M1) chez des patients déficitaires en $\mathrm{GH}(\mathrm{n}=10)$.

Résultats et Analyse statistique : Après un mois de sevrage en $\mathrm{GH}$, la diminution significative du taux plasmatique de l'IGF-1 ($12,12 \pm 2,36 \mathrm{nmol} / \mathrm{L} P<0,01) \mathrm{s}^{\prime}$ accompagne d'une diminution du poids et de I'IMC $\left(-1,31 \pm 0,45 \mathrm{~kg}\right.$ et $-0,78 \pm 0,41 \mathrm{~kg} / \mathrm{m}^{2} P<0,05$ respectivement) associée à une modification de la composition corporelle : diminution de la masse maigre et augmentation de la masse grasse $(-1,17 \pm 0,41 \mathrm{~kg}$ et $+0,36 \pm 0,16 \mathrm{~kg} P<0,05$ respectivement). On observe également après un mois de sevrage une modification des paramètres d'insulino-résistance avec une diminution du HOMA-index $(-0,45 \pm 0,19 P=0,05)$ associée à une diminution significative de l'insulinémie à jeun et du peptide $C(-2,04 \pm 0,78 \mathrm{mUl} / \mathrm{L}$ et $-0,15 \pm 0,05 \mathrm{nmol} / \mathrm{L} P<0,05 \mathrm{respectivement})$. Les taux plasmatiques de triglycérides (TG) et d'apolipoprotéine (Apo) C-III à jeun diminuent de façon significative après un mois de sevrage en $\mathrm{GH}(-0,41 \pm 0,16 \mathrm{mmol} / \mathrm{L}$ et $-23,07 \pm 6,93 \mathrm{mg} / \mathrm{L} P<0,05$ respectivement) accompagnés par une diminution des taux d'acides gras libres $(-0,16 \pm 0,07 \mathrm{mmol} / \mathrm{L} P=0,074)$.

A ces modifications observées au niveau plasmatique, s'ajoutent au niveau de la fraction LRT à jeun, une diminution significative des taux de TG, cholestérol et phospholipides ainsi que des apos B, C-II et C-III. De façon concomitante, après sevrage, les taux de TG et d'apo C-III mesurés dans la fraction LRT en période postprandiale diminuent significativement lors du repas test. Les variations des taux de TG observées dans les fractions plasmatiques et LRT après sevrage en GH sont corrélées de façon négative aux taux plasmatiques de HDL et d'adiponectine et de façon positive aux variations de TG, cholestérol, phospholipides, apos B, CII et C-III dans la fraction LRT (corrélations non paramétriques de Spearman: $r>0,45$ et $P<0,05$ ).

Conclusion : De nombreuses modifications du profil lipidique et notamment des triglycérides et des apolipoprotéines C-II et C-III dans la fraction LRT sont observées chez les patients déficitaires en GH à jeun après un mois de sevrage. Ces résultats pourraient suggérer un rôle direct de la GH dans la régulation du métabolisme des LRT pouvant notamment participer à la perturbation des taux de triglycérides plasmatiques dans cette pathologie.

Conflits d'intérêts : Aucun conflit à déclarer 

de réalimentation.

B. Ben Mehel ${ }^{1,}{ }^{*}$, S. Bousbahi ${ }^{1}$, P. Gérard ${ }^{2}$ et Soumia Bousbahi

${ }^{1}$ Laboratoire de Physiologie de la Nutrition et Sécurité Alimentaire, Université Ahmed Ben Bella Oran 1, Oran, Algérie, ${ }^{2}$ MICALIS, INRA, Jouy en Josas, Philippines

Introduction et but de l'étude : Les aliments fermentés traditionnels représentent environ un tiers de la nourriture dans le monde. En Algérie, le blé fermenté traditionnel appelé Hamoum (BFH) une denrée alimentaire ancestrale consommé sous forme de couscous. Ce blé est obtenu après une fermentation naturelle dans un grenier souterrain appelé Matmora.

Historiquement, la FWH était considérée comme un aliment ayant des propriétés médicinales dans la prévention et le traitement de nombreuses complications physiopathologiques intestinales. Cependant, la relation entre le blé fermenté et le microbiote intestinal est rarement élucidée dans le contexte de la santé de l'intestin.

Le but de notre étude est de vérifier si le BFH rétablira l'équilibre de la flore bactérienne intestinale et la restructuration de la barrière intestinale après une translocation bactérienne intestinale dans un modèle de rats malnutris.

Matériel et méthodes : 28 jeunes rats Wistar mâles d'un poids de 30 à $35 \mathrm{~g}$ et âgés de quatre semaines ont été choisis comme modèle animal. Le groupe contrôle positif (groupe malnutri) ont été nourris avec un régime de malnutrition protéique (2\% de caséine) pendant 25 jours; le groupe témoin négatif (groupe normal) a été nourri avec un régime standard (20\% de caséine) pendant 25 jours. Les groupes restants pour la phase de réalimentation ont reçu pour la première fois le régime de malnutrition protéique ( $2 \%$ de caséine) pendant 25 jours avant d'être nourris pendant 25 jours avec un régime enrichi en BFH et un régime conventionnel (groupe Std).

Résultats et Analyse statistique : Nos résultats montrent augmentation de la pullulation bactérienne caecale et un taux de translocation bactérienne élevé dans les ganglions lymphatiques mésentériques, la rate et le foie dans le groupe malnutri. Dans le groupe réalimenté avec le BFH, nous avons constaté une diminution de la pullulation bactérienne caecale, une translocation bactérienne fortement diminuée dans les MLN dans la rate et une absence totale dans le foie. Les analyses histologiques ont montré que la morphométrie des villosités intestinales et le nombre des lymphocytes intra-épithéliaux étaient bien rétablis dans le groupe BFH à l'inverse du groupe malnutri

Conclusion : Nous somme arrivé à montrer que la supplémentation en BFH diminue significativement la translocation bactérienne intestinale et permet une meilleure restructuration la barrière muqueuse durant la phase de réalimentation par le BFH chez les rats malnutris. Le BFH pourrait être utilisé comme adjuvant nutritionnel dans les physiopathologies intestinales. D’autres études sont en cours pour une valorisation biotechnologique.

Remerciements : Mes sincères remerciements pour le docteur Philippe Gérard et Catherine Philippe de I'Unité de recherche MICALIS de I'INRA Jouy en Josas.

Conflits d'intérêts : Aucun conflit à déclarer 


\section{SANTE PUBLIQUE}

\section{P202}

Des dispositifs de cuisine connectés pour influencer l'homéostasie nutritionnelle : un essai contrôlé randomisé de faisabilité à domicile

M.-F. Vaillant ${ }^{12,{ }^{*}, \text { H. Terrisse }}{ }^{3}$, S. Artemova ${ }^{3}$, C. Rolland ${ }^{3}$, M. Joyeux-Faure 2456 , J.-C. Borel ${ }^{2456}$, O. Marion ${ }^{7}$, P. Dancer ${ }^{7}$, M. Sicard ${ }^{7}$, P. Casas-Agustench ${ }^{8}$, C. Andres-Lacueva ${ }^{8}$, A. J. Lloyd ${ }^{9}$, J. Draper ${ }^{9}$, C. Pison ${ }^{210}$, C. Moinard $^{10}$

${ }^{1}$ Laboratoire de Bioénergétique Fondamentale et Appliquée, INSERM U1055, Université Grenoble Alpes, ${ }^{2} \mathrm{CHU}$ Grenoble Alpes, ${ }^{3}$ TIMC-IMAG, UMR CNRS5525, ${ }^{4}$ Université Grenoble Alpes, ${ }^{5}$ INSERM1042, Grenoble, ${ }^{6} \mathrm{AGIR}$ à dom, Meylan, ${ }^{7}$ Groupe SEB, Ecully, France, ${ }^{8}$ Biomarkers and Nutrimetabolomic Laboratory, Department of Nutrition, Food Sciences and Gastronomy, Food Technology Reference Net (XaRTA), Nutrition and Food Safety Research Institute (INSA), Campus Torribera, Faculty of Pharmacy and Food Sciences, University of Barcelona, CIBER de Fragilidad y Envejecimiento Saludable (CIBERFES), Instituto de Salud Carlos III, Barcelona, Espagne, ${ }^{9}$ Metabolomics Centre, Université d'Aberystwyth, Aberystwyth, Royaume-Uni, ${ }^{10}$ Laboratoire de Bioénergétique Fondamentale et Appliquée, INSERM U 1055, Université Grenoble Alpes, Grenoble 9, France

Introduction et but de l'étude : Les campagnes des pouvoirs publics peinant à corriger les mauvaises habitudes alimentaires, nous proposons d'évaluer l'intérêt d'outils de cuisine connectés à le faire sans contrainte dans le cadre d'une étude pilote de faisabilité. Matériel et méthodes : Essai contrôlé, randomisé selon la méthode de Zelen, monocentrique (ClinicalTrials.gov NCT03171571) incluant des volontaires sains (représentatifs de la population française), avec des dispositifs de cuisson connectés (GI) ou feuille de conseils alimentaires (Groupe contrôle GC). La faisabilité et l'acceptabilité évaluées par l'exhaustivité des recueils obtenus à 0 , 6 et 12 mois, à domicile et en consultation, étaient le critère de jugement principal. Les changements à 1 an dans le score Alternate Healthy Eating Index-2010 (AHEI-2010), les variations anthropométriques, la composition corporelle, la pression artérielle, la qualité de vie SF36, l'actimétrie, les paramètres sanguins, les biomarqueurs urinaires et le microbiote constituaient les critères secondaires.

Résultats et Analyse statistique : Résultats positifs concernant l'exhaustivité des données obtenues sur 20 sujets (16 femmes), non-fumeurs, en charge des repas à domicile, âge moyen (écart type ET) $=39,3(3,2)$ ans, indice de masse corporelle $=27,3(1,3)$ $\mathrm{kg} / \mathrm{m} 2$, randomisés dans le GI $(\mathrm{n}=11)$ ou GC $(\mathrm{n}=9)$.

Après un an : pas de différences significatives entre GI vs GC pour le gain d'1 quintile de l'AHEI-2010, les données anthropométriques, de pression artérielle, les paramètres sanguins, le nombre de pas et le SF 36. Cependant, la durée médiane du sommeil a augmenté de près d'1h dans le $\mathrm{Gl}:+0,9 \mathrm{~h} / \mathrm{j}[0,3-1,2]$ vs $-0,0[-0,4-0,3]$ pour $\mathrm{GC}(\mathrm{p}=0,041)$. L'analyse du microbiote a montré un effet des outils connectés (augmentation des Firmicutes pour les abondances relatives, $p=0,020$ ) pour le Gl.

Conclusion : Nos résultats préliminaires ont démontré la faisabilité et l'acceptabilité d'une évaluation multi-échelle complète à domicile pour mesurer l'impact des appareils de cuisson connectés sur les habitudes alimentaires et la santé. Les résultats préliminaires originaux devraient être confirmés par l'essai contrôlé randomisé multicentrique en cours sur 160 sujets en France et Royaume-Uni (NCT03169088).

Remerciements : Financement EIT Health

Conflits d'intérêts : M.-F. Vaillant: Aucun conflit à déclarer, H. Terrisse: Aucun conflit à déclarer, S. Artemova: Aucun conflit à déclarer, C. Rolland: Aucun conflit à déclarer, M. Joyeux-Faure est employé(e) de AGIR à dom (home care provider), J.-C. Borel est employé(e) de AGIR à dom (home care provider), O. Marion est employé(e) de Groupe SEB, P. Dancer est employé(e) de Groupe SEB, M. Sicard est employé(e) de Groupe SEB, P. Casas-Agustench: Aucun conflit à déclarer, C. Andres-Lacueva: Aucun conflit à déclarer, A. Lloyd: Aucun conflit à déclarer, J. Draper: Aucun conflit à déclarer, C. Pison: Aucun conflit à déclarer, C. Moinard: Aucun conflit à déclarer 
Il est possible de réduire la teneur en sel des menus des restaurants scolaires grâce à des interventions coordonnées auprès des personnels de cuisine et des fournisseurs d'aliments industriels

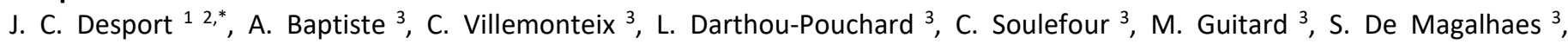
V. Javerliat ${ }^{3}$, C. Bachelerie ${ }^{4}$, C. Jalladeau ${ }^{4}$, A. Lecointre ${ }^{4}$, P. Fayemendy ${ }^{12}$, P. Jésus ${ }^{12}$

${ }^{1}$ unité de nutrition, CHU Dupuytren, ${ }^{2}$ Inserm UMR 1094, Limoges, ${ }^{3}$ réseau LINUT, Isle, ${ }^{4}$ Caisse des écoles, Ville de Limoges, Limoges, France

Introduction et but de l'étude : La consommation de chlorure de sodium (sel) chez l'enfant est supérieure aux recommandations et, du fait de son retentissement sur la pression artérielle, sa réduction est un objectif de santé publique. C'est une mesure efficace chez l'enfant, avec la possibilité d'une prolongation de l'effet à l'âge adulte. L'objectif principal de l'étude était, en agissant au niveau des secteurs de cuisine et de logistique, de diminuer de $20 \%$ la teneur en sel des menus de trois restaurants scolaires (écoles maternelles et primaires) de la Ville de Limoges.

Matériel et méthodes : L'intervention, menée par un réseau de santé, comportait quatre phases : mesure des contenus en sel de trois repas successifs dans trois écoles, formations et discussions avec les personnels de cuisine et les personnels en charge de la logistique alimentaire, interventions auprès des fournisseurs de denrées alimentaires industrielles, nouvelles mesures à distance des contenus en sel de trois repas successifs dans les mêmes établissements. L'analyse statistique utilisait les tests de MannWhitney et Kruskal-Wallis.

Résultats et Analyse statistique : Initialement, les contenus en sel des repas étaient dans tous les cas supérieurs aux apports recommandés pour un repas principal. Les formations étaient bien perçues et permettaient de confronter les pratiques et de faire 10 propositions d'action. Les interventions auprès des fournisseurs permettaient d'obtenir des denrées alimentaires moins salées. L'évaluation finale montrait, versus les évaluations initiales, une baisse des contenus en sel $(p<0,05$ aussi bien en maternelles qu'en primaires), la baisse était de $32,9 \%$ pour les maternelles et de $32,3 \%$ pour les primaires. Les contenus en sel étaient très proches des valeurs recommandées.

Conclusion : Cette action a permis une réduction des contenus en sel dans des restaurants scolaires supérieure à l'objectif initial. Elle est la première décrite de ce type. Ceci suggère qu'une telle intervention peut être une aide importante pour obtenir une baisse de la consommation journalière en sel chez l'enfant.

Remerciements : aux personnels et élus de la Ville de Limoges

Conflits d'intérêts : J. C. Desport a reçu une subvention/soutien de subvention ARS Nouvelle Aquitaine, A. Baptiste a reçu une subvention/soutien de subvention ARS Nouvelle Aquitaine, C. Villemonteix a reçu une subvention/soutien de subvention ARS Nouvelle Aquitaine, L. Darthou-Pouchard a reçu une subvention/soutien de subvention ARS Nouvelle Aquitaine, C. Soulefour a reçu une subvention/soutien de subvention ARS Nouvelle Aquitaine, M. Guitard a reçu une subvention/soutien de subvention ARS Nouvelle Aquitaine, S. De Magalhaes a reçu une subvention/soutien de subvention ARS Nouvelle Aquitaine, V. Javerliat a reçu une subvention/soutien de subvention ARS Nouvelle Aquitaine, C. Bachelerie a reçu une subvention/soutien de subvention ARS Nouvelle Aquitaine, $C$. Jalladeau a reçu une subvention/soutien de subvention ARS Nouvelle Aquitaine, A. Lecointre a reçu une subvention/soutien de subvention ARS Nouvelle Aquitaine, P. Fayemendy a reçu une subvention/soutien de subvention ARS Nouvelle Aquitaine, P. Jésus a reçu une subvention/soutien de subvention ARS Nouvelle Aquitaine 
Adhérence au régime Méditerranéen, indice de masse corporelle et risque de diabète de type 2 : Résultats de l'étude UK Biobank

P. M. Andre ${ }^{1,}{ }^{*}$, G. Proctor ${ }^{2}$, F. Rodriguez-Artalejo ${ }^{3}$, E. Lopez-Garcia ${ }^{3}$, D. Gomez-Cabrero ${ }^{2}$, P. Bros ${ }^{4}$, E. Neyraud ${ }^{4}$, E. Garcia-Esquinas ${ }^{3}$, M. Morzel ${ }^{4}$, C. Féart $^{1}$

${ }^{1}$ Bordeaux Population Health Research Center, Univ. Bordeaux, Inserm, Bordeaux, France, ${ }^{2}$ Mucosal \& Salivary Biology Division, King's College, London, Royaume-Uni,

${ }^{3}$ CIBERESP and Department of Preventive Medicine and Public Health, School of Medicine, Universidad Autónoma de Madrid, Madrid, Espagne, ${ }^{4}$ Centre des Sciences du Goût et de l'Alimentation, AgroSup Dijon, CNRS, INRA, Université de Bourgogne FrancheComté, Dijon, France

Introduction et but de l'étude : Au cours des dernières décennies, l'incidence du diabète de type II (DT2) a augmenté de manière significative à travers le monde, entrainant une augmentation de la morbidité et de la mortalité associées. En termes de prévention, les preuves s'accumulent quant à l'association entre l'adhérence à un régime de type Méditerranéen et un moindre risque de DT2. Dans cette étude, nous nous sommes intéressés à l'effet potentiellement médiateur de l'indice de masse corporelle (IMC) en tant que mécanisme sous-jacent à cette relation.

Matériel et méthodes : Les participants de la UK Biobank ayant des données cliniques sur le statut diabétique et ayant répondu à au moins un questionnaire nutritionnel ont été inclus. Le statut diabétique était auto-déclaré et les participants ayant un diagnostic de diabète prévalent, gestationnel ou incertain (ç.à.d. variant au cours des suivis) ont été exclus. Le score MEDAS a été utilisé pour évaluer l'adhérence à un régime de type Méditerranéen à l'inclusion, basé sur les seuils de consommation de 11 groupes d'aliments (huile d'olive, légumes, fruits, viande rouge, beurre ou crème, boissons sucrées, vin, légumineuses, poisson, pâtisseries et noix) ainsi qu'une question additionnelle sur la consommation préférentielle de viande rouge ou blanche. Une analyse de médiation causale basée sur un modèle de Cox à risques proportionnels a été utilisé pour évaluer les relations entre MEDAS score et risque de DT2.

Résultats et Analyse statistique : L'échantillon d'étude était composé de 6362 participants, dont 113 ont développé un diabète incident durant une période de suivi moyenne de 4,6ans. A l'inclusion, le score MEDAS moyen était de 4,6, variant de 0 à 11 . Concernant l'effet total, l'incidence de DT2 était réduite de $12 \%$ chez les participants ayant un score MEDAS plus élevé. Cependant, l'effet direct du score MEDAS sur le risque de DT2 n'était pas significatif après ajustement sur les potentiels facteurs de confusion dont l'IMC (RR 0,91 IC95\% [0,82 - 1,02]). Les analyses de médiation ont suggéré que l'IMC expliquait environ 3\% de l'effet du score MEDAS sur le risque de diabète (RR 0,97 IC95\% [0,96-0,97]).

Conclusion : Les effets bénéfiques, et désormais bien connus, d'une plus grande adhérence à un régime de type Méditerranéen sur le risque de DT2 pourraient en partie être médiés par la modulation de l'IMC chez les participants de la UK Biobank.

Remerciements : Le projet SALAMANDER est financé dans le cadre du JPI ERA-HDHL "Biomarkers for Nutrition and Health"

Conflits d'intérêts : Aucun conflit à déclarer 
Introduction et but de l'étude : Le sélénium (Se) est un élément trace essentiel. A faible dose, il est d'une importance fondamentale en nutrition humaine. A dose plus élevée, il devient toxique. Des apports alimentaires insuffisants aboutissent à des carences pouvant avoir des conséquences graves sur la santé humaine. Dès lors déterminer le statut sélénié d'une population donnée, revient à prévenir de nombreuses pathologies pouvant constituer de vrais problèmes de santé publique.

L'objectif de ce travail est de déterminer le taux du Se plasmatique de 60 individus fumeurs et de 80 témoins non-fumeurs de la ville de Tlemcen (extrême ouest Algérien). Ceci dans le but de vérifier si ces tabagiques sont protégés contre les effets du stress oxydant.

Matériel et méthodes : Au total 140 individus âgés entre 30 et 40 ans, en bonne santé ont été sélectionnés pour l'étude. Soixante fumeurs et 80 non-fumeurs. Le dosage du sélénium a été réalisé par voltamétrie cathodique inverse à impulsion différentielle.

Le test paramétrique de Student est réalisé pour comparer entre le groupe des fumeurs et des non-fumeurs. Le coefficient de corrélation de Pearson est utilisé afin de rechercher une éventuelle relation entre le nombre de cigarettes fumées par jour et le taux de Se plasmatique.

Résultats et Analyse statistique : Ce travail montre que la population de fumeurs présente un taux de Se plasmatique moyen de $70 \pm 12 \mu \mathrm{g} / \mathrm{L}$, alors que celui des non-fumeurs est de $76 \pm 14,23 \mu \mathrm{g} / \mathrm{L}$, la différence $n^{\prime}$ est pas significative $(P>0,05)$. Par contre une corrélation négative et significative a été observée entre le taux de Se chez les fumeurs et le nombre de cigarettes fumées par jour.

Conclusion : Ce taux de Se plasmatique des cas et des témoins est insuffisant pour assurer une fonction optimale de l'enzyme antioxydante, glutathion peroxydase, enzyme clé dans la réduction du peroxyde d'hydrogène ou des hydroperoxydes lipidiques et donc dans la protection contre les effets délétères du stress oxydant. En effet cette enzyme nécessite un taux de sélénium plasmatique d'au moins $100 \mu \mathrm{g} / \mathrm{L}$ pour assurer pleinement sa fonction.

Le statut sélénié est corrélé au nombre de cigarettes fumés par jour chez les tabagiques Tlemceniens.

Références : [1] Brigelius-Flohé R. Introduction to serial reviews on selenium and diabetes type2- An unexpected link. Free Radic Biol Med. 2013; 65:1536-37. DOI:

10.1016/j.freeradbiomed.2013.07.018.

[2] Césarini JP (2004) Le sélénium : actualités. Édition John Libbey Eurotext, Collection pathologie science formation, Montrouge : p 95.

[3] Kipp AP, Strohm D, Brigelius-Flohé R, Schomburg L, Bechthold A, Leschik-Bonnet E, Heseker H. German Nutrition Society (DGE) (2015) Revised reference values for selenium intake. J Trace Elem Med Biol 32: 195-99. DOI:10.1016/j.jtemb.2015.07.005

Conflits d'intérêts : Aucun conflit à déclarer 
Association entre profils métabolomiques plasmatiques par RMN et risque à long terme de développer un cancer de la prostate. L. Lécuyer ${ }^{1,}{ }^{*}$, A. Victor Bala ${ }^{2}$, N. Bouchemal ${ }^{2}$, M. Nawfal Triba ${ }^{2}$, A. Demidem ${ }^{3}$, A. Rossary ${ }^{3}$, P. Galan ${ }^{1}$, S. Hercberg ${ }^{14}$, V. Partula $^{1}$, L. Le Moyec ${ }^{5}$, P. Latino-Martel ${ }^{1}$, E. Kesse-Guyot ${ }^{1}$, M. Deschasaux ${ }^{1}$, M.-P. Vasson ${ }^{36}$, P. Savarin ${ }^{2}$, M. Touvier ${ }^{1}$

${ }^{1}$ EREN, CRESS, U1153 Inserm / U1125 Inra / Cnam / Université Paris 13, Université Paris 13 - SMBH, Bobigny Cedex, ${ }^{2}$ Equipe SBMB - CSPBAT - UMR 7244, Université Paris 13, Bobigny, ${ }^{3}$ Unité de Nutrition Humaine (UNH), Inra UMR 1019, CRNH, Université Clermont Auvergne, Clermont-Ferrand, ${ }^{4}$ Département de santé publique, Hôpital Avicenne, Bobigny Cedex, ${ }^{5}$ UBIAE, INSERM, Université Evry, Université Paris-Saclay, Evry, ${ }^{6}$ Centre Anticancer Jean-Perrin, CHU Clermont-Ferrand, Clermont-Ferrand, France

Introduction et but de l'étude : Le cancer de la prostate est l'un des principaux cancers chez l'homme dans le monde. Les mécanismes impliqués dans son étiologie sont encore mal compris. La métabolomique est un puissant outil permettant d'élucider de nouvelles voies mécanistiques mis en jeu et d'identifier de nouveaux biomarqueurs caractéristiques d'un profil à plus haut risque de développer un cancer de la prostate à long terme. Cette étude prospective a donc pour but d'étudier si des profils métabolomiques établis à partir d'un prélèvement sanguin sur des hommes à priori en bonne santé pourraient contribuer à l'identification des hommes à plus haut risque de développer un cancer de la prostate dans la prochaine décennie et de mieux comprendre les mécanismes impliqués dans la mise en place de ce cancer.

Matériel et méthodes : Un cas-témoin niché prospectif a été réalisé dans la cohorte SU.VI.MAX, incluant 162 cas de cancer de la prostate diagnostiqués pendant les 13 ans de suivi, et 162 témoins appariés. Les profils métabolomiques RMN non ciblés ont été réalisés sur des échantillons de plasma pré-diagnostic prélevés à l'inclusion en utilisant les séquences NOESY 1D et CPMG d'un spectromètre Bruker Avance III de $500 \mathrm{MHz}$. Des modèles de régressions logistiques conditionnelles multivariées ont été réalisés sur chaque variable RMN.

Résultats et Analyse statistique : Les hommes ayant un taux plasmatique moins élevé en urée et plus élevé en valine, albumine, tyrosine, phénylalanine, 3-methylhistidine, histidine, glutamine, albumine lysyl et créatine avaient un plus haut risque de développer un cancer de la prostate dans les 13 qui suivaient. Le métabolite le plus associé au risque du cancer de la prostate était I'histidine (OR=1.46 [1.12-1.88], $\mathrm{p}=0.004)$.

Conclusion : Cette étude a mis en évidence une association entre les profils métabolomiques RMN et le risque de développer un cancer de la prostate à long terme. Ces résultats fournissent des éléments qui pourront permettre de mieux comprendre les mécanismes complexes impliqués dans la carcinogenèse de la prostate. S'ils sont répliqués dans une cohorte indépendante, ils pourront contribuer au développement de stratégies pour l'identification des personnes à plus haut risque de développer un cancer de la prostate.

Trial registration: SU.VI.MAX, clinicaltrials.gov NCT00272428. Registered 3 January 2006

Remerciements : Fondation de France / Institut National du cancer (INCa) / Institut Fédératif de Recherche Biomédicale Federative (IFRB) / Projet labellisé NACRe Partenariat.

Conflits d'intérêts : Aucun conflit à déclarer 
Association prospective entre le potentiel inflammatoire du régime alimentaire et le risque de symptômes dépressifs incidents M. Adjibade ${ }^{1,}{ }^{*}$, C. Lemogne ${ }^{234}$, M. Touvier ${ }^{1}$, S. Hercberg ${ }^{15}$, P. Galan ${ }^{1}$, K. E. Assmann ${ }^{1}$, C. Julia ${ }^{1}$, E. Kesse-Guyot ${ }^{1}$

${ }^{1}$ Equipe de Recherche en Epidémiologie Nutritionnelle (EREN), Université Paris 13, Centre d'Epidémiologie et Statistiques Sorbonne Paris Cité, Inserm (U1153), Inra (U1125), Cnam, COMUE Sorbonne Paris Cité, Bobigny, 'Université Paris Descartes, Sorbonne Paris Cité, Faculté de Médecine, ${ }^{3} \mathrm{AP}-\mathrm{HP}$, Hôpitaux Universitaires Paris Ouest, Service de Psychiatrie de l'Adulte et du Sujet Agé, ${ }^{4}$ Inserm (U894), Centre de Psychiatrie et Neurosciences, Paris, ${ }^{5}$ Département de Santé Publique, Hôpital Avicenne, Bobigny, France

Introduction et but de l'étude : Il a été suggéré que l'inflammation (pouvant être modulée par l'alimentation) joue un rôle important dans l'étiologie de la dépression, mais les études prospectives portant sur l'association entre le potentiel inflammatoire du régime alimentaire et la dépression en population générale sont limitées. L'objectif de la présente étude était donc d'examiner l'association prospective entre le potentiel inflammatoire du régime alimentaire et le risque de symptômes dépressifs dans une population d'adultes français.

Matériel et méthodes : L'étude a porté sur un échantillon de 26730 hommes et femmes (âgés de 18 ans ou plus) de la cohorte NutriNet-Santé, qui avaient des données alimentaires valides, avaient rempli le questionnaire Center for Epidemiologic StudiesDepression Scale (CES-D) au moins 2 fois au cours du suivi (avec un maximum de 3 points disponibles par participant) et qui n'avaient pas de symptômes dépressifs lors de la première évaluation de la symptomatologie dépressive (CES-D< 17 pour les hommes et $<23$ pour les femmes). Les cas incidents de symptômes dépressifs étaient les participants qui présentaient des symptômes dépressifs au moins une fois au cours du suivi. Le potentiel inflammatoire du régime alimentaire a été mesuré à I'aide d'une version alternative du Dietary Inflammatory Index original, nommée Alternate Dietary Inflammatory Index (ADII), un score élevé reflétant une alimentation proinflammatoire. Les associations entre l'ADIl et le risque de symptômes dépressifs ont été évaluées à l'aide des ratios de risques instantanés (HR) et leurs intervalles de confiance (IC) à 95\%, estimés en utilisant des modèles de Cox à risque proportionnel pour les données censurées par intervalles.

Résultats et Analyse statistique : Au cours du suivi, 2221 cas de symptômes dépressifs incidents ont été identifiés. Après ajustement sur différents facteurs de confusion potentiels, les participants qui étaient dans le quatrième quartile de l'ADII (reflétant un régime alimentaire plus " pro-inflammatoire ") avaient $15 \%$ (IC à $95 \%=2 \%-31 \%$ ) de plus de risque de développer des symptômes dépressifs, comparés aux participants qui étaient dans le premier quartile. Un effet modulateur de l'indice de masse corporelle (IMC $<25 \mathrm{vs} \geq 25 \mathrm{~kg} / \mathrm{m}^{2}$ ) a été observé, avec des associations qui n'étaient significatives que chez les participants présentant un IMC 25 (HR=1,29; IC à 95\%=1,04-1,60).

Conclusion : Les résultats de cette étude suggèrent que la promotion d'une alimentation « saine " présentant des propriétés antiinflammatoires est importante pour la prévention des symptômes dépressifs, en particulier chez les personnes présentant un IMC supérieur au seuil de 25 .

Conflits d'intérêts : M. Adjibade: Aucun conflit à déclarer, C. Lemogne a un conflit d'intérêt avec Lundbeck, Astra Zeneca, DaiichiSankyo, Janssen, Lundbeck, Otsuka Pharmaceuticals et Servier (il a reçu des honoraires pour la composition du conseil de Lundbeck et a été invité à parler aux symposiums d'Astra Zeneca, Daiichi-Sankyo, Janssen, Lundbeck, Otsuka Pharmaceuticals et Servier), M. Touvier: Aucun conflit à déclarer, S. Hercberg: Aucun conflit à déclarer, P. Galan: Aucun conflit à déclarer, K. Assmann: Aucun conflit à déclarer, C. Julia: Aucun conflit à déclarer, E. Kesse-Guyot: Aucun conflit à déclarer 
Association prospective entre la consommation d'aliments ultra-transformés et le risque de symptômes dépressifs incidents M. Adjibade ${ }^{1, *}$, C. Julia ${ }^{12}$, B. Allès ${ }^{1}$, M. Touvier ${ }^{1}$, C. Lemogne ${ }^{345}$, B. Srour ${ }^{1}$, S. Hercberg ${ }^{12}{ }^{2}$, P. Galan ${ }^{1}$, K. E. Assmann ${ }^{1}$, E. Kesse-Guyot ${ }^{1}$

${ }^{1}$ Equipe de Recherche en Epidémiologie Nutritionnelle (EREN), Université Paris 13, Centre d'Epidémiologie et Statistiques Sorbonne Paris Cité, Inserm (U1153), Inra (U1125), Cnam, COMUE Sorbonne Paris Cité, ²Département de Santé Publique, Hôpital Avicenne, Bobigny, ${ }^{3}$ Université Paris Descartes, Sorbonne Paris Cité, Faculté de Médecine, ${ }^{4}$ AP-HP, Hôpitaux Universitaires Paris Ouest, Service de Psychiatrie de l'Adulte et du Sujet Agé, ${ }^{5}$ Inserm (U894), Centre de Psychiatrie et Neurosciences, Paris, France

Introduction et but de l'étude : Au cours des dernières décennies, une augmentation de la consommation d'aliments ultratransformés a été observée dans de nombreux pays, en particulier dans les pays occidentaux. Une consommation élevée de ces aliments a été associée à un excès de poids et à un risque plus élevé de diverses pathologies, mais aucune étude n'a porté sur leurs associations avec le risque de dépression. Cette étude avait donc pour objectif d'examiner l'association entre la proportion d'aliments ultra-transformés dans le régime alimentaire et le risque de symptômes dépressifs incidents dans la cohorte NutriNetSanté.

Matériel et méthodes : L'étude comprenait 26730 participants âgés de 18 ans ou plus, initialement exempts de symptômes dépressifs et ayant des données alimentaires valides. Les symptômes dépressifs ont été évalués à l'aide du seuil sexe spécifique proposé pour la version française de l'échelle Center for Epidemiologic Studies-Depression Scale (CES-D $\geq 17$ pour les hommes et $\geq 23$ pour les femmes) et les cas incidents de symptômes dépressifs étaient les participants qui présentaient des symptômes dépressifs au moins une fois au cours du suivi (avec un maximum de 3 points disponibles par participant). La proportion (en poids) d'aliments ultra-transformés dans le régime alimentaire a été calculée pour chaque participant, en utilisant la classification NOVA appliquée aux consommations alimentaires. Les associations entre la proportion d'aliments ultra-transformés dans le régime alimentaire et le risque de symptômes dépressifs ont été évaluées à l'aide des modèles de Cox à risque proportionnel pour les données censurées par intervalles, et les ratios de risques instantanés (HR) et leurs intervalles de confiance (IC) à 95\% ont été estimés.

Résultats et Analyse statistique : Un total de 221 cas incidents de symptômes dépressifs a été identifié au cours du suivi. Après ajustement sur divers facteurs de confusion potentiels, une augmentation de $10 \%$ de la consommation d'aliments ultratransformés dans le régime alimentaire a été associée à une augmentation de $21 \%$ (IC à $95 \%=15 \%-27 \%$ ) du risque de survenue des symptômes dépressifs. Dans les analyses concernant la proportion d'aliments ultra-transformés par groupe alimentaire, seuls

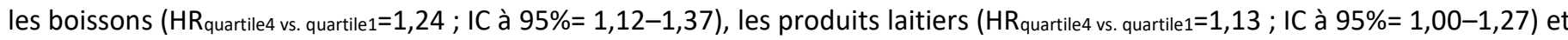

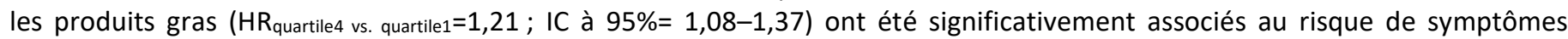
dépressifs.

Conclusion : Les résultats de cette étude montrent l'importance de la prise en compte de cet aspect non nutritionnel du régime alimentaire, dans un contexte de promotion de la santé mentale.

Conflits d'intérêts : M. Adjibade: Aucun conflit à déclarer, C. Julia: Aucun conflit à déclarer, B. Allès: Aucun conflit à déclarer, M. Touvier: Aucun conflit à déclarer, C. Lemogne a un conflit d'intérêt avec Lundbeck, Astra Zeneca, Daiichi-Sankyo, Janssen, Lundbeck, Otsuka Pharmaceuticals et Servier (il a reçu des honoraires pour la composition du conseil de Lundbeck et a été invité à parler aux symposiums d'Astra Zeneca, Daiichi-Sankyo, Janssen, Lundbeck, Otsuka Pharmaceuticals et Servier), B. Srour: Aucun conflit à déclarer, S. Hercberg: Aucun conflit à déclarer, P. Galan: Aucun conflit à déclarer, K. Assmann: Aucun conflit à déclarer, E. Kesse-Guyot: Aucun conflit à déclarer 
Association entre consommation de charcuterie et symptômes d'asthme

R. M. Andrianasolo ${ }^{1,}{ }^{*}$, S. Hercberg ${ }^{12}$, M. Touvier ${ }^{1}$, N. Druesne-Pecollo ${ }^{1}$, M. Adjibade ${ }^{1}$, E. Kesse-Guyot ${ }^{1}$, P. Galan ${ }^{1}$, R. Varraso $^{34}$ ${ }^{1}$ Equipe de Recherche en Epidémiologie Nutritionnelle (EREN), UMR U1153 Inserm/U1125/Cnam/Univ Paris13, ${ }^{2}$ Département de Santé Publique, Hôpital Avicenne, Bobigny, ${ }^{3}$ Université Versailles St-Quentin-en-Yvelines, UMR-S 1168, F-78180, Montigny le Bretonneux, ${ }^{4}$ Inserm, U1168, VIMA: Aging and chronic diseases. Epidemiological and public health approaches, F-94807, Villejuif, France

Introduction et but de l'étude : La consommation de charcuterie a été classée comme cancérogène par l'OMS. En ce qui concerne la santé respiratoire, la consommation fréquente de charcuterie a été associée à une augmentation des symptômes, des exacerbations, et de l'incidence des bronchopneumopathies chroniques obstructives. A l'heure actuelle, peu d'études ont été conduites sur l'association entre la consommation de charcuterie et l'asthme, et leurs résultats sont divergents. Par ailleurs, la force de l'association entre la consommation de charcuterie et l'asthme pourrait également dépendre d'autres facteurs pouvant influencer l'équilibre oxydants/antioxydants en faveur des oxydants. Notre étude vise à mieux comprendre l'association entre la consommation de charcuterie et le score de symptôme de l'asthme, ainsi qu'à clarifier le rôle combiné du surpoids/obésité, du tabagisme, d'une moins bonne qualité nutritionnelle de l'alimentation et d'une consommation élevée de charcuterie sur le score de symptôme d'asthme.

Matériel et méthodes : En 2017, 35380 participants de la cohorte NutriNet-Santé ont répondu à un questionnaire détaillé sur la santé respiratoire. L'asthme était défini par le score de symptôme d'asthme (nombre de réponses positives à cinq questions). La consommation de charcuterie, estimée à partir d'au moins 3 enregistrements alimentaires de $24 \mathrm{~h}$ sur 2 ans de suivi, a été classée en 4 catégories : $0,<2,2-5$ ou $>5$ portions / semaine, 1 portion correspondant à 50 grammes. L'association entre consommation de charcuterie et le score de symptôme d'asthme a été évaluée à l'aide de régression binomiale négative. Nous avons également évalué le rôle combiné de 4 comportements à risque - un indice de masse corporelle (IMC) $\geq 25 \mathrm{~kg} / \mathrm{m}^{2}$ (vs. $\left.<25\right)$, être fumeurs "vie » (vs. non-fumeurs), avoir une « moins bonne qualité nutritionnelle globale de l'alimentation estimée à partir du score Alternate Healthy Eating Index-2010 (vs. «meilleure») et consommer >5 portions/semaine de charcuterie (vs. $\leq 5$ ) - sur le score de symptôme de l'asthme par une régression binomiale négative.

Résultats et Analyse statistique : Les participants étaient âgés en moyenne de 54 ans ( \pm 14 ), 49\% étaient fumeurs « vie ", 32\% avaient un IMC $\geq 25 \mathrm{~kg} / \mathrm{m}^{2}$ et $27 \%$ rapportaient au moins 1 symptôme d'asthme dans les 12 derniers mois. Après ajustement sur différents facteurs de confusion, la consommation de charcuterie était positivement et significativement associée au score de symptôme de l'asthme : les odds ratio (OR) (IC 95\%) étaient de 1,15 (1,04-1,27) chez les femmes et de 1,23 (1,01-1,50) chez les hommes, pour une consommation de $>5$ vs 0 portions de charcuterie/semaine. Comparativement aux participants ne présentant aucun des 4 comportements à risque, les OR pour les participants présentant simultanément les 4 comportements à risque étaient de $2,18(1,91-2,48)$ chez les femmes et de $2,70(2,10-3,45)$ chez les hommes.

Conclusion : La consommation élevée de charcuterie est associée à une augmentation des symptômes d'asthme, et son rôle est d'autant plus important quand sa consommation était combinée à d'autres comportements à risque tels que le surpoids/obésité, le tabagisme et une mauvaise qualité nutritionnelle de l'alimentation.

Conflits d'intérêts : Aucun conflit à déclarer 
Association entre le score individuel FSA mesurant la qualité globale de l'alimentation et les symptômes d'asthme R. M. Andrianasolo ${ }^{1,}{ }^{*}$, C. Julia ${ }^{12}$, R. Varraso ${ }^{34}$, M. Egnell ${ }^{1}$, M. Touvier ${ }^{1}$, E. Kesse-Guyot ${ }^{1}$, S. Hercberg ${ }^{12}$, P. Galan ${ }^{1}$

${ }^{1}$ Equipe de Recherche en Epidémiologie Nutritionnelle (EREN), UMR U1153 Inserm/U1125/Cnam/Univ Paris13, ${ }^{2}$ Département de Santé Publique, Hôpital Avicenne, Bobigny, ${ }^{3}$ Université Versailles St-Quentin-en-Yvelines, UMR-S 1168, F-78180, Montigny le Bretonneux, ${ }^{4}$ Inserm, U1168, VIMA: Aging and chronic diseases. Epidemiological and public health approaches, F-94807, Villejuif, France

Introduction et but de l'étude : La France a adopté en 2017 un étiquetage nutritionnel simplifié sur la face avant des emballages (Nutri-Score) destiné à informer les consommateurs de façon simple sur la qualité nutritionnelle des aliments. Il est basé sur l'utilisation d'un système de profil nutritionnel (NPS) mise au point par la UK Food Standard Agency (FSA) et modifié par le Haut Comité de la Santé Publique en France et appelé FSA-NPS. Au niveau individuel, le score FSAm-NPS DI (FSA Dietary Index) reflète la qualité nutritionnelle globale de l'alimentation. Notre objectif était d'étudier, dans une cohorte prospective, l'association entre la qualité globale de l'alimentation évaluée par le score FSAm-NPS DI (qui sous-tend le calcul du Nutri-Score) et le score de symptôme de l'asthme.

Matériel et méthodes : 34323 participants (25 823 femmes et 8500 hommes) de la cohorte NutriNet-Santé ont été inclus dans l'analyse. La qualité nutritionnelle globale du régime a été évaluée à l'aide du score FSAm-NPS DI. L'asthme était défini par le score de symptôme d'asthme (basé sur le nombre de réponses positives à cinq questions sur la présence de symptômes d'asthme dans les 12 derniers mois). L'association entre score FSAm-NPS DI et le score de symptôme d'asthme a été évaluée à l'aide de régression binomiale négative.

Résultats et Analyse statistique : L'âge moyen des participants était de 54 ans ( \pm 14 ), 27\% rapportaient au moins un symptôme d'asthme. Après ajustement sur plusieurs facteurs de confusion potentiels, un score FSAm-NPS DI plus élevé, reflétant une moins bonne qualité nutritionnelle de l'alimentation, était associé à un score de symptôme de l'asthme plus élevé avec un odds ratio (IC $95 \%)$ de $1,27(1,17-1,38)$ chez les femmes et $1,31(1,13-1,53)$ chez les hommes.

Conclusion : Un choix alimentaire de moindre qualité nutritionnelle, tel que reflété par un score FSAm-NPS DI plus élevé, est associé à une augmentation des symptômes d'asthme. Ces résultats contribuent à valider le score FSAm, ils renforcent également la pertinence de l'approche de santé publique qui vise à orienter les consommateurs vers des choix alimentaires plus sains en mettant en place un système d'étiquetage nutritionnel sur la face avant des emballages, basé sur le score FSAm-NPS, comme le Nutri-Score.

Conflits d'intérêts : Aucun conflit à déclarer 
Les technologies innovantes pour l'évaluation de la prise alimentaire par capture d'image : un outil pour la recherche clinique et épidémiologique ?

L. Albaladejo ${ }^{1, *}$, M.-F. Vaillant ${ }^{23}$, P. Gillois ${ }^{34}$, J.-L. Bosson ${ }^{34}$

${ }^{1}$ Université Grenoble Alpes, Grenoble, France, ${ }^{2}$ Laboratoire de Bioénergétique Fondamentale et Appliquée, INSERM U1055, Université Grenoble Alpes, ${ }^{3} \mathrm{CHU}$ Grenoble Alpes, ${ }^{4} \mathrm{TIMC}-\mathrm{IMAG}$, UMR CNRS5525, Université Grenoble Alpes, Grenoble, France

Introduction et but de l'étude : La méthode de référence de l'évaluation des apports nutritionnels est l'enquête alimentaire traditionnelle confrontée à un problème de quantification et de sous-déclaration. L'utilisation des technologies innovantes devient une solution à considérer pour l'évaluation nutritionnelle. Nous avons effectué une revue systématique des articles publiés entre 2007 et 2017 sur les outils d'évaluation des apports nutritionnels utilisant la capture d'image sur smartphone. Il s'agissait aussi d'évaluer leur fiabilité dans les études épidémiologiques.

Matériel et méthodes : Identification sur la plateforme Pubmed d'articles en anglais sur les critères d'inclusion suivant : études sur populations de tous âges et ethnies, avec ou sans pathologie, utilisant les photos pour analyser la prise alimentaire. N'ont pas été incluses les études analysant l'influence de la prise de photo sur le comportement alimentaire, les technologies n'utilisant pas la capture d'image, les protocoles utilisant un catalogue d'images.

Critère principal de l'étude : Capture d'images des plats par photo

Critère secondaire : Comparaison entre la méthode de référence et la nouvelle technologie des résultats obtenus sur l'estimation qualitative et quantitative des plats

Résultats et Analyse statistique : 564 articles ont été identifiés par mots-clés, 69 retenus par leurs titre et abstract, 17 ont été finalement analysés :

- Trois études portaient sur l'acquisition d'images et estimation de quantités de manière automatique. Cette procédure nécessite uniquement que l'utilisateur prenne une photo. L'application va automatiquement estimer le volume et reconnaître les aliments, par des algorithmes de traitement d'image, facilités par un marqueur fiduciaire. Une analyse nutritionnelle peut également être effectuée par un logiciel avec systèmes de codage

- Quatorze études utilisaient la capture d'images seule suivie d'une analyse par un professionnel de la nutrition. L'utilisateur prend des photos de ses repas ou collations avant et après consommation. Il est également invité à décrire les aliments, les quantités, les marques, les méthodes de cuisson par écrit ou oralement. Un spécialiste de la nutrition évalue et analyse les apports énergétiques

Ces études ont utilisé des protocoles hétérogènes. La fiabilité de l'estimation calorique par capture d'image a été comparée par rapport au gold standard (test à l'eau doublement marquée) et aux méthodes classiques. Les résultats pour la capture d'image se rapprochent du gold standard, mieux que par les méthodes classiques.

Conclusion : Les méthodes de capture d'image sont encore peu courantes dans la recherche et pratique clinique. La méthode la plus utilisée en épidémiologie consiste à utiliser une capture d'image unique analysée par un professionnel compétent. Les études parues ne comparent pas suffisamment les performances technologiques aux méthodes standards d'estimation de l'apport nutritionnel. Ces outils doivent être améliorés, notamment pour corriger les matières grasses ou sucres ajoutés sous-estimés, pour être utiles en recherche épidémiologique et clinique.

Conflits d'intérêts : Aucun conflit à déclarer 
${ }^{1}$ Filière Nutrition et diététique, Haute Ecole de Santé, HES-SO Genève, Carouge, ${ }^{2}$ Département de médecine communautaire, de premier recours et des urgences, Hôpitaux Universitaires de Genève, Genève, ${ }^{3}$ Départemetnt de Médecine Interne, Centre Hospitalier Universitaire Vaudois, Lausanne, Suisse

Introduction et but de l'étude : La qualité globale de l'alimentation a une importance considérable dans le maintien de la santé, mais il n'existe pas, en Suisse, d'outil validé permettant de la caractériser rapidement. Historiquement, ce sont surtout les apports en nutriments qui étaient calculés afin de détecter les risques de carences en macro- et micronutriments. Dans une société de surabondance alimentaire cette approche est insuffisante, car il est possible de couvrir ses besoins en nutriments tout en consommant une diète inadéquate. Le but de cette étude était de développer et valider un Score d'alimentation saine (SCASA) sous forme d'un questionnaire auto-administré.

Matériel et méthodes : Les items ont été formulés sur la base d'une revue de littérature et de la méthode RAND permettant un consensus formalisé d'experts. Une validation faciale de la version 1 du questionnaire (18 items) a été réalisée auprès de 15 volontaires. La validation de construit de la version 2 du questionnaire (17 items ; modalités réponse sous forme de choix entre 3 et 4 valeurs) a été effectuée sur la base de 6 plans alimentaires : 3 de qualité optimale et 3 de qualité défavorable pour la santé. L'analyse a considéré le pourcentage du score maximal obtenu pour chaque plan. La fiabilité a été évaluée dans un échantillon issu de la population $(n=65)$ en comparant le résultat de SCASA et le résultat obtenu sur la base d'un carnet alimentaire de 5 jours (gold-standard). La concordance était évaluée par le kappa de Cohen, considéré comme suffisant à partir de 0,2.

Résultats et Analyse statistique : La validation faciale a montré une excellente compréhensibilité du questionnaire. Les plans alimentaires optimaux ont obtenu des scores de $93 \%$ à $95 \%$ et les plans défavorables des scores de $44 \%$ à $46 \%$. Concernant la fiabilité, 7 items (41\%) présentaient un accord suffisant: fruits, farineux complets, produits laitiers, sources de protéines, poisson, boissons sucrées, bonbons ; 7 items (41\%) présentaient un accord insuffisant: légumes, légumineuses, viande, viande rouge, charcuteries, douceurs \& snacks, fruits oléagineux. Le kappa n’a pas pu être calculé pour 3 items (18\%): matières grasses (manque de détails dans les carnets alimentaires); farineux et alcool (manque de variance).

Conclusion : La cohérence des scores obtenus pour les plans alimentaires indique une bonne validité de construit. Les scores relativement élevés des plans défavorables peuvent être liés au fait que les aliments entrainant une diminution du score étaient regroupés en 4 items, alors que les autres aliments étaient détaillés en 13 items. Le kappa insuffisant de 7 items peut être expliqué par la difficulté à évaluer de manière globale les groupes d’aliments concernés, par un biais de désirabilité sociale (aliments sucrés et snacks) et par un biais de temporalité (consommation de viande accrue lors du début de la saison des grillades). Cette analyse a donné lieu à des modifications de formulation des items et une validation à grande échelle est prévue.

Conflits d'intérêts : Aucun conflit à déclarer 
PNNS-GS2: Développement et validation d'un score de qualité diététique reflétant les recommandations nutritionnelles françaises de 2017

D. Chaltiel ${ }^{1, *}$, M. Adjibade ${ }^{1}$, V. Deschamps ${ }^{2}$, M. Touvier $^{1}$, S. Hercberg ${ }^{13}$, C. Julia ${ }^{13}$, E. Kesse-Guyot ${ }^{1}$

${ }^{1}$ Equipe de Recherche en Épidémiologie Nutritionnelle, ${ }^{2}$ Equipe de Surveillance en Epidémiologie Nutritionnelle, Centre De Recherche Épidémiologie et Statistique Sorbonne, ${ }^{3}$ Service de Santé Publique, Hôpital Avicenne, AP-HP, Bobigny, France

Introduction et but de l'étude : Les recommandations nutritionnelles ayant été revues en mars 2017, il est apparu nécessaire de mettre à jour le Programme National Nutrition Santé - Guidelines Score (PNNS-GS), score de qualité nutritionnelle du régime développé selon les recommandations précédentes datant de 2001. Cette étude visait donc à développer et valider le PNNS-GS2, le nouveau score de qualité diététique basé sur les nouvelles recommandations.

Matériel et méthodes : Notre échantillon était constitué de 80965 participants recrutés parmi les adultes français au sein de la cohorte prospective NutriNet-Santé. Les données collectées comprenaient des enregistrements diététiques des $24 \mathrm{~h}$ sur 2 ans, des données sociodémographiques ainsi que (dans un sous-échantillon de 16938 personnes) des indicateurs clinico-biologiques. Les seuils et les pondérations des composantes du PNNS-GS2 ont été développées collégialement par des experts en nutrition ayant participé à la mise à jour des recommandations. Le score comporte 22 composantes pour une valeur théorique allant de -17 à 13.5 points. Nous avons décrit les données nutritionnelles, sociodémographiques, cliniques et biologiques en fonction du quintile de PNNS-GS2. Les validités d'apparence, de contenu, de construit et de critère ont également été évaluées.

Résultats et Analyse statistique : La moyenne du PNNS-GS2 dans notre échantillon était de 2,1 (SD=3,1) chez les femmes et $-0,3$ $(S D=3,6)$ chez les hommes. Un PNNS-GS2 élevé (et donc une meilleur adhérence aux nouvelles recommandations) était positivement associé avec (différence moyenne Q5-Q1) un âge élevé ( $\Delta$ femmes $=+8,4 / \Delta$ hommes $=+4,7$ ans), une meilleure éducation ( $\Delta$ femmes $=+3,9 / \Delta$ hommes $=+7,4 \%$ avec un niveau universitaire), une plus grande activité physique $(\Delta$ femmes $=+13,3 / \Delta$ hommes $=+3,5 \%$ avec $\geq 60 \mathrm{~min} / \mathrm{j}$ ) et un plus grand pourcentage de non-fumeur ( $\Delta$ femmes $=+9,7 / \Delta$ hommes $=+13,7 \%)$. Un PNNS-GS2 élevé était également associé positivement à un apport plus élevé en fibres $(\Delta \mathrm{femmes}=+8,7 / \Delta$ hommes $=+10,7 \mathrm{~g} / \mathrm{j})$ ou la vitamine $\mathrm{C}(\Delta$ femmes $=+36,6 / \Delta$ hommes $=+43,8 \mathrm{mg} / \mathrm{j})$, et négativement avec la pression artérielle moyenne $(\Delta$ femmes=-3/ $\Delta$ hommes=-2,8 $\mathrm{mmHg}$ ) et les concentrations plasmatiques de LDL-cholestérol $(\Delta \mathrm{femmes}=-$ $0,07 / \Delta$ hommes $=-0,06 \mathrm{~g} / \mathrm{L}$ ) et de triglycérides ( $\Delta$ femmes $=-0,1 / \Delta$ hommes $=-0,16 \mathrm{~g} / \mathrm{L}$ ). Tous les tests étaient significatifs $(p<0,05)$.

Conclusion : Les associations observées entre le PNNS-GS2 et les facteurs sociodémographiques, nutritionnels et clinicobiologiques étudiés sont cohérentes et attestent de sa validité. Des études ultérieures seront nécessaires pour estimer son association avec des événements de mortalité et de morbidité.

Conflits d'intérêts : Aucun conflit à déclarer 
Analyse de l'association prospective entre le score d'adéquation aux recommandations nutritionnelles françaises de 2017 (PNNS-GS2) et l'apparition de surpoids et d'obésité dans la cohorte NutriNet-Santé

D. Chaltiel ${ }^{1, *}$, V. Deschamps ${ }^{2}$, M. Touvier ${ }^{1}$, S. Hercberg ${ }^{13}$, C. Julia ${ }^{13}$, E. Kesse-Guyot ${ }^{1}$

${ }^{1}$ Equipe de Recherche en Épidémiologie Nutritionnelle, ${ }^{2}$ Equipe de Surveillance en Epidémiologie Nutritionnelle, Centre De Recherche Épidémiologie et Statistique Sorbonne, ${ }^{3}$ Service de Santé Publique, Hôpital Avicenne, AP-HP, Bobigny, France

Introduction et but de l'étude : Le nouveau score d'adéquation aux recommandations nutritionnelles françaises de mars 2017 (PNNS-GS2) a été préalablement validé. Cette validation doit toutefois être poursuivie par l'évaluation de la valeur prédictive de ce nouveau score sur les événements de santé. Le surpoids et l'obésité étant des facteurs de risque majeurs de morbi-mortalité très liés à l'alimentation, cette étude visait donc à analyser leur association prospective avec le PNNS-GS2. Un objectif secondaire était de comparer ces résultats à ceux obtenus avec le PNNS-GS, reflétant les précédentes recommandations.

Matériel et méthodes : L'échantillon était constitué de participants recrutés parmi les adultes français au sein de la cohorte prospective NutriNet-Santé ( $\mathrm{N}=31804$ pour le surpoids et 42471 pour l'obésité). Le PNNS-GS et le PNNS-GS2 ont été calculés à partir des données alimentaires recueillies au cours des 2 premières années de suivi, puis les poids ont été enregistrés lors des réponses à différents questionnaires. L'association entre les scores et l'incidence de surpoids ou d'obésité a été modélisée par un modèle de Cox. Les événements étaient définis comme avoir un IMC devenant supérieur à 25 ou 30, pour le surpoids ou l'obésité respectivement. La comparaison au PNNS-GS s'est faite par l'intermédiaire du mPNNS-GS, score modifié sans la composante " activité physique ".

Résultats et Analyse statistique : Les moyennes des scores étaient de 2,2 (SD=3,2) pour le PNNS-GS2 et 8.2 (SD=1.6) pour le mPNNS-GS dans l'échantillon "surpoids", et de $1.9(\mathrm{SD}=3,3)$ pour le PNNS-GS2 et 8.2 (SD=1.6) pour le mPNNS-GS dans l'échantillon " obésité ". Le PNNS-GS2 est significativement associé à l'incidence de surpoids et d'obésité dans notre cohorte, en quintiles comme en continu, comme le montrent les Hazard Ratios du tableau suivant (modèles ajustés sur âge (échelle de temps), sexe, taille, apport énergétique, activité physique, catégorie socio-professionnelle, tabagisme, cohabitation, revenus et consommation d'alcool, en gras si significatifs), et ce de façon plus marquée que le mPNNS-GS.

\begin{tabular}{|c|c|c|c|c|c|c|c|}
\hline & Score & $\begin{array}{c}\text { HR } \\
{[95 \% I C]} \\
\text { Q1 }\end{array}$ & $\begin{array}{c}\text { HR [95\%IC] } \\
\text { Q2 }\end{array}$ & $\begin{array}{c}\text { HR [95\%IC] } \\
\text { Q3 }\end{array}$ & $\begin{array}{c}\text { HR [95\%IC] } \\
\text { Q4 }\end{array}$ & $\begin{array}{c}\text { HR [95\%IC] } \\
\text { Q5 }\end{array}$ & $\begin{array}{l}\text { HR }[95 \% I C] \\
\text { Continu }\end{array}$ \\
\hline \multirow[t]{2}{*}{ Obésité } & PNNSGS2 & 1.00 & $0.91[0.79-1.04]$ & $0.70[0.60-0.82]$ & $0.57[0.49-0.68]$ & $0.43[0.36-0.51]$ & $0.90[0.88-0.92]$ \\
\hline & mPNNSGS & 1.00 & $1.05[0.90-1.23]$ & $1.08[0.93-1.26]$ & $1.05[0.88-1.24]$ & $1.03[0.87-1.21]$ & $1.01[0.97-1.04]$ \\
\hline \multirow[t]{2}{*}{ Surpoids } & PNNSGS2 & 1.00 & $0.81[0.73-0.89]$ & $0.74[0.66-0.82]$ & $0.61[0.54-0.68]$ & $0.48[0.43-0.54]$ & $0.92[0.91-0.93]$ \\
\hline & mPNNSGS & 1.00 & $1.04[0.94-1.15]$ & $1.01[0.91-1.12]$ & $1.02[0.91-1.15]$ & $0.96[0.86-1.07]$ & 0.99 [0.97-1.02] \\
\hline
\end{tabular}

Les tests de tendance linéaire sur les quintiles de PNNS-GS2 étaient significatifs pour le surpoids et l'obésité ( $p<0.0001$ ). Concernant le mPNNS-GS, les tests étaient non-significatifs pour le surpoids ( $p=0.445$ ) comme pour l'obésité ( $p=0.792)$.

Conclusion : L'association forte du PNNS-GS2 avec les incidences de surpoids et d'obésité renforcent sa validité de construit. Sa supériorité apparente sur le mPNNS-GS contribue à la pertinence des nouvelles recommandations nutritionnelles. L'association avec d'autres événements de santé devra être testée.

Conflits d'intérêts : Aucun conflit à déclarer 

l'industrie agroalimentaire au Maroc

\author{
A. Derouiche ${ }^{12,{ }^{*}}$, H. Makhlouki ${ }^{3}$, A. Belhouari ${ }^{3}$, K. Mouhtadi $^{3}$, Y. Elkardi ${ }^{3}$, A. Jafri ${ }^{4}$, C. Martin ${ }^{1}$
}

${ }^{1}$ Institut Danone Maroc, ${ }^{2}$ Goupe de Recherche Nutrition Humaine LBS URAC34, université Hassan2 FSBM Casablanca Maroc, ${ }^{3}$ Groupe de Recherche Nutrition Humaine LBS URAC34, Université Hassan2 FSBM Casablanca Maroc, ${ }^{4}$ Faculté des sciences et techniques de la santé, Université Mohammed VI des sciences de la santé, Casablanca, Maroc

Introduction et but de l'étude : L'éducation nutritionnelle vise l'amélioration volontaire des comportements alimentaires et l'adoption des habitudes saines. Dans un environnement de travail, l'éducation nutritionnelle relève de la responsabilité sociale de l'employeur de promouvoir des choix alimentaires plus sains. Cette étude vise à évaluer l'impact de l'éducation nutritionnelle sur le comportement alimentaire, le mode de vie et la composition corporelle d'un groupe de salarié d'une industrieagroalimentaire.

Matériel et méthodes : Un groupe de 44 travailleurs se sont portés volontaires pour participer à cet essai de six mois. Les participants étaient agées de 28 à 58 ans (43\% de femmes et $57 \%$ d'hommes). Tous les participants ont rempli un questionnaire d'évaluation par des scores au début et à la fin de l'étude. Le questionnaire était divisé en trois sections: groupes alimentaires, comportement alimentaire et mode de vie. Les mesures anthropométriques (taille, poids, IMC, masse musculaire, masse grasse, circonférence de la hanche et tour de taille) ont été surveillées pendant l'étude. Des séances d'éducation nutritionnelle ont porté sur quatre sujets, dont le mode de vie sain. Les participants étaient libres d'assister aux ateliers

Résultats et Analyse statistique : Seulement quinze participants ont assisté à toutes les sessions. Les résultats ont montré une diminution de $28,6 \%$ d'Obésité et une augmentation de 32,6\%de surpoids mais sans atteindre la significativité. Les changements des scores du comportement alimentaire n'étaient pas significatifs, alors que ceux des groupes alimentaires et du mode de vie ont changé de manière significative ( $p=0,053$ et $p=0,042$ respectivement)

Conclusion : Cette étude révèle l'engagement des travailleurs dans l'éducation nutritionnelle, ce qui pourrait contribuer à améliorer leurs choix alimentaires, leur santé et leur productivité.

Mots-clés: éducation nutritionnelle, industrie, travailleurs, Maroc

Remerciements : Nous remercions les salariés et leurs responsables d'avoir permis le déroulement de cette étude

Conflits d'intérêts : Aucun conflit à déclarer 
Évaluation de la consommation de l'iode et du sodium dans une population d'adultes Marocains âgée de 18 à 25 ans. A. Derouiche ${ }^{1,{ }^{*}}$, M. Elarbaoui ${ }^{1}$, F. Achibane ${ }^{1}$, H. Makhlouki ${ }^{1}$, S. Loukda ${ }^{1}$, Y. ElKardi ${ }^{1}$, H. Taki $^{1}$, R. Saile $^{1}$, A. Jafri ${ }^{2}$, B. Ellahi $^{3}$ ${ }^{1}$ Groupe de Recherche Nutrition Humaine LBS URAC34, Université Hassan2 FSBM Casablanca Maroc, ${ }^{2}$ Faculté des sciences de la santé, Université Mohammed VI, Casablanca, Maroc, ${ }^{3}$ Faculté de la santé et des services sociaux, Université de Chester, Chester, Royaume-Uni

Introduction et but de l'étude : La surconsommation du sel peut induire plusieurs maladies non transmissibles tel que l'HTA, cancer de l'estomac, l'ostéoporose le diabète de type2, l'obésité ...ce pendant ce condiment est le véhicule principal de l'iode de consommation au Maroc et sa carence constituée un problème de santé publique dans ledit pays. De ce fait, le plan d'action mené au Maroc pour la réduction de la consommation du sel doit tenir compte de la couverture des besoins en lode à travers le sel. L'objectif de ce travail est l'évaluation des apports quotidiens en sodium et en iode dans une population d'adultes âgés de 18 à 25 ans.

Matériel et méthodes : Un échantillon de 120 étudiants volontaires a été recruté. Apres les mesures anthropométriques des participants, chaque étudiant a collecté ses urines de $24 \mathrm{~h}$ selon la méthodologie de l'OMS. Apres la mesure des volumes des collettes, un prélèvement de $30 \mathrm{ml}$ a été effectué de chaque échantillon est stocké à -20 degrés jusqu'à l'analyse de l'iode, le sodium, le potassium, la créatinine et le magnésium

Résultats et Analyse statistique : Nos résultats d'analyse de l'excrétion urinaire en lode ont montré que 37,5\% personnes étaient sous la normale $57,5 \%$ avaient des valeurs normales, alors que $5 \%$ étaient en surcharge en lode $300 \mu \mathrm{g}$. Concernant l'excrétion de sodium par 24 heures elle a montré une moyenne de 3,1 g /jour, soit 7,7 g de sel / jour; distribuée en 9g et $8.4 \mathrm{~g} / \mathrm{jour}$ chez les hommes et les femmes respectivement. L'analyse des résultats de la distribution des niveaux de consommation quotidienne en sel a montré que 15,20\% consommaient moins de $5 \mathrm{~g} /$ jour, tandis que $84,8 \%$ consommaient plus de $5 \mathrm{~g} /$ jour, dont 17,1\% consommaient plus du double des recommandations de l'OMS fixées à $5 \mathrm{~g} / \mathrm{j}$.

Conclusion : Une carence en iode a été constatée chez $37,5 \%$ de notre population, alors que $84 \%$ consommaient plus de $5 \mathrm{~g}$ de chlorure de sodium, ce qui nécessite des campagnes de sensibilisation et des politiques visant à réduire la consommation de sodium et en tenant compte des apports en lode à travers le sel fortifiée en ce dernier.

MOTS-CLÉS: lode, sel, maladies apparentées, collecte d'urine, mesures anthropométriques, rappel de 24h, Maroc.

Remerciements : Nous remercions l'ensembles des étudiants des Masters Biologie et Santé, Master Biotechnologie Démarche Qualité, Master International MISS ainsi que les étudiants de la licence de la Faculté des Sciences Ben M'Sik Université Hassan2 Casablanca qui ont participé volontairement à cette étude.

Conflits d'intérêts : Aucun conflit à déclarer 
L'exclusion du gluten dans la population française : caractéristiques socio-démographiques, motivations et profils alimentaires L. Perrin ${ }^{1}$, B. Allès ${ }^{1,}$, , C. Buscail ${ }^{12}$, S. Hercberg ${ }^{12}$, C. Julia ${ }^{1}$, E. Kesse-Guyot ${ }^{1}$

${ }^{1}$ Equipe de Recherche en Epidémiologie Nutritionnelle (EREN), Université Paris $13,{ }^{2}$ Département de Santé Publique, Hôpital Avicennes, Bobigny, France

Introduction et but de l'étude : Dans les pays occidentaux, le régime sans gluten s'est récemment développé chez les personnes en bonne santé. Cependant, il existe peu d'informations sur les caractéristiques sociodémographiques, et plus spécifiquement le profil alimentaire de ces consommateurs. L'objectif de cette étude était de déterminer la prévalence de l'exclusion du gluten dans la population française adulte, de décrire les motivations des personnes qui l'excluent et comparer leurs caractéristiques sociodémographiques et leurs profils alimentaires à ceux qui ne l'excluent pas.

Matériel et méthodes : L'échantillon de notre étude était composé de 20456 participants de la cohorte NutriNet-Santé qui avaient répondu à un questionnaire optionnel sur les exclusions alimentaires et ne possédaient pas de données manquantes sur les caractéristiques sociodémographiques et les données alimentaires. Les individus qui avaient déclarés une maladie cœliaque étaient exclus de l'échantillon. Pour être davantage représentatif de la population française, nous avons pondéré nos données avec les données de recensement de la population française. Dans nos analyses, la consommation alimentaire et les apports en nutriments ont été estimés à partir d'une ANCOVA ajustée sur l'énergie, l'âge et le sexe. Par la suite, nous avons réalisé une Analyse en Composantes Principales (ACP) sur 18 groupes alimentaires. Trois profils alimentaires ont été identifiés et une régression logistique polytomique a ensuite été réalisée pour mesurer l'association entre les régimes alimentaires (quintiles, Q) et la pratique d'exclusion du gluten (totale, partielle, sans exclusion).

Résultats et Analyse statistique : Dans notre échantillon, 10,3\% des individus excluaient le gluten, dont 1,7\% totalement. Les individus excluant le gluten étaient majoritairement plus âgés, des femmes, ayant un niveau d'éducation faible, et un plus grand nombre d'exclusions alimentaires, dont le lactose. La principale motivation d'exclusion du gluten était le bien-être physique (exclusion totale : $25,6 \%$ et partielle : $38,9 \%$ ) et la perception que c'est plus sain (exclusion totale : $22,1 \%$ et partielle : $27,8 \%$ ). Ils avaient un profil globalement plus sain : ils étaient non-fumeurs, consommaient davantage de fruits et légumes, et moins d'alcool et de produits gras. Leurs apports en nutriments étaient également plus favorables. Après ajustement sur de nombreux facteurs de confusion, le profil alimentaire « sain » était positivement associé à l'exclusion totale du gluten (OR Q5 vs Q1 = 14.44, IC à 95\% $=[8.62-24.19], \mathrm{p}<.0001)$.

Conclusion : Nos résultats ont mis en évidence que les personnes qui excluent le gluten présentent un profil alimentaire globalement plus sain. Cette étude apporte de nouvelles informations sur la consommation alimentaire et les comportements des personnes excluant le gluten et pourra servir de critère à de futures études s'intéressant aux impacts potentiels d'un régime sans gluten chez les individus en bonne santé.

Conflits d'intérêts : Aucun conflit à déclarer 

M. Egnell ${ }^{1,}{ }^{*}$, P. Crosetto ${ }^{2}$, T. D'Almeida ${ }^{1}$, E. Kesse-Guyot ${ }^{1}$, M. Touvier ${ }^{1}$, B. Ruffieux ${ }^{23}$, S. Hercberg ${ }^{14}$, L. Muller $^{2}$, C. Julia $^{14}$ ${ }^{1}$ Equipe de Recherche en Epidémiologie Nutritionnelle, Université Paris 13, Bobigny, ${ }^{2}$ UMR 1215 GAEL, INRA, ${ }^{3}$ Institut Polytechnique de Grenoble, Grenoble, ${ }^{4}$ Département de Santé Publique, Hôpital Avicenne, Bobigny, France

Introduction et but de l'étude : Les logos nutritionnels en face avant des emballages ont pour objectif d'améliorer les choix alimentaires, et pourraient donc potentiellement participer à la prévention des maladies chroniques liées à la nutrition. Ces logos nutritionnels ont en effet été montrés comme étant associés à une amélioration de la qualité nutritionnelle des achats ainsi que de la qualité des régimes, elle-même associée ensuite au risque d'apparition de maladies chroniques. Cependant, l'impact potentiel direct des logos nutritionnels en face avant des emballages sur la mortalité par maladies chroniques n'a encore jamais été estimé. Ainsi, l'objectif de l'étude était d'estimer l'impact potentiel de cinq logos nutritionnels - Nutri-Score, Health Star Rating system (HSR), Feux tricolores Multiples (FTM), Apports de Références (ARs) et SENS - sur la mortalité par maladies chroniques liées à la nutrition à l'aide d'un modèle de simulation.

Matériel et méthodes : Des données issues d'une expérimentation en laboratoire (Equipe GAEL INRA) ont été utilisées afin d'évaluer les effets de ces cinq logos sur la qualité nutritionnelle des achats. Les différences relatives de compositions nutritionnelles des paniers d'achats entre une situation d'achats de référence sans logo et une situation d'achats avec un des cinq logos sur les emballages, ont été ensuite transposées à des données de consommations alimentaires issues d'une étude observationnelle (Etude de cohorte NutriNet-Santé, Université Paris 13), afin de déterminer les apports nutritionnels d'un régime de 'référence' et d'un régime 'avec logo'. Enfin, une étude de macro-simulation basée sur le modèle PRIME a été menée afin d'estimer l'impact de la modification des apports nutritionnels entre les deux régimes liée à l'utilisation d'un logo sur la mortalité par maladies chroniques (modèle PRIME, Université d'Oxford).

Résultats et Analyse statistique : Le modèle PRIME simule le nombre de morts évitées par maladies chroniques liées à la nutrition, résultant de la différence du nombre de morts évitées entre une distribution baseline (régime de 'référence') et une distribution counterfactual (régime 'avec logo'). L'utilisation des logos nutritionnels conduirait à une diminution significative de la mortalité par maladies chroniques comparée à la structure de mortalité dans la population française en 2014, avec des résultats contrastés selon le logo. Approximativement 3,4\% des morts causées par des maladies chroniques liées à la nutrition seraient évitées par le Nutri-Score, suivi du HSR (2,8\%), des ARs $(1,9 \%)$, des FTM (1,6\%) puis le SENS (1,1\%). Les maladies les plus impactées seraient principalement les maladies cardiovasculaires et les cancers liées à la nutrition.

Conclusion : Le Nutri-Score, avec son format graduel, résumé, et l'utilisation de couleurs à sémantique forte, semble être le logo nutritionnel en face avant des emballages le plus efficace pour participer à la diminution de la mortalité par maladies chroniques liées à la nutrition.

Conflits d'intérêts : Aucun conflit à déclarer 
Compréhension objective de différents formats de logos nutritionnels en face avant des emballages : résultats d'une étude comparative expérimentale internationale

M. Egnell ${ }^{1, *}$, S. Pettigrew ${ }^{2}$, Z. Talati $^{2}$, S. Hercberg ${ }^{13}$, C. Julia ${ }^{13}$

${ }^{1}$ Equipe de Recherche en Epidémiologie Nutritionnelle, Université Paris 13, Bobigny, France, ${ }^{2}$ School of Psychology, Université de Curtin, Perth, Australie, ${ }^{3}$ Département de Santé Publique, Hôpital Avicenne, Bobigny, France

Introduction et but de l'étude : Dans un objectif de prévention des maladies chroniques liées à la nutrition, les logos nutritionnels en face avant des emballages ont été identifiés comme des outils efficaces pour aider les consommateurs à mieux identifier la qualité nutritionnelle des produits et les encourager à faire des choix plus sains. Il a été démontré que le format graphique d'un logo pouvait influencer son efficacité auprès du consommateur, pourtant peu d'études ont comparé plusieurs formats de logos sur la compréhension objective par les consommateurs, en incluant notamment de nouveaux logos, et au sein de contextes culturels différents. L'objectif était donc d'étudier la compréhension objective de 5 logos (Health Star Rating système(HSR), Feux Tricolores Multiples(FTM), Nutri-Score, Apports de Référence(ARs) et symboles Warning), dans 12 pays.

Matériel et méthodes : La compréhension objective des 5 logos a été étudiée via une enquête en ligne en Allemagne, Argentine, Australie, Bulgarie, Canada, Danemark, Espagne, Etats-Unis, France, Mexique, Royaume-Uni, et Singapour. Les participants étaient randomisés dans l'un des 5 groupes: HSR, FTM, Nutri-Score, ARs ou Warning. La compréhension des logos était mesurée à travers leur capacité à classer des séries de 3 produits d'une même catégorie, selon leur qualité nutritionnelle, dans une situation contrôle sans logo puis dans la situation avec logo. Trois catégories de produits ont été testées dans le cadre de l'étude : pizzas, gâteaux et céréales petit-déjeuner. Les effets des logos sur la compréhension étaient évalués à l'aide de régressions logistiques ordinales, comparant entre les différents logos, l'amélioration de la capacité des consommateurs à correctement classer les produits comparé au sans logo.

Résultats et Analyse statistique : Comparé à l'absence de logo, les 5 logos augmentaient le nombre de bonnes réponses concernant le classement des produits, avec des performances hétérogènes selon les formats. Au global, comparé aux ARs (logo avec le moins de bonnes réponses), le Nutri-Score était le logo associé à l'amélioration la plus importante de la capacité des consommateurs à classer correctement les produits (OR=3.07[2.75-3.43], p-value<0.0001), suivi des FTM (OR=1.77[1.59-1.98], pvalue<0.0001), HSR (OR=1.37[1.23-1.53], p-value<0.0001), puis des Warning (OR=1.28[1.15-1.43], $p$-value<0.0001). Le Nutri-Score était aussi le logo le mieux compris quel que soit le pays, avec des ORs allant de 2.14[1.48-3.10] ( $p$-value=0.001) en Argentine jusqu'à 4.45[3.02-6.56] ( $p$-value<0.0001) à Singapour. Des tendances similaires étaient observées par catégorie de produits. En excluant les sujets ayant déclaré ne pas avoir vu le logo lors de l'enquête, le Nutri-Score était toujours le logo avec les meilleurs résultats, suivi des Warning.

Conclusion : Malgré les différences culturelles, le Nutri-Score avec son format résumé, graduel et coloré semble être le logo le plus efficace pour aider les consommateurs à identifier la qualité nutritionnelle des produits, dans la très grande majorité des pays inclus dans l'étude quelle que soit la catégorie de produits testée.

Conflits d'intérêts : Aucun conflit à déclarer 

A. Demidem ${ }^{3}$, M.-P. Vasson ${ }^{3} 5$, V. Partula ${ }^{2}$, M. Deschasaux ${ }^{2}$, B. Srour ${ }^{2}$, P. Latino-Martel ${ }^{2}$, E. Kesse-Guyot ${ }^{2}$, S. Durand ${ }^{3}$, E. Pujos-Guillot ${ }^{3}$, C. Manach ${ }^{36}$, T. Mathilde ${ }^{26}$

${ }^{1}$ Contribution équivalente (co-premiers), ${ }^{2}$ EREN, CRESS, Université Paris 13, Bobigny, ${ }^{3}$ INRA, UNH, Clermont Auvergne University, Clermont-Ferrand, ${ }^{4}$ Département de santé publique, Hôpital Avicenne, Bobigny, ${ }^{5}$ Centre Anticancer Jean-Perrin, CHU ClermontFerrand, Clermont-Ferrand, ${ }^{6}$ Contribution équivalente (co-derniers), France

Introduction et but de l'étude : Notre alimentation se traduit au niveau plasmatique par la présence de divers métabolites exogènes et endogènes. L'identification de profils de métabolites plasmatiques liés à la nutrition permettrait d'améliorer les mesures d'exposition alimentaire en épidémiologie nutritionnelle et de mieux comprendre les effets biologiques de certains composants alimentaires et notamment ceux de la qualité de l'alimentation sur la santé. Notre objectif était donc de déterminer les associations entre différents profils alimentaires et les profils de métabolites plasmatiques.

Matériel et méthodes : Cette étude incluait 160 femmes de la cohorte SU.VI.MAX ( $\geq 10$ R24). Ces femmes ont été sélectionnées selon leur niveau d'adéquation aux recommandations du Programme National Nutrition Santé ; 80 femmes au sein du 10e décile de la distribution du score PNNS-GS, appariées avec 80 femmes du 1er décile. Les échantillons de plasma récoltés à l'inclusion, ont été analysés en spectrométrie de masse non ciblée. Les données ont été analysées avec des méthodes de régressions logistiques conditionnelles ( $1^{\mathrm{e}}$ vs $10^{\mathrm{e}}$ décile du score PNNS-GS) et des analyses de corrélations avec les composantes du score PNNS-GS et avec des patterns issus d'une Analyse en Composante Principale (ACP) réalisée sur 29 groupes d'aliments.

Résultats et Analyse statistique : Au total, 30 ions étaient associés aux données nutritionnelles, l'identification de ces ions est en cours. 10 ions étaient associés au score PNNS-GS (FDR<0,1); parmi eux, l'acide pipécolique était associé à une bonne adéquation aux recommandations nutritionnelles alors que le 2-deoxy-D-glucose était associé à une mauvaise adéquation aux recommandations. 8 ions étaient associés à au moins une composante de l'ACP (FDR $<0,1$ et coefficient $>|0,2|) ; 4$ associés négativement au profil alimentaire "Western", dont l'acide pipécolique ; 5 associés positivement au profil alimentaire " Healthy ", dont l'acide pipécolique et 1 associé négativement à ce dernier profil ; 1 associé négativement au profil alimentaire "Produits sucrés». L'acide pipécolique était également associé (FDR<0,1 et coefficient $>|0,2|$ ) à la composante "fruits et légumes » du score PNNS-GS.

Conclusion : Cette étude met en évidence des associations entre profils métabolomiques et profils alimentaires obtenus par deux méthodes complémentaires. Les premiers résultats permettent de révéler des signatures métabolomiques liées à des profils alimentaires différents. L'identification de ces signatures pourrait contribuer à terme à l'amélioration de l'évaluation des expositions nutritionnelles dans les études épidémiologiques et à une meilleure compréhension des mécanismes impliqués dans les relations nutrition-santé.

Remerciements : Institut National du Cancer (INCa) / Institut Fédératif de Recherche biomédicale (IFRB) / Canceropôle Ile-deFrance / Projet labellisé NACRe Partenariat

Conflits d'intérêts : Aucun conflit à déclarer 

M. Egnell ${ }^{1,}{ }^{*}$, E. Kesse-Guyot ${ }^{1}$, P. Galan ${ }^{1}$, M. Touvier ${ }^{1}$, M. Rayner ${ }^{2}$, J. Jewell ${ }^{3}$, J. Breda ${ }^{34}$, S. Hercberg ${ }^{15}$, C. Julia $^{15}$

${ }^{1}$ EREN, Université Paris 13, Bobigny, France, ${ }^{2}$ Centre on Population Approaches for Non-Communicable Disease Prevention, University of Oxford, Oxford, Royaume-Uni, ${ }^{3}$ Nutrition, Physical Activity and Obesity Programme, WHO Regional Office for Europe Copenhagen, Copenhagen, Danemark, ${ }^{4}$ Office for the Prevention and Control of Noncommunicable Diseases, WHO Regional Office for Europe, Moscow, Moscow, Russie, Fédération De, ${ }^{5}$ Département de Santé Publique, Hôpital Avicenne, Bobigny, France

Introduction et but de l'étude : En Europe, 3 logos nutritionnels en face avant des emballages, 2 mis en place par des gouvernements (Nutri-Score et Feux Tricolores Multiples (FTM)) et l'un créé par un consortium d'entreprises agro-alimentaires (Evolved Nutrition Label (ENL)), sont en discussion. Contrairement au Nutri-Score et aux FTM basés sur le contenu nutritionnel au $100 \mathrm{~g}$ d'un aliment, l'ENL est basé sur une portion d'aliment, se traduisant alors par un étiquetage plus favorable spécifiquement pour les aliments consommés en petites quantités. Or, l'effet de ces 3 logos sur la sélection des tailles de portion est inconnu. L'objectif de l'étude était donc d'évaluer l'effet de l'ENL, des FTM et du Nutri-Score sur la sélection des tailles de portions, pour des aliments de moins bonne qualité nutritionnelle, en comparaison à l'absence de logo.

Matériel et méthodes : Chaque participant, issu de la cohorte Nutri-Net Santé, était exposé à 3 catégories alimentaires pour lesquelles une consommation en quantité limitée est recommandée (biscuits, fromages, pâtes à tartiner), et 4 produits par catégorie où les 4 situations d'étiquetage (3 logos et l'absence de logo comme contrôle) étaient représentées. L'ordre d'apparition des produits ainsi que les combinaisons produit*logo étaient randomisées. Pour chaque produit, le participant choisissait une taille de portions, parmi 4 photos standardisées de taille croissante, et le nombre de portions qu'ils consommeraient dans une occasion réelle de consommation. Des tests de Kruskall-Wallis étaient réalisés pour comparer les tailles de portions moyennes choisies entre les différents logos et le contrôle sans logo, ainsi qu'entre les logos. L'effet des logos sur la portion était analysé à l'aide de modèles de régression logistique ordinale.

Résultats et Analyse statistique : Comparé à l'absence de logo, le Nutri-Score conduisait à la sélection d'une portion significativement plus faible (OR=0.76(0.74-0.76), p-value<0.0001), suivi des FTM (OR=0.83(0.82-0.84), $p$-value $<0.0001)$. Au global, I'ENL (OR=0.99(0.98-1.00), p-value=0.2) n'était pas significativement différent du contrôle. Les effets du Nutri-Score et des FTM étaient similaires dans les 3 catégories d'aliments, avec la sélection de portions significativement plus petites. En revanche, les effets de l'ENL étaient différents en fonction de la catégorie considérée : alors qu'il conduisait à une portion plus faible pour les fromages $(\mathrm{OR}=0.84(0.83-0.87)$, p-value<0.0001), il était également associé à une augmentation significative de la taille de portion pour les pâtes à tartiner $(\mathrm{OR}=1.19(1.15-1.22), \mathrm{p}$-value<0.0001).

Conclusion : Le Nutri-Score, suivi des FTM, semblent être des outils efficaces pour augmenter la capacité du consommateur à identifier la qualité nutritionnelle des aliments et l'encourager à choisir des portions plus faibles pour des produits de moins bonne qualité nutritionnelle. En revanche, l'ENL semble avoir à un effet très limité pour diminuer les portions des produits de moins bonne qualité nutritionnelle comparé à l'absence de logo, et tendrait même à accroître la taille de portion pour les pâtes à tartiner.

Conflits d'intérêts : M. Egnell: Aucun conflit à déclarer, E. Kesse-Guyot: Aucun conflit à déclarer, P. Galan: Aucun conflit à déclarer, M. Touvier: Aucun conflit à déclarer, M. Rayner: Aucun conflit à déclarer, J. Jewell est employé(e) de The World Health Organization (WHO) Regional Office for Europe. The authors are responsible for the views expressed in this publication and they do not necessarily represent the decisions or stated policy of WHO. , J. Breda est employé(e) de The World Health Organization (WHO) Regional Office for Europe. The authors are responsible for the views expressed in this publication and they do not necessarily represent the decisions or stated policy of WHO. , S. Hercberg: Aucun conflit à déclarer, C. Julia: Aucun conflit à déclarer 
La prévalence du syndrome métabolique comparée à l'activité physique chez la population de Marrakech, au sud-ouest du Maroc

Z. Hannoun ${ }^{1,}{ }^{*}$, I. Samara ${ }^{2}$, K. Harraqui ${ }^{1}$, R. Attoumane ${ }^{1}$, O. Bensmail ${ }^{3}$, F. El arabi ${ }^{4}$, A. Bour ${ }^{5}$

${ }^{1}$ Doctorante, Faculté des sciences - Université Ibn Tofail - Kenitra, Maroc, Kenitra, ${ }^{2}$ Docteur, Centre hospitalier provincial Prince Moulay Abdellah, Sale, ${ }^{3}$ Doctorant, Faculté des sciences - Université Ibn Tofail - Kenitra, Maroc, Kenitra, ${ }^{4}$ Nutritionniste, Hôpital Mohamed Sekkat, Casablanca, ${ }^{5}$ Professeur, Faculté des sciences - Université Ibn Tofail - Kenitra, Maroc, Kenitra, Maroc

Introduction et but de l'étude : Le syndrome métabolique est un groupe de facteurs de risque de maladies cardiovasculaires où le manque d'activité physique est un des facteurs favorisant son apparition. Le but de cette étude était de déterminer la prévalence du syndrome métabolique par rapport à l'activité physique dans une population de Marrakech, au Maroc.

Matériel et méthodes : L'étude a été menée à l'hôpital régional Ibn zohr de Marrakech. La collecte de données a été réalisée à l'aide d'un questionnaire développé et validé localement et qui comprend les données socio démographiques. L'indice de masse corporel (IMC) a été calculé pour évaluer le degré d'obésité de chaque sujet. Pour déterminer le niveau d'activité physique, nous avons utilisé la version courte du questionnaire IPAQ; ensuite les paramètres sanguins ont été dosés par l'Automate de biochimie DIRUI CS-600. Toutes les analyses statistiques ont été effectuées en utilisant le logiciel SPSS.

Résultats et Analyse statistique : Au total, 300 sujets ont participé à l'étude dont $\mathbf{5 6 . 6 \%}$ des femmes et $\mathbf{4 3 . 3} \%$ des hommes avec un sex-ratio de 0,87 . L'âge moyen de notre population était de $51 \pm 7.78$ ans. Cinquante trois des participants (17.6\%) avaient un syndrome métabolique, avec une prédomminance des femmes : 43 femmes $(81.1 \%)$ et 10 hommes (18.8\%). 43.3\% de la population étudiée ont une activité physique faible, $39.6 \%$ ont une activité modérée et seulement $17 \%$ s'avèrent exercer une activité physique intense, sans différence significative entre les hommes et les femmes.

Conclusion : Le syndrome métabolique s'installe lentement mais sûrement au Maroc. Il constitue une menace d'une augmentation spectaculaire des troubles cardiovasculaires, du diabète de type 2 et de leurs conséquences. La sédentarité est l'un des facteurs lié à l'émergence du syndrome métabolique. Mettre en œuvre des stratégies de prévention et encourager à l'activité physique et au mode de vie sain, minimiseront sûrement les graves problèmes de santé publique au pays.

Conflits d'intérêts : Aucun conflit à déclarer 
Rapport au plaisir alimentaire chez les mangeurs urbains en France

S. Thiron ${ }^{1, *}$

${ }^{1}$ Consultante en socio-anthropologie de l'alimentation, Indépendant, Montpellier, France

Introduction et but de l'étude : Les maux de la société moderne tels que l'obésité ou les problèmes environnementaux sont associés à une dérégulation de la consommation, notamment sur le plan alimentaire. Les mangeurs auraient une inclination trop poussée à s'adonner au plaisir. Pourtant, les réflexions sur le rôle du plaisir alimentaire évoluent depuis quelques années (Dupuy, 2014 ; Régnier et Ferguson, 2014). II n'est plus considéré comme un obstacle à la régulation des comportements, et pourrait devenir un allié de la santé publique tant qu'il reste mesuré (Cornil, Chandon, Touati, 2018).

Cette étude vise à explorer la relation entre santé et plaisir alimentaire chez les mangeurs urbains, et la diversité des rapports au plaisir à partir de ses manifestations quotidiennes.

Matériel et méthodes : Une enquête qualitative a été menée en 2018 à Paris et Montpellier, auprès de 25 adultes ayant entre 23 et 51 ans, 8 hommes et 17 femmes, de tous niveaux de diplômes, appartenant à des catégories sociales moyennes ou supérieures. Une première phase de focus groups (5 groupes regroupant 23 participants) a été suivie d'une phase ethnographique (7 entretiens semi-directifs avec observation à domicile, dont 5 personnes ayant participé aux focus groups).

Résultats et Analyse statistique : Aujourd'hui, de nouveaux enjeux entourent le plaisir alimentaire :

- La contrainte nutritionnelle : le respect d'un certain équilibre nutritionnel,

- La contrainte physiologique : ce qui fait du bien au corps (confort digestif, peau...),

- La contrainte environnementale : la consommation d'aliments respectueux de la planète,

- La contrainte sociale : une forme de solidarité inter- et intra-générationnelle.

Ainsi, le plaisir n'est pas uniquement soumis aux stimulations de l'environnement et aux envies individuelles, mais reste bien encadré. La moralisation du plaisir n'est pas en voie de s'amenuiser. Le plaisir n'est acceptable et accepté que dans le cadre du respect de ces contraintes, qui ne sont plus uniquement d'ordre sanitaire, et doivent répondre aux enjeux sociétaux du XXle siècle. Pour satisfaire ces contraintes, les mangeurs structurent leur rapport au plaisir au travers de rendez-vous ritualisés, dépendant du lieu, du moment, du contexte social et de l'aliment. Ces rendez-vous permettent de réguler le rapport au plaisir sur le long terme, d'éviter les faux pas et les frustrations, en suivant des "cycles du plaisir" sur l'année, la semaine ou encore la journée. Ils apportent du sens, en réenchantant un quotidien alimentaire soumis à des contraintes de plus en plus fortes.

Conclusion : Le plaisir reste soumis à la pression morale dans la société française. Aussi, le mangeur développe des stratégies pour structurer son plaisir conformément à ces contraintes, au travers de rendez-vous qui lui permettent d'équilibrer son rapport au plaisir sur le long terme.

\section{Références :}

Cornil Y., Chandon P., Touati N., 2018, Plaisir épicurien, plaisir viscéral et préférence de tailles de portions alimentaires, Cahiers de Nutrition et de Diététique, 53(2), 77-85

Dupuy A., 2014, Thématisation du plaisir alimentaire et visées utilitaristes, Sociologie et sociétés, 46(2), 253-275

Régnier F., Ferguson P., 2014, Présentation : Manger - négocier le plaisir et la nécessité. Sociologie et sociétés, 46(2), 5-15

Remerciements :

à Anne Dupuy, maîtresse de conférences en sociologie à l'Université Toulouse Jean Jaurès, pour son expertise.

à Danone Research et à Danone Produits Frais France, pour les moyens financiers fournis.

Conflits d'intérêts : S. Thiron a reçu une subvention/soutien de Danone Produits Frais France 
E. Chazelas ${ }^{1}{ }^{*}$, B. Srour ${ }^{1}$, E. Desmetz ${ }^{1}$, E. Kesse-Guyot ${ }^{1}$, C. Julia ${ }^{12}$, V. Deschamps ${ }^{3}$, N. Druesne-Pecollo ${ }^{1}$, P. Galan $^{1}$, S. Hercberg ${ }^{12}$, P. Latino-Martel ${ }^{1}$, M. Deschasaux ${ }^{1}$, M. Touvier ${ }^{1}$

${ }^{1}$ Equipe de Recherche en Epidémiologie Nutritionnelle EREN, UMR U1153 Inserm / U1125 Inra / Cnam / Université Paris 13 Centre de Recherche en Epidémiologie et Biostatistiques Sorbonne Paris Cité, ${ }^{2}$ Département de Santé Publique, Hôpital Avicenne, ${ }^{3}$ Equipe de Surveillance en Epidémiologie Nutritionnelle ESEN, Santé Publique France, Bobigny, France

Introduction et but de l'étude : Selon le Global Burden of Disease, la consommation de boissons sucrées a augmentée d'environ $40 \%$ entre 1990 et 2016. Les boissons sucrées et édulcorées ont étés associées au risque de pathologies cardiométaboliques, mais concernant le lien direct avec le cancer, les données de la littérature sont encore limitées. Notre objectif était d'évaluer les associations entre la consommation de boissons sucrées et édulcorées et le risque de cancer.

Matériel et méthodes : Les analyses ont été effectuées sur 101257 sujets adultes (âge moyen $42.2 \pm 14.4$ ans; temps de suivi moyen 5.1 ans) inclus dans la cohorte prospective NutriNet-Santé (2009-2017). La consommation de boissons sucrées et édulcorées a été évaluée grâce à des enregistrements de $24 \mathrm{~h}$ répétés, élaborés afin de déterminer la consommation habituelle de 3300 items d'aliments et de boissons différents. Les associations entre la consommation de boissons sucrées et édulcorées et le risque de cancer (au global, sein, prostate et colorectal) ont été étudiées grâce à des modèles de Cox à risques proportionnels, ajustés sur les facteurs de confusion connus.

Résultats et Analyse statistique : Une augmentation de $100 \mathrm{~mL}$ de la consommation de boissons sucrées était significativement associée au risque de cancer au global $(n=2193$ cas; $H R=1.08$, intervalle de confiance à $95 \%: 1.04$ à 1.12 , $P<.0001)$ et de cancer du sein ( $n=683$ cas; $H R=1.11$, intervalle de confiance à $95 \%: 1.04$ à $1.19, P<0.002$ ). La consommation de boissons édulcorées $n$ 'était pas associée au risque de cancer. Les sous-analyses ont montré qu'une augmentation de $100 \mathrm{~mL}$ de la consommation de jus de fruit $100 \%$ pur jus était significativement associée au risque de cancer au global ( $H R=1.08$, intervalle de confiance à $95 \%: 1.02$ à $1.15, \mathrm{P}=0.01)$.

Conclusion : Dans cette étude prospective de grande échelle, une augmentation de $100 \mathrm{~mL}$ de la consommation de boissons sucrées était associée à une augmentation significative du risque de cancer au global de $8 \%$ et de cancer du sein de $11 \%$. Les jus de fruits $100 \%$ pur jus étaient aussi associés à une augmentation de risque de cancer au global de $8 \%$. Étant donné la consommation importante de boissons sucrées dans les pays occidentaux, ces résultats suggèrent que ces dernières représentent un facteur de risque modifiable clé dans le cadre de la prévention des cancers.

Conflits d'intérêts : Aucun conflit à déclarer 
Effets des logos nutritionnels en face avant des emballages sur la qualité nutritionnelle des achats des étudiants : essai randomisé contrôlé à trois bras

M. Egnell ${ }^{1,}{ }^{*}$, I. Boutron ${ }^{2}$, S. Péneau ${ }^{1}$, P. Ducrot ${ }^{3}$, M. Touvier ${ }^{1}$, P. Galan ${ }^{1}$, C. Buscail ${ }^{14}$, R. Porcher ${ }^{2}$, P. Ravaud $^{2}$, S. Hercberg ${ }^{14}$, E. Kesse-Guyot ${ }^{1}$, C. Julia ${ }^{14}$

${ }^{1}$ EREN, CRESS, Université Paris 13, Bobigny, ${ }^{2}$ METHODS, CRESS, Hôpital Hôtel-Dieu, Paris, ${ }^{3}$ Santé Publique France, Saint-Maurice, ${ }^{4}$ Département de Santé Publique, Hôpital Avicenne, Bobigny, France

Introduction et but de l'étude : Les étudiants, du fait de leur gain d'autonomie, sont considérés comme une population vulnérable et à risque d'avoir une alimentation déséquilibrée. Les logos nutritionnels en face avant des emballages, apportant une information simplifiée sur le contenu nutritionnel des aliments, sont des outils efficaces pour encourager des choix plus sains. L'objectif de l'étude était de comparer l'effet du Nutri-Score (un logo recommandé par les autorités de santé publique en France) aux Apports de Références (ARs, un implémenté par l'industrie depuis 2006) et à l'absence de logo, sur la qualité nutritionnelle des achats des étudiants.

Matériel et méthodes : Un essai randomisé contrôlé à trois bras parallèles a été mené en 2016, à l'aide d'un supermarché expérimental en ligne. Les participants étaient répartis aléatoirement dans l'un des trois bras : (1) le bras expérimental, où le NutriScore était apposés sur les emballages des produits transformés, ou l'un des 2 bras contrôles (2) avec les ARs ou (3) sans logo. 1866 étudiants âgés de 18 à 25 ans et impliqués dans les achats alimentaires habituellement ont été recrutés par mail au sein d'universités, inclus et invités à réaliser une session d'achats en ligne sur le supermarché expérimental. Le critère de jugement principal était la qualité nutritionnelle globale du panier d'achat, estimée à l'aide du profil nutritionnel de la Food Standard Agency adapté par le HCSP (score FSAm-NPS, un score élevé traduit une moins bonne qualité nutritionnelle). Les critères de jugement secondaires étaient le contenu énergétique et nutritionnel du panier. L'ensemble les produits des paniers ont été inclus dans les analyses. La qualité globale et le contenu nutritionnels des paniers ont été comparés dans les trois bras à l'aide d'ANOVAs et des comparaisons post-hoc deux à deux ont été réalisées à l'aide de tests de Tukey.

Résultats et Analyse statistique : Le score FSAm-NPS moyen des paniers était respectivement $2,02(3,56)(\mathrm{N}=623)$ dans le bras Nutri-Score, $2,69(3,44)(\mathrm{N}=651)$ dans le bras ARs et $2,45(3,28)(\mathrm{N}=592)$ dans le bras sans logo $(\mathrm{p}=0.002)$. Ces résultats traduisent une meilleure qualité nutritionnelle avec le Nutri-Score comparé aux ARs avec une différence moyenne de -0.67[-1.12;-0.21] points, mais il n'y avait pas de différence significative entre le Nutri-Score et l'absence de logo avec une différence moyenne de $0.43[-0.89 ; 0.03]$ points, ni entre les ARs et l'absence de logo (0.23[-0.22;0.69] points). Toutes les variables secondaires différaient significativement entre les groupes. Les comparaisons 2 à 2 ont montré un contenu plus faible des paniers en calories et acides gras saturés, et plus élevé en fruits et légumes dans le bras Nutri-Score comparé aux 2 autres bras.

Conclusion : Le Nutri-Score, avec son format graduel et coloriel, semble avoir un impact bénéfique sur la qualité nutritionnelle des achats alimentaires des étudiants, plus à risque d'avoir une alimentation déséquilibrée.

Conflits d'intérêts : Aucun conflit à déclarer 
Risque et bénéfice nutritionnels associés à la consommation de viande rouge en France.

J. De Oliveira Mota ${ }^{1,}{ }^{*}$, P. Tounian ${ }^{2}$, S. Guillou ${ }^{1}$, F. Pierre $^{3}$, J.-M. Membré ${ }^{1}$

${ }^{1}$ Secalim, INRA, Oniris, Université Bretagne Loire, Nantes, ${ }^{2}$ Service de nutrition et gastroentérologie pédiatriques, Hôpital Trousseau, APHP, Sorbonne Université, Paris, ${ }^{3}$ INRA, ToxAlim, Université de Toulouse, INRA, ENVT, INP-Purpan, UPS, Toulouse, France

Introduction et but de l'étude : La consommation de viande rouge pourrait s'avérer être un problème de santé publique, notamment en raison de son lien avec l'augmentation du risque de cancer colorectal. En effet, l'Organisation Mondiale de la Santé et le World Cancer Research Fund ont classé la viande rouge comme étant probablement cancérogène pour l'homme (groupe $2 \mathrm{~A}$ de I'OMS). Cependant, la viande représente la principale source de fer correctement biodisponible et participe de ce fait à la prévention de carence martiale. L'objectif de l'étude était de quantifier les risques et bénéfices nutritionnels associés à la consommation de viande rouge, puis de les exprimer en années de vie en bonne santé perdues ou gagnées (DALY), afin de pouvoir comparer l'impact des différents scénarii de consommation sur la santé.

Matériel et méthodes : Un modèle mathématique probabiliste a été développé, par classe d'âge et par genre, afin de quantifier le risque de cancer colorectal et le bénéfice lié à la réduction de la carence en fer entraînés par la consommation de viande rouge. Les effets de la variabilité due à l'hétérogénéité entre individus, et de l'incertitude, liée au manque de connaissance, sur les résultats du modèle ont également été caractérisés.

Résultats et Analyse statistique : En utilisant ce modèle, le rapport risque / bénéfice associé à la consommation de viande rouge a été calculé pour différents scénarii de consommation.

Conclusion : Cette étude pourra aider à la prise de décision par les autorités et les consommateurs afin de déterminer la quantité optimale de viande rouge à consommer pour profiter des bénéfices tout en minimisant les risques. Plus généralement, les résultats de cette étude pourraient servir de base pour intégrer des risques ou bénéfices supplémentaires associés à la consommation de viande tels que les risques cardiovasculaires ou la limitation de la sarcopénie.

Conflits d'intérêts : Aucun conflit à déclarer 
Apports en macro- et micronutriments et caractéristiques sociodémographiques et cliniques des consommateurs de produits laitiers chez les personnes âgées de la cohorte Trois-Cités-Bordeaux.

H. Pellay ${ }^{1, *}$, C. Helmer ${ }^{1}$, C. Boyer ${ }^{2}$, C. Marmonier ${ }^{2}$, C. Samieri ${ }^{1}$, C. Féart ${ }^{1}$

${ }^{1}$ Univ. Bordeaux, Inserm, Bordeaux Population Health Research Center, UMR 1219, Bordeaux, ${ }^{2}$ CNIEL, Direction des Affaires Scientifiques et Techniques, Service Recherche Nutrition-Santé, Paris, France

Introduction et but de l'étude : Les recommandations nutritionnelles actuelles accordent une place majeure à la consommation de produits laitiers (PL) chez les personnes âgées, grâce notamment à leur richesse en macronutriments.

L'objectif de cette étude était d'identifier, parmi un échantillon de personnes âgées de la population générale, les consommateurs réguliers de PL et de décrire leurs apports en macro- et micronutriments ainsi que leurs caractéristiques sociodémographiques et cliniques.

Matériel et méthodes : L'échantillon d'étude était constitué des participants bordelais de la cohorte Trois-Cités, âgés de 65 ans et plus à l'inclusion, ayant répondu à un fréquentiel alimentaire et à un rappel des $24 \mathrm{~h}$. Les consommateurs réguliers déclaraient consommer des PL au moins une fois par jour. L'IMC, la présence d'une hypertension, d'une hypercholestérolémie, d'un diabète, les performances cognitives globales et la symptomatologie dépressive étaient mesurés, alors que les autres caractéristiques cliniques étaient auto-déclarées (tabagisme, angine de poitrine, troubles du rythme cardiaque, insuffisance cardiaque, artérite, infarctus du myocarde, ostéoporose, dyspnée, asthme et maladie de Parkinson).

Résultats et Analyse statistique : L'analyse portait sur 1596 participants âgés de 74,2 ans en moyenne, dont 62\% de femmes. Parmi eux, 94\% étaient considérés comme des consommateurs réguliers de PL. Ces derniers avaient un âge moyen et un niveau d'étude comparables aux non-consommateurs réguliers, et étaient plus souvent mariés (57\% vs $45 \%$ ). Concernant les apports nutritionnels, les consommations journalières de calcium (877 mg vs $691 \mathrm{mg}$ ) et de phosphore (1119 mg vs $1013 \mathrm{mg}$ ) étaient significativement plus élevées chez les consommateurs réguliers de PL vs les autres. Concernant les caractéristiques cliniques, les consommateurs réguliers de PL déclaraient significativement moins souvent souffrir d'insuffisance cardiaque (6\% vs $11 \%)$ et de dyspnée (13\% vs $20 \%$ ) que les autres. II n'y avait pas de différence significative pour les autres comorbidités.

Conclusion : Cette étude transversale souligne qu'une consommation régulière de PL contribue significativement aux apports en calcium et phosphore chez les personnes âgées. La consommation quotidienne de PL est associée à moins de désordres cliniques chez les personnes âgées mais des analyses longitudinales sont nécessaires pour évaluer l'intensité de cette relation. De même, l'étude des sous-types de PL et des profils alimentaires clarifiera la part des bénéfices des PL dans l'alimentation globale.

Conflits d'intérêts : Aucun conflit à déclarer 
Évaluation du statut en iode et détermination des carences iodés chez des enfants Marocains âgés de 6 à 18 ans A. Rami ${ }^{1,{ }^{*}}$, N. Saeid ${ }^{1}$, M. Idrissi ${ }^{1}$, M. Taghzaoui ${ }^{1}$, A. El Hamdouchi ${ }^{1}$, F. Z. Mouzouni ${ }^{2}$, K. El Kari ${ }^{1}$, M. El Mzibri ${ }^{1}$, H. Aguenaou ${ }^{1}$ ${ }^{1}$ Unité Mixte de Recherche en Nutrition et Alimentation CNESTEN, ${ }^{2}$ Direction de l'Epidémiologie et de contrôle des Maladies non Transmissibles, Université Ibn Tofail, Kénitra, Rabat, Maroc

Introduction et but de l'étude : La carence en iode a des effets néfastes sur la croissance et le développement humains depuis l'enfance et jusqu'à l'âge adulte. Groupés sous le nom, des troubles dus aux carences en iode (TDCl). Environ 29,8\% enfants en âge scolaire à travers le monde souffrent de TDCl. Afin de contrôler la carence en iode Maroc a adopté depuis 1995, la stratégie universelle d'iodation du sel. L'objectif de la présente étude et d'évaluer le statut en iode chez des enfants Marocains et de déterminer la prévalence de la carence en iode.

Matériel et méthodes : C'est une étude transversale 280 enfants sont invités pour participer à l'étude. L'apport en iode est évalué par l'analyse de la concentration de l'iode dans les urines de $24 \mathrm{~h}$. Le pourcentage des apports en produits riches en iode sont déterminés à l'aide d'un questionnaire sur la fréquence alimentaire (QFA).

Résultats et Analyse statistique : La médiane de l'excrétion urinaire (24h) et de la concentration en iode sont respectivement de $77 \mu \mathrm{g} /$ jour et de $96 \mu \mathrm{g} / \mathrm{L}$. Environ $72,5 \%$ des enfants sont carencés en iode $58 \%$ présentent une carence légère et $14,5 \%$ ont une carence modérée. Une différence significative, est observée en fonction du sexe et de l'âge, ainsi la carence est plus évoluée chez les garçons et les enfants âgés de 6 à 8 ans. Le statut en iode chez les enfants déficients ne change pas avec le niveau de consommation des produits laitiers et des œufs, et les résultats ne montrent aucune association significative $(p>0,05)$. Cependant, une consommation importante de poisson était significativement associée à un bon statut en iode $(p=0,044)$.

Conclusion : Malgré la mise en œuvre de la stratégie Nationale d'iodation du sel les enfants Marocains souffrent toujours de carences en iode; ce qui peut être dues en partie au non-respect des normes sur l'iodation du sel, et aussi à une faible consommation de produits riches en iode. Ceci nous mène à revoir la stratégie de lutte contre les TDCl en améliorant le processus de l'iodation du sel, ainsi que la promotion de la consommation de produits riches en iode.

Conflits d'intérêts : Aucun conflit à déclarer 


\section{P229}

La qualité de l'alimentation contribue-t-elle à expliquer les différences socioéconomiques de la prévalence du syndrome métabolique dans les Antilles Françaises?

Z. Colombet ${ }^{1, *}$, M. Pérignon ${ }^{1}$, B. Salanave ${ }^{2}$, E. Landais ${ }^{3}$, Y. Martin-Prével ${ }^{3}$, B. Allès ${ }^{4}$, S. Drogué ${ }^{1}$, M.-J. Amiot-Carlin ${ }^{1}$, C. Méjean ${ }^{1}$ ${ }^{1}$ MOISA, Univ Montpellier, CIRAD, CIHEAM-IAMM, INRA, Montpellier SupAgro, Montpellier, ${ }^{2}$ Santé publique France, Direction des maladies non transmissibles et traumatismes, Equipe de surveillance et d'épidémiologie nutritionnelle (ESEN), Saint-Maurice, France, Université Paris 13, Sorbonne Paris Cité, Centre de Recherche en Epidémiologies et Biostatistiques, Bobigny, ${ }^{3}$ IRD (Institut de Recherche pour le Développement), NUTRIPASS Unit, IRD-UM-SupAgro, 911 av. Agropolis, 34394 Montpellier, ${ }^{4}$ EREN, Centre de Recherche en Epidémiologie et Statistiques Sorbonne Paris Cité UMR U1153 Inserm / U1125 Inra / Cnam / Univ Paris 13, Bobigny, France

Introduction et but de l'étude : L'obésité et les maladies chroniques représentent des problèmes de santé majeurs dans les Antilles françaises où une grande partie de la population est précaire. Pourtant, les inégalités socioéconomiques des maladies chroniques y sont peu explorées et la contribution de la qualité de l'alimentation pouvant expliquer ces inégalités n'a jamais été évaluée. Nous avons donc étudié les associations entre les indicateurs de statut socioéconomique et la prévalence du syndrome métabolique (MetS) dans les Antilles Françaises et l'effet médiateur de la qualité de l'alimentation.

Matériel et méthodes : Les apports alimentaires ont été estimés par des rappels de $24 \mathrm{~h}$ chez 1144 participants ( $\geq 16$ ans) inclus dans l'étude Kannari, enquête à visée représentative de la population générale résidant en Guadeloupe et Martinique. La qualité de l'alimentation a été évaluée par le « Diet Quality Index-International » (DQI-I) [de 0 à 100 points]. La prévalence du MetS a été estimée selon les critères du Joint Interim Statement, à partir de mesures biologiques et anthropométriques. Les associations entre les indicateurs de position socioéconomique (niveau d'éducation, être allocataire d'une aide sociale, situation par rapport à l'emploi, foyer avec enfants, famille monoparentale) et la prévalence du MetS, ainsi que l'effet médiateur de la qualité de l'alimentation ont été évalués grâce à des régressions logistiques multivariables, ajustées sur le département (Guadeloupe ou Martinique), le sexe, l'âge et l'indice de masse corporelle.

Résultats et Analyse statistique : Les prévalences du MetS chez les Guadeloupéens et les Martiniquais étaient de $18 \%$ et $28 \%$ respectivement. Les participants ayant un niveau d'éducation primaire ou secondaire $\left(O R_{\text {primaire vs. } \geq b a c}=1,9 ; I C 95 \%=[1,0-3,6]\right.$ et $\left.\mathrm{OR}_{\text {secondaire vs. } \geq \mathrm{bac}}=2,7 ; \mathrm{IC} 95 \%=[1,4-5,1]\right)$ et les allocataires d'une aide sociale $(\mathrm{OR}=2,2 ; \mathrm{IC} 95 \%=[1,1-4,2])$ présentaient un risque plus élevé de MetS comparés aux participants de niveau d'éducation supérieur et aux non-allocataires. La qualité de l'alimentation expliquait $11 \%$ de la variation du risque de MetS dû au niveau d'éducation et seulement $2 \%$ de la variation dû au fait d'être allocataire d'aide sociale.

Conclusion : La qualité de l'alimentation contribue peu à expliquer les inégalités socioéconomiques du MetS observées en Guadeloupe et Martinique. L'étude de l'influence d'autres facteurs du mode de vie (tabagisme, alcool, activité physique) est nécessaire pour mieux comprendre les mécanismes des inégalités socioéconomiques de santé et ainsi guider les actions de santé publique futures.

Conflits d'intérêts : Aucun conflit à déclarer 
Estimation des facteurs de risque des maladies cardiovasculaires dans une population représentative de Casablanca K. Mohtadi ${ }^{1}{ }^{*}$, R. Msaad $^{1}$, R. Essadik ${ }^{1}$, H. Lebrazi ${ }^{1}$, A. Kettani ${ }^{1}$, H. Taki $^{1}$, R. Saile $^{1}$

${ }^{1}$ Laboratoire de Biologie et Santé (URAC 34), Faculté des Sciences Ben M'Sik, Université Hassan II de Casablanca, Casablanca, Maroc

Introduction et but de l'étude : Les maladies cardiovasculaires étaient la cause principale de mortalité sur l'échelle mondiale. Le contrôle des facteurs de risque cardiovasculaires modifiables peut réduire la mortalité qui leur est due. L'objectif de notre étude était d'identifier certains de ces facteurs de risque cardiovasculaires dans une population représentative de Casablanca.

Matériel et méthodes : Notre étude a été portée sur 609 sujets dont 363 femmes et 246 hommes, âgés de 18 à 65 ans. Les données ont été recueillies à travers un questionnaire comportant les caractéristiques sociodémographiques, les antécédents médicaux et certains facteurs liés au mode de vie, tels que l'activité physique et le tabagisme. Nous avons également évalué les mesures anthropométriques et le profil biochimique. Notre population a été subdivisée en trois groupes en fonction de leur indice de masse corporelle (IMC): obèses, groupe en surpoids et groupe témoin.

Résultats et Analyse statistique : L'analyse a montré que l'âge moyen de la population était de 54,47 $\pm 10,89$ ans et que 58,8\% étaient des femmes. Alors que la prévalence du diabète et de l'hypertension ont étaient respectivement de 38,1\% et de $35,8 \%$ avec une domination féminine. L'indice athérogénique du plasma (AIP) le plus élevé était présent; en particulier chez les personnes en surpoids et obèses; il était respectivement de $31,1 \%$ et $28,2 \%$. La prévalence de l'hypercholestérolémie était de $9,77 \%$ chez les obèses, $11,20 \%$ chez les sujets en surpoids et de $2,87 \%$ chez les sujets avec un IMC normal. L'activité physique pratiquée était plus élevée chez les groupes en surpoids et chez les groupes obèses (24,7\% et $16 \%$ respectivement) que chez les personnes ayant un IMC normal (9,5\%). On constate que 35,20\%, 13,98\% et 1,81\% de la population étudiée ont respectivement 3, 4 et 5 facteurs de risque de maladie cardiovasculaire alors que 5,26\% n'en ont pas, on observe aussi que les femmes en ont plus (59,7\% vs $40,3 \%$ ). Conclusion : II existe une prévalence élevée de facteurs de risque cardiovasculaires chez la population générale de Casablanca, et ce, due à de mauvaises habitudes telles que le mode de vie sédentaire, le diabète et / ou I'hypertension.

Conflits d'intérêts : Aucun conflit à déclarer 
Apports alimentaires en sodium et en potassium chez des adultes Tunisiens : enquête pilote réalisée dans le cadre de l'évaluation de la stratégie nationale visant à réduire la consommation de sel

R. Doggui ${ }^{1,}{ }^{*}$, M. El Ati-Hellal ${ }^{2}$, P. Traissac ${ }^{3}$, A. Mensi ${ }^{4}$, S. Sassi ${ }^{4}$, M. M. Abassi ${ }^{5}$, H. Ben Gharbia ${ }^{6}$, M. Thabet $^{4}$, J. El Ati $^{1}$

${ }^{1}$ Laboratoire de Recherche en Surveillance et Epidémiologie Nutritionnelles en Tunisie, Institut National de Nutrition et de Technologie Alimentaire, ${ }^{2}$ Laboratoire de toxicologie, Unité de toxicologie, recherche et environnement, Centre d'assistance médicale urgente, Tunis, Tunisie, ${ }^{3}$ UMR NUTRIPASS, IRD-Université de Montpellier-Montpellier Supagro, Montpellier, France, ${ }^{4}$ Laboratoire de Surveillance et Epidémiologie Nutritionnelles en Tunisie, Institut National de Nutrition et de Technologie Alimentaire, Tunis, ${ }^{5}$ Laboratoire de Recherche en Surveillance et Epidémiologie Nutritionnelles, Institut National de Nutrition et de Technologie Alimentaire, Tunisi, ' Laboratoire de Recherche en Surveillance et Epidémiologie Nutritionnelles en Tunisie, Institut National de Nutrition et de Technologie Alimentaire en Tunisie, Tunis, Tunisie

Introduction et but de l'étude : Au regard de la forte prévalence de l'hypertension en Tunisie (30,6 \%), la stratégie nationale de prévention de l'obésité et des maladies chroniques associées inclut des mesures spécifiques visant à la réduction de la consommation de sel. De plus, un faible apport en potassium concomitant à une consommation excessive de sodium prédispose à l'hypertension. Les objectifs de ce travail sont donc, dans la zone d'intervention pilote de Bizerte, de: i) décrire la consommation de sodium et de potassium ainsi que leurs sources alimentaires; ii) évaluer l'adéquation de l'apport sodique.

Matériel et méthodes : L'enquête a été menée, avant le début de l'intervention dans la ville de Bizerte en 2014 : étude transversale sur un échantillon représentatif des adultes (20-64 ans) vivant dans la ville de Bizerte, soit un total de 226 sujets sélectionnés. Des urines de 24 heures ont été collectées pour la détermination de la consommation de sodium et de potassium (gold standard). De manière parallèle, un rappel de 24 heures a permis d'explorer les groupes alimentaires qui contribuaient le plus à l'ingéré quotidien en ces minéraux. Le seuil recommandé par l'Organisation Mondiale de la Santé (OMS) pour la consommation de sel est de $5 \mathrm{~g} /$ jour ( $2 \mathrm{~g} /$ jour de sodium). La régression linéaire multivariable a été utilisée pour explorer l'association entre les ingérés en sodium et les covariables.

Résultats et Analyse statistique : Au total, 194 sujets ont participé à l'étude. L'excrétion quotidienne de sodium était de 138,3 \pm $46,5 \mathrm{mmol} /$ jour ce qui correspond à un apport journalier en sel de $8,7 \pm 2,1 \mathrm{~g} /$ jour. Plus de $87,1 \%$ des sujets avaient un apport en sel qui dépassait $10 \mathrm{~g} / \mathrm{jour}$. L'excrétion du sodium était plus importante chez les hommes que chez les femmes (150,8 vs. 128,0 ; $P=0,001)$. L'analyse ajustée par régression linéaire a révélé que la consommation de sel était plus élevée chez les sujets avec un indice de masse corporelle $\geq 30 \mathrm{~kg} / \mathrm{m}^{2}(+1,1 \mathrm{~g} / \mathrm{jour}$ avec IC à $95 \%:[0,1-2,0])$. Une faible excrétion de potassium a été identifiée (61,0 $\pm 22,7 \mathrm{mmol} / \mathrm{jour}$ ) et seulement 10,3\% des sujets avaient un apport adéquat. Les principaux groupes d'aliments qui contribuaient à l'ingéré quotidien en sodium étaient le pain (42\%) et le sel ajouté $(35,5 \%)$. Le potassium provenait surtout des légumes (41\%), du pain (11,3\%) et des fruits $(9,1 \%)$.

Conclusion : L'étude montrait une coexistence d'un apport élevé en sodium et faible en potassium au sein des sujets de l'étude au regard des recommandations de l'OMS, ce qui pourrait constituer un facteur de risque de l'hypertension. Actuellement, la stratégie mise en place dans cette région pilote vise à la réduction de la teneur en sel du pain. Un monitoring mensuel a révélé une réduction significative de cette teneur durant la première phase de l'étude, au-delà du seuil de $20 \%$ fixé comme objectif intermédiaire.

Conflits d'intérêts : Aucun conflit à déclarer 
Apports en sucres libres de la population française et qualité de l'alimentation des forts-consommateurs : résultats de l'enquête CCAF 2016

\author{
C. Deshayes ${ }^{1}$, E. Sauvage ${ }^{2}$, P. Reiser ${ }^{1, *}$, P. Hebel $^{2}$ \\ ${ }^{1}$ CEDUS, ${ }^{2}$ CREDOC, Paris, France
}

Introduction et but de l'étude : Les sucres libres (SL) sont définis comme les sucres ajoutés, et les sucres naturellement présents dans le miel, les sirops, les jus de fruits. Dans un objectif de prévention de l'obésité et de la carie, l'Organisation Mondiale de la Santé (OMS) recommande une consommation de SL inférieure à $10 \%$ de l'apport énergétique total (AET). L'objectif de cette étude est d'actualiser les données de consommation de SL de la population française et d'étudier l'association entre les niveaux de consommation de SL et la qualité de l'alimentation.

Matériel et méthodes : Les données sont issues de l'enquête CCAF 2016 du CREDOC portant sur un échantillon représentatif de 1735 adultes (+18 ans) et 1354 enfants (3-17 ans) ayant complété un carnet alimentaire de 7 jours. La table de composition nutritionnelle Ciqual 2016 a été utilisée pour calculer les apports nutritionnels, complétée par l'estimation des teneurs en SL à partir d'une méthodologie en 10 étapes[1]. Les apports en SL ont été comparés à la recommandation de l'OMS. Les individus ont été classés selon qu'ils respectent ou dépassent la recommandation (appelés respectivement $S L<10 \%$ et $S L \geq 10 \%$ ), puis en terciles pour le groupe $S L \geq 10 \%$. La qualité de l'alimentation a été évaluée à partir de l'indice PANDIET mesurant l'adéquation des apports en macro- et micronutriments aux recommandations[2].

Résultats et Analyse statistique : Chez les adultes, la moyenne des apports en sucres libres est de 10,2 +/- 0,1 \% de l'AET (50,7 +/$0,8 \mathrm{~g} / \mathrm{j})$. Chez les enfants la moyenne est de $14,8+/-0,1 \%(61,6+/-0,8 \mathrm{~g} / \mathrm{j})$. Dans cette population, les apports en SL diminuaient avec l'âge ( $p$-value $<0.0001$ ), de manière constante tout au long de la vie passant de $16,3 \%$ pour les 3-6 ans à 8,6 \% de l'AET chez les 76 ans et plus. La consommation moyenne, adultes comme enfants, apparaît stable depuis l'enquête INCA 2 (2006-2007) : 9,5 $\%$ de l'AET ou $53,6 \mathrm{~g} / \mathrm{j}$ chez les adultes ; $13,4 \%$ de l'AET ou $66,8 \mathrm{~g} / \mathrm{j}$ chez l'adolescent et $15,8 \%$ de l'AET ou $60,4 \mathrm{~g} / \mathrm{j}$ chez les plus jeunes[3].

Chez les enfants, la qualité de l'alimentation n'est pas différente entre les groupes $S L<10 \%$ et SL $\geq 10 \%$. Cependant, dans l'analyse par terciles, la qualité de l'alimentation apparaît moins bonne pour le dernier tercile $S \mathrm{~L} \geq 10 \%$ par rapport au groupe respectant la recommandation. Chez les adultes, la qualité de l'alimentation du groupe $S L \geq 10 \%$ est moins bonne que celle du groupe $S L<10 \%$. Une fois décomposé en terciles, c'est uniquement le dernier tercile qui apparaît comme différent.

Conclusion : En moyenne les adultes français respectent la recommandation d'apports en sucres libres de l'OMS, mais les enfants la dépassent. L'étude montre que ce sont seulement les très forts consommateurs de SL (dernier tercile) qui ont une qualité de l'alimentation significativement moins bonne que les consommateurs respectant la recommandation.

Références : [1] Louie JC, et al. A systematic methodology to estimate added sugar content of foods. Eur J Clin Nutr. 2015;69(2):154-161

[2] Verger EO, et al. Simple changes within dietary subgroups can rapidly improve the nutrient adequacy of the diet of French adults. J. Nutr. 2014, 144, 929-936

[3] Azaïs-Braesco V, Maillot M. Apports en sucres et principaux contributeurs dans la population française. Cahiers de Nutrition et de Diététique, Yaourt et santé 52, 2017, S58-S65

Conflits d'intérêts : C. Deshayes est employé(e) de CEDUS, E. Sauvage est employé(e) de CREDOC, P. Reiser est employé(e) de CEDUS, P. Hebel est employé(e) de CREDOC 

M. Maillot ${ }^{1}$, N. Darmon ${ }^{2, *}$

${ }^{1}$ MS-Nutrition, Marseille, ${ }^{2}$ INRA, Montpellier, France

Introduction et but de l'étude : Le HCSP a rendu le 16 février 2017 un avis relatif à la révision des repères alimentaires pour les adultes du futur Programme national nutrition santé 2017-2021. Par rapport aux repères actuellement en vigueur, on note i) l'ajout de nouveaux repères (légumineuses, charcuteries, fruits à coques, céréales complètes), ii) une précision des repères sur les matières grasses (distinction des huiles riches en ALA) et les viandes (distinction des viandes blanches et rouges), et iii) une diminution du repère pour les produits laitiers qui passent de 3 à 2 portions/j. L'objectif de cette étude était d'évaluer l'impact du respect des nouveaux repères HCSP sur la composition nutritionnelle des diètes, et de comparer cet impact selon que le repère pour les produits laitiers est fixé à 2 ou à 3 portions/j.

Matériel et méthodes : Les repères du HCSP ont été traduits en repères chiffrés (quantités minimales et/ou maximales recommandées, en grammes ou en nombre de portions/j). Pour chacune des diètes observées des 1863 adultes de l'étude (>18ans) de l'enquête INCA2, l'approche de modélisation de diètes individuelles a été appliquée pour obtenir deux nouvelles diètes (iso-énergétiques avec l'observée) respectant les nouveaux repères du HCSP : la première avec 2 portions/j (modèle HCSP) et la seconde avec 3 portions/j (modèle HCSP3) de produits laitiers. La qualité nutritionnelle des diètes modélisées a été comparée à celle des diètes observées sur la base des teneurs nutritionnelles moyennes, d'indicateurs de qualité globale (Densité Energétique-DE, Mean Adequacy Ratio-MAR, Mean Excess Ratio-MER) et du pourcentage d'inadéquation pour chaque nutriment (i.e., pourcentage de diètes ayant une teneur inférieure à la valeur nutritionnelle de référence, Besoin Nutritionnel Moyen ou Apport Satisfaisant).

Résultats et Analyse statistique : Quel que soit le modèle, il a été possible de construire une diète modélisée respectant l'ensemble des repères pour $98 \%$ des diètes. Par rapport aux diètes observées, les diètes HCSP et HCSP3 avaient une meilleure qualité nutritionnelle globale : baisse de la densité énergétique $(183 ; 153$; et $152 \mathrm{kcal} / 100 \mathrm{~g}$ pour observé, HCSP et HCSP3, respectivement), et du MER $(18,1 ; 6,7$ et 7,8 \%/j), et hausse du MAR (de 84,4; 89,9 et 90,0\%/j). Les vitamines $C$, B9 et B6 étaient les nutriments les plus améliorés par les deux modèles. Pour la vitamine B2 et le calcium, le modèle HCSP dégradait la situation alors que le modèle HCSP3 l'améliorait ( $38 ; 46$ et $26 \%$ d'inadéquation pour la vitamine B2 et 51 ; 58 et $16 \%$ pour le calcium). Les deux modèles augmentaient (i.e. dégradaient) le pourcentage d'inadéquation pour le zinc et l'iode, mais de façon plus modérée avec le HCSP3. Quel que soit le modèle, le pourcentage d'inadéquation restait très élevé (supérieur à $80 \%$ ) pour les fibres, les acides gras oméga-3 à longue chaine EPA+DHA et la vitamine D.

Conclusion : Le respect des repères HCSP améliore globalement la qualité nutritionnelle. Cette amélioration serait meilleure si les produits laitiers étaient portés à 3 portions/j.

Conflits d'intérêts : M. Maillot a reçu une subvention/soutien de CNIEL, est employé(e) de MS-Nutrition, N. Darmon a reçu une subvention/soutien de CNIEL 
Association prospective entre divers scores nutritionnels et les pathologies cardiovasculaires : l'adéquation au régime Méditerranéen est-elle davantage associée aux pathologies cardiovasculaires que l'adéquation aux recommandations nutritionnelles?

A. Trébuchet ${ }^{1}$, C. Julia ${ }^{12}$, L. Fézeu ${ }^{1}$, M. Touvier ${ }^{1}$, D. Chaltiel ${ }^{1}$, S. Hercberg ${ }^{12}$, P. Galan ${ }^{1}$, M. Adjibade ${ }^{1,}{ }^{*}$, E. Kesse-Guyot $^{1}$

${ }^{1}$ Equipe de Recherche en Epidémiologie Nutritionnelle (EREN), Université Paris 13, Centre d'Epidémiologie et Statistiques Sorbonne Paris Cité, Inserm (U1153), Inra (U1125), Cnam, COMUE Sorbonne Paris Cité, ²Département de Santé Publique, Hôpital Avicenne, Bobigny, France

Introduction et but de l'étude : Le rôle de la nutrition dans les pathologies cardiovasculaires (MCV) est une question majeure de santé publique. L'association entre l'adéquation au régime méditerranéen et le risque de MCV a été largement documentée, mais aucune étude n'a évalué la supériorité de ce régime par rapport aux recommandations nutritionnelles nationales. Notre objectif était donc d'évaluer l'association prospective entre différents scores nutritionnels et l'incidence des MCV.

Matériel et méthodes : L'étude a porté sur 94113 participants de la cohorte NutriNet-Santé, suivis de mars 2009 à mars 2018. Les critères d'inclusion étaient: avoir complété au moins 3 enregistrements alimentaires de $24 \mathrm{~h}$ au cours des deux premières années de suivi, et avoir les données nécessaires pour le calcul des scores nutritionnels. Trois scores nutritionnels qui mesurent respectivement l'adéquation au régime méditerranéen et aux recommandations nutritionnelles françaises et américaines ont été calculés : le Literature-Based Adherence Score to the Mediterranean Diet (MEDI-LITE), la version modifiée du Programme National Nutrition Santé Guideline Score (mPNNS-GS) et l'Alternative Healthy Eating Index-2010 (AHEI-2010). Les MCV regroupaient : l'angioplastie, l'angor, l'infarctus du myocarde, le syndrome coronarien aigu et les accidents vasculaires cérébraux. Nous avons examiné les associations entre les différents scores nutritionnels et les MCV à l'aide des ratios de risques instantanés (HR) et leurs intervalles de confiance (IC) à 95\%, estimés en utilisant des modèles de Cox à risques proportionnels. Le C-index a été utilisé pour estimer la capacité des différents scores nutritionnels à prédire le risque de MCV.

Résultats et Analyse statistique : Au total, 1399 événements de MCV ont été référencés au cours du suivi. Après ajustement sur les différents facteurs de confusion potentiels, les participants qui étaient dans le quartile le plus élevé des scores MEDI-LITE et AHEI-2010 avaient significativement moins de risque d'avoir des MCV, comparés aux participants qui étaient dans le quartile le plus bas (le HR était de 0,79 (IC à 95\% = 0,67-0,93) pour le MEDI-LITE et de 0,75 (IC à 95\% = 0,63-0,89) pour l'AHEI-2010). L'association entre le mPNNS-GS et les MCV n'était pas significative. Des associations similaires ont été observées après suppression des événements précoces de MCV, des participants ayant moins de 6 enregistrements alimentaires de 24h et lorsque les accidents ischémiques transitoires (AIT) étaient considérés comme MCV. Dans ce dernier cas, l'association entre le mPNNS-GS et le risque de MCV était significative. La capacité prédictive des scores nutritionnels considérés était très similaire (la statistique C était de 0,7664 pour l'AHEI-2010; 0,7680 pour le mPNNS-GS et 0,7681 pour le MEDI-LITE).

Conclusion : Les résultats de la présente étude ont confirmé l'effet bénéfique du régime méditerranéen concernant le risque des $\mathrm{MCV}$ et suggèrent qu'une meilleure adéquation aux recommandations nutritionnelles pourrait également jouer un rôle important dans la prévention des MCV.

Conflits d'intérêts : Aucun conflit à déclarer 
Prévalence des troubles du comportement alimentaire au cours de la période 2000-2018 : une revue systématique de la littérature

M. Galmiche ${ }^{123,^{*}, \text { P. Déchelotte }}{ }^{245}$, G. Lambert ${ }^{1}$, M. P. Tavolacci ${ }^{256}$

${ }^{1}$ Targedys SA, ${ }^{2}$ Nutrition, Inflammation et Dysfonction de l'Axe Intestin Cerveau, Inserm UMR 1073, ${ }^{3}$ Université de Rouen, Institute for Research and Innovation in Biomedicine, ${ }^{4}$ Nutrition, CHU-Hôpitaux de Rouen, ${ }^{5}$ Université de rouen, Institute for Research and Innovation in Biomedicine (IRIB), ${ }^{6} \mathrm{CIC} 1204$ INSERM, CHU de Rouen, Rouen, France

Introduction et but de l'étude : Les troubles du comportement alimentaire (TCA) sont des perturbations sévères, qui peuvent conduire à de multiples complications tant psychologiques que somatiques et sont susceptibles d'impacter la qualité de vie et la mortalité du patient. Les TCA les mieux caractérisés sont l'anorexie, la boulimie et l'hyperphagie boulimique. Les TCA constituent un réel problème de santé publique. L'objectif de ce travail est de donner une vue exhaustive des études s'intéressant à la prévalence des différents TCA et d'étudier l'évolution de ces prévalences au cours de la période d'études.

Matériel et méthodes : La recherche dans la littérature scientifique a été menée suivant les directives PRISMA et limitée aux études publiées en anglais ou français entre 2000 et 2018. Tous les types de prévalence ont été intégrés dans cette revue: instantanée, sur 12 mois, à vie. Selon ces critères d'inclusion, cette recherche a permis de sélectionner 94 études avec un diagnostic précis de TCA (déterminé par interview ou selon une classification internationale: ICD et DSM) et 27 études avec un diagnostic de TCA classés en catégories larges (déterminé grâce au SCOFF ou EAT).

Résultats et Analyse statistique : Dans les 94 études avec un diagnostic précis, les moyennes pondérées de prévalences à vie de l'ensemble des TCA étaient 8,4\% pour les femmes et 2,2\% pour les hommes. Pour les prévalence sur 12 mois de l'ensemble des TCA, les moyennes pondérées étaient de $3,8 \%$ pour les femmes et $0,7 \%$ pour les hommes. Pour les prévalence instantanée de l'ensemble des TCA, les moyennes pondérées étaient de 5,7\% pour les femmes et de 2,2\% pour les hommes.Selon les continents, les moyennes pondérées de prévalences instantanées étaient de 4,6\% en Amérique, 2,2\% en Europe et 3,5\% en Asie. Par ailleurs, 27 autres études ont reporté la prévalence des TCA classés en catégories larges; les moyennes pondérées pour le total des TCA étaient de $19,4 \%$ chez les femmes (6,5\%>36\%) et de $13,8 \%$ pour les hommes (3,6\% - 27,1\%).Pour finir, la moyenne pondérée des prévalences instantanées (avec diagnostic précis) augmenterait au cours de la période d'étude, progressant de 3,5\% de 2000-2006 à 7,8\% de 2013-2018.

Conclusion : Cette revue a permis de mettre en évidence que le manque de standardisation des études de prévalence des TCA (outils diagnostic/ d'évaluation) et l'évolution des différentes classifications rendent complexes l'intégration de toutes les données de prévalence. Néanmoins, les études les plus récentes confirment que les TCA sont de plus en plus répandues dans le monde entier, en particulier chez les femmes. En effet, durant la période 2000-2018, une tendance à l'augmentation de la prévalence des TCA est observée.

Cette revue souligne donc le véritable défi permanent que constitue les TCA pour la santé publique et les professionnels de santé. Remerciements : Soutien de la société TargEDys SA et du ministère de I'Industrie et de la Technologie pour le financement du contrat de thèse MG PhD CIFRE

Conflits d'intérêts : Aucun conflit à déclarer 
Restructurer la consommation de sources protéiques par des modifications de tailles de portion pour augmenter l'adéquation nutritionnelle : ce qui fonctionne pour tout le monde et ce qui dépend du profil de consommation initial

E. De Gavelle ${ }^{1,{ }^{*}}$, J.-F. Huneau ${ }^{1}$, H. Fouillet ${ }^{1}$, F. Mariotti ${ }^{1}$

${ }^{1}$ UMR PNCA, AgroParisTech, INRA, Université Paris Saclay, Paris, France

Introduction et but de l'étude : Les profils de consommation de protéines alimentaires sont en transition dans les pays occidentaux, mais il existe peu de données concernant l'impact de ces modifications sur l'adéquation nutritionnelle de l'alimentation. Les repères de consommation proposent des objectifs finaux sans préciser la manière de les atteindre. Ils sont génériques et ne prennent pas en compte la diversité des profils de consommation des individus. Notre objectif était d'identifier les modifications simples de la consommation de sources protéiques qui augmentent le plus l'adéquation nutritionnelle globale, et d'analyser dans quelle mesure ces modifications dépendent des profils de consommation protéique.

Matériel et méthodes : Nous avons identifié différents profils diététiques de consommation de sources de protéines chez 1678 adultes de l'étude INCA2 (2006-2007), représentative de la population française. Pour chaque individu, nous avons identifié l'augmentation de taille de portion d'un aliment protéique associée à la diminution de taille de portion d'un autre aliment protéique qui permettait d'augmenter le plus l'adéquation nutritionnelle (en utilisant le score probabiliste PANDiet). Pour chaque individu, cette étape a été itérée 20 fois selon deux scénarios : soit en manipulant l'apport d'aliments déjà consommés par l'individu (scénario 1, S1), soit en permettant l'introduction d'aliments qui sont consommés par $>10 \%$ des individus appartenant au profil de consommation protéique de l'individu (scénario $2, \mathrm{S2}$ ).

Résultats et Analyse statistique : Sur l'ensemble de la population, les principales modifications de portions d'aliments protéiques consistaient à réduire les portions de charcuterie (S1 et S2), de sandwichs et de fromage (S2), tout en augmentant les portions de poissons gras, de volaille maigre (S1 et S2) et de légumineuses (S2). Cependant, ces changements différaient selon le profil de consommation protéique initial de l'individu. Par exemple, si la consommation de charcuterie a diminué dans tous les profils (S1 et S2) et la consommation de pâtes a augmenté dans tous les profils (S1), dans S2, la consommation de légumineuses a augmenté chez les « consommateurs de volaille » et les « consommateurs de poissons » uniquement et la consommation de viande maigre (hors volaille) chez les « consommateurs de produits à emporter » et les " consommateurs de lait » uniquement. L'amélioration de l'adéquation nutritionnelle globale était semblable d'un profil initial à un autre, mais elle était la résultante de modifications différentes de l'adéquation nutritionnelle pour des nutriments spécifiques, qui dépendaient du profil. Par exemple, les apports en fibres ont augmenté chez les « consommateurs de volaille » et « consommateurs de poisson » uniquement.

Conclusion : Au-delà de modifications génériques de la consommation de sources protéiques dans l'ensemble de la population adulte française, le profil de consommation protéique initial est essentiel pour identifier les groupes alimentaires les plus susceptibles d'améliorer l'adéquation nutritionnelle globale et le profil des nutriments dont l'adéquation peut facilement être augmentée par une réorganisation de la consommation des aliments protidiques.

Conflits d'intérêts : Aucun conflit à déclarer 
Vécu, ressenti et prise en charge des différentes intolérances alimentaires non-allergiques (gluten, lactose, FODMAP) en médecine générale. Une étude descriptive auprès de 188 médecins généralistes en Rhône-Alpes-Auvergne.

M. Cart-Tanneur ${ }^{1}$, P.-L. Braud ${ }^{1}$, B. Prost ${ }^{2, *}$

${ }^{1}$ Faculté de Médecine Lyon Sud, Université Claude Bernard Lyon 1, ${ }^{2}$ Hépato-gastroentérologie, Centre Hospitalier St Joseph St Luc, Lyon, France

Introduction et but de l'étude : Alors que les médias et patients évoquent de plus en plus l'existence d'intolérances alimentaires non-allergiques (IANA) au gluten, lactose et FODMAP, il ne semble pas exister de recommandations claires facilement accessibles aux médecins généralistes (MG) pour prendre en charge ces patients. Le médecin traitant est pourtant souvent le premier médecin consulté et celui vers qui le patient revient une fois les explorations complémentaires digestives et allergologiques réalisées et négatives. Le but de l'étude était d'évaluer les comportements et le ressenti des médecins généralistes face aux patients se plaignant d'IANA au gluten, lactose et FODMAP.

Matériel et méthodes : Il s'agit d'une étude observationnelle descriptive quantitative. Un questionnaire en ligne a été élaboré puis transmis via des listes de diffusion à plus de 500 médecins généralistes de la région Rhône-Alpes-Auvergne.

Résultats et Analyse statistique : 188 réponses ont été obtenues. 87\% des MG jugent leurs connaissances insuffisantes et presque $100 \%$ sont intéressés par des formations sur le sujet. Dans la grande majorité des cas (78\%), ces problématiques sont abordées au cours d'une consultation pour un autre motif médical que l'IANA en elle-même. L'hypersensibilité au gluten est surtout évoquée par le patient (58\%) tandis que l'intolérance au lactose est souvent connue du MG (données de la littérature scientifique). Plus d'1/3 des MG n'ont jamais entendu parler des FODMAP.

Un tiers des praticiens estime que ces régimes sont potentiellement utiles mais presque $50 \%$ estime qu'il s'agit aussi d'un effet de mode. La moitié des praticiens a déjà prescrit un régime d'éviction, principalement au lactose. Ce régime est cependant initié par le patient (sans avis médical au préalable) dans la majorité des cas, et dans $95 \%$ des cas pour le gluten ce qui peut être préjudiciable pour le diagnostic de la maladie cœliaque.

Les MG déclarent globalement ne pas se sentir très à l'aise devant un patient évoquant une IANA en raison d'un manque de connaissances/formation pour la moitié d'entre eux, du poids des médias et de l'effet de mode pour 10\%, de la subjectivité des symptômes et/ou d'un profil psychologique particulier du patient pour 7\%. Les MG jugent la composante psychosomatique de ce type de plainte comme assez importante (note médiane de 6.5/10).

Conclusion : Les résultats mettent en avant le manque de connaissances sur le sujet par la grande majorité des MG interrogés. Ils déclarent en moyenne ne pas être à l'aise face à ces patients et intéressés par des informations scientifiques sur le sujet. L'intolérance au lactose est la plus connue et son éviction la plus prescrite. L'implication des médecins généralistes dans les IANA semble aussi compliquée par le poids des médias et l'effet de mode amenant souvent les patients à initier et gérer leur(s) régime(s) d'éviction seuls, parfois avant l'exclusion d'une pathologie organique sous-jacente.

Conflits d'intérêts : Aucun conflit à déclarer 
Développement d'un modèle d'estimation de l'excrétion urinaire de sodium de 24-heures en utilisant le rappel alimentaire de 24-heures chez des enfants marocains.

M. Idrissi ${ }^{1,{ }^{*}}$, N. Saeid ${ }^{1}$, A. Rami ${ }^{1}$, T. Marouan ${ }^{1}$, A. El Hamdouchi ${ }^{1}$, S. Mounach ${ }^{2}$, M. El Mzibri ${ }^{1}$, K. El Kari ${ }^{1}$, H. Aguenaou ${ }^{1}$

${ }^{1}$ Unité Mixte de Recherche en Nutrition et Alimentation CNESTEN-Université Ibn Tofail, Kénitra, ${ }^{2}$ Direction de l'Epidémiologie et de contrôle des Maladies non Transmissibles/ Ministère de la Santé, Rabat, Maroc, Rabat, Maroc

Introduction et but de l'étude : Plusieurs méthodes peuvent être utilisées pour évaluer les apports en sodium, mais la collecte des urines de $24 \mathrm{~h}$ reste la méthode standard proposées par l'OMS, qui peut donner des mesures plus fiables, seulement son utilisation reste très difficile et peut engendrer des pertes au niveau de la collecte vu les critères d'exclusion urinaire qui s'en suivent, ainsi des méthodes alternatives comme la collecte des données sur la consommation alimentaire sont proposées dans la lumières ou ces derniers sont validés par la méthode standard. Ainsi le but de la présente étude est de développer et de valider une équation de prédiction de l'excrétion urinaire de 24-heure (estimation de l'apport en sodium) obtenu par le rappel alimentaire de 24-heures et les données anthropométriques.

Matériel et méthodes : Dans l'ensemble 240 enfants âgés de 6 à 14 ans, sont recrutés au niveau de trois écoles dans la région de Rabat-Kénitra. Seulement 112 enfants ont pu fournir à la fois les urines de 24-heures valides et le questionnaire sur le rappel alimentaire de 24-h (X 3).Une équation de régression est développée pour prédire l'excrétion de sodium des urines de 24-heures à partir des données du rappel alimentaire de 24-heures $(X 3)$ et des facteurs connus par leur influence sur l'excrétion de sodium urinaire de 24-heures et qu'ont montré une contribution significative dans la capacité de prédiction du modèle nouvellement développé. L'excrétion urinaire de sodium de 24-heures mesurée a été comparée avec celle prédite par notre modèle utilisant la méthode graphique de Bland-Altman.

Résultats et Analyse statistique : l'apport en sodium est de l'ordre de 1857,5 mg/24h (1066,5-3036,5 mg/24h). Le meilleur modèle observé est celui développé à partir de l'apport en sodium obtenu par le rappel alimentaire de 24-heures, l'âge, et le poids corporel. La corrélation de Spearman et de Pearson entre l'excrétion urinaire de sodium de 24-heures mesurée et prédite est de l'ordre de 0,491 et 0,52, respectivement. Le graphique de Bland-Altman a montré une dépendance entre la différence (valeur observée moins valeur prédite) et la moyenne (valeur observé + valeurs prédite /2). Pour avoir une distribution de différence plus consistante tout au long des valeurs moyennes des deux méthodes et donc une interprétation plus correcte de niveau de concordance entre les deux méthodes la différence est présentée (sur le graphique de Bland-Altman) sous forme de logarithme. Le graphique de Bland-Altman a indiqué une bonne concordance au niveau du groupe entre l'excrétion moyenne de sodium de 24-heure observée et celle estimée, avec un bais faible, égal à -132,96 mg/jour (95\% Cl: -253,59;-12,33 mg/jour).

Conclusion : Cette étude suggère que notre modèle nouvellement développé pourrait être utile pour prédire l'excrétion urinaire moyenne de sodium de 24 heures chez des enfants ayant des caractéristiques pareilles que notre population.

Conflits d'intérêts : Aucun conflit à déclarer 
Niveau d'activité physique et qualité de vie d'un groupe de femmes libanaises âgées entre 40 et 60 ans.

A. El Hajj ${ }^{1}$, N. Wardy ${ }^{1}$, S. Haider ${ }^{1}$, O. Nada El ${ }^{1}$, L. Khabbaz ${ }^{1}$, T. Wahanian Papazian ${ }^{1,{ }^{*}}$ et Les etudiantes en Master Nutrition Humaine et licence promo 2018

${ }^{1}$ Faculté de Pharmacie, Département de Nutrition, Université Saint Joseph, Beirut, Liban

Introduction et but de l'étude : L'activité physique (AP) est un moyen efficace pour prévenir ou atténuer les symptômes climatériques et améliorer ainsi la qualité de vie (QDV) des femmes en période de ménopause.

L'objectif principal de cette étude est d'examiner l'association entre le niveau d'AP et l'impact de plusieurs facteurs (sociodémographiques et anthropométriques) sur la QDV d'un groupe de femmes libanaises âgées entre 40 et 60 ans.

Matériel et méthodes : Il s'agit d'une étude corrélationnelle transversale menée sur un échantillon de 1113 femmes libanaises âgées entre 40 et 60 ans durant la période Décembre 2017- Avril 2018. Le questionnaire d'évaluation de la QDV durant la période de ménopause (MENQOL) et le questionnaire d'évaluation d'AP (IPAQ court) ont été remplis par une nutritionniste lors d'une entrevue en face à face. Les mesures anthropométriques étaient prises par l'équipe chercheuse.

Résultats et Analyse statistique : Les participantes avaient respectivement une moyenne d'âge et d'IMC de $49.53 \pm 5.74$ ans et $26.69 \pm 5.37 \mathrm{Kg} / \mathrm{m}^{2} .45 .4 \%$ des participantes avaient un faible niveau d'activité physique. Les scores moyens du MENQOL les plus élevés étaient au niveau des domaines physique et psychosocial $(p=0.000)$. Les femmes péri-ménopausées avaient les scores moyens les plus élevés au niveau des domaines vasomoteur, physique et psychosocial $(p=0.000)$, tandis que les femmes postménopausées souffraient plus au niveau du domaine sexuel $(p=0.000)$. Les femmes ayant un niveau d'activité physique modéré ou élevé avaient une meilleur QDV ( $p=0.000)$. Le statut ménopausique, le niveau d'éducation, l'indice de densité, l'indice de masse corporelle, le statut marital, le tabagisme et l'alcool sont également impliqués comme facteurs associés à la fréquence des symptômes liés à la ménopause.

Conclusion : Cette étude réalisée sur un échantillon vaste hétérogène a montré que l'AP a un impact positif sur la QDV des femmes libanaises en période de ménopause, d'où l'interêt de stresser sur son rôle crucial auprès des femmes.

Remerciements : Dr Nada El Osta pour les analyses statistiques

Les participantes

L'équipe chercheuse

Conflits d'intérêts : Aucun conflit à déclarer 
Consommation des produits ultra-transformés chez les pesco-végétariens, les végétariens et les véganes et les déterminants sociodémographiques associés dans l'étude de cohorte NutriNet-Santé

J. Gehring ${ }^{1, *}$, M. Touvier ${ }^{1}$, J. Baudry ${ }^{1}$, C. Julia ${ }^{12}$, C. Buscail ${ }^{1}$, S. Hercberg ${ }^{12}$, E. Kesse-Guyot ${ }^{1}$, B. Allès ${ }^{1}$

${ }^{1}$ EREN, Université Paris $13,{ }^{2}$ Département de santé Publique, Hôpital Avicenne, Bobigny, France

Introduction et but de l'étude : Le développement du marché des produits de substitution, transformés ou ultra-transformés (UPFs), à destination des végétariens suggèrent qu'ils en consomment de plus en plus afin de remplacer les aliments d'origine animale riches en protéine. La part d'UPFs dans l'alimentation des végétariens et des véganes serait donc en augmentation. Le but de cette étude était de décrire la part d'UPFs dans l'alimentation d'omnivores, de pesco-végétariens, de végétariens et de véganes, ainsi que les caractéristiques sociodémographiques et anthropométriques associées, chez des adultes de la cohorte NutriNet-Santé.

Matériel et méthodes : La population étudiée a été segmentée en 4 types de régimes alimentaires : 19812 omnivores, 646 pescovégétariens, 500 végétariens et 254 véganes. Un indicateur de consommation d'UPFs a été calculé pour chaque individu selon la part de l'apport énergétique correspondant aux UPFs, en utilisant la classification NOVA. Les consommations moyennes de 29 groupes alimentaires, ajustés sur le sexe, l'âge et l'apport énergétique total, ont été comparées selon les types de régimes alimentaires et l'indicateur d'UPFs. Dans le sous-échantillon ne contenant que des participants suivant un régime végétarien ( $n=1$ 400), I'association entre la consommation d'UPFs et les variables sociodémographiques et anthropométriques, ainsi que la durée et l'âge au début du régime, a été estimée par un modèle de régression linéaire multiple.

Résultats et Analyse statistique : Comparativement aux omnivores, les végétariens étaient plus jeunes, plus susceptibles d'être des femmes, d'avoir un niveau d'activité physique moyen ou élevé, un niveau d'études supérieur, d'avoir un IMC plus bas et de vivre seuls sans enfant. Les UPFs représentaient 33,0\%, 32,5\%,37,0\% et 39,5\% respectivement pour les omnivores, les pescovégétariens, les végétariens et les véganes. Au-delà du total UPFs consommés, il existait des différences concernant certains groupes alimentaires entre les 3 types de régimes végétariens. Les grands consommateurs d'UPFs parmi les 3 types de végétarisme ont une consommation plus faible de fruits, légumes, céréales non cuites, noix et graines et plus élevée de galettes végétariennes,

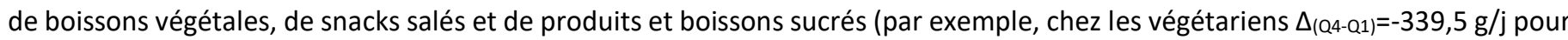
la consommation de fruits et $\Delta\left(\mathrm{Q}_{4}-\mathrm{Q}_{1}\right)=+19.8 \mathrm{~g} / \mathrm{j}$ pour celle des galettes végétariennes). Enfin, il a été montré qu'une augmentation de la durée du régime ou de l'âge de l'individu au début du régime étaient associés à une plus faible consommation d'UPFs.

Conclusion : Les régimes végétariens peuvent être distingués en fonction de la proportion d'UPFs dans leur régime alimentaire. Cela indique que tous les végétariens et véganes n'ont pas nécessairement une alimentation favorable à la santé. Ainsi, la part d'UPFs dans l'alimentation permettra de définir une nouvelle typologie de végétariens, qui pourra être utilisée dans des études sur le lien entre végétarisme et santé.

Conflits d'intérêts : Aucun conflit à déclarer 
${ }^{1}$ MOISA, Univ Montpellier, INRA, CIRAD, CIHEAM-IAMM, Montpellier SupAgro, Montpellier, ${ }^{2}$ Equipe de Recherche en Epidémiologie Nutritionnelle EREN, UMR U1153 Inserm / U1125 Inra / Cnam / Université Paris 13 Centre de Recherche en Epidémiologie et Biostatistiques Sorbonne Paris Cité, Bobigny, France, ${ }^{3}$ Université Libre de Bruxelles, Ecole de Santé publique, Centre de Recherche en Epidémiologie, Biostatistique et Recherche Clinique, Bruxelles, Belgique

Introduction et but de l'étude : Bien qu'il ait été montré que le chômage puisse avoir un effet délétère sur l'état de santé, peu d'études se sont intéressées à son impact sur les comportements alimentaires, en particulier avec une approche longitudinale. L'objectif de notre étude était d'évaluer l'effet de la durée de chômage sur les apports alimentaires d'adultes.

Matériel et méthodes : Cette étude a été menée auprès de 3955 adultes inclus dans l'étude NutriNet-Santé entre 2009 et 2010 , et pour lesquels les consommations alimentaires ont été estimées à l'inclusion et chaque année de suivi, par des enregistrements alimentaires de 24 h répétés. Parmi cette population, nous avons identifié 3 groupes d'individus ayant un emploi à l'inclusion, selon leur statut d'emploi durant le suivi : 207 ont connu une période de chômage $\geq 1$ an (CLD), 98 ont vécu un chômage $<1$ an (CCD) et 2198 sont restés actifs au cours des 5 ans de suivi.

Résultats et Analyse statistique : Des modèles mixtes à effets aléatoires du temps et du statut d'emploi et ajustés sur l'âge, le sexe, le niveau d'éducation et les apports en énergie ont été utilisés pour évaluer les changements alimentaires liés au chômage. A l'inclusion, les deux groupes de chômeurs (CLD et CCD) avaient un score d'adéquation aux recommandations du PNNS (CLD : $\beta$ $=-0,24$, IC $95 \%=[-0,34 ;-0,09] ;$ CCD : $\beta=-0,23$, IC $95 \%=[0,43 ;-0,06])$ et des consommations de «fruits et légumes » (CLD : $\beta=$ $-0,25$, IC $95 \%=[-0,38 ;-0,12]) ; C C D: \beta=-0,22$, IC $95 \%=[-0,40 ;-0,04])$ et de " viande rouge, charcuterie et volaille » (CLD : $\beta=-$ $0,15$, IC $95 \%=[-0,27 ;-0,03]) ;$ CCD : $\beta=-0.12$, IC $95 \%=[-0,29 ;-0,06])$, plus faibles que les personnes restées actives pendant la même période. Comparé aux actifs, le groupe CCD avait un apport en sodium plus faible à l'inclusion $(\beta=-0,13, \mathrm{IC} 95 \%=[-0,25$; $-0,05]$ ) tandis que le groupe CLD avaient des apports en vitamines $B 9$ et $C$ plus faibles (respectivement, $\beta=-0,14, I C 95 \%=[-0,26$; $-0,03]) \beta=-0,18$, IC $95 \%=[-0,31 ;-0,05])$ ) et des apports en énergie et en produits gras sucrés plus élevés (respectivement, $\beta=$ $0,15$, IC $95 \%=[0,03 ; 2,64]) ; \beta=0,12$, IC $95 \%=[0,02 ; 0,2]))$. En revanche, il n'y avait pas d'association statistiquement significative entre le statut d'emploi et une évolution au cours du temps des consommations, excepté pour les fruits et légumes et les produits gras sucrés. Chez le groupe CLD, une légère diminution des apports en produits gras sucrés et une faible augmentation de la consommation de fruits et légumes étaient observés au cours du suivi.

Conclusion : Notre étude a mis en évidence que, sur une période de cinq ans, les personnes ayant connu le chômage, quelle que soit sa durée, ont une alimentation moins favorable à la santé dès le début de cette période que ceux qui restent actifs tout au long du suivi, probablement en lien avec des conditions socioéconomiques plus difficiles (catégorie socioprofessionnelle plus basse, revenus plus faibles). Néanmoins, l'impact du chômage sur l'alimentation n'est pas clairement identifié. D'autres changements du mode de vie tels que le tabagisme et l'activité physique doivent être investigués pour comprendre les effets potentiellement néfastes du chômage sur la santé.

Conflits d'intérêts : Aucun conflit à déclarer 
Introduction et but de l'étude : Au cours de leur cycle de vie, les mangeurs actualisent leurs pratiques alimentaires connaissent des " bifurcations" (Hughes, 1996) qui réorganisent leurs habitudes alimentaires. L'arrivée du premier enfant est une étape décrite comme représentant une " période charnière " (Poulain, 2015), où l'enfant est au cœur des préoccupations parentales et où se mettent en place de nouvelles responsabilités individuelles et parentales. Les travaux montrent ce moment comme favorable à de plus fortes exigences concernant la qualité de l'alimentation des enfants (Anderson, 2001 ; Dupuy et Rochedy, Sous presse). Cependant, les recherches se focalisent sur les pratiques alimentaires des enfants et ne s'intéressent que peu à ce qui se passe du côté des parents, alors même que l'arrivée d'un premier enfant cumule des facteurs - endogènes et exogènes (Lamine, 2008) - de changement des pratiques et augmente certaines contraintes. L'objet de cette étude sociologique est de décrire et expliquer les changements dans les pratiques alimentaires parentales lors de l'arrivée du premier enfant.

Matériel et méthodes : Une enquête ethnographique a été menée auprès de 16 familles (hétéro, mono et homoparentales) avec un seul enfant âgé de moins de 18 mois dans trois villes. Pour chacune des familles, il a été réalisé dans des entretiens ( $\mathrm{n}=46$ ) individuels avec l'un et l'autre des parents, puis des entretiens en couple. En parallèle de ces entretiens, des observations ont été réalisées dans ces familles $(n=16)$ au moment de l'approvisionnement, la préparation ou encore les temps du repas.

Résultats et Analyse statistique : Nous observons comment la pression d'être un "bon parent " ainsi que la compression des activités et des temps sociaux réorganisent la gestion du quotidien et par prolongement les pratiques alimentaires des parents. Ainsi, ce travail identifie les freins et les contraintes aux « bonnes " pratiques alimentaires qui pèsent sur eux malgré l'envie de bien manger. De plus, ce travail met en avant les pratiques alimentaires quotidiennes entre nouvelles expérimentations, négociations et rejets des normes dans leur organisation. Enfin, ce travail souligne également les déterminants sociaux et les inégalités sociales de ces ajustements individuels et familiaux.

Conclusion : Nous montrons comment l'arrivée d'un enfant au sein de la cellule familiale bouleverse différentes dimensions des pratiques alimentaires parentales en fonction de l'âge et des pratiques alimentaires de leur jeune enfant.

Références : Anderson A-S., 2001, "Pregnancy as a time for dietary change?", Proceedings of the Nutrition Society, 60: $497-504$. Dupuy A. \& Rochedy A., Sous presse. " Diversification alimentaire, recherche de naturalité et contraintes saisonnières dans la prime enfance. "Surtout à cette saison, on complète par un fruit cru" » in Julien M.-P., Adamiec C. \& Régnier F. (dir) La saisonnalité : Rennes : PUR.

Hughes E-C., 1996[1950], "Carrières, cycles et tournants de l'existence ", in Hughes Everett C., Le Regard sociologique, Textes rassemblés et présentés par Chapoulie J-M, EHESS, Paris : 165-173.

Lamine C., 2008, Les Intermittents du bio : pour une sociologie pragmatique des choix alimentaires émergents, Paris, Editions de la Maison des Sciences de l'Homme.

Poulain J-P., 2015, « Alimentation et cycle de vie », in Esnouf C., Fioramonti J. et Laurioux B. (dir), L'alimentation à découvert, Paris, CNRS Editions : 27-28.

Conflits d'intérêts : Aucun conflit à déclarer 
Z. Colombet ${ }^{1,}{ }^{*}$, M. Pérignon ${ }^{1}$, B. Salanave ${ }^{2}$, E. Landais ${ }^{3}$, Y. Martin-Prével ${ }^{3}$, B. Allès ${ }^{4}$, M.-J. Amiot-Carlin ${ }^{1}$, N. Darmon ${ }^{1}$, C. Méjean ${ }^{1}$

${ }^{1}$ MOISA, Univ Montpellier, CIRAD, CIHEAM-IAMM, INRA, Montpellier SupAgro, Montpellier, 2'Santé publique France, Direction des maladies non transmissibles et traumatismes, Equipe de surveillance et d'épidémiologie nutritionnelle (ESEN), Saint-Maurice, France, Université Paris 13, Sorbonne Paris Cité, Centre de Recherche en Epidémiologies et Biostatistiques, Bobigny, ${ }^{3}$ IRD (Institut de Recherche pour le Développement), NUTRIPASS Unit, IRD-UM-SupAgro, 911 av. Agropolis, 34394 Montpellier, ${ }^{4}$ EREN, Centre de Recherche en Epidémiologie et Statistiques Sorbonne Paris Cité UMR U1153 Inserm / U1125 Inra / Cnam / Univ Paris 13, Bobigny, France

Introduction et but de l'étude : Dans les Caraïbes et plus spécifiquement dans les Antilles Françaises, des prévalences élevées d'obésité et de maladies chroniques coexistent avec des taux de pauvreté très importants. Pour identifier les processus menant aux inégalités sociales de santé, il est nécessaire d'explorer l'influence des différents indicateurs de statut socioéconomique sur la qualité de l'alimentation. En effet, plusieurs études constatent que les différents indicateurs socioéconomiques ne sont pas interchangeables et peuvent avoir un effet cumulatif sur la qualité de l'alimentation. Nous avons donc étudié les associations indépendantes entre chaque indicateur de statut socioéconomique et la qualité de l'alimentation dans les Antilles françaises.

Matériel et méthodes : Les apports alimentaires ont été estimés par des rappels de $24 \mathrm{~h}$ chez 1144 participants ( $\geq 16$ ans) de l'étude Kannari, enquête transversale à visée représentative de la population générale résidant en Guadeloupe et Martinique. La qualité de l'alimentation a été mesurée avec le mPNNS-GS [maximum de 13,5 points], mesurant l'adhésion aux repères de consommation du PNNS, et le " Diet Quality Index-International » (DQI-I) [de 0 à 100 points] qui prend en compte plusieurs dimensions de la qualité de l'alimentation (diversité, adéquation, modération et équilibre). Les associations entre les scores de qualité de l'alimentation et les indicateurs de statut socioéconomique (niveau d'éducation, allocataire d'aide sociale, situation par rapport à l'emploi, foyer avec enfants, famille monoparentale) ont été évaluées grâce à des régressions linéaires multivariées, ajustées sur le département de résidence (Guadeloupe ou Martinique), le sexe, l'âge et le statut matrimonial.

Résultats et Analyse statistique : En moyenne, le mPNNS-GS était de 8,3 points [de 4 à 11,5 points] et le DQI-I était de 60,8 points [de 39 à 82 points]. Les participants ayant un niveau d'études équivalent au baccalauréat avaient un mPNNS-GS plus faible que ceux ayant un niveau d'études supérieur $(8,0$ (SEM : 0,2$)$ vs. 8,4 points (SEM : 0,1$) ; p=0,01)$ mais aucune différence significative n'était retrouvée avec les participants ayant un niveau d'études primaire ou secondaire. Les personnes sans emploi ou n'ayant jamais eu d'emploi avaient un DQI-I plus faible que les personnes ayant un emploi $(60,7$ (SEM : 0,7$)$ vs. 62,5 points (SEM : 0,4) ; $p=0,02)$.

Conclusion : Dans cette étude peu de différences socioéconomiques de la qualité de l'alimentation ont été observées, probablement en raison d'une sous-représentation des populations les plus précaires. Identifier les groupes de population les plus à risque d'avoir une alimentation défavorable à la santé reste nécessaire dans les Antilles françaises afin de mieux cibler les actions de santé publique.

Conflits d'intérêts : Aucun conflit à déclarer 
Z. Colombet ${ }^{1,}{ }^{*}$, M. Pérignon ${ }^{1}$, B. Salanave ${ }^{2}$, E. Landais ${ }^{3}$, Y. Martin-Prével ${ }^{3}$, B. Allès ${ }^{4}$, M.-J. Amiot-Carlin ${ }^{1}$, N. Darmon ${ }^{1}$, C. Méjean ${ }^{1}$

${ }^{1}$ MOISA, Univ Montpellier, CIRAD, CIHEAM-IAMM, INRA, Montpellier SupAgro, Montpellier, 2'Santé publique France, Direction des maladies non transmissibles et traumatismes, Equipe de surveillance et d'épidémiologie nutritionnelle (ESEN), Saint-Maurice, France, Université Paris 13, Sorbonne Paris Cité, Centre de Recherche en Epidémiologies et Biostatistiques, Bobigny, ${ }^{3}$ IRD (Institut de Recherche pour le Développement), NUTRIPASS Unit, IRD-UM-SupAgro, 911 av. Agropolis, 34394 Montpellier, ${ }^{4}$ EREN, Centre de Recherche en Epidémiologie et Statistiques Sorbonne Paris Cité UMR U1153 Inserm / U1125 Inra / Cnam / Univ Paris 13, Bobigny, France

Introduction et but de l'étude : La sous-déclaration énergétique, c'est-à-dire des personnes ayant un apport énergétique déclaré inférieur à leur besoin énergétique minimal, est un véritable problème dans les études d'épidémiologie nutritionnelle car elle peut modifier les associations entre l'alimentation et l'état de santé. Les rares études menées dans les Antilles françaises montrent un fort pourcentage de sous-déclarants énergétiques, cependant, la seule étude les ayant caractérisés utilisait des équations non adaptées à des sujets obèses. Or, de fortes prévalence d'obésité sont observées dans les Antilles françaises. Nous avons donc caractérisé les participants sous-déclarants avec des équations adaptées et identifié les caractéristiques individuelles associées à cette sous-déclaration afin de la prévenir lors de futures études nutritionnelles dans les Antilles françaises.

Matériel et méthodes : L'étude portait sur les données de l'étude Kannari, étude épidémiologique transversale à visée représentative en Guadeloupe et Martinique en 2013-2014. La méthode proposée par Black a été utilisée pour l'identification des sous-déclarants énergétiques et les équations de Mifflin ont été utilisées pour le calcul du basal metabolic rate (BMR) en fonction du poids, de la taille et du sexe. Les apports énergétiques ont été estimés grâce à des rappels de 24h. Les caractéristiques individuelles (âge, sexe, situation professionnelle, niveau d'éducation, indice de masse corporelle (IMC)) associées à la sousdéclaration ont été évaluées par des modèles de régressions logistiques, ajusté sur l'apport énergétique et le département de résidence (Guadeloupe ou Martinique).

Résultats et Analyse statistique : Parmi les 1341 participants ayant un rappel de 24h, 15\% ont été identifiés comme sousdéclarants énergétiques. Les hommes avaient une probabilité plus élevée de sous-déclarer leurs consommations alimentaires que les femmes $\left(O R=9,3 ;\right.$ IC95\% = [5,7 - 15,2]). La probabilité de sous-déclaration diminuait avec l'âge $\left(O R_{46-60}\right.$ vs. $\leq 45$ ans $=0,5 ;$ IC95\% $=[0,3-0,8]$ et $O R>60$ vs. $\leq 45$ ans $=0,2 ; I C 95 \%=[0,1-0,5])$ et augmentait avec le niveau d'éducation $\left(O R_{\text {secondaire vs. primaire }=1,7 ; I C 95 \%}\right.$ $=[1,0-2,8]$ et ORsupérieur vs. primaire $=2,1 ;$ IC95\% $=[1,3-3,6]$ ) et avec l'IMC (ORsurpoids vs. normal $=2,8 ;$ IC95\% = $[1,7-4,7]$ et ORobésité vs. normal $=5,3 ;$ IC95\% $=[3,1-8,9])$.

Conclusion : Malgré une attention particulière apportée aux rappels de $24 \mathrm{~h}$ lors de l'enquête Kannari, de nombreux participants étaient sous-déclarants. L'identification des caractéristiques individuelles de la sous-déclaration a permis d'identifier des souspopulations plus à susceptibles d'être sous-déclarants qui devront faire l'objet d'une attention particulière lors des futures études nutritionnelles dans les Antilles françaises.

Conflits d'intérêts : Aucun conflit à déclarer 
Effet de l'alimentation maternelle sur le gain de poids au cours de la grossesse.

F. Tebbani ${ }^{1,}{ }^{*}$, H. Oulamara ${ }^{1}$, A. Agli ${ }^{1}$

${ }^{1}$ Nutrition humaine, Institut de nutrition de l'alimentation et des technologies agroalimentaires, Constantine, Algérie

Introduction et but de l'étude : Parmi les principaux déterminants du gain pondéral gestationnel se trouve la consommation alimentaire.

Evaluer les apports maternels en énergie et en nutriments des femmes de notre population à chaque trimestre de grossesse, de les comparer aux apports nutritionnels conseillés (ANC) et apprécier leur effet sur le gain pondéral gestationnel.

Matériel et méthodes : C'est une étude prospective et longitudinale d'une cohorte de 300 femmes enceintes âgées de 19 à 43 ans, réalisée durant toute la période de la grossesse aux centres de consultations et de suivi prénatales à Constantine (Algérie) du décembre 2013 au Juin 2016. L'estimation des apports en énergie, macro et micronutriments a été effectuée par un enregistrement alimentaire de trois jours et répétée à chaque trimestre de grossesse. La taille et le poids de départ et le poids en fin de grossesse ont été mesurés. Le gain pondéral total a été calculé. L'âge, la parité, le niveau d'instruction et le niveau de vie ont été recueillis. Les statistiques ont été effectuées en utilisant les logiciels Statview ${ }^{\mathrm{TM}}$ et SPSS.

Résultats et Analyse statistique : La majorité des femmes (75,0\%) ont un gain pondéral inadéquat en fin de grossesse (47,3\% de gain insuffisant et $27,7 \%$ de gain excessif) et seulement $25,0 \%$ d'entre elles ont un gain de poids normal. L'apport calorique, vitaminique et en minéraux des femmes ayant un gain pondéral insuffisant est inférieur à celui des femmes avec un gain normal et un gain excessif lors des trois trimestres de grossesse $(p<0,0001)$. Egalement, pour toutes les catégories de gain pondéral, les apports moyens en énergie et en nutriments sont plus faibles au premier trimestre puis augmentent au deuxième trimestre et se stabilisent au troisième trimestre. Entre le premier et le troisième trimestre, 86 à $100 \%$ des femmes ont des apports en vitamines (B9, A et D) et en minéraux ( $\mathrm{Ca}$, Fe, Mg et $\mathrm{Zn}$ ) nettement inférieurs aux ANC.

Conclusion : Une surveillance de l'alimentation est un moyen efficace de lutter contre un gain de poids inadéquat (excessif ou insuffisant), soit en évitant son apparition, soit en limitant son aggravation.

Références : IOM. Weight Gain During Pregnancy: Reexamining the Guidelines; Committee to Reexamine IOM Pregnancy weight Guidelines; Sponsor Briefing May 27, 2009.

LAGIOU P, TAMIMI RM, MUCCI LA, ADAMI H-O, HSIEH C-C, TRICHOPOULOS D. Diet during pregnancy in relation to maternal weight gain and birth size. Eur J Clin Nutr 2004; 58: 231-237.

OLAFSDOTTIR AS, SKULADOTTIR GV, THORSDOTTIR I, HAUKSSON A, STEINGRIMSDOTTIR L. Maternal diet in early and late pregnancy in relation to weight gain. International Journal of Obesity 2006, 30(3), 492-499.

Conflits d'intérêts : Aucun conflit à déclarer 
Le rôle des herbes et épices sur l'appréciation de plats à base de légumineuses.

M. Vannereux ${ }^{1, *}$, A. Dougkas ${ }^{1}$, A. Giboreau ${ }^{1}$

${ }^{1}$ Centre de Recherche, Institut Paul Bocuse, Ecully, France

Introduction et but de l'étude : Il est largement reconnu qu'un régime plus riche en aliments d'origine végétale, telle que les légumineuses, permettrait de favoriser davantage la santé des consommateurs. Cependant, malgré leurs bienfaits, leur consommation reste faible. Sachant que le goût est le premier facteur pris en compte par les consommateurs, l'aromatisation par les herbes et épices se positionne comme une stratégie intéressante pour augmenter leur appréciation et leur consommation. À ce jour, il existe pourtant peu d'études menées sur ce sujet. Ainsi, ce travail s'intéresse au « goût » de plats à base de légumineuses, sous l'angle gustatif (variation du sel) et sous l'angle olfactif (variation des herbes et épices) et leur effet sur la perception des consommateurs en situation réelle de prise de repas. L'objectif de cette étude est de mesurer l'effet des herbes et épices sur l'amélioration des caractéristiques sensorielles de plats à base de légumineuses en interaction avec leur teneur en sel.

Matériel et méthodes : Etape 1. Etude des préférences

Quatre recettes avec différentes combinaisons d'herbes et d'épices ont été formulées par des chefs cuisiniers. Puis ces recettes ont été évaluées par des consommateurs en situation réelle de repas. 128 consommateurs âgés entre 18 et 35 ans (61 femmes, 67 hommes) ont participé à un déjeuner expérimental mené en situation réelle de consommation au restaurant. Chaque participant a goûté et donné son appréciation de 4 recettes proposées simultanément en tant qu'apéritif (Curcuma, Gingembre, Paprika et Épinard), selon un ordre équilibré de présentation. L'appréciation du goût général a été mesurée avec une échelle de Likert en 9 points, suivie d'une citation spontanée des attributs positifs et négatifs puis d'un test de classement de préférences

Etape 2. Etude des interactions entre épices et sel.

La recette préférée a ensuite été déclinée en quatre variantes plus ou moins riches en sel et en épices, selon un design $2 \times 2$ (taux faible / fort en sel $x$ taux faible / fort en épices). Un test par paire a alors été mené auprès de 115 personnes (57 femmes, 58 hommes) dans le but de déterminer si les recettes étaient perçues différemment en termes de niveau de sel et d'herbes et épices. Résultats et Analyse statistique : Lors de la $1^{\text {ère }}$ étape, la recette Épinard a obtenu une moyenne significativement supérieure aux autres (Test de Student $5 \%, p<0,001$ ). Ce résultat a été confirmé par le test de classement où cette même recette a été placée comme significativement préférée par les consommateurs (Test de Friedman $5 \%, p<0,001$ ).

Lors de la $2^{\text {ème }}$ étape, les résultats ont montré que la différence entre les échantillons pris 2 à 2 était facilement perceptible (Loi binomiale $5 \%, p<0,001)$. Les épices permettent donc de compenser la réduction en sel et d'offrir des recettes riches en " goût ", au sens intégré du terme (saveurs + arômes).

Conclusion : Ces études ont permis de sélectionner une recette préférée des consommateurs et montré que les variations de sel et d'herbes et épices sont aisément perceptibles par les consommateurs. L'aromatisation des plats à base de légumineuses par des herbes et épices semble ainsi être un levier intéressant pour augmenter l'appréciation et la consommation de légumineuses.

Remerciements : Cette étude est sponsorisée par l'Institut des Sciences de McCormick

Conflits d'intérêts : M. Vannereux: Aucun conflit à déclarer, A. Dougkas a reçu une subvention/soutien de McCormick Science Institute, A. Giboreau: Aucun conflit à déclarer 
Facteurs influant sur le gain pondéral insuffisant au Cours de la grossesse.

F. Tebbani ${ }^{1,}{ }^{*}$, H. Oulamara ${ }^{1}$, A. Agli ${ }^{1}$

${ }^{1}$ Nutrition humaine, Institut de nutrition de l'alimentation et des technologies agroalimentaires, Constantine, Algérie

Introduction et but de l'étude : Etudier le gain pondéral chez la femme enceinte selon les recommandations de l'Institut Américain de Médecine (IOM 2009) et identifier les facteurs de risque de gain pondéral insuffisant chez les femmes gestantes à Constantine (Algérie).

Matériel et méthodes : Une étude prospective et longitudinale d'une cohorte de 300 femmes enceintes âgées de 19 à 43 ans, a été réalisée durant toute la période de la grossesse aux centres de consultations et de suivis prénatals à Constantine (Algérie) du décembre 2013 au Juillet 2016. Leur poids était mesuré au premier, deuxième et troisième trimestre. Le gain pondéral gestationnel (GPG) était classé comme inférieur, conforme ou supérieur aux recommandations de l'IOM. Les facteurs influant sur le GPG, tels que I'IMC pré-gestationnel, les connaissances des femmes en matière de GPG, les signes sympathiques, les habitudes et consommations alimentaire et le stress maternel ont été étudiés. Les statistiques ont été effectuées en utilisant les logiciels Statview ${ }^{\mathrm{TM}}$ et SPSS.

Résultats et Analyse statistique : La majorité des femmes (65,4\%) ont un gain pondéral total insuffisant en fin de grossesse et seulement $34,6 \%$ d'entre elles ont un gain de poids conforme aux recommandations de l'IOM. Le gain de poids insuffisant est plus fréquent chez les femmes obèses avant grossesse $(p<0,0001)$, ayant déclaré ignorer le gain idéal $(p=0,01)$, présentant des signes sympathiques $(p=0,01)$, ayant des aversions alimentaires $(p=0,0086)$ et ont des apports vitaminiques et en minéraux insuffisants $(p<0,01)$. Ce gain est également fréquent chez les femmes ayant une durée faible de sommeil et avec un environnement stressant.

Conclusion : Le gain pondéral gestationnel est associé à un certain nombre de facteurs et ces relations varient en fonction de chaque trimestre de grossesse.

Références : TOVAR A, GUTHRIE LB, PLATEK D, STUEBE A, HERRING S J, \& OKEN E. (2011) Modifiable predictors associated with having a gestational weight gain goal. Maternal \& Child Health Journal, 15, 1119-1126.

HASTOY A, LIEN TRAN P, LAKESTANI O, BARAU G, GERARDIN P, BOUKERROU M. (2015) L'hyperémèse gravidique : quelles conséquences sur la grossesse? Journal de Gynécologie Obstétrique et Biologies de la Reproduction 2015. 44, $154-163$.

Conflits d'intérêts : Aucun conflit à déclarer 
Effet de l'alimentation maternelle sur le poids du nouveau ne.

F. Tebbani ${ }^{1}$, H. Oulamara ${ }^{1, *}$, A. Agli ${ }^{1}$

${ }^{1}$ Nutrition humaine, Institut de nutrition de l'alimentation et des technologies agroalimentaires, Constantine, Algérie

Introduction et but de l'étude : La grossesse est une période d'augmentation des besoins métaboliques. Les vitamines, les minéraux et les oligo-éléments sont des déterminants majeurs de la santé de la femme enceinte et du fœutus.

Evaluer les apports maternels en oligoéléments et vitamines dans les premier, deuxième et troisième trimestres de grossesse et apprécier leur effet sur le poids de naissance.

Matériel et méthodes : C'est une étude prospective et longitudinale réalisée auprès 226 femmes enceintes durant toute la période de la grossesse aux centres de consultations et de suivi prénatales à Constantine (Algérie) du décembre 2013 au Juin 2016. Nous avons analysé les apports maternels en fer, en éléments minéraux et en vitamines en les comparants aux apports normalement conseillés (ANC), puis par analyse multivariée, nous avons étudié la corrélation entre ces apports et le poids de naissance. Les statistiques ont été effectuées en utilisant les logiciels Statview ${ }^{\mathrm{TM}}$ et SPSS.

Résultats et Analyse statistique : Cette étude a noté l'effet positif de certains facteurs maternels sur le poids de naissance, tels que : I'âge maternel, la parité, l'IMC pré-gravidique et le terme de grossesse. Les apports quotidiens moyens en minéraux (fer, calcium, zinc et magnésium) et vitamines (B9, B1 et E) étaient inférieurs aux apports recommandés (ANC). En revanche, les apports moyens en vitamine $C$ en $2^{\text {ième }}$ et $3^{\text {ième }}$ trimestres de grossesse correspondaient aux ANC. Seuls les apports en magnésium en premier trimestre $(p=0,02)$, calcium en $3^{\mathrm{e}}$ trimestre $(p=0,06)$ et la vitamine $B 9$ en $1^{\mathrm{e}}$ et $3^{\mathrm{e}}$ trimestre $(p=0,07 ; p=0,004)$ respectivement, étaient significativement corrélés avec le poids de naissance.

Conclusion : Les apports en oligoéléments et vitamines dans notre population d'étude sont diminués par rapport aux ANC. La correction du régime des femmes enceintes est un besoin urgent. Ainsi, en encourageant la qualité plutôt que la quantité pour éviter des carences on oligoéléments et vitamines nuisibles au développement et à la croissance du fœtus.

Références : Costello AMdL, Osrin D (2004). Micronutrients status during pregnancy and outcomes for newborn infants in developing countries. Am J Clin Nutr; 79 : 933-934.

Zazzo JF (1995). Oligoéléments et grossesse. Repr Hum Horm;8 :539-45.

Ramakrishnan U (2004). Nutrition and low birth weight : from research to practice. Am J Clin Nutr; 79 : $17-21$

Conflits d'intérêts : Aucun conflit à déclarer 
Les acides gras trans monoinsaturés dans l'alimentation des Français : une étude qualitative.

E. J. Guillocheau ${ }^{12,{ }^{*}}$, C. Penhoat ${ }^{1}$, A. Godet ${ }^{1}$, D. Catheline ${ }^{1}$, P. Legrand ${ }^{1}$, V. Rioux ${ }^{1}$

${ }^{1}$ Laboratoire de Biochimie-Nutrition humaine, Agrocampus-Ouest, Rennes, ${ }^{2}$ Direction des Affaires Scientifiques et Techniques, Centre national interprofessionel de l'économie laitière (CNIEL), Paris, France

Introduction et but de l'étude : Dans quels aliments trouve-t-on des acides gras trans monoinsaturés (AGT) à l'heure actuelle en France ? Si les Etats-Unis ont interdit l'utilisation d'huiles partiellement hydrogénées à l'horizon 2018, aucune législation n'a vu le jour en Europe : I'incertitude demeure sur les AGT industriels (AGTi). Par ailleurs, les AGT naturellement présents dans les produits laitiers et les viandes de ruminants (AGTn) sont difficilement distingables de leurs homologues industriels lorsqu'il s'agit de mesurer les taux dans les aliments. Alors, que trouve-t-on dans les aliments : AGTi ou AGTn ?

Cette question est cruciale au sens où des bénéfices ont été montrés concernant les AGTn, contrairement à leurs homologues industriels. D'autre part, l'OMS a récemment rappelé l'importance de supprimer les AGTi de l'alimentation. Pourtant, très peu de laboratoires ont la capacité de différencier AGTi et AGTn : cette étude qualitative se veut donc être une première en France et en Europe, ne négligeant aucun aliment, et faisant la différence claire entre AGTi et AGTn à l'aide de méthodes très fines.

Matériel et méthodes : Nous avons acheté des aliments couramment consommés par la population française, dans des supermarchés de la région rennaise : produits laitiers (laits, yaourts, fromages), viandes (veau, agneau, bœuf), mais aussi des biscuits et des glaces. Dans la mesure du possible, les impacts saisonniers ont été évalués, ainsi que le type d'agriculture (bio vs. conventionnelle).

Les lipides de ces aliments ont été extraits, puis dérivés en esters méthyliques d'acides gras. Une première analyse par chromatographie en phase gazeuse couplée à un spectromètre de masse (GC-MS) a été effectuée. En cas de présence d'AGT, nous avons procédé à une isolation de la fraction trans par chromatographie au nitrate d'argent, puis une nouvelle analyse de cette fraction par GC-MS afin de déterminer les isomères de position des C16:1 trans et des C18:1 trans.

Résultats et Analyse statistique : Les margarines, aliments emblématiques de l'ère des AGTi, sont dépourvues d'AGT (<0,5\% des acides gras totaux). Les aliments de type biscuits ou glaces, eux aussi suspects, contiennent des AGT uniquement lorsque leur composition se base sur de la matière grasse laitière : ainsi, ces aliments ont bien un profil C16:1 trans et C18:1 trans identique à ceux des produits laitiers. De manière intéressante, des différences de profils C16:1 trans et de $\mathrm{C} 18: 1$ trans sont constatées entre produits laitiers et certains échantillons de viande.

Conclusion : Les AGTi sont désormais absents de l'alimentation française ; les produits de ruminants seront les seuls contributeurs à l'apport en AGT à terme. La nutrition animale jour vraisemblablement un rôle dans la différence entre produits laitiers et viandes de ruminants: il conviendra d'évaluer précisément la contribution de ces viandes dans l'apport en AGT pour tirer des conclusions. Les analyses d'aliments se poursuivront de manière à couvrir le maximum d'aliments consommés, et à construire une base de données permettant d'estimer les apports en AGT en France.

Conflits d'intérêts : E. Guillocheau est employé(e) de Centre national interprofessionnel de l'économie laitière (CNIEL), C. Penhoat: Aucun conflit à déclarer, A. Godet: Aucun conflit à déclarer, D. Catheline: Aucun conflit à déclarer, P. Legrand: Aucun conflit à déclarer, V. Rioux: Aucun conflit à déclarer 
Introduction et but de l'étude : La parodontite est une maladie inflammatoire des tissus de soutien de la dent (le parodonte) très fréquente dans la population générale adulte. Elle est une maladie d'une origine multifactorielle strictement liée à une dysbiose du microbiote orale. Des données récentes suggèrent que les habitudes alimentaires pourraient influencer le développement et la gravité de la parodontite. Néanmoins, à ce jour, l'association entre la consommation de différents types d'alcool et la parodontite au niveau de la population française (de tout âge) n'est pas connue. Cette étude vise à analyser les données de l'ecohorte NutriNet-Santé pour évaluer l'association entre la consommation de boissons alcoolisées et le risque de parodontite.

Matériel et méthodes : L'étude a porté sur 35390 adultes faisant partie de l'e-cohorte NutriNet-Santé (https://www.etudenutrinet-sante.fr/) et ayant rempli un questionnaire sur la santé bucco-dentaire entre 2011 et 2012. Le risque de parodontite a été évalué en calculant le score de dépistage parodontal (Periodontal Screening Score, PESS) à partir de 4 questions sélectionnées. Un score ${ }^{3} 5$ correspond à un risque élevé de parodontite sévère. Les données concernant la consommation d'alcool ont été obtenues à partir des questionnaires semi-quantitatifs et des enquêtes alimentaires de 24h. Des analyses transversales ont été réalisées à l'aide de modèles de régression multivariés.

Résultats et Analyse statistique : La population étudiée avait un âge moyen de 49,04 $\pm 13,94$ ans et était composée principalement de femmes (75,8\%). Au total, 7263 (20,5\%) sujets présentaient un risque élevé de parodontite sévère. Après ajustement sur les facteurs de confusion connus (âge, sexe, IMC, statut socioéconomique), la consommation d'alcool était significativement plus élevée chez les adultes à haut risque de parodontite sévère par rapport au groupe à faible risque de parodontite (11,19 versus $7,97 \mathrm{~g} / \mathrm{j}$ d'éthanol ; $p=0,011$ ). En moyenne, le groupe à haut risque de parodontite consommait du vin 3,75 jours par semaine, tandis que le groupe à faible risque de parodontite consommait du vin seulement 2,86 jours par semaine ( $p=0.025$ ). Une consommation excessive d'alcool (correspondant à $>30 \mathrm{~g} / \mathrm{jour}$ pour les hommes et $>20 \mathrm{~g} / \mathrm{jour}$ pour les femmes) associée au tabagisme augmentait significativement le risque d'avoir une parodontite sévère (OR : 7,30; IC à 95 \%:6,10-8,73; $p<0,0001)$.

Conclusion : Ces résultats permettent d'actualiser les données sur le risque de la parodontite sévère en France, même si cet échantillon pourrait être sujet d'un biais de sélection. Les résultats corroborent une association entre la consommation d'alcool et le risque de parodontite, notamment en fonction du statut tabagique.

Conflits d'intérêts : Aucun conflit à déclarer 


\section{P252}

\section{Inventaire de plats et mets traditionnels consommés durant le mois de Ramadan}

M. Bencharif ${ }^{1, *}$, E. Boudjouada ${ }^{2}$, Y. Benabbas ${ }^{3}$

${ }^{1}$ Département de Nutrition, Laboratoire de Nutrition et Technologies Alimentaires (LNTA), INATAA, Université Frères Mentouri Constantine 1, Algérie, ${ }^{2}$ Département de Nutrition, INATAA, Université Frères Mentouri Constantine 1 , Algérie, ${ }^{3}$ Médecine Interne, CHU de Constantine, Constantine, Algérie

Introduction et but de l'étude : Le mois de Ramadan est le temps d'une modification transitoire du rythme de vie et des habitudes alimentaires. Les aliments traditionnels sont des produits patrimonials, s'appuyant sur les savoirs et les savoirs-faires acquis de génération en génération. L'objectif de cette étude était de dresser un inventaire de plats et mets traditionnels consommés durant le mois de Ramadan et de les caractériser d'un point de vue nutritionnel et de santé.

Matériel et méthodes : Une enquêté auprès de 400 ménages réparti sur cinq régions de l'Algérie (Est, Ouest, Centre, Sud et Kabylie). Un questionnaire a permis de recueillir les recettes détaillées, le moment et la fréquence de consommation de plats et mets traditionnels consommés durant le Ramadan. Nous avons déterminé les apports nutritionnels et énergétiques de chacun des plats et mets répertoriés.

Résultats et Analyse statistique : L'enquête de consommation a permis de recueillir 62 plats et mets traditionnels regroupés en 7 groupes d'aliments : 6 types de pains, 6 soupes, 11 pâtes traditionnels, 20 Tadjines, 4 salés, 14 gâteaux et 3 desserts consommés durant le mois de Ramadan.

Concernant l'apport énergétique moyen respective pour $100 \mathrm{~g}$ de pains, de soupes, de pâtes traditionnels, de Tadjines, de salés, de gâteaux et de desserts était de 332,41 kcal, 117,08 kcal, 379,64 kcal, 232,69 kcal, 340,10 kcal, 808,90 kcal, 385,37 kcal, avec un apport moyen égal à 2596,19 kcal dans le cas d'une consommation de $100 \mathrm{~g}$ seulement de chaque type.

La caractérisation de ces plats a permis d'apporter des renseignements sur les pratiques culinaires des enquêtés durant le Ramadan.

La plupart des plats et des mets traditionnels étaient nationaux et communs pour toutes les régions. Les algériens consommaient varié mais pas toujours équilibré.

La détermination des apports nutritionnels de ces plats traditionnels a révélé que la plupart d'entres-eux avaient des potentiels nutritionnels intéressants mais étaient diminués à cause de l'utilisation en quantité trop importante de matière grasse, de sucre et de sel. Ce qui peut poser de sérieux problèmes de santé dans le cas d'une consommation tout au long de l'année (lithiases biliaires, accidents cardio-vasculaires, obésité, diabète, tension artérielle,...).

Conclusion : Il serait intéressant de faire appel à l'éducation nutritionnelle à l'échelle national, particulièrement avant le mois de Ramadan et de faire des recommandations à la destination de la population dans le but d'améliorer les pratiques culinaires concernant les plats traditionnels et en diminuant la part de ceux considérés comme mauvais (gras, sucre, sel).

Conflits d'intérêts : Aucun conflit à déclarer 
Utilisation d'un journal alimentaire numérique à champ libre : évaluation préliminaire de l'exploitabilité des données récoltées avec une approche semi-supervisée.

S. Akkoyunlu ${ }^{1, *}$, N. Darcel ${ }^{2}$, C. Manfredotti $^{1}$, A. Cornuéjols ${ }^{1}$, F. Delaere $^{3}$

${ }^{1}$ UMR MIA-Paris, ${ }^{2}$ UMR PNCA, AgroParisTech, Paris, ${ }^{3}$ Danone Nutricia Research, Palaiseau, France

Introduction et but de l'étude : Un carnet alimentaire permet une analyse fine des consommations et de leur dynamique mais constitue un processus long et fastidieux pour les sujets comme pour l'expérimentateur. De plus en plus d'outils numériques ergonomiques facilitent l'acquisition des données par le sujet, mais sont souvent dénués d'une classification d'aliments hiérarchisée, ou sont assimilables à un carnet ouvert. Une analyse des aliments ou des nutriments ingérés requiert alors une phase lourde de codage des aliments par l'expérimentateur. L'objectif de cette étude est d'évaluer la performance d'un algorithme de codage automatique des aliments issus d'un carnet alimentaire électronique ouvert.

Matériel et méthodes : Les données de consommation alimentaires sont récoltées à l'aide d'une application mobile, MyFitnessPal. L'utilisateur note ses consommations librement ou peut scanner le code barre de l'aliment pour obtenir son nom commercial. La classification $\mathrm{CIQUAL}^{1}$ (Anses) est organisée suivant une hiérarchie à 4 niveaux : groupe, sous-groupe, sous-sous-groupe et aliment. Notre objectif est d'assigner un code à chaque acte de consommation - en priorité, un code d'aliment puis de sous-sous-groupe, de sous-groupe et enfin de groupe.

L'algorithme consiste à utiliser le nom du produit (exemple : "Danone Activia") comme requête pour trouver les aliments similaires selon leur dénomination dans la base CIQUAL. Les résultats de la requête sont classés selon un score de pertinence basé sur les occurrences des mots. Si la majorité des résultats font partie d'un même sous-sous-groupe, alors nous assignons le code correspondant à l'aliment. Sinon, nous passons au sous-groupe puis au groupe. Si aucun consensus n'est obtenu, l'aliment ne peut être catégorisé. Dans ce cas, la base OpenFoodFacts ${ }^{2}$, base open source de produits alimentaires non hiérarchisée, est utilisée. Si les informations sur la catégorie existent (exemple : \{"produits laitiers", "yaourt"\}) alors nous les utilisons pour trouver la catégorie correspondante dans la base CIQUAL. Les catégories proposées par l'algorithme ont ensuite été vérifiées manuellement pour en estimer la performance.

Résultats et Analyse statistique : Un journal tenu durant un mois contenant 1474 actes de consommation comptant 442 aliments différents est utilisé. En combinant les résultats sur les deux bases, nous obtenons 431 correspondances (97\%) dont 337 (78\%) ont été jugées bonnes a posteriori. Parmi les bonnes correspondances, 46 (14\%) sont des codes aliment, 156 (46\%) des codes sous-sous-groupe, 120 (36\%) des codes sous-groupe et enfin 15 (4\%) des codes groupe. Les 11 (2\%) non-correspondances correspondent à des aliments mal orthographiés ou inexistants dans les deux bases.

Conclusion : En limitant les actions de recherche par le sujet, l'utilisation d'un carnet alimentaire électronique à champ libre est une alternative intéressante aux outils actuels de recueil alimentaire. Le problème du codage des aliments peut être allégé en utilisant un algorithme de codage automatique. Ce dernier peut être amélioré en tenant compte des fautes d'orthographe.

Références : 1 . https://ciqual.anses.fr/

2. https://fr.openfoodfacts.org/

Conflits d'intérêts : Aucun conflit à déclarer 
${ }^{1}$ Laboratoire de Nutrition et Technologies Alimentaires (LNTA), INATAA, Université Frères Mentouri Constantine 1, Algérie, ${ }^{2}$ Institut de la Nutrition, de l'Alimentation et des Technologies Agro-Alimentaires (INATAA), Université Frères Mentouri Constantine 1, Algérie, Constantine, Algérie

Introduction et but de l'étude : L'adolescence est une période vulnérable, un passage obligatoire, de transition sensible où les choix de vie qui se dessinent vont influencer fortement la santé à l'âge adulte. Notre objectif était de caractériser l'état nutritionnel d'une population d'adolescents algériens.

Matériel et méthodes : Une étude descriptive et transversale a été réalisée dans trois établissements publics de la ville de Skikda au cours de l'année scolaire 2017/2018. La fiche d'enquête regroupait des renseignements généraux sur les élèves, leur anthropométrie, alimentation et activité physique. Le traitement des données a été réalisé avec le logiciel Epi-Info 6.04.

Résultats et Analyse statistique : L'étude a concerné 300 élèves (133 garçons, 167 filles). Leur moyenne d'âge était de $14,36 \pm 2,37$ ans (10-19ans) dont $5 \%$ ont déclaré fumer du tabac. Leur poids était de $49,13 \pm 13,74 \mathrm{~kg}$, leur taille de $1,57 \pm 0,13 \mathrm{~m}$ et leur indice de masse corporelle de $20,73 \pm 3,32 \mathrm{~kg} / \mathrm{m}^{2}$. La prévalence du surpoids (inclus l'obésité) était de $20,57 \%$. Aucune différence n'a été constatée par genre et selon le niveau d'instruction ( $p>0,05) .38,00 \%$ des adolescents avaient des antécédents familiaux de diabète, $19,33 \%$ de maladies respiratoires chroniques et $12,33 \%$ de maladies cardiovasculaires. $13,00 \%$ avaient un poids de naissance supérieur à $4000 \mathrm{~g}$. Dans notre étude le surpoids (obésité inclus) n'était pas significativement associé à la durée et au type d'allaitement maternel. La fréquence de consommation du groupe produits gras et sucrés était de 5,68 $3,14 \mathrm{fois} / \mathrm{jour}$. Les adolescents déclaraient pratiquer du sport une fois par semaine dans les établissements scolaires. En dehors de l'école, $24,66 \%$ faisaient du sport dans une structure adaptée et $43,33 \%$ en plein air. En semaine, les adolescents passaient en moyenne $8,5 \mathrm{~h}$ en sommeil, 6,79h à étudier, 1,33h à regarder la télévision, 0,50h à jouer à la console de jeu. En week-end, les adolescents passaient plus de temps au sommeil, devant un écran et à jouer $(p<0,05)$.

Conclusion : Il est important de maintenir un mode de vie sain dès le jeune âge, en subissant un dépistage périodique des maladies non transmissibles. La détection et la gestion des maladies à un stade précoce peuvent prévenir les complications et les décès prématurés.

Conflits d'intérêts : Aucun conflit à déclarer 


\section{INDEX DES AUTEURS}

JFN 2018 - Livre des résumes | Page 352 


\begin{tabular}{|c|c|c|}
\hline NOM & PRENOM & RESUME(S) $\mathbf{N}^{\circ}$ \\
\hline ABASSI & $\begin{array}{l}\text { Mohamed } \\
\text { Mehdi }\end{array}$ & P231 \\
\hline ABOUBACAR & Mohamed & CO18, C034, P116 \\
\hline ABOUSSALEH & Youssef & P006, P007 \\
\hline ACHAMRAH & Najate & $\begin{array}{l}\text { CO27, CO29, CO30, } \\
\text { CO32, P038, P059, } \\
\text { P060, P193 }\end{array}$ \\
\hline ACHIBANE & Fatimaazahra & P155, P216 \\
\hline ADAMO & Vincent & P177 \\
\hline ADJIBADE & Moufidath & $\begin{array}{l}\text { CO70, P207, P208, } \\
\text { P209, P213, P234 }\end{array}$ \\
\hline ADOUI & Faiza & P023 \\
\hline AGLI & Abdenacer & $\begin{array}{l}\text { P024, P142, P245, } \\
\text { P247, P248 }\end{array}$ \\
\hline AGUENAOU & Hassan & P100, P228, P238 \\
\hline AGUESSE & Audrey & P126 \\
\hline AINAD TABET & Soraya & P190 \\
\hline AIT & Sabrina & $\mathrm{CO} 38$ \\
\hline AIT BOUDAOUD & Amel & P138 \\
\hline AIT-BENALI & Sarah & P088 \\
\hline AKÉ & Michèle & P174 \\
\hline AKIR & Nicolas & P116 \\
\hline AKKOYUNLU & Sema & P253 \\
\hline ALACHAHER & Fatima Zohra & P076 \\
\hline ALBALADEJO & Laura & P211 \\
\hline ALBERT & Matthew & $\mathrm{COO1}$ \\
\hline ALBERTO & Jean-Marc & P188 \\
\hline ALLAM & Ouassila & P024, P142 \\
\hline ALLÈS & Benjamin & $\begin{array}{l}\text { CO61, P007, P148, } \\
\text { P208, P217, P229, } \\
\text { P240, P243, P244 }\end{array}$ \\
\hline AMAMOU & Asma & CO28, P195, P196 \\
\hline AMIOT-CARLIN & Marie-Josèphe & P229, P243, P244 \\
\hline AMMARIA & Behar & P072 \\
\hline AMRI & Ez-Zoubir & $\mathrm{CO} 73$ \\
\hline ANDRE & Perrine & P204 \\
\hline ANDRÉ & François & $\mathrm{CO} 17$ \\
\hline ANDREEVA & Valentina & P026, P251 \\
\hline ANDRÈS & Christian R & P105 \\
\hline ANDRES-LACUEVA & Cristina & P202 \\
\hline ANDRIANASOLO & Roland & C061, P209, P210 \\
\hline ANDRIEUX & Séverine & P152 \\
\hline ANOTA & Amélie & CO14 \\
\hline ANTOINE & Valery & $\mathrm{CO} 13$ \\
\hline APEL & Katharina & $\mathrm{CO} 64$ \\
\hline ARAUJO & Carla & P135 \\
\hline
\end{tabular}

\begin{tabular}{|c|c|c|}
\hline NOM & PRENOM & RESUME(S) $\mathbf{N}^{\circ}$ \\
\hline ARMAND & Martine & CO54, P090, P182 \\
\hline ARNAL & Manon & CO31, P049 \\
\hline AROULANDOM & Joseph & P183 \\
\hline ARTEMOVA & Svetlana & P202 \\
\hline ARVANITAKIS & Marianna & P107 \\
\hline ASSAD-BUSTILLOS & Melissa & P001, P010 \\
\hline ASSMANN & Karen & $\begin{array}{l}\text { CO01, CO70, P207, } \\
\text { P208 }\end{array}$ \\
\hline ASTA & Sandrine & P124 \\
\hline ATKINSON & Fiona & P092 \\
\hline ATTALIN & Vincent & P052 \\
\hline ATTOUMANE & Rachmat & P222 \\
\hline AUBERTIN-LEHEUDRE & Mylène & P069, P083 \\
\hline AUBRY & Régis & CO14 \\
\hline AUDY & Julie & $\mathrm{CO} 44$ \\
\hline AYAD & N. & P101 \\
\hline AYED & Khadija & P036, P165 \\
\hline AYER & Audrey & $\mathrm{COO4}$ \\
\hline AZHAR & Saïda & $\mathrm{CO} 29, \mathrm{CO} 45, \mathrm{CO} 76$ \\
\hline AZZOUT-MARNICHE & Dalila & P094, P096, P157 \\
\hline BACHELERIE & Cécile & P203 \\
\hline BACHIRI & Zeineb & P115 \\
\hline BACHMANN & Patrick & P040, P061, P066 \\
\hline BADO & André & P191 \\
\hline BAGNOUD & Géraldine & P055, P106 \\
\hline BAHAR & Ammaria & P071 \\
\hline BAHLOULI & Wafa & CO28, P193, P194 \\
\hline BAILLY & Nathalie & P118 \\
\hline BAKKOUCHE & Salah Eddine & P105 \\
\hline BALAS & Laurence & $\mathrm{CO} 75$ \\
\hline BALINT & Brittany & $\mathrm{CO} 20$ \\
\hline BANOS & Anne & P135 \\
\hline BAPTISTE & Aurélie & P203 \\
\hline BARBET & Virginie & CO06 \\
\hline BARD & Jean Marie & P089 \\
\hline BAREILLE & Nathalie & $\mathrm{CO} 25$ \\
\hline BARIK & Aicha & P131 \\
\hline BARNOUD & Didier & $\mathrm{CO} 37, \mathrm{CO} 38, \mathrm{CO} 66$ \\
\hline BARONCINI & Marc & COO8 \\
\hline BARSAM & Elise & P050 \\
\hline BATAILLE & Julie & P063 \\
\hline BAUDRY & Charlotte & P185 \\
\hline BAUDRY & Julia & P007, P148, P240 \\
\hline BEAUDEAU & François & $\mathrm{CO} 25$ \\
\hline
\end{tabular}




\begin{tabular}{|c|c|c|c|c|c|}
\hline NOM & PRENOM & RESUME(S) $\mathbf{N}^{\circ}$ & NOM & PRENOM & RESUME(S) $\mathbf{N}^{\circ}$ \\
\hline $\mathrm{BECHE}$ & Cindy & P167 & BERTRAND & Nicolas & $\mathrm{CO} 23$ \\
\hline BEHAR & Ammaria & P073, P130 & BERTRAND & Valérie & P181 \\
\hline BEKHTAOUI & Meriem & P077 & BERZOU & Sadia & P012 \\
\hline BELHADJ & Moussa & P072, P130 & BESNARD & Philippe & $\mathrm{CO} 04, \mathrm{CO} 07, \mathrm{P} 166$ \\
\hline BELHOUARI & Abderahman & P215 & BIESSY & Carine & CO09 \\
\hline BÉLIARD & Sophie & P200 & BILLIAUWS & Lore & CO52, P063 \\
\hline BELLAHRECHE & Zineb & P140 & BINDELS & Laure & CO02, P187 \\
\hline BELLISLE & France & P032 & BLACHIER & François & CO05, P099 \\
\hline BELMONTE & Liliana & $\begin{array}{l}\text { CO29, CO27, CO32, } \\
\text { P194 }\end{array}$ & $\begin{array}{l}\text { BLACHNIO- } \\
\text { ZABIELSKA4 }\end{array}$ & Agnieszka & P146 \\
\hline BELTRAN & Stéphane & P105 & BLAIS & Anne & P094, P099, P157 \\
\hline BEN GHARBIA & Houda & P231 & BLANCHARD & Anne & CO43 \\
\hline BEN MEHEL & Benakriche & P201 & BLANCHARD & Claire & CO04 \\
\hline BEN NACEUR & Khadija & P087 & BLASCO & Hélène & P105 \\
\hline BEN OTHMAN & Rym & P098 & BLAT & Sophie & P185 \\
\hline BEN SALAH & Elyes & P134 & BLOT & Adeline & $\mathrm{CO} 42, \mathrm{CO} 55$ \\
\hline BEN YOUSSEF & Sarra & P013 & BLUTEAU & Alice & CO17 \\
\hline BENABBAS & Youcef & P252 & BOBIN-DUBIGEON & Christine & P089 \\
\hline BENADOUDA & Soraya & P186 & BOEHM & Vanessa & CO52 \\
\hline BENAHMED & Ahmed & P124 & BOIDIN-CESCHINI & Laetitia & P097 \\
\hline BENAICHETA & Nora & P078 & & & $\mathrm{CO} 33, \mathrm{CO} 42, \mathrm{PO} 85$ \\
\hline BENAKRICHE & Ben Mehel & P192 & BOIRIE & Yves & $\begin{array}{l}\text { P121, P151, P153, } \\
\text { P158, P161 }\end{array}$ \\
\hline BENALIOUA & Najwa & P043, P156 & BOIS & Corinne & CO47 \\
\hline BENAZZOUG & Yasmina & P088 & BOISSEAU & Nathalie & P161 \\
\hline BENCHARIF & Meriem & P022, P252, P254 & BÔLE-FEYSOT & Christine & CO46, P193, P196 \\
\hline \begin{tabular}{|l} 
BENKARA \\
BENKIRANE
\end{tabular} & \begin{tabular}{|l|} 
Yassine \\
Hasnae
\end{tabular} & \begin{tabular}{|l|} 
P041 \\
P100
\end{tabular} & BONAFOS & Béatrice & $\begin{array}{l}\text { CO75, P144, P145, } \\
\text { P146 }\end{array}$ \\
\hline BENLEBNA & Melha & CO75 & BONAL & Véronique & P063 \\
\hline BENLIAN & Pascale & P097 & BONGARD & Vanina & P091 \\
\hline BENSLAMA & Yasmine & P071, P072, P073 & BONHOMMO & Sandrine & P049 \\
\hline BENSMAIL & Omar & P222 & BONNABRY & Pascal & P177 \\
\hline BENTALEB & Maroua & P022 & BOREL & Jean-Christian & P202 \\
\hline BERGEAT & Damien & P198 & BOREL & Patrick & CO17 \\
\hline BERGER & Mette & P055, P106 & BORG & Christophe & P109 \\
\hline BERGEROT & Damien & CO43 & BOROS & Léa & P027 \\
\hline BERGOIN & Charlotte & $\mathrm{CO} 38$ & BOROT & Sophie & P110 \\
\hline BERHAIEM & Sami & P069 & BOSSON & Jean-Luc & P211 \\
\hline BERNARD & Arnaud & CO04, CO07, P166 & BOTTINI & Silvia & CO60 \\
\hline BERNARD & Jonathan & CO54, P090, P182 & BOTZ & Alexandra & P051 \\
\hline BERNARD & Monique & P182 & BOUCHARD & Philippe & P251 \\
\hline BERRICHE & Olfa & P035, P098 & BOUCHEMAL & Nadia & CO01, P206 \\
\hline BERTIN & Eric & P147 & BOUCHENAK & Malika & P186 \\
\hline BERTOLINO & Virginie & P137 & BOUCHOUD & Lucie & P177 \\
\hline BERTRAND & Monelle & P114 & BOUDERBA & Saida & \begin{tabular}{|l|} 
P143 \\
\end{tabular} \\
\hline
\end{tabular}




\begin{tabular}{|c|c|c|c|c|c|}
\hline NOM & PRENOM & RESUME(S) $\mathbf{N}^{\circ}$ & NOM & PRENOM & RESUME(S) No \\
\hline BOUDERBALA & Sherazede & P075, P077 & BRONOWICKI & Jean-Pierre & P188 \\
\hline BOUDJEMA & Karim & P168 & BROS & Pauline & P204 \\
\hline BOUDJOUADA & Esma & P252 & BROUSSAUDIER & Alice & P084 \\
\hline BOUDRY & Gaëlle & P185 & BRUGAILLÈRES & Pauline & P176 \\
\hline BOUËTTÉ & Gwenhaël & CO64 & BRULE & Marine & CO57 \\
\hline BOUGHELLOUT & Halima & \begin{tabular}{|l|} 
P023 \\
\end{tabular} & BRUN & Jean-Frédéric & P069, P074 \\
\hline BOUHALLAB & Saïd & $\mathrm{CO} 23$ & BUCHE-FOISSY & Cécile & P086 \\
\hline BOUILLOT & Jean Luc & CO03 & BUFFIÈRE & Caroline & $\mathrm{CO} 15$ \\
\hline BOUKAÏBA & Emilie & P033 & BUGHIN & François & P074 \\
\hline BOUKHAYATIA & Fatma & P035, P087, P098 & BUISSON & Charline & P187 \\
\hline BOUKORTT & Farida & P078 & BUJAN & L. & $\mathrm{P} 113$ \\
\hline BOUKOUFA & Abdelhamid & P068 & BURGEVIN & Marion & P103 \\
\hline BOULAHSSASS & Rabia & CO13 & BURGHELEA & Alexandra & P189 \\
\hline BOULET-CRAIG & Aubrée & P112 & BURNAT & Catherine & P050 \\
\hline BOULETE & Illona & CO46 & BUSCAIL & Camille & CO01, P217, P225, \\
\hline BOULEUC & Carole & CO14 & & & \\
\hline BOULGHITI & M. & P101 & BUZZO & Solange & P066 \\
\hline BOUMAIZA & Jihene & P165 & CACÉRĖS & Francis & $\mathrm{CO} 16$ \\
\hline BOUNEDJAR & Adda & P115 & CAHU & Armelle & P198 \\
\hline BOUR & Abdellatif & P132, P222 & CAILLAUD & Denis & $\mathrm{CO} 33$ \\
\hline BOUREGHDA & Imad & P186 & CAILLAUD & Laura & P021 \\
\hline BOURRON & Olivier & P134 & CAILLET & Philippe & $\mathrm{CO} 13$ \\
\hline BOUSBAHI & Soumia & P201 & CALABRESE & Anastasia & P200 \\
\hline BOUTATA & Fatima Zohra & P254 & CALDEFIE-CHEZET & Florence & P127 \\
\hline BOUTELOUP & Corinne & CO37 & CALVEZ & Julian & P099 \\
\hline BOUTINON & Amandine & P021 & CALVEZ & Juliane & CO16, P096 \\
\hline BOUTRON & Isabelle & P225 & CAMIER & Aurore & CO47, P011 \\
\hline BOUVART & Laura & P179 & CAMPILLO-GIMENEZ & Boris & P136 \\
\hline BOUZIANE-NEDJADI & Karim & P186 & CANI & Patrice & $\mathrm{CO} 2$ \\
\hline BOUZIANI & Amina & P100 & CANO & Noël & CO65 \\
\hline BOYER & Constance & P227 & CANON & Francis & $\mathrm{CO} 57$ \\
\hline BRAC DE LA PERRIÈRE & Clémence & P136 & CAPDUPUY & Claudie & P135 \\
\hline BRACK & Olivier & P092 & CAPEL & Frédéric & P091, P121 \\
\hline BRAMI & Gilles & P093 & CAPELLE & Julie & P122, P169 \\
\hline BRANCHU & Julie & P086 & CARAYON & Aude & P121 \\
\hline BRAUD & Pierre-Louis & P237 & CARBONNELLE & Delphine & P189 \\
\hline BREDA & João & P221 & CARDENOUX & Charlotte & P158 \\
\hline BREIL & Béatrice & P086 & CARETTE & Claire & CO43, P138 \\
\hline BRETON & Jonathan & $\mathrm{CO} 27$ & CARIOU & Bertrand & $\mathrm{CO} 4$ \\
\hline BREUILLARD & Charlotte & P120, P129 & CARIS-VEYRAT & Catherine & $\mathrm{CO} 15$ \\
\hline BREYTON & Anne-Esther & CO40 & CARLIN & Gabrielle & P170 \\
\hline BRINDISI & Marie-Claude & P160 & CARRA & Maria Clotilde & P251 \\
\hline BRISSARD & Léa & CO78 & CARRARD & Isabelle & P212 \\
\hline & & & CARTIER & Christel & $\mathrm{CO} 53$ \\
\hline
\end{tabular}




\begin{tabular}{|c|c|c|c|c|c|}
\hline NOM & PRENOM & RESUME(S) $\mathbf{N}^{\circ}$ & NOM & PRENOM & RESUME(S) $\mathbf{N}^{\circ}$ \\
\hline CARTON & Thomas & P089 & CHAZELAS & Eloi & CO61, P224 \\
\hline CART-TANNEUR & Marie & P237 & CHEHIMI & Marwa & CO77 \\
\hline CARUGHI & Arianna & P032 & CHEMINAT & Marie & $\mathrm{CO} 47$ \\
\hline CARVALHO & Livia & P083 & CHERRAD & Hayet & P075, P077 \\
\hline CASAGRANDE & Corinne & CO09 & \multirow{2}{*}{ CHESNEAU } & \multirow{2}{*}{ Guillaume } & \multirow{2}{*}{$\begin{array}{l}\mathrm{CO} 21, \mathrm{CO} 22, \mathrm{CO} 25, \\
\mathrm{CO} 26, \mathrm{P} 018\end{array}$} \\
\hline CASANOVA & Nathalie & $\mathrm{COO7}$ & & & \\
\hline CASAS & François & $\mathrm{CO} 75$ & CHIHA & Fouad & P041 \\
\hline CASAS-AGUSTENCH & Patricia & P202 & CHOISET & Yvan & P010 \\
\hline CASPAR-BAUGUIL & S. & P113 & CHRISTMANN & Daniel & P057 \\
\hline CASTAGNA & Eloïse & P030 & CHRISTODOULOU & Aris & P002 \\
\hline CASTELLANO & Blandine & P126 & CLEMENT & Karine & $\mathrm{CO} 39$ \\
\hline CASTETBON & Katia & CO63, P241 & CLOUZEAU & Haude & P064, P065 \\
\hline CASTRONOVO & Vincenzo & $\mathrm{CO} 62$ & COËFFIER & Moïse & $\begin{array}{l}\mathrm{CO} 27, \mathrm{CO} 28, \mathrm{CO} 30, \\
\text { P193, P194 }\end{array}$ \\
\hline CATHELINE & Daniel & CO74, P249 & COLIN & Michel & P019, P021 \\
\hline CATHELINE & Gwénaëlle & $\mathrm{CO} 71$ & COLLET & Xavier & $\mathrm{CO} 04, \mathrm{CO} 07, \mathrm{CO} 17$ \\
\hline CAZANAVE & Aude & $\mathrm{CO} 53$ & COLOMBET & Zoé & P229, P243, P244 \\
\hline CAZAUX & Diane & P110 & COLSON & Cecilia & $\mathrm{CO} 73$ \\
\hline CELA & Jérémy & $\mathrm{P} 212$ & COMBE & Emmanuel & CO55 \\
\hline CENICEROS ROZALEN & Isabel & $\mathrm{CO} 65$ & COMÉRA & Christine & $\mathrm{CO} 53$ \\
\hline CENTENO & Delphine & P220 & CONSTANT & Aymery & P162 \\
\hline CHABANET & Claire & P160 & COOPMAN & Stéphanie & CO51 \\
\hline CHABUT & Claire & P044 & CORCIA & Philippe & P105 \\
\hline CHACON & Raphaël & P172 & CORNUÉJOLS & Antoine & P253 \\
\hline CHAH WAKILIAN & Anne & $\mathrm{CO} 13$ & COSTA & David & P164 \\
\hline CHALLINE & Alexandre & P138 & COSTES & Frédéric & $\mathrm{CO} 33$ \\
\hline CHALTIEL & Dan & P213, P214, P234 & COUDERC & Anne-Laure & $\mathrm{CO} 13$ \\
\hline CHAMBARON & Stéphanie & P025, P160 & COUDRAY & Charles & CO75, P144, P145, \\
\hline CHAMBON & Christophe & P009 & & & P146 \\
\hline CHAMBRIER & Cécile & $\mathrm{CO} 38$ & COUÉ & Marine & P126 \\
\hline CHANTEREAU & Hélène & $\mathrm{COO3}$ & $\begin{array}{l}\text { COUGNARD- } \\
\text { GRÉGOIRE }\end{array}$ & Audrey & $\mathrm{CO} 72$ \\
\hline CHAOUAD & Billel & P104 & COULON-BIDET & Elodie & P081 \\
\hline CHAPEAU & Anne-Laure & $\mathrm{CO} 23$ & COUPAYE & Muriel & $\mathrm{COO7}$ \\
\hline CHAPRON & Morgane & P016 & COURATIER & Philippe & $\mathrm{CO} 36$ \\
\hline CHARCHOUR & $\operatorname{Rim}$ & \multirow{3}{*}{$\begin{array}{l}\text { CO47, CO48, CO54, } \\
\text { P011, P090, P173, } \\
\text { P182 }\end{array}$} & COURTEIX & Daniel & P161 \\
\hline \multirow[t]{2}{*}{ CHARLES } & \multirow[t]{2}{*}{ Marie-Aline } & & COUSTOLS-VALAT & Magali & P114 \\
\hline & & & COUTURIER & Karine & P129 \\
\hline CHARRIÈRE & Mélanie & P055, P106 & CRISTOL & Laurence & $\mathrm{CO} 13$ \\
\hline CHATEAU & Yann & P123 & CROHIN & Gaetan & P118 \\
\hline CHATEL & Jean-Marc & P082 & CROSETTO & Paolo & P218 \\
\hline CHATELLIER & Vincent & $\mathrm{CO} 25$ & CYNOBER & Luc & $\mathrm{CO} 18, \mathrm{CO} 34, \mathrm{P} 102$, \\
\hline CHAUMEL & Florence & P050 & & & P116 \\
\hline CHAUMONTET & Catherine & P094, P157, P170 & CZERNICHOW & Sébastien & CO43, P138 \\
\hline CHAUVIN & Alain & P168 & DA COSTA & Anais & $\mathrm{CO} 10$ \\
\hline
\end{tabular}




\begin{tabular}{|c|c|c|}
\hline NOM & PRENOM & RESUME(S) $\mathbf{N}^{\circ}$ \\
\hline DACLAT & Rita & P128 \\
\hline DAGHER & Nour & P199 \\
\hline DAHMANI & Yasmina & P140 \\
\hline DAILEY-CHWALIBÓG & Trenton & $\mathrm{CO} 49, \mathrm{CO} 50$ \\
\hline DALI SAHI & Majda & P205 \\
\hline DALI TEMALA & Ghada & P013 \\
\hline DALI-SAHI & Majda & $\begin{array}{l}\text { P047, P048, P058, } \\
\text { P067, P071, P073, } \\
\text { P072, P130 }\end{array}$ \\
\hline DALLE & Céline & P220 \\
\hline DALMASSO & Luc & P169 \\
\hline D'ALMEIDA & Tania & P218 \\
\hline D'ALU & Michèle & P054 \\
\hline DAMADE & Richard & P199 \\
\hline DAMELINCOURT & Jean-Luc & P029 \\
\hline DAMERON & Maël & P015 \\
\hline DANCER & Paul & P202 \\
\hline DANEL & Nicolas & CO37 \\
\hline DANIEL & Noëmie & CO44 \\
\hline DANOUSSOU & Divya & P138 \\
\hline DARCEL & Nicolas & $\begin{array}{l}\text { P027, P031, P170, } \\
\text { P253 }\end{array}$ \\
\hline DARDENNE & $\mathrm{N}$. & CO62 \\
\hline DARDEVET & Dominique & CO19, P082 \\
\hline DARMAUN & Dominique & P184 \\
\hline DARMON & Nicole & $\begin{array}{l}\text { P008, P233, P243, } \\
\text { P244 }\end{array}$ \\
\hline \begin{tabular}{|l|} 
DARTHOU- \\
POUCHARD \\
\end{tabular} & Lucile & P203 \\
\hline DASTUGUE & Aurélie & CO04, P166 \\
\hline DAUFFY & Sandra & P053 \\
\hline DAUW & Gaëlle & P056 \\
\hline DAVID & Jérémie & CO19, P082 \\
\hline DAVIDENKO & Olga & P027, P031 \\
\hline DAVIDOU & Sylvie & P002 \\
\hline DAVILA & Anne-Marie & P170 \\
\hline DAVISSE-PATURET & Camille & CO48, P011 \\
\hline DAYOT & Françoise & P053 \\
\hline DE AGOSTINI & Maria & CO54, P090 \\
\hline DE AVEZEDO & Marcela & P082 \\
\hline DE BANDT & David & CO43 \\
\hline DE BANDT & Jean Pascal & \begin{tabular}{|l}
$\mathrm{CO} 18, \mathrm{CO} 34, \mathrm{P} 102$, \\
P116
\end{tabular} \\
\hline DE BRAUER & Camille & \begin{tabular}{|l|} 
P199 \\
\end{tabular} \\
\hline DE DECKER & Laure & CO13 \\
\hline DE FLINES & Jenny & P117 \\
\hline
\end{tabular}

\begin{tabular}{|c|c|c|}
\hline NOM & PRENOM & RESUME(S) No \\
\hline DE GAVELLE & Erwan & P014, P031, P236 \\
\hline DE LA FARGE & Florence & $\mathrm{CO53}$ \\
\hline $\begin{array}{l}\text { DE LAUZON- } \\
\text { GUILLAIN }\end{array}$ & Blandine & $\begin{array}{l}\text { CO47, CO48, P011, } \\
\text { P173 }\end{array}$ \\
\hline DE LUCA & Arnaud & CO37, P179 \\
\hline DE MAGALHAES & Simon & P203 \\
\hline DE MAHIEU & Magali & P118 \\
\hline DE OLIVEIRA MOTA & Juliana & P226 \\
\hline DE PLACE & Alice & $\mathrm{CO53}$ \\
\hline DE SAINT-VINCENT & Sarah & P121 \\
\hline DEBOSSU & Nadine & P147 \\
\hline DÉCHELOTTE & Pierre & $\begin{array}{l}\mathrm{CO} 27 \mathrm{CO} 28, \mathrm{CO} 29, \\
\mathrm{CO} 30, \mathrm{CO} 32, \mathrm{CO} 45, \\
\mathrm{CO} 46, \mathrm{P} 159, \mathrm{P} 181, \\
\mathrm{P} 193, \mathrm{P} 194, \mathrm{P} 197, \\
\mathrm{P} 235\end{array}$ \\
\hline DEFOORT & Catherine & CO17 \\
\hline DEGÉE & S. & $\mathrm{CO} 62$ \\
\hline DEGLAIRE & Amélie & P172, P185 \\
\hline DEHAK & Rabha & CO51 \\
\hline DEHBI & Fatima & P111 \\
\hline DEKAJ & Saranda & P051 \\
\hline DELAERE & Fabien & P253 \\
\hline DELAMARRE & Josselin & P168 \\
\hline DELARUE & Jacques & P019, P021 \\
\hline DELARUE & Julien & P020, P031, P033 \\
\hline DELAY & Julie & $\mathrm{CO} 29, \mathrm{CO} 32$ \\
\hline DELCOURT & Cécile & $\mathrm{CO} 71, \mathrm{CO} 72$ \\
\hline DELLA VALLE & Guy & P001 \\
\hline DELLA-VALLE & Guy & P010 \\
\hline DELRIEU & Lidia & P040 \\
\hline DELSOGLIO & Marta & P038, P059, P060 \\
\hline DELTEIL & Corine & P170 \\
\hline DELYFER & Marie-Noëlle & $\mathrm{CO} 72$ \\
\hline DELZENNE & Nathalie & CO02, P187 \\
\hline DEMERS-MATHIEU & Véronique & $\mathrm{CO} 44$ \\
\hline DEMIDEM & Aicha & P206, P220 \\
\hline DEMONTEIL & Lauriane & P175 \\
\hline D'ENGREMONT & Christelle & P109, P110 \\
\hline DENIZEAU & Mirrdyn & P016 \\
\hline DENNOUNI-MEDJATI & Nouria & \begin{tabular}{|l|} 
P067, P072, P073, \\
P047, P130, P205
\end{tabular} \\
\hline DEPEINT & Flore & P086 \\
\hline DEPEZAY & Laurence & P030 \\
\hline DEPOUX & Stéphanie & CO31 \\
\hline
\end{tabular}




\begin{tabular}{|c|c|c|c|c|c|}
\hline NOM & PRENOM & RESUME(S) $\mathbf{N}^{\circ}$ & NOM & PRENOM & RESUME(S) $N^{\circ}$ \\
\hline DEQUANTER & Didier & P131 & DOS REIS & Charlene & P057 \\
\hline DERBOUZ & Zoubir & P115 & DOUGKAS & Anestis & P246 \\
\hline \multirow{2}{*}{ DEROISSART } & \multirow{2}{*}{ Camille } & \multirow{2}{*}{$\begin{array}{l}\text { CO29, CO45, CO76, } \\
\text { P197 }\end{array}$} & DOUSSET & Bertrand & P138 \\
\hline & & & DRAI & Jocelyne & $\mathrm{CO55}$ \\
\hline DEROUICHE & Abdelfettah & $\begin{array}{l}\text { P043, P155, P156, } \\
\text { P215, P216 }\end{array}$ & DRAPEAU & Vicky & P151 \\
\hline DESBLÈS & Chloé & P158 & DRAPER & John & P202 \\
\hline DESBORDES & Florine & P084 & DROGUÉ & Sophie & P229 \\
\hline DESCHAMP & Chantal & P015 & DROUIN & Gaetan & $\mathrm{CO} 74$ \\
\hline DESCHAMPS & Valérie & P214, P213, P224 & DROUIN & Simon & P112 \\
\hline \multirow{2}{*}{ DESCHASAUX } & \multirow{2}{*}{ Mélanie } & \multirow{2}{*}{\begin{tabular}{|l|} 
CO01, CO09, CO61, \\
P206, P220, P224 \\
\end{tabular}} & DRUESNE-PECOLLO & Nathalie & P026, P209, P224 \\
\hline & & & DUBAIL & Iharilalao & $\mathrm{CO} 18$ \\
\hline DESEURE & Fiona & P097 & DUBERN & Béatrice & CO03, CO39, P178 \\
\hline DESGORCES & François & P041 & DUBOURG & Laurence & $\mathrm{CO} 38$ \\
\hline DESHAYES & Chloé & P232 & DUCLOS & Cedric & P135 \\
\hline DESMARCHELIER & Charles & $\mathrm{CO} 17$ & DUCLOS & Martine & P042, P151 \\
\hline DESMAZIÈRES & Zoé & $\mathrm{CO} 34$ & DUCROT & Pauline & P225 \\
\hline DESMETZ & Elisa & P224 & DUFFY & Darragh & $\mathrm{COO1}$ \\
\hline \multirow{2}{*}{ DESPORT } & \multirow{2}{*}{ Jean Claude } & \multirow{2}{*}{$\begin{array}{l}\text { CO31, CO36, CO66, } \\
\text { P049, P084, P141, } \\
\text { P203 }\end{array}$} & DUFOURG & Marie-Noëlle & $\mathrm{CO} 47$ \\
\hline & & & DULAC & Maude & P083 \\
\hline DETZEN & Laurent & P251 & DUMOULIN & Gilles & P163 \\
\hline DEVIOT & Xavier & P044 & DUPERTUIS & Yves & P059, P060 \\
\hline DEVOILLE & Laurent & $\mathrm{CO} 53$ & DUPONT & Didier & CO15, P172, P185 \\
\hline DEVROEY & Marianne & P119 & DUPONT-ROUSSEL & Jeanine & P200 \\
\hline DEWEZ & France & P124 & DURAND & Annie & P187 \\
\hline DHAUSSY & Amélie & $\mathrm{COO4,CO07}$ & DURAND & Jean Philippe & P102 \\
\hline DI PALMA & Mario & $\mathrm{CO} 11, \mathrm{CO} 12$ & DURAND & Stéphanie & P220 \\
\hline DIAB-ASSAF & Mona & P189 & \begin{tabular}{|l|} 
DURAND \\
\end{tabular} & Thierry & CO75, P146 \\
\hline DIBI & Sylvain & P174 & DUSOULIER & Marion & P175 \\
\hline DIDA & Nawal & P186 & ECKERT & Philippe & P055, P106 \\
\hline DIDA-TALEB & Nawal & P012 & EDDE & Manon & $\mathrm{CO} 71$ \\
\hline DIEBOLD & Frédéric & CO37, P052, P137 & EDELINE & Julien & P136 \\
\hline DIJOUX & Jason & P159 & EGNELL & Manon & $\begin{array}{l}\text { P148, P210, P218, } \\
\text { P219, P221, P225 }\end{array}$ \\
\hline DIMET & Jérôme & P081 & EISENDRATH & Pierre & P131 \\
\hline DINAR & Aurélie & $\mathrm{CO} 16$ & EL ANSARI & Nawal & P034, P080 \\
\hline DIOLOGENT & Elodie & P159 & EL ARABI & Fatine & P222 \\
\hline DISSE & Emmanuel & $\mathrm{CO} 40, \mathrm{CO} 42$ & EL ARBAOUI & Maria & P155 \\
\hline DJEMAI & Haidar & P041 & EL ATI & Jalila & \begin{tabular}{|l|} 
P095, P231 \\
\end{tabular} \\
\hline DO REGO & Jean-Claude & $\begin{array}{l}\text { CO28, CO45, CO46, } \\
\text { CO76, P193, P194 }\end{array}$ & EL ATI-HELLAL & Myriam & P095, P231 \\
\hline DO REGO & Jean-Luc & $\mathrm{CO} 27$ & EL BAKKOURI & Jalila & P111 \\
\hline DOGGUI & Radhouene & P095, P231 & EL HAJJ & Aya & P239 \\
\hline & & CO45, CO46, CO76, & EL HAJJ BOUTROS & Guy & P083 \\
\hline DOMINIQUE & Manon & P197 & EL HAMDOUCHI & Asmaa & P100, P228, P238 \\
\hline DONNEAU & A.-F. & $\mathrm{CO} 62$ & EL KARI & Khalid & P100, P228, P238 \\
\hline
\end{tabular}




\begin{tabular}{|c|c|c|c|c|c|}
\hline NOM & PRENOM & RESUME(S) No & NOM & PRENOM & RESUME(S) $\mathbf{N}^{\circ}$ \\
\hline EL MGHARI & Ghizlane & P034, P080 & FERRARI & Pietro & CO09 \\
\hline EL MZIBRI & Mohammed & P100, P228, P238 & FERRIERES & Jean & P091 \\
\hline EL OUARRADI & Nassiba & P034, P080 & FERVERS & Béatrice & P040 \\
\hline ELARBAOUI & Maria & P216 & FESSI & Sabrine & P251 \\
\hline EL-HAFAIA & Naouel & P116 & FETISSOV & Sergueï & $\mathrm{CO} 45$ \\
\hline ELIA & Josiane & P189 & FEUGIER & Nathalie & $\mathrm{CO} 42$ \\
\hline ELJAAFARI & Assia & $\mathrm{CO} 77$ & FÉZEU & Léopold & CO61, P234 \\
\hline \multirow{2}{*}{ ELKARDI } & \multirow{2}{*}{ Younes } & \multirow{2}{*}{$\begin{array}{l}\text { P043, P155, P156, } \\
\text { P215, P216 }\end{array}$} & FILLALI & Wasfi & P114 \\
\hline & & & FILLON & Alicia & P042, P151 \\
\hline ELLAHI & Basma & P216 & FINGAL & Chantal & P066 \\
\hline ENAUD & Raphael & P064, P065 & FIORLETTA & Ingrid & P066 \\
\hline ENNAIFER & Sonia & P165 & FLAMANT & Cyril & P184 \\
\hline ENNIGROU & Samir & P013 & FLEURIET & Ophélie & P137 \\
\hline ESSADIK & Rajaa & P043, P156, P230 & FLISS & Ismail & $\mathrm{CO} 44$ \\
\hline ESVAN & Maxime & CO64 & FLORENT & Vincent & CO08, P152 \\
\hline ETHEVE & Stéphane & $\mathrm{CO} 72$ & FOLLIARD & Sophie & P053 \\
\hline EVEN & Patrick & P094, P157 & FOLOPE & Vanessa & CO29, CO30, P159 \\
\hline FADEUR & Marjorie & P117 & FOLOPPE & Valérie & $\mathrm{CO} 32$ \\
\hline FADLI & Meriem & P101 & FONDANECHE & Sylvie & P050 \\
\hline FAIKA & Ben Mami & P087 & FONTAA & Vincent & P154 \\
\hline FALEWEE & Marie-Noëlle & P123 & FONTAINE & Eric & P045, P120 \\
\hline $\begin{array}{l}\text { FALEWÉE } \\
\text { FANTINO }\end{array}$ & $\begin{array}{l}\text { Juliette } \\
\text { Marc }\end{array}$ & $\begin{array}{l}\mathrm{P} 126 \\
\mathrm{P} 032\end{array}$ & FORHAN & Anne & $\begin{array}{l}\text { CO48, CO54, P090, } \\
\text { P182 }\end{array}$ \\
\hline $\begin{array}{l}\text { FANTINO } \\
\text { FARDELLONE }\end{array}$ & $\begin{array}{l}\text { Marc } \\
\text { Patrice }\end{array}$ & \begin{tabular}{|l|} 
P032 \\
P093
\end{tabular} & ЕО & Hólòn & $\mathrm{CO} 41, \mathrm{CO} 49, \mathrm{CO} 50$ \\
\hline FARDET & Anthony & P002 & FUUILLE I & Helene & P014, P031, P236 \\
\hline FARGES & Marie-Chantal & P127 & FOURATI & Salma & P134 \\
\hline FARIGON & Nicolas & CO33, CO42, P153 & FOURET & Gilles & $\begin{array}{l}\text { CO75, P144, P145, } \\
\text { P146 }\end{array}$ \\
\hline FARINA & Eddy & $\mathrm{P} 212$ & FOY & Gabrielle & P044 \\
\hline FAUTREL & Alain & $\mathrm{CO} 74$ & FRANCO & Anita & P112 \\
\hline FAVRE & Doris & P055, P106 & FRANDAS-CHAUTY & Anne & P184 \\
\hline FAVRE & Eva & P055, P106 & FRANK & Kelly & P002 \\
\hline FAYFMFNDY & Philinne & CO31, C036, CO66, & FREYSSENET & Damien & P040 \\
\hline theiviciod & 17mpse & P203 & GABORIT & Patrice & CO55 \\
\hline FÉART & Catherine & CO71, CO72, P227, & GAETA & Giuliano & P025 \\
\hline FEARI & Catherine & P204 & GAMET & Sulvie & CO75, P144, P145, \\
\hline FEBVEY-COMBES & Olivia & P040 & GAILLEI & & P146 \\
\hline FEENEY & MJ & P032 & GAIRE & Kévin & CO06 \\
\hline FEILLET-COUDRAY & Christine & $\begin{array}{l}\text { CO75, P144, P145, } \\
\text { P146 }\end{array}$ & & & $\begin{array}{l}\text { CO01, CO61, CO70, } \\
\text { P026, P206, P207, }\end{array}$ \\
\hline FEIN & Francine & P109 & GALAN & Pilar & P208, P209, P210, \\
\hline FELTIN & Nicolas & $\mathrm{CO53}$ & & & $\mathrm{P} 225, \mathrm{P} 234$ \\
\hline FERNANDEZ & Pauline & P030 & GALINIER & Anne & P114 \\
\hline FERNANE & Amirouche & \begin{tabular}{|l|l} 
P088 \\
\end{tabular} & GALMICHE & Marie & $\mathrm{CO} 29, \mathrm{CO} 32, \mathrm{P} 235$ \\
\hline FERON & Gilles & $\begin{array}{l}\text { CO57, P001, P010, } \\
\text { P028 }\end{array}$ & GANDY & Raphael & P171 \\
\hline
\end{tabular}




\begin{tabular}{|c|c|c|}
\hline NOM & PRENOM & RESUME(S) $\mathbf{N}^{\circ}$ \\
\hline GARABIGE & Valérie & P066 \\
\hline GARCIA-ESQUINAS & Esther & P204 \\
\hline GASCOIN & Géraldine & P184 \\
\hline GATÉ & Mathilde & CO30 \\
\hline GAUBIL-KALADJIAN & Isabelle & P147 \\
\hline GAUDE & Agathe & P045 \\
\hline GAUDFERNAU & Fleur & P011, P027 \\
\hline GAUDICHON & Claire & $\begin{array}{l}\text { CO05, CO16, P094, } \\
\text { P096, P157 }\end{array}$ \\
\hline GAUDIN & Clémentine & P189 \\
\hline GAUDRAT & Bulle & P152 \\
\hline GAUJOUX & Sébastien & P138 \\
\hline GAULTIER & Eric & CO53 \\
\hline GAUTIER & Yentl & P198 \\
\hline GAVLAK & Benoit & P147 \\
\hline GAY & Julie & P135 \\
\hline GAYRARD & Véronique & CO53 \\
\hline GAYRAUD & Guillaume & $\mathrm{CO} 10$ \\
\hline GEHRING & Joséphine & P240 \\
\hline GENDRE & Louis-Adrien & P153 \\
\hline GENIN & Pauline & P042 \\
\hline GENTES & Elodie & P153 \\
\hline GENTON & Laurence & P038 \\
\hline GEORGES & Julien & P168 \\
\hline GÉRARD & Philippe & P185, P201 \\
\hline GERAUD & Marion & P133 \\
\hline GERBAIX & Maude & P161 \\
\hline GERMAIN & Antoine & $\mathrm{CO} 26$ \\
\hline GERVAIS & Roselyne & P044 \\
\hline GESAN-GUIZIOU & Geneviève & CO55 \\
\hline GHENNAI & Anissa & P143 \\
\hline GHOUINI & Ahmed & P068, P101 \\
\hline GHOUL & Adel & P104 \\
\hline GHUKASYAN & Gevorg & CO74 \\
\hline GIALLOUROU & Natasa & $\mathrm{CO} 27$ \\
\hline GIBOREAU & Agnès & P246 \\
\hline GILLET & Valérie & P163 \\
\hline GILLOIS & Pierre & P211 \\
\hline GIRAL & Philippe & P128 \\
\hline GIRARD & Nicolas & P139 \\
\hline GIRARDET & Jean-Philippe & P178 \\
\hline GIRAUDET & Christophe & P121 \\
\hline GIRAUDET & Rémi & P027 \\
\hline GLEIZE & Béatrice & CO15 \\
\hline
\end{tabular}

\begin{tabular}{|c|c|c|}
\hline NOM & PRENOM & RESUME(S) $\mathbf{N}^{\circ}$ \\
\hline GODART & Nathalie & P062 \\
\hline GODET & Ambre & P249 \\
\hline GOEPP & Marie & P127 \\
\hline GOICHON & Alexis & $\begin{array}{l}\text { CO27, CO28, P193, } \\
\text { P194, P195 }\end{array}$ \\
\hline GOLDWASSER & François & CO11, CO12, P102 \\
\hline GOMEZ-CABRERO & David & P204 \\
\hline GONNEAU-LEJEUNE & Julie & $\mathrm{CO} 37$ \\
\hline GOSSERIES & Olivia & P117 \\
\hline GOTTRAND & Frédéric & CO51 \\
\hline GOUDABLE & Joelle & $\mathrm{CO} 42$ \\
\hline GOUJON & Géraldine & P050 \\
\hline GOUNOT & Daniel & P025 \\
\hline GOURC & Christophe & P046 \\
\hline GOURDY & Pierre & CO59 \\
\hline GOUX & Aurélie & CO40, P092 \\
\hline GRAF & Christophe & P038 \\
\hline GRANDIN & Flore & $\mathrm{CO53}$ \\
\hline GRANDJEAN & Valérie & $\mathrm{CO} 60$ \\
\hline GRAVELAT & Martine & $\mathrm{CO} 31$ \\
\hline GREIL & Annick & $\mathrm{CO} 33$ \\
\hline GREY & Dan & P171 \\
\hline GRIGIONI & Sébastien & $\begin{array}{l}\mathrm{CO} 29, \mathrm{CO} 30, \mathrm{CO} 32, \\
\text { P159 }\end{array}$ \\
\hline GRILLOT & Julienne & P109, P110 \\
\hline GRODARD & Ghislain & $\mathrm{CO} 14$ \\
\hline GRODSTEIN & Francine & CO67 \\
\hline GROSJEAN & Patrycja & P154 \\
\hline GRULET & Justine & P147 \\
\hline GUEANT & Jean-Louis & CO20, P188 \\
\hline GUEDJ & Anne-Marie & P164 \\
\hline GUENZET & Akila & P012 \\
\hline GUÉRIN & Charlène & $\begin{array}{l}\text { CO28, CO45, P193, } \\
\text { P195 }\end{array}$ \\
\hline GUÉRIN & Maryse & P128 \\
\hline GUÉRIN & Olivier & $\mathrm{CO} 35$ \\
\hline GUÉRIN-DEREMAUX & Laetitia & P096 \\
\hline GUESDON & Benjamin & $\mathrm{CO} 49, \mathrm{CO} 50$ \\
\hline GUESSOUS & Idris & P212 \\
\hline GUIBERT & Hélène & P141 \\
\hline GUIDAL & Valentin & P039 \\
\hline GUILLARD & Adèle & $\mathrm{CO} 53$ \\
\hline GUILLAUME & Benoît & P124 \\
\hline GUILLAUME & Michèle & CO62 \\
\hline GUILLET & Christelle & CO42, P121, P161 \\
\hline
\end{tabular}




\begin{tabular}{|c|c|c|}
\hline NOM & PRENOM & RESUME(S) $\mathbf{N}^{\circ}$ \\
\hline GUILLEVIC & Mathieu & CO26, P018 \\
\hline GUILLIN & Florence & P096 \\
\hline GUILLOCHEAU & Etienne & CO74, P249 \\
\hline GUILLOU & Hervé & CO59 \\
\hline GUILLOU & Sandrine & P226 \\
\hline GUIMBER & Dominique & C051, P179 \\
\hline GUINHUT & Marie & P062 \\
\hline GUITARD & Marjolaine & P203 \\
\hline GUNTER & Marc & CO09 \\
\hline GURIEC & Nathalie & P019 \\
\hline HADDI & Abir & P190 \\
\hline HADJ KADI & Amira & P186 \\
\hline HAIDER & Sahar & P239 \\
\hline HAMANI-MEDJAOUI & Imane & P149 \\
\hline HAMDI & S. & P113 \\
\hline HAMDI & Zeineb & P251 \\
\hline HAMON & Pascaline & $\mathrm{CO} 23$ \\
\hline HAMOUS & Amina & P186 \\
\hline HANACHI & Mouna & P062 \\
\hline HANF & Matthieu & P184 \\
\hline HANKARD & Regis & P179 \\
\hline HANNOUN & Zineb & P132, P222 \\
\hline HARB & Zeinab & P188 \\
\hline HAREK & Yahia & $\begin{array}{l}\text { P072, P073, P130, } \\
\text { P205 }\end{array}$ \\
\hline HARIVEL & Virginie & P171 \\
\hline HARNAY & Stéphanie & P015 \\
\hline HARRAQUI & Khouloud & P132, P222 \\
\hline HATIRA & Yousra & P087 \\
\hline HATRON & Pierre-Yves & P097 \\
\hline HATTE & Victor & P127 \\
\hline HAZARD & Diane & P159 \\
\hline HEBEL & Pascale & P232 \\
\hline HEBERT & Christophe & P123 \\
\hline HECQ & Jean-Daniel & P056 \\
\hline HEIDEGGER & Claudia-Paula & P059, P060 \\
\hline HEITZ & Damien & CO13 \\
\hline HEJBLUM & Boris & CO68, CO69 \\
\hline HELMER & Catherine & $\begin{array}{l}\text { C068, CO69, CO71, } \\
\text { P227 }\end{array}$ \\
\hline HEMERY & Youna & P016 \\
\hline
\end{tabular}

\begin{tabular}{|c|c|c|}
\hline NOM & PRENOM & RESUME(S) $\mathbf{N}^{\circ}$ \\
\hline HERCBERG & Serge & $\begin{array}{l}\text { CO01, CO09, CO61, } \\
\text { CO63, CO70, P007, } \\
\text { P026, P148, P206, } \\
\text { P207, P208, P209, } \\
\text { P210, P213, P214, } \\
\text { P217, P218, P219, } \\
\text { P220, P221, P224, } \\
\text { P225, P234, P240, } \\
\text { P241, P251 }\end{array}$ \\
\hline HERMIER & Dominique & CO41 \\
\hline HERRMANN & François & P038 \\
\hline HEUDE & Barbara & $\begin{array}{l}\text { CO48, CO54, P090, } \\
\text { P182 }\end{array}$ \\
\hline HEYD & Bruno & P109 \\
\hline HICHAMI & Aziz & $\mathrm{CO} 78$ \\
\hline HIEL & Sophie & COO2 \\
\hline HIOLLE & Manon & CO15 \\
\hline HOGE & Axelle & CO62 \\
\hline HOUDEAU & Eric & CO53 \\
\hline HOUNKPONOU & Hanane & P193 \\
\hline HOUTI & Leila & P149 \\
\hline HSAINI & Asmae & P079 \\
\hline HUBERT & Hervé & $\mathrm{CO} 35$ \\
\hline HUMBERT & Patricia & P147 \\
\hline HUNEAU & Jean-François & $\begin{array}{l}\text { CO41, CO49, CO50, } \\
\text { P014, P031, P236 }\end{array}$ \\
\hline HUVELIN & Jean-Michel & P189 \\
\hline HUYBRECHTS & Inge & COO9 \\
\hline ICARD-VERNIÈRE & Christèle & P017 \\
\hline IDRISSI & Mohamed & P100, P228, P238 \\
\hline ILLNER & Anne-Kathrin & P029, P086 \\
\hline INGUENEAU & Cécile & P113, P114 \\
\hline IPPOLITI & Camilla & P117 \\
\hline ISACCO & Laurie & P163 \\
\hline ISSANCHOU & Sylvie & P176 \\
\hline JAFRI & Ali & $\begin{array}{l}\text { P043, P111, P155, } \\
\text { P156, P215, P216, }\end{array}$ \\
\hline JALI & Eva & CO65 \\
\hline JALLADEAU & Clotilde & P203 \\
\hline JAMELEDDINE & Saloua & P036, P165 \\
\hline JAMOUSSI & Henda & P035, P098 \\
\hline JAN & Gwénaël & $\mathrm{CO} 24$ \\
\hline JARBEAU & Marine & CO28, P194, P195, \\
\hline JARRY & Anne-Charlotte & P191 \\
\hline JARZAGUET & Marianne & P082 \\
\hline JAVERLIAT & Valérie & P203 \\
\hline
\end{tabular}




\begin{tabular}{|c|c|c|c|c|c|}
\hline NOM & PRENOM & RESUME(S) $\mathbf{N}^{\circ}$ & NOM & PRENOM & RESUME(S) $\mathbf{N}^{\circ}$ \\
\hline JAZIRI & Narjess & P013 & \multirow{6}{*}{ KESSE-GUYOT } & \multirow{6}{*}{ Emmanuelle } & \multirow{6}{*}{$\begin{array}{l}\text { CO01, CO09, CO61, } \\
\text { CO70, P007, P148, } \\
\text { P206, P207, P208, } \\
\text { P209, P210, P213, } \\
\text { P214, P217, P218, } \\
\text { P220, P221, P224, } \\
\text { P225, P234, P240 }\end{array}$} \\
\hline JEANTET & Romain & P172 & & & \\
\hline JEGATHEESAN & Prasanthi & CO34, P116 & & & \\
\hline JELTSCH & Pauline & P028 & & & \\
\hline JENAB & Mazda & CO09 & & & \\
\hline \multirow{3}{*}{ JÉSUS } & \multirow{3}{*}{ Pierre } & \multirow{3}{*}{$\begin{array}{l}\text { CO31, CO36, CO66, } \\
\text { P049, P084, P105, } \\
\text { P141, P203 }\end{array}$} & & & \\
\hline & & & KETTANI & Anass & P043, P156, P230 \\
\hline & & & KHABBAZ & Lydia & P239 \\
\hline JEWELL & Jo & P221 & KHAMMASSI & Marwa & P151 \\
\hline JISSENDI & Patrice & COO8 & KHAN & Amira & CO78 \\
\hline JOFFRE & Florent & CO55 & KHAN & Naim Akhtar & CO78 \\
\hline JOLY & Francisca & CO52, P063, P191 & KHELFAT & K. & P101 \\
\hline JOLY & Lise & P045 & KHEROUA & Omar & P190, P192 \\
\hline JOUINOT & Anne & P102 & KHIARI & Marwa & P035, P087, P098 \\
\hline $\begin{array}{l}\text { JOUMARD- } \\
\text { CUBIZOLLES }\end{array}$ & Laurie & CO55 & \multirow[t]{2}{*}{ KHODOROVA } & \multirow[t]{2}{*}{ Nadezda } & \multirow{2}{*}{$\begin{array}{l}\text { CO16, CO05, CO41, } \\
\text { CO49, CO50, P094, } \\
\text { P096 }\end{array}$} \\
\hline JOUVE & Chrystèle & P091 & & & \\
\hline JOUVEAU & Sylvain & P084 & KOCEIR & El Hadj Ahmed & P143 \\
\hline JOUY & Nicolas & P144, P145 & $\mathrm{KOCH}$ & Stéphane & P109 \\
\hline JOVER & Bernard & P146 & KODDE & Andrea & P170 \\
\hline JOYEUX-FAURE & Marie & P202 & KONTARIS & Ioannis & P025 \\
\hline JUCHET-MARTIN & Mélanie & $\mathrm{CO} 18, \mathrm{CO} 34$ & KOROBELNIK & Jean-François & CO72 \\
\hline JUIN & Hervé & CO26 & KOUASSI & Karen-Aristide & P174 \\
\hline \multirow{5}{*}{ JULIA } & \multirow{5}{*}{ Chantal } & \multirow{5}{*}{$\begin{array}{l}\mathrm{CO} 01, \mathrm{CO} 09, \mathrm{CO} 61, \\
\text { P148, P207, P208, } \\
\text { P210, P213, P214, } \\
\text { P217, P218, P219, } \\
\text { P221, P224, P225, } \\
\text { P234, P240, P251 } \\
\end{array}$} & KOUBE & Ingrid & P119 \\
\hline & & & KRAJINOVIC & Maja & P112 \\
\hline & & & KRINITZKI & Sacha & P173 \\
\hline & & & KROUF & Djamil & P012 \\
\hline & & & KRUSEMAN & Maaike & P212 \\
\hline KACHECOUCHE & Youcef & P072 & KRZNARIC & Zeljko & CO65 \\
\hline KACHEKOUCHE & Youssouf & $\begin{array}{l}\text { P047, P048, P058, } \\
\text { P067 }\end{array}$ & KSIAZEK & Elea & P175 \\
\hline KADAWATHAGEDARA & Manik & CO47 & KSOURI & Riadh & P165 \\
\hline KAI & Joanna & P102 & KUPCZYK & Kinga & CO65 \\
\hline KAIDOMAR & Michel & P137 & LABOURÉ & Hélène & P028 \\
\hline KAPEL & Romain & CO16 & LACAZE & Laurence & CO65, P136, P168 \\
\hline KARELIS & Anthony & P069 & LACOMBE & Karine & P167 \\
\hline KAROLY & Edward & P091 & LACORTE & Jean-Marc & $\begin{array}{l}\text { CO39, CO43, P128, } \\
\text { P134 }\end{array}$ \\
\hline KARSEGARD & Véronique & P038 & LACOUR & Camille & P007 \\
\hline KARSENTY & Alexandra & $\mathrm{CO} 03, \mathrm{CO} 39$ & LACROSSE & Dominique & P054, P056 \\
\hline KASSA & N. & P101 & LAGROST & Laurent & CO04, CO07, P188 \\
\hline KATSAHIAN & Sandrine & P138 & LAGUERRE & Aurélie & P028 \\
\hline KELEVINA & Tatiana & P055, P106 & LAIRON & Denis & P007, P148 \\
\hline KELOUA & Safia & P178 & LAMARCHE & Frédéric & P120 \\
\hline KENNAB & Naima & P101 & LAMARRE & Adèle & $\mathrm{CO} 29, \mathrm{CO} 32$ \\
\hline KERHOAS & Nathalie & $\mathrm{CO} 21, \mathrm{CO} 22$ & LAMBERT & Céline & P158 \\
\hline
\end{tabular}




\begin{tabular}{|c|c|c|c|c|c|}
\hline NOM & PRENOM & RESUME(S) $\mathbf{N}^{\circ}$ & NOM & PRENOM & RESUME(S) $\mathbf{N}^{\circ}$ \\
\hline \multirow{2}{*}{ LAMBERT } & \multirow{2}{*}{ Grégory } & \multirow{2}{*}{\begin{tabular}{|l|} 
CO29, CO45, CO46, \\
P197, P235 \\
\end{tabular}} & LE MOIGNE & Amandine & P191 \\
\hline & & & LE MOYEC & Laurence & P206 \\
\hline $\begin{array}{l}\text { LAMBERT- } \\
\text { PORCHERON }\end{array}$ & Stéphanie & CO40, CO55 & LE ROUX & Linda & P172 \\
\hline LAMIREAU & Thierry & P064, P065 & LE ROY & Tiphaine & $\mathrm{CO} 2$ \\
\hline LAMPURÉ & Aurélie & CO63, P241 & LE RUYET & Pascale & P185 \\
\hline LAN & Annaïg & CO05 & LE SOLLIEC & Marie-Anne & $\mathrm{CO} 29, \mathrm{CO} 45, \mathrm{CO} 76$ \\
\hline LANCHAIS & Kassandra & P085 & LE VACON & Françoise & P089 \\
\hline LANDAIS & Edwige & P229, P243, P244 & LEBACQUER & Olivier & P121 \\
\hline LANGEVIN & Brigitte & P007 & LEBRAZI & Halima & P043, P156, P230 \\
\hline LANGLAIS & Sabrina & P045 & LEBRUN & Chantal & $\mathrm{CO} 17$ \\
\hline LANIEL & Julie & P112 & LECERF & Jean-Michel & P097 \\
\hline LANTHIER & Nicolas & $\mathrm{CO} 2$ & LECLERCQ & Sophie & $\mathrm{COO2}$ \\
\hline LANTZ & Olivier & COO1 & LECOINTRE & Aurélie & P203 \\
\hline LAOUALY & Ibrahim & P016 & LÉCUYER & Lucie & CO01, P206, P220 \\
\hline LARDINOIS & Mathilde & P054 & LEDOUX & Séverine & $\mathrm{CO} 4, \mathrm{CO} 07$ \\
\hline LARDJAM-HETRAF & Sarah & P149 & LEFEVRE-ARBOGAST & Sophie & CO68, CO69 \\
\hline LASCOUTS & Emilie & CO65 & LEFRANC-MILLOT & Catherine & P096 \\
\hline \multirow{2}{*}{ LATINO-MARTEL } & \multirow{2}{*}{ Paule } & \multirow{2}{*}{\begin{tabular}{|l|} 
CO01, CO09, P206, \\
P220, P224 \\
\end{tabular}} & LE-GOUEVEC & Francis & P168 \\
\hline & & & LEGRAND & Antoine & P124 \\
\hline LAUGERETTE & Fabienne & P187 & LEGRAND & Philippe & P249, $\mathrm{CO} 4$ \\
\hline LAUREYS & Steven & P117 & \multirow{2}{*}{ LEGRAND } & \multirow{2}{*}{ Romain } & \multirow{2}{*}{$\begin{array}{l}\text { CO29, CO45, CO46, } \\
\text { C076, P197 }\end{array}$} \\
\hline LAURIN & Emilie & CO44 & & & \\
\hline LAUTRETTE & Géraldine & $\mathrm{CO} 36$ & LÉKÉ & André & CO37, P180 \\
\hline LAUVERJAT & Madeleine & $\mathrm{CO} 38$ & \begin{tabular}{|l} 
LELANDAIS \\
\end{tabular} & Hélène & P159 \\
\hline LAVEFVE & Laura & P086 & LEMAIRE & Charlène & P153 \\
\hline LAVENAT & Marie & P164 & LEMAIRE & Marion & P185 \\
\hline LAVERDIÈRE & Caroline & P112 & LEMALE & Julie & P178, P183 \\
\hline LAVIGNE & Thierry & P057 & LEMIERE & Celine & P159 \\
\hline LAVILLE & Martine & $\mathrm{CO} 40, \mathrm{CO} 42, \mathrm{CO} 55$ & LEMOGNE & Cédric & P207, P208 \\
\hline LAZZATI & Andréa & P138 & LEMOINE & Anaïs & P183 \\
\hline LE BACQUER & Olivier & P085 & LEON & Fatima & $\mathrm{CO} 46$ \\
\hline LE BARZ & Mélanie & $\mathrm{CO} 44$ & LÉON & Fatima & $\mathrm{CO} 45, \mathrm{CO} 76$ \\
\hline LE BEYEC - LE BIHAN & Johanne & CO39, CO43, P191 & LÉPINE & Olivier & P126 \\
\hline LE FLAHEC & Catherine & CO31 & LEPOUSÉ & Doriane & P039 \\
\hline LE FOLL & Gaelle & P159 & LEROI & Thomas & P154 \\
\hline LE GALL & Maude & CO43, P191 & LEROY & Pascal & P014 \\
\hline LE GARF & Sébastien & P039, P150 & LERUYET & Pascale & $\mathrm{CO} 42$ \\
\hline LE GRAND & Jennifer & P063 & LESAICHOT & Quentin & P158 \\
\hline LE GUENNEC & Delphine & P127 & LESCUT & Dominique & CO37, CO51 \\
\hline LE HUËROU-LURON & Isabelle & P185 & LESPINE & Anne & $\mathrm{CO} 17$ \\
\hline LE MAY & Cédric & $\mathrm{CO} 4, \mathrm{CO} 07, \mathrm{CO} 17$ & LESSIRE & Michel & $\mathrm{CO} 26$ \\
\hline LE MENN & Gwenaelle & P150 & LEUILLET & Sebastien & P089 \\
\hline \multirow{2}{*}{ LE MINOUS } & \multirow{2}{*}{$\begin{array}{l}\text { Anne- } \\
\text { Emmanuelle }\end{array}$} & \multirow{2}{*}{ P015 } & LEURANGUER & Jérome & P015 \\
\hline & & & LÉVEILLÉ & Pauline & P112 \\
\hline
\end{tabular}




\begin{tabular}{|c|c|c|}
\hline NOM & PRENOM & RESUME(S) $\mathbf{N}^{\circ}$ \\
\hline LEVY & Emile & P112 \\
\hline LEY & Delphine & CO51 \\
\hline L'HUILLIER & Clément & CO28, P193, P194 \\
\hline LIENHARDT-ROUSSIE & Anne & P141 \\
\hline LIORET & Sandrine & CO47, P011, P173 \\
\hline LIPPÉ & Sarah & P112 \\
\hline LIROT & Corinne & P045 \\
\hline LLOYD & Amanda & P202 \\
\hline LOBINET & Emilie & P114 \\
\hline LOÏ & Cécile & P116 \\
\hline LOIZON & Emmanuelle & P187 \\
\hline LOPEZ-GARCIA & Esther & P204 \\
\hline LOUGDA & Sanaa & P155 \\
\hline LOUIS & Hubert & P107 \\
\hline LOUKDA & Sanae & P216 \\
\hline LOUVET & Camille & $\mathrm{CO} 42$ \\
\hline LOW-YANWEN & Dorrain & CO68 \\
\hline LUBRANO & Jean & P133 \\
\hline LUCAS & Nicolas & $\begin{array}{l}\text { CO29, } \mathrm{CO} 45, \mathrm{CO} 46 \\
\text { CO76, P197 }\end{array}$ \\
\hline LUSSON & Hélène & P053 \\
\hline LUU HUYEN & Trang & P089 \\
\hline LYAN & Bernard & P220 \\
\hline MADOUASSE & Aurélien & $\mathrm{CO} 25$ \\
\hline MAGASSA & Salimata & $\mathrm{CO} 18, \mathrm{CO} 34$ \\
\hline MAGNE & Gérard & CO31 \\
\hline MAHE & Pascal & P015 \\
\hline MAHJOUB & Faten & P035, P098 \\
\hline MAILLOT & Matthieu & P233 \\
\hline MAIRESSE & Guillaume & $\begin{array}{l}\mathrm{CO} 21, \mathrm{CO} 22, \mathrm{CO} 25 \\
\mathrm{CO} 26, \mathrm{P} 018,\end{array}$ \\
\hline MAKDAD & Meryem & P132 \\
\hline MAKHLOUF & Anne-Marie & CO65 \\
\hline MAKHLOUKI & Houria & P155, P215, P216 \\
\hline MALHERBE & Christian & P117 \\
\hline MALLEK & Aicha & P140 \\
\hline MALPUECH-BRUGÈRE & Corinne & CO55, P091 \\
\hline MANACH & Claudine & CO68, P220 \\
\hline MANFREDOTTI & Cristina & P253 \\
\hline MANTHA & Olivier & CO41 \\
\hline MAQUART & Guillaume & P166 \\
\hline MARANINCHI & Marie & P200 \\
\hline MARCANGELI & Vincent & P083 \\
\hline MARCHAL & Timothée & P139 \\
\hline
\end{tabular}

\begin{tabular}{|c|c|c|}
\hline NOM & PRENOM & RESUME(S) $\mathbf{N}^{\circ}$ \\
\hline \begin{tabular}{|l|} 
MARCHAL \\
BRESSENOT \\
\end{tabular} & Aude & P188 \\
\hline MARCIL & Valerie & P112 \\
\hline MARDUEL & Agnès & P175 \\
\hline MARES & Pierre & P164 \\
\hline MARESCHAL & Julie & P038 \\
\hline MARETTE & André & $\mathrm{CO} 44$ \\
\hline MARGARITIS & Irène & P003 \\
\hline MARGIER & Marielle & $\mathrm{CO} 17$ \\
\hline MARIN & Benoit & $\mathrm{CO} 36$ \\
\hline MARION & Oberli & P202 \\
\hline MARION-LETELLIER & Rachel & P193, P195, P196 \\
\hline MARIOTTI & François & $\begin{array}{l}\text { CO41, CO49, CO50, } \\
\text { P014, P031, P236 }\end{array}$ \\
\hline MARLIER & Luc & P025 \\
\hline MARMONIER & Corinne & P091, P227 \\
\hline MAROUAN & Taghzaoui & P238 \\
\hline MARQUES VIDAL & Pedro & P212 \\
\hline MARTIN & Agnès & P040 \\
\hline MARTIN & Boutiere & P120, P129 \\
\hline MARTIN & Chloé & $\mathrm{P} 215$ \\
\hline MARTIN & Christophe & $\cos$ \\
\hline MARTIN & Corinne & P050 \\
\hline MARTINEZ & Arianne & P055, P106 \\
\hline MARTIN-PRÉVEL & Yves & P229, P243, P244 \\
\hline MARTY & Michèle & $\mathrm{CO} 74$ \\
\hline MAS & Marine & P160 \\
\hline MASGRAU & Aurélie & $\mathrm{CO} 42$ \\
\hline MASSEBOEUF & Nathalie & P052, P137 \\
\hline MASSICARD & Mickael & P167 \\
\hline MASURIER & Julie & P151, P158 \\
\hline MATHE & Daniel & $\mathrm{CO} 31$ \\
\hline MATHÉ & Véronique & $\mathrm{CO} 41, \mathrm{CO} 49, \mathrm{CO} 50$ \\
\hline MATHIEU & Marie-Eve & P151 \\
\hline MATHILDE & Touvier & P220 \\
\hline MAURICE & Bastien & P020, P033 \\
\hline MAYER LACROSNIERE & Sophie & $\mathrm{CO} 03$ \\
\hline \begin{tabular}{|l|} 
MEDIENE- \\
BENCHEKOR
\end{tabular} & Sounnia & P149 \\
\hline MEDJATI-DENNOUNI & Noria & $\begin{array}{l}\text { P047, P048, P058, } \\
\text { P071 }\end{array}$ \\
\hline MEIGNAN & Thomas & $\mathrm{CO} 25$ \\
\hline MEINGAN & Philippe & $\mathrm{CO} 11$ \\
\hline MÉJEAN & Caroline & \begin{tabular}{|l|} 
CO61, CO63, P008, \\
P229, P241, P243, \\
P244
\end{tabular} \\
\hline
\end{tabular}




\begin{tabular}{|c|c|c|}
\hline NOM & PRENOM & RESUME(S) $\mathbf{N}^{\circ}$ \\
\hline MEKIRI & Sarah & CO05 \\
\hline MELCHIOR & Jean-Claude & P062 \\
\hline MELZI & \begin{tabular}{|l} 
Mohamed \\
Aimene \\
\end{tabular} & P115 \\
\hline MEMBRÉ & Jeanne-Marie & P226 \\
\hline MÉNARD & Olivia & P185 \\
\hline MENSI & Azza & P231 \\
\hline MENTAVERRI & Romuald & P093 \\
\hline MERAD BOUDIA & Hamza & P047, P048, P067 \\
\hline MERCIER & Jacques & P074 \\
\hline MERET & Caroline & P159 \\
\hline MERKT & Elise & P051 \\
\hline MERLE & Benedicte & CO72 \\
\hline MERLIN & Jean-François & P166 \\
\hline MERTENS & Cecile & $\mathrm{CO} 13$ \\
\hline MESTARI & Fouzi & P134 \\
\hline METZ & Lore & P042, P161 \\
\hline MEUGNIER & Emmanuelle & P187 \\
\hline MEUNIER & Jean-Pierre & CO35 \\
\hline MEUNIER & Nathalie & CO15 \\
\hline MEYER & Clara & P045 \\
\hline MEYNIER & Alexandra & $\mathrm{CO} 40$ \\
\hline MEZIANE & Abderahim & P047, P048 \\
\hline MEZIANE & Fatima Zahra & P071 \\
\hline MICHALSKI & Marie-Caroline & CO55, P187 \\
\hline MICHAUD & Laurent & Co51 \\
\hline MICHEAU & Pierre & CO68, P220 \\
\hline MICHEL & Cécile & P123 \\
\hline MICHEL & Céline & P054, P056 \\
\hline MICHELOTTI & Gregory & P091 \\
\hline MIGNOT & Coralie & P025 \\
\hline MIGUET & Maud & P151 \\
\hline MILARD & Marine & P187 \\
\hline MIOLANNE & Magalie & CO42, P153, P158 \\
\hline $\begin{array}{l}\text { MISHELLANY- } \\
\text { DUTOUR }\end{array}$ & Anne & CO42 \\
\hline MISSET & Blanche & P084 \\
\hline MIZOURI & Ramla & P035, P087, P098 \\
\hline MOHAMED & Belomaria & P006 \\
\hline MOHAMED-ELARBI & Brahim & P198 \\
\hline MOHTADI & Karima & P043, P156, P230 \\
\hline MOINARD & Christophe & P120, P129, P202 \\
\hline MOIRAND & Romain & P162 \\
\hline MONDOT & Stanislas & CO01 \\
\hline
\end{tabular}

\begin{tabular}{|c|c|c|}
\hline NOM & PRENOM & RESUME(S) $\mathbf{N}^{\circ}$ \\
\hline MONIER & Arnaud & P199 \\
\hline MONNOT & Marie-Claude & CO06 \\
\hline MONRIBOT & Anthia & P063 \\
\hline MONTAGNER & Alexandra & CO59 \\
\hline MONTEIRO & Carlos & CO61 \\
\hline MORAIS & José & P083 \\
\hline MORAND & Christine & P220 \\
\hline MOREAU & Manon & P099 \\
\hline MOREIRA & Sarah & CO06 \\
\hline MOREL & Sophia & P112 \\
\hline MORELLE & Magali & P040 \\
\hline MORIN & Aline & $\mathrm{CO} 27$ \\
\hline MORIN & Béatrice & $\begin{array}{l}\text { CO31, CO66, P049, } \\
\text { P084, P141 }\end{array}$ \\
\hline MORIN & Mathieu & CO53 \\
\hline MORO & Joanna & P094 \\
\hline MORREAU & Laura & CO43 \\
\hline MORZEL & Martine & P204 \\
\hline MOSONI & Laurent & CO19 \\
\hline MOTHE-SATNEY & Isabelle & P039, P150, \\
\hline MOUGIN & Fabienne & P163 \\
\hline MOUHTADI & Karima & P215 \\
\hline MOULAHOUM & Anissa & P104 \\
\hline MOUNACH & Samir & P238 \\
\hline MOUQUET-RIVIER & Claire & P016, P017 \\
\hline MOUSSA & Hainikoye & P016 \\
\hline MOUZOUNI & Fatima Zahra & P228 \\
\hline MOVASSAT & Jamileh & CO60 \\
\hline MSAAD & Rajaa & P043, P156, P230 \\
\hline MULLER & Laurent & P218 \\
\hline MULLIEZ & Aurélien & CO33, $\mathrm{CO} 65$ \\
\hline MUNOZ & Carolina & CO57 \\
\hline MURDACA & Joseph & P039, P150 \\
\hline MURPHY & Neil & CO09 \\
\hline MUTTER & Didier & P057 \\
\hline NADA EL & Osta & P239 \\
\hline NAIMI & Sabrine & CO44 \\
\hline NAKIB & Samir & CO34, P116 \\
\hline NASSER & Yara & P049 \\
\hline NATUREL & Jacques & P050 \\
\hline NAU & Françoise & CO15, P172 \\
\hline NAWFAL TRIBA & Mohamed & P206 \\
\hline NAZARE & Julie-Anne & $\mathrm{CO} 40$ \\
\hline NAZIH & El-Hassan & P089, P189 \\
\hline
\end{tabular}




\begin{tabular}{|c|c|c|}
\hline NOM & PRENOM & RESUME(S) $\mathbf{N}^{\circ}$ \\
\hline N'BRA & Alexandre & P174 \\
\hline NEELS & Jaap & P039, P150 \\
\hline NEGAOUI & Hanane & P190 \\
\hline NÉROME & Simone & P063 \\
\hline NEUFCOURT & Lola & P092 \\
\hline NEVEUX & Nathalie & CO34, P102, P116 \\
\hline NEYRAUD & Eric & P204 \\
\hline NEYRINCK & Audrey & CO02, P187 \\
\hline NGUYEN & Jean-Marie & P074 \\
\hline NIAMBA & Claude-Narcisse & P086 \\
\hline NICKLAUS & Sophie & P011, P175, P176 \\
\hline NICOL & Marie & $\mathrm{CO} 36$ \\
\hline NILSSON & Ida & CO08 \\
\hline NIOT & Isabelle & CO06 \\
\hline NISOLLE & Michelle & CO62 \\
\hline NOAILLETAS & Véronique & P084 \\
\hline NOBIS & Séverine & CO27, CO45, P194 \\
\hline NOËL & Dominique & P054, P056 \\
\hline NOIREZ & Philippe & P041, P069, P083 \\
\hline NORMAND & Sylvie & CO40 \\
\hline NOVY & Karel & P197 \\
\hline O FETISSOV & Sergueï & $\mathrm{CO} 46$ \\
\hline OCCHIPINTI & Cynthia & P066 \\
\hline ODERDA & Leslie & $\mathrm{CO03}$ \\
\hline O'NEILL & Claire & P183 \\
\hline OPPERT & Jean Michel & CO39 \\
\hline ORSET & Caroline & P014 \\
\hline OSHIMA & Taku & P059, P060 \\
\hline OTHMANI-MECIF & Khira & P088, P104 \\
\hline OUCHCHANE & Lemlih & CO55 \\
\hline OUGUERRAM & Khadija & P126 \\
\hline OUHAIBI-DJELLOULI & Hadjira & P149 \\
\hline OULAMARA & Hayet & $\begin{array}{l}\text { P024, P142, P245, } \\
\text { P247, P248 }\end{array}$ \\
\hline OZDENER & Hakan & CO78 \\
\hline PAGES & Vincent & P125 \\
\hline PAILLARD & Anaïs & P122, P169 \\
\hline PAILLAUD & Elena & $\mathrm{CO} 13$ \\
\hline PALAYER & Isabelle & $\mathrm{CO} 13$ \\
\hline PALIER & Juliette & P001, P010 \\
\hline PALMIER-FORESTIER & Charlotte & P153 \\
\hline PAMOUKDJIAN & Frederic & CO13 \\
\hline PANTET & Olivier & P055, P106 \\
\hline PAQUOT & Nicolas & P117 \\
\hline
\end{tabular}

\begin{tabular}{|c|c|c|}
\hline NOM & PRENOM & RESUME(S) $\mathbf{N}^{\circ}$ \\
\hline PARANT & Olivier & P114 \\
\hline PARIZET & Laure & P051 \\
\hline PARTULA & Valentin & CO01, P206, P220 \\
\hline PATIN & Franck & P105 \\
\hline PELLAY & Hermine & P227 \\
\hline PELLOUX & Véronique & $\mathrm{CO} 39$ \\
\hline PÉNEAU & Sandrine & P225 \\
\hline PENHOAT & Armelle & P187 \\
\hline PENHOAT & Clémence & CO74, P249 \\
\hline PERALDI & Catherine & $\mathrm{CO} 38$ \\
\hline PEREIRA & Bruno & $\mathrm{CO} 15, \mathrm{CO} 42, \mathrm{P} 158$ \\
\hline PERETTI & Noel & P179 \\
\hline PÉRIGNON & Marlène & $\begin{array}{l}\text { P008, P299, P243, } \\
\text { P244 }\end{array}$ \\
\hline PÉROL & Olivia & P040 \\
\hline PERRET & Frédéric & P163 \\
\hline PERRIEN & Emmanuelle & P053 \\
\hline PERRIER & Marie & CO31 \\
\hline PERRIMON & Marjorie & P014 \\
\hline PERRIN & Laëtitia & P217 \\
\hline PERROT & Loïc & $\mathrm{CO} 33$ \\
\hline PESSEMESSE & Laurence & $\mathrm{CO} 75$ \\
\hline PÉTÉRA & Mélanie & $\mathrm{P} 220$ \\
\hline PETIT & André & $\mathrm{CO} 30$ \\
\hline PETIT & Emilien & CO54, P090, P182 \\
\hline PETIT & Karina & P189 \\
\hline PETTIGREW & Simone & P219 \\
\hline PEYRON & Marie Agnès & P009 \\
\hline PEYRONNET & Sophie & P063 \\
\hline PHAN & Franck & P134 \\
\hline PIALOUX & Vincent & P040 \\
\hline PIANELLI & Giorgia & P016 \\
\hline PICARD-HAGEN & Nicole & $\mathrm{CO53}$ \\
\hline PICHARD & Claude & $\begin{array}{l}\text { CO65, P038, P059, } \\
\text { P060 }\end{array}$ \\
\hline PICHOT & Benoît & P181 \\
\hline PIEDCOQ & Julien & CO16, P094, P157 \\
\hline PIERART & Valérie & P131 \\
\hline PIERRE & Fabrice & P226 \\
\hline PIFFERI & Fabien & $\operatorname{co56}$ \\
\hline PIGEYRE & Marie & $\mathrm{COOB}$ \\
\hline PIGNEUR & Frédéric & $\mathrm{CO} 11, \mathrm{CO} 12$ \\
\hline PILLEUL & Frank & P040 \\
\hline PILON & Geneviève & CO44 \\
\hline
\end{tabular}




\begin{tabular}{|c|c|c|c|c|c|}
\hline NOM & PRENOM & RESUME(S) N & NOM & PRENOM & RESUME(S) No \\
\hline PILOQUET & Hugues & P179 & RANDUINEAU & Gwénaëlle & P198 \\
\hline PINGUET & J. & P009 & RAOUL & Sterenn & P027 \\
\hline PIQUEMAL & Fabien & P135 & RAOULT & Violette & P179 \\
\hline PIQUEMAL & Régis & P122, P169 & RASO & Jérémie & P188 \\
\hline PIQUET & Marie-Astrid & P133, P135, P167 & RAVAUD & Philippe & P225 \\
\hline PISCUC & Maria & $\mathrm{CO} 05$ & RAYNAL & Ketsia & CO55 \\
\hline PISON & Christophe & P202 & RAYNARD & Bruno & CO11, CO12, P061, \\
\hline POGGIOGALLE & Eleonora & P121 & & & P066, P139 \\
\hline POIDEVIN & Perrine & $\mathrm{P} 180$ & $\begin{array}{l}\text { RAYNAUD DE } \\
\text { MAUVERGER }\end{array}$ & Eric & P074 \\
\hline POINTEREAU & Philippe & P007, P148 & RAYNAUD-SIMON & Agathe & $\mathrm{CO} 13, \mathrm{CO} 35$ \\
\hline POIRÉE & Brigitte & P066 & RAYNER & Mike & P221 \\
\hline POIRIER & Hélène & CO06 & REBIÈRE & François & P084 \\
\hline POITOU-BERNERT & Christine & CO39 & REBOUISSOUX & Laurent & P064, P065 \\
\hline POLAKOF & Sergio & CO19 & REBOUL & Emmanuelle & CO15, CO17 \\
\hline POLIZZI & Arnaud & $\mathrm{CO} 59$ & RÉGNIER & Marion & CO59 \\
\hline PONS & Magali & P061, P066 & REISER & Philippe & $\mathrm{P} 232$ \\
\hline PORCHER & Raphaël & P225 & REMY & Gaetane & P054 \\
\hline \multirow{2}{*}{ POTIER } & \multirow{2}{*}{ Julien } & \multirow{2}{*}{ P050 } & RESPONDEK & Frédérique & CO04, COOA \\
\hline & & & REZGANI & Imen & P087 \\
\hline POUGET & Mélanie & P153 & RIBEIRO-PARENTI & Lara & P191 \\
\hline POUILLART & Philippe & P086 & RIBET & David & $\mathrm{CO} 28$ \\
\hline PRADIGNAC & Alain & P051, P154, P057 & RIBOLI & Elio & CO09 \\
\hline PREISER & Jean-Charles & $\begin{array}{l}\text { CO65, P107, P118, } \\
\text { P119 }\end{array}$ & RICHARD & D. & P009 \\
\hline PREUX & Pierre Marie & $\mathrm{CO} 36$ & RICHARD & Ruddy & $\mathrm{CO} 15$ \\
\hline PREVOT & Vincent & $\mathrm{COOB}$ & $\mathrm{RICHOU}$ & Marlène & P074 \\
\hline PRIÉ & Perrine & P159 & RIEGERT & Myriam & P154 \\
\hline PRIGENT & Anne Yvonne & P019, P021 & RIEHL & Elise & P051 \\
\hline PROCTOR & Gordon & P204 & RIEUBON & Julie & P066 \\
\hline PROST & Bénédicte & P237 & RIEUSSET & Jennifer & P146 \\
\hline PROTHE & Carole & P110 & RIGAL & Amélie & P158 \\
\hline PROUST-LIMA & Cécile & $\mathrm{CO} 67, \mathrm{CO} 68, \mathrm{CO} 69$ & RIGAUDIÈRE & \begin{tabular}{|l} 
Jean-Paul \\
\end{tabular} & P091, P121 \\
\hline PUJOL & Sophie & P046 & RIGONDET & Roxane & P158 \\
\hline PUJOS-GUILLOT & Estelle & P220 & RIGUET & Mathilde & P159 \\
\hline QUÉRÉ & Clémence & P029 & RIMBERT & Agnès & $\mathrm{CO} 29, \mathrm{CO} 32, \mathrm{CO} 30$ \\
\hline QUILLARD & Muriel & $\mathrm{CO} 29$ & RIOU & Coralie & P019 \\
\hline QUILLIEN & Véronique & $\mathrm{CO} 74$ & RIOUX & Vincent & CO74, P249 \\
\hline QUINTANA-MURCI & Lluis & $\mathrm{COO1}$ & RIQUELME & Marie & P153 \\
\hline RABESONA & Hanitra & P010 & RISSELIN & Virginie & P159 \\
\hline RADOI & Loredana & $\mathrm{COO7}$ & RIVES-LANGE & Claire & P138 \\
\hline RAILLAT & Julie & P109, P110 & ROBAEY & Philippe & P112 \\
\hline RAIMBAULT & Adeline & P159 & ROBERT & Julien & $\mathrm{CO} 35$ \\
\hline RAMASSAMY & Radji & CO34, P116 & ROBERT & Maud & $\mathrm{CO} 42$ \\
\hline RAMI & Anass & P100, P228, P238 & ROBIN & Aude & P137 \\
\hline
\end{tabular}




\begin{tabular}{|c|c|c|c|c|c|}
\hline NOM & PRENOM & RESUME(S) $\mathbf{N}^{\circ}$ & NOM & PRENOM & RESUME(S) $\mathbf{N}^{\circ}$ \\
\hline ROBQUIN & Nadine & P050 & SALMI & Takwa & P058 \\
\hline ROCHE & Johanna & P163 & SALMIA & Wafia & P023 \\
\hline ROCHEDY & Amandine & P242 & SAMARA & Ilyas & P222 \\
\hline ROCHEFORT & Gaël & P099 & SAMI & Ouidad & CO07 \\
\hline RODIER & Francis & P112 & \multirow{2}{*}{ SAMIERI } & \multirow{2}{*}{ Cécilia } & \multirow{2}{*}{$\begin{array}{l}\mathrm{CO} 67, \mathrm{CO} 68, \mathrm{CO} 69, \\
\mathrm{CO} 71, \mathrm{CO} 72, \mathrm{P} 227\end{array}$} \\
\hline RODRIGUEZ & Julie & CO02 & & & \\
\hline RODRIGUEZ- & \multirow{2}{*}{ Fernando } & \multirow{2}{*}{ P204 } & SANCHEZ & Phelipe & P121 \\
\hline ARTALEJO & & & SAND & Renaud & P118 \\
\hline RODRIGUEZ-GUEANT & Rosa-Maria & $\mathrm{CO} 20$ & SANTE-LHOUTELLIER & Suzanne & P009 \\
\hline ROGGHE & Pierre-Arnaud & P125 & SARFATI & Gilles & $\mathrm{CO} 34$ \\
\hline ROHR & Serge & P154 & SASSI & Sonia & P231 \\
\hline ROLLAND & Carole & P202 & SAUR & Flo & P113 \\
\hline ROLLET & Pascaline & CO63, P241 & SAUVAGE & Elena & P232 \\
\hline RONDEAUX & Julie & $\mathrm{CO} 29, \mathrm{CO} 45$ & SAUVINET & Valérie & P187 \\
\hline ROSSARY & Adrien & P127, P206, P220 & SAVARIN & Philippe & CO01, P206 \\
\hline ROTOVNIK KOZJEK & Nada & CO65 & SAVARY-AUZELOUX & Isabelle & CO15, C019, P082 \\
\hline ROUAUD & Agnes & $\mathrm{CO} 13$ & SAVOYE & Guillaume & P195, P196 \\
\hline ROUGÉ & Stéphanie & P127 & SAYD & Thierry & P009 \\
\hline ROUILLY & Vincent & CO01 & SCHALCH & Wolfgang & CO72 \\
\hline ROULAND & Matthieu & P195 & SCHINDLER & Maximilian & P038 \\
\hline ROUSSEAU & Amélie & P152 & SCHNEIDER & Stéphane & CO66 \\
\hline ROUSSEAU & Anne-Sophie & P039, P150 & SCHUBERT & Louis & CO52 \\
\hline ROUSSEAU & Fabien & P125 & SCHUCK & Pierre & P172 \\
\hline ROUX & Pascale & P066 & SCHWARTZ & Camille & P176 \\
\hline ROY & Denis & CO44 & SCHWEBEL & Hérvé & P177 \\
\hline ROYER-GARABIGE & Valérie & CO14, P139 & SEBAI & Imen & P013 \\
\hline ROYO & Julie & CO56 & \begin{tabular}{|l|} 
SEBEDIO \\
\end{tabular} & Jean-Louis & P091 \\
\hline ROZÉ & Jean-Christophe & P184 & SECONDA & Louise & P007, P148 \\
\hline RUFFIEUX & Bernard & P218 & SEGUY & David & CO35 \\
\hline RUILLIER & Diane & P154 & SEMIANE & Nesrine & P140 \\
\hline SABOURDY & Véronique & CO31 & SENELLART & Hélène & P053 \\
\hline SADEGHIPOUR & Farshid & P177 & SENESSE & Pierre & P066 \\
\hline SAEID & Naima & P100, P228, P238 & SEPTIER & Chantal & CO57, P001, P028 \\
\hline SAIDI & Djamel & P190, P192 & SERAIRI BEJI & Raja & P036, P165 \\
\hline SAILE & Rachid & P043, P155, P156, & SI HASSEN & Wendy & CO63, P241 \\
\hline SAILLARD & Tinhaine & P216, P230 & SIBILLE & Brigitte & P039, P150 \\
\hline \begin{tabular}{|l} 
SAINTES \\
\end{tabular} & Charlotte & 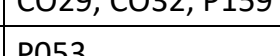 & SIBOUAKAZ & Dina & P088 \\
\hline \begin{tabular}{|l} 
SAINT-EVE \\
\end{tabular} & Anne & \begin{tabular}{|l|} 
P020, P033 \\
\end{tabular} & SICARD & Mariette & P202 \\
\hline SAINTON & Bérangère & P122 & SICURANI & Joseph & P123 \\
\hline & & CO28, P193, P194. & SIMON & Hélène & CO11 \\
\hline SALAMEH & Emmeline & P195 & SIMON & Laure & P184 \\
\hline SALANAVE & Benoît & P229, P243, P244 & SINNETT & Daniel & P112 \\
\hline SALEM & Joe- Elie & P128 & SIREJACOB & Yoann & CO32 \\
\hline SALLES & Jérôme & P121 & SLIM & Karem & CO42, P153 \\
\hline
\end{tabular}




\begin{tabular}{|c|c|c|c|c|c|}
\hline NOM & PRENOM & RESUME(S) $\mathbf{N}^{\circ}$ & NOM & PRENOM & RESUME(S) $N^{\circ}$ \\
\hline SLIMANI & Lamia & P111 & \multirow{2}{*}{ THIBAULT } & \multirow{2}{*}{ Ronan } & \multirow{2}{*}{$\begin{array}{l}\text { CO64, CO65, P136, } \\
\text { P162, P168, P198 }\end{array}$} \\
\hline SMATI-GRANGEON & Sarra & CO59 & & & \\
\hline SOLEM LAVIEC & Heidi & $\mathrm{CO} 13$ & THIBAUT & Aurore & $\mathrm{P} 117$ \\
\hline SOLER & Louis-Georges & P014, P020, P033 & & Laurine & P030 \\
\hline SOM & Mickael & P136, P162 & THIRON & Sophie & P223 \\
\hline SOMMER & Isabelle & P177 & THISSEN & Jean Paul & $\mathrm{COO2}$ \\
\hline SOTHIER & Monique & $\mathrm{CO} 40$ & THIVEL & David & $\begin{array}{l}\text { P042, P151, P158, } \\
\text { P163 }\end{array}$ \\
\hline SOUAT & Sanaa & P111 & \multirow{2}{*}{$\begin{array}{l}\text { THOMAS } \\
\text { THOMAS }\end{array}$} & \multirow{2}{*}{ Aline } & CO71 \\
\hline SOUBERBIELLE & Jean Claude & P093 & & & P136 \\
\hline SOUCHON & Isabelle & P020, P033 & THOMAS & Stéphanie & $\mathrm{COO1}$ \\
\hline SOULEFOUR & Céline & P203 & THURET & Sandrine & $\mathrm{CO} 68, \mathrm{CO} 69$ \\
\hline SOUMOY & Laura & P056 & TICHIT & Christine & $\mathrm{CO63}$ \\
\hline SOURISSEAU & Huguette & CO36, P049 & TIMMERMANS & M. & CO62 \\
\hline SROUR & Bernard & $\begin{array}{l}\text { CO09, CO61, P208, } \\
\text { P220, P224 }\end{array}$ & TIRELLE & Pauline & CO28, P193 \\
\hline STALINS & Philippine & P119 & TISSOT & Valentin & $\mathrm{CO} 11$ \\
\hline ST-GELAIS & Daniel & $\mathrm{CO} 44$ & TOLÉDANO & Alain & $\mathrm{CO} 11, \mathrm{CO} 12$ \\
\hline SULTAN & Serge & P112 & TOMÉ & Daniel & $\begin{array}{l}\text { CO16, P094, P157, } \\
\text { P170 }\end{array}$ \\
\hline SWANN & Jonathan & $\mathrm{CO} 27$ & TORRES & Marion & $\mathrm{COO1}$ \\
\hline SZABO DE EDELENYI & Fabien & P026 & TOUILLAUD & Marina & P040 \\
\hline$\frac{\text { SZALAI }}{\text { SZOPINSKI }}$ & $\begin{array}{l}\text { Amandine } \\
\text { Monique }\end{array}$ & $\begin{array}{l}\mathrm{P} 107 \\
\mathrm{CO} 31\end{array}$ & TOUNIAN & Patrick & $\begin{array}{l}\text { CO03, CO39, P178, } \\
\text { P183, P226 }\end{array}$ \\
\hline$\frac{\text { SZOPINSKI }}{\text { TABAR }}$ & $\begin{array}{l}\text { Monique } \\
\text { V. }\end{array}$ & $\mathrm{CO} 62$ & TOURNIER & Carole & P001, P175 \\
\hline TABOZ & Youness & P100 & & & CO01, CO09, CO61, \\
\hline TAGHLIT & Abdennour & P088 & & & P207, P208, P209, \\
\hline TAGHZAOUI & Marouan & P228 & TOUVIER & Mathilde & P210, P213, P214, \\
\hline TAHRI & El Hassan & P156 & & & P218, P221, P224, \\
\hline TAILLARD & Véronique & P164 & & & $\begin{array}{l}\text { P225, P234, P240, } \\
\text { P241 }\end{array}$ \\
\hline TAKI & Hassan & $\begin{array}{l}\text { P043, P155, P156, } \\
\text { P216, P230 }\end{array}$ & TRAISSAC & Pierre & P095, P231 \\
\hline TALATI & Zenobia & P219 & TRAMUNT & Blandine & CO59 \\
\hline TANNOURY & Mona & P189 & TRASRIEUX & Laurent & P051 \\
\hline TAVOLACCI & Marie Pierre & CO29, CO32, P235 & TRÉBUCHET & Aurore & P234 \\
\hline TEBBANI & Fouzia & P245, P247, P248 & TRÉDAN & Olivier & P040 \\
\hline TEMSSEK & Aroua & P087 & TREIBER & Tobias & P197 \\
\hline TENNOUNE-EL & Naouel & $\mathrm{CO} 34$ & TREILLE & Serge & P124 \\
\hline HAFAIA & & & TREMBLAY & Angelo & P151 \\
\hline TEOT & Luc & P046 & TRIBA & Mohamed & $\mathrm{COO1}$ \\
\hline TERRISSE & Hugo & P202 & TURCO & Célia & P109, P110 \\
\hline TESSIER & Romain & $\mathrm{CO} 16$ & ULMANN & \begin{tabular}{|l|} 
Guillaume \\
\end{tabular} & P102 \\
\hline TESTU & Sophie & P108 & UMORET & Remy & P188 \\
\hline THABET & Meriem & P231 & URDACI & Maria & $\mathrm{CO} 44$ \\
\hline THARREY & Marion & P008 & \begin{tabular}{|l} 
VAILLANT \\
\end{tabular} & Marie-France & CO37, P202, P211 \\
\hline THIBAULT & Hélène & P141 & VALENTINO & Muriel & P159 \\
\hline
\end{tabular}




\begin{tabular}{|c|c|c|}
\hline NOM & PRENOM & RESUME(S) $\mathbf{N}^{\circ}$ \\
\hline VALERO & Simon & $\mathrm{CO} 13$ \\
\hline VALÉRO & René & P200 \\
\hline VAL-LAILLET & David & P162, P168, P198 \\
\hline VAN DE HEIJNING & Bert & P170 \\
\hline VAN DEN BERGHE & Laurie & $\mathrm{CO} 40$ \\
\hline VAN DER WERF & Hayo M G & $\mathrm{CO} 21$ \\
\hline VAN GOSSUM & André & P107 \\
\hline VAN GOSSUM & Marc & P131 \\
\hline VAN LISSUM & Marion & P019, P021 \\
\hline VAN OUYTSEL & Pauline & P107 \\
\hline $\begin{array}{l}\text { VANDENBERGHE- } \\
\text { DESCAMPS }\end{array}$ & Mathilde & P001 \\
\hline VANDERLINDEN & Thierry & P125 \\
\hline VANESSE & Virginie & P054, P056 \\
\hline VANNEREUX & Marine & P246 \\
\hline VANSTEENE & Damien & CO11, P053, P061 \\
\hline VARIN & Thibault & CO44 \\
\hline VARRASO & Raphaëlle & P209, P210 \\
\hline VASSEUR & Florence & P167 \\
\hline VASSON & Marie-Paule & $\begin{array}{l}\text { CO10, P206, P220, } \\
\text { P127 }\end{array}$ \\
\hline VASTEL & Elise & P133, P167 \\
\hline VATIN & Severine & P147 \\
\hline VAYSSIÈRE & Christophe & CO53 \\
\hline VERBRUGGE & Anne-Marie & P117 \\
\hline VERBRUGGHE & Sylvie & P081 \\
\hline VERGONJEANNE & Marion & CO31, CO36 \\
\hline VERNEREY & Dewi & P109 \\
\hline VIAUD & Juliette & P136 \\
\hline VICTOR-BALA & Agnès & CO01, P206 \\
\hline VIDAL & Hubert & CO77 \\
\hline VIDAL & Sandra & CO05 \\
\hline VIDÉ & Joris & P144, P145 \\
\hline VIENOT & Angélique & P109, P110 \\
\hline VIGNAU & Jean & CO08 \\
\hline VIGOR & Claire & P146 \\
\hline VILLEMONTEIX & Carole & P203 \\
\hline VINOY & Sophie & CO40, P092 \\
\hline VIOTTI & Julien & P123 \\
\hline VIX & Michel & P154 \\
\hline VO VAN-REGNAULT & Gwenn & P003 \\
\hline VOISIN & Angélique & P141 \\
\hline VORS & Cécile & CO55 \\
\hline VOURC'H & Patrick & P105 \\
\hline
\end{tabular}

\begin{tabular}{|l|l|l|}
\hline NOM & PRENOM & RESUME(S) N \\
\hline VOWINCKEL & Jakob & P197 \\
\hline VUITTON & Lucine & P109 \\
\hline WAEYTENS & Suzanne & P118 \\
\hline WAGNER & Maude & CO67 \\
\hline WAHANIAN \\
PAPAZIAN & Tatiana & P239 \\
\hline WALRAND & Stéphane & P085, P121, P161 \\
\hline WARD & Heather & CO09 \\
\hline WARD & Robert & CO77 \\
\hline WARDY & Nina & P239 \\
\hline WEENEN & Hugo & P175 \\
\hline WEIDERPASS & Elisabete & CO09 \\
\hline WOMENI & Hilaire & P017 \\
\hline YAKER & Linda & P195, P196 \\
\hline YANNI & Antoine & P131 \\
\hline YAO & Jean Simon & P174 \\
\hline YASMINA & Benazzoug & P104 \\
\hline YOUNES & Hassan & P029, P086 \\
\hline YSSAAD & Djamila & P192 \\
\hline ZAGHET & Océane & P064, P065 \\
\hline ZAHRA & Hajer & P087, P098 \\
\hline ZERROUK & Fouzia & P104 \\
\hline ZOUNGRANA & Stéphanie & P017 \\
\hline ZRIBI & Sabrine & P035 \\
\hline
\end{tabular}

